Storie dell'arte contemporanea 4 Atlante delle Biennali 1

\title{
Storie della Biennale di Venezia
}

a cura di

Stefania Portinari e Nico Stringa

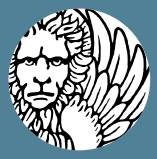

Edizioni

Ca'Foscari

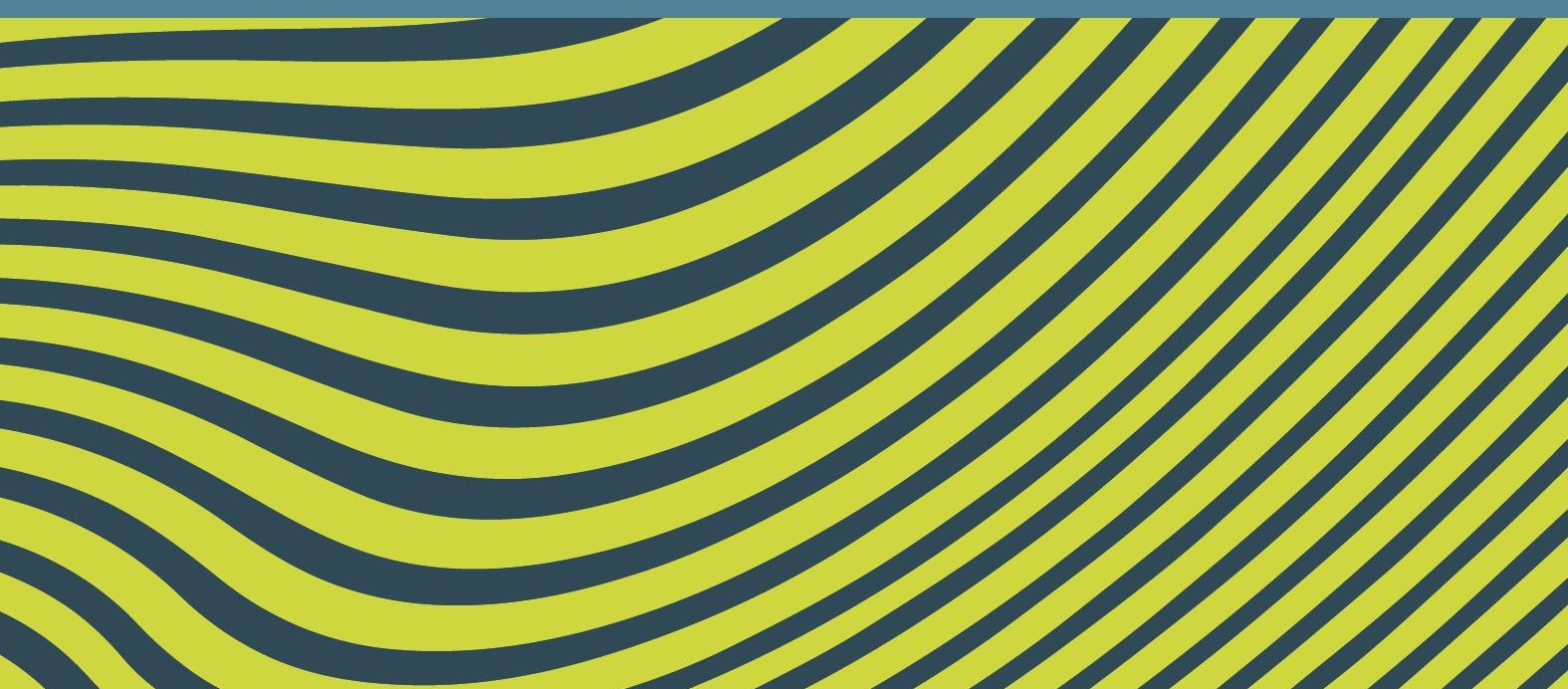



Storie della Biennale di Venezia

Storie dell'arte contemporanea Atlante delle Biennali

Serie diretta da

Nico Stringa e Stefania Portinari

$4 \mid 1$

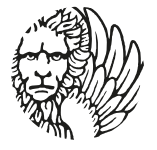

Edizioni

Ca'Foscari 


\section{Storie dell'arte contemporanea Atlante delle Biennali}

Direzione scientifica Nico Stringa (Università Ca' Foscari Venezia, Italia) Stefania Portinari (Università Ca' Foscari Venezia, Italia)

Comitato scientifico Luca Massimo Barbero (Fondazione Giorgio Cini Venezia, Italia) Giuseppina Dal Canton (già Università degli Studi di Padova, Italia) Stefania Portinari (Università Ca' Foscari Venezia, Italia) Jean-François Rodriguez (già Università degli Studi di Verona, Italia) Sileno Salvagnini (Accademia di Belle Arti di Venezia, Italia) Nico Stringa (Università Ca' Foscari Venezia, Italia) Valerio Terraroli (Università degli Studi di Verona, Italia)

Comitato di lettura Guido Bartorelli (Università degli Studi di Padova, Italia) Riccardo Caldura (Accademia di Belle Arti di Venezia) Massimo De Grassi (Università degli Studi di Trieste, Italia) Silvia Grandi (Alma Mater Studiorum Università degli Studi di Bologna, Italia)

\section{Direzione e redazione}

Dipartimento di Studi Umanistici

Università Ca' Foscari Venezia

Palazzo Malcanton Marcorà

Dorsoduro 3484/D|30123 Venezia

artecontemporanea@unive.it

Storie dell'arte contemporanea | Atlante delle Biennali

e-ISSN 2724-136X

ISSN 2704-9973

URL http://edizionicafoscari.unive.it/it/edizioni/collane/storie-dellarte-contemporanea/ 


\section{Storie della Biennale di Venezia}

a cura di Stefania Portinari e Nico Stringa

Venezia

Edizioni Ca' Foscari - Digital Publishing 2019 
Storie della Biennale di Venezia

Stefania Portinari e Nico Stringa (a cura di)

(C) 2019 Stefania Portinari, Nico Stringa per il testo

(C) 2019 Edizioni Ca' Foscari - Digital Publishing per la presente edizione

(ㄷ)(1)

Quest'opera è distribuita con Licenza Creative Commons Attribuzione 4.0 Internazionale This work is licensed under a Creative Commons Attribution 4.0 International License

Qualunque parte di questa pubblicazione può essere riprodotta, memorizzata in un sistema di recupero dati o trasmessa in qualsiasi forma o con qualsiasi mezzo, elettronico o meccanico, senza autorizzazione, a condizione che se ne citi la fonte.

Any part of this publication may be reproduced, stored in a retrieval system, or transmitted in any form or by any means without permission provided that the source is fully credited.

Edizioni Ca' Foscari - Digital Publishing

Università Ca' Foscari Venezia | Dorsoduro 3246, 30123 Venezia

http://edizionicafoscari.unive.it|ecf@unive.it

1 a edizione dicembre 2019

ISBN 978-88-6969-366-3 [ebook]

ISBN 978-88-6969-367-0 [print]

Storie della Biennale di Venezia / A cura di Stefania Portinari e Nico Stringa 1. ed. - Venezia: Edizioni Ca' Foscari - Digital Publishing, 2019. - 440 p.; 23 cm. (Storie dell'arte contemporanea; 4, 1). - ISBN 978-88-6969-367-0.

URL https://edizionicafoscari.unive.it/it/edizioni/libri/978-88-6969-367-0/

DOI http://doi.org/10.30687/978-88-6969-366-3 
Storie della Biennale di Venezia

a cura di Stefania Portinari e Nico Stringa

\section{Sommario}

\section{L'Archivio della Biennale}

Non solo memoria

Debora Rossi

Introduzione

Stefania Portinari, Nico Stringa

STORIE DELLA BIENNALE: NUOVE RICERCHE

Comunicazione e graphic design

della Biennale di Venezia (1895-1950)

Cristina Fontana

Le donne artiste alla Biennale d'arte di Venezia

Le scultrici e le prime edizioni (1895-1912)

Clarissa Coidessa

Gli artisti veneziani alla Biennale (1895-1905)

Organizzatori ed espositori: l'antinomia dei ruoli

Matilde Ferrarin

1914. Galileo Chini a Venezia

Massimo De Grassi

I pittori veneti alle 'Biennali di Pallucchini' (1948-1956)

Le partecipazioni e la ricezione della stampa

Elisa Rampazzo

Palma Bucarelli e la Biennale di Venezia (1948-1968)

Acquisizioni della Direttrice per la Galleria Nazionale d'Arte Moderna di Roma 
Umbro Apollonio e l'Archivio della Biennale di Venezia (1948-1972)

Vittorio Pajusco

Dell'Archivio Storico delle Arti Contemporanee «la Biblioteca n'era il principio»

Giorgia Marchesin

Narcissus Garden for Sale: «one piece 2 dollars»

Yayoi Kusama alla Biennale di Venezia nel 1966

Stefania Portinari

Il gioco di nessuno

La forma biennale tra Venezia e Parigi nella fotografia

del dopoguerra fino agli anni Ottanta

Francesca Dolzani

Jeff Koons alla XLIV Esposizione Internazionale d'Arte La Biennale di Venezia (1990)

Sabina Laura De Stefano

"What kind of dirt do you need?"

Da San Diego e Tijuana a Venezia: Border Art oltre i confini Andrea Masala

«All We Are Saying Is Give Pizza Chance»

L'effetto YBA e l'irruzione di una nuova generazione alle Biennali degli anni Novanta del Novecento

Diego Mantoan

Arte relazionale alla Biennale di Venezia dal 1999 al 2017

Francesca Amadi

Dak'Art. Biennale de l'Arte Africain Contemporain 
MAPPING PAVILIONS

L'Argentina alla Biennale d'Arte di Venezia

Paola Natalia Pepa

La Spagna alle prime Biennali veneziane

del secondo dopoguerra

La ricezione della stampa

Romina Viggiano

Breve storia di una liberazione

La Spagna alla Biennale di Venezia dal 1979 al 1999

Giulia Crespi

Padiglioni che denunciano, riflettono e astraggono

Un'analisi critica e trasversale della partecipazione spagnola alla Biennale di Venezia (2003-2011)

Anita Orzes

Retelling the History of the Mexico Pavilion

at La Biennale di Venezia

Carolina Nieto Ruiz

Il Padiglione coreano a Venezia: una questione identitaria

Samantha Chia

Oltre l'Adriatico

La partecipazione albanese all'Esposizione Internazionale d'Arte di Venezia

Margherita Fochessati

Nota sui cataloghi della Biennale

Andrea Masala 


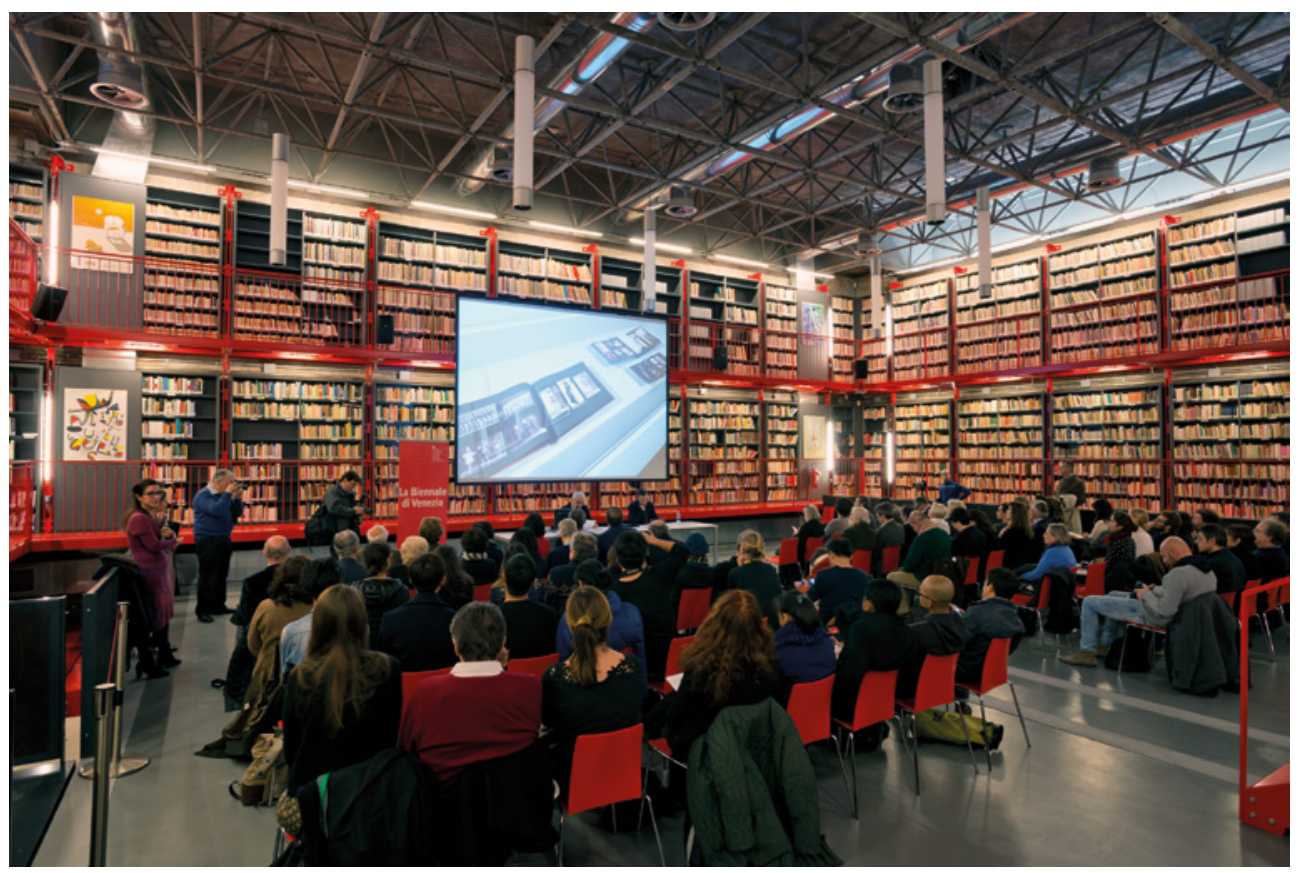

Figura 1 Biblioteca della Biennale. Courtesy Archivio Storico della Biennale di Venezia - ASAC (Foto: Andrea Avezzù) 


\section{L’Archivio della Biennale Non solo memoria}

\section{Debora Rossi}

Vice Direttore Generale della Biennale di Venezia,

Responsabile Organizzativo dell'Archivio Storico

L'Archivio Storico delle Arti contemporanee della Biennale di Venezia costituisce da sempre un riferimento importante per tutti gli studenti e ricercatori sul tema della storia dell'Istituzione stessa e delle arti contemporanee più in generale.

Dagli anni 2000 i Fondi hanno trovato casa in due nuove sedi: la Biblioteca sistemata al Padiglione Centrale ai Giardini, i restanti Fondi al Parco Scientifico Tecnologico - Vega - di Marghera.

I materiali sono stati tutti riordinati e catalogati e oggi più di 3.000 utenti tra Giardini e Marghera frequentano annualmente le nostre sedi.

Quando l'Archivio nacque nel 1928 lo scopo dei promotori era di recuperare e conservare materiali di diverso tipo e origine, riguardanti le opere degli artisti partecipanti. Il metodo utilizzato, oltre alla raccolta di libri e cataloghi provenienti dall'Italia e dall'estero, fu quello di avviare contatti con gli artisti per ricevere documentazione e materiale fotografico sulla loro attività.

Secondo questa visione iniziale, così come oggi, l'Archivio storico della Biennale ambiva a rappresentare la sua attività ma al tempo stesso quella degli artisti.

Tracce di questa impostazione si ritrovano anche nell'assetto attuale: il Fondo storico che è costituito dai materiali d'archivio propriamente detti, ovvero dai documenti che testimoniano e documentano la storia dell'istituzione si può certamente considerare compiuto. Diversa è invece la natura e funzione delle Collezioni che lo comple- 
tano, per lo più frutto dell'azione di singoli curatori/conservatori susseguitisi nel tempo, ognuno interessato a promuovere, quasi come un bricolage di famiglia, la raccolta di specifico materiale che, con spirito diverso, concorre a formare una specie di Wunderkammer alla maniera di Aby Warburg, ${ }^{1}$ naturalmente con una connaturata costante incompletezza.

Nel riordinare l'Archivio in alcuni casi abbiamo posto prudenza, come per una parte del Fondo storico, lasciando spesso i faldoni nell'ordine trovato perché la modalità di accumulazione, così come la loro denominazione, risultava importante in quanto contenuto, essendone divenuta quasi parte integrante. ${ }^{2}$

Allo stesso modo, nell'avviare la digitalizzazione non si è intervenuti in modo indiscriminato ma si è posta attenzione solo sui documenti da mettere in sicurezza. ${ }^{3}$

Per tutte le restanti collezioni si sfrutta il massimo della tecnologia disponibile per garantirne la massima fruizione. ${ }^{4}$

L'Archivio storico della Biennale rappresenta oggi la storia passata ma anche la sua trasformazione presente e costituisce un luogo di riferimento per i Direttori artistici nel progettare le mostre del futuro.

Si presenta come uno spazio vivo, dove a partire dallo studio dei documenti e dalla storia con la guida degli stessi Direttori artistici, selezionati giovani laureati possono approfondire i programmi correnti e imparare a scrivere saggi critici e scientifici; per questo oggi l'Archivio è anche Biennale College - ASAC - Scrivere in residenza.

1 Warburg, Aby [1994] (2002). Mnemosyne. L'atlante delle immagini. Torino: Aragno.

2 Si veda il caso delle cosiddette «Scatole nere», serie di fascicoli che raccolgono i documenti relativi alle prime Biennali (1895-1942), costituiti proprio da contenitori neri che hanno finito nel tempo per indicare i contenuti stessi e che pertanto, in sede di riordino, sono stati conservati in nuove scatole dello stesso colore.

3 Come nel caso della serie «Copialettere», ovvero le minute della corrispondenza dal 1898 al 1983: dato il supporto molto fragile si è deciso di ritirarli dalla consultazione e di trarne file digitali corredati dall'indice dei destinatari.

4 Così per il restauro e riversamento in digitale della collezione di video d'artista attuata con il supporto tecnico dell'Università di Udine. 


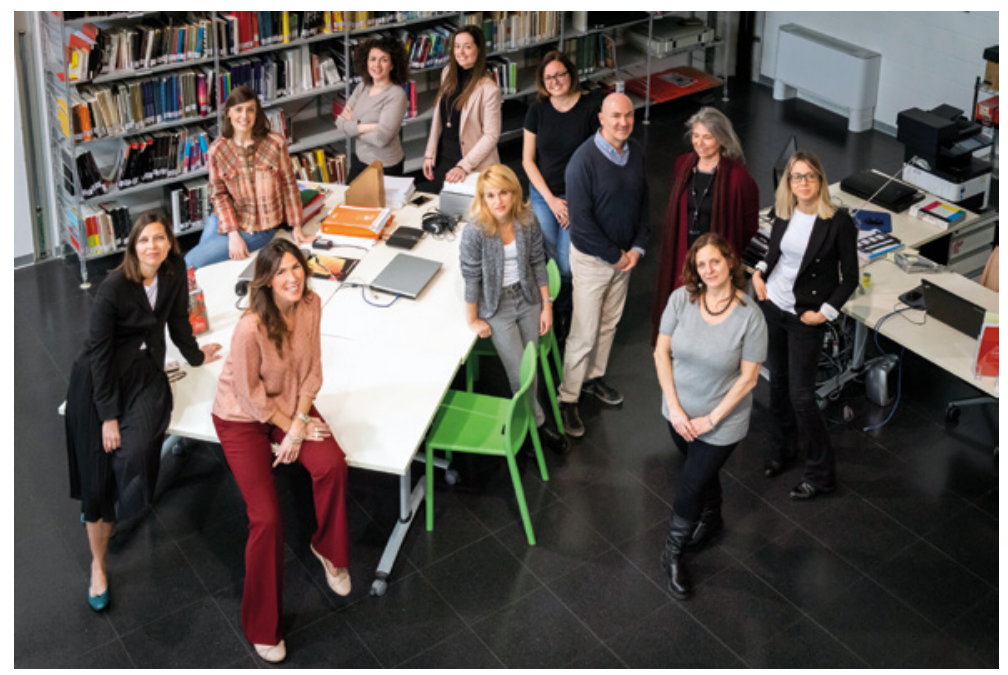

Figura 2 Staff dell'Archivio Storico della Biennale. Courtesy Archivio Storico della Biennale di Venezia - ASAC (Foto: Lorenzo Marzi) 


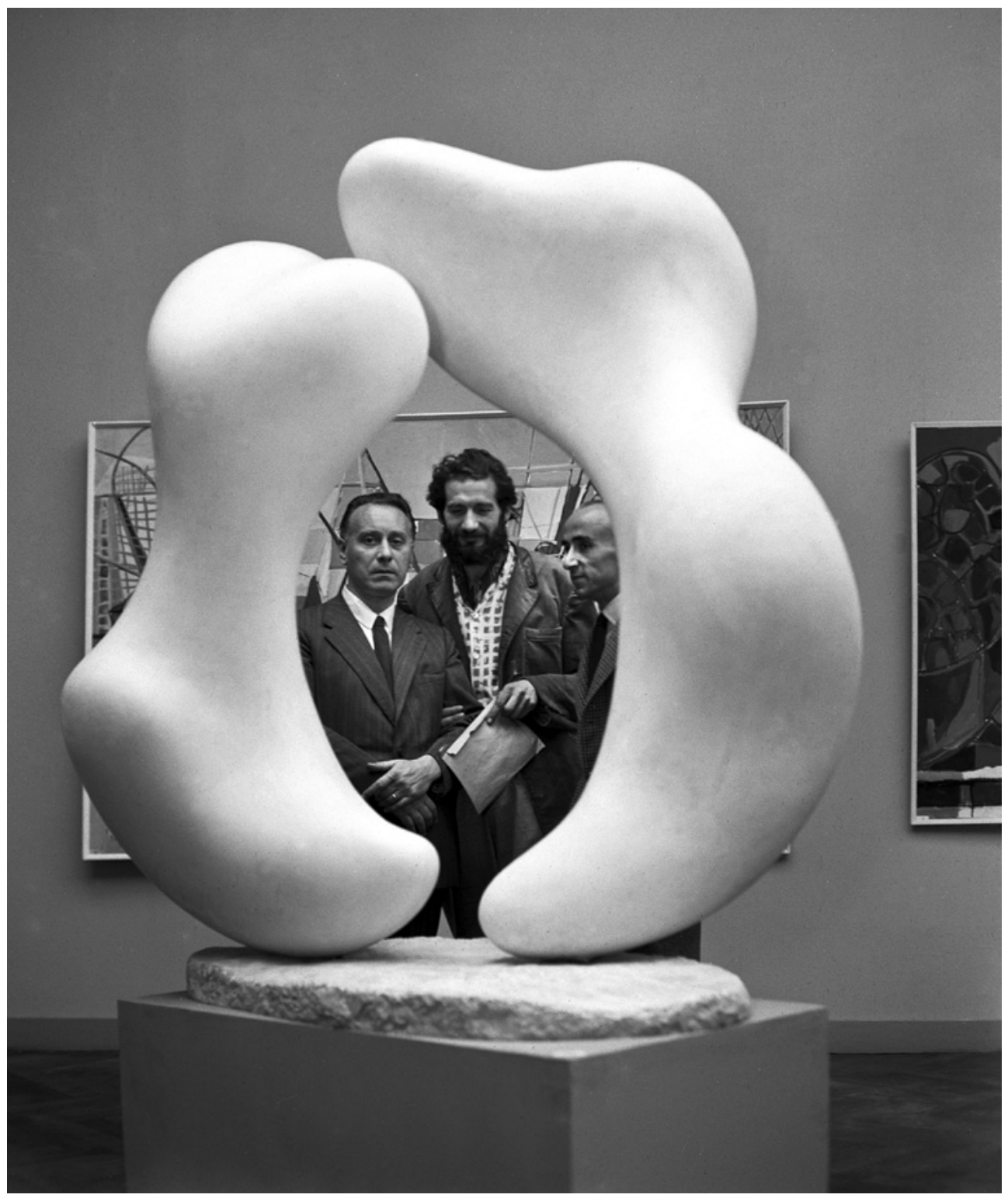

Renato Birolli, Emilio Vedova e Felice Casorati davanti alla scultura Estasi di Salvatore, Biennale 1950 Courtesy Archivio Cameraphoto Epoche Venezia. Archivio Cameraphoto/@ Vittorio Pavan 


\section{Introduzione}

Stefania Portinari, Nico Stringa

Università Ca' Foscari Venezia, Italia

Storie della Biennale di Venezia è il primo volume che apre la sottocollana «Atlante delle Biennali», per mappare sia la storia di quell'Esposizione Internazionale d'Arte che è stata il modello di riferimento di tutte le altre, che il presente.

Questa nuova piattaforma di studi, collocata all'interno della collana «Storie dell'arte contemporanea» delle Edizioni Ca' Foscari, intende porsi come uno strumento per i nostri studi in atto con reti di ricerca internazionali, ma anche per esiti di convegni, pubblicazioni scelte di postgraduates e dottorandi, indagini nel campo delle Digital Humanities.

Questo libro è in parte il risultato del Postgraduate Symposium Storie della Biennale di Venezia tenutosi il 6 e 7 dicembre 2016 presso la nostra Università Ca' Foscari Venezia, nell'aula aula magna Silvio Trentin di Ca' Dolfin, da noi curato a seguito anche degli assegni di ricerca conferiti su argomenti legati al tema e con il co-finanziamento del Dipartimento di Studi Umanistici. Sotto l'egida della presenza del prof. Tommaso Trini dell'Accademia di Belle Arti di Brera, curatore e testimone importante di momenti fondanti della Biennale, e del prof. Stefano Chiodi dell'Università di Roma Tre, autore di preziose ricerche di riferimento e in quel momento recente curatore del volume Alberto Boatto. Ghenos Eros Thanatos e altri scritti sull'arte. 1968-1985 (2016), hanno presentato le loro ricerche Matilde Ferrarin, Cristina Fontana, Paola Natalia Pepa, Carolina Nieto Ruiz, Elvira Shlyakhtina, Giulia Crespi, Anita Orzes, Laura Poletto, Francesca Dolzani.

A queste si sono aggiunti i saggi di alcuni colleghi che ci hanno fatto dono di loro studi e gli esiti di recenti tesi di laurea particolarmente significative. 
Platea dell'umanità è stato il titolo assegnato da Harald Szeemann alla Biennale da lui curata nel 2001, intendendo che fosse un «luogo verso il quale si guarda e da dove si è guardati, un luogo nel quale il pubblico è spettatore, protagonista e misura delle cose, un luogo di incontro tra artista, opera e osservatore»: ${ }^{1}$ allo stesso modo ci auguriamo che questa nuova impresa editoriale possa essere uno specchio dei nostri studi in dialogo serrato con quelli dei colleghi e dei nostri studenti, con uno sguardo speciale rivolto alla Fondazione La Biennale di Venezia e all'ASAC, il suo archivio storico, ai quali va ogni nostro più vivo ringraziamento. 


Storie della Biennale: nuove ricerche 


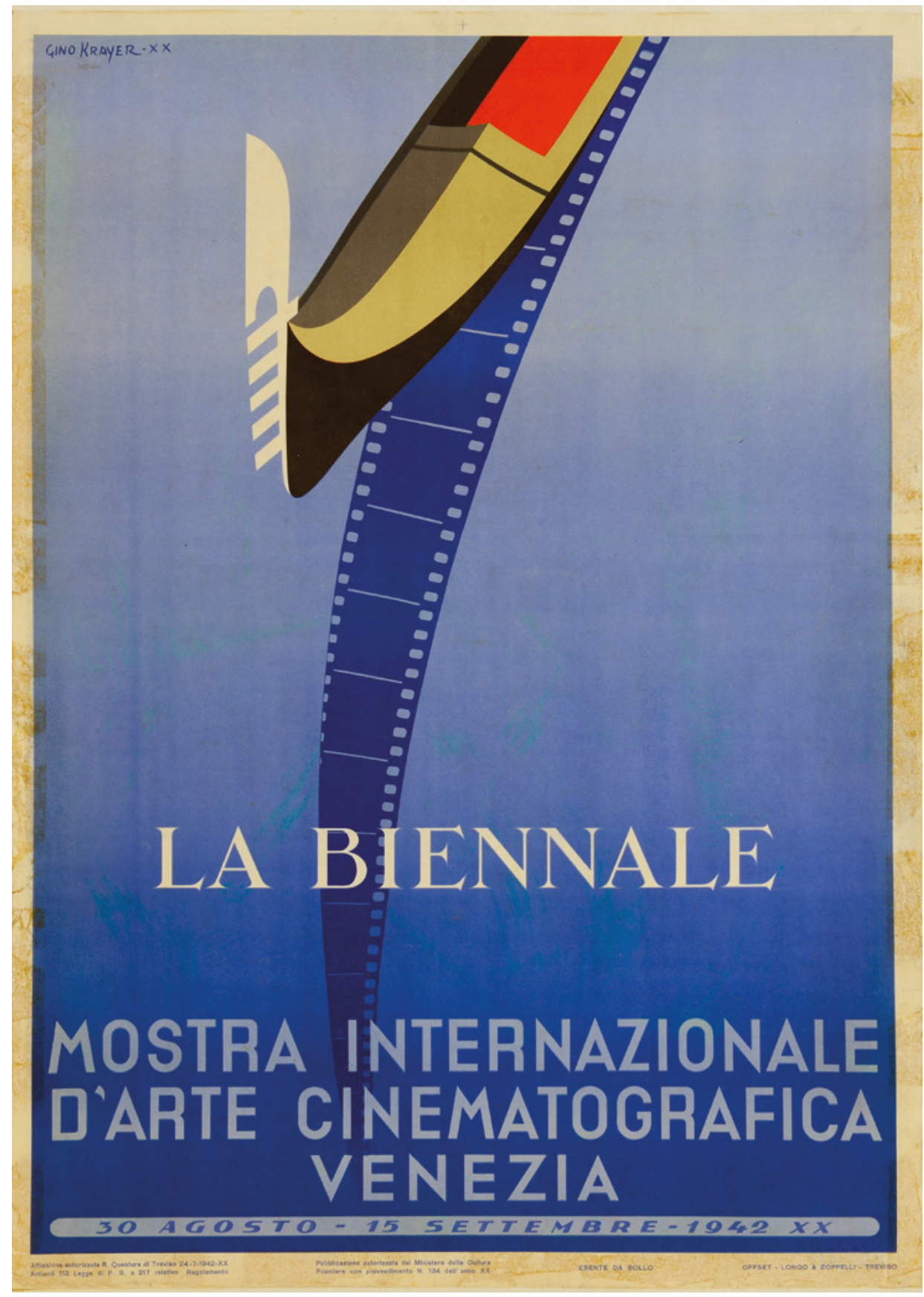

Gino Krayer, Manifesto per la Mostra Internazionale d'Arte Cinematografica di Venezia, 1942 (Treviso, Museo nazionale Collezione Salce) - Polo museale del Veneto, su concessione del Ministero per i beni e le attività culturali e per il turismo 


\title{
Comunicazione e graphic design della Biennale di Venezia (1895-1950)
}

Cristina Fontana

Fondazione Teatro Comunale Città di Vicenza, Italia

\begin{abstract}
Between 1985 and 1950 Venice Biennale was very focused on advertising and posters. In those early years advertising was organized by Antonio Fradeletto, Romolo Bazzoni and Augusto Sezanne, the official graphic designer of the pre-war biennials, that created also the Biennale brand. Over the years, communication strategies of the Institution developed due to personalities such as Pica, Maraini and Pallucchini. Biennale posters were fundamental to give visibility to the institution in the world. An accurate graphic design and an allusion to Venice contributed to spread a truly significant image of the exhibition.
\end{abstract}

Keywords Biennale. Posters. Advertising. Graphics. Communication.

La Biennale di Venezia ha potuto trasmettere il proprio messaggio fin dagli esordi mediante l'adozione di strategie di promozione. Nel periodo d'anni compreso tra il 1895 e il 1950 l'istituzione fu in grado di assumere consapevolmente le metodologie di una propaganda moderna grazie a una studiata pianificazione e logica pubblicitaria, la vasta diffusione di materiale carta-

Questo saggio prende avvio da Fontana, Cristina (2014). Manifesti e pubblicità della Biennale di Venezia dal 1895 al 1950 [tesi di laurea magistrale]. Relatore Nico Stringa; correlatore Stefania Portinari. a.a. 2013/2014. Venezia: Università Ca' Foscari Venezia; e Fontana, Cristina (2012). Augusto Sezanne: modernità e tradizione, i manifesti della Biennale dal 1895 al 1920 [tesi di laurea triennale]. Relatore Francesca Castellani; correlatore Elena Scantamburlo, a.a. 2011/2012. Venezia: Università IUAV; oltre che dalla partecipazione al convegno Storie della Biennale di Venezia, a cura di Nico Stringa e Stefania Portinari (Venezia, Università Ca’ Foscari Venezia, Ca’ Dolfin, 6-7 dicembre 2016).

Storie dell'arte contemporanea 4 | Atlante delle Biennali 1

ISSN 2704-9973

ISBN [ebook] 978-88-6969-366-3 | ISBN [print] 978-88-6969-367-0

Edizioni 
ceo promozionale, l'aggiornamento e l'uso di diversi mezzi di comunicazione. ${ }^{1}$

Tra i vari aspetti che caratterizzano l'organizzazione delle prime Biennali, ancora poca attenzione si attribuisce alla strategia di comunicazione che fu adottata ai suoi esordi, in un tempo in cui l'istituzione seppe avvalersi della cosiddetta réclame sfruttando le novità della cartellonistica (che in Italia giunsero con qualche ritardo rispetto agli altri Paesi europei) per ottenere il successo dell'Esposizione, forgiando un'immagine che, oltre a rappresentarla, si impose al suo pubblico borghese. La Biennale utilizzò infatti la comunicazione abbinata alla ricerca grafica per attrarre il pubblico, farsi riconoscere a livello internazionale e rilanciare il 'mito di Venezia', nel tentativo di riportare in auge la città.

Nel 1894, in seguito alla decisione di Riccardo Selvatico (l'allora sindaco e presidente dell'Esposizione) e dei membri del Comitato Ordinatore, venne istituito un Comitato per la Propaganda e per la Stampa che doveva occuparsi della pubblicità e dell'accoglienza dei «giornalisti italiani e stranieri ed a facilitare il loro soggiorno nella [...] città» (Bazzoni 1962, 17; cf. Di Martino 1995, 13), per trasmettere al pubblico notizie e informazioni sull'attività della Biennale. Gli sviluppi dell'apparato propagandistico dell'Esposizione si devono far rientrare in una prima stagione della pubblicità in cui l'innovazione grafica era considerata con sospetto (Scantamburlo 2009, 68-9). Tuttavia i promotori della Biennale erano interessati alla divulgazione e avevano compreso quanto questo aspetto potesse risultare efficace. Per questo motivo si avvalsero fin da subito di una specifica veste grafica, presentando nel budget preventivo di spesa 60.000 lire per pubblicità, una spesa consistente per il tempo, dimostrando che si trattò di un investimento coraggioso ma fondamentale.

Riccardo Selvatico si prodigò per gestire la larga diffusione nelle città italiane e straniere di réclame, cartoline, opuscoli, avvisi comunali, manifesti, segnalibri, francobolli e locandine. Propose anche la vendita di scatole di cerini acquerellate, in quanto «pubblicità molto utile e popolare» (Scantamburlo 2009, 68; 2017, 53-4). Questi strumenti si rafforzarono nel tempo e il Municipio di Venezia riceveva numerose richieste di pubblicazione di réclame (da quotidiani, case editrici, imprese private, teatri, hotel, aziende di navigazione) da ap-

1 Sulla grafica della Biennale sono state organizzate alcune mostre di manifesti, tra le quali una a Casale di Monferrato nel 1983, a Cuneo nel 1996, oltre che a Venezia, Italia: 150 / Biennale 116. Tutti i manifesti di 116 anni di vita in mostra (Venezia, Ca' Giustinian, 25 febbraio-20 maggio 2011) (senza catalogo): cf. tra gli altri volumi Immagine di una rassegna. I manifesti della Biennale di Venezia = Catalogo della mostra (Casale Monferrato, Galleria degli Stucchi, 9-22 giugno 1983). Venezia: s.n., 1983. La Biennale di Venezia attraverso i suoi manifesti. Cento anni di grafica = Catalogo della mostra (Cuneo, Ridotto del Cinema Monviso, 31 maggio-16 giugno 1996). Venezia, 1996. 
porre in catalogo, che dimostrano quanto il mezzo pubblicitario suscitasse sempre maggiore interesse. ${ }^{2}$ Molti erano gli editori e le tipografie che contattavano la Biennale per collaborare come stampatori del catalogo o di tutto il materiale grafico. Nonostante questo, essa rimase legata alle Officine lito e fotografiche più moderne ed innovative: la Carlo Ferrari (una delle stamperie che si occupò del catalogo per molti anni) e la Giacomelli, entrambe di Venezia, la Chappuis di Bologna, l'Istituto d'Arti Grafiche di Bergamo e la Ricordi di Milano, addette anche alla tiratura dei manifesti.

Il lavoro del Comitato Ordinatore era intenso e sempre esposto a continui accidenti e cambiamenti, dato che occorreva verificare quali fossero le stamperie migliori da ingaggiare, effettuare un attento confronto dei prezzi e il controllo della tiratura, il cui esito non era sempre soddisfacente. ${ }^{3}$ Romolo Bazzoni, l'amministratore della Biennale, affiancava in questo compito Fradeletto, occupandosi della supervisione dei sistemi della pubblicità, corrispondendo con i cartellonisti e con le officine grafiche, seguendo l'ideazione, l'evoluzione (nella fase di progetto e stampa) e la distribuzione del manifesto in Italia e all'estero.

Nei decenni di sviluppo del mezzo pubblicitario, che comprendono almeno tutta l'età giolittiana, il target group a cui era indirizzata la réclame si restringeva alla borghesia (Ceserani 1988, 10-11; 1980, 57).

Il manifesto fu il mezzo pubblicitario principale di cui si avvalse la Biennale: il suo utilizzo è da ricondurre allo sviluppo dell'arte grafica, soprattutto condotta attraverso lo stile dell'Art Nouveau, all'innovazione dei sistemi di stampa e alla diffusione delle cartiere e delle officine tipo-litografiche (Porzio 1982, 23-30; Villani 1966, 25; Rossi 1965, 19). Augusto Sezanne fu il cartellonista ufficiale della Biennale dal 1895 al 1920, facendo eccezione per il 1905 e il 1909, quando fu sostituito da Ettore Tito e da Adolfo De Carolis.

Fiorentino di nascita, ma formatosi all'Accademia di Belle Arti di Bologna, Sezanne si dedicò a diversi settori dell'attività artistica, distinguendosi come cartellonista, pittore, decoratore, architetto, restauratore e illustratore. La sua personalità, aperta alla sperimentazione di linguaggi differenti, lo rendeva idoneo alle necessità della nuova istituzione veneziana, che desiderava imporsi con vitalità nel rinnovato panorama artistico dell'epoca. Ricoprì anche un ruolo fondamentale all'interno della Biennale in qualità di promotore e membro del Comitato Ordinatore fino al 1899, avviando una stretta collaborazione con l'istituzione che si rivelerà, però, più proficua dal punto di vista dell'attività grafico-pubblicitaria. I suoi manifesti

2 Venezia, La Biennale di Venezia, Archivio Storico delle Arti Contemporanee (ASAC), Fondo Storico (FS), Scatole Nere (SN), b. 37: Varie.

3 ASAC, FS, SN, b. 7: Municipio 1896-1897. 
consentirono all'Esposizione di crearsi una immagine rappresentativa, che la distinse dalla concorrenza, concedendole quella solidità che le permise di mantenersi vivida nel tempo. Sezanne può essere infatti considerato un precursore del grafico pubblicitario moderno avendo dato vita al 'marchio' Biennale: realizzò il logo che sarebbe stato riprodotto su tutto il materiale pubblicitario dell'Esposizione Internazionale d'Arte rielaborando l'iconografia del leone in moleca e progettò tutti i materiali promozionali. Fissò dunque l'icona della Biennale utilizzando l'immagine di Venezia, che divenne la sua identità grafica. Nei suoi manifesti ritroviamo infatti i simboli storici della città lagunare: il Leone di San Marco, la galea veneziana, la gondola, i Mori della Torre dell'Orologio, la Quadriga, la Zecca, le Prigioni, la Libreria del Sansovino, la Basilica Marciana, il Palazzo Ducale, Punta della Dogana, il Campanile di San Marco, il Ponte di Rialto e la chiesa di Santa Maria della Salute.

L'artista era appoggiato da Fradeletto, che fu accorto nei confronti dei mezzi di comunicazione, ma si confrontava costantemente anche con Bazzoni. Nel corso degli anni sviluppò anche una più consapevole riflessione teorica sul metodo della cartellonistica, convincendosi che «il manifesto doveva essere sempre nuovo», istantaneo ed essenziale. ${ }^{4}$ Oltre che con un rimando alle antiche tradizioni rinascimentali, i manifesti si presentavano con un linguaggio influenzato dall'Art Noveau; per certi versi Sezanne era ancora legato al gusto descrittivo di fine Ottocento, che caratterizza la produzione grafica italiana dell'epoca, influenzata da movimenti artistici che generano spesso risultati ibridi (Bossaglia 1967, 21, 30-1; Fanelli 1981, 101). La stilizzazione formale e quella sintesi dei colori predicata da Vittorio Pica, sull'esempio del cartellonismo europeo, probabilmente non era sentita come adatta alla rappresentazione della città lagunare: unica al mondo, lontana dalle grandi metropoli, lambita dal mare, affezionata al suo passato storico, la Biennale voleva far emergere queste caratteristiche per richiamarsi al fascino della città. I manifesti di Sezanne desiderano dunque evocare le radici culturali della Serenissima tramite i simboli che la incarnano per eccellenza, nel precoce tentativo di far corrispondere l'immagine di Venezia con la Biennale, facendola diventare l'alter ego dell'Esposizione (Scantamburlo 2017, 53-62). L'artista insomma consegnò ai suoi successori un'immagine dell'istituzione codificata e stabilita.

Dopo la Prima guerra mondiale, l'organizzazione dell'Esposizione mise in campo nuove sinergie gestionali: Grimani e Fradeletto fu-

4 Nel 1898, infatti, Sezanne scriveva a Fradeletto de «l'inutilità degli avvisi grandi, la necessità che l'avviso sia un'opera d'arte come disegno, e come riproduzione, in modo tale che sia quasi ricercato, l'efficacia dei piccoli avvisi che si insinuano e penetrano garbatamente negli occhi del pubblico senza annoiarlo»: ASAC, FS, SN, b. 8: O.P.Q.R.S.T: Corrispondenza alfabetica, lettera di A. Sezanne a A. Fradeletto, 14 aprile 1898. 
rono sostituiti da Giovanni Bordiga (cf. Di Martino 1995, 32; Rabitti $1995,31)$ e da Vittorio Pica, critico d'arte e letterato, esperto in arte grafica e cartellonistica (Pica 1994, 27-98; Picone Petrusa 1994, 10). Dal punto di vista del materiale archivistico, le informazioni inerenti agli anni compresi tra il 1920 e il 1926 si presentano lacunose e scarse di fonti riguardo alle scelte intraprese dalla Biennale. Se da una parte la comunicazione pubblicitaria si affacciava a modalità espressive nuove, sia nell'ambito dei linguaggi utilizzati che della grafica, dall'altra manteneva invariate le caratteristiche del periodo storico precedente (Mori 2007, 61). Convivono quindi per tutti gli anni Venti orientamenti differenti, che risentono dell'influenza della propaganda fascista ma anche delle avanzate tecniche pubblicitarie americane, che a una grafica attraente affiancavano lo studio dell'andamento del mercato e l'indagine psicologica del consumatore (Valeri 1986, 53; Bourlot 2005, 275). L'Italia, guardando all'organizzazione della pubblicità così come veniva condotta all'estero, alla quale si cominciavano a dedicare congressi europei e mondiali, nel 1924 costituì il Sindacato Nazionale di Pubblicità (Valeri 1986, 59$60)$. Per quanto riguarda la storia della Biennale, le linee guida seguite erano le stesse degli anni precedenti: la pubblicità era sempre una voce fondamentale nel bilancio dell'Istituzione, tra le più alte dopo le spese strutturali destinate all'edificio espositivo (gli eventuali allestimenti e adattamenti delle sale) e gli stipendi dei dipendenti. ${ }^{5}$

Nel 1927, con la nomina di Antonio Maraini a segretario generale dell'Esposizione, la gestione della propaganda assunse una dimensione maggiore nel tentativo di raggiungere un più vasto pubblico. Rispetto a Pica si poneva come un uomo più pragmatico, con precedenti importanti come organizzatore e allestitore di mostre (Spadini 1991, 72; De Sabbata 2006, 48, 54). Maraini nel 1928 fece istituire l'Istituto Storico di Arte Contemporanea (dal 1930 denominato Archivio Storico delle Arti Contemporanee) nel tentativo di rinnovare l'immagine della Biennale anche come centro culturale stabile, facendo redarre anche il Bollettino dell'Esposizione come «organo dei propri atti a fine di pubblicità». ${ }^{6}$ Maraini era consapevole dell'importanza della pubblicità perché, come si può trarre da un verbale della Commissione Straordinaria del 1931, pensava che, «oltre al fatto spirituale, un'esposizione deve essere un fatto commerciale». Da un'organizzazione «municipale» assistiamo al passaggio a una pianificazione vasta e capillare della comunicazione dato che, come scrisse Maraini a Bazzoni nel 1928, «oggi non è più possibile trascurare questa parte, che tutte le aziende sempre più sviluppano e che ha realmen-

5 ASAC, FS, SN, b. 44: Pratiche varie 1928.

6 ASAC, FS, SN, b. 133: lettera di A. Maraini a R. Bazzoni, 12 dicembre 1928. 
te importanza soprattutto nei risultati finanziari». ${ }^{7}$ Ad occuparsi delle questioni pubblicitarie era Bazzoni, ${ }^{8}$ seguito dall'attenta supervisione di Maraini (mediante una fitta corrispondenza, dato che egli si trovava spesso fuori Venezia) e di Elio Zorzi, che curava i rapporti con la stampa nazionale e internazionale. ${ }^{9}$

Cruciale per il potenziamento del sistema comunicativo della Biennale fu il Regio Decreto del 13 gennaio 1930 nr. 33, che la trasformò in ente autonomo, svincolandola dal controllo comunale per assoggettarla a quello statale (Di Martino 1995, 36; Bazzoni 1962, 124-30). Nuovo presidente dell'Esposizione divenne il conte Giuseppe Volpi di Misurata che, veneziano di nascita, era stato anche ministro delle Finanze di Mussolini dal 1925 al 1928 (Gagliardi 2008, 447) e divenne un ulteriore punto di riferimento per l'organo promozionale dell'Ente mediante i contatti nell'ambito turistico e imprenditoriale, che permisero di ottenere collaborazioni importanti.

La Biennale fu in grado di rafforzare la propria posizione, intensificando e ampliando le proposte culturali anche a altre discipline (Rabitti 1995, 34; Pallucchini 2011, 608-9). A partire dal 1930 furono avviate altre manifestazioni quali il Festival Internazionale di Musica Contemporanea, il Festival Internazionale del Cinematografo (1932) e quello del teatro (1934), senza dimenticare l'esperienza del Congresso d'Arte Contemporanea del 1932 e le due edizioni dei Convegni di Poesia del 1932 e del 1934 (Bazzoni 1962, 159-68; Di Martino 1995, 37). Era quindi indispensabile gestire i meccanismi di propaganda in maniera efficiente e ricercare immagini che rappresentassero le nuove sezioni. Grazie a personalità come Volpi e Maraini, l'attività di promozione dell'ente fu gestita in maniera piuttosto autonoma. La Biennale cominciò ad avvalersi di consulenze fornite da organismi esterni quali agenzie e concessionarie esclusive della pubblicità (es. Rudolf Mosse, Giuseppe Borghi, Gabriele Balla, Unione Pubblicità Italiana, SPI),$^{10}$ che nascevano sfidando i tempi e le ristrettezze economiche, specializzate soprattutto in piccoli stampati e annunci, valendosi di tecnici esperti (cf. Valeri 1986, 67-8; Alberti 2007, 98). $\mathrm{Si}$ accordavano cooperazioni anche con associazioni e agenzie turistiche (es. ENIT, CIT, Wagons-Lits e Cook) ${ }^{11}$ e con l'imprenditoria lo- 
cale (es. CIGA, Compagnia Italiana dei Grandi Alberghi). ${ }^{12}$ La stampa quotidiana era utilizzata per tenere al corrente il pubblico sulle attività dell'ente, articoli e inserti sulla Biennale venivano pubblicati su giornali e riviste italiane e straniere.$^{13}$ I Paesi coinvolti dalla propaganda erano numerosi in Europa, estendendosi anche alle città del Nord e Sud Africa, del Sud America e dell'Australia. ${ }^{14}$

Maraini solitamente impostava anche una sorta di guida pubblicitaria e inviava alla stampa italiana e estera avvisi e fotografie. Nel 1934 venne dato alle stampe il volume La Biennale di Venezia, storie e statistiche, a cura dell'ufficio stampa, che tratteggiava il profilo dell'istituzione, ripercorrendone gli avvenimenti salienti. ${ }^{15} \mathrm{Il}$ volume fu venduto in alcune librerie e dato in omaggio ai direttori dei più importanti giornali italiani. ${ }^{16}$ Numeri speciali furono dedicati poi periodicamente alla Biennale e comparivano sul mensile $L e$ Tre Venezie, ${ }^{17}$ con cui Zorzi collaborava in qualità di giornalista, su Emporium $^{18}$ o su riviste di turismo e cultura quali Le Vie d'Italia e Il Mondo. ${ }^{19}$ La Biennale sviluppò anche una forma di pubblicità associata con la Triennale di Milano, che si appoggiava costantemente all'ente veneziano per organizzare il proprio sistema pubblicitario e le manifestazioni. Carlo Alberto Felice, membro del direttorio e segretario della Triennale (Pansera 1978, 41), manteneva una regolare corrispondenza con Maraini, Bazzoni e Zorzi, chiedendo informazioni su tutte le attività che concernevano l'organizzazione dell'Esposizione, tra cui la promozione. ${ }^{20} \mathrm{Nel} 1930$, a causa della concomitanza delle due manifestazioni, gli enti culturali decisero di organizzare congiuntamente la pubblicità, come avrebbero fat-

12 ASAC, FS, SN, b. 88: Biennale propaganda, lettera del consigliere delegato della CIGA a E. Zorzi, Venezia, 16 febbraio 1934.

13 ASAC, FS, SN, b. 60: Corrispondenza: X Y Z, lettera di E. Zorzi a R. Bazzoni, Venezia, 26 febbraio 1929.

14 ASAC, FS, SN, b. 90: S.I. Rudolf Mosse.

15 ASAC, FS, SN, b. 88: Biennale propaganda, Corriere Adriatico, Ancona, 10 maggio 1934.

16 ASAC, FS, SN, b. 97: Libro La Biennale.

17 ASAC, FS, SN, b. 90: Pubblicità, lettera di G. Giuriati alla Direzione della Biennale, Venezia, 6 aprile 1934

18 ASAC, FS, SN, b. 90: Pubblicità urgente, sottofasc. "Offerte di pubblicità», lettera del procuratore dell'Istituto d'Arti Grafiche di Bergamo a R. Bazzoni, 17 luglio 1934. Cf. anche: ASAC, FS, SN, b. 112: fasc. «Ordinazione. Manifesti e cartelli», e ASAC, FS, US, b. 1: fasc. «XXV Esposizione Internazionale d'Arte del 1950», sottofasc. «Inserzioni pubblicitarie».

19 ASAC, Carte del Capo Ufficio Stampa Elio Zorzi, b. 5.

20 ASAC, FS, US, b. 1: XXII Biennale - Stampa 1940, lettera di C.A. Felice a R. Bazzoni (Milano, 6 febbraio 1940). Cf. anche: la corrispondenza con C.A. Felice in ASAC, FS, SN, b. 73: fasc. «F»; ASAC, FS, SN, b. 74: fasc. «Corrispondenza: T»; ASAC, FS, SN, b. 87: fasc. «Stampa e Propaganda»; ASAC, FS, SN, b. 111: fasc. «Triennale di Milano». 
to anche nel 1934, nel 1935 (per il Padiglione del Turismo, all'Esposizione Universale di Bruxelles) nel 1936 e nel 1940. Ci si accordava sulle proposte, dopo aver stabilito la cifra del budget comune, e si pianificava l'intera campagna allo scopo di ottenere entrambo dei vantaggi valendosi anche della pubblicità realizzata dall'altro. ${ }^{21}$ La Biennale impiegò più mezzi di comunicazione per raccogliere consenso tra il pubblico, appoggiandosi spesso a agenzie di Milano che, insieme a Torino e Genova, era il polo della pubblicità (Valeri 1986, 65-6). L'istituzione si serviva del telegramma, dello strillonaggio, della pubblicità ambulante mediante uomini-sandwich, della distribuzione diretta, della pubblicità stradale per mezzo di insegne di grandi dimensioni e di pubblicità luminosa. ${ }^{22}$ Si confrontò anche con i mezzi di comunicazione di massa, attraverso l'utilizzo della radio (cf. Panicali 1979, 49) e del cinema.

Il pubblico si diversificava e per coinvolgere un numero di persone più ampio possibile e farle partecipare a eventi che prima ritenevano irraggiungibili o non potevano conoscere, l'informazione assunse una forma semplificata, con slogan comprensibili a tutti (Losito 1994, 17, 23-4; Wright 1976, 21-3). Fino a quel momento, la Biennale era riuscita a raccogliere e a mantenere numerosi contatti nel mondo dell'informazione e della pubblicità e a potenziare le strategie comunicative che, dopo la Seconda guerra mondiale, erano consolidate. Al termine del conflitto, a Maraini e a Volpi subentrarono Rodolfo Pallucchini, nuovo segretario generale, e Giovanni Ponti, come commissario straordinario. Mentre si proponevano di perseguire nuovi orientamenti artistici (Rabitti 1995, 37-8; Pallucchini 2011, 608-9), il sistema pubblicitario rimase il medesimo, intensificandosi in funzione degli sviluppi tecnologici e culturali, con il supporto di Bazzoni in qualità di consulente..$^{23}$

Dal punto di vista grafico, la rappresentazione di Venezia si mantenne come simbolo istituzionalizzato e segno riconoscibile dell'Esposizione. A partire dagli anni Venti, ad eccezione del manifesto di Sezanne realizzato per la XII Esposizione, vengono modificate le modalità di scelta dei cartellonisti. Tra il 1920 ed il 1950, sebbene con qualche irregolarità, furono emessi dei bandi di concorso dalla

21 ASAC, FS, SN, b. 111. ASAC, FS, SN, b. 112: fasc. «Ordinazione. Manifesti e cartelli». Cf. anche ASAC, FS, SN, b. 111: fasc. «Triennale di Milano», lettera di R. Bazzoni a C.A. Felice, 30 settembre 1935.

22 ASAC, FS, SN, b. 90: fasc. «Pubblicità», lettera di G. Cavallo al G. Volpi, 14 maggio 1934. Lettera al Comitato della Prima Esposizione Internazionale d'Arte Cinematografica di Venezia, 31 luglio 1932; in ASAC, FS, SN, b. 90: fasc. «Pubblicità». ASAC, FS, SN, b. 90: fasc. «Pubblicità», lettera dell'amministratore M. Piacentini della Ditta Lentilla al Comitato della Biennale, 23 ottobre 1933.

23 ASAC, FS, US, b. 2: fasc. «Aziende di pubblicità» e fasc. «Testi di volantini o di altre pubblicazioni». 
Segreteria dell'Esposizione. Il primo venne istituito nel 1922, durante il mandato di Pica. I bandi di concorso raccoglievano l'adesione di molti artisti italiani impegnati nel panorama contemporaneo dell'arte, attivi nella partecipazione alle esposizioni nazionali (la Biennale, la Quadriennale di Roma e la Triennale di Milano) ${ }^{24} \mathrm{e}$ internazionali più importanti all'epoca. La Biennale si avvalse di un ventaglio di personalità artistiche che, nonostante la formazione, lo stile e il temperamento differente, erano attive nei diversi ambiti della pittura, della grafica, delle arti applicate, decorative e dell'architettura, disponibili alle sperimentazioni e alle contaminazioni che scaturivano tra i vari campi dell'arte. Un bravo cartellonista, infatti, doveva essere una persona colta e consapevole della società in cui si trovava ad operare. La progettazione del messaggio pubblicitario venne affidata sempre più spesso alla grafica d'autore e, negli anni Trenta, fu oggetto d'interesse di molti, grazie al perfezionamento dei sistemi di stampa, che condussero alla nascita di nuove riviste d'arte illustrate (Pallottino 1980, 262). Furono organizzate anche le prime manifestazioni culturali che sancivano l'importanza dell'illustrazione e della grafica, come la Fiera Internazionale del Libro di Firenze del 1922 e le Biennali di Monza, che vi dedicavano sale specializzate.

L'importanza della comunicazione grafica nelle biennali del dopoguerra diventò ancora maggiore e furono ampliati i contatti con le officine tipo-litografiche. Tra le industrie grafiche non era un caso raro che si stabilissero delle cooperazioni allo scopo di abbattere la spesa della riproduzione e della stampa del materiale commissionato dalla Biennale..$^{25}$ Per aumentare la curiosità del pubblico a ogni realizzazione del manifesto la Biennale diramava dei comunicati stampa che riportavano una breve descrizione del cartellone e che venivano pubblicati sui giornali e sulle riviste d'arte. Essi erano inoltre esenti dalla tassa di bollo, per agevolare le pratiche burocratiche e finanziarie..$^{26}$ I manifesti venivano diffusi in Italia e all'estero: l'immagine della Biennale giunse in Europa, Stati Uniti e Canada, perfi-

24 I cartellonisti dei manifesti della Biennale (tra anni Venti e anni CInquanta) erano artisti noti, impegnati attivamente anche nell'ambito della pittura. Si confrontino, ad esempio, gli elenchi contenuti in ASAC, FS, SN, b. 112: fasc. «Vecchi elenchi 1936» e b. 124: fasc. «Inviti italiani».

25 ASAC, FS, Serie Teatro (da qui in poi ST), b. 2: fasc. «Affissioni».

26 ASAC, FS, SN, b. 88: fasc. «Concessioni doganali», lettera anonima al Ministero delle Finanze (Venezia, 15 marzo 1932). Cf. anche la lettera della Direzione degli Spettacoli all'IGAP (Venezia, 23 giugno 1937): «La Biennale di Venezia è esente dalla tassa di bollo per due leggi consecutive del 1918 e del 1923: questa esenzione fu confermata con foglio 21 Gennaio 1937»; in ASAC, FS, ST, b. 2: fasc. «Affissioni». 
no Africa e Australia. ${ }^{27}$ In questo modo, la Biennale e Venezia furono 'viste' sui mezzi di trasporto, nei luoghi turistici e nei locali di pubblico ritrovo, ${ }^{28}$ facendo del cartellone pubblicitario il medium ideale per ottenere un vasto riconoscimento internazionale.

Nel periodo di gestione di Vittorio Pica nello stile dei manifesti convivevano elementi compositi ed eterogenei, caratterizzati da propaggini dello stile secessionista e Art Nouveau, che confluirono nel Déco degli anni Venti (Bossaglia 1980, 145). Non si sentiva ancora la necessità di mutare repentinamente, poiché l'immagine tradizionale rimaneva rassicurante. I manifesti della Biennale realizzati in questo arco d'anni sono ancora legati al mondo della pittura rispetto ai risultati formali più sintetici e razionali che si svilupperanno negli anni Trenta (Mori 2007, 61-3). I documenti relativi a questo periodo sono molto scarsi, in termini di corrispondenze con gli artisti e con le tipografie. Ciò che sappiamo riguarda la pubblicazione, il 30 aprile del 1921, del primo bando di concorso (che presentava le linee guida che i partecipanti dovevano rispettare) - aperto a tutti gli artisti italiani - per ottenere l'ambita commissione del manifesto. Gli esiti sono immagini che riportano Venezia con le sue navi e il molo di San Marco di Giulio Cisari (1922), le donnine trasportate in gondola sul Canal Grande di Marcello Dudovich (1924), Palazzo Ducale e i simboli dei quattro venti delle fortune marinare della Serenissima di Brenno Del Giudice (1926). A partire dagli anni Trenta, invece, con la nomina di Maraini, la considerazione estetica del manifesto si integra ad una strategia visuale più matura, che tiene maggiormente in considerazione degli effetti commerciali e di marketing del cartellone. Questa nuova fase storica della Biennale è attraversata anche dalle vicende della politica fascista che però, nonostante l'imposizione dell'autarchia, non riuscì a sopprimere la forza dello sviluppo della pubblicità. La Biennale, dunque, continuò con successo la sua strategia promozionale, avvalendosi di una numerosa varietà di cartellonisti, provenienti da esperienze diverse. La creazione dei festival della Biennale richiedeva una corrispondente valorizzazione grafica, così come la definizione del nome 'festival' e del titolo della rassegna assunsero una particolare importanza:

27 ASAC, FS, SN, b. 79: fasc. «Tipografi e litografi». Cf. anche le lettere di A. Tivoli a R. Bazzoni, 5 agosto 1928 e [settembre] 1928; in ASAC, FS, SN, b. 49: fasc. «Corrispondenza: T»; e la lettera dell'IGAP del 5 luglio 1937; in ASAC, FS, ST, b. 2: fasc. "Affissioni» e ASAC, FS, SN, b. 90: fasc. «Pubblicità».

28 ASAC, FS, SN, b. 79: fasc. "Pubblicità XVI - 1928», lettera dall'Associazione Nazionale Combattenti, 31 gennaio 1928. Cfr anche: ASAC, FS, SN, b. 49: fasc. "Corrispondenza: S»; b. 70: fasc. «Pubblicità»; la lettera di L. Ferrari alla Biennale, 30 maggio 1936, per la diffusione dei manifesti nei principali alberghi e stazioni ferroviarie delle Dolomiti; in ASAC, FS, SN, b. 124: fasc. "Offerte di pubblicità per Parigi»; il Modulo del Ministero delle Comunicazioni Ferrovie dello Stato Sezione Movimento e Traffico Venezia; in ASAC, FS, SN, b. 81 e ASAC, FS, ST, b. 2: fasc. «Affissioni». 
A proposito del festival (vocabolo) non c'è nessuna ragione di cambiare l'intestazione della manifestazione - scriveva in un appunto il Segretario Maraini - Festival è una parola internazionale, ormai da tutti accettata e consacrata all'uso, e che esprime da sola tutto il nostro programma. Mutarla danneggerebbe la manifestazione [...], creerebbe confusioni proprio superflue. (Scantamburlo 2009, 72)

L'allusione incessante a Venezia si fondeva così con il logo dell'esposizione d'arte e con quello dei festival, nel tentativo di creare l'immagine coordinata dell'istituzione. La veste grafica dei manifesti risentì anche dell'influenza del graphic design del tempo: la figura del leone subì un processo di semplificazione, soprattutto dopo il 1934, venendo affiancato da due fasci littori che compariranno di frequente anche nella produzione cartellonistica. ${ }^{29} \mathrm{Il}$ leone in moleca sezanniano, con cui l'istituzione si era identificata fin dagli esordi, dopo la statalizzazione dell'Ente non poteva più essere confuso con quello del Comune di Venezia (di cui era stato emanazione diretta), assumendo la dimensione unica di 'marchio Biennale'. I bandi di concorso (istituiti regolarmente a partire dal 1934) erano aperti alla partecipazione dei soli artisti iscritti al sindacato fascista. Nel 1936, inoltre, sedici manifesti della Biennale furono esposti alla Prima Mostra nazionale del Cartellone e della Grafica pubblicitaria a Roma, che era stata promossa proprio dal Sindacato nazionale fascista di Belle Arti e dalla Confederazione fascista professionisti ed artisti. ${ }^{30}$

A livello stilistico venne abbandonata la linea sinuosa di fine anni Venti preferendo una maggiore geometria delle forme, che rinvia alla velocità e ai temi della grafica futurista. I tagli si fecero prospettici, i colori squillanti e si cerca una simmetria e tendenza alla bidimensionalità, evocando anche l'architettura e l'arte del Novecento Italiano (Benzi 2004, 22-30). La fisionomia dell'iconografia della Biennale è arricchita comunque da cifre stilistiche differenti, caratterizzate da tendenze espressive a volte contraddittorie, in un contesto aperto e fluido come era quello dell'industria culturale (Pallottino 1982, 435). I manifesti della Biennale, mantenendosi coerenti e radicati agli elementi e agli attributi tradizionali affermatisi nel tempo, riflettono gli orientamenti dei cartellonisti del periodo che furono Giulio Rosso (1928), Gorgon Tanozzi (1930), Bruno Bramanti (1932), Carlo Bisi (1934), Giulio Cisari (1936), Carlo Dalla Zorza (1936), Giuseppe Riccobaldi (1938)

29 ASAC, FS, SN, b. 108: fasc. «Risposte agli inviti»; cf. anche la lettera di A. Maraini a R. Bazzoni, 2 febbraio 1933; in ASAC, FS, SN, b. 133: fasc. «Maraini». Per quanto riguarda l'iconografia del fascio littorio, a titolo di esempio, si cf. la lettera di A. Maraini a R. Bazzoni, 11 febbraio 1933; in ASAC, FS, SN, b. 133: fasc. «Maraini».

30 ASAC, FS, SN, b. 109: fasc. «Corrispondenza: M». 
e Giacinto Mondaini (1940 e 1942). I simboli fascisti si trovano soprattutto nel manifesto di Riccobaldi, con il leone vittorioso di piazza San Marco, e in quelli tenebrosi di Mondaini con i caratteri cubitali della massiccia numerazione romana e il monumento di Colleoni.

$\mathrm{Al}$ termine della guerra, il manifesto, come la radio, cinema e televisione, ne uscì rinnovato, influenzato dalle sperimentazioni della grafica industriale e anche dallo stile pittorico dell'action painting e del tachisme (Rossi 1992, 113). Si spalancarono i contatti con gli Stati Uniti permettendo a molti grafici d'oltreoceano di lavorare con l'Italia. I bandi di concorso emessi dal 1947 erano rivolti agli artisti italiani e stranieri, lasciando piena libertà nella scelta del soggetto da rappresentare. Nel manifesto di Sergio Marzari (1948), ma soprattutto in quello di Paola Levi Montalcini (1950), vengono eliminati i dettagli, procedendo verso una maggiore sintesi e semplificazione formale, quasi astratta.

Poco invece sappiamo del materiale grafico del Festival Internazionale di Musica Contemporanea e di Teatro perché è scarsa la documentazione rimasta (soprattutto dopo il 1934), probabilmente andata perduta o non ancora pervenuta all'ASAC. L'unico esempio di manifesto che possiamo considerare propriamente 'musicale' fu quello realizzato da Giuseppe Piombanti Ammanati, uno dei quattro vincitori del concorso del 1934. ${ }^{31}$ Piombanti «coglie una suonatrice di liuto in stile schiettamente novecentesco e la solleva fra il campanile e la basilica giocando con toni rossi e azzurri». ${ }^{32}$ L'immagine del manifesto rimanda anche al Déco, che caratterizza molti lavori dell'artista, prima di volgere a uno stile Novecento e postNovecento (Servolini 1955, 652).

I manifesti realizzati a partire dal 1936 (anno in cui la rassegna divenne annuale) non sono pervenuti all'ASAC, in cui non si trovano nemmeno documenti che attestino la loro esecuzione. ${ }^{33}$ Consultando la Collezione Salce di Treviso, però, si rinviene un manifesto del 1939 disegnato da Riccobaldi e stampato dall'IGAP. Potrebbe trattarsi del cartellone ufficiale della manifestazione, senza escludere che fosse un semplice bozzetto in concorso. Al centro è rappresentata una maschera veneziana sorridente e in primo piano una nota musicale che rimanda al profilo di un'imbarcazione e costituisce l'iniziale della parola 'Venezia'.

Come per il Festival di Musica e per l'Esposizione Internazionale d'Arte, anche per il Primo Convegno Internazionale di Teatro (co-

31 ASAC, FS, SN, b. 91: fasc. «Manifesti - Concorso», lettera di G. Piombanti a R? Bazzoni, 21 ottobre 1933.

32 I cartelloni della XIX Biennale, in ASAC, FS, SN, b. 85: fasc. «Programmino».

33 ASAC, FS, Serie Musica (da qui in poi SM), b. n. 1, 2, 3: fasc. «Musica 1950»; ASAC, FS, ST, b. 2: fasc. «Affissioni», lettera anonima a S. Pelloj, 12 giugno 1937; ASAC, FS, SA, b. 21: fasc. «Astolfo De Maria», lettera di A. Maraini ad A. De Maria, 11 agosto 1937 
sì nominato agli esordi nel 1934) furono realizzati cartelloni, veline, locandine, stampati tipografici e depliant diffusi assieme al materiale promozionale della Biennale. Il primo manifesto ufficiale del Festival del Teatro fu realizzato da Giacinto Mondaini, che disegnò «una snella figura in bauta [...], che ricorda il gusto di Alessandro Longhi e che arresta col vivo contrasto dei suoi toni l'attenzione del pubblico innanzi alla leggenda». ${ }^{34}$ Mondaini, dunque, immortala uno degli elementi caratteristici del patrimonio culturale veneziano, la bauta, una maschera di origine settecentesca (Scarsella 1998, 46-52). Il manifesto venne riutilizzato anche per i festival degli anni successivi (1936, 1937, 1939, 1941), variando i colori dello sfondo e l'impostazione delle diciture, una scelta probabilmente adottata per non aggravare la situazione finanziaria del festival e delle altre attività della Biennale che, rispetto al teatro, si erano consolidate con maggiore successo. ${ }^{35}$

Non sono invece per ora rinvenibili informazioni riguardo ai manifesti realizzati dopo il 1941. Sembrerebbe, a partire da una corrispondenza, che dopo il secondo conflitto mondiale fossero stati emessi dei bandi di concorso, di cui non si è rinvenuta documentazione. ${ }^{36}$

A partire dal 1932 la Biennale di Venezia cominciò a riscuotere un grandissimo successo anche grazie al Festival Internazionale d'Arte Cinematografica, che doveva distinguersi graficamente dalle altre manifestazioni. Iconograficamente i manifesti del cinema richiamano Venezia e il Lido, accostati a rappresentazioni di pellicole e bobine, che diventano il simbolo della manifestazione. A realizzare il manifesto della prima edizione fu Nuchovich, che rappresentò una bobina dietro a cui si staglia la colonna marciana. Nel 1934 il vincitore del concorso fu il padovano Gianpaolo Lazzaro che rappresentò «un film spiegato in senso verticale, che rivela in negativo una testa di donna vista di fianco oltre la quale traspare in pallida luce serale il profilo di Venezia lontana», non senza un rimando al fascio littorio. ${ }^{37}$

La bobina divenne il soggetto principale del festival, comparendo anche nei successivi manifesti: Astolfo De Maria (1935 e 1936) la rappresenta avvolta attorno al gruppo scultoreo di Bernardo Falcone posto sulla torre della Dogana Da Mar (Dal Canton 1996, 75) e nel

34 ASAC, FS, SN, b. 91: fasc. «Manifesti - Concorso», lettera di G. Mondaini a R. Bazzoni, 22 ottobre 1933. ASAC, FS, SN, b. 85: fasc. «Programmino», I cartelloni della XIX Biennale.

35 ASAC, FS, SN, b. 136; ASAC, FS, ST, b. 2: fasc. «Affissioni»; ASAC, FS, SN, b. 106: fasc. «Ministro Stampa e Propaganda per Manifestazioni collaterali», lettera della Direzione degli Spettacoli all'IGAP, 23 giugno 1937; ASAC, FS, ST, b. 2: fasc. «Corrispondenza - Fornitori e preventivi - Assicurazioni», lettera delle Industrie Omassini \& Pascon alla Biennale, 13 giugno 1936.

36 ASAC, FS, Serie Cinema (da qui in poi SC), b. 18: fasc. «Manifesto IX Mostra», lettera di Adinolfi a E. Zorzi, 6 marzo 1948.

37 ASAC, FS, SN, b. 85: fasc. «Programmino», I cartelloni della XIX Biennale. 
secondo manifesto, realizzato sempre per l'edizione del 1935 (ristampato nel 1936 con una variante di colori), attorno a un elemento sferico che assume le fattezze di un pianeta. Riccobaldi riprende nel 1937 l'iconografia del globo, in un manifesto che viene ristampato modificato nel colore del lettering. Nel 1939, 1940 e 1941 viene recuperato quello del pittore veneziano Gino Krayer, modificandone solo i colori.

Durante questo periodo la Biennale sospendeva l'attività dell'esposizione d'arte a causa delle tensioni politiche che portarono allo scoppio della Seconda guerra mondiale e continuò ad organizzare annualmente solo il Festival del Cinema, a cui parteciparono le nazioni neutrali e quelle alleate a Italia e Germania. ${ }^{38}$ I manifesti di questo arco d'anni riverberano il progressivo controllo che il Regime assunse sulla manifestazione cinematografica, che era connessa fin dall'inizio con il progetto mussoliniano di apertura internazionale (Adagio 1998, 397-8).

Nel 1939 Krayer tenta recuperareo l'iconografia del dominante e ieratico leone di San Marco e mantenendo il riferimento diretto alla manifestazione mediante la rappresentazione della pellicola, che si srotola dalla colonna. Nel 1942 Krayer si mantenne cartellonista del festival del cinema, presentando una brillante interpretazione della gondola: il suo riflesso nell'acqua assume le sembianze della pellicola cinematografica, che risalta dalle sfumature dello sfondo blu oltremare.

Dopo che la manifestazione fu sospesa a causa della guerra, tra il 1942 ed il 1945, l'entusiasmo della riapertura, nel 1946, è rappresentato dal cavalluccio marino che Krayer fa emergere dai flutti della laguna. I manifesti dell'artista si imposero per quasi un decennio sull'immagine del Festival del Cinema: nel 1947, infatti, ritorna con qualche modifica compositiva e coloristica, l'idea della gondola del 1942. È interessante notare che il numero dell'edizione non è progressivo, ma riprende quello riportato nel manifesto del 1940, quasi a voler tornare indietro nel tempo, per cancellare e riscrivere un pezzo di storia culturale. Nel 1948 il vincitore fu Graziano Gasparini, che realizzò anche la copertina del catalogo del festival..$^{39}$ Rispetto ai precedenti, rappresentò una figura umana stilizzata, ma ritorna il motivo tanto caro della sfera (somigliante a un mappamondo) che qui corrisponde alla testa dell'omino. In mano reca delle bobine cinematografiche, alcune srotolate al vento all'incedere dei passi. Nel 1949 a vincere il bando di concorso, fu l'artista Pio Maturo, che rappresentò il cinematografo come un cerchio bianco, attorno

38 ASAC, FS, SC, b. 18: fasc. «Cineteca e Biblioteca», La Mostra Internazionale d'Arte Cinematografica di Venezia.

39 «Gasparini si afferma in campo nazionale», Ultimissime, 3 maggio 1948. 
a cui ruotano le bandiere colorate delle nazioni. ${ }^{40}$ Come per i manifesti della Biennale d'arte, le forme si spostano verso una maggiore sintesi, risultato delle influenze moderne e di una maggiore consapevolezza grafica. Il manifesto ideato da Maturo sembra ripreso nel 1950, sebbene in veste differente, da Gino Morandis, che rappresenta la pellicola cinematografica circondata da un anello di bandiere. ${ }^{41}$

Venezia, nella sua dimensione seducente e comunicativa, esercita la propria capacità di attrazione su tutto l'apparato pubblicitario della Biennale, e divenne la base su cui si fondò la corporate image dell'Istituzione e la liaison grafica tra le diverse tipologie di manifestazioni. Come affermava il famoso cartellonista Leonetto Cappiello: «tutti i manifesti che ricordate sono rimasti nella vostra memoria per la forma dell'immagine inventata dall'artista, che è diventata inscindibile dal prodotto e dal suo nome» (Rauch 2006, 27). La Biennale non rimase estranea a questa dinamica, creando e consolidando un'immagine distinguibile con immediatezza, grazie al costante rimando all'affascinante mondo lagunare con cui si identificava, che la rese nel tempo capitale della cultura e dell'arte internazionale.

40 ASAC, FS, AV, b. 27: fasc. «M - 1949-1950», Concorso per il Manifesto della X Mostra d'Arte Cinematografica. ASAC, FS, US, b. 2: fasc. «Francobollo Commemorativo», Comunicato stampa del 21 marzo 1949.

41 ASAC, FS, AV, b. 25: fasc. «Prof. Giorgio Morandi», lettera di R. Pallucchini a G. Morandis (s.l., 9 gennaio 1950); ASAC, FS, US, b. 2: fasc. «È stato scelto il cartellone per la XI Mostra del Cinema», comunicato sull'esito del bando di concorso (Venezia, 4 febbraio 1950). 


\section{Bibliografia}

Adagio, Carmelo (1998). «Mostra del cinema di Venezia». De Bernardi, Alberto; Guarracino, Scipione (a cura di), Il fascismo. Dizionario di storia, personaggi, cultura, economia, fonti e dibattito storiografico. Milano: Bruno Mondadori, 397-8.

Alberti, Alessia (2007). «Le origini della moderna comunicazione commerciale in Italia attraverso le riviste specializzate negli anni venti». Salsi, Claudio (a cura di), Pubblicità e Arte. Grafica internazionale dall'affiche alla Pop Art. Milano: Skira, 98.

Bazzoni, Romolo (1962). 60 anni della Biennale di Venezia. Venezia: Lombroso. Benzi, Fabio (2004). «ll déco. Origini e definizione di uno stile "moderno"». Benzi, Fabio (a cura di), Il Deco in Italia. Milano: Electa, 22-30.

Bourlot, Alberto (2005). «Pubblicità». Colombo, Fausto (a cura di), Atlante della Comunicazione. Milano: Hoepli, 275.

Bossaglia, Rossana (1967). «Grafica Italiana del Liberty». Critica d'Arte, 14(90). Bossaglia, Rossana (1980). «Arti decorative e Decò». Barilli, Renato; Solmi, Franco (a cura di), La Metafisica: gli Anni Venti. Volume secondo. Bologna: Grafis, 145.

Ceserani, Gian Paolo (1980). Vetrina della Belle Epoque. Bari: Laterza

Ceserani, Gian Paolo (1981). Vetrina del Ventennio 1923-1943. Roma-Bari: Laterza.

Ceserani, Gian Paolo (1988). Storia della pubblicità in Italia. Roma: Laterza.

Dal Canton, Giuseppina (1996). «ll percorso artistico del "bizzarro Astolfo"». Astolfo De Maria 1891-1946 = Catalogo della mostra (Venezia, Palazzo Fortuny, 23 marzo-19 maggio 1996). Milano: Electa, 75.

De Sabbata, Massimo (2006). Tra diplomazia e arte: le Biennali di Antonio Maraini (1928-1942). Udine: Forum.

Di Martino, Enzo (1995). La Biennale di Venezia 1895-1995. Cento anni di arte e cultura. Milano: Giorgio Mondadori.

Fanelli, Giovanni (1981). Il disegno Liberty. Bari: Laterza.

Farioli, Elisabetta (1981). «L'Aemilia Ars: alcune precisazioni». Solmi, Franco; Dezzi Bardeschi, Marco (a cura di), Alfonso Rubbiani: $i$ veri e falsi storici $=$ Catalogo della mostra (Bologna, Galleria d'Arte Moderna, febbraio-marzo 1981). Casalecchio di Reno: Grafis, 267-8.

Gagliardi, Alessio (2008). «Imprenditori e regime». Isnenghi, Mario; Albanese, Giulia (a cura di), Gli italiani in guerra. II Ventennio fascista: dall'impresa di Fiume alla seconda guerra mondiale (1919-1940). Torino: Utet, 447.

Losito, Gianni (1994). Il potere dei media. Roma: Carocci.

Mori, Giovanna (2007). «Esempi di grafica pubblicitaria dagli anni Venti ai primi anni Trenta alla Raccolta Bertarelli». Salsi, Claudio (a cura di), Pubblicità e Arte. Grafica internazionale dall'affiche alla Pop Art. Milano: Skira, 61-3.

Pallottino, Paola (1980). «Quaranta illustratori del Novecento: panorama dei centri di produzione di immagini in Italia negli Anni Venti». Barilli, Renato; Solmi, Franco (a cura di), La Metafisica: gli Anni Venti. Volume secondo. Bologna: Grafis, 262.

Pallottino, Paola (1982). «L'illustrazione». Gli Anni Trenta: arte e cultura in Italia = Catalogo della mostra (Milano, Galleria Vittorio Emanuele, 27 gennaio-28 aprile). Milano: Mazzotta, 435.

Pallucchini, Rodolfo (2011). «Storia della Biennale». Tomasella, Giuliana (a cura di), Rodolfo Pallucchini. Scritti sull'arte contemporanea. Verona: Scripta, 608-9.

Panicali, Anna (1979). «L'EIAR e la propaganda radiofonica». Immagine di popolo e organizzazione del consenso in Italia negli anni Trenta e Quaranta $=\mathrm{Ca}$ - 
talogo della mostra (Venezia, Museo d'Arte Moderna di Ca' Pesaro, novembre-dicembre 1979). Venezia: Marsilio, 49.

Pansera, Anty (1978). Storia e cronaca della Triennale. Milano: Longanesi.

Pica, Vittorio (1994). «ll Manifesto». Picone Petrusa, Mariantonietta (a cura di), Il Manifesto. Arte e comunicazione nelle origini della pubblicità. Napoli: Liguori, 10.

Picone Petrusa, Mariantonietta (1994). "Vittorio Pica e l'arte del manifesto». Picone Petrusa, Mariantonietta (a cura di), Il Manifesto. Arte e comunicazione nelle origini della pubblicità. Napoli: Liguori.

Porzio, Domenico (1982). «Invenzione ed evoluzione tecnica». Porzio, Domenico (a cura di), La Litografia: duecento anni di storia, arte, tecnica. Milano: Mondadori, 23-30.

Rabitti, Chiara (1995). «Gli eventi e gli uomini: breve storia di un'istituzione». Venezia e la Biennale: i percorsi del gusto = Catalogo della mostra (Venezia, Galleria d'Arte Moderna di Ca' Pesaro, 10 giugno-15 ottobre 1995). Milano: Fabbri Editori, 31-8.

Rauch, Andrea (2006). Graphic desig. La storia, i protagonisti e i temi dall'Ottocento ai giorni nostri. Milano: Mondadori.

Romano, Sergio (1979). Giuseppe Volpi. Industria e finanza tra Giolitti e Mussolini. Milano: Bompiani.

Rossi, Attilio (1965). «ll manifesto italiano». Il manifesto italiano nel centenario del manifesto litografico $=$ Catalogo della mostra (Milano, Palazzo della Permanente, marzo-aprile 1965). Milano: Ente manifestazioni milanesi, 19.

Rossi, Attilio (1992). I manifesti. Milano: Sonzogno.

Scantamburlo, Elena (2008). «Los Manifesti della Mostra del Cinema di Venezia (1932-1942)». AGR: Colleccionistas de Cine, 10(39), 32-45.

Scantamburlo, Elena (2009). «Tra arte e pubblicità: i manifesti e la grafica delle Biennali di Maraini (1928-1942)». Dal Canton, Giuseppina; Trevisan, Babet (a cura di), Donazione Eugenio Da Venezia. Quaderno n. $18=$ Atti della Giornata di Studio che si è tenuta al Museo Civico di Rovereto l'11 dicembre 2008. Venezia: Fondazione La Biennale di Venezia, ASAC Archivio Storico delle Arti Contemporanee; Fondazione Querini Stampalia; Museo Civico di Rovereto, 67-83.

Scantamburlo, Elena (2017). "Augusto Sezanne e i primi manifesti della Biennale d'Arte di Venezia: 1895-1899». Castellani, Francesca; Charans, Eleonora (a cura di), Crocevia Biennale $=$ Atti del convegno di studio. Milano: Scalpendi, 53-62.

Scarsella, Alessandro (1998). Le maschere veneziane. Le fantastiche e tradizionali figure del Carnevale più antico del mondo. Roma: Newton Compton.

Servolini, Luigi (1955). Dizionario italiano degli incisori italiani moderni e contemporanei. Milano: Gorlich.

Spadini, Pasqualina (1991). "Antonio Maraini artista e critico del ventennio». Officina della Critica. Libri, cataloghi e carte d'archivio = Catalogo della mostra (Roma, Biblioteca della Galleria Nazionale d'Arte Moderna, 20 dicembre 1991-4 marzo 1992). Milano: Electa, 72.

Valeri, Antonio (1986). Pubblicità italiana. Storia, protagonisti e tendenze dicento anni di comunicazione. Milano: Edizioni del Sole 24 Ore.

Villani, Dino (1966). La pubblicità e i suoi mezzi. Milano: Giuffrè.

Wright, Charles (1976). La comunicazione di massa. Prospettiva sociologica. 3a ed. Roma: Armando. 


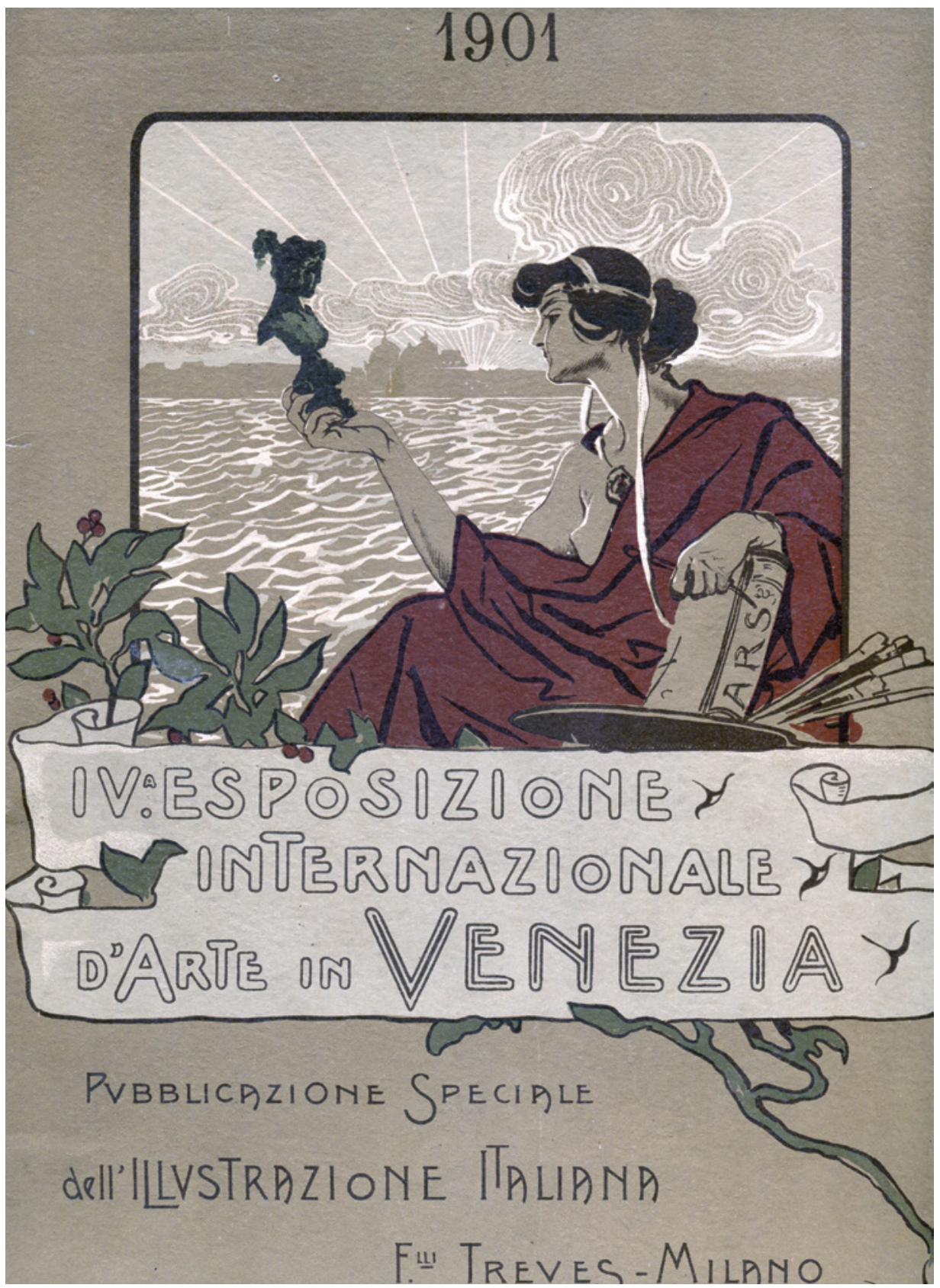

Figura 1 Copertina del numero speciale dell'Illustrazione Italiana dedicato alla IV Biennale del 1901. Venezia, collezione privata 


\title{
Le donne artiste alla Biennale d'arte di Venezia Le scultrici e le prime edizioni (1895-1912)
}

\section{Clarissa Coidessa}

Università Ca' Foscari Venezia, Italia

\begin{abstract}
This study on the theme of female participation at Venice Biennale from 1895 to 1995 contains data that allow us to outline the history of their participation, also in comparison of the male one. In order to limit the number of the analised artists and in order to carry out a more in-depth analysis, the first ten editions of Venice Biennale (1895-1912) were chosen: for this reason, the sculpture sector was also preferred as case study. A specific situation of the Venetian exhibition has been discovered. The artistic training of some sculptors and the study of art history made it possible to understand the role of women.
\end{abstract}

Keywords Women artists. Sculpture. Venice Biennale. 1895-1912.

Sommario 1 Analisi, studio e dati di una partecipazione al femminile. - 2 La Biennale e la scultura al femminile. - 3 Le scultrici alla Biennale d’Arte di Venezia, 1895-1912.

\section{Analisi, studio e dati di una partecipazione al femminile}

Il tema della partecipazione femminile all'Esposizione veneziana si rivela un ambito insidioso e di non facile osservazione a causa della difficile posizione che la donna artista ha occupato nel mondo dell'arte. La nascita dell'Esposizione Internazionale d'Arte della città di Venezia si colloca in un momento stori-

Questo saggio prende avvio da Coidessa, Clarissa (2019). Donne artiste alla Biennale d'arte di Venezia. Analisi e studio di una partecipazione ancora tutta da scoprire [tesi di laurea magistrale]. Relatore Nico Stringa; correlatore Stefania Portinari, a.a. 2017/2018. Venezia: Università Ca' Foscari Venezia. 
co particolare, a cavallo fra Ottocento e Novecento, in cui nonostante il numero delle giovani donne che si affacciano al mondo dell'arte sia in crescita sono ancora poche coloro che hanno il coraggio e la possibilità di perseguire una carriera in cui, come segnala Maria Antonietta Trasforini, persistono prepotentemente

i segni del genere, ovvero di quelle costruzioni storiche del maschile e del femminile che collocano gli individui in posizioni asimmetriche rispetto al linguaggio, al potere e ai significati. (Trasforini 2017, 27)

Si è inteso dunque indagare la presenza delle donne artiste alla Biennale d'arte di Venezia prendendo in considerazione un ampio arco temporale di cento anni che si estende dal 1895, anno di apertura della rassegna veneziana, al 1995. Contemplando esclusivamente il settore delle arti visive, l'obiettivo perseguito è stato quello di scoprire chi furono le partecipanti e tentare di comprendere quale fosse la posizione da loro occupata all'interno di questo specifico contesto.

Il primo passo compiuto è stato un lungo lavoro di analisi e compilazione dei dati effettuato sul numero delle donne artiste, che ha permesso di censire per la prima volta in maniera organica le espositrici presenti nelle edizioni della rassegna veneziana. Questo ha portato alla loro individuazione nel panorama generale dei partecipanti e alla raccolta delle informazioni relative alla loro consistenza. ${ }^{1}$ Un secondo risultato ottenuto è stata la creazione di due tipologie di schede: le prime raccolgono le informazioni relative alle opere presentate dalle artiste in ogni singola edizione della Biennale di Venezia dal 1895 al 1995. Le seconde riportano l'elenco dei nomi delle espositrici (in ordine alfabetico), la data di partecipazione, il numero delle presenze all'Esposizione veneziana e, quando rinvenuti, i dettagli anagrafici di ognuna di loro.

Sono 1.648 le donne individuate. Come si evince dal primo grafico [graf. 1], che mostra la totalità dei partecipanti alla Biennale di Venezia suddivisi per genere, visibile ed incontestabile è la netta e preponderante superiorità degli artisti uomini rispetto alle donne. Se le artiste come detto sono 1.648, gli uomini sono 12.607. Facendo un calcolo sul totale delle esposizioni registrate, risulta che gli uomini abbiano esposto per il 91,04\% mentre le donne per l'8,28\%.

Nel grafico si può notare, inoltre, la presenza di una terza categoria, quella dei nomi To be defined (sigla TBD), derivante da alcune situazioni in cui la determinazione del genere di ogni singolo esponen-

1 In questa analisi si è deciso di non prendere in considerazione collettivi di artisti stranieri la cui problematicità non consente di reperire i nomi dei membri costituenti. Cf. anche Vergine 1980; Pancotto 2006; Pajusco 2017. 
Clarissa Coidessa

Le donne artiste alla Biennale di Venezia

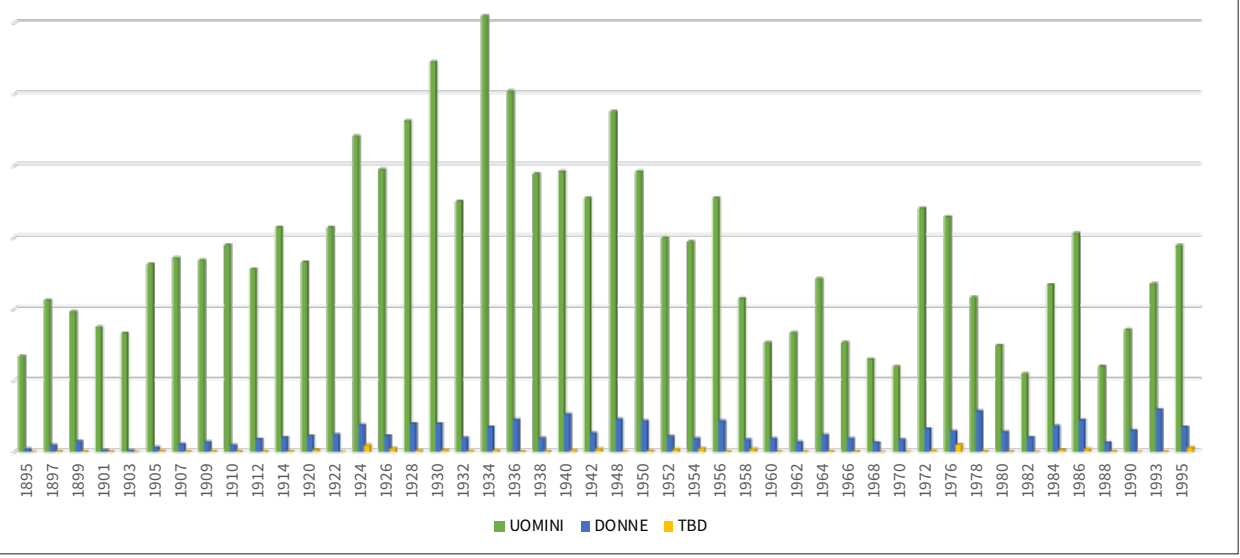

Grafico 1 Totale dei partecipanti suddivisi secondo le categorie di genere, 1895-1995

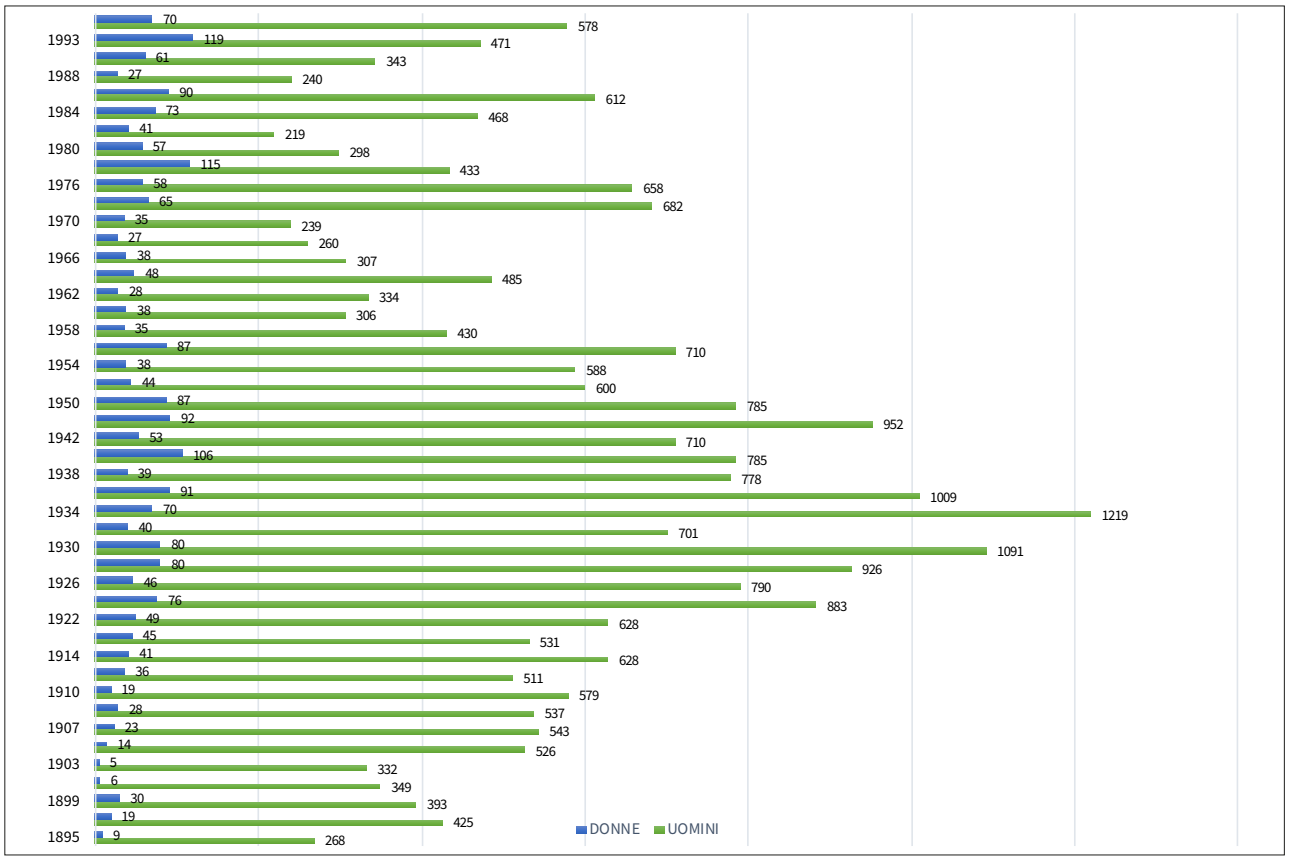

Grafico 2 Rapporto artisti uomini-artiste donne negli anni, 1895-1995 
Clarissa Coidessa

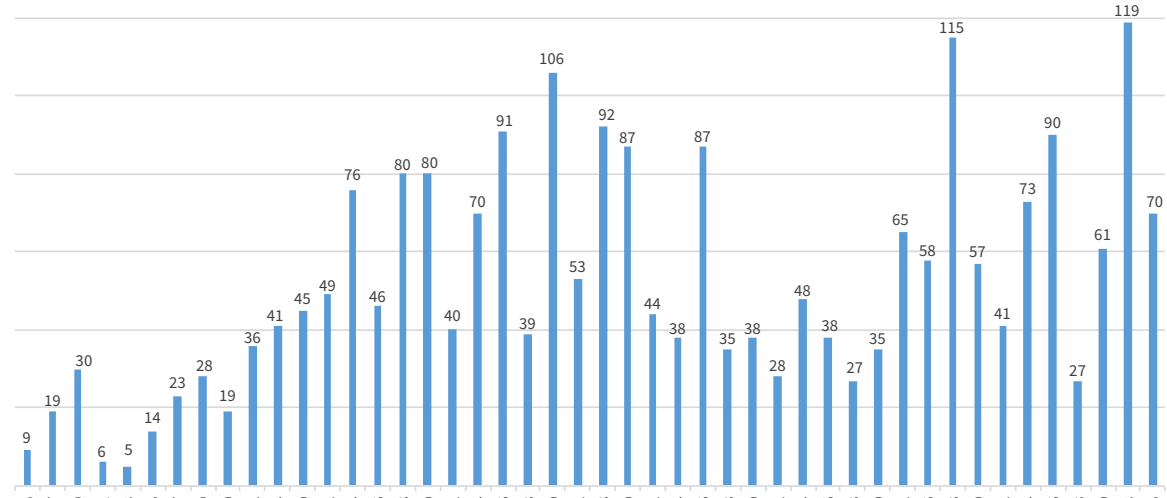

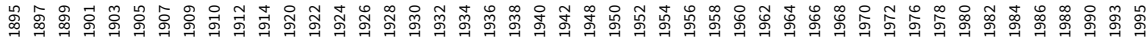

Grafico 3 Le donne artiste, diagramma a barre verticali (1895-1995)

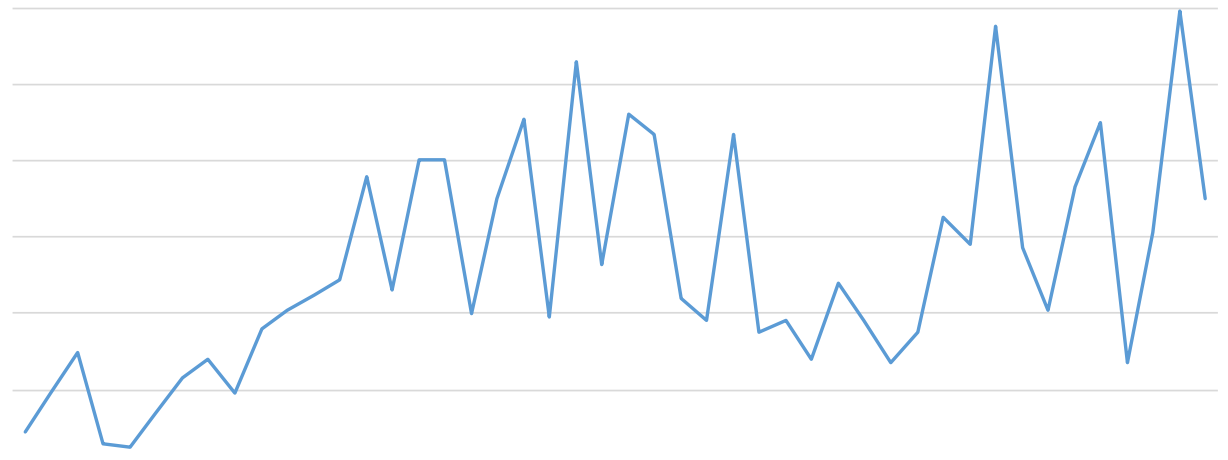

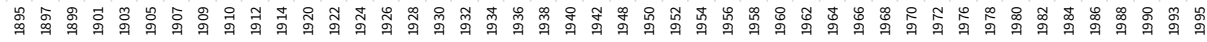


Clarissa Coidessa

Le donne artiste alla Biennale di Venezia

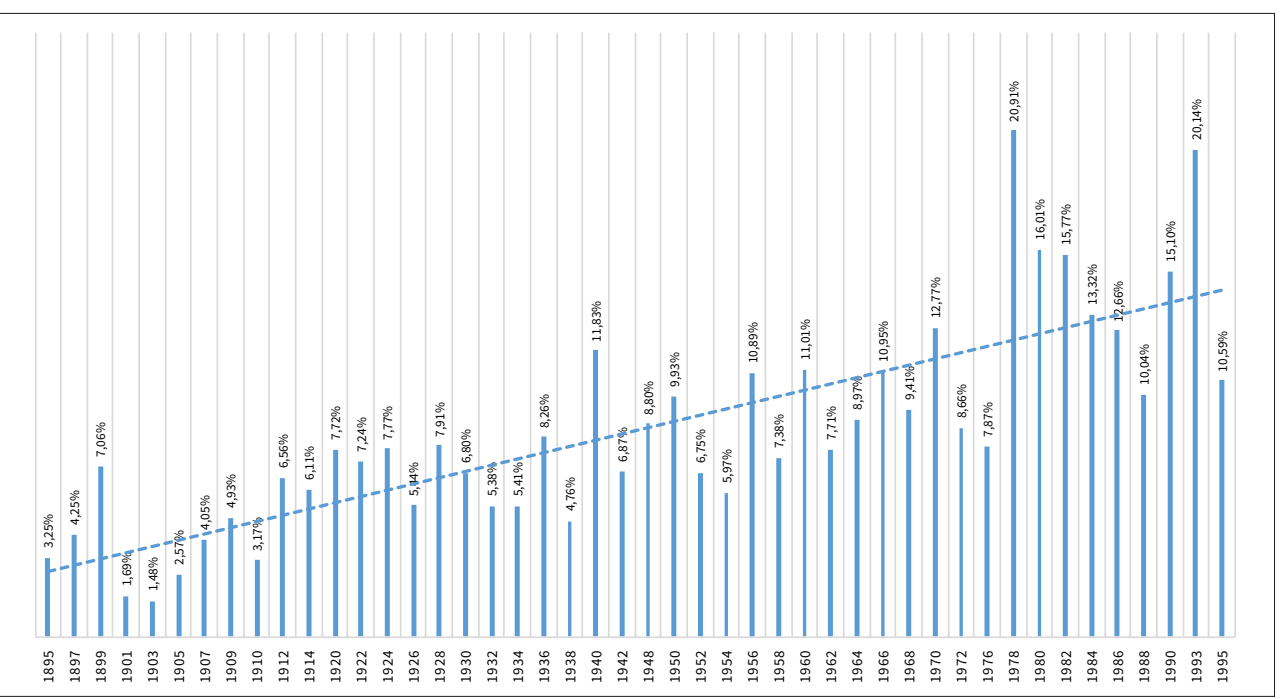

Grafico 5 Percentuali delle donne artiste sul totale dei partecipanti, 1895-1995

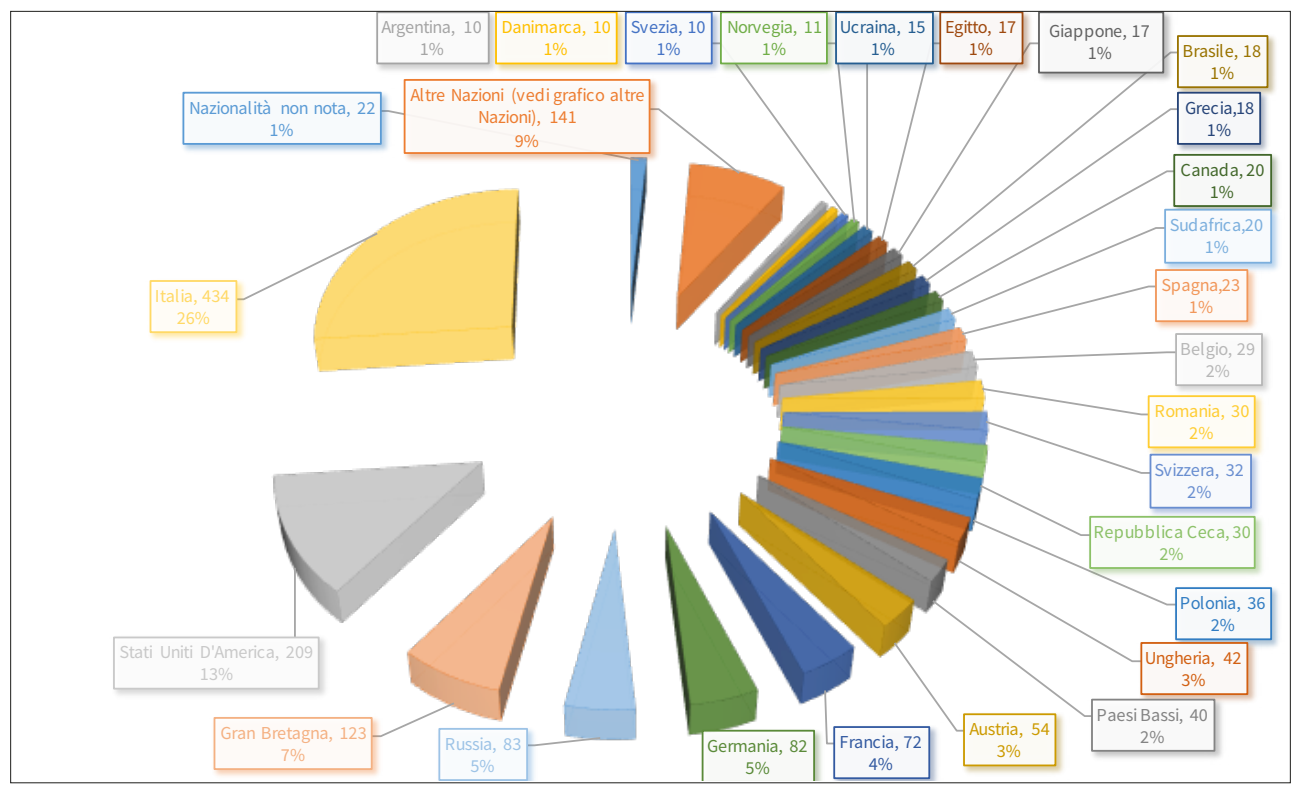

Grafico 6 Percentuali delle donne artiste per nazione, 1895-1995 
te si è resa impossibile. Tale gruppo rappresenta una bassa se non minima percentuale: si tratta mediamente del $0,68 \%$ sul totale, un numero che dunque non influisce notevolmente sull'analisi di dati.

Osservando la variazione annuale dei partecipanti uomini e donne alla Biennale d'arte di Venezia [graf. 2], le annate 1928, 1940, 1948, 1956, 1978, 1990 e 1993 risultano momenti storici particolarmente favorevoli per le artiste: vi si registra un aumento pari al doppio o una crescita definibile notevole rispetto all'annata precedente, non riscontrabile invece nel caso della partecipazione maschile. Nel 1978, in particolare, gli uomini sono più che dimezzati rispetto all'edizione del 1976 mentre le artiste aumentano considerevolmente: si passa da 58 a 115 espositrici. $^{2}$

Se i dati riportati fino ad ora si riferivano alla duplice presenza maschile e femminile all'interno dell'Esposizione d'Arte della Città di Venezia, il diagramma a barre verticali [graf. 3] e il successivo diagramma a linee [graf. 4] si concentrano esclusivamente sui dati relativi alle donne artiste che esposero dal 1895 al 1995.

Si può notare, ad una prima visione, quanto discontinua e variabile sia la presenza delle espositrici alla rassegna veneziana. Il grafico è caratterizzato da un andamento tendenzialmente sinusoidale: si registra un generale aumento fino al 1950, fatta eccezione di annate numericamente più basse che interrompono la tendenza crescente quali il 1926, 1932, 1938 e il 1942. Segue poi un andamento piuttosto basso fino al 1970 ed infine vi è una generale e tendenziale risalita, caratterizzata da picchi nelle edizioni della Biennale del 1978 (115 artiste) e del 1993 (119 artiste), anno che registra il culmine della presenza della donna alla Biennale di Venezia.

Di notevole interesse è soffermarsi sulle percentuali delle artiste sul totale degli espositori. Il grafico [graf. 5] mostra come non si giunga mai alla soglia del 50\%. Il picco massimo si registra nel 1978 con una percentuale femminile del solo $20,91 \%$. Sono dati significativi.

La linea di tendenza presente all'interno del grafico dimostra tuttavia che, nonostante la percentuale delle donne artiste dal $1895 \mathrm{al}$ 1995 sia bassa, l'andamento è crescente col passare degli anni. Si registrano dei livelli più spiccatamente alti nelle edizioni della Biennale del 1978 (20,91\%), del 1980 (16,01\%), del 1982 (15,77\%), del 1990 $(15,01)$ ed infine del $1993(20,14 \%)$.

Il grafico sulle percentuali delle donne artiste per nazione, 18951995 [graf. 6] e il grafico sulle percentuali della categoria «Altre nazioni», 1895-1995 [graf. 7], accertano infine l'internazionalità delle donne artiste all'Esposizione veneziana.

2 Le annate 1901, 1932, 1938, 1942, 1952 e 1980 registrano invece un dimezzamento della presenza femminile. 


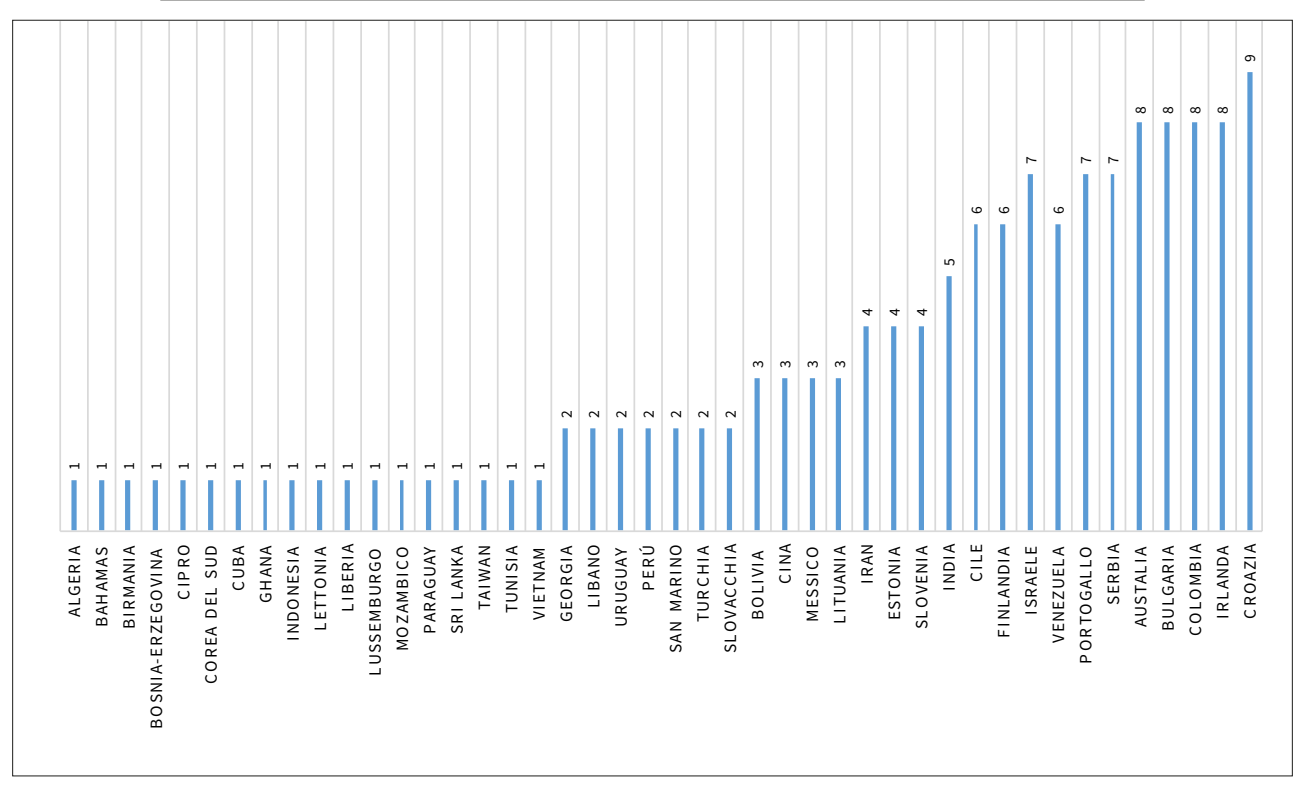

Grafico 7 Percentuali della categoria «Altre nazioni», 1895-1995

Le nazioni maggiormente rappresentate negli anni furono l'Italia (26\%), gli Stati Uniti d'America (13\%); la Gran Bretagna (7\%) e infine la Russia e la Germania (5\%). ${ }^{3}$

I risultati mostrati fino ad ora si sono avvalsi di un approccio di tipo quantitativo, che rappresenta un aspetto fondamentale e imprescindibile della ricerca.

La storia della partecipazione femminile alla Biennale di Venezia non può rimanere tuttavia solo un fatto numerico. L'indagine è stata così affiancata da un approccio di tipo qualitativo, volto a scoprire un mondo immenso, fatto di storie e vicende artistiche al femminile che si intrecciarono e vissero tramite le opere nelle sale della rassegna veneziana.

Il percorso non è stato facile ma è stato perseguito con la volontà di definire la posizione occupata dalle artiste all'epoca della loro partecipazione alla Biennale, esaltando il valore e l'arricchimento che esse vi apportarono.

In questo tentativo, vista l'ampiezza temporale presa in considerazione e l'alto numero di espositrici, la migliore idea è parsa quella di concentrarsi esclusivamente sulle prime dieci edizioni che han- 
no caratterizzato l'Esposizione Internazionale della città di Venezia, dal 1895 al 1912, esaminando le 189 donne che vi hanno preso parte.

Dal punto di vista storico e temporale è parso corretto cominciare la ricerca dal principio, dall'apertura della Biennale nel 1895. Le dieci annate scelte hanno permesso poi di contare su una visione generale abbastanza ampia che ha reso possibile compiere analisi e confronti sui dati. Gli anni compresi fra il 1895 e il 1912 si situano, infine, in un periodo particolare per la storia della donna artista. Sono questi, anni caratterizzati da forti cambiamenti che vedono le donne impegnate in una faticosa battaglia per la propria affermazione nel mondo dell'arte. Il numero di figure femminili che vi si dedica registra un forte aumento e

il lavoro dell'arte cominci[a] a rappresentare per molte, e di diverse classi sociali, una passione da trasformare in lavoro, una strada per il cambiamento della propria vita. (Trasforini 2017, 68)

L'aumento dell'alfabetizzazione delle donne, che si registra alla fine dell'Ottocento, porta le artiste ad una nuova consapevolezza: la necessità di ambire ad una migliore formazione, «ad un riconoscimento pubblico [volto all'affermazione di un proprio] status professionale» (Trasforini 2017, 67).

Rimane tuttavia indiscutibile che tra la fine dell'Ottocento e l'inizio del Novecento venisse ancora compiuta una differenza, come scrive Martina Corgnati, fra

'Arte' con la 'a' maiuscola e 'arte delle donne', una categoria a parte, non omogenea e non comparabile alla prima. (Corgnati 2004, 2)

Quale dunque si è rivelata la posizione delle donne che hanno esposto alla Biennale di Venezia nelle sue prime dieci edizioni? Quale il loro status di artiste?

Le 189 espositrici sono state, escludendo un piccolo gruppo di aristocratiche dilettanti, ${ }^{4}$ professioniste dell'arte che si sono distinte nel panorama artistico della propria nazione e al di fuori di essa. Molte hanno avuto riconoscimenti ma tutte hanno dimostrato bravura e capacità di sperimentare il vasto repertorio di tecniche artistiche loro disponibile a dispetto di quell'

4 Sono cinque le artiste amatoriali, il cui avvicinamento all'arte costituiva uno dei numerosi tasselli dell'ineccepibile educazione richiesta per una signorina di rango elevato. Nel 1895 si incontra Sofia Cacherano di Bricherasio (Torino 1867-Miradolo 1950), nel 1899 la contessa Maria Camerini Scola (Rovigo 1864-Creazzo 1937) e Nerina Pisani Volpi di Misurata (Firenze 1875-Roma 1942). Segue l'edizione del 1903 con Dada Albrizzi, quella del 1909 che vanta la partecipazione della contessa delle Fiandre Marie Louise Alexandrine Karoline Von Hohenzollern-Sigmaringen (Inzighofen 1845-Bruxelles 1912) ed infine la Biennale del 1912 con la baronessa Carla Celesia di Vagliasco (Firenze 1868-Collesalvetti 1939). 
infido stereotipo che attraversa la storia dell'arte e si rafforza nell'800 [...] che presenta le donne come prive di creatività, incapaci di significativi contributi e influenze nel campo artistico [...] negandone l'eterogeneità, la diversità e dunque la specificità del loro produrre e delle loro opere. (Trasforini 2017, 33)

La maggior parte di esse studiarono e si formarono presso le accademie, gli atelier di artisti e le scuole private, furono premiate con prestigiosi riconoscimenti e quasi tutte furono membri di associazioni o società artistiche. ${ }^{5}$ Fra loro, molte aprirono un proprio atelier. Ne sono un esempio Dora Hitz e Tini Rupprecht che esposero alla Biennale del 1897, le sorelle Macdonald che si incontrano in quella del 1899, le americane Cecilia Beux, Elizabeth Elliot Shippen Green e Bessie Vonnoh Potter che parteciparono nel 1909 e infine la polacca Olga Boznanska e Theresa Feodorowna Ries che esposero l'anno successivo. L'apertura di un atelier assumeva un ruolo determinante, da intendere come «il simbolo di una nuova consapevolezza del proprio status di artista» (Bartolena 2003, 76). Infine, alcune fra le donne artiste presenti all'Esposizione veneziana si dedicarono all'insegnamento dell'arte o fondarono scuole d'arte. ${ }^{6}$ Un simile interesse per l'arte non poteva appartenere quindi ad una dilettante, categoria in cui le artiste venivano inserite, ma essere proprio solo di chi aveva scelto l'arte come propria ragione di vita.

\section{La Biennale e la scultura al femminile}

Un campo che si è dimostrato particolarmente interessante e ricco di rivelazioni è stato senza dubbio quello della scultura. L'Esposizione Internazionale della Città di Venezia rappresenta, in quest'ambito, un caso di grande eccezionalità.

Nella faticosità del lavoro e nella durezza dei materiali da trattare, sono da sempre stati individuati gli ostacoli che per lunghi anni hanno giustificato il tentativo di interdire alle donne il genere della scultura. Quest'ultima era considerata un mestiere da uomo, non adatto alle signorine per bene.

5 Da sempre le associazioni hanno avuto un ruolo importante di aggregazione sociale. Sono luoghi in cui si facevano «circolare informazioni sulle innovazioni artistiche [...] [e si presentavano come occasione] di valutazione e autovalutazione degli artisti. L'appartenenza delle donne ad essi era un fattore dunque di fondamentale importanza segno della possibilità concessa all'artista di essere perfettamente inserita nel suo tempo» (Trasforini 2017, 45).

6 Tra le insegnanti troviamo i nomi di Aletta Jacoba Von Thol che espose alla Biennale del 1897, Jassie Marion King che partecipò a quella del 1899 e Käte Kollowitz che prese parte all'edizione del 1909. Fondarono invece scuole d'arte Dora Hitz (Biennale 1897), Ernestine Schutze Naumburg (Biennale 1901) e Ketty Gilson (Biennale 1909). 
In generale, a sfavore della scultura delle donne giocavano [...] la prevalenza dello studio del nudo e dell'anatomia, il costo dei materiali, oltre alla necessità di un corredo di attrezzi più da grande laboratorio che da piccolo studio, come la necessità di un atelier con trespoli, gessi, creta. (Trasforini 2017, 126)

A cavallo tra Ottocento e Novecento erano quindi poche coloro che avevano il coraggio e la volontà di intraprendere un percorso che si prospettava arduo e faticoso, situazione che rimarrà invariata in tutta la prima metà del Novecento fino agli anni Sessanta, momento in cui le scultrici saranno pienamente libere di creare e di acquisire visibilità e credibilità anche in questo settore dell'arte (Bartolena 2003, 152).

Andando a indagare le donne artiste che si dedicarono alla scultura nelle prime dieci edizioni della rassegna veneziana, è stato inaspettato scoprire che, nonostante il loro esiguo numero, esse siano state, senza ombra di dubbio, numericamente superiori alle espositrici dei settori del decorativo.

È questa una situazione decisamente rilevante, considerata l'idea generalmente diffusa che l'arte decorativa rappresentasse il genere prediletto dalle donne artiste e che la scultura al contrario non lo fosse. A cavallo fra Ottocento e Novecento il decorativo era visto come una caratteristica intrinsecamente propria della donna e trovava la sua ragione d'essere nel ruolo occupato dalla figura femminile nella società d'allora. Oltre che madri e mogli, le donne avevano il compito di occuparsi della casa, luogo a loro destinato.

Le donne [...] ornano la casa con la propria presenza ma anche con le risorse del loro spontaneo talento. Sono decorative e decoratrici; un ruolo, quest'ultimo, che cominciano a non esercitare più soltanto tra le pareti domestiche. (Altea 2012, 32)

Linda Nochlin si domandava a tal proposito se le donne non fossero state «dirottate nelle cosiddette arti minori o decorative perché queste erano considerate meno esigenti e certo [...] meno prestigiose» (Harris Sutherland-Nochlin 1979, 55). E possibile e quasi certo che ciò sia accaduto: se un'artista dell'epoca desiderava diplomarsi nel modo dell'arte, l'unico settore loro aperto era quello delle arti decorative (Harris Sutherland-Nochlin 1979, 48).

Il grafico sulle percentuali delle donne artiste che espongono opere di Arte Decorativa, 1895-1995 [graf. 8] e il Grafico sulle percentuali delle donne artiste che espongono opere di Scultura, 1895-1995 [graf. 9], permettono di comprendere, attraverso un loro confronto, la singolare situazione che caratterizza la Biennale di Venezia.

Analizzando il settore delle Arti decorative si nota la loro assenza durante la prima edizione dell'Esposizione veneziana. Tale scelta era stata compiuta per distinguersi dall'Esposizione Nazionale Arti- 
Clarissa Coidessa

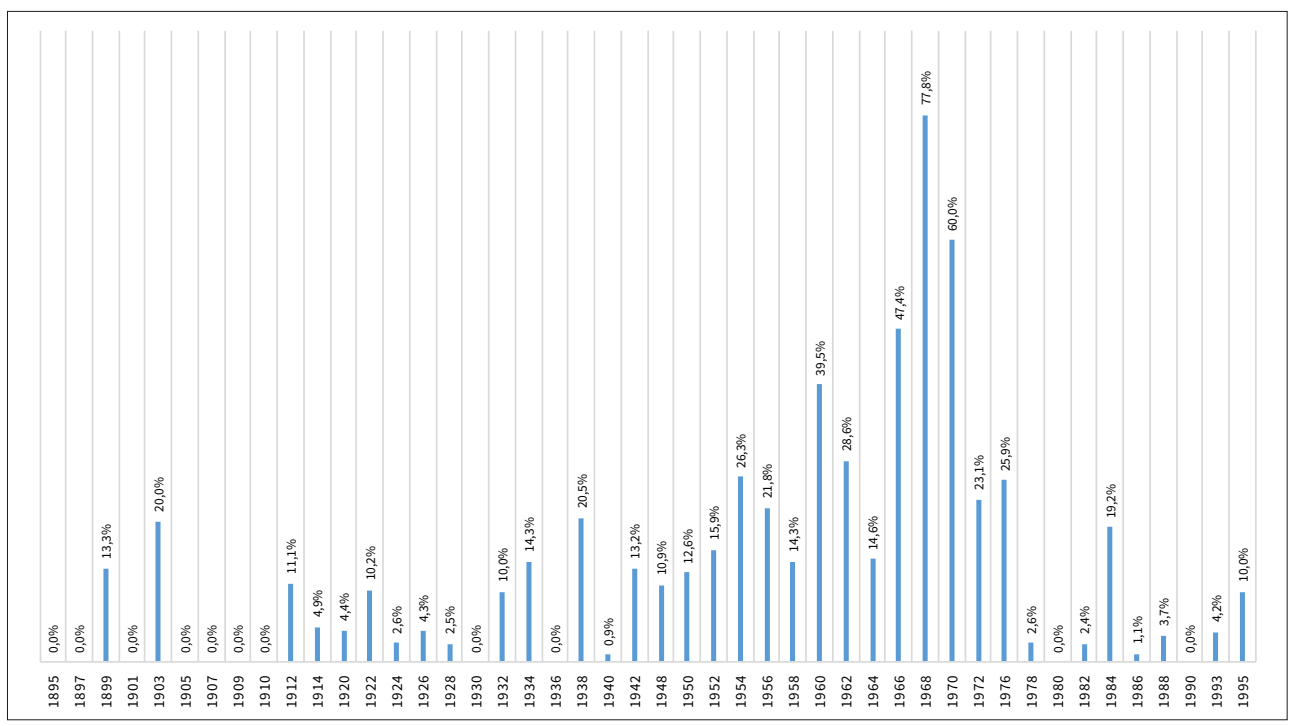

Grafico 8 Percentuali delle donne artiste che espongono opere di Arte Decorativa, 1895-1995

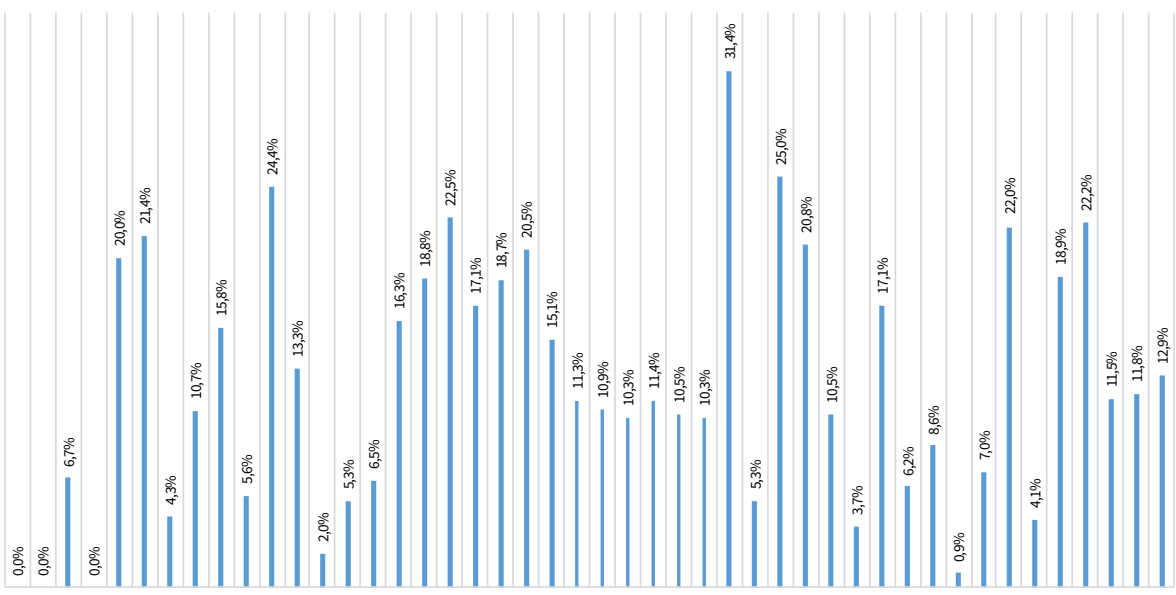

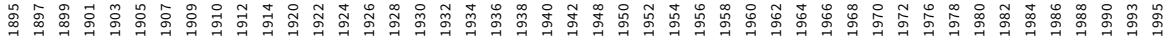


stica organizzata ai Giardini nel 1887, mostra che aveva lasciato largo spazio al decorativo (Scotton 1995, 123). È invece il 1899 che dà avvio all'esposizione femminile di opere d'arte decorativa.

Osservando l'arco temporale fino al 1912, si assiste alla mancanza di espositrici nelle edizioni del 1897, del 1901 e in quelle dal 1905 al 1910.

Le percentuali delle artiste sono da considerarsi quindi basse ed è stato sorprendente notare che lo rimarranno anche nelle annate successive fino al 1995. Si registrano numeri più alti solamente dal 1965 al 1970, in cui si supera la soglia del 50\% nelle edizioni del 1968 e del 1970, con la percentuale massima registrata all'Esposizione d'arte del 1968 (77,8\%).

Guardando invece al campo della scultura, se nelle prime due edizioni non vi sono artiste che espongono opere di questo genere, è l'annata del 1899 che apre la strada ad una loro partecipazione. Con una breve assenza nel 1901, dall'Esposizione veneziana del 1903 fino al 1912 quella delle scultrici sarà una presenza costante, priva di assenze fino al 1995.

\section{Le scultrici alla Biennale d'Arte di Venezia, 1895-1912}

Le artiste che, nelle prime dieci edizioni della Biennale di Venezia, si dedicarono alla scultura furono tredici.

Nel 1899 si incontrano Isa Albazzi e la contessa Maria Camerini Scola (Rovigo 1864-Creazzo 1937). Nel 1903 espose Therese Feodorowna Ries (Mosca 1874-Lugano 1950), che presenterà due sculture anche nel 1910.

Alla Biennale del 1905 parteciparono Eliza Beetz (Schaerbeek 1875-Neuilly-sur-Seine 1949), Charlotte Gabrielle Besnard (Parigi 1855-Parigi 1930) e Ilse Twardowsky-Conrat (Vienna 1880-Monaco di Baviera 1942).

Il 1907 fu l'anno di Irma von Duczynska (Leopoli 1870-Monaco 1932). L'Ottava Esposizione Internazionale d'Arte del 1909 vide invece la partecipazione di tre scultrici: Martha Bauer, Eberle Mary Abastenia St. Leger (Webster City 1878-Webster City 1942) e Bessie Vonnoh Potter (Saint Louis 1872-New York 1955), mentre la Biennale del 1910, di Elza Kalmár Kövesházi (Vienna 1876-Budapest 1956) e Melicent Stone (Havant 1868-1922). La X. Esposizione Internazionale d'Arte infine registrò la presenza di Maria Antonietta Pogliani (Castell'Alfero 1886-Roma 1956) e nuovamente quella dell'inglese Melicent Stone.

Il numero delle artiste nominate è indiscutibilmente basso. Se da un lato questo è collegabile, come detto, alla difficile accettazione da parte del pubblico e della società della donna scultrice e alle difficoltà e agli ostacoli che coloro che si dedicavano a quest'ar- 
te incontravano nel proprio cammino, dall'altro può essere tuttavia riconducibile ad una specificità propria della Biennale di Venezia. Nelle prime edizioni alla scultura è riservata nel complesso una minore attenzione rispetto alla pittura (Fergonzi 1995, 103). Solitamente, le esposizioni nazionali ed internazionali ottocentesche ponevano le opere di scultura nei corridoi di ingresso degli edifici, creando un effetto di monumentale grandezza. L'Esposizione Internazionale d'Arte della Città di Venezia decise invece di virare verso una scelta opposta. Venne evitato di «riservare alla sola scultura l'ambiente più ampio e scenografico del Padiglione centrale» (Fergonzi 1995, 103) ponendola in dialogo con i dipinti, numericamente superiori. Le opere scultoree si trovavano così ad avere rilievo minore rispetto a quelle pittoriche.

Nell'osservare in modo più ravvicinato le figure delle artiste scultrici e nell'indagare la loro storia, ${ }^{7}$ colpisce notevolmente l'eccellente qualità e la bellezza delle loro opere, unite ad un grande carattere e a una forte personalità che permise loro di brillare nel firmamento degli artisti espositori.

Esemplare è il caso di Charlotte Gabrielle Dubray Besnard, che in vita godette di un grande successo raggiunto con duro lavoro e caparbietà.

Per la propria formazione artistica, l'artista parigina mise in atto un efficace e rigoroso programma che le permise di eguagliare la preparazione e il percorso compiuto dai colleghi maschi all'Accademia di Belle Arti di Parigi, il cui ingresso fu interdetto alla donne fino al 1897. Le sue parole d'ordine erano «étudier, puis se faire remarquer au Salon, pour enfin décrocher des commandes» (Moreau-Sionneau 2013, 4). La fase dello studio avveniva attraverso la copia attenta e meticolosa delle opere del padre Gabriel-Vital Dubray (1813-1892), facendo proprio un particolare stile accademico che caratterizza i lavori della prima giovinezza.

La grande forza di volontà e il carattere di Charlotte Dubray Besnard, le permisero così di farsi strada nel campo dell'arte scultorea, giungendo, come sperato, a partecipare in numerose occasioni al celebre Salon che si teneva con cadenza annuale nelle sale del Louvre e ad essere premiata nel 1900 con la medaglia d'argento all'Esposizione Universale di Parigi per l'opera Saint François (Moreau-Sionneau 2013,6). Tale risolutezza si coglie nelle parole della critica d'arte francese, Camille Mauclair, che ne delineò la personalità affermando che ella fosse «très féminine avec un cerveau quasi masculin» (Mauclair 1914, 124).

7 Non è stato possibile delineare il profilo delle artiste Isa Albazzi, Martha Bauer e Melicent Stone a causa della mancanza di informazioni sulle prime e delle esigue notizie rinvenute sulla terza. 
Nel momento in cui Charlotte Dubray Besnard espose alla Biennale di Venezia ella aveva quindi già raggiunto un ampio riconoscimento presso un'importante esposizione, al pari dei colleghi uomini.

Nell'ottava sala dell'Esposizione d'Arte di Venezia, la sala francese, Charlotte Besnard espose due sculture: Busto della signora G. e Busto della Signora Aman-Jean, sua cara amica.

In quest'ultima opera, la Besnard lavora al genere del ritratto ad occhi chiusi, testimone del passaggio, compiuto dall'artista parigina, da una fase d'impronta accademica ad una di più libera sperimentazione di novità iconografiche. Esso «n'a plus rien à voir avec un portrait réaliste. La pose originale traduit au contraire un degré d'intimité réelle et illustre le goût qu'ont les artistes idéalistes» (Moreau-Sionneau 2013, 10). L'amica è colta in una posa dolce e sognante, con il capo reclinato verso destra. Ė una rappresentazione di grande delicatezza che esprime l'affetto e l'amorevolezza provata per la protagonista.

Nella lista dei nomi degli artisti che esposero alla Biennale di Venezia, il cognome Besnard compare più di una volta: oltre al marito Albert Besnard (1849-1934), illustre pittore e incisore parigino, ${ }^{8} \mathrm{si}$ distingue anche il nome di Philippe Besnard, terzogenito della coppia, che partecipò all'Esposizione veneziana nel 1930 e nel 1934 con opere di scultura. L'intera famiglia Besnard, ad eccezione del primogenito Robert, fece dell'arte la propria professione. La secondogenita, Germain seguì le impronte della madre divenendo scultrice mentre Jean si dedicò alle arti decorative con la realizzazione di ceramiche.

Quello di Charlotte si presenta quindi come il caso di un'artista che riuscì a coniugare perfettamente vita professionale e vita familiare, minando il pregiudizio diffuso secondo il quale il problema della donna artista nasceva dall'idea che quest'ultima non fosse capace di conciliare il ruolo di artista con quello di moglie e di madre (Corgnati 2004, 1).

È di notevole interesse soffermarsi anche sul ruolo ricoperto dalla Besnard come moglie di un famoso e rinomato artista.

Le donne artiste che si sono legate sentimentalmente ad un proprio collega sono numerose. Nell'ambito dell'Esposizione Internazionale d'Arte di Venezia ne sono un esempio Sientje Van Houten Mesdag, moglie del pittore olandese Hendrik Willem Mesdaga, e Etha Fles, compagna di vita di Medardo Rosso, che esposero alla Biennale nel 1895; Juliette Wytsman, compagna di Rodolphe Wytsman, e Gesina Mesdag Van Calcar, moglie del pittore Taco Mesdag, che parteciparono a quella del 1899.

8 Albert Besnard partecipò a numerose edizioni della Biennale veneziana: fu presenza costante dal 1895 al 1909, edizione in cui gli venne dedicata una mostra individuale. Espose inoltre nel 1912, 1922, 1924 ed infine nel 1934. 
Tali relazioni se da un lato permettevano alle artiste di approfittare della notorietà di cui godeva il marito per avere una maggiore visibilità nelle mostre, dall'altro le relegava in una posizione marginale e limitata vissuta all'ombra del successo del proprio consorte. La storia della scultrice Beetz-Charpentier che espose, come la Besnard, alla Biennale di Venezia nel 1905, ${ }^{9}$ ne è un esempio. Scarse sono le notizie reperite sulla sua formazione ed affermazione come artista. Eliza viene ricordata principalmente come moglie in seconde nozze dello scultore Alexandre Charpentier, suo maestro. ${ }^{10}$

Il caso di Charlotte Besnard è invece diverso. È stata avanzata l'ipotesi che lei non abbia occupato un ruolo secondario nella relazione con il marito ma che abbia avuto, al contrario, una certa influenza sulla sua carriera, le sue relazioni e sul suo successo a Londra. ${ }^{11}$

La grinta, lo spirito imprenditoriale e di autopromozione, unite alle notevoli capacità dimostrate in ogni campo ${ }^{12}$ hanno reso Charlotte Gabrielle Besnard una grande artista, riconosciuta nel panorama artistico europeo e francese di fine Ottocento e inizio Novecento.

Nel gruppo delle scultrici presenti nelle dieci edizioni prese in esame, un'altra figura che si rivela intrigante è quella della russa Therese Feodorowna Ries. L'artista espose alla Biennale in due occasioni: nel 1903 presentando tre opere intitolate Busto del conte Giovanni Hellmer, Busto dello scultore Prof. Hellmer, Il bacio e nel 1910 con La caduta, lavoro in marmo e L'anima ritorna a Dio, in gesso.

L'artista condivide con le scultrici Ilse Twardowsky-Conrat, Irma von Duczynska ed Elza Kalmár Kövesházi, ${ }^{13}$ la città di Vienna come luogo prescelto per la propria formazione artistica. Dopo aver passato una difficile giovinezza a Mosca, segnata dalla perdita di un figlio, dal divorzio con il marito e dall'espulsione dall'Accademia di Belle arti di Mosca a causa dell'irriverenza del suo carattere, Therese Feodorowna Ries decise nel 1894 di trasferirsi nella capitale austriaca per affinare le sue doti di scultrice (Johnson 2012, 205). Poiché l'Accademia di belle arti viennese non accettava ancora la

9 Eliza Beetz-Charpentier presentò alla Biennale veneziana, nella V Sala internazionale, tre sculture una targa di bronzo caratterizzata da dei ritratti del marito.

10 Elisa Beetz-Charpentier, Notice Artiste n² 2686, Musée d'Orsay, 2006-2019.

11 Tale ipotesi è stata presentata dalla studiosa Chantal Beauvalot, che ha individuato nelle conoscenze intessute dalla Besnard con gli aristocratici inglesi, l'arma del rapido successo del marito (Moreau-Sionneau 2013, 15).

12 La Besnard organizzò l'Exposition des femmes peintres al Salon du Lyceum di Parigi nel 1908 e fu autrice di articoli dedicati all'insegnamento dell'arte nelle scuole. (Moreau-Sionneau 2013, 13-14).

13 Ilse Twardowsky-Conrat partecipò con un ritratto in marmo alla Sesta Esposizione Internazionale d'Arte del 1905 mentre l'artista Irma von Duczynska vi espose nel 1907 con l'opera in bronzo intitolata Fratelli. L’ungherese Elza Kalmár Kövesházi infine prese parte alla Biennale veneziana del 1910 con tre bronzi dal titolo Fanciullo che danza, Danzatrice greca, Salomè e alle successive edizioni del 1924, 1928 e 1932. 
presenza femminile nei corsi che vi venivano impartiti, ${ }^{14}$ la ventenne Ries convinse lo scultore Edmund von Hellmer a prenderla sotto la sua ala:

he at first demurred, but when he saw a photograph of her Sleepwalker, he gave her studio space at the Academy. (Johnson 2012, 205)

Si evince quindi che le doti innate di Therese dovevano essere notevoli, e che tali capacità le permisero prontamente di raggiungere grande fama e notorietà nell'ambiente viennese. Fanno sorridere a questo proposito le parole dello scrittore Karl Kraus, che, in occasione dell'apertura dell'atelier dell'artista russa al Palazzo Liechtenstein nel 1906, esclamò:

Too much Feodorowna Ries! A windstorm of publicity is blowing the Viennese leaflet-woods. (Johnson 2012, 1)

L'apice del successo era stato tuttavia raggiunto dall'artista anni prima, verso la seconda metà degli anni Novanta dell'Ottocento, quando, dopo una visita al suo studio, fu invitata da Klimt e dagli altri membri della Secessione ad esporre le proprie sculture al Palazzo della Secessione di Vienna (Johnson 2012, 1). ${ }^{15}$

Guardando le opere create dalla Feodorowna si può certamente comprendere la ragione del suo grandioso successo: le sculture , d'impronta rodiniana, colpiscono per la forza che emanano, per l'espressività della materia che le compone e le movimenta. Se un'opera come La strega aveva destato scompiglio e scosso gli animi per la forte caratterizzazione del volto che era molto lontano dal consueto canone di bellezza, i capolavori esposti dall'artista alla Biennale di Venezia affascinano.

L'abbraccio che lega i due innamorati ne Il bacio è appassionato e struggente, l'opera funeraria L'anima ritorna a Dio è invece conturbante. Una figura seminuda con le braccia chiuse sul petto, è accolta fra quelle di una più grande che avvolge il suo volto, nascondendolo alla vista. Ciò che colpisce è la rappresentazione non dettagliata, sfuggente, quasi astratta di Dio e il vortice che si crea nel momento in cui l'uomo e la sua anima ritornano a lui.

14 L'accademia aprì le sue porte alle artiste solo nel 1921. Si noti in questo il forte ritardo rispetto alla Russia (Johnson 2012, 205).

15 Si racconta inoltre che August Rodin «un giorno, a Parigi, dinanzi alle opere della Ries, le strinse la mano, dichiarandole: 'noi siamo parenti'» (Malagola Carlo, «Una scultrice russa. Theresa Feodorowna Ries», Airs et Labor. Musica e musicisti. Rivista mensile illustrate, 61(7), 15 luglio 1906). 
The artist has ingeniously broken with all conventions. All grave angels and urn carriers are set aside. But she has found something powerful to replace these, portraying neither earthly departure nor sorrowful end, but rather a shining new beginning: the union of the soul with God. ${ }^{16}$

Le tracce della scultrice russa iniziano a perdersi durante la fine degli anni Trenta del Novecento, quando lo studio di Therese Feodorowna Ries fu confiscato dai tedeschi e molte delle sue opere furono danneggiate o distrutte durante la guerra. È questo un destino che ha accomunato numerosi artisti in quei momenti difficili. Ne è un esempio la storia della scultrice viennese Ilse Twardowsky-Conrat che, nel 1942, decise di togliersi la vita dopo aver ricevuto un secondo ordine di espulsione. ${ }^{17}$ Colpisce come la versatilità artistica e l'ammirazione che le sue opere avevano suscitato, abbiano potuto essere così bruscamente interrotte dalla sua morte.

La presenza delle artiste americane Eberle Mary Abastenia St. Leger e Bessie Vonnoh Potter, permette infine di volgere uno sguardo anche alla situazione della scultura negli Stati Uniti.

L'America a differenza dell'Europa era un paese in cui fu più facile per le artiste dedicarsi a questo genere d'arte.

In questo settore [...] le donne venivano incoraggiate a diventare scultrici proprio perché la scultura era indicata come un nuovo campo dell'arte americana, non ancora eccessivamente associata ad artisti maschi. (Trasforini 2017, 125)

L'attività di Eberle Mary Abastenia St. Leger si inscrive principalmente nella città di New York, luogo in cui si trasferì nel 1899 e da cui trasse ispirazione per l'iconografia dei propri lavori. L'artista, originaria dell'Iowa, divenne famosa per la creazione di piccole ed eleganti sculture in bronzo cariche di energia e di vita che raffiguravano bambini e donne delle classi sociali meno abbienti, colti nei momenti di vita quotidiana. L'opera Ragazza che pattina del Metropolitan Museum of Art, ne è un esempio di eccezionale valore. Una bambina è fermata nel bronzo nel momento in cui sta sfrecciando spensierata su un paio di pattini. Il vento le smuove i capelli, sottolineando il senso di movimento e di libertà che la scultura emana. L'artista racconta: 
The children of the East Side play without restrains; their griefs and their joys are expressed with absolute abandon... [T] heir natural emotions are not retrained by the pretty curtseys taught by governesses... They laugh loudly. They shout. They race on roller skates and dance unrestrainedly. [...] They are real-real as they can be. They express life. (Marter 2001, 629)

Ragazza che pattina del Metropolitan Museum of Art, presenta il titolo uguale a quello dell'opera che venne esposta da Eberle alla Biennale di Venezia del 1909. Non è tuttavia sicuro che si tratti dello stesso capolavoro poiché l'artista americana realizzò una serie di sei-otto bronzi dedicati al tema della ragazza sui pattini, lavorando a questa iconografia per ben due decenni. ${ }^{18}$

Nel momento in cui Eberle Mary Abastenia St. Leger partecipò alla Biennale veneziana, l'artista aveva raggiunto già una notevole notorietà in America, nonostante le sue opere di critica e di denuncia sociale e «her social radicalism positioned her work outside public taste» (Morgan 2018).

Nel 1906 era stata ammessa alla National Sculpture Society e l'anno successivo si era legata alla Galleria Machbeth, dove tenne numerose esposizioni (Marter 2001, 627). L'artista compì numerosi viaggi in Europa visitando l'Italia e Parigi tra il 1907 e il 1913, come d'abitudine per la formazione artistica delle americane che nel loro paese non potevano trovare capolavori dell'arte che fungessero da modelli di studio e dove mancava soprattutto una «tradizione di insegnamento artistico accademico» (Trasforini 2017, 104). Risale invece al 1895 il soggiorno che Bessie Vonnoh Potter compì nella capitale francese e che significò molto per l'artista americana: qui ella visitò lo studio di August Rodin, le cui opere avevano già largamente influenzato il suo stile di modellazione, in occasione del Chicago World's Fair in cui era esposto il Ritratto di Paul Trubetsoky dello scultore parigino (Morgan 2018).

Bessie Vonnoh Potter condivise con Eberle Mary Abastenia St. Leger una vita ricca di successi e di notorietà. Prima della partecipazione all'Esposizione veneziana, Bessie aveva aperto uno studio a Chicago e aveva vinto la medaglia d'oro al St. Louis World's Fair. Nel 1896, l'Art Institute of Chicago aveva acquistato numerose sue opere che rappresentarono «the first sculptures by a woman in its collection» e nel 1921, Bessie Vonnoh Potter fu accolta e nominata prima scultrice donna membro accademico permanente della National Academy of Design.

18 Altri esemplari dello stesso tema iconografico si trovano nei seguenti musei: Des Moines Art Center; The Museum Of Art, Rhode Island School of Design, Providence; Whitney Museum of American Art, New York (Marter 2001, 629). 
I suoi bronzi rappresentano in maniera delicata e squisita giovani donne avvolte in voluminosi vestiti, eleganti danzatrici e madri che teneramente stringono a sé o sono circondate dai propri figli come si può ammirare nell'opera in bronzo Maternità esposta dalla Vonnoh Potter alla Ottava Biennale veneziana del 1909.

Tali iconografie familiari colpirono la critica e il pubblico per la loro dolcezza e conferirono a Bessie Vonnoh Potter un posto speciale nel panorama artistico americano. Esemplari sono le parole dedicatele da un autore anonimo:

It would be useless and unprofitable to compare Mrs. Vonnoh with other American sculptors, because her work occupies its own special and well-defined place, just as the works of Partridge, SaintGaudens, French, Barnard and MacMonnies have their own respective characteristics sharply drawn and accentuated. In her own field - the field of the statuette - Mrs. Vonnoh has no superiors in this country. ${ }^{19}$

Accomunate da una vicina ricerca artistica e da una comune passione per il bronzo, Eberle Mary Abastenia St. Leger e Bessie Vonnoh Potter, con i loro successi e la propria indipendenza artistica prepararono il terreno per i futuri sviluppi dell'arte scultorea americana al femminile.

\section{Bibliografia}

Altea, Giuliana (2012). Il fantasma del decorativo. Milano: il Saggiatore.

Bartolena, Simona (2003). Arte al Femminile. Donne artiste dal Rinascimento al XXI secolo. Milano: Mondadori Electa.

Corgnati, Martina (2004). Artiste. Dall'Impressionismo al nuovo millennio. Milano: Bruno Mondadori.

Fergonzi, Flavio (1995). «La scultura alla Biennale di Venezia (1895-1948)». Venezia e la Biennale 1995, 103-10.

Harris Sutherland, Ann; Nochlin, Linda (1979). Le grandi pittrici 1550-1950. Milano: Feltrinelli.

Hassler, Donna J.; Marter, Joan M.; Tolles, Thayer (2001). American Sculpture in The Metropolitan Museum of Art. A Catalogue of Works by Artists Born between 1865 and 1885, vol. 2. New York: The Metropolitan Museum of Art.

Johnson, M. Julie (2012). The Memory Factory: The Forgotten Women Artists of Vienna 1900. West Lafayette (IN): Purdue University Press.

Marter, Joan (2001). "Girl Skating, 1906». Hassler, Marter, Tolles 2001, cat. no. 288.

Mauclair, Camille (1914). Albert Besnard. L'homme et l'oeuvre. Parigi: Delagrave.

19 Leininger-Miller Theresa, «Bessie Potter Vonnoh: Sculptor of Women», NineteenthCentury Art Worldwide, 8(2), 2009, https://www.19thc-artworldwide.org/autumn@9/ bessie-potter-vonnoh-sculptor-of-women (2019-07-13). 
Méneux, Catherine; Pernoud, Emmanuel; Wat, Pierre (2013). Actes de la Journée d'études Actualité de la recherche en XIXe siècle, Master 1 (2012-2013). Paris. Pubblicato online sul sito dell'HiCSA nel gennaio 2014.

Moreau-Sionneau, Hélèn (2013). «Charlotte Besnard (1854-1931): être femme sculpteur et épouse d'artiste en vogue, au tournant du XXe siècle». Méneux 2013, 1-18.

Morgan, Ann Lee (2018). The Oxford Dictionary of American Art \& Artists. Oxford: Oxford University Press.

Pajusco, Vittorio (2017). «Bice Levi Minzi (Bice Rossi Minzi) e l'Esposizione Internazionale Femminile di Torino del 1913». Stringa, Nico; Portinari, Stefania (a cura di), Gli artisti di Ca' Pesaro. L'Esposizione d'arte del 1913. Venezia: Edizioni Ca' Foscari, 159-83. DOI http://doi.org/10.14277/6969-197-3/SAC-1-8.

Pancotto, Pier Paolo (2006). Artiste a Roma nella prima metà del '900. Roma: Palombi.

Scotton, Flavia (1995). «Arti applicate: dalla fondazione al Padiglione Venezia». Venezia e la Biennale 1995, 123-8.

Trasforini, Maria Antonietta (2017). Nel segno delle artiste. Donne, professioni d'arte e modernità. Bologna: il Mulino.

Venezia e la Biennale: i percorsi del gusto = Catalogo della mostra (Venezia, Galleria d'Arte Moderna di Ca' Pesaro, 10 giugno-15 ottobre 1995). Milano: Fabbri Editori.

Vergine, Lea (1980). L'altra metà dell'avanguardia 1910-1940. Pittrici e scultrici nei movimenti delle avanguardie storiche. Milano: Mazzotta. 



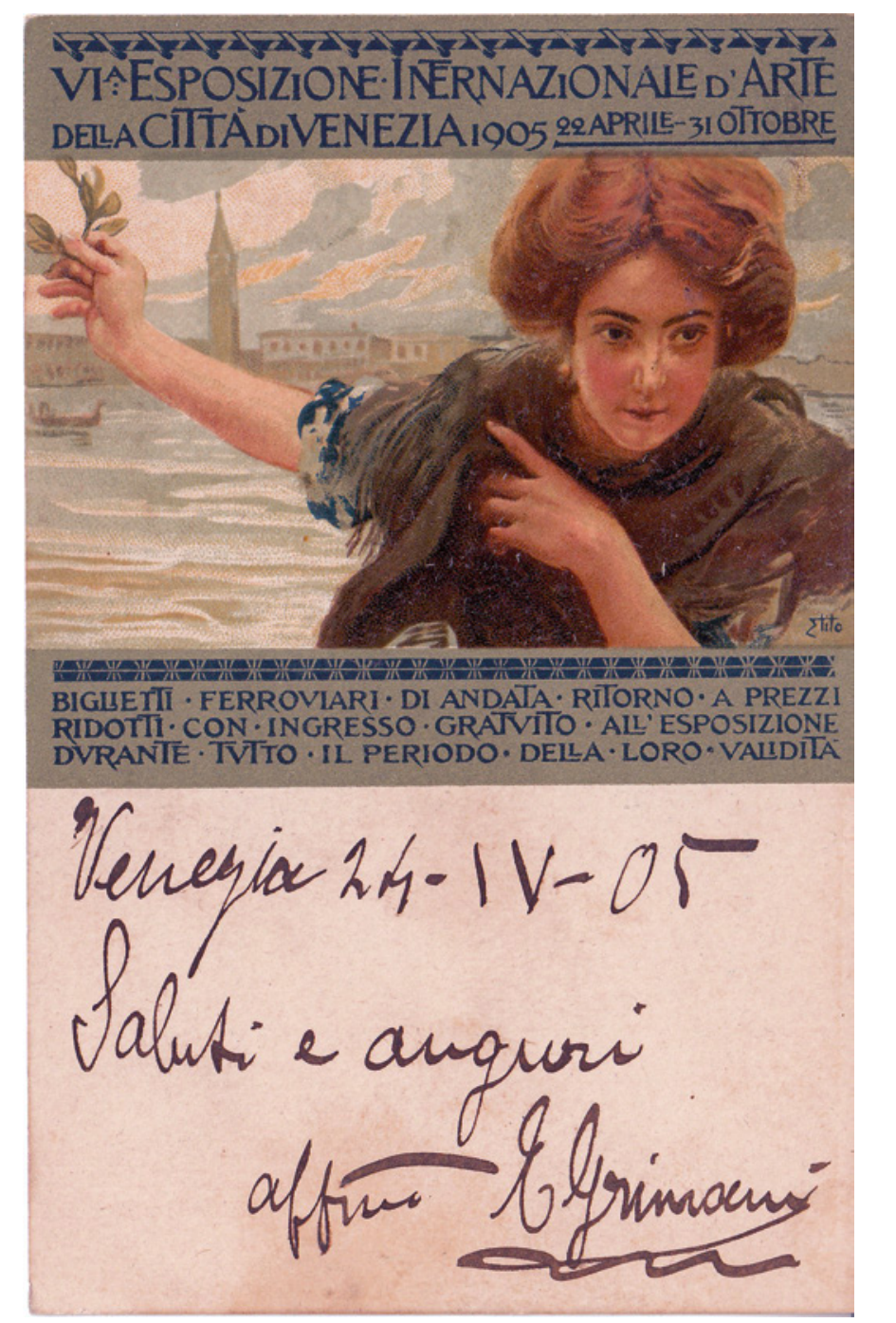

Figura 1 Cartolina della VI Biennale inviata a Lodovico Mocenigo dal sindaco di Venezia
Filippo Grimani, 24 aprile 1905 (illustrazione di Ettore Tito). Collezione Claudio Romeo 


\title{
Gli artisti veneziani alla Biennale (1895-1905) Organizzatori ed espositori: l'antinomia dei ruoli
}

Matilde Ferrarin

Università Ca’ Foscari Venezia, Italia

\begin{abstract}
Venice Biennale, created by the will and the organizational skills of the Venetian artists, was actually managed exclusively by Antonio Fradeletto. The new figure of the "General Secretary" has distinguished for the first time the role of artists, interrupting the tradition of 'self-rule' of painters and sculptors used to managing any artistic event. This essay analyses this important moment of transition, reporting events that took place in its administration during the first years of the Biennale. Through magazines periodicals of the time we report the critical fortune that these works of art had in the local and national press, in an attempt to verify if the creators of the Biennale succeeded in the difficult task of emerging and getting noticed in such a vast and international artistic context.
\end{abstract}

Keywords Venetian artists. Antonio Fradeletto. Venice Biennale. Organization of exhibitions.

Si è soliti far iniziare la storia della Biennale, la più longeva tra le grandi istituzioni espositive, nelle sale del caffè Florian. D’altronde i grandi caffè avevano assunto fin dal secolo precedente un ruolo sociale nuovo: ai loro tavoli sono nati manifesti politici e letterari, sono stati organizzati complotti

Questo saggio prende avvio da Ferrarin, Matilde (2016). Gli artisti veneziani alla Biennale (18951905). Organizzatori ed espositori: l'antinomia dei ruoli [tesi di laurea magistrale]. Relatore Nico Stringa; correlatore Stefania Portinari, a.a. 2015/2016. Venezia: Università Ca' Foscari Venezia; e dalla partecipazione al convegno Storie della Biennale di Venezia, a cura di Nico Stringa e Stefania Portinari (Venezia, Università Ca' Foscari Venezia, Ca' Dolfin, 6-7 dicembre 2016). 
e rivoluzioni, tanto che, come ha affermato Piero Bargellini (1944) «non si potrebbe scrivere una pagina di storia né letteraria né artistica dell'Ottocento senza citare il nome di un caffè». Tuttavia le radici dell'ideazione di un ente come la Biennale e le ragioni profonde della sua realizzazione sono invece connesse a un ambito molto più vasto e complesso: sono ancorate all'ampio progetto politico dell'allora sindaco Riccardo Selvatico, impegnato a rinvigorire la città di Venezia attraverso un programma in gran parte sviluppato su riforme culturali; sono ben fondate nell'ambiente accademico veneziano, formato da docenti e allievi impegnati a portare a Venezia nuovi stimoli, tessendo un fitto groviglio di relazioni con l'estero e con le altre esperienze espositive italiane; sono infine indissolubilmente legate alla figura di Antonio Fradeletto, dibattuto perno attorno al quale ha ruotato l'intero organismo della Biennale fino al primo dopoguerra ${ }^{1}$.

Per poter valutare con chiarezza il ruolo che artisti e personaggi veneziani hanno avuto nell'origine dell'Esposizione Internazionale di Venezia è indispensabile analizzare, senza alcuna esclusione, tutti e tre questi importanti aspetti. La Biennale, fenomeno culturale e di costume spesso considerato un «calderone onnicomprensivo» (Romanelli 1995, 11), viene spesso estrapolato dal suo originale panorama culturale e studiato come un ente svincolato da qualunque legame con la sua città. Bisogna invece ritornare proprio al clima culturale, politico e sociale di Venezia per trovare le reali ragioni della nascita di una così longeva istituzione. Bisogna analizzare la Biennale anche come l'azione di un piccolo gruppo di uomini, accomunati dalla passione per Venezia e protagonisti, in diversa misura, del cambiamento della loro città e di un punto di svolta della storia dell'arte.

Un sindaco-poeta, desideroso di dare un apporto significativo al decollo della città, è il primo protagonista di questa vicenda. Il periodo di gestazione dell'Esposizione Internazionale d'Arte della Città di Venezia risale infatti al mandato del sindaco laico e progressista Riccardo Selvatico nella prima metà degli anni Novanta dell'Ottocento. Commediografo e poeta, prima ancora che politico, Selvatico era stato un intellettuale di spicco nella scena culturale veneziana della seconda metà del XIX secolo e capostipite di una famiglia di artisti (entrambi i figli, Lino e Luigi, sarebbero divenuti noti pittori d'inizio Novecento). E sentì come una missione personale il rilancio culturale ed economico di Venezia e indubbiamente l'ideazione e attuazione dell'Esposizione fu il suo successo più illustre e durevole in entrambi gli ambiti.

Il suo programma progressista era particolarmente articolato e diretto a sostenere lo sviluppo industriale e commerciale della città, includendo la laicizzazione della scuola, la creazione di nuove scuo- 
le professionali, la razionalizzazione del servizio medico e sanitario e la diminuzione del carico tributario gravante sui ceti più poveri. Il progetto dell'Esposizione si dispone in questa complessa e multiforme azione di governo e Riccardo Selvatico motivò così l'idea della mostra: «Che l'Esposizione riesca al duplice scopo di giovare al decoro ed all'incremento dell'arte e di creare ed avviare qui un mercato artistico dal quale la Città può sperare un vantaggio non lieve» (Romanelli 1995, 23). Egli progettava così un piano d'azione che univa la necessità di «far fruttare la storia», come il sindaco stesso auspicava, per rendere cioè la tradizione e il folclore un valore anche economico, a un'intuizione altrettanto acuta, quella per la quale una città come Venezia potesse trasformarsi in un'officina di modernità.

La Biennale era dunque una colonna portante sulla quale poggiava il disegno di Selvatico di far emergere le potenzialità culturali di una nuova Venezia.

È altrettanto fondamentale far emergere l'importanza del ruolo che gli artisti veneziani, docenti all'Accademia di Belle Arti o comunque vicini ad essa, hanno avuto nella pianificazione e poi nell'iniziale amministrazione della nuova esposizione. Sono i veneziani, pittori e scultori dell'Accademia di Belle Arti, docenti e allievi, personaggi appartenenti alla Società Promotrice e al Circolo Artistico, i veri protagonisti della storia della Biennale alle sue origini, coinvolti in diversa misura nella nascita di un'idea, nella sua attuazione, nella gestione e nell'amministrazione di un ente destinato in poco tempo a crescere. Possiamo dunque ritornare tra le sontuose stanze del caffè Florian, in piazza San Marco, dove effettivamente artisti, docenti, intellettuali e personalità di spicco dell'ambiente culturale veneziano si recavano per trascorrere qualche ora dopo gli studi, gli impegni amministrativi, le conferenze e le lezioni.

Riccardo Selvatico era l'esponente più autorevole di quel piccolo movimento creativo e discuteva delle sue idee progressiste ai tavolini del caffè con gli artisti Mario De Maria, Alessandro Zezzos, Bartolomeo Bezzi, Emilio Marsili e Augusto Sezanne. Le occasionali riunioni diventarono in poco tempo organizzate e attive e al piccolo manipolo del caffè Florian si aggregarono parecchi altri tra gli artisti veneziani più accreditati e di maggiore fama, tra cui Luigi Nono, Guglielmo Ciardi, Cesare Laurenti, Antonio Dal Zotto (Stella 1912, 15), un gruppo accomunato dalla passione per l'arte e l'amore per una città che a oltre un secolo dalla fine della Serenissima era ancora alla ricerca di un'identità, di un nuovo primato che non poteva più essere né economico, né politico, né militare. Ma quando l'idea originaria cominciò a prendere sempre di più una forma concreta, l'amministrazione comunale si impegnò a fare in modo che pittori e scultori veneziani fossero relegati a ruoli marginali e a rendere la Biennale per quanto possibile autonoma dalla loro gestione, distinguendosi così definitivamente dalle esperienze estere (Stringa 2008). 
Fu Antonio Fradeletto, professore di letteratura che sporadicamente aveva partecipato all'iniziale scambio di idee e alla nascita del progetto, a prendere in mano la gestione dell'ente. Ciò che sicuramente sorprende, e che rende la sua figura discussa e studiata, è la sua provenienza da un contesto di studi e una preparazione professionale estranei al mondo dell'arte. Docente di lingua e letteratura italiana e di storia alla Regia Scuola Superiore di Commercio in Venezia (1881-1930), Fradeletto non aveva compiuto studi artistici, non si occupava di arte e di rado era stato coinvolto nell'ambiente dell'Accademia veneziana di Belle Arti. Eppure, nonostante questa iniziale estraneità al mondo artistico, era stata unanimemente considerata la persona in grado di assume tutte le responsabilità della nascente Esposizione Internazionale.

Fradeletto si era dedicato nella prima giovinezza agli studi classici, per poi iscriversi alla facoltà di Lettere e Filosofia all'Università di Padova. Agli anni degli studi universitari risale il primo cimento nell'attività sulla quale avrebbe costruito, negli anni a venire, la propria reputazione: le conferenze. Conseguita brillantemente la laurea (1880), dopo pochi giorni dalla proclamazione aveva già ottenuto la cattedra di lettere italiane presso la Regia Scuola Superiore di Commercio di Venezia, sua città natale. Fradeletto fu in grado fin da subito di sfruttare la sua figura professionale di docente e si fece ben presto largo nell'ambiente culturale veneziano. Grazie alla sua rinomata abilità oratoria, infatti, divenne in breve tempo uno dei maggiori conferenzieri italiani, molto richiesto nei circoli letterari, nelle accademie e nei teatri. Ma Fradeletto era coinvolto anche nell'educazione e nell'istruzione popolare: si prodigava a favore dell'insegnamento elementare e medio, delle palestre marziali, della società di tiro a segno, e la sua presenza diveniva a poco a poco costante anche all'interno dei luoghi minori di fruizione culturale.

Docente, organizzatore culturale e conferenziere di successo, Fradeletto era unanimemente riconosciuto come un factotum sullo scenario della cultura veneziana (Ceschin 2001, 106). Indispensabile era stato anche un ingegnoso intreccio di conoscenze importanti, ed in particolare il sodalizio con Riccardo Selvatico che, vedendo aumentare l'impegno organizzativo della nascente Biennale, lo nominò al suo fianco, come segretario generale dell'Esposizione. Le sue qualità di mediatore e di coordinatore, la sua attenzione agli stimoli degli ambienti culturali vicini ed esteri compensarono le scarse competenze in ambito prettamente artistico. Nonostante le polemiche, inevitabilmente presenti durante tutto il suo mandato, come scrive Romolo Bazzoni, «egli s'insediò nella saletta della biblioteca del Comune, poco discosta dal gabinetto del Sindaco, che era divenuto anche l'Ufficio di Presidenza» (Bazzoni 1962, 22), e vi rimase per undici biennali, fino al 1914. 
Fradeletto, con un rigore e un'irremovibilità da uomo di Stato (sarebbe stato deputato a Roma dal 1900 al 1920) non si sarebbe lasciato intimidire dai numerosi attacchi, vedendo in essi il più delle volte delle palesi strumentalizzazioni, e avrebbe attraversato a testa alta un ventennio cruciale nella storia della cultura, certo non solo veneziana. Se lo storico veneziano Mario Isnenghi (1986) lo ha definito un «funzionario della modernità», come scrive Alessandro Stella tracciando la storia dei primi anni di esistenza dell'ente:

senza l'intelligente autocrazia di Fradeletto che impedì l'imperversare delle forze distruttrici, e seppe cedere e resistere a tempo e luogo, l'Esposizione avrebbe avuto l'immatura fine di molte a noi note istituzioni artistiche italiane. (Stella 1912, 11)

Nei tumultuosi dieci anni a cavallo tra i due secoli, nel delicato momento di avvio della Biennale, le prime proposte su come organizzarla vennero proprio dagli artisti veneziani, in particolare dall'emulazione di alcune loro esperienze pregresse.

Un modello diretto fu in particolare il Verein bildender Künstler Münchens (Unione degli artisti di Monaco), più nota con il nome di Secession (Secessione) e le sue esposizioni d'arte che si tenevano dal 1893. ${ }^{2}$ Queste esposizioni esclusive, piccole ma raffinate, che si tenevano in un edificio appositamente realizzato, riscuotevano un grande successo in termini economici (May 2011). Gran parte degli artisti veneziani coinvolti nella nascita della Biennale avevano partecipato e sperimentato direttamente lo stimolante contesto delle esposizioni internazionali, entrando in contatto anche con quelle di Parigi, Dresda, Berlino, Vienna e Budapest. In particolare Bartolomeo Bezzi, che fin dal 1883 partecipava regolarmente alle mostre di Monaco, aveva espresso l'importanza di sviluppare a Venezia una politica artistica di respiro internazionale, e di operare affinché anche l'Italia mettesse in atto tutte le tattiche necessarie a consentire all'arte nazionale di disporsi in relazione con quella straniera e di trarne beneficio, portando avanti il proposito di «giovare al decoro e all'incremento dell'arte e di creare un mercato artistico dal quale la città potesse ricavare un non lieve vantaggio». ${ }^{3}$

Una volta presa la decisione di rendere la mostra di carattere internazionale, si movimentarono con grande impegno molti altri artisti veneziani, che provvidero a stabilire i contatti con i colleghi

2 Che la Secessione di Monaco sia il principale modello di riferimento della Biennale lo afferma anche P.G. Molmenti nel discorso conclusivo della manifestazione, il 3 novembre 1895: «La chiusura della Internazionale», Gazzetta di Venezia, 4 novembre 1895 3 ASAC, FS, AV, Scatole nere 001, 1894-1895: «Lavoro preliminare per le Esposizioni biennali veneziane». 
d'oltralpe sfruttando le relazioni di amicizia e di collaborazione intrecciate in tanti anni di mostre all'estero (Stringa 2008). Come recita l'introduzione della Prima Esposizione Internazionale d'Arte della Città di Venezia, del 1895:

Una Mostra internazionale dovrà attivare maggiormente il pubblico con la fama degli illustri stranieri che vi concorreranno, porgerà a tutti gli intelligenti che non sono in grado d'intraprendere lunghi viaggi il modo di conoscere e paragonare gli indirizzi estetici più diversi, e arricchirà il patrimonio intellettuale dei giovani artisti paesani, i quali dall'opera dei loro confratelli d'altre nazioni si sentiranno tratti a concepimenti più larghi. (10)

Ma quando l'idea originaria cominciò a prendere sempre più una forma concreta, il segretario Fradeletto e l'Amministrazione comunale si impegnarono a rendere la Biennale per quanto possibile autonoma dalla gestione degli artisti locali. La Presidenza decise di neutralizzare gli artisti locali riunendoli in un piccolo consiglio interno: il Comitato Ordinatore. Tale rappresentanza venne mantenuta esclusivamente nelle mani degli artisti veneziani (anche se in modo travagliato) per tutti i primi dieci anni di vita della Biennale, ma continuerà a lamentare la totale mancanza di potere decisionale all'interno della gestione della mostra.

In effetti il Comitato Ordinatore, che avrebbe dovuto fungere soprattutto da consiglio interno in grado di formulare nuove proposte e suggerimenti per l'ordinamento, finì per svolgere esclusivamente il compito di collocare le opere nelle sale. In pratica, Antonio Fradeletto e Riccardo Selvatico caricarono sulle proprio spalle tutto l'onere della grande impresa.

L'edizione del 1897 vide però un considerevole cambiamento ai vertici dell'amministrazione dell'Ente: la presidenza della Seconda Esposizione Internazionale d'Arte venne infatti assunta da Filippo Grimani, eletto nuovo sindaco di Venezia proprio nell'estate del 1895, quando la Prima Biennale era ancora in corso. L'uscita di scena della giunta ispiratrice della manifestazione e la salita in carica di quella conservatrice guidata dal conte Grimani fu certamente uno dei momenti più delicati per la vita della neonata Biennale.

Il buon esito di tale cambiamento fu dovuto certamente al fatto che il sindaco e la sua Giunta, approdati al comando di una Biennale così ben avviata e comprendendo che il cambiamento ai vertici dell'amministrazione stava ponendo l'ente in una posizione pericolosa, non si arrischiarono a proporre alcuna sostanziale modifica al regolamento e alla composizione dei Comitati, almeno per quel primo anno. Approfittarono anzi dell'esperienza di Antonio Fradeletto, che nella precedente edizione si era dimostrato abilissimo organizzatore di ogni aspetto della mostra e mantennero la presenza degli artisti venezia- 


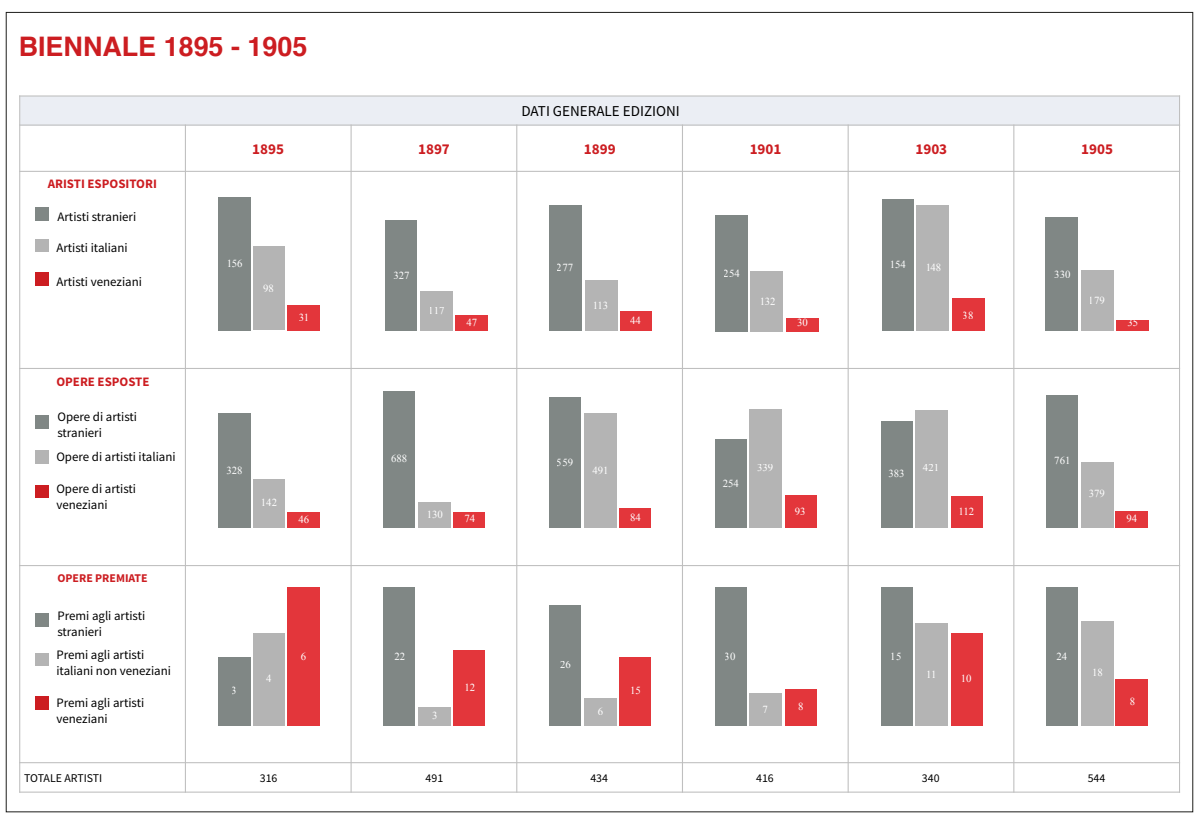

ni, preziosa garanzia di continuità con la prima vincente edizione. ${ }^{4}$

Possiamo presupporre che nell'amministrazione della Seconda edizione della Biennale veneziana sia intercorso tra gli artisti, la Presidenza e la Segreteria un rapporto pressoché stabile ed improntato, almeno in apparenza, ad un generale equilibrio. Eppure risulta evidente che tra gli artisti si stava progressivamente insinuando un sotterraneo clima di fermento, provato dal fatto che a Venezia, proprio nel 1897, venne fondata la Gazzetta degli Artisti, un giornale che si proponeva di difendere gli interessi degli artisti. La nascita di un'iniziativa di questo tipo testimoniava l'esigenza degli artisti locali di ritornare a porsi come soggetto della gestione dell'arte, rivendicando un'autorità che evidentemente all'interno degli uffici amministrativi stavano perdendo. Qualunque tipo di malcontento e di polemica riguardante il mondo dell'arte diventava così pubblico e veniva diffuso su scala nazionale per essere condiviso e valutato dagli artisti di tutta Italia.

La definitiva dimostrazione che una certa vena di insoddisfazione non doveva essere mancata tra i membri del Comitato Ordinatore, viene dal fatto che, proprio nell'arco di tempo che intercorse tra

4 Cf. Alessandro Stella, «Una nuova Esposizione», Gazzetta degli Artisti, 7 gennaio 1897, 25 nota 6: «Vi è soltanto una vera unanimità, nel volere conservata ai successi della prossima Esposizione la Segreteria, a cui sta a capo l'egregio prof. Antonio Fradeletto». 
la fine della Seconda Esposizione e l'inizio della Terza, essi furono tra i principali promotori di un'iniziativa che si rivelò un 'passo falso', una mossa azzardata che comprometterà irrimediabilmente il loro ruolo amministrativo all'interno dell'Ente. Si tratta dell'episodio della Corporazione dei pittori e scultori italiani, ${ }^{5}$ nata dall'operosità di artisti «allo scopo di dare maggiore impulso al movimento artistico d'Italia e tener alto il decoro delle patrie tradizioni ${ }^{6}$ quindi di intervenire e intromettersi nella gestione pratica delle esposizioni. L'aggregazione, sviluppatasi come gruppo italiano, era nata in realtà a Venezia, proprio da alcuni membri del Comitato Ordinatore della Biennale. Come riporta Alessandro Stella:

Si è ufficialmente costituita con proprio Statuto la Corporazione dei pittori e degli scultori italiani. Essa comprenderà i nomi migliori dell'arte nazionale. Ė retta da un consiglio centrale, residente a Venezia e composto per la prima gestione dai pittori Bezzi, De Maria, Tito. Ha delegati nelle varie regioni italiane e all'estero. $(1912,37)$

Dunque, se ci eravamo limitati a supporre una situazione di inappagamento e irrequietezza da parte degli artisti veneziani, la fondazione dell'associazione corporativa ci ha fornito la prova definitiva che il malcontento si basava sull'effettiva consapevolezza da parte degli artisti del loro ruolo secondario e marginale. Gli artisti aderenti alla Corporazione agivano con la formula di un vero e proprio 'ricatto' delle istituzioni: essi avrebbero acconsentito a esporre i propri lavori a condizione che gli organismi deputati alla gestione delle opere accontentassero le loro richieste. In caso contrario, la Corporazione si sarebbe astenuta in blocco, privando la nazione delle più alte rappresentanze artistiche allora militanti in Italia. I vertici dell'Esposizione furono così costretti a riservare al sodalizio uno spazio espositivo dedicato. Questa concessione scatenò le polemiche della stampa e degli altri artisti italiani, che accusarono la Presidenza di aver mostrato in qualche modo una certa predisposizione verso i corporati. Causa di polemica era anche il fatto che ben cinque membri del Comitato Ordinatore erano anche dei corporati, una situazione che poteva far sorgere sospetti intorno alle accettazioni delle opere. ${ }^{7} \mathrm{Ne}$ seguirono le forzate e inevitabili dimissioni dal Comitato Ordinato-

5 La vicenda della Corporazione rimane ancora poco nota: una fonte attendibile è l'articolo «L'arte italiana e la Corporazione degli artisti», Nuova Antologia, marzo 1899, 146-66.

6 Cf. Gazzetta degli Artisti, 7 gennaio 1899, 3.

7 Cf. «Per l'arte soltanto», Marzocco, 29 gennaio 1899: «Si può dare molto da arzigogolare ai maligni quando si sa che la grande maggioranza degli artisti membri del Comitato Ordinatore dell'Esposizione appartiene alla Corporazione, che questa è sorta appunto l'anno in cui sono tolti i premi e quelle somme sono destinate agli acquisti [...] 
re dei membri appartenenti alla Corporazione: Pietro Fragiacomo, Antonio Rotta, Vincenzo De Stefani, Emilio Marsili, Bartolomeo Bezzi e Guglielmo Ciardi.

Antonio Fradeletto riconobbe nelle agitazioni del 1899 la perfetta occasione per prendere definitivamente le redini della Biennale. Dopo l'allontanamento nel 1897 di Riccardo Selvatico e Giovanni Bordiga, l'eliminazione del Comitato Ordinatore rendeva ora il segretario generale libero di agire indisturbato. Questa repentina presa di potere venne unanimemente riconosciuta come un glorioso atto di salvataggio, senza il quale la Biennale probabilmente non avrebbe superato queste prime tumultuose edizioni di fine secolo. Ancora Stella riporta:

Fradeletto in un'occasione determinata dalla imprudente condotta degli artisti, non si peritò di ricorrere ad un vero colpo di stato facendo onore al principio che una autocrazia intelligente è preferibile ad ogni altra specie di governo, quando i popoli non sanno giovarsi onestamente della libertà. La riforma audace è consistita nella soppressione legittimata del comitato e ordinatore nell'accentramento dei poteri nella presidenza, composta dal conte Filippo Grimani ed Antonio Fradeletto segretario. $(1912,35)$

Se i qui pro quo legati al complotto della Corporazione avevano incrinato i rapporti con gli organizzatori della mostra, quel che avvenne durante l'apertura dell'Esposizione del 1901 ebbe l'effetto per alcuni di loro di una vera e propria cesura. Un gruppo di artisti si stava infatti organizzando la sotterranea promozione di una grande esposizione d'arte internazionale a Milano per il 1904. I sostenitori della Biennale gridarono ovviamente allo scandalo, e si scagliarono contro la minaccia di una concorrenza all'Esposizione di Venezia.

Il primato organizzativo della Biennale fu insidiato anche da altre città italiane desiderose di offrire ospitalità ad una mostra d'arte importante tra le quali Firenze, che aveva cominciato ad invitare gli artisti stranieri che notoriamente esponevano a Venezia e perfino alcuni membri veneziani dei Comitati. Fradeletto riuscì a dissuadere i componenti del Comitato Ordinatore che verso Venezia avevano un debito morale, ma quel che è certo è che da quel momento in poi per alcuni artisti veneziani le porte degli uffici della Biennale si chiusero definitivamente. Con la consueta maschera di difensore dell'Istituzione veneziana poté così allontanare con una scusa quanto mai valida Bartolomeo Bezzi, uno degli artisti che avevano dato origine alla Biennale, partecipe alla creazione della mostra fin dagli incon-

e che molti appartenenti alla Corporazione e membri del Comitato hanno quasi nelle mani le sorti dell'Esposizione». 
tri al caffè Florian e membro del Comitato tra i più attivi e interessati. ${ }^{8}$ Si noterà l'assenza nei successivi Comitati anche di De Stefani, Fragiacomo e Marsili, storici membri e fondatori della Biennale.

Il caso della partecipazione degli artisti veneziani all'organizzazione di mostre ed esposizioni 'concorrenti' costituiva un chiaro segnale dell'insofferenza rispetto all'egemonia della Biennale e alle chiusure dell'ambiente presidenziale. Consapevoli che il loro ruolo all'interno della gestione dell'Ente veneziano si stava facendo sempre più marginale, gli artisti evidentemente sentirono il bisogno di cercare altrove una mostra che potesse accogliere le loro capacità gestionali. Nell'edizione successiva, con la morte dell'ideatore dell'Esposizione, Riccardo Selvatico, avvenuta nell'agosto del 1901, e con l'allontanamento di molti membri del Comitato Ordinatore, Fradeletto rimarrà l'unico superstite della Prima edizione.

La Quinta Biennale non risultò meno burrascosa: lo scontento nato dal verdetto di selezione, che escludeva 823 opere su 963, suscitò una controversia quanto mai accesa, in parte per la numerosità delle opere rifiutate, in parte per la rilevanza degli artisti esclusi. L'ostinazione delle polemiche costrinse Fradeletto a inventare un nuovo articolo del regolamento e a incaricare il Comitato Ordinatore di scegliere le migliori opere escluse per allestire una Sala dei Rifiutati. Nell'analizzare e confrontare i documenti riguardanti il verdetto della Giuria e la Sala dei Rifiutati è emerso un dato chiarificatore: la Biennale del 1903 si trovò a chiudere le porte a una corposa percentuale di opere di artisti veneziani. Tale percentuale di veneziani annoverava i nomi di Italico Brass, Millo Bortoluzzi, Felice Castegnaro, Giuseppe Ciardi, Trajano Chitarin, Guido Maria Stella, Emanuele Brugnoli, Eugenio de Blaas, Giuseppe Duodo, Emo Mazzetti, San Fior Silvio Giovanni, Romolo Tessari, Mario Zoppellari ed altri. Insomma, alcuni degli artisti che avevano ispirato, spronato e sostenuto la nascita dell'Esposizione nella propria città si trovavano ora, a causa di un regolamento eccessivamente rigido, esclusi dalla stessa. ${ }^{9} \mathrm{La}$ Sa-

8 Bartolomeo Bezzi partecipa all’Esposizione di Milano. Cf. «I dolori del Signor Bezzi», Gazzetta degli Artisti, 3 aprile 1901, 33: "Se Milano vorrà la sua Internazionale, sarà al Signor Bartolomeo Bezzi, proprio a lui, membro del Comitato di Venezia, che si dovrà la noja delle maggiori difficoltà. [...] Si aspettava forse dai suoi colleghi e dai Veneziani un indirizzo di felicitazioni?!!! Se trasportando le sue tende a Milano dimenticò gli obblighi che lo legavano a Venezia, per quanto gli sia amaro, mandi giù nello stomaco il giudizio. Si faccia o non si faccia l'Internazionale di Milano, non importa. Resta assodato che il Signor Bezzi andò in quella città per fare proseliti ad un'idea che lo mise in conflitto coi doveri della sua posizione a Venezia!»

9 Cf. «Tribuna degli Artisti», Gazzetta degli Artisti, 2 maggio 1903, 82. Una lettera anonima inviata alla redazione dimostra il risentimento degli artisti veneziani: «Oh amico mio, oh nobile e grande Riccardo, se tu vedessi a che hanno ridotto l'opera tua! I nostri non sono odi, non sono asti. È la coscienza del nostro valore, o, se non volete, delle nobili fatiche, che ci fa parlare; è l'anima duramente offesa che ci obbliga a protestare. Un vecchio pittore veneziano che non si firma per non venire anche seppellito!». 
la $\mathrm{K}$, dove questi artisti esclusi furono invitati a esporre le loro opere, doveva esprimere proprio il tentativo di Fradeletto di recuperare dall'interno la scomoda situazione di quell'anno e dimostrava che tra le sue preoccupazioni era comunque presente quella di mantenere il delicato equilibrio con la componente veneziana dell'Esposizione.

Chiusa la Quinta Esposizione, gli artisti veneziani desiderosi di far valere le loro esigenze decisero di unirsi e di scendere in campo. Si generò in breve tempo una situazione di tensione che non sfuggì ai commentatori dell'epoca, come riporta un articolo su Il Marzocco che immagina cosa avrebbe potuto succedere se si fosse dato corso alla rabbia dei rifiutati:

Chi fosse venuto ne avrebbe viste delle belle. Per lo meno la Bastiglia, cioè l'Esposizione, incendiata, rasa al suolo, senza più pietra su pietra, anzi, gesso su gesso, e i membri delle varie giurie, con a capo l'eloquente segretario, l'onorevole Fradeletto, trascinati captivi, per essere poi gettati ad bestias. ${ }^{10}$

In seguito ad un'assemblea gli artisti locali stesero un violento ordine del giorno, tendente a ridare ad essi una più ampia responsabilità nell'ordinamento della mostra. Si trattava di un vero e proprio attacco frontale alla Segreteria condotto da coloro che, per periodi più o meno lunghi, avevano fatto parte dell'entourage della Biennale: Emilio Marsili, Guglielmo e Beppe Ciardi, Alessandro Zezzos, Mario De Maria, Urbano Nono, Giuseppe Vizzotto Alberti tra gli altri. L'ordine del giorno venne comunicato al presidente dell'Esposizione e pubblicato sui giornali. La Presidenza rispose con una pubblica denuncia verso l'ambiguità e l'ipocrisia degli artisti veneziani i quali, pur opponendosi con malignità all'ordinamento dell'Esposizione, non rinunciavano a esporvi le loro opere. Ma gli artisti proseguirono con accanimento la protesta, diramando un opuscolo di sedici pagine intitolato Per una diretta partecipazione degli Artisti all'ordinamento delle Esposizioni Internazionali d'Arte della Città di Venezia. ${ }^{11}$ Essi speravano soprattutto che la diffusione di tale fascicolo potesse aiutarli a raccogliere il consenso e il supporto degli altri artisti italiani, rendendoli partecipi di un problema che avrebbe dovuto interessare l'intera comunità artistica. Tuttavia l'opuscolo, e con esso le preoccupazioni degli artisti veneziani, non ebbe la risonanza auspicata e le proteste rimasero circoscritte alla città lagunare. Risulta

10 Cf. Mario Morasso, «L'Esposizione di Venezia e gli artisti. Il dissidio e le cause», Il Marzocco, 29 maggio 1904.

11 L'opuscolo venne sottoscritto da Eugenio de Blaas, Millo Bortoluzzi, Trajano Chitarin, Guglielmo Ciardi, Zaccaria Dal Bò, Marius De Maria, Vincenzo De' Stefani, Roberto Ferruzzi, Emilio Marsili, Luigi Mion, Luigi Rosa, Raffaele Tafuri, Guglielmo Talamini, Giuseppe Vizzotto-Alberti, Alessandro Zezzos. 
dunque evidente che il comunicato stesse cercando di diffondere il malcontento di un ristretto numero di artisti locali, che si proclamavano «gli ideatori e iniziatori» di un'impresa di cui avrebbero voluto essere «i veri continuatori»:

Accadde purtroppo che la collaborazione degli artisti, così larga ed efficace in principio, andò restringendosi ad un piccolo numero di amici consiglieri della Presidenza. Ed anzi, a parlare più esatto, una sola volontà direttrice e moderatrice finì col concentrare in sé - troppo autocraticamente - ogni potere ed ogni deliberazione. (Per una diretta partecipazione 1904, 10)

Queste parole sono chiarificatrici della situazione di progressiva emarginazione degli artisti veneziani. Come diretta conseguenza di questa tensione, per la Biennale del 1905 la Presidenza decise di togliere agli artisti veneziani l'importanza di appartenere ad un Comitato esclusivo e li fece confluire in un unico grande Consiglio nel quale gli artisti stranieri avevano il compito di diramare gli inviti all'estero, mentre gli italiani si occupavano di convocare gli artisti delle singole regioni. Il compito del collocamento delle opere nelle sale internazionali fu quindi tolto agli artisti veneziani e affidato ad un nuovissimo Comitato di Collocamento. Questo rivoluzionario provvedimento privò definitivamente gli artisti veneziani della loro posizione di superiorità e distinzione e li mise finalmente allo stesso livello di tutti gli altri artisti italiani e stranieri partecipanti all'amministrazione dell'Esposizione. Il 1905 esercitò una vera e propria cesura: non accadde più, nel futuro della Biennale, che agli artisti veneziani venisse concesso uno spazio così rilevante e disgiunto.

Possiamo concludere dunque, al termine di questa analisi della gestione organizzativa delle prime sei Esposizioni, che proprio con la Biennale si è delineata e ha preso forma una specifica e peculiare figura dell'ambito artistico, quella dell'organizzatore dell'evento. Mentre le esposizioni precedenti in Italia e all'estero erano state nelle mani di comitati artistici che conducevano le iniziative, a Venezia, con Antonio Fradeletto, era nata la figura del segretario generale, che assumeva un ruolo dominante nell'organizzazione complessiva delle mostre. Tale figura non si limitava a coordinare la complessa trama organizzativa, ma imponeva le sue decisioni, curava personalmente la corrispondenza con artisti e istituzioni, studiava i rapporti tra invitati e sottoposti a giuria, orientava le selezioni, i premi e gli acquisti. La figura del segretario generale distinse per la prima volta il ruolo degli artisti da quello degli esperti, interrompendo la tradizione di 'autogoverno' dei pittori e degli scultori abituati a ideare, preparare e gestire qualunque evento artistico. Proviamo ora ad osservare l'iter parallelo degli artisti espositori. Tale percorso è complesso e comunque meno lineare di quanto possa ap- 
parire scorrendo i cataloghi dell'Esposizione e affidandosi ad alcuni luoghi comuni sulla pittura locale. In particolare poniamo l'attenzione sui delicati equilibri che hanno caratterizzato la presenza degli artisti veneziani in queste prime esposizioni, individuando i tentativi della Presidenza di concedere largo spazio ai pittori locali senza attirare accuse di favoritismi. In effetti già durante la stipulazione di un regolamento si era compresa la necessità di rendere chiaro il rapporto che gli artisti locali dovevano avere con l'Esposizione, per evitare ogni insinuazione di vantaggio. E così il regolamento, riportato nelle prime pagine del catalogo, recitava al punto cinque: «per un sentimento che sarà facilmente apprezzato, il comitato Ordinatore si astiene dal rivolgere speciale invito agli artisti veneziani, veneti, o italiani dimoranti a Venezia, i quali dovranno pertanto assoggettarsi al verdetto della giuria di accettazione» (Biennale 1 1895, 4). Tale regola di certo sembrava svantaggiare gli artisti veneziani, come anche il punto sette del regolamento, che impediva di accogliere alla mostra opere esposte in Italia e che in pratica favoriva gli artisti stranieri rispetto agli italiani.

Nonostante l'apparente imparzialità, bisogna riconoscere che gli artisti veneziani risultarono in fin dei conti addirittura privilegiati sia a livello di accettazione delle opere che a livello delle vendite. Certamente, un dato sicuro è che per tutte e sei le edizioni prese in esame gli artisti veneziani sono risultati in numero nettamente superiore rispetto a tutte le altre rappresentanze italiane e straniere. Dalla Prima edizione fino alla Sesta la critica si è lamentata del fatto che, passeggiando tra le sale dell'Esposizione, la presenza degli artisti veneziani risultasse troppo incombente. Insomma, per ciascuna Biennale, gli articoli, i saggi e i commenti della stampa hanno fatto emergere una costante polemica sulla sbilanciata percentuale di veneziani che occupava le sale dell'Esposizione. Anche quando nel $1901 \mathrm{fu}$ istituita la suddivisione in sale regionali per garantire maggiore equità e una distribuzione più omogenea, essi finirono per occupare due sale, mentre le altre regioni soltanto una.

Un'accurata analisi dei dati ci permette di analizzare anche il percorso altalenante della distribuzione dei premi, degli acquisti e delle medaglie agli artisti locali. Possiamo individuare anche in questo caso un indubbio vantaggio degli artisti veneziani ai quali, nelle prime edizioni, erano perfino riservati alcuni premi. Nel 1899 gli artisti locali premiati in denaro o tramite acquisti furono addirittura dodici, contro i soli tre artisti italiani non veneziani.

Anche in questo caso, la svolta avvenne nel 1901: la situazione di quell'edizione venne disciplinata da una nuova presenza nella Giuria di Premiazione, Antonio Fradeletto, il quale, riuscito finalmente ad entrare anche nell'ambito della premiazione, deve aver imposto un criterio più dosato di distribuzione dei premi, di nuovo nel tentativo di confutare le voci che da tempo lamentavano una predisposizione per gli artisti veneti. 
Se si cerca di individuare le tendenze stilistiche, le influenze e gli scambi intercorsi all'interno della Biennale, dall'esame delle opere e della relativa documentazione, così come della critica coeva, è emerso che l'iter di buona parte degli artisti veneziani, che hanno vissuto e lavorato a cavallo dei due secoli, è stato quasi sempre movimentato da articolati rapporti: anche quando gli artisti non ebbero occasione di uscire dall'area triveneta, vedendo in loco l'arte degli altri Paesi poterono arricchire le loro conoscenze visitando la Biennale che fin dalle prime edizioni si prefiggeva di offrire «a tutti gli intelligenti che non sono in grado di intraprendere lunghi viaggi, il modo di conoscere e di paragonare gli indirizzi estetici più diversi» e di far sì che «i giovani artisti paesani, dall'opera di loro confratelli d'altre nazioni», si sentissero «tratti a concepimenti più larghi» (Biennale 1 1895, 10). Infatti, se bisogna ammettere che la Biennale fu in larga misura una passerella di molta arte accademica e ufficiale, non bisogna però generalizzare e occorre invece riconoscere che il rapporto tradizione-avanguardia vi giocò in maniera non univoca, con molte, anche se caute, aperture al rinnovamento. Considerando quindi che le Biennali erano iniziate nella seconda metà degli anni Novanta, cerchiamo di individuare come i pittori veneziani si siano rapportati alle tendenze prevalenti in quello scorcio di secolo: il postimpressionismo, il simbolismo e, per quanto riguarda le arti decorative, l'Art nouveau. Il nostro esame dei primi dieci anni della Biennale rivela numerosi tentativi di rinnovamento linguistico e di allineamento con le menzionate correnti europee sia da parte di alcuni artisti legati all'ufficialità e all'accademia, sia da parte dei più aperti e rivoluzionari.

La Prima Biennale fu dominata dal gusto tedesco e da quello inglese, con propensioni per il simbolismo. Le opere degli artisti veneziani oscillavano tutte tra il realismo prevalente e l'esordiente simbolismo (cf. Pica 1909, 163). Esemplificativi della prima tendenza da un lato Morocomio (1895) di Silvio Rotta, ancora impegnato sul fronte di un realismo sociale che descriveva con lucidità critica le condizioni delle classi più disagiate e delle istituzioni contemporanee, l'opera di Vettore Antonio Cargnel, Averte Faciem Tuam a peccati meis (1895), sempre di impronta realistica e Alessandro Milesi con Fabbricatori di penitenze (1895), che volle commuovere il pubblico rappresentando la miseria di alcuni operai condannati a un lavoro faticoso e degradante: fabbricare corone di penitenza. ${ }^{12}$ Dall'altro lato Un saluto (1895) di Pietro Fragiacomo, un paesaggio sentimentale intonato alla malinconia, preannunciava il 'paesaggio-stato d'animo' caro alla stagione simbolista. Anche il dittico di Laurenti La Parabola

12 Cf. Pompeo Dini, «L'Esposizione Internazionale di Belle Arti in Venezia», Natura ed Arte, 10 maggio 1895, 268. 
(1895) si dimostrava tipico della tendenza a conferire valori simbolici a una pittura prevalentemente descrittiva. ${ }^{13}$

Due anni dopo i buoni auspici con i quali si era conclusa la Prima Biennale si erano avverati: quasi tutti gli artisti locali dimostrarono di aver approfittato dell'insegnamento dell'esposizione passata e di aver elaborato gli elementi contenuti nei grandi modelli stranieri. ${ }^{14}$ Il naturalismo tardo impressionista di Anders Zorn, Max Liebermann, Joaquín Sorolla non lasciò indifferenti alcuni artisti, come ad esempio Tito e Milesi, ma fu in particolare la pittura dei danesi, degli svedesi, dei norvegesi e dei tedeschi che, secondo parte della critica, aveva lasciato il segno più profondo sugli artisti veneziani. L'opera di Ettore Tito, L'Estate (1897), nella quale l'assonanza evidente con l'arte d'oltralpe si notava allora soprattutto nell'intonazione cromatica, apparve tra le più rappresentative. Il segno forte dei norvegesi unito al colore più scuro ed evidente dei danesi fu riproposto anche da Fragiacomo nel suo Al vento (1987). Cesare Laurenti si mostrò altrettanto aperto alle influenze esterne partecipando alla Biennale del 1897 con Fioritura nuova (1987), che dimostrava una scelta stilistica vicina al decorativismo floreale che andava diffondendosi a livello europeo..$^{15}$ Perfino nei soggetti si rilevò una ricerca della verità tipica della pittura tedesca e olandese che si esprimeva in particolare attraverso dipinti di contenuto sociale. Ne era un chiaro esempio il dipinto Sposalizio (1987) di Alessandro Milesi. ${ }^{16}$

Se la critica si dimostrò entusiasta di questa prima proficua apertura verso l'arte internazionale, già nell'edizione del 1899 questa attenzione verso quanto si produceva nel resto del mondo cominciò ad essere considerata eccessiva fra i veneti, accusati per la prima volta di una "smania di imitare i modelli stranieri». ${ }^{17}$ In particolare venne notata una grande influenza esercitata dalla pittura scozzese di cui risentirono soprattutto le opere dei paesaggisti ed in particola-

13 Angelo Muraro, «L'Esposizione d'Arte Internazionale», Nuove Veglie Veneziane, 1 giugno 1895, 887.

14 Antonio Munaro 1897, 75: «E ne approfitterò per dire anche dell'influenza che l'Esposizione del 95 ebbe sui pittori italiani, i veneziani in particolare, i quali non pare che prima avessero studiato su Monet, su Besnard, su Israéls, su Dettmann, su Kroyer, se solo dal '95 al '97 seppero - alcuni con giusto criterio, altri con minore intensità e maturità di giudizio - interpretarne lo spirito e assumerne talune parvenze. Anzi per ciò si può dire - e io fino all'apertura di questa nova Internazionale non lo credevo - che la prima è stata non solo diletto e mercato, ma anche ammaestramento».

15 Cf. Guido Martinelli, «Le grandi esposizioni internazionali: l’Esposizione artistica di Venezia», Emporium, agosto 1897, 139.

16 Cf. Mario Pilo, «La Seconda Esposizione Internazionale d'Arte della Città di Venezia», Gazzetta Letteraria, 11 settembre 1897, 4.

17 Mario Pilo, «La terza Esposizione Internazionale d'Arte della Città di Venezia», Gazzetta Letteraria, 2 settembre 1899, 31. 
re quelle di Pietro Fragiacomo e Guglielmo Ciardi. ${ }^{18}$ I notturni di De Maria vennero invece paragonati a quelli dei norvegesi e degli svedesi, indiscussi maestri di tali soggetti.

Nel 1901 la suddivisione della sezione italiana per sale regionali ebbe tra gli altri scopi quello di ridare risalto alla continuità delle scuole ottocentesche locali rispetto alle novità linguistiche ispirate a correnti internazionali. La conferma veniva proprio dall'importanza attribuita ad alcuni artisti legati al passato ed alla tradizione, come Luigi Nono, di cui venne allestita una mostra personale. Ma parecchi dei pittori veneti, e in particolare veneziani, stavano ormai mostrando già da alcuni anni una palese influenza dall'arte d'oltralpe, tanto da rinunciare ad alcuni tradizionali caratteri locali per presentarsi «camuffati da Scozzesi, da Scandinavi o da Tedeschi» (cf. Ximenes $1901,28)$. Gran parte della stampa sconfessava con sempre maggiore accanimento un eccessivo avvicinamento alla pittura straniera. Divenne celebre in questo senso il giudizio che Vittorio Pica diede sui pittori veneti parlando di una vera e propria 'ossessione nordica'. ${ }^{19}$ Cominciò proprio in quell'anno il lento declino di alcuni dei più importanti artisti locali. L'apertura all'estero di Ettore Tito per esempio divise i commentatori e nella Biennale del 1901 nessuna delle cinque opere da lui esposte fu acquistata per la Galleria di Ca' Pesaro.

Nemmeno nel 1903 le accuse ai veneziani si placarono: «Hanno copiato gli stranieri, si sono suggestionati alla visione di artisti di altri paesi e di altri climi e si sono snaturati; neppure uno dei veneziani è completamente sincero», scrisse il quotidiano La libertà ${ }^{20}$ In effetti per quell'anno le critiche si fecero più aspre che mai: Cesare Laurenti era «guasto della smania di voler dipingere secondo le ricette straniere», ${ }^{21}$ Ettore Tito presentava una «Venere contorta e sbagliata, da scartare» (cf. Franchi 1903, 21), e «volgare assai» ${ }^{22}$ e Luigi Nono era ormai «in un doloroso periodo di decadenza». ${ }^{23}$

Una vera e propria svolta avvenne nel 1905, quando si riconobbe agli artisti veneziani un ritorno ai canoni dell'arte locale nella scelta dei soggetti, nei colori e negli effetti di luce. Probabilmente il clima internazionale che da ormai dieci anni si respirava nella laguna

18 Cf. Ugo Ojetti, «L'esposizione di Venezia, i Paesisti Italiani», Corriere della Sera, 15-16 settembre 1899.

19 Cf. Vittorio Pica, «L'arte mondiale alla IV Esposizione di Venezia», Emporium, 1901, agosto, 25.

20 La Libertà, 17 agosto 1903.

21 Cf. Alessandro Stella, «La Quarta Esposizione Internazionale della città di Venezia», Natura ed Arte, 10(16), 15 luglio 1901, 222.

22 Cf. Ugo Ojetti, «L'Esposizione di Venezia. I ritratti», Corriere della Sera, 6 giugno 1903.

23 Cf. Alessandro Stella, «La Quarta Esposizione Internazionale della città di Venezia», Natura ed Arte, 10(16), 15 luglio 1901, 222. 
veneta aveva spinto i pittori veneziani, dopo un periodo di imitazione e di apprendimento dell'arte nordica, a ricercare gli elementi che potessero identificare la loro arte, rendendola evidentemente distinta e riconoscibile all'interno di un ambiente così cosmopolita. Ma se la critica in passato aveva osteggiato l'imitazione dell'arte straniera, nel 1905 lamentò un'eccessiva ripetizione degli stessi soggetti. Ettore Tito, ad esempio si presentò quell'anno con dei semplici quadri di genere come L'Alzaia (1905), La frutta (1905), Dopo la pioggia (1905), La botte (1905) e Tempo favorevole (1905), ripetendo con insistenza ormai eccessiva i suoi usuali tipi di lavandaie che stendono il bucato al vento, i carrettieri che accompagnano i cavalli da traino, i ragazzi che giocano o le donne e i bimbi che passeggiano lungo i canali. Luigi Nono col Rosario del sabato (1905), continuava ad insistere «nel suo misticismo patetico» (Ximenes 1905, 11), come anche Silvio Rotta che con Carità (1905) riproponeva il tema della «poveraglia affamata» (Pica 1905, 114). L'artista Ettore Ximenes proseguiva, chiedendosi se

Dieci anni di esposizioni internazionali a Venezia hanno giovato all'arte italiana? Hanno influito per migliorare la produzione artistica nazionale? Che abbiano influito beneficamente sulla cultura degli artisti e dei non artisti è un fatto incontestabile, ma che siano sortiti risultati sensibilmente apprezzabili, non si può in coscienza affermare. (11)

Ma certamente dieci anni di mostre internazionali avevano portato in Italia e a Venezia una ventata di modernità e avevano spinto i pittori locali a un inevitabile e evidente rinnovamento. 


\section{Bibliografia}

Bargellini, Piero (1944). Caffè Michelangiolo, Firenze: Vallecchi.

Bazzoni, Romolo (1962). 60 anni di Biennale di Venezia. Venezia: Lombroso.

Ceschin, Daniele (2001). La voce di Venezia. Antonio Fradeletto e l'organizzazione della cultura tra Otto e Novecento. Padova: Il Poligrafo.

Dal Canton, Giuseppina (2000). «Una fonte per la storia dell'arte a Venezia fra Ottocento e Novecento». Resini, Daniele; Zerbi, Myriam (a cura di), Venezia fra Ottocento e Novecento nelle fotografie di Tommaso Filippi = Catalogo della mostra (Stra, Museo Nazionale di Villa Pisani, 30 marzo-3 novembre 2013). Venezia: Ire, 29-37.

De Paulis, Giovanni (1905). Impressioni d'un ipercritico sulla VI Esposizione Internazionale d'arte a Venezia. Aquila: A. Perfilla.

Di Martino, Enzo; Rizzi, Paolo (1982). Storia della Biennale, 1895-1982. Milano: Electa.

Franchi, Anna (1903). Quinta esposizione di Venezia. Firenze: Lumachi.

Isnenghi, Mario (1986). «La cultura». Franzina, Emilio, Venezia. Bari-Roma: Laterza, 381-480.

La Biennale di Venezia (1996). La Biennale di Venezia: le Esposizioni Internazionali d'arte, 1895-1995: artisti, mostre, partecipazioni nazionali, premi. Milano: Electa.

Lamberti, Maria Mimita (1995). «Il contesto delle prime mostre, dalla fine del secolo alla guerra mondiale: artisti e pubblico ai Giardini». Venezia e la Biennale, 39-47.

Masau, Maria; Pavanello, Giuseppe (a cura di) (1995). Arte d'Europa tra due secoli: 1895-1914, Trieste, Venezia e le Biennali. Milano: Electa.

May, Jan Andreas (2011). «30 aprile 1895. La nascita della Biennale». Israël, Uwe (a cura di), Venezia, i giorni della storia. Roma: Viella, 233-50.

Munaro, Antonio (1897). La Seconda Esposizione Internazionale D’arte: note critiche. Venezia: Ferdinando Ongania.

Ojetti, Ugo (1897). L'arte moderna a Venezia: esposizione mondiale del 1897. Roma: Enrico Voghera.

Pallucchini, Rodolfo (1979). «Significato e valore della "Biennale" nella vita artistica veneziana e Italiana». Storia della civiltà veneziana, vol. 3. Firenze: Sansoni, 157-88.

Paralupi, Rufo (1899). L'arte internazionale a Venezia. Bologna: Libreria Editrice Fratelli Treves.

Pavanello, Giuseppe (1983) «La pittura dell'ottocento». Venezia nell'Ottocento: immagini e mito. Milano: Electa, 13-94.

Pellegrini, Paola (1992). La prima esposizione internazionale d'arte della città di Venezia del 1895: storia della mostra dalla prima idea alla sua realizzazione, 1893-1895 [tesi di laurea]. Roma: Università La Sapienza, Facoltà di Lettere e Filosofia, a.a. 1991/1992.

Per una diretta partecipazione (1904). Per una diretta partecipazione degli artisti all'Ordinamento delle Esposizioni internazionali d'arte della Città di Venezia. Venezia: Nuova Tipografia Commerciale.

Pica, Vittorio (1897). L’Arte Mondiale a Venezia. Napoli: Pierro.

Pica, Vittorio (1901). L'arte mondiale alla IV Esposizione di Venezia. Bergamo: Istituto italiano d'arti grafiche.

Rabitti, Chiara (1995). "Gli eventi e gli uomini: breve storia di un'istituzione». Venezia e la Biennale, 26-38. 
Romanelli, Giandomenico (1995). «Biennale 1895, nascita, infanzia e prime imprese di una creatura di genio». Venezia e la Biennale 1995, 21-5.

Stella, Alessandro (1912). Cronistoria della Esposizione internazionale d'arte della città di Venezia: 1895-1912. Venezia: G. Fabbris.

Stringa, Nico (2008). «La Biennale di Venezia, tracce per un secolo di storia». La pittura nel Veneto. Il Novecento, vol. 2. Milano: Electa, 655-670.

Stringa, Nico (a cura di) (2009). La pittura nel Veneto. Il Novecento. Dizionario degli artisti. Milano: Electa.

Stringa, Nico (a cura di) (2016). L'Accademia di Belle Arti di Venezia. L'Ottocento. Crocetta del Montello: Antiga.

Stringa, Nico; Pavanello, Giuseppe (2006). La pittura nel Veneto. Il Novecento, vol. 1. Milano: Electa.

Toniato, Toni (a cura di) (1995). Modernità alla specchio: 1860-1960. Venezia: Supernova.

Venezia e la Biennale (1995). Venezia e la Biennale: $i$ percorsi del gusto = Catalogo della mostra (Venezia, Galleria d'Arte Moderna di Ca' Pesaro, 10 giugno-15 ottobre 1995). Milano: Fabbri Editori.

Ximenes, Edoardo (1905). Sesta Esposizione Internazionale d'Arte in Venezia. Pubblicazione speciale dell'lllustrazione Italiana. Milano: F.lli Treves. 


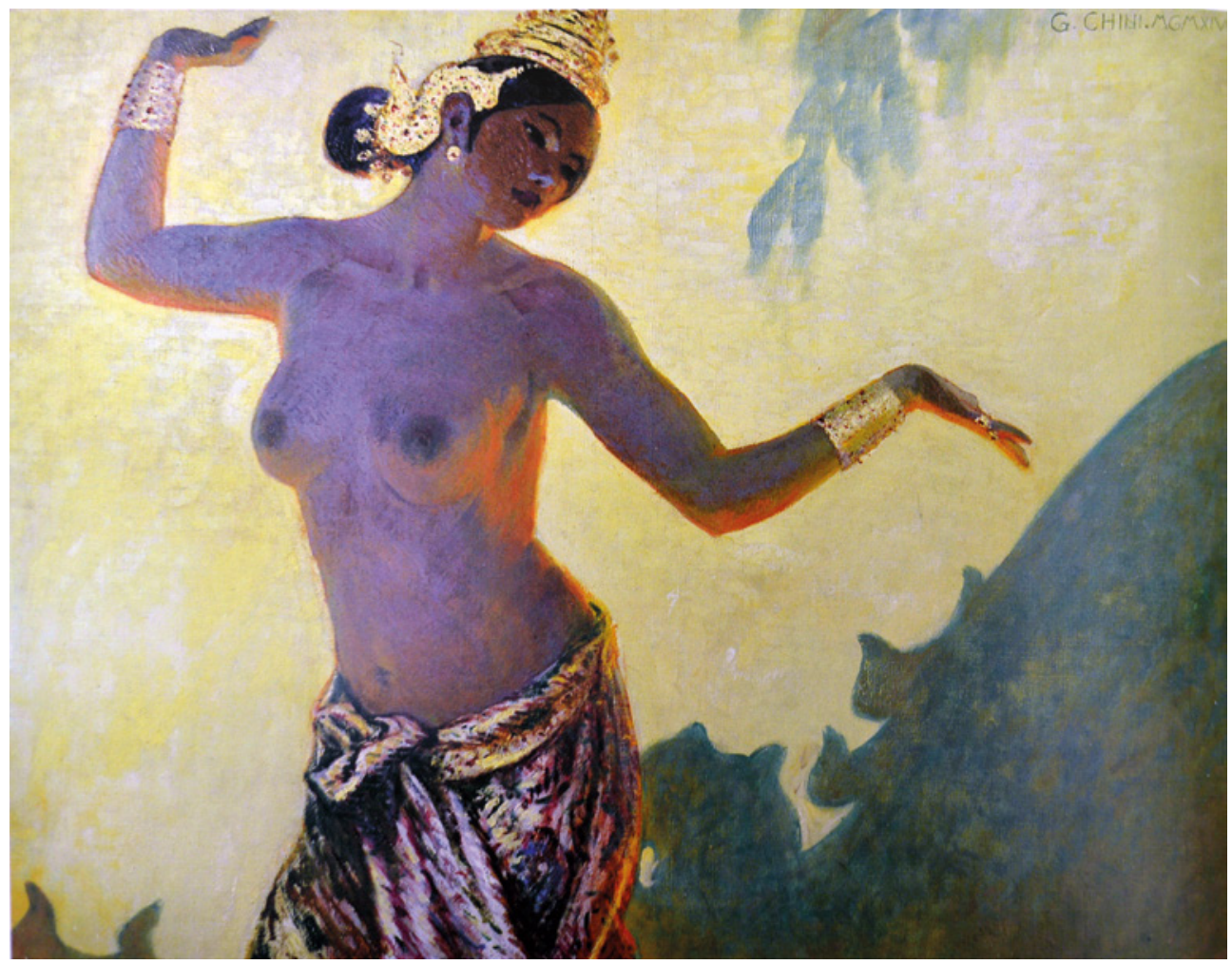

Figura 1 Galileo Chini, Danzatrice Monn, 1914.

Collezione privata 


\title{
1914. Galileo Chini a Venezia
}

\author{
Massimo De Grassi \\ Università degli Studi di Trieste, Italia
}

\begin{abstract}
Antonio Fradeletto, in his role as General Secretary of the Biennale, made agreements with Galileo Chini to set up a personal exhibition at Venice Biennale in 1914. In that exhibition he would summarise his newly concluded human and professional experience in Siam, not so much in terms of a celebration of his vast decorative interventions, as in those of his very personal reinterpretation of what had been the perception of a reality that appeared, and was, very far from the imagination of the public of the Venetian event. Alongside this sort of visual summary of that little-known East, Chini had had the opportunity to create the decoration of the International Hall destined to host the works of Ivan Mestrovic, and at that juncture, despite the winds of war were now blowing impetuously over the whole of Europe or perhaps just for that reason, he had proposed a soothing reading, all focused on the themes of Spring.
\end{abstract}

Keywords Galileo Chini. Biennale di Venezia 1914. Antonio Fradeletto. Ivan Mestrovic. Bangkok.

Tanto Bazzoni che Pica mi hanno tenuto parola del desiderio vostro di avere nel venturo anno a Venezia una sala per esporvi le impressioni ed i ricordi di codesto paese tanto caratteristico. Io sono disposto ad accogliere di buon grado la vostra proposta, anzitutto pel valore dell'artista e poi per l'interesse che desteranno le figurazioni di costumi e di paesi così diversi dai nostri ${ }^{1}$

con queste parole Antonio Fradeletto, nella sua carica di segretario generale della Biennale di Venezia stringeva definitivamente accordi con Galileo Chi-

1 Camaiore, Archivio Chini (https://www.galileochini.it/?archivio_chini=ricerca-in-archivio); per la trascrizione completa si veda l'Appendice «Documento 1 ». Per una parziale trascrizione: Margozzi 2014. Sulla partecipazione di Chini alla Biennale cf. anche Durante 2006. 
ni per allestire una mostra personale all'edizione del 1914 che riassumesse la sua appena conclusa esperienza umana e professionale nel Siam, non tanto nei termini di una celebrazione dei suoi vastissimi interventi decorativi, quanto in quelli della sua personalissima rilettura di quella che era stata la percezione di una realtà che appariva, ed era, molto lontana dall'immaginario del pubblico della manifestazione veneziana.

Accanto a questa sorta di riassunto visivo di quell'oriente così poco conosciuto, Chini aveva avuto modo di realizzare la decorazione della sala Internazionale destinata a ospitare le opere di Ivan Mestrovic, e in quel frangente, nonostante i venti di guerra soffiassero ormai impetuosi su tutta l'Europa o forse proprio per quello, aveva proposto una rasserenante lettura, tutta indirizzata sui temi della Primavera. ${ }^{2}$ Due fronti quindi molto diversi su cui il giocare la propria reputazione e il proprio successo.

Così Chini ricorda quei frangenti nella sua autobiografia, vergata però a molti anni di distanza, brevemente, e non senza qualche piccola imprecisione:

in quella esposizione ero stato invitato da Vittorio Pica, che era succeduto ad Antonio Fradeletto, a riordinare e comporre il Salone di Onore ed a fare una mostra personale di alcuni dipinti riproducenti cose che avevo fatto al Siam. Il Salone di Onore era diviso in pannelli decorativi rappresentanti fantasie cromatiche di gusto assolutamente sintetico, anche per far figurare le sculture che lo iugoslavo Ivan Mestrovic esponeva. (Chini 2014, 69)

In realtà i contatti di Chini per l'allestimento della mostra, da lui stesso promossa, erano stati sempre tenuti con Fradeletto, visto che Vittorio Pica gli era subentrato solo pochissimi mesi prima dell'apertura dei cancelli dell'esposizione

Procedendo con ordine, vale la pena di indagare sulla ragione che aveva spinto la segreteria della Biennale a chiedere con insistenza se effettivamente Chini avesse avuto la possibilità materiale di esporre le sue tele siamesi. Già il 20 gennaio 1913, Antonio Fradeletto, ancora pienamente in carica come segretario generale dell'istituzione veneziana, chiedeva assicurazioni riguardo la tempistica di consegna del materiale da esporre:

Ma per riservarvi la sala io devo avere l'assicurazione che Voi arriverete in tempo per preparare tutti i quadri necessari, e mi occorre conoscere all'incirca lo spazio che vi sarà necessario. Se vi

2 Sul ciclo chiniano della Biennale del 1914 si veda soprattutto: Margozzi 2004; 2014, con nota bibliografica. 
sono delle opere di grandi dimensioni, come mi accenna Bazzoni, sarà anzi meglio che Voi mi indichiate le misure delle pareti. ${ }^{3}$

In una successiva lettera indirizzata alla moglie di Chini, datata alla fine di giugno, Fradeletto chiedeva notizie di Galileo e soprattutto del suo rientro in patria:

In una lettera, pervenutaci or è qualche tempo, il Suo egregio Marito ci diceva che con tutta probabilità egli avrebbe fatto ritorno in Italia entro il corrente mese di Giugno. Noi ci rivolgiamo pertanto a Lei, gentile Signora, affine di sapere se il caro e valente amico nostro è effettivamente rientrato in patria o s'egli prolunga ancora il suo soggiorno nel lontano Siam e quando, in ogni caso, potremo aver il piacere rivederlo. ${ }^{4}$

Di fatto, una volta rientrato in patria Chini, i contatti saranno tenuti sul campo da Romolo Bazzoni, come dimostra una lettera indirizzata da quest'ultimo al pittore il 29 novembre 1913:

Ho riferito all'onor. Fradeletto il nostro colloquio e gli ho descritto anche le belle cose vedute nel vostro studio. Egli è contento che Voi abbiate materiale sufficiente per formare una bella saletta ed è perfettamente d'accordo con me che le vostre opere debbano essere esposte in un ambiente apposito e non unite con altre di artisti diversi come si era parlato da principio.

Siamo dunque intesi: sala speciale non troppo vasta ma con uno sfondo sufficiente perché si possa ammirar bene il grande quadro della festa notturna che starà nel centro.

Siamo d'accordo anche che Voi manderete a Venezia un numero di opere superiore a quelle che si esporranno e che faremo qui la scelta. ${ }^{5}$

La scelta definitiva poi operata sarà una sorta di antologia tematica, articolata intorno ai nuclei nevralgici della sua produzione siamese: ${ }^{6}$ quadri di figura, soprattutto danzatrici e attrici; indagini sul paesaggio, a cominciare dalla propria abitazione per proseguire con le suggestioni coloristiche di opere come L'ora nostalgica sul Nennam e soprattutto della grande tela con La festa dell'ultimo giorno dell'anno cinese a Bangkok, di gran lunga la più celebre e riprodotta, che apre un ulteriore filone, quello relativo all'illustrazione dei costumi civili

3 Camaiore, Archivio Chini (Appendice «Documento 1»).

4 Camaiore, Archivio Chini (Appendice «Documento 2»).

5 Camaiore, Archivio Chini (Appendice «Documento 3»).

6 Su questi aspetti della produzione siamese: Galileo Chini (1873-1956) 2007. 


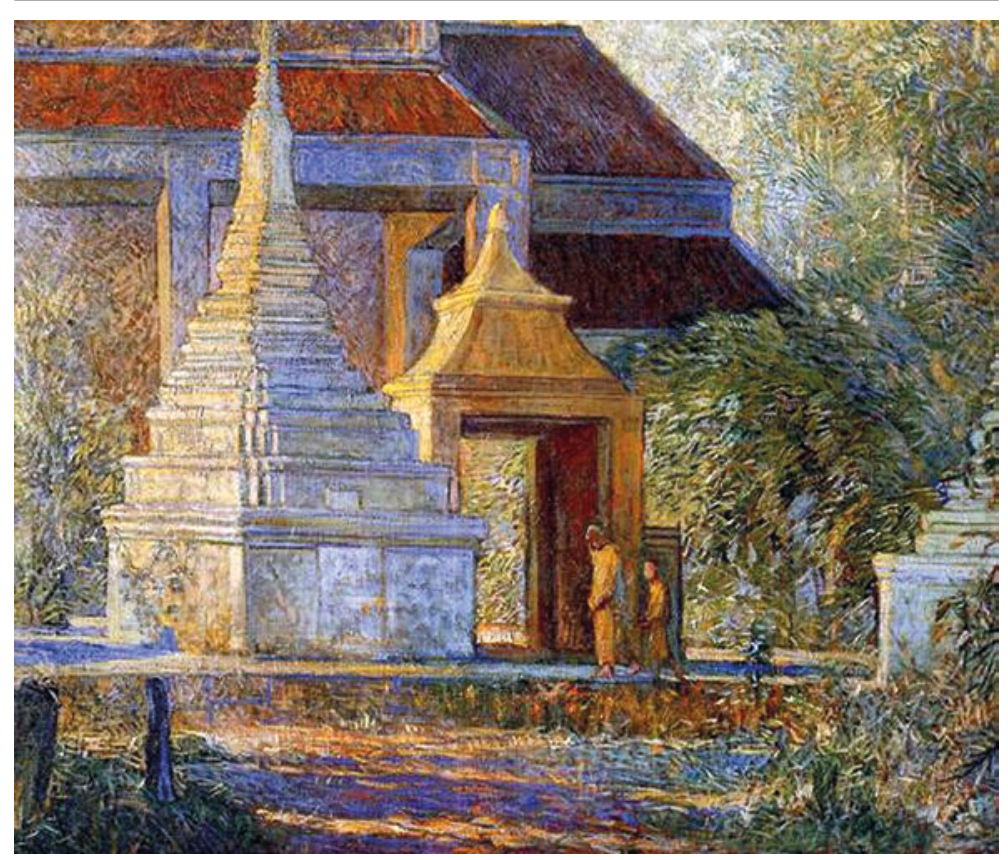

Figura 2 Galileo Chini, Vecchio cimitero diSam Phaya, 1913. Collezione privata

e religiosi della città. Chiudevano poi il percorso due nature morte che comprendevano una vasta scelta di pregiata oggettistica locale.

Una ricchezza tematica ben descritta nel contributo anonimo apparso sulle pagine del fascicolo La XI Esposizione Internazionale d'Arte della Città di Venezia, che costituisce anche, e di gran lunga, il contributo più esteso riguardo questa sala:

Galileo Chini ci è già apparso nella vasta decorazione del salone centrale. Nella sala XXV egli si presenta ancora con una mostra di quindici dipinti, tutti ritraenti costumi paesaggi e figure del Siam. Nel Siam il Chini si recò recentemente per decorare la sala del trono nel palazzo reale; e con grande efficacia coloristica vi dipinse, parte a fresco e parte a calce, tre mezze cupole, una grande lunetta e la grande cupola dello scalone, e diresse con l'aiuto di due giovani condotti da Firenze, l'ornamentazione delle altre parti dell'edificio. Del suo soggiorno nel Siam sono ricordo le quindici tele qui esposte, con le quali il Chini ha armonizzato la sala, decorandola con motivi di stile orientale. Codeste tele, come abbiamo già detto sono di figura, di paesaggio e di costumi; vi sono anche due piccoli quadri di natura morta. Fra i quadri di figu- 
ra conviene ricordare quelli, intitolati L'idolo, Ninnoli d'oriente e "Me Su" l'attrice e riproducenti tre strane ed interessanti figure di donne siamesi. Quella del quadro L'idolo è ritta in piedi, completamente nuda, in un'attitudine d'immobilità jeratica; ha braccialetti collana ed orecchini d'oro; e pur d'oro è una specie di raggiera, che le adorna il capo. La donna del quadro Ninnoli d'oriente è seduta; dai fianchi ai piedi è ricoperta d'una gonna di seta; nudo ha il petto; dietro a lei sono piatti e vasi scintillanti. "Me Su" l'attrice e in piedi; ha una gonna, che le scende soltanto fino ai ginocchi; ed è in un'attitudine di moto e di vita.

Sei sono i quadri di prospettiva e di paesaggio; vi hanno predominio le tonalità chiare e vi si ammirano generalmente giuochi festosi di luce tra le fronde. Ricordiamo La mia veranda a Bangkok, Il mio cortile a Bangkok e Il vecchio cimitero di San Phaya [fig. 2]; l'edificio funebre sorge in mezzo alla letizia del verde; due figure silenziose stanno per varcare la porta sacra; e un sentimento di pace triste e solenne è diffuso in tutta la tela. L'ora nostalgica sul Nennam è dominato invece da una forte tonalità rossa; il tramonto ha coperto tutto il cielo di porpora, e la porpora si riflette nelle acque placide del fiume; sopra una zattera due figure umane stanno assistendo al meraviglioso spettacolo naturale, e i celebri versi danteschi sorgono spontanei nello spirito a commentare il titolo e la significazione del quadro [fig. 4].

Di maggiore interesse sono forse i quadri, che riproducono i costumi popolari e soprattutto religiosi del Siam. La bisca del gran Cinese a Bangkok rappresenta una sala illuminata da luci artificiali con numerose figure di giuocatori occupati dalla loro passione travolgente. Nel quadro intitolato $\mathrm{Nel}$ tempio si vedono entro una luce incerta alcuni fedeli inginocchiati; dall'alto scende e sembra come sospesa sul loro capo una mano gigantesca di metallo, la mano terribile e misteriosa del dio. La casa di Ghotamo è intitolato un trittico, le cui parti sono La festa, La pace e L'indolenza. La prima parte ci conduce nel tempio; i fedeli sono seduti ai piedi di due grandi pilastri; nel fondo, in alto, illuminato dalle luci che salgono dal basso, risplende l'enorme idolo di metallo. Nella seconda parte siamo fuori dal tempio; fra due pilastri stanno due figure silenziose. La terza parte del trittico ci riconduce alla porta del tempio; alcuni uomini sono sdraiati sui gradini, nel torpore afoso del giorno orientale. La festa dell'ultimo giorno dell'anno cinese a Bangkok s'intitola il quadro di maggiori dimensioni di questa raccolta di Galileo Chini [fig. 3]. Esso occupa il centro della parete di fronte alla porta e attira subito e tiene ferma l'attenzione con la violenza de' suoi colori e delle sue luci. Passano da destra a sinistra con movimenti impetuosi grandi figure di uomini, che portano ed alzano nell'aria palloni multicolori, palloni verdi, palloni gialli, palloni rossi internamente illuminati. La luce irrompe 
da ogni parte ed invade tutto il quadro, mercè una stupefacente abilità, che prova nuovamente il valore dell'artista, che al palazzo delle Esposizioni veneziane ha dato le due fastose decorazioni del salone della cupola e del salone centrale. ${ }^{7}$

Come quasi sempre in quelle circostanze i dipinti esposti erano anche in vendita, e dagli appunti manoscritti a margine della copia del catalogo ufficiale della manifestazione conservata presso gli archivi mestrini dell'ASAC se ne conoscono anche i valori di partenza, strettamente correlati alle dimensioni delle tele. ${ }^{8}$ Dai registri delle vendite e dall'archivio dell'artista risulta un'unica vendita, quella delle due nature morte acquistate da casa Savoia per 1600 lire. ${ }^{9}$

Nella scelta di allestire una mostra così particolare e così personale, rafforzata anche dalla decisione di esporre alla pressoché contemporanea mostra della Secessione Romana la Danzatrice Moon [fig. 1] maturata pittoricamente nelle stesse circostanze. ${ }^{10}$ rientravano indubbiamente i consolidati rapporti personali di Chini con la direzione della Biennale, ma di certo una trattativa portata avanti praticamente al buio, senza cioè conoscere la natura delle opere da esporre, presupponeva anche una totale fiducia nell'artista e soprattutto una forte curiosità per quella produzione. In tutto questo di certo rientrava di certo il vivo interesse che la trasferta di Chini aveva suscitato nel mondo artistico italiano, se non altro per la destinazione esotica e

7 Galileo Chini in La XI Esposizione Internazionale d'Arte della Città di Venezia, 1914, aprile-maggio, s.p.

8 Si trascrivono di seguito la presentazione in catalogo e i dati relativi alle quotazioni proposte: «cresciuto in reputazione, fu chiamato ad ornare la Sala del Trono per il Re del Siam. Quest'opera, eseguita tutta da italiani, è il monumento europeo di maggior mole e di più alto senso d'arte che vi sia in tutta l'Asia e si deve agli architetti Rigotti e Tamagno, agli ingegneri Allegri e Gollo. Il Chini vi dipinse una grande efficacia coloristica, parte a fresco e parte a calce, tre mezze cupole, una grande lunetta dello Scalone e diresse l'ornamentazione delle altre parti dell'edificio, con l'aiuto di due giovani condotti da Firenze. All'Accademia non ci potei andare per studiare, ci andai per insegnare (era stato nominato professore all'Istituto di Belle Arti di Roma) ma il Siam mi impedi di proseguire... E del suo soggiorno nel Siam è un magnifico riflesso questa Mostra, come delle sue virtù decorative è nuovo documento la trasformazione felice del nostro Salone centrale/ 1 Nel tempio. 700/ 2 Fronde e luci. 1500/ 3 Natura morta. 1000/ 4 L'idolo. 5000/ 5 Ninoli d'oriente. 3000/ 6 "Men su" l'attrice. 5000/ 7 Natura morta. 1000/ 8 Vecchio cimitero di San Phaya. 2000/ 9 La festa dell'ultimo giorno dell'anno cinese a Bangkok. 10000/10 "Wat Sam-Chat". 2500/ 11 La mia veranda a Bangkok. 700/ 12 La casa di Ghotamo./ a) la fede. b) la pace. c) l'indolenza 1000 - 4500/13 Il mio cortile a Bangkok. 700/ 14 L'ora nostalgica sul Nen-nam. 1500/ 15 La bisca del gran Cinese a Bangkok 700/ Mobilio eseguito espressamente dalla Ditta F. Spicciani \& Fratelli di Lucca».

9 Camaiore, Archivio Chini (Appendice «Documento 4»). Le due opere si trovano attualmente nelle collezioni di Ca' Pesaro - Galleria Internazionale d'Arte Moderna di Venezia nr. inv. 0534 e 0535.

10 L'opera sarà riprodotta anche nel catalogo ufficiale della mostra: Secessione 1914, tav. XXII. Sul dipinto anche: Carrera 2014, 164. 
Massimo De Grassi

1914. Galileo Chini a Venezia

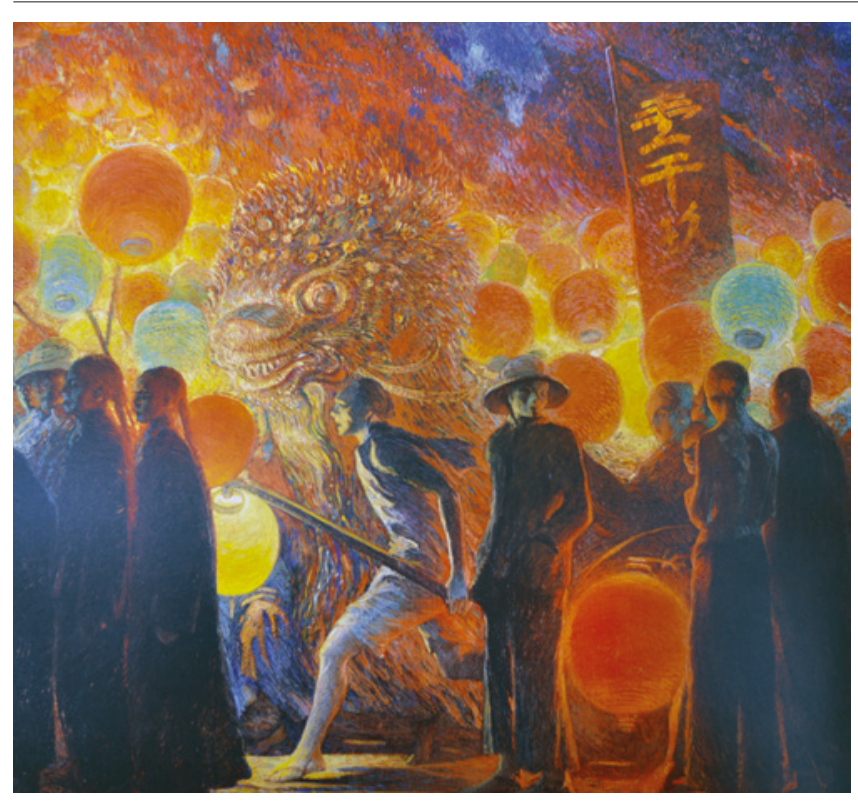

Figura 3 Galileo Chini, La festa dell'ultimo giorno dell'anno cinese, 1912-13. Collezione privata

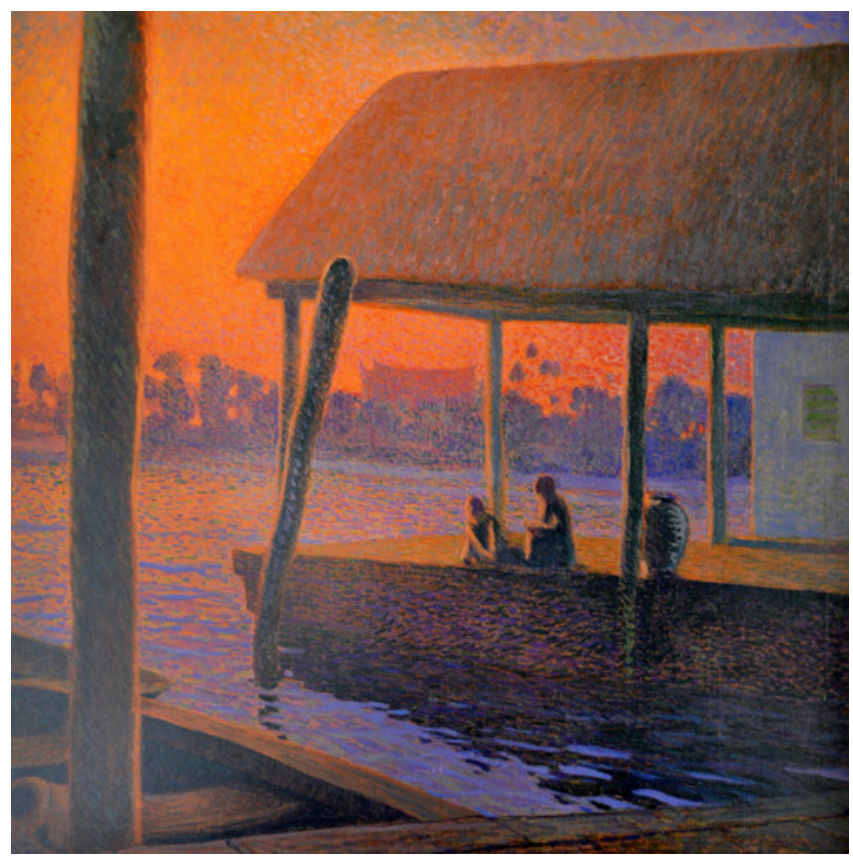

Figura 4 Galileo Chini, L'ora nostalgica sul Nen-nam, 1912-13. Collezione privata 
per la portata della commissione ricevuta. All'epoca in realtà il regno del Siam era una realtà ormai consolidata per molti artisti e professionisti italiani, anche se nessuno del peso e della notorietà di Chini.

Da almeno vent'anni infatti operavano nell'attuale Thailandia una serie di professionisti soprattutto di origine piemontese che avevano nel tempo contribuito in modo decisivo alla svolta occidentalizzante che prima Rama V e poi il figlio Rhama VI avevano voluto dare alla capitale del regno (cf. Filippi 2008). Il viaggio di Chini rappresentava di fatto una sorta di punto d'arrivo di una complessa serie di eventi, che avevano avuto transito anche, pochi mesi prima della partenza del pittore toscano, dal grande Padiglione siamese all'Esposizione Internazionale di Torino del 1911, progettato da quello stesso Mario Tamagno che nel contempo a Bangkok stava preparando le superfici di affreschi della nuova maestosa Sala del Trono, Ananta Samakhon, commissionata dal re Chulalongkorn nel 1907, una splendida costruzione in stile neorinascimentale italiano, destinata a essere dipinta da Chini (cf. Filippi 2008, 107-20).

Il soggiorno siamese di Galileo Chini si era collocato quindi in un contesto piuttosto ricco di interscambi culturali e soprattutto professionali intercorsi tra l'Italia e il regno dell'estremo oriente. Un contesto arricchito e strutturato intorno a presenze ormai sedimentate da decenni sul territorio dell'attuale Thailandia. Presenze che si avviano intorno al processo di europeizzazione che la capitale siamese stava attivando sin dalla fine del secolo precedente (cf. Ferri De Lazara, Piazzardi, Cassio 1996).

Tra gli artisti fino a quel momento presenti in loco però la figura di Chini aveva sicuramente un peso specifico diverso, soprattutto sul piano di quella notorietà internazionale guadagnata in virtù di comprovate capacità di articolare efficacemente grandi spazi decorativi coniugando modernità e capacità narrative, allegoria e storia, fantasie e realtà. Capacità che evidentemente mancavano negli altri pittori che negli anni precedenti avevano fatto parte di quella piccola diaspora di artisti e professionisti piemontesi attratti nel regno orientale dalla possibilità di nuove affermazioni e di guadagni difficilmente ottenibili in patria, dove le possibilità di affermazione avevano ben altra consistenza ma, nel caso specifico, né Cesare Ferro né altri sembravano in grado di articolare un'impresa così impegnativa come quella cui sarà chiamato Chini.

Sotto questo aspetto Cesare Ferro, che pure era pittore quotato e poteva vantare una più strutturata presenza sul territorio, non aveva i requisiti necessari non avendo di fatto quasi mai affrontato la grande decorazione, e solo occasionalmente la pittura murale, come nel caso dei pannelli decorativi con miti silvani per la villa Ambara a Bangkok, completati intorno al 1908 (Moncassoli, Auneddu 1998, 56-66).

La scelta di Chini, volendo rimanere nell'ambito della cultura figurativa italiana, era di fatto una scelta obbligata, almeno per il pro- 
getto di Tamagno, concentrato appunto sul recupero della tradizione rinascimentale italiana.

Nello specifico, l'interesse della corte siamese per l'arte italiana si era concretizzato anche negli acquisti operati dal re siamese alla Biennale veneziana del 1907, quando Rama IV aveva acquistato un dipinto di Augusto Noci e il costosissimo gruppo in bronzo di Clemente Origo; certo non opere d'avanguardia (ma comunque di un gusto più aggiornato dei contemporanei acquisti di Casa Savoia) e collocate nell'alveo di una consolidata produzione di artisti noti a livello internazionale (Margozzi 2006, 33-8).

In questo senso l'esperienza di Chini nella Sala del trono assume per l'orizzonte culturale della casa regnante tailandese il valore di una grande innovazione, anche per la capacità dell'artista di ibridare con estrema efficacia con la tradizione figurativa locale, soprattutto per quanto riguarda gli aspetti prettamente decorativi. L'enorme spazio a disposizione comprendeva tre mezze cupole, una grande lunetta e la vasta cupola dello scalone. Nell'intraprendere quest'impresa Chini comincerà da un'attenta analisi ambientale, visitando il territorio in lungo e in largo per comprendere a fondo l'arte e l'atmosfera culturale locale, oltre che i monumenti artistici ai quali ispirarsi, per entrare nell'atmosfera orientale. Oltre alla decorazione del salone principale, Chini prenderà parte anche alla scelta dei decori negli altri ambienti dell'edificio, orientandone lo stile strutturato su di una raffinata e inedita combinazione di sintassi decorativa italiana intessuta con elementi linguistici tipicamente siamesi.

L'incontro tra motivi decorativi tipici della tradizione locale con la sapiente organizzazione spaziale e le capacità narrative di Chini aveva prodotto un risultato che sembra essere una perfetta celebrazione delle aspettative di Rama V, che miravano a una celebrazione dinastica che avesse dignità visiva di livello internazionale, grazie soprattutto alle capacità professionali e artistiche della colonia italiana di Bangkok, la cui esperienza trovava nella sala del trono una sorta di punto d'arrivo delle sinergie culturali messe in campo nei decenni precedenti. Sul piano culturale si trattava dell'affermazione ormai consolidata e definitiva di un'italianità conclamata. Oltre agli echi internazionali, l'esperienza di Chini avrà notevoli riverberi anche in patria.

Chini si tratterrà a Bangkok fino all'estate del 1913, e oltre alla grandiosa decorazione del palazzo del trono realizzerà anche ritratti del sovrano e della sua corte oltre che numerosi dipinti da cavalletto, che colgono con intima partecipazione vari aspetti della vita orientale. Rientrato in Italia, portando con sé una ricercata collezione di oggetti siamesi e cinesi, non dimenticherà mai le atmosfere del Siam, che continueranno a ispirare molti suoi dipinti fino agli anni '40. In questo senso importantissime saranno le sue scenografie per il primo memorabile allestimento della Turandot di Giacomo Puccini, 
ispirate proprio alla corte siamese, e le stesse decorazioni che Chini realizzerà per la sua famiglia negli interni della sua Casa delle Vacanze a Lido di Camaiore.

Del valore di quell'esperienza era ben conscio Fradeletto che, evidentemente informato a proposito della produzione da cavalletto cui l'artista aveva messo mano nel suo lungo soggiorno siamese, gli aveva proposto già nel 1913 di allestire una sala personale alla successiva Biennale dedicata soltanto a quelle opere, senza nemmeno essere a conoscenza della natura specifica di quei lavori. Una prova di fiducia naturalmente collegata alla ormai lunga consuetudine che legava la direzione della rassegna veneziana all'artista faentino, capace di assecondare al meglio le necessità decorative della mostra, come del resto aveva dimostrato con la decorazione della sala ottagona del Padiglione Italia affrescata in occasione dell'edizione del 1909 della mostra veneziana.

Alla richiesta, evidentemente accolta con entusiasmo, si era poi aggiunta quella di realizzare dei pannelli decorativi per la Sala internazionale destinati a fare da corona alle opere di Ivan Mestrovic. Fradeletto, in una lettera del dicembre 1913, aveva apprezzato le soluzioni proposte per la Sala Mestrovic. ${ }^{11}$

Le due anime della ricerca chiniana di quel momento si trovavano così idealmente a contatto, distanziate soltanto di pochi metri. Se però le grandi tele ispirate alla Primavera troveranno ampi riscontri sulla stampa, più difficile sarà la ricezione delle tele a tema siamese, divise tra prospetti schiettamente etnografici, seduzioni esotiche permeate di sgargianti cromatismi e più meditate tele introspettive, dove l'osservazione della realtà locale mostrava un più alto grado di consapevolezza.

La critica italiana mostrerà però scarso interesse per queste prove: se manifestazioni di interesse arrivano dalla Francia, con la richiesta da parte di Gustave Poulies di materiale fotografico per un articolo sulla Gazzette des Beaux Arts, ${ }^{12}$ poi non utilizzato, e analoghe richieste arrivano da giornali illustrati italiani, ${ }^{13}$ i giudizi dei critici più autorevoli rimangono sospesi se non negativi. Ugo Ojetti, sulle auterevoli colonne de Il Corriere della Sera, lo considera «più decoratore che pittore di cavalletto», e anche la prova che oggi viene considerata come la più rappresentativa di quella stagione, «la grande tela rossa e gialla della Festa dell'ultimo dell'anno a Bangkok, sulla maggiore parete della saletta dedicata ai suoi ricordi del Siam [...] è solo un fastoso pannello decorativo». Ojetti poi stigmatizza anche lo sperimentalismo tecnico con cui di fatto Chini aveva affrontato i 
vari soggetti degli altri dipinti, che effettivamente oscillano tra un convenzionale divisionismo e un bozzettismo naturalistico; quest'ultimo perfettamente funzionale alla natura di reportage di alcune di quelle tele. Così si era espresso in proposito Ojetti:

la sua pittura fluente e veloce muta tanto spesso che non si sa più dove sia il vero Chini: l'Attrice $\mathrm{Me}$-Su ha perfino dei ricordi, certo involontari, di Blanche. Pure, se egli sostasse un momento e approfondisse un tema, ad esempio la Pace di quella notte lunare azzurra e verde nella Casa di Gothamo, egli arriverebbe anche a dipingere un quadro degno del suo grande ingegno irrequieto. ${ }^{14}$

L'analisi più compiuta rimane comunque quella di Arduino Colasanti sulle pagine di Emporium dove, pur rimarcando l'eccessivo secessionismo della sala centrale, sottolineato anche da Ojetti, ${ }^{15}$ individuava nella mostra personale «un interessante riflesso del suo soggiorno nel Siam», grazie al quale:

nella rievocazione di quel lontano mondo orientale ha saputo darci molti saggi convincenti della sua libera e fervida fantasia in una serie di rappresentazioni di intenzioni nobilmente decorative e di un'armoniosa finezza di colore. Nel ritratto di "Me-Su" l'attrice, nel Vecchio Cimitero di San Phaya, nella Festa dell'ultimo giorno dell'anno cinese a Bangkok, in Fronde e luci è la suggestione affascinante e nostalgica di certi canti stranieri e barbarici. Ma dove il giovane pittore toscano ha trovato una nota veramente profonda è nel Trittico La Casa di Ghotamo, poetica figurazione del tempo di Budda in una pallida luce lunare, mentre nel cielo terso le prime stelle assentono, tremando in ritmo all'implorazione dei fedeli inginocchiati. ${ }^{16}$

Con queste premesse, gli esiti del viaggio di Chini in Siam sono anche il segno di un Orientalismo maturo e consapevole, una sorta di primitivismo mancato. Il pittore toscano non era partito alla ricerca di una cultura incontaminata con cui confrontarsi, parte da professionista affermato con l'intento di 'contaminare' quella situazione culturale con la propria arte, come prima di loro avevano fatto Antonio Fontanesi e Vincenzo Ragusa partendo per il Giappone; ma come

14 Ugo Ojetti, «L'undecima biennale veneziana. Gl'italiani», Corriere della Sera, 12 giugno 1914

15 Ugo Ojetti, «L'undecima biennale veneziana. Francesi, spagnoli, inglesi, belgi», Corriere della Sera, 23 aprile 1914.

16 Arduino Colasanti, «Esposizioni italiane: la Mostra Internazionale d'Arte a Venezia. I», Emporium, 40(235), 1914, 26. 
quest'ultimo, e al contrario di Fontanesi, Chini interiorizza profondamente quell'esperienza, all'origine soltanto professionale.

La scelta di proporre una sala personale alla Biennale veneziana dedicata proprio a quell'esperienza, pur facilitata da un rapporto privilegiato con la direzione dell'istituto veneziano, ha davvero il crisma della scoperta e della fascinazione di un orizzonte figurativo fino a quel momento toccato soltanto attraverso le sollecitazioni stilistiche che erano arrivate a lui filtrate dal vocabolario internazionale dell'Art Noveau. Non si trattava poi di un primitivismo vero e proprio, la cultura figurativa thay non aveva infatti nulla di primitivo nell'accezione corrente del termine e le suggestioni arrivavano a Chini soprattutto dalle situazioni locali e al più dalle arti di movimento, dal teatro e dalla ricchezza visiva dei cerimoniali di corte. Si potrebbe quasi definire un primitivismo alla rovescia, se il termine di paragone è Gauguin. Ma diventa qualcosa di molto più simile al rapporto di Matisse con il vicino Oriente e il Nord Africa, dove le suggestioni sono più mediate e sottili, come appunto quelle di Chini.

Rispetto a Matisse, almeno in questa prima fase, Chini è più un reporter e meno un assimilatore. Il peso di questa assimilazione di contenuti si svelerà del tutto più tardi, nei bozzetti per la Turandot e in certi problematici dipinti dei decenni successivi, quando quegli elementi diventeranno una sorta di ideale bene rifugio pittorico da utilizzare per dimenticare e estraniarsi dal quotidiano. 


\section{Appendice}

\section{Documento 1}

Venezia, 20 gennaio 1913

\section{Carissimo Chini}

La vostra gentile Signora, dietro mia richiesta, mi ha favorito il vostro indirizzo.

Desideravo scrivervi anzitutto perché non vi tornerà discara una mia parola di saluto in codesto lontano soggiorno e poi per parlare un pochi o dei nostri progetti per la prossima esposizione del 1914. Tanto Bazzoni che Pica mi hanno tenuto parola del desiderio vostro di avere nel venturo anno a Venezia una sala per esporvi le impressioni ed i ricordi di codesto paese tanto caratteristico. Io sono disposto ad accogliere di buon grado la vostra proposta, anzitutto pel valore dell'artista e poi per l'interesse che desteranno le figurazioni di costumi e di paesi così diversi dai nostri. Ma per riservarvi la sala io devo avere l'assicurazione che Voi arriverete in tempo per preparare tutti i quadri necessari, e mi occorre conoscere all'incirca lo spazio che vi sarà necessario. Se vi sono delle opere di grandi dimensioni, come mi accenna Bazzoni, sarà anzi meglio che Voi mi indichiate le misure delle pareti.

Dunque coraggio e all'opera; io confido nel successo di questa vostra Esposizione.

Attendo un vostro cenno a volta di corriere e Vi saluto affettuosamente.

Vostro aff: A. Fradeletto

Cordiali saluti dall'amico Bazzoni

Scrivetemi anche quando avverrà il vostro ritorno. 


\section{Documento 2}

Venezia, 26 giugno 1913

Gentilissima Signora

In una lettera, pervenutaci or è qualche tempo, il Suo egregio Marito ci diceva che con tutta probabilità egli avrebbe fatto ritorno in Italia entro il corrente mese di Giugno. Noi ci rivolgiamo pertanto a Lei, gentile Signora, affine di sapere se il caro e valente amico nostro è effettivamente rientrato in patria o s'egli prolunga ancora il suo soggiorno nel lontano Siam e quando, in ogni caso, potremo aver il piacere rivederlo.

In attesa di un Suo cortese riscontro, Le porgiamo, gentile Signora, l'espressione del nostro distinto ossequio

Il segretario generale

A. Fradeletto

\section{Documento 3}

Venezia, 29 novembre 1913

\section{Carissimo Chini}

Ho riferito all'onor. Fradeletto il nostro colloquio e gli ho descritto anche le belle cose vedute nel vostro studio. Egli è contento che Voi abbiate materiale sufficiente per formare una bella saletta ed è perfettamente d'accordo con me che le vostre opere debbano essere esposte in un ambiente apposito e non unite con altre di artisti diversi come si era parlato da principio.

Siamo dunque intesi: sala speciale non troppo vasta ma con uno sfondo sufficiente perché si possa ammirar bene il grande quadro della festa notturna che starà nel centro.

Siamo d'accordo anche che Voi manderete a Venezia un numero di opere superiore a quelle che si esporranno e che faremo qui la scelta. Vi ringrazio ancora per le cortesie usatemi e Vi prego di ricordarmi devotamente alla Vostra gentile Signora ed ai Vostri bravi figliuoli. Tante cose cordiali a Voi e tanti affettuosi saluti dal

Vostro aff.mo Romolo Bazzoni 


\section{Documento 4}

Venezia li 8 giugno 1914

Egregio Signore,

Il ministero della Real Casa mi ha testè rimesso la somma di Lire 1600 (mille seicento), al pagamento dei due quadri "Natura morta" da lei venduti a S.M. Il Re.

Detratto l'importo della percentuale dovuta all'Esposizione, rimangono Lire 1440 che le mando a mezzo Vaglia del Banco di Napoli.

La prego di ritornarmi - firmata nel bollo - l'acclusa quietanza e, frattanto, aggradirà i miei migliori saluti

Dev.mo Romolo Bazzoni

\section{Documento 5}

Venezia, 26 XII. 913.

Carissimo Chini,

auguri cordiali a Voi e alle famiglie Vostre! Desideravo, tornando da Roma, farci una visitina. Ma ho dovuto protrarre la mia permanenza alla Capitale fino a tutto il 23. Così per essere in grado di passare la vigilia di Natale coi miei, sono ritornato direttamente a Venezia. Spero di venire costà entro il prossimo mese.

Intanto vi ringrazio per l'ideata trasformazione del Salone centrale. Il nostro Bazzoni mi fece vedere il disegno, illustrandomelo con parecchi schiarimenti. È un colpo di bacchetta magica. Applausi!

Avrei solo un dubbio circa il collocamento di quadri al di sotto dei pannelli decorativi. Ma il Bazzoni mi rassicura, dicendomi che la tonalità dei pannelli è così blanda e tenue ch'essi non procureranno effetto sulle opere sottoposte.

Grazie ancora. Abbiatevi una stretta affettuosa di mano.

Vostro

A. Fradeletto 


\section{Documento 6}

Caro Chini,

Incaricato dalla Gazette des Beaux Arts di scrivere gli articoli sull'Esposizione di Venezia, tengo molto a riprodurre uno dei suoi quadri del Siam: ad esempio, L'idolo, o la Fede, o tutt'altro d'importante, e possibilmente un pannello decorativo del Salone Centrale. Le chiederò perciò di mandarmi al più presto le fotografie di cui potrebbe lei disporre.

Grazie, e cordialmente sua, Gustave Poulies

\section{Documento 7}

Chmo Sig.re preparo per l'Almanacco del Bemporad un gruppo di foto delle opere più notevoli della XI Mostra veneziana, che andranno in Cronaca Artistica con poche righe di testo. Fra queste fot. - non più di 10 - ne vorrei mettere una di un quadro suo, appartenente alla Sala personale. Le ho riservato a posta lo spazio. Vuole Mandarmela? Mi rimetto a Lei per la scelta del quadro. Ma la prego di favorirmi con cortese prontezza. Vide, sui quattro quotidiani della Società Editrice Romana, quello che scrissi dell'opera sua?

Gradisca i miei cordiali ossequi e mi abbia

dev.mo A. Lancellotti 


\section{Bibliografia}

Benzi, F. (2014). Il tarlo polverizza anche la quercia. Le memorie di Galileo Chini. Firenze: Maschietto Editore.

Carrera, M. (2014). «Galileo Chini, Danzatrice Moon». Frezzotti, S. (a cura di), Secessione e avanguardia. L'arte in Italia prima della Grande Guerra 1905-1915 = Catalogo della mostra (Roma, Galleria nazionale d'Arte Moderna, 31 ottobre 2014-15 febbraio 2015). Milano: Electa.

Durante, Lia (2006). «Galileo Chini alla Biennale di Venezia». Durante, Lia (a cura di), Restauri. Galileo Chini e altre opere della collezione permanente. Venezia: La Biennale, 22-37.

Ferri De Lazara, L.; Piazzardi, Paolo; Cassio, A. (1996). Italiani alla corte del Siam - Italians at the Court of Siam. Bangkok: Amarin Printing and Publishing.

Filippi, F.B. (2008). Da Torino a Bangkok. Architetti e ingegneri nel Regno del Siam. Venezia: Marsilio.

Galileo Chini (1873-1956) (2007). Galileo Chini (1873-1956). Un pittore italiano alla corte del Re del Siam = Catalogo della mostra (Roma, Galleria Antonacci, 16 maggio-30 giugno 2007). Roma: Francesca Antonacci.

Margozzi, M. (2004). Galileo Chini. La primavera= Catalogo della mostra (Roma, Galleria Nazionale d'Arte Moderna, 15 dicembre 2004-15 febbraio 2005). Viareggio: Idea Books.

Margozzi, M. (2006). «Galileo Chini e il Siam». Benzi, F.; Margozzi, M. (a cura di), Galileo Chini dipinti, decorazione, ceramica, teatro, illustrazione = Catalogo della mostra. Milano: Electa.

Margozzi, M. (2014). La Primavera di Galileo Chini da Venezia a Montecatini 19142014. Firenze: Maschietto.

Moncassoli, M.L.; Auneddu, G. (1998). Arte italiana alla corte del Siam. Cesare Ferro pittore 1904-1925. Torino: Anisa Attività.

Secessione (1914). Seconda Esposizione Internazionale d'Arte "della Secessione" = Catalogo Illustrato. Roma. 


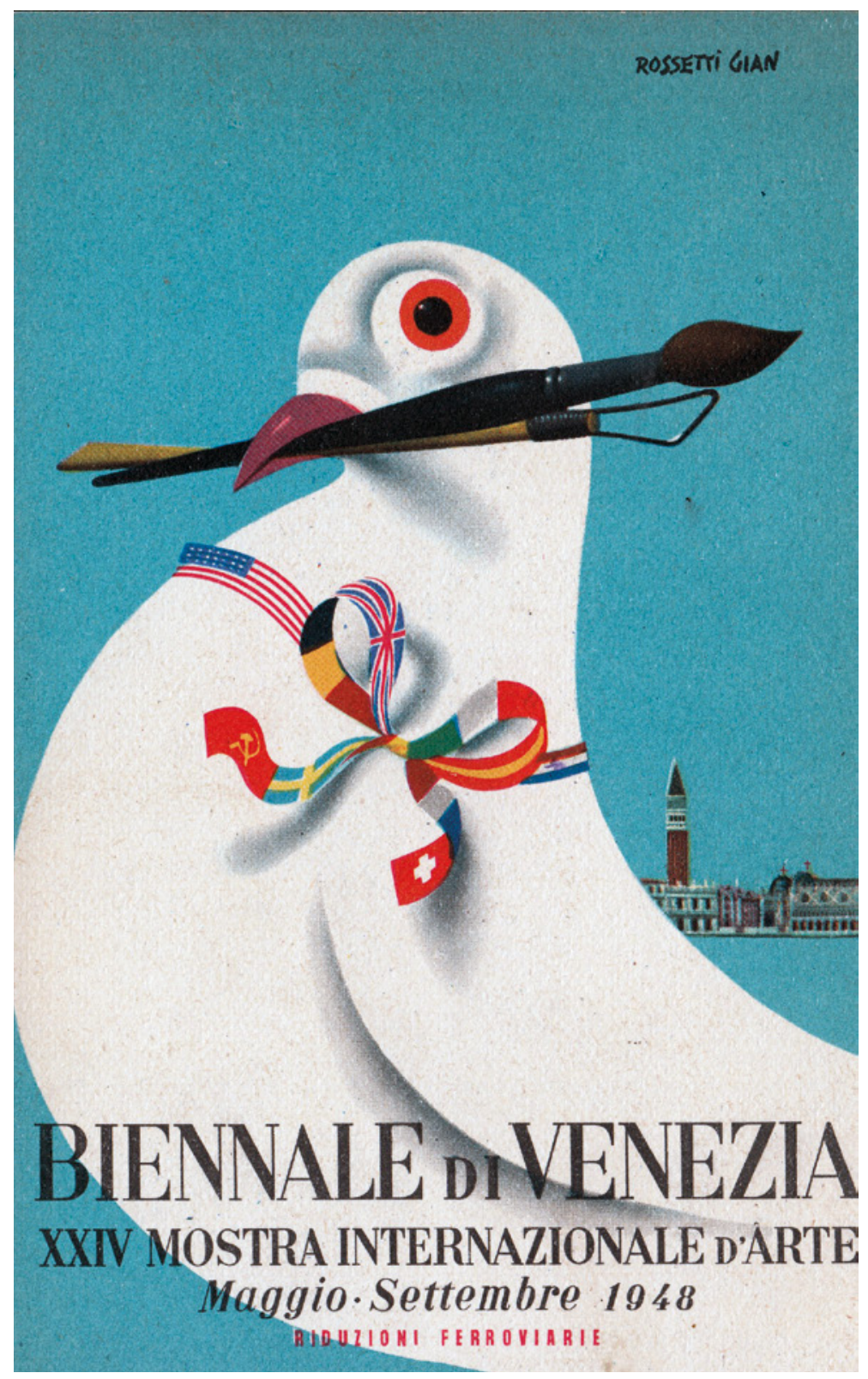

Figura 1 Cartolina della XXIV Biennale del 1948 (illustrazione di Gian Rossetti). Collezione Claudio Romeo 


\title{
I pittori veneti alle 'Biennali di Pallucchini' (1948-1956) Le partecipazioni e la ricezione della stampa
}

\author{
Elisa Rampazzo
}

\begin{abstract}
Venice Biennale was created to magnify this wonderful city also through the encreasing of arts, so a participation of painters born or living in the Veneto region - who originally 'invented', with politicians and intellectuals, the Biennale - at the beginning was always considerable. This essay focuses on the analysis of their presence at the Biennale between 1948 and 1956, when Rodolfo Pallucchini was General Secretary of the institution. It is examined through a statistical method that allows a more complete view of this mapping. Another topic is the reception on the press, that highlights the diatribe between artists and critics.
\end{abstract}

Keywords Venetian painting. Venice Biennale. Rodolfo Pallucchini. Press review.

Sommario 1 Le partecipazioni.- 2 La rassegna stampa.

\section{Le partecipazioni}

Venezia durante la Seconda guerra mondiale era stata risparmiata da bombardamenti e rappresaglie, grazie allo status di città culturale e monumentale, ed era diventata luogo di rifugio per molti artisti. Gli anni Cinquanta

Questo saggio prende avvio da Rampazzo, Elisa (2018). I pittori veneti alle 'Biennali di Pallucchini' (1948-1956): le partecipazioni attraverso la ricezione della stampa [tesi di laurea magistrale]. Relatore Stefania Portinari; correlatore Nico Stringa, a.a. 2016/2017. Venezia: Università Ca' Foscari Venezia.

Storie dell'arte contemporanea 4 | Atlante delle Biennali 1

ISSN 2704-9973

ISBN [ebook] 978-88-6969-366-3 | ISBN [print] 978-88-6969-367-0 
rappresentano un momento di fervore culturale, con la ripresa delle attività, delle istituzioni e delle iniziative. Con la Biennale del 1948, la prima dopo il conflitto, Venezia riuscì a recuperare il ruolo di riferimento artistico internazionale e divenire il simbolo di rinascita per la città stessa. Dopo il periodo di forzato isolamento culturale, per gli artisti era importante aggiornarsi e poter liberamente testimoniare le proprie idee, anche politiche, e presentare le proprie ricerche.

Quella edizione e le quattro successive furono accomunate dalla presenza di Rodolfo Pallucchini come segretario generale, che resse la carica fino alla XXVIII edizione del 1956, coprendo quindi un arco di tempo quasi decennale. Il suo ruolo in queste Biennali fu talmente importante che a tutt'oggi le edizioni del dopoguerra sono indicate come le 'Biennali di Pallucchini' (cf. Bandera 1999; Pilo 2001). Un’altra carica istituzionale di grande rilievo e centrale per la rinascita dell'Esposizione Internazionale fu rappresentata dal commissario straordinario e presidente dell'ente, il veneziano Giovanni Ponti, primo sindaco di Venezia dopo la Liberazione. A collaborare con loro per la riuscita delle cinque edizioni della Biennale fu creata una Commissione per l'arte figurativa composta da un nutrito numero di artisti e critici.

Fondamentale per la riuscita delle Biennali del dopoguerra fu fornire al pubblico una visione ampia e eterogenea dell'arte degli ultimi decenni. Per riuscire nell'intento, Pallucchini aprì gli spazi agli sviluppi emergenti in ambito contemporaneo, lasciando margine alle correnti attuali. Pallucchini stesso definì le cinque edizioni «Biennali di aggiornamento» (Pallucchini 1979), intendendo sia il recupero d'arte di inizio Novecento trascurata nel corso del conflitto, sia il rinnovamento artistico che stava portando un cambiamento nella visione dell'arte stessa. Nell'introduzione alla XXIV Biennale del 1948 il segretario generale sostenne infatti l'esigenza di un aggiornamento richiamando i principi cardine della Prima Biennale del 1895 nata per volere del sindaco Riccardo Selvatico, del primo segretario generale dell'Esposizione Antonio Fradeletto e degli artisti veneziani dell'Accademia di Belle Arti (Pallucchini 1948).

A partire dalla sua creazione, accanto all'esigenza di affermazione internazionale, esisteva un'anima fortemente veneziana, composta dall'ambiente artistico locale che esigeva di emergere e di imporsi. L'antinomia tra l'Esposizione dal marcato segno internazionale e l'imprescindibile contesto lagunare accompagna tutto il corso della Biennale, determinando un legame complesso e molto spesso ricco di ostacoli.

I pittori veneti, per nascita o residenza, costituiscono una parte rilevante del totale delle partecipazioni in queste edizioni e attraverso un'analisi grafica si vuole far emerge questo dato. Come strumento di raccolta dei dati impiegati si fa riferimento alla consultazione dei cataloghi delle mostre oggetto di analisi. Con i primi quattro grafici si analizzano i numeri relativi alle presenze e alle opere sul totale degli artisti che parteciparono alle Biennali del dopoguerra, successi- 
vamente si pone l'attenzione sull'operato dei pittori veneti - intendendo con tale ampia accezione artisti che sono nati in Veneto, ma anche che vi operarono a lungo o ebbero significativi legami con il territorio durante gli anni della loro partecipazione a queste Biennali al punto da sentirsi essi stessi parte del milieu artistico soprattutto veneziano -, facendo emergere i nomi degli artisti che diedero un maggior contributo in termini di presenze e di numero di opere esposte.

Il grafico 1 pone a confronto le diverse edizioni dell'Esposizione Internazionale d'Arte riportando la partecipazione degli artisti: in particolare sono indicati il totale degli artisti presenti nelle diverse edizioni, suddivisi tra stranieri, indicati con il colore rosso, e italiani, indicati con il colore rosa; seguono in colore blu i pittori italiani non veneti e in colore azzurro i pittori italiani veneti, la cui somma fornisce il totale dei pittori italiani presenti.

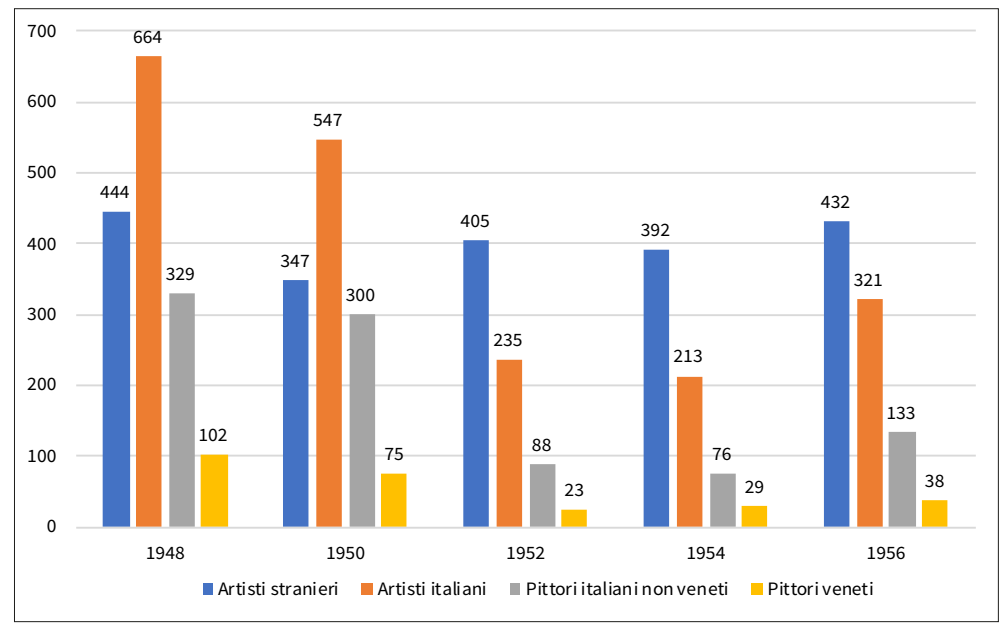

Grafico 1 Numero di artisti e pittori per edizione

Per quanto riguarda il numero degli artisti stranieri, si può notare che il loro numero non varia molto, rimanendo compreso tra i 444 artisti che parteciparono all'edizione del 1948 e i 347 artisti presenti alla Biennale del 1950.

Un andamento più dinamico lo si riscontra nella partecipazione italiana: si evidenzia, infatti, un numero elevato di presenze per l'edizione del 1948 con 664 artisti, seguito dall'edizione successiva con 547 artisti presenti. In queste due edizioni il totale degli artisti italiani supera il totale degli artisti internazionali.

Il numero delle partecipazioni italiane si dimezza con le Biennali successive del 1952 e del 1954, che riscontrano una partecipazione di 253 e 213 artisti, per poi risalire leggermente con l'edizione del 1956 con 321 artisti. 
Il numero totale di pittori italiani segue un andamento proporzionale alle presenze degli artisti italiani. Il dato sulle presenze dei pittori italiani, suddivisi tra pittori veneti e pittori non veneti, risulta maggiormente indicativo se reso in termini di percentuale usufruendo del supporto del grafico 2 .

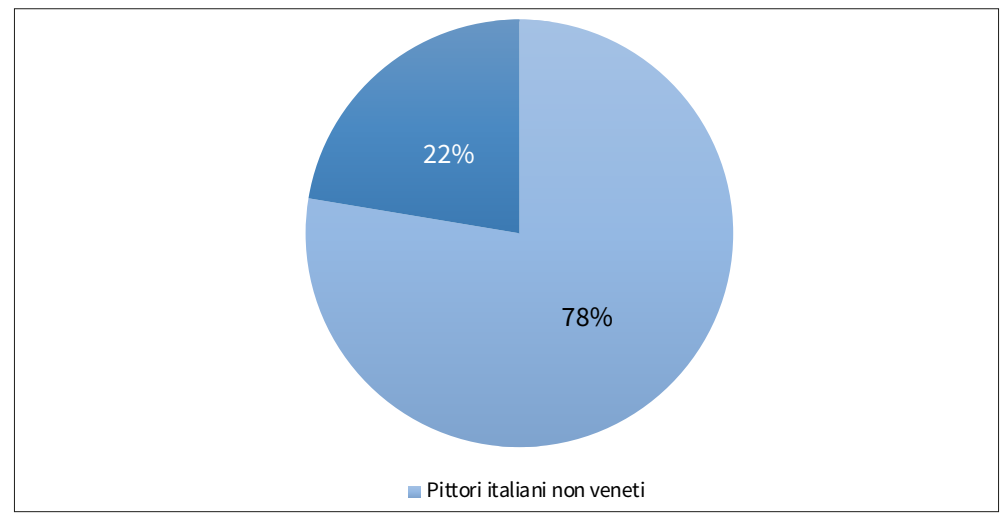

Grafico 2 Percentuale pittori veneti alle 'Biennali di Pallucchini'

Analizzando il totale di presenze alle 'Biennali di Pallucchini' emerge il dato significativo del $22 \%$ di pittori veneti sul totale di pittori italiani. Ciò significa che più di $1 / 5$ dei pittori presenti con le loro opere nelle sale della Biennale era nato o svolgeva la propria professione in Veneto. Nel 1954 si è ottenuto il dato maggiore di presenze registrando il 28\% di pittori veneti; mentre il dato di partecipazione più basso si è registrato nell'edizione del 1950 con una percentuale del $20 \%$ sul totale dei pittori italiani.

La stessa tipologia di analisi è stata eseguita nel grafico 3, comparando il numero di opere che gli artisti internazionali (in colore rosso) e italiani (in rosa) portarono alle Biennali in esame, la cui somma costituisce il numero totale delle opere presenti nelle differenti edizioni. In blu e azzurro è invece stato accostato il numero delle opere di pittori italiani non veneti e delle opere di pittori italiani veneti, la cui somma genera il totale delle opere di pittori italiani. 


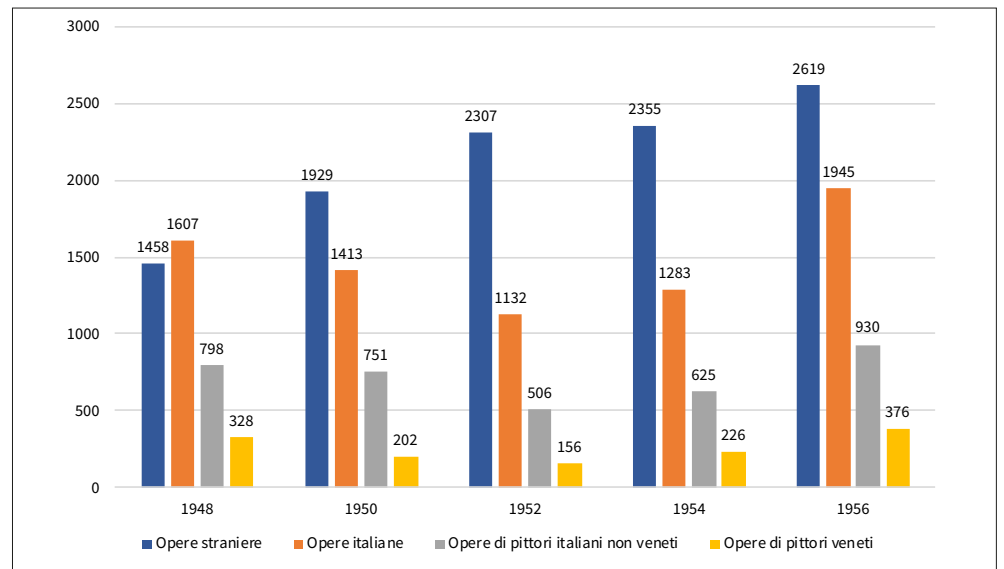

Grafico 3 Numero di opere e di pitture per edizione

Le opere degli artisti stranieri nel corso delle edizioni subì un incremento notevole, passando dalle 1.458 opere del 1948, in cui erano al di sotto del numero delle opere di artisti italiani, alle 2.619 opere dell'edizione del 1956, in cui superarono con uno scarto di più di 600 opere il numero delle italiane.

Anche in questo caso si può notare che il numero di pitture italiane segue proporzionalmente il numero delle opere italiane nelle varie edizioni. Per quanto riguarda il dato sulle pitture venete in comparazione a quelle eseguite da pittori italiani non veneti si può osservare nel grafico 4.

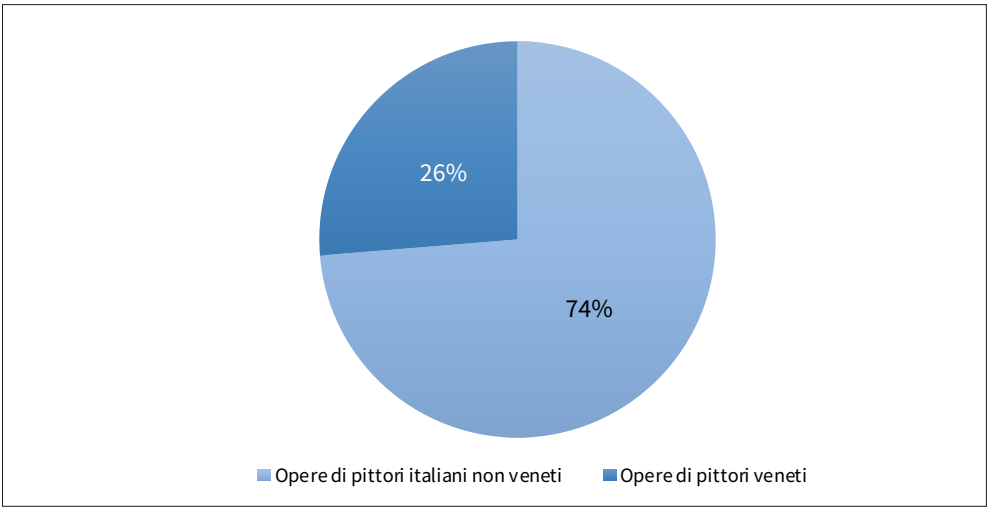

Grafico 4 Percentuale di pitture di artisti veneti alle 'Biennali di Pallucchini' 
In questo caso si riscontra una percentuale del $26 \%$ di pitture di artisti veneti sul totale di pitture di artisti italiani. Ė un dato ancor più significativo, che evidenzia che più di una su quattro delle opere esposte nel Padiglione italiano nelle varie edizioni era stata creata da un pittore veneto o operante in laguna.

Concentrando l'attenzione sull'apporto dei pittori veneti alle 'Biennali di Pallucchini', emergono alcuni dati significativi riguardanti le partecipazioni alle diverse edizioni e il numero delle opere che i pittori presentarono nel Padiglione italiano. In particolare, l'intento dei seguenti grafici è segnalare i nomi degli artisti che si distinsero in termini di presenze e di numerosità di opere.

Per quanto riguarda la partecipazione degli artisti, si è voluto evidenziare, nel grafico 5, il numero dei pittori veneti che parteciparono a una sola edizione, a due edizioni, a tre edizioni o a tutte e cinque le edizioni dell'Esposizione Internazionale d'Arte sotto la guida di Pallucchini.

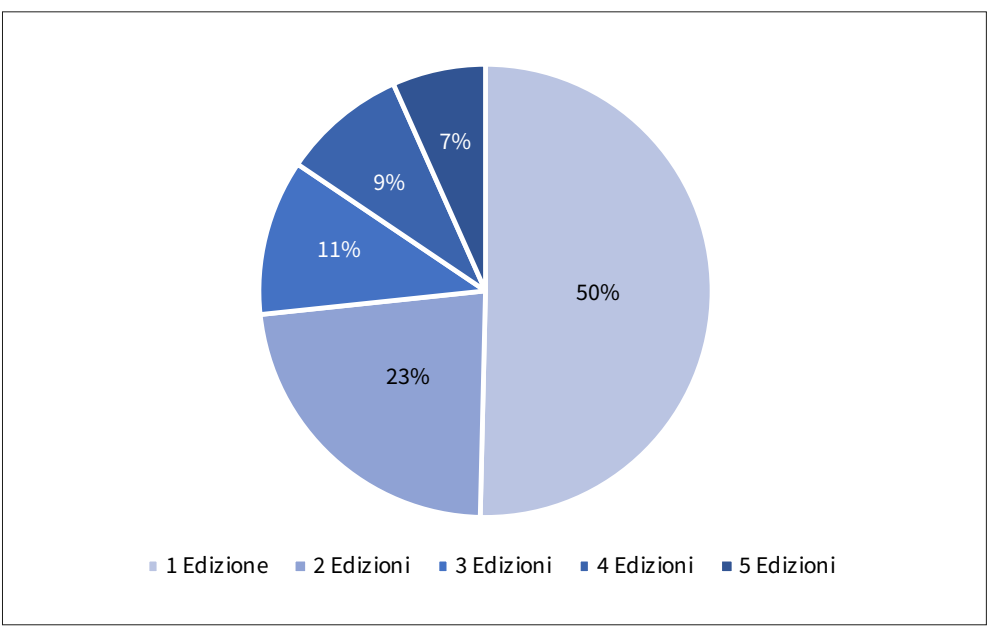

Grafico 5 Percentuale delle partecipazioni dei pittori veneti alle 'Biennali di Pallucchini'

Si evidenzia in modo significativo che il numero di pittori veneti presenti a una sola edizione fu esattamente la metà, raggiungendo il $50 \%$, ossia 68 pittori su un totale di 135. Un altro dato significativo è che solamente il $7 \%$ dei pittori veneti fu presente a tutte e cinque le edizioni in esame. Questi ultimi sono oggetto di un'ulteriore analisi riportata nel grafico 6 . 


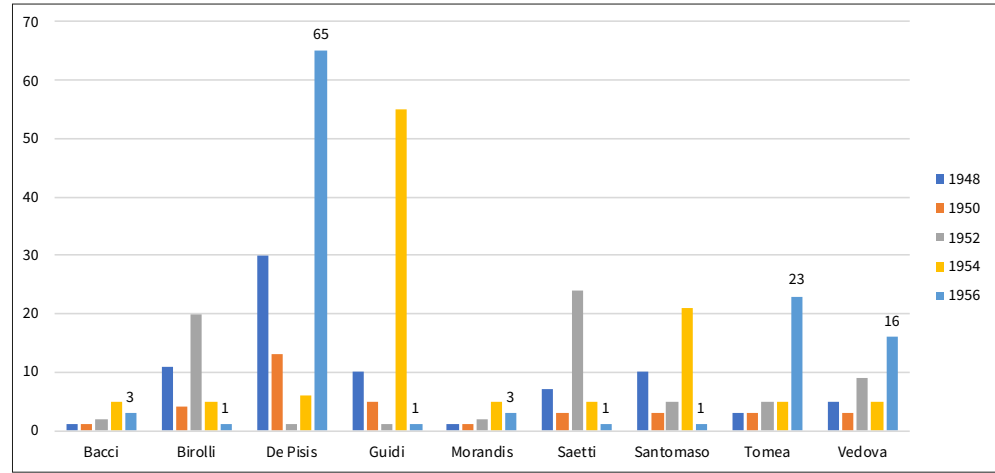

Grafico 6 Numero di opere di artisti veneti presenti a tutte le edizioni delle 'Biennali di Pallucchini'

Per un totale di 135 pittori veneti presenti alle Biennali dal 1948 al 1956, gli artisti che parteciparono a tutte e cinque le edizioni furono nove. Poiché naturalmente questo significa notevole visibilità di critica e di pubblico nel contesto artistico internazionale, si tratta di artisti allora molto noti quali Edmondo Bacci, Renato Birolli, Virgilio Guidi, Gino Morandis, Bruno Saetti, Giuseppe Santomaso, Fiorenzo Tomea, Emilio Vedova. Questa partecipazione consentì a due di loro, Saetti e Santomaso, di aggiudicarsi anche l'ambito Premio di pittura per un artista italiano.

Edmondo Bacci e Gino Morandis furono presenti nelle cinque edizioni esponendo un totale di 12 opere ciascuno. Entrambi riscossero un modesto successo aderendo al Movimento spazialista veneziano. Nell'Esposizione del 1952 esposero affiancati nella sala XIV, a testimonianza del legame artistico che li univa. Bacci fu presente con ammissione da parte della Giuria a tutte le edizioni della Biennale in esame, tranne che su invito alla Biennale del 1952 con la possibilità di presentare due disegni. Morandis, invece, venne ammesso dalla Giuria alle edizioni del 1948, 1950 e 1956, mentre fu presente su invito per le edizioni del 1952 e 1954.

Il ventinovenne Emilio Vedova fu presente per la prima volta alla Biennale nell'edizione del 1948, aderendo al gruppo del Fronte Nuovo delle Arti. Presente sempre su invito da parte della Commissione alle cinque edizioni in esame, totalizzò un numero di 38 opere esposte e nel 1956 si aggiudicò una personale di 16 opere.

Fiorenzo Tomea partecipò a tutte le 'Biennali di Pallucchini' esponendo un totale di 39 opere. Fu sempre presente su invito nel corso delle cinque edizioni riscuotendo molto successo; nell'edizione del 1956 gli venne dedicata una personale di 23 opere.

Giuseppe Santomaso fu uno degli artisti che si contraddistinse maggiormente nel corso delle Biennali del dopoguerra esponendo 40 
opere nelle cinque Esposizioni. Il suo legame con la Biennale fu sempre forte sin dalla prima edizione del dopoguerra, tanto che nel 1952 si aggiudicò un posto all'interno della Commissione esecutiva. L'edizione a cui sicuramente riscosse maggior successo fu quella del 1954 che gli valse il riconoscimento di uno dei Gran Premi, il Premio per un pittore italiano. Nella Biennale successiva, a dimostrazione della fama ottenuta, venne inserito nella mostra Presenze, in cui espose assieme ad altri grandi maestri del suo tempo (cf. Stringa 2017).

Il docente dell'Accademia di Belle Arti di Venezia Bruno Saetti si aggiudicò il primo premio per un pittore nell'Esposizione del 1952, alla quale era stato invitato a esporre una personale di 24 opere. Presente in tutte le edizioni su invito, all'epoca era considerato uno dei maggiori esponenti del panorama artistico italiano.

Il veronese Renato Birolli, con 41 opere presentate nel corso delle cinque edizioni delle Biennali in oggetto, è stato senza dubbio uno degli artisti maggiormente attivi e apprezzati dalla critica.

I due artisti che si contraddistinsero maggiormente per l'apporto non solo numerico ma anche per notorietà nel corso delle 'Biennali di Pallucchini' furono Filippo de Pisis e Virgilio Guidi. Entrambi 'veneziani' ma di adozione - e in particolare per de Pisis solo per circa cinque anni, ma il loro legame davvero intenso con la città ci porta ad annoverarli tra queste presenze. ${ }^{1}$ Le Biennali del dopoguerra rappresentarono per entrambi un'occasione di celebrazione e riconoscimento delle loro carriere. Virgilio Guidi presentò 72 opere nel corso delle cinque edizioni e di particolare rilievo fu la personale di 55 opere alla Biennale del 1954. Si tratta di una delle maggiori mostre mai realizzate all'interno della Biennale per un artista ancora in vita. Il legame tra Filippo de Pisis e la Biennale iniziò invece prima della Seconda guerra mondiale, tanto che nel 1948 gli venne subito dedicata una mostra personale in cui poté esporre 30 opere. Nei cataloghi della Biennale del 1948 e del 1950 de Pisis era segnalato come residente nella città lagunare, ma negli anni successivi si dovette trasferire a Brugherio per essere ricoverato in una casa di cura. Una seconda personale era in programma per l'edizione del 1956, che si tramutò in retrospettiva a causa della sua morte avvenuta pochi mesi prima dell'inizio della Biennale.

Attraverso questo campione di artisti, si può notare la compresenza di varie personalità e correnti. Si va da pittori ampiamente ri-

1 In particolare de Pisis ha residenza a Venezia solo dal 1943 al 1948, ma è per noi particolarmente interessante che Pallucchini avesse scritto un testo per lui in una cartella di grafica edita dalla Galleria del Cavallino nel 1944 (in occasione della sua personale) e alla Biennale del 1948, nell'anno del suo ricovero a villa Margherita a Brugherio, gli sia dedicata una sala personale con una retrospettiva di opere dal 1926 al 1948 presentata proprio dallo stesso Pallucchini. Anche Afro viene qui un poco irritualmente considerato tra i 'veneti' per la sua vicinanza all'ambiente e in particolare al Gruppo degli Otto Pittori Italiani che vede la sua presentazione ufficiale alla Biennale del 1952. 
conosciuti come de Pisis, Guidi e Saetti, ai componenti dei collettivi artistici che caratterizzano il secondo dopoguerra come Birolli, Santomaso e Vedova per il Fronte Nuovo delle Arti ed il successivo Gruppo degli Otto e Bacci, Morandis e nuovamente Guidi per il ramo veneziano dello Spazialismo. Gli artisti maggiormente presenti a queste edizioni appartenevano alla generazione di mezzo che si stava artisticamente affermando proprio nei primi anni del dopoguerra. In questo modo la Biennale dimostra di essere un'istituzione attenta a comprendere i diversi stili e l'eterogeneità delle correnti artistiche.

Mantenendo una visione d'insieme sul totale degli artisti veneti e le opere da essi presentate, si prenda in considerazione il grafico 7.

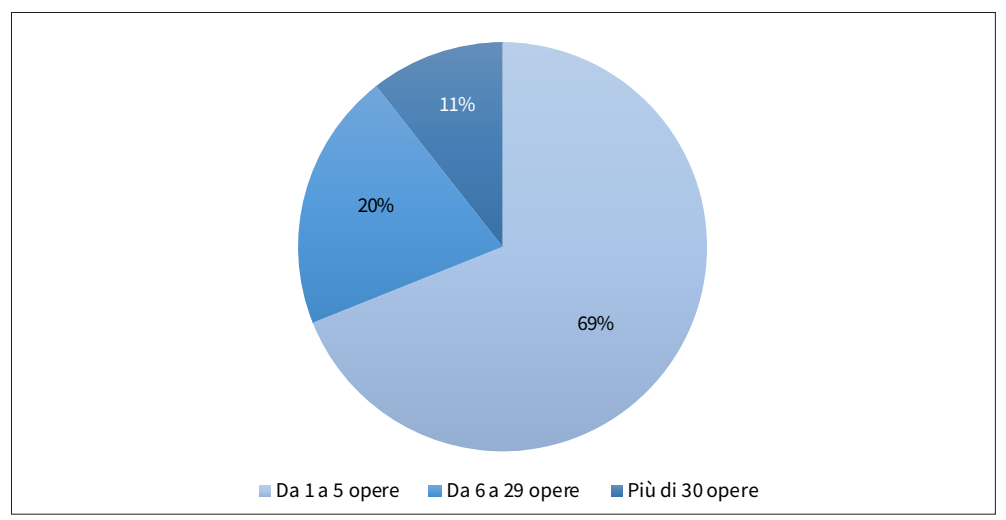

Grafico 7 Rapporto tra numero di pittori veneti e numero di opere presentate nelle 5 edizion delle 'Biennali di Pallucchini'

Il grafico esamina le 1.288 opere presentate dai 135 pittori veneti nel corso delle 'Biennali di Pallucchini', mettendo in evidenza il numero di opere presentato singolarmente da ogni artista. Il dato percentuale che emerge con più evidenza è che il 69\%, che equivale a 91 pittori veneti, presentò nei dieci anni d'Esposizione da 1 a 5 opere; il $20 \%$, ossia 27 pittori veneti, parteciparono con un numero di opere compreso tra 6 e 29 opere; mentre solo l'11\%, quindi 14 pittori veneti, esposero più di 30 opere nel corso delle edizioni.

Nel prossimo grafico si riscontra invece nello specifico quali artisti esposero il maggior numero di opere tra tutte e cinque le edizioni delle Biennali dal 1948 al 1956. Non sono presenti nel grafico gli artisti che, anche se rientrano nel $11 \%$ del grafico precedente, erano presenti alle Biennali in oggetto unicamente con una mostra retrospettiva. Data la dovuta premessa, poniamo l'attenzione sul grafico 8. 


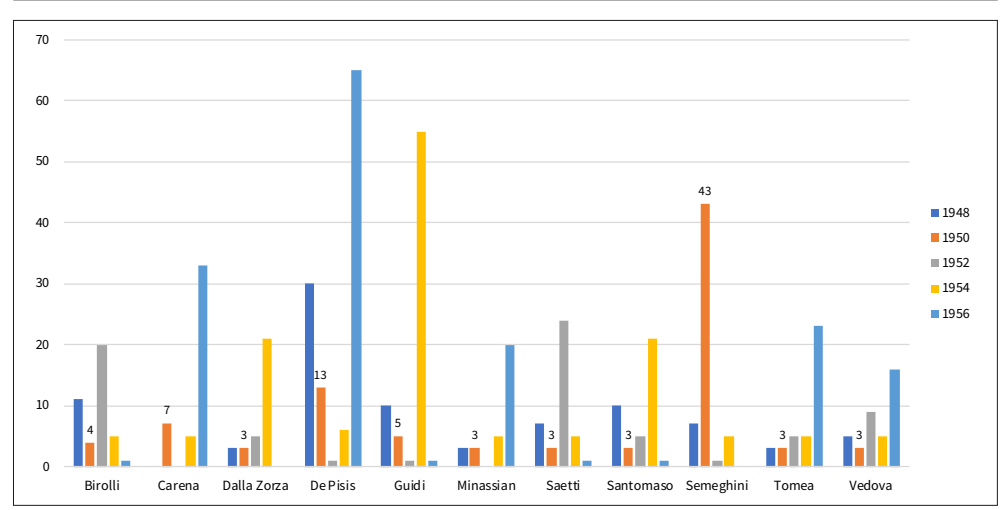

Grafico 8 Artisti che rappresentarono più di 30 opere alle 'Biennali di Pallucchini'

Comparando il grafico 8 con il grafico 6, in cui sono rappresentati gli artisti presenti a tutte e cinque le edizioni, si può notare che compaiono nuovamente Birolli, de Pisis, Guidi, Saetti, Santomaso, Tomea e Vedova, mentre non sono presenti nel nuovo grafico Bacci e Morandis, poiché non hanno totalizzato più di 30 opere presenti in totale, nonostante la presenza a tutte le edizioni in esame. In questo grafico, gli artisti che sono maggiormente rappresentati sono nuovamente de Pisis e Guidi.

Gli artisti che raggiunsero un numero considerevole di opere esposte nonostante non fossero presenti a tutte e cinque le edizioni della Biennale furono Pio Semeghini, Felice Carena, Carlo Dalla Zorza e Leone Minassian.

Pio Semeghini presentò 56 opere complessivamente, soprattutto grazie alla mostra personale di 43 opere organizzata per la Biennale del 1950. Fu molto impegnato anche nella vicenda organizzativa dell'Esposizione: infatti prese parte alla Commissione per le arti figurative nell'edizione del 1948. Anche Felice Carena si contraddistinse per l'elevato numero di opere presentate, grazie in particolare alla personale del 1956 di 33 opere riuscì a presentare al pubblico 45 dipinti nel corso delle Biennali in esame. Tra gli artisti che esposero più opere troviamo poi uno dei maggiori interpreti dei paesaggi lagunari, ossia il veneziano Carlo Dalla Zorza, con 32 opere. Leone Minassian, originario di Costantinopoli, visse a Venezia e in queste Biennali espose un totale di 31 opere.

Possiamo dunque comprendere che si trattasse di pittori che operavano a Venezia da molti anni e che raggiunsero una notevole fama a livello nazionale, in particolare i docenti dell'Accademia Semeghini e Carena, ormai settantenni. Ma anche i più giovani Dalla Zorza e Minassian si stavano affermando a livello nazionale.

Questa analisi si può esemplificare anche in questo elenco alfabetico che segue, con i 135 pittori veneti, di nascita o di residenza, che parteciparono alle edizioni dell'Esposizione Internazionale d'Arte 
dal 1948 al 1956: per ogni edizione viene riportato il numero di opere esposte da ciascun artista.

Con questa mappatura si è voluto fornire un metodo per risalire in modo immediato agli artisti che presero parte alle 'Biennali di Pallucchini' e al numero di opere che presentarono.

\begin{tabular}{|c|c|c|c|c|c|c|}
\hline \multicolumn{7}{|c|}{ Elenco pittori veneti con numero di opere per edizione } \\
\hline \multicolumn{2}{|c|}{ Pittori } & \multicolumn{5}{|c|}{ Numero di opere } \\
\hline Cognome & Nome & $\begin{array}{l}\text { ed. XXIV } \\
1948\end{array}$ & $\begin{array}{l}\text { ed. XXV } \\
1950\end{array}$ & $\begin{array}{l}\text { ed. XXVI } \\
1952\end{array}$ & $\begin{array}{l}\text { ed. XXVII } \\
1954\end{array}$ & $\begin{array}{l}\text { ed. XXVIII } \\
1956\end{array}$ \\
\hline Bacci & Edmondo & 1 & 1 & 2 & 5 & 3 \\
\hline Bagattini & Vittorino & & 1 & & & \\
\hline Barbaro & Saverio & & 1 & & & 3 \\
\hline Basaldella & Afro & & & $9 P$ & 5 & $11 \mathrm{P}$ \\
\hline Bergamin & Maria & & 1 & & & \\
\hline Bergamini & Aldo & 3 & 1 & & & \\
\hline Biasion & Renzo & 1 & 3 & & & \\
\hline Birolli & Renato & $11 \mathrm{M}$ & 4 & $20 P$ & 5 & $1 \mathrm{M}$ \\
\hline Boccato & Ugo & 1 & & & & \\
\hline Boldrini & Gustavo & & & & & 3 \\
\hline Borsato & Renato & & & & 5 & 3 \\
\hline Brass & Italico & $13 \mathrm{R}$ & & & & \\
\hline Breddo & Gastone & 5 & 3 & 5 & 5 & \\
\hline Brunello & Luigi & 1 & & & & \\
\hline Butera & Remigio & 3 & 2 & 5 & & \\
\hline Cadorin & Guido & 1 & 1 & $1 \mathrm{M}$ & & \\
\hline $\begin{array}{l}\text { Cagnaccio di } \\
\text { San Pietro }\end{array}$ & & $5 \mathrm{R}$ & & & & \\
\hline Cancian & Sante & $5 \mathrm{R}$ & & & & \\
\hline Candiani & Luigi & 1 & 1 & & & \\
\hline Capisani & Matelda & & & & & 3 \\
\hline Carena & Felice & & 7 & & 5 & $33 \mathrm{P}$ \\
\hline Carli & Valeria & & 1 & & & \\
\hline Carraro & Mario & & 1 & & & \\
\hline $\begin{array}{l}\text { Carrer } \\
\text { Battaglia }\end{array}$ & Attilio & & 1 & & & \\
\hline Carrer & Guido & 1 & 1 & & & \\
\hline Casarini & Pino & 1 & & & & \\
\hline Casarotti & Alberto & & 1 & & & \\
\hline Cavaglieri & Mario & 5 & 3 & & & \\
\hline Cavallet & Oscar & 1 & 1 & & & \\
\hline Celeghin & Orazio & 1 & 1 & & & 3 \\
\hline Cobianco & Luigi Vincenzo & 1 & 1 & & & 3 \\
\hline Coletti & Nando & 1 & 1 & & & \\
\hline Legenda $\quad M$ & mostra $\mathrm{P}=$ Per & sonale & $=$ Retrosp & ttiva & & \\
\hline
\end{tabular}




\begin{tabular}{|c|c|c|c|c|c|c|}
\hline \multicolumn{7}{|c|}{ Elenco pittori veneti con numero di opere per edizione } \\
\hline \multicolumn{2}{|c|}{ Pittori } & \multicolumn{5}{|c|}{ Numero di opere } \\
\hline Cognome & Nome & $\begin{array}{l}\text { ed. XXIV } \\
1948 \\
\end{array}$ & $\begin{array}{l}\text { ed. XXV } \\
1950 \\
\end{array}$ & $\begin{array}{l}\text { ed. XXVI } \\
1952 \\
\end{array}$ & $\begin{array}{l}\text { ed. XXVII } \\
1954\end{array}$ & $\begin{array}{l}\text { ed. XXVII } \\
1956 \\
\end{array}$ \\
\hline Consolo & Paola & $5 \mathrm{R}$ & & & & \\
\hline Conversano & Romano & 1 & & & & \\
\hline Crepet & Angelo Mario & & & & & 3 \\
\hline Cristofoletti & Ciro & 1 & & & & \\
\hline Cristofoletti & Eugenio & 1 & 1 & & & \\
\hline Cusin & Federico & 1 & & & & \\
\hline Dalla Zorza & Carlo & 3 & 3 & 5 & $21 \mathrm{P}$ & \\
\hline Dal Prà & Amleto & 1 & & & & \\
\hline Darzino & Bruno & 1 & 1 & & & \\
\hline Da Venezia & Eugenio & 3 & 1 & & & 3 \\
\hline De Grandis & Luigina & & 1 & & & \\
\hline Deluigi & Mario & 4 & 1 & 5 & 5 & \\
\hline De Pisis & Filippo & $30 P$ & 13 & $1 \mathrm{M}$ & 6 & $65 \mathrm{R}$ \\
\hline De Stefani & Girolamo & & & & & 3 \\
\hline Dinon & Mario & 2 & 1 & & & 3 \\
\hline Disertori & Mario & 2 & 1 & & & \\
\hline Farina & Guido & 3 & 1 & & & \\
\hline Fasan & Antonio & 2 & 3 & & 5 & \\
\hline Favai & Gennaro & 2 & & & & \\
\hline Favretto & Giacomo & & $21 \mathrm{R}$ & & & \\
\hline Ferro & Antonio & 1 & 1 & & & \\
\hline Fontanarosa & Rosetta & 1 & & & & \\
\hline Franzoni & Aldo & 1 & & & & \\
\hline Frumi & Lotte & 2 & & & 3 & 3 \\
\hline Fulgenzi & Toni & 1 & 1 & & & \\
\hline Furlotti & Bruno & 1 & & & & \\
\hline Galletti & Giuseppe & & 1 & & & \\
\hline Gaspari & Luciano & 3 & 3 & 5 & & 3 \\
\hline Gasparini & Bruna & 1 & 1 & & & \\
\hline Gianniotti & Teodoro & & & & $9 R$ & \\
\hline Gianquinto & Alberto & & & & & 3 \\
\hline Guidi & Virgilio & 10 & 5 & $1 \mathrm{M}$ & $55 \mathrm{P}$ & $1 \mathrm{M}$ \\
\hline Hollesch & Carlo & & 1 & & 3 & \\
\hline Jodi & Casimiro & 1 & & & & \\
\hline Lanaro & Dino & 1 & 2 & & & 3 \\
\hline Lazzaro & Dino & 1 & 1 & & & \\
\hline Leoncini & Marcello & 1 & & & & \\
\hline Lovisetto & Aldo & & 1 & & & \\
\hline Lucatello & Albino & & & & & 3 \\
\hline Legenda & nostra $\mathrm{P}=\mathrm{Pe}$ & sonale & $=$ Retrosp & ttiva & & \\
\hline
\end{tabular}




\begin{tabular}{|c|c|c|c|c|c|c|}
\hline \multicolumn{7}{|c|}{ Elenco pittori veneti con numero di opere per edizione } \\
\hline \multicolumn{2}{|c|}{ Pittori } & \multicolumn{5}{|c|}{ Numero di opere } \\
\hline Cognome & Nome & $\begin{array}{l}\text { ed. XXIV } \\
1948 \\
\end{array}$ & $\begin{array}{l}\text { ed. XXV } \\
1950 \\
\end{array}$ & $\begin{array}{l}\text { ed. XXVI } \\
1952 \\
\end{array}$ & $\begin{array}{l}\text { ed. XXVII } \\
1954\end{array}$ & $\begin{array}{l}\text { ed. XXVIII } \\
1956\end{array}$ \\
\hline Majoli & Giovanni & 1 & & & & \\
\hline Martini & Alberto & & & 2 & & $114 \mathrm{R}$ \\
\hline Minassian & Leone & 3 & 3 & & 5 & $20 P$ \\
\hline Moggioli & Umberto & $5 \mathrm{R}$ & & & & \\
\hline Morandis & Gino & 1 & 1 & 2 & 5 & 3 \\
\hline Mori & Neno & 3 & 3 & & 5 & 3 \\
\hline Mušič & Anton Zoran & 2 & 3 & & 5 & $12 \mathrm{P}$ \\
\hline Nardi & Antonio & 1 & & & & \\
\hline Novati & Marco & 2 & 1 & 5 & 5 & \\
\hline Padoa & Laura & 1 & 1 & & & 3 \\
\hline $\begin{array}{l}\text { Paganin } \\
\text { Vescovi }\end{array}$ & Dolores & 1 & & & & \\
\hline Paolucci & Giorgio Daio & & & & 5 & 3 \\
\hline Pendini & Fulvio & 1 & 1 & 2 & 3 & \\
\hline Perina & Giulio & & 1 & & & \\
\hline Perissinotti & Lino & 1 & & & & \\
\hline Perotti & Francesco & 3 & 3 & & 5 & \\
\hline Pizzinato & Armando & $5 \mathrm{M}$ & 3 & 5 & 5 & \\
\hline Polato & Aldo & 1 & & & & \\
\hline Polato & Attilio & 1 & & & & \\
\hline Poli & Ebe & & 1 & & & \\
\hline Pomi & Alessandro & 1 & & & & \\
\hline Ponti & Pino & 1 & & & & \\
\hline Pontini & Giovanni & & & & & 3 \\
\hline Pornaro & Alessandro & 1 & & & & \\
\hline Potenza & Primo & 1 & & & & 3 \\
\hline Rampin & Saverio & & 1 & & & \\
\hline Ravenna & Juti & 5 & 3 & & & \\
\hline Rizzetto & Ezio & 3 & & & & 3 \\
\hline Roma & Gina & 1 & 1 & & & 3 \\
\hline Rossi & Gino & $55 \mathrm{R}$ & & & & \\
\hline Saetti & Bruno & 7 & 3 & $24 \mathrm{P}$ & 5 & $1 \mathrm{M}$ \\
\hline Santomaso & Giuseppe & $10 M$ & 3 & 5 & $21 \mathrm{P}$ & $1 \mathrm{M}$ \\
\hline Scarpa & Gino & 1 & & & & \\
\hline Scarpa Croce & Luigi & 1 & 1 & & & \\
\hline Scattola & Ferruccio & 3 & & & & \\
\hline Seibezzi & Fioravante & 3 & 3 & 5 & & \\
\hline Semeghini & Pio & 7 & $43 \mathrm{P}$ & $1 \mathrm{M}$ & 5 & \\
\hline Spadon & Vera & 1 & 1 & & & \\
\hline Legenda $M$ & mostra $\mathrm{P}=\mathrm{P}$ & sonale & $=$ Retrosp & ettiva & & \\
\hline
\end{tabular}




\begin{tabular}{|c|c|c|c|c|c|c|}
\hline \multicolumn{7}{|c|}{ Elenco pittori veneti con numero di opere per edizione } \\
\hline \multicolumn{2}{|c|}{ Pittori } & \multicolumn{5}{|c|}{ Numero di opere } \\
\hline Cognome & Nome & $\begin{array}{l}\text { ed. XXIV } \\
1948 \\
\end{array}$ & $\begin{array}{l}\text { ed. XXV } \\
1950 \\
\end{array}$ & $\begin{array}{l}\text { ed. XXVI } \\
1952 \\
\end{array}$ & $\begin{array}{l}\text { ed. XXVII } \\
1954\end{array}$ & $\begin{array}{l}\text { ed. XXVIII } \\
1956\end{array}$ \\
\hline Spadon & Wilma & 1 & 1 & & & \\
\hline Springolo & Nino & 5 & 3 & & & \\
\hline Tavella & Aldo & & 1 & & & \\
\hline Tomea & Fiorenzo & 3 & 3 & 5 & 5 & $23 P$ \\
\hline Tommasini & Annamaria & 3 & & & & \\
\hline Tonello & Armando & 1 & 1 & & & \\
\hline Tosi & Salvatore & 1 & 1 & & & \\
\hline Trentini & Guido & 2 & 1 & & & \\
\hline Trentini & Nurdio & 1 & 1 & & & \\
\hline Tursi & Francesco & $3 R$ & & & & \\
\hline $\begin{array}{l}\text { Urbani De } \\
\text { Gheltof }\end{array}$ & Giuseppe & 1 & & & & \\
\hline Valenzin & Giorgio & 1 & 1 & & & \\
\hline Varagnolo & Mario & 2 & 1 & & 5 & 3 \\
\hline Variola & Angelo & 1 & & & & \\
\hline Varnier & Delfino & 1 & & & & \\
\hline Vedova & Emilio & $5 \mathrm{M}$ & 3 & $9 P$ & 5 & $16 \mathrm{P}$ \\
\hline Vianello & Vinicio & 1 & & & & \\
\hline Villa & Rino & 1 & & & & \\
\hline Vitturi & Albano & 1 & & & & \\
\hline Zandomeneghi & Federico & & & $32 \mathrm{R}$ & & \\
\hline Zanutto & Angelo & 2 & & & & \\
\hline Zanutto & Renzo & & 1 & & & \\
\hline Zorzi & Cesare & 1 & & & & \\
\hline Zotti & Carmelo & & & & & 3 \\
\hline Zuccheri & Luigi & & 1 & & & \\
\hline Legenda & mostra $\mathrm{P}=1$ & onale & $=$ Retrosp & ttiva & & \\
\hline
\end{tabular}

\section{La rassegna stampa}

La ricezione della stampa è stata compiuta sui fondi dell'Archivio Storico della Biennale. Gli articoli della Raccolta Documentaria di Arti Visive della Biennale di Venezia per l'arco temporale 1948-1957 furono raccolti in maniera accurata e selezionata dal personale della Biennale dell'epoca, che li suddivise per anno e per provenienza. Certamente la raccolta può non rappresentare una rassegna stampa completa, tuttavia consente una mappatura e un riferimento parziale esaustivo per farne riverbero di una considerazione generale.

Il totale degli articoli analizzati che citano al loro interno gli artisti veneti è di 187; un numero notevole che lascia comprendere quanto 
la Biennale fosse presente sulla stampa italiana. I dati che emergono dalla selezione della Raccolta Documentaria mostrano un panorama ampio e articolato, che non riguarda strettamente l'area veneziana ma coinvolge tutta la penisola italiana. Sono 35 gli articoli editi a Roma, provenienti dalla maggior parte dei quotidiani nazionali; mentre sono 23 gli articoli di giornali pubblicati a Venezia, con una risonanza per lo più locale. Tra i quotidiani con il maggior numero di articoli in cui si citano pittori veneti sono: Il Gazzettino di Venezia con 12 articoli e L'Unità con 13 articoli. I quotidiani Minosse di Venezia e L'Arena di Verona contano 7 articoli ciascuno.

Ė Silvio Branzi, giornalista e critico d'arte, a redigere il maggior numero di articoli sulla Biennale e sulle partecipazioni venete. Nel 1950 propone un articolo su Minosse intitolato Artisti cittadini in progresso, il cui intento è di «rintracciare tutti i nostri amici di casa», facendo riferimento ad artisti che vivono e operano a Venezia, all'interno del Padiglione italiano. ${ }^{2}$ Si complimenta con il pittore Deluigi per «l'identità» dei suoi dipinti che si riconosce «a prima vista», così come per quelli di Morandis. Un giudizio positivo lo riserva anche per le opere proposte da Santomaso, che si contraddistinguono per «l'elevatura qualitativa delle soluzioni cromatiche». Un giudizio più severo viene destinato ai pittori Mario Dinon e Gastone Breddo. Tra le pittrici presenta Maria Bergamin come la rivelazione della XXV Biennale, mentre si è sentito «sconfortato» dal lavoro di Laura Padoa. Tra i quadri migliori della Biennale individua la pittura di Bacci, Seibezzi, Gaspari, Vedova, Santomaso e Carena.

Con la sua analisi Branzi fornisce una visione ampia dell'operato artistico dei pittori venenti all'Esposizione. Sempre suo è Troppe le opere esposte nel Padiglione dell'Italia, pubblicato ne Il Gazzettino nel $1950 .^{3}$ Branzi inserì all'interno dell'articolo un paragrafo dedicato all'analisi dei «Veneti e no», in cui cita brevemente l'operato di numerosi pittori veneti (Bergamini, Birolli, Breddo, Campigli, Candiani, Carena, Celeghin, Cesetti, Cobianco, Da Venezia, Dalla Zorza, de Pisis, Disertori, Farina, Guberti, Mori, Mušič, Paolucci, Pizzinato, Poli, Saetti, Scarpa Croce, Seibezzi, Springolo, Tomea, Vedova, Zanutto). Sempre sul periodico Minosse trova spazio per la XXVI Biennale un articolo intitolato «Veneziani che s'impongono». In questa edizione gli artisti che richiamano l'attenzione del giornalista sono Vedova e Deluigi. Per quanto riguarda Breddo sostiene che sia stato «male collocato e trascurato» aggiungendo che «si sia presentato seriamente e da pittore, con tutte le culture, se volete, ma non rinun-

2 Silvio Branzi, «Artisti cittadini in progresso», Minosse, 9 luglio 1950.

3 Silvio Branzi, «Troppe le opere esposte nel Padiglione dell'Italia», Il Gazzettino, 11 ottobre 1950.

4 Silvio Branzi, «Veneziani che s'impongono», Minosse, 28 giugno 1952. 
ciando al sentimento». Ritiene Santomaso «coerente e deciso», Remigio Butera «fedelissimo al suo modulo», mentre riscontra una delusione in Pizzinato e Cadorin. Di Guidi sostiene che «tra i maestri è il vero maestro» con «uno dei più bei quadri di tutta la Biennale». Aggiunge una nota polemica all'articolo domandandosi se «dopo aver visto centinaia di quadri inutili» si è «pensato ai nostri artisti esclusi come Celeghin, Cobianco, Mušič, Minassian».

Per quanto riguarda l'analisi del totale degli artisti veneti presenti nelle cinque edizioni del dopoguerra della Biennale, emerge che sono 64 gli artisti citati sui periodici presenti nella Raccolta Documentaria di Arti Visive della Biennale di Venezia. ${ }^{5}$

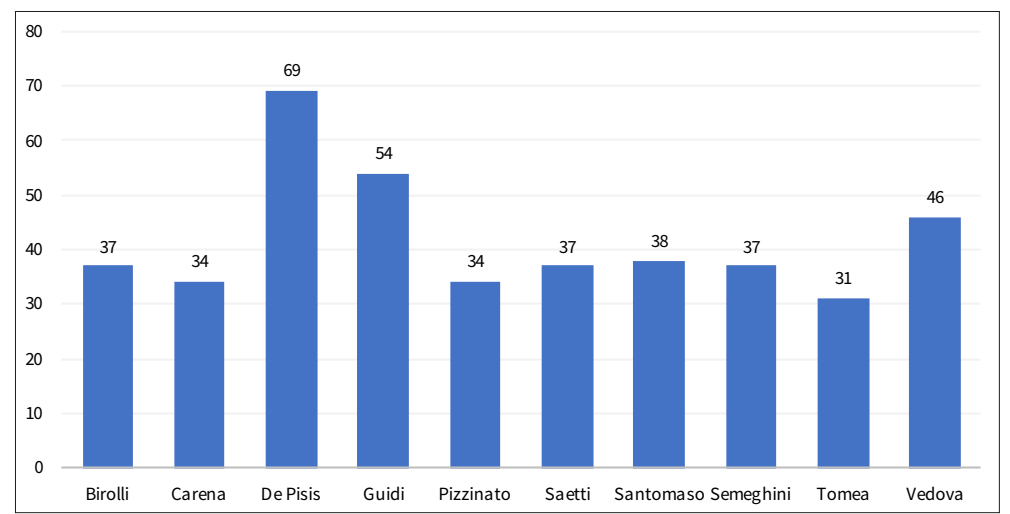

Grafico 9 Pittori veneti più citati sui stampa

Il grafico 9 vuole evidenziare i nomi dei dieci artisti con il maggior numero di citazioni all'interno della Raccolta Documentaria. Sono gli artisti di maggior rilievo nel panorama non soltanto locale ma anche nazionale; molti di loro inoltre esposero in mostre personali. Paragonandolo con il grafico 8 proposto precedentemente, si nota la quasi totale corrispondenza negli artisti. In entrambi i grafici ritornano Birolli, Carena, de Pisis, Guidi, Saetti, Santomaso, Semeghini, Tomea e Vedova. Ovviamente non è un caso che gli artisti con il maggior numero di opere siano anche quelli che riuscirono a riscuotere maggior risalto da parte della critica. Si evidenzia anche una correlazione tra il numero totale di opere proposte e gli articoli dedicati agli artisti: infatti, in entrambi i grafici compare al primo posto Filippo de Pisis, che totalizza sia il maggior numero di opere esposte sia di articoli a lui dedicati; è seguito, in entrambi i grafici, da Virgilio Guidi.

5 Il numero delle presenze evidenziate non vuole essere un indicatore dell'importanza del pittore nel panorama artistico, ma vuole fornire un dato di ricerca. 
La maggior parte degli articoli presenti all'interno della Raccolta Documentaria tratta le vicende generali che caratterizzarono le Esposizioni, in particolare si concentrano sul periodo della vernice nel mese di giugno con l'inaugurazione dell'Esposizione da parte del presidente della Repubblica, dando particolare rilievo alle partecipazioni straniere, in particolare agli artisti del Padiglione francese. Un'altra argomentazione all'interno della stampa nazionale sono le retrospettive dedicate agli artisti da poco scomparsi. Tra quelle dedicate agli artisti italiani si nota un accrescimento dell'attenzione nell'edizione del 1956 per la presenza delle retrospettive di Filippo de Pisis e Arturo Tosi.

Molti articoli, inoltre, sono incentrati sulla discussione relativa alle presenze numeriche degli artisti alle varie edizioni della Biennale. La polemica venne mossa dagli artisti a seguito della decisione della Commissione per le arti figurative di alleggerire e «sprovincializzare» il Padiglione italiano e la Biennale nel suo complesso, con l'idea di riscuotere un maggior successo di critica e di rilievo internazionale. Questa scelta fu perseguita da Pallucchini per tutto il corso della sua carica decennale, divenendo uno degli scopi del suo mandato. Si nota, infatti, un'evoluzione dalla prima Biennale del 1948, in cui vennero invitati e ammessi numerosi artisti seguendo il principio di varietà e inclusione a seguito del conflitto, all'ultima Biennale dell'era Pallucchini del 1956, nella quale il loro numero era stato notevolmente ridotto, ma ancora generava accese proteste da parte della critica poiché percentualmente superiore rispetto a quello dei Padiglioni stranieri.

La partecipazione degli artisti era sottoposta alla decisione della Commissione che poteva scegliere di ammettere a esporre tramite invito diretto o tramite accettazione della candidatura sottoponendo le opere alla valutazione della Giuria. La Commissione per le arti figurative giocava quindi un ruolo primario nella presenza degli artisti alla Biennale e le numerose modifiche nella sua conformazione comportarono altrettanti cambiamenti di giudizi e scelte nelle ammissioni. Non sorprende quindi se nacquero numerose proteste da parte degli artisti coinvolti, che chiesero nel corso delle edizioni maggior chiarezza e rappresentatività all'interno della Commissione e della Giuria giudicante.

Per quanto riguarda la prima edizione del dopoguerra, quella del 1948, gli artisti ammessi per invito furono 407, mentre gli artisti scelti per Giuria furono 231. Ciò che destò maggior disapprovazione da parte della critica, oltre all'eccessiva presenza numerica, fu l'ammissione con la possibilità di presentare un numero limitato di opere per artista, che pareva insufficiente per dimostrare adeguatamente il valore artistico di ciascuno.

La Commissione della XXV Biennale del 1950 proseguì lungo la linea impostata nell'edizione precedente; si assistette a un leggero 
calo di presenze ma non sufficiente ad attenuare le polemiche mosse dalla critica. Gli artisti italiani infatti partecipanti a questa edizione risultano 547: quelli invitati furono ridotti a 297 e quelli ammessi per Giuria furono aumentati a 250, come da numero stabilito per regolamento. ${ }^{6}$

I critici continuavano a mettere in evidenza la grande quantità di artisti presenti, la maggior parte con una sola opera ciascuno, il che provocava - a loro avviso - il disinteresse dei visitatori nel percorrere le sale del Padiglione.

A conclusione della Biennale, Silvio Branzi intitolava il suo articolo Troppe le opere esposte nel Padiglione dell'Italia ${ }^{7}$ facendo comprendere la centralità della tematica e promuovendo l'esigenza di una sempre più urgente riforma. Già alla fine della XXV Biennale compariva un articolo di Pallucchini sulla rivista La Biennale di Venezia in cui analizzava e affrontava a posteriori la tematica, come si comprende direttamente dal titolo Qualità contro numero. ${ }^{8}$ Pallucchini concordava con la critica sulla necessaria diminuzione delle smodate presenze del Padiglione italiano, poiché andavano inevitabilmente ad abbassare il livello artistico dell'Esposizione. Auspicava di vedere un radicale cambiamento nelle modalità di selezione per le Biennali successive, non essendo ancora sufficientemente soddisfatto dei risultati ottenuti nell'edizione che si stava per concludere. A tal proposito, nel giugno 1951 seguì un entusiasta articolo su Le Tre Venezie dedicato alla neonata Biennale d'Arte Triveneta indetta dalla città di Padova, individuata da Pallucchini come una valida rassegna con lo «scopo di offrire un panorama ampio e circostanziato delle forze artistiche operanti in una determinata regione» che avrebbe potuto riunire i migliori artisti operanti nel contesto locale (Tomasella 2011). Questa risultava quindi utile, secondo Pallucchini, sia per la scoperta dei giovani artisti che avrebbero avuto l'opportunità di emergere sia per conoscere l'operato degli artisti che vivevano in centri più isolati. Si trattava di un banco di prova anche per tutti gli artisti che non venivano invitati, selezionati o ammessi alla Biennale di Venezia, ma che comunque avevano l'opportunità di far conoscere la loro arte al pubblico e ai collezionisti. La nuova rassegna rispecchiava esattamente gli auspici di Pallucchini per un ritrovato coordinamento tra le istituzioni espositive, lasciando alla Biennale veneziana la possibilità di

6 Le opere presentate ed esaminate dalla Giuria, eletta interamente dagli artisti concorrenti, furono 3.698 presentate da 1.608 artisti. Gli artisti scelti furono 250 con la possibilità di presentare una sola opera ciascuno.

7 Silvio Branzi, «Troppe le opere esposte nel Padiglione dell'Italia», Il Gazzettino, 11 ottobre 1950.

8 Rodolfo Pallucchini, «Qualità contro numero», La Biennale di Venezia, 2, 1950, 7. 
dedicarsi a un contesto maggiormente internazionale, non avendo più l'obbligo, come sottolinea Marangon, di registrare le espressioni locali (Marangon 1999).

Il cambiamento nella selezione degli artisti auspicato dalla critica stava trovando realizzazione nella preparazione della successiva Esposizione; è infatti la XXVI Biennale del 1952 a scuotere maggiormente gli animi. Gli artisti si sentivano minacciati dagli sviluppi che la Commissione stava attuando, tanto che il collettivo Ordine della Valigia, che raggruppava pittori operanti in laguna, promosse un Referendum redatto da Gino Damerini da sottoporre a tutti gli artisti che volevano partecipare alla XXVI edizione della Biennale (Marangon 1999). Come sottolinea Giuseppina Dal Canton, gli artisti rivendicavano la necessità di restringere il numero e l'ampiezza delle retrospettive a favore dell'allargamento delle presenze attive nel territorio per una maggior rappresentazione dell'arte odierna (Dal Canton 1999). Nel saggio Orientamenti per la XXVI Biennale, presente nella rivista La Biennale di Venezia, ${ }^{9}$ Pallucchini citava «il referendum lanciato agli artisti italiani da un gruppo di pittori veneziani» dell'Ordine della Valigia, riproponendo e smontando le undici domande poste dal questionario. Tra le domande l'Ordine della Valigia ne poneva una riguardante le 250 opere ammesse dalla Giuria alla Biennale appena terminata, sostenendo che la limitazione andasse a svantaggio di artisti meritevoli ingiustamente esclusi. La risposta di Pallucchini fu la seguente:

Evidentemente chi ha formulato il questionario pecca di quel facile ottimismo tanto di moda tra le due guerre, ritenendo che, oltre ai duecentonovantasette invitati e i duecentocinquanta ammessi dalla giuria, la nostra terra sia prodiga di chissà quanti altri artisti. In nessun altro tempo, anche nei più felici momenti, ai quali ricorrono spesso con tanta nostalgia i laudatori del passato, il nostro paese è stato mai così ricco di artisti!

La discussione tra Rodolfo Pallucchini e Gino Damerini trovò seguito nelle pagine de Il Gazzettino e è solo una delle numerose querelle che Pallucchini dovette affrontare nel corso della preparazione della XXVI Biennale. ${ }^{10}$

Il fattore di cambiamento che incise più drasticamente sulla presenza degli artisti veneziani alla Biennale del 1952 fu l'eliminazione

9 Rodolfo Pallucchini, «Orientamenti per la XXVI Biennale», La Biennale di Venezia 4, 1951, aprile, 3-4.

10 Per approfondire la controversia si veda: Mugnone s.d.; Rodolfo Pallucchini, «Orientamenti per la XXVI Biennale», La Biennale di Venezia, 4, 1951, aprile; Gino Damerini, «L'Ordine della Valigia risponde al prof. Pallucchini», Il Gazzettino, 3 giugno 1951; e Tomasella 2011. 
del criterio di ammissione tramite la selezione operata da una Giuria. L'intento della XXVI Biennale è chiaro e evidente: presentare al pubblico meno artisti, che verranno selezionati solo su invito con la possibilità di presentare non meno di 5 opere ciascuno. Si tratta di un notevole rinnovamento rispetto alle precedenti edizioni, che portò numerosi artisti veneti a trovare una soluzione per non essere penalizzati dal nuovo metodo di partecipazione.

Tanta era però la preoccupazione degli artisti veneziani che formarono un comitato e il 10 ottobre del 1951 chiesero alle autorità cittadine e dell'ente che venisse designato dal sindaco un rappresentante degli artisti veneziani tra la neonata Commissione esecutiva che si occupava degli inviti (Rabitti 1990). La petizione venne presa in considerazione dall'ente e gli artisti veneziani per eleggere il loro candidato si ritrovarono il 2 novembre a Ca' Giustinian in un'assemblea presieduta da Diego Valeri. Il giorno seguente comparve su «Il Gazzettino» l'articolo dedicato all'Assemblea degli artisti veneziani per il rappresentante alla Biennale ${ }^{11}$ che riportava i punti salienti della riunione, mettendo in evidenza gli artisti veneziani che maggiormente si esposero per la questione: Vinicio Vianello, Armando Pizzinato e Eugenio Da Venezia, che espressero opinioni differenti su come eleggere il rappresentante. Dopo discussioni e prese di posizione in una "assemblea così 'scapigliata' come è d'obbligo per gli artisti», al punto che «anche la votazione è diventata un problema», come riportato nel Gazzettino, la comunicazione ufficiale dei risultati venne presentata solo il 3 dicembre, data ormai così tarda che giustificava per Giovanni Ponti la vanificazione di ogni richiesta e l'esclusione di qualsiasi possibile rappresentante della città alla Biennale.

Nonostante le polemiche e le proteste da parte degli artisti locali nei confronti della Commissione esecutiva, negli Orientamenti per la XXVI Biennale di Pallucchini si può percepire la soddisfazione di essere riusciti a proporre una nuova Biennale con un numero ridotto di inviti e una maggiore possibilità di visibilità degli artisti invitati. ${ }^{12}$ Questa rivoluzione fu possibile grazie alla sperimentazione di un sistema ciclico a «rotazione» nella scelta degli artisti, già utilizzato per i Padiglioni stranieri, che consisteva nel non richiamare gli artisti che avevano già esposto nelle Biennali degli anni precedenti. In questo modo, scrive sempre Pallucchini, «la Commissione si è trovata d'accordo nella tanto auspicata selezione di valori, in modo da eliminare quel senso di inflazione numerica, che ha tanto nociuto alle Biennali del 1948 e del 1950, specialmente nei riguardi del-

11 «Assemblea degli artisti veneziani per il rappresentante alla Biennale», Il Gazzettino, 3 novembre 1951

12 Rodolfo Pallucchini, «Orientamenti per la XXVI Biennale», La Biennale di Venezia, 4, 1951, aprile. 
la comprensione straniera della nostra arte». ${ }^{13}$

Esemplificativo del sentimento che univa gli artisti nei confronti dei cambiamenti in atto fu l'articolo di Elio Zorzi sulla rivista «Epoca». Con un'intervista a Rodolfo Pallucchini dal titolo La Biennale quest'anno ha soltanto 19.800 nemici, Zorzi ironizza sul numero di artisti esclusi dall'Esposizione veneziana definendoli «nemici». ${ }^{14}$ Nell'introduzione al catalogo Pallucchini chiariva che la questione, «come di consueto, ha fatto sollevare tante polemiche e spargere fiumi d'inchiostro a penne più o meno in buona fede. Va da sé che è impossibile presentare ogni due anni tutto quanto si fa in Italia nel campo dell'arte. Bisognerebbe moltiplicare almeno per cinque la capienza del Padiglione centrale: col risultato che l'interesse del pubblico verrebbe diluito fino alla stanchezza» (Pallucchini 1952). Gli artisti invitati, quindi la totalità degli artisti italiani presenti alla Biennale, furono 235, a differenza delle 664 del 1948 e delle 574 del 1950. I pittori presenti erano 111, di cui solamente 22 veneti.

La XXVII Biennale del 1954 prevedeva un criterio di selezione su invito ancora più ristretto ma, a seguito delle forti contestazioni, si decise di ripristinare l'ammissione per concorso e di inserire nella Sottocommissione per le arti figurative un maggior numero di artisti. Nell'introduzione al catalogo Pallucchini giustificò il reinserimento dell'ammissione per concorso con lo «scopo di allargare il panorama dell'arte italiana d'oggi con opere di artisti poco noti, per lo più giovani sfuggiti al vaglio degli inviti, ma necessariamente non inferiori al livello qualitativo degli invitati», ma ammetteva anche che questo avesse abbassato il livello dell'Esposizione Internazionale, poiché permetteva che «una grandissima maggioranza di opere» si presentasse «con un livello dilettantistico» (Pallucchini 1954).

Gli artisti presentarono oltre 3.000 opere all'ammissione per Giuria; di queste ne furono scelte 132, per un totale di 213 opere di artisti italiani presenti nel Padiglione. Dal tono particolarmente polemico nei confronti degli organizzatori della XXVII Biennale è l'articolo di Roberto Giani comparso su «Il Giornale di Sicilia» in cui fa ben comprendere dal titolo quale sia la questione: Sono troppi gli artisti veneziani. ${ }^{15}$ Il sottotitolo riporta chiaramente «Sembra che soltanto Venezia possa rappresentare il nostro paese e che i milanesi, i romani, i napoletani e i siciliani non siano italiani». L'intento provocatorio dell'articolo fa comprendere come fosse percepita in Italia la questione delle partecipazioni degli artisti, attribuendo la responsabilità

13 Rodolfo Pallucchini, «Orientamenti per la XXVI Biennale», La Biennale di Venezia, 4, 1951, aprile.

14 Elio Zorzi, «La Biennale quest'anno ha soltanto 19.800 nemici», Epoca, 3, 1952, marzo.

15 Roberto Giani, «Sono troppi gli artisti veneziani», Il Giornale di Sicilia, 22 giugno 1954. 
alla composizione dell'Ente accusato di favorire gli artisti veneziani rispetto a quelli del resto d'Italia.

Come riportato nei grafici, le percentuali evidenziano che nel 1954 si ha il dato maggiore di presenze di pittori veneti, con un $28 \%$ sul totale dei pittori italiani partecipanti. Si tratta di un numero preponderante che effettivamente fa discutere la stampa sul fatto che i veneti appaiano come rappresentanti preferiti nel panorama artistico nazionale: «di qualunque tendenza si tratti, meglio che agli altri artisti». Nell'edizione del 1954 venne assegnato inoltre il Gran Premio per la pittura proprio a un veneziano, Giuseppe Santomaso, e la vittoria generò ancora maggiori discussioni. Su questo Giani afferma: «Si parla tanto di assegnare il premio per la pittura a Santomaso, attorno già ferve una polemica astiosa ma giusta».

Per quanto riguarda la Biennale del 1956, l'ultima con Pallucchini in carica, si vide un inasprimento dei toni da parte del segretario generale. Nell'introduzione al catalogo Pallucchini lamentava il sempre maggior numero di artisti che proponevano le loro opere al giudizio della Giuria per l'ammissione attraverso concorso. Si registrarono infatti 4.272 opere presentate, delle quali ne furono scelte 663. Gli artisti italiani ammessi grazie alla Giuria furono 237 (di cui 125 pittori), mentre quelli chiamati su invito furono solamente $84 .^{16}$

Questa eclatante sproporzione e la crescita degli ammessi grazie alla Giuria vennero attribuiti da Pallucchini alla Sottocommissione che era infatti «composta in maggioranza di artisti, e per di più scelti fra liste di nomi presentate da organizzazioni sindacali». Proseguiva denunciando la «larghezza eccessiva» della sezione dedicata agli ammessi presente nel Padiglione italiano, che «in conseguenza si presentano con affollamento che nuoce alla lettura delle opere». Non gli restava che suggerire di meditare «sulla necessità di sganciare da una mostra come quella veneziana, dove l'arte italiana viene messa a confronto con selezioni rappresentative di ogni paese, quell'ammissione per concorso che in pratica, soprattutto quando gli addetti ai lavori si presentano legati da responsabilità sindacali, significa, tranne rare eccezioni, un appesantimento del livello generale dell'esposizione» (Pallucchini 1956). Si nota in queste parole la stanchezza maturata dal segretario generale, ormai prossimo alla conclusione del suo operato. Nell'introduzione è presente un continuo rimando all'edizione della Biennale del 1948, come se Pallucchini volesse ormai evocare un riepilogo, valutando la sua attività proprio nel momento in cui la porta a conclusione.

16 Si passa dalla mancata possibilità di candidarsi attraverso concorso alla Biennale del 1952, alle 132 opere ammesse dalla Giuria nell'edizione del 1954 e si finisce con l'accettazione di 663 opere nell'edizione del 1956. 


\section{Bibliografia}

Bandera, Maria Cristina (1999). Il carteggio Longhi - Pallucchini, Le prime Biennali del dopoguerra, 1948-1956. Milano: Charta.

Barbero, Luca Massimo (a cura di) (1996). Spazialismo: arte astratta Venezia 1950-1960 = Catalogo della mostra (Vicenza, Basilica Palladiana, 12 ottobre 1996-19 gennaio 1997). Venezia: Il Cardo.

Barbero, Luca Massimo (a cura di) (1997). Venezia '50-'60: l'officina del contemporaneo $=$ Catalogo della mostra (Venezia, Palazzo Fortuny, 15 giugno-9 novembre 1997). Milano: Edizioni Charta.

Bazzoni, Romolo (1962). 60 anni di Biennale di Venezia. Venezia: Lombroso.

Bianchi, Giovanni (2013). «Venezia '50-'60: le stagioni dell'arte». Finnegans, ottobre, 48-52.

Budillon Puma, Pascale (1995). La biennale di Venezia dalla guerra alla crisi: 1948-1968. Bari: Palomar.

Castellani, Alessia (2006). Venezia 1948-1968: politiche espositive tra pubblico e privato. Padova: Cleup.

Dal Canton, Giuseppina (1984). «Arte a Venezia: 1946-1956». Rosand, David (a cura di), Interpretazioni veneziane: studi di storia dell'arte in onore di Michelangelo Muraro. Venezia: Arsenale.

Dal Canton, Giuseppina (1999). «Le Biennali degli anni Cinquanta e gli artisti veneti». Venezia 1950-59 1999.

Donaggio, Adriano (1997). «Forma e riforma. La Biennale e le sue leggi: 100 anni di storia». La Biennale, Venezia, l'arte contemporanea = Atti del convegno (Venezia, San Giorgio Maggiore, Fondazione Bevilacqua La Masa, 14 dicembre 1996). Venezia: Grafiche Veneziane.

Marangon, Dino (1999), «Cronaca veneziana 1948-59. Eventi e protagonisti». Venezia 1950-59 1999.

Mugnone, Giuseppe (s.d.). Il Magnifico Ordine della Valigia e i suoi 27 pittori veneziani. Venezia: Galleria Venezia.

Pallucchini, Rodolfo (1948). «Introduzione alla XXIV. Biennale». Biennale 241948.

Pallucchini, Rodolfo (1950). «Introduzione». Biennale 251950.

Pallucchini, Rodolfo (1952). «Introduzione». Biennale 261952.

Pallucchini, Rodolfo (1954). «Introduzione». Biennale 271954.

Pallucchini, Rodolfo (1956). «Introduzione». Biennale 281956.

Pallucchini, Rodolfo (1979). «Al Lettore». Arte Veneta, 32, 1978.

Pallucchini, Rodolfo (1979). «Significato e valore della 'Biennale' nella vita artistica veneziana e Italiana». Storia della civiltà veneziana, vol. 3. Firenze: Sansoni.

Pavanello, Giuseppe; Stringa, Nico (2006). La pittura nel Veneto: Il Novecento, t. 1. Milano: Electa.

Pavanello, Giuseppe; Stringa, Nico (2008). La pittura nel Veneto: /l Novecento, t. 2. Milano: Electa.

Pilo, Giuseppe Maria (a cura di) (2001). Una vita per l'arte veneta = Atti della Giornata di Studio in onore e ricordo di Rodolfo Pallucchini (Venezia, Università Ca' Foscari Venezia, Auditorium Santa Margherita, 10 novembre 1999). Monfalcone: Edizioni della Laguna.

Rabitti, Chiara (1990). «Eugenio da Venezia e la Biennale». Eugenio Da Venezia, La Donazione alla Querini Stampalia = Catalogo della mostra (Venezia, Fondazione Querini Stampalia, 9 novembre-9 dicembre 1990). Milano: Electa. 
«Rodolfo Pallucchini e le arti del Novecento» (2012) = Atti del convegno di studi (Venezia, Fondazione Cini, 3-4 novembre 2008). Saggi e memorie di storia dell'arte, 35-2011.

Stringa, Nico (a cura di) (2006). Venezia '900: da Boccioni a Vedova = Catalogo della mostra (Treviso, Casa dei Carraresi, 27 ottobre 2006-8 aprile 2007). Venezia: Marsilio.

Stringa, Nico (2017). Giuseppe Santomaso. Catalogo ragionato dei dipinti. Torino: Allemandi.

Tomasella, Giuliana (a cura di) (2011). Rodolfo Pallucchini. Scritti sull'arte contemporanea. Venezia: Scripta Edizioni.

Venezia 1950-59 (1999). Venezia 1950-59: Il rinnovamento della pittura in Italia $=$ Catalogo della mostra (Ferrara, Palazzo dei Diamanti, 26 settembre 1999-9 gennaio 2000). A cura di Maria Grazia Messina. Ferrara: Industria Grafica.

Venezia e la Biennale: i percorsi del gusto = Catalogo della mostra (Venezia, Galleria d'Arte Moderna di Ca' Pesaro, 10 giugno-15 ottobre 1995). Milano: Fabbri Editori. 



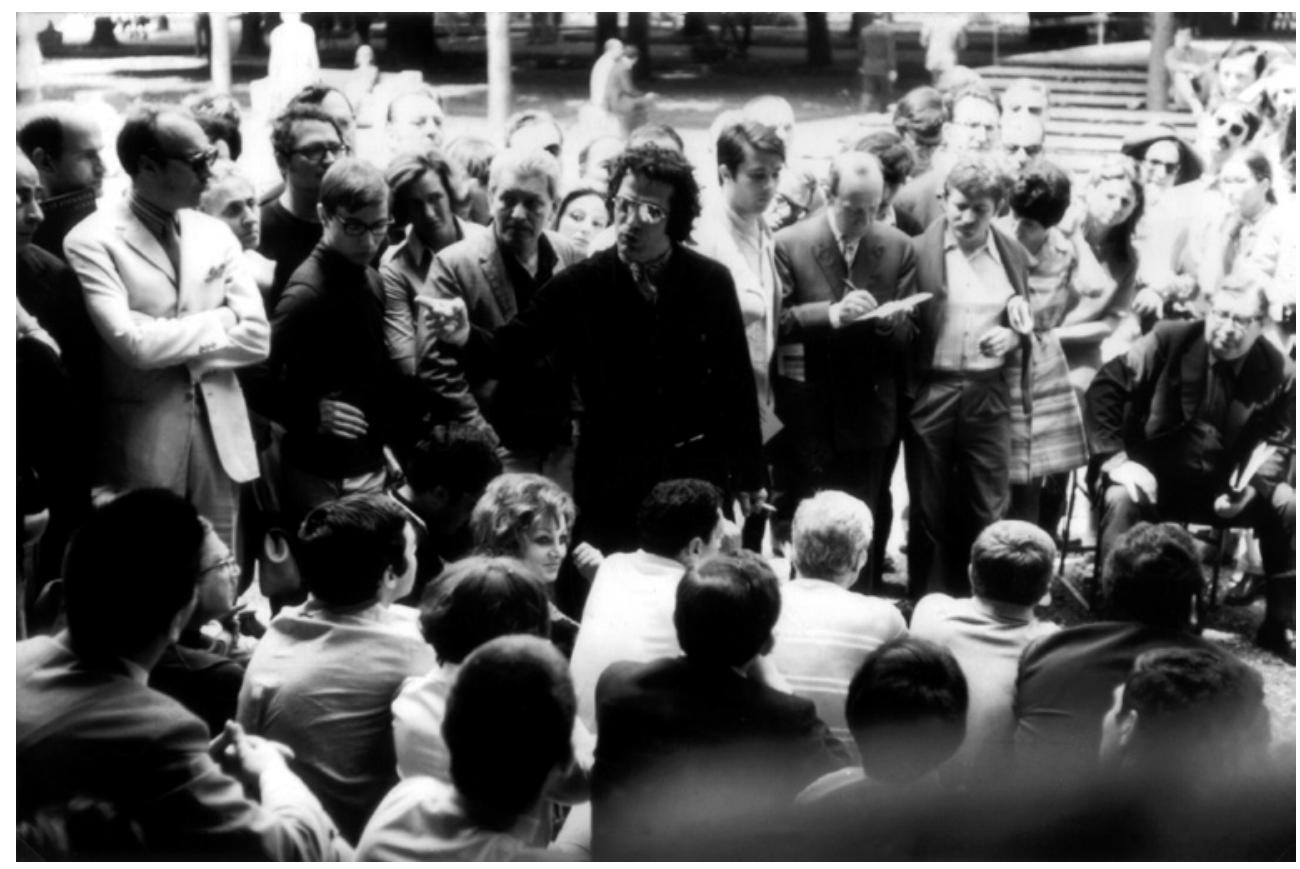

Pino Pascali alla Biennale di Venezia, 1968. Fotografia d'Archivio. Fondazione Pino Pascali, Polignano a Mare. Donazione Famiglia Pascali 


\title{
Palma Bucarelli e la Biennale di Venezia (1948-1968) Acquisizioni della Direttrice per la Galleria Nazionale d'Arte Moderna di Roma
}

\author{
Giorgia Cicalini
}

\begin{abstract}
Venice Biennale had a role also in enhancing and expanding the collections of the Galleria Nazionale d'Arte Moderna (GNAM) in Rome between 1948 and 1968: though the purchases funded by the Ministry of Education, the director Palma Bucarelli has indeed been able to pursuit the aim of seeing her museum growing exponentially. Between many difficulties, due to low funding given by Italian State, from Venice Biennale registers of purchased works it becomes increasingly clear that there is an interest in filling the gaps in museum's collections in order to expand its boundaries beyond the national sphere and towards the contemporaneity.
\end{abstract}

Keywords Palma Bucarelli. Galleria Nazionale d'Arte Moderna di Roma (GNAM). Venice Biennale. Art market.

Palma Bucarelli è tra i protagonisti della cultura artistica italiana del secondo dopoguerra: sostenitrice dell'arte contemporanea in un momento storico complesso, lega la sua vita in modo imprescindibile alla Galleria Nazionale d'Arte Moderna di Roma, di cui è direttrice dal 1940 al 1975, divenendo un

Questo saggio trae origine dalle ricerche compiute in occasione della tesi di laurea magistrale Cicalini, Giorgia (2017). Palma Bucarelli e la Biennale di Venezia (1948-68): acquisizioni, prestiti e interventi critici della Direttrice della Galleria Nazionale d'Arte Moderna di Roma [tesi di laurea magistrale]. Relatore Nico Stringa; correlatore Stefania Portinari. a.a. 2016/2017. Venezia: Università Ca' Foscari Venezia. 
personaggio in vista nell'ambiente culturale e nella Roma mondana degli anni Cinquanta e Sessanta, svolgendo con eleganza e tenacia l'arduo compito di dirigere un museo da riportare in auge dopo anni di direzioni 'nazionaliste'. ${ }^{1}$

I suoi primi anni come direttrice sono ricordati per il coraggio nel gestire la salvaguardia del patrimonio durante la Seconda guerra mondiale, supervisionando e seguendo di persona il trasporto delle opere, per metterle al sicuro dall'esercito e dai bombardamenti. Come scrive Rachele Ferrario, «una delle sue qualità migliori è stata quella di intercettare di volta in volta il sentire della propria epoca, e dell'arte del suo tempo» (Ferrario 2011, 59): nel corso dei trentacinque anni del suo mandato infatti si distinse per l'estrema modernità con cui trasformò la Galleria Nazionale, al suo arrivo limitata dai vincoli del nazionalismo fascista, da 'contenitore' di opere a museo moderno e attivo per lo sviluppo culturale in Italia, oltre che punto di riferimento internazionale. Tra il 1955 e il 1956 ad esempio tornano ad arricchire la collezione internazionale della Galleria le opere acquistate dallo Stato alle Biennali di Venezia dal 1909 negli anni Trenta che, per una convenzione firmata nel 1934 dall'allora direttore Roberto Papini, erano state spostate da Roma alla Galleria Internazionale d'Arte Moderna di Venezia che era stata giudicata più adatta a ospitare opere venete e straniere in virtù del preteso carattere 'cosmopolita' della città di Venezia; mentre nella Galleria di Roma pareva più opportuno esporre le opere degli italiani. La direttrice ha motivo dunque di richiedere le opere cedute per criteri 'nazionalistici', estranei al suo nuovo concetto di museo contemporaneo, per il quale intende invece inserire una significativa rappresentanza di movimenti artistici internazionali. Sebbene il gruppo di opere che arriva da Ca' Pesaro sia consistente, tuttavia non è del tutto eccellente, fatta eccezione per Le tre età (1905) di Gustav Klimt e un dipinto di Utrillo (Camerlengo 2009).

Per aumentare il prestigio del museo anche rispetto alle maggiori istituzioni mondiali, un obiettivo raggiungibile non solo tramite l'incremento delle collezioni, soprattutto alla luce delle esigue risorse economiche a lei disponibili, Palma Bucarelli organizza un'intensa attività espositiva cercando di portare in Galleria i più noti artisti e movimenti dell'epoca, un'operazione di cui sono testimonianza le mostre Esposizione d'arte contemporanea 1944-45 (1944); Arte astratta e concreta in Italia (1951); Picasso (1953); Scipione (1954); Mondrian (1956); Pollock (1958); Capolavori del Museo Guggenheim di New York (1958); Modigliani (1959); Il gruppo De Stijl - l'arte olandese (196061); Moore (1961); Bauhaus (1961); Rothko (1962); Pascali (1969); Klee (1970); Manzoni (1971); Morandi (1972); Capogrossi (1975).

1 La GNAM era stata istituita nel 1883 e fino al 1911 aveva sede in Palazzo delle Esposizioni. 
Le sue 'tendenze astrattiste' e le scelte espositive causano non pochi scandali nel mondo politico e intellettuale dell'epoca, tra cui risaltano nel 1959 lo scandalo dei falsi di Modigliani² e il 'caso Burri', che le costò un'ingiunzione parlamentare per aver esposto il Grande Sacco (1952), considerato solo una 'vecchia e sporca' tela. ${ }^{3}$ Nel 1971 poi viene presentata una nuova interrogazione parlamentare dal deputato democristiano Guido Bernardi, durante la grande retrospettiva di Piero Manzoni: nonostante le intenzioni dell'artista vengano esposte nel catalogo curato da Germano Celant (1971), il politico vuole mettere sotto accusa la condotta della direttrice e ne approfitta per evidenziare come a suo avviso non si riesca a percepire l'aspetto dissacrante dell'opera di Piero Manzoni nei confronti della società dei consumi e di un mercato dell'arte in cui si compra 'a scatola chiusa', e denuncia solo il contenuto scandaloso delle celebri scatolette di Merda d'artista (1961) o «m... d'artista», come i giornali dell'epoca si limitano a scrivere, facendo sorridere Palma Bucarelli. Queste polemiche sono sintomo di come anche la classe dirigente del Paese avesse una mentalità ancora poco aperta all'arte più innovativa, ma l'effetto ottenuto fu sempre contrario a quello desiderato: donarono anzi grande pubblicità alle mostre, che registrarono notevoli successi.

Tra successi e polemiche, l'imperativo nella gestione della direttrice sarà sempre quello che «un museo d'arte moderna può anche essere istituzionalmente nazionale, ma è e non può non essere culturalmente internazionale», come scriveva Giulio Carlo Argan in Museo perché museo come (1980, 39), e di conseguenza l'impegno per l'aggiornamento della sua Galleria non poteva prescindere dalla partecipazione attiva e costante alla manifestazione artistica internazionale italiana per eccellenza: la Biennale di Venezia.

Nei rapporti intercorsi con la Biennale, si nota come Palma Bucarelli lavori 'dietro le quinte' allo scopo di inserire la Galleria nel panorama culturale internazionale attraverso acquisti, prestiti, interventi istituzionali e critici in forma di presentazioni in catalogo: scrive

2 Il critico Virgilio Guzzi visita la mostra dedicata all'artista prima dell'inaugurazione e individua due falsi, costringendo così Bucarelli a rimuoverli dalle pareti per evitare inconvenienti. Ma le polemiche arrivano comunque, poiché i dipinti erano già presenti nel catalogo ufficiale curato dal critico Nello Ponente, ormai stampato. Così, accusata di essersi affidata a persone non qualificate per la costruzione di una mostra così importante, e di non aver riconosciuto i falsi, Palma Bucarelli si difende in modo diplomatico: la stampa era arrivata ad allestimento non concluso, dunque i quadri dovevano essere ancora controllati; inoltre i due presunti falsi provenivano da due importanti gallerie di Zurigo e Parigi (cf. Ferrario 2010, 200).

3 Nonostante le opere di Burri siano già state esposte in Galleria, nelle collettive di arte astratta e concreta e nelle mostre annuali dell'Art Club, l'onorevole Umberto Terracini, esponente del PCI, presenta comunque un'ingiunzione parlamentare chiedendo quale fosse la cifra pagata «per assicurarsi la proprietà della vecchia, sporca e sdrucita tela da imballaggio [...] messa in cornice da tale Alberto Burri» (cf. Ferrario 2010, 205). 
infatti nel 1958 per Turcato, nel 1960 per Fautrier, nel 1962 su Giacometti e nel 1968 di Pascali.

La sua partecipazione alle manifestazioni veneziane coincide con la 'reggenza' di Rodolfo Pallucchini (1948-56) e Gian Alberto Dell'Acqua (1958-68). Le prime cinque Biennali del dopoguerra, che Pallucchini guida come segretario generale, si concentrano sulla revisione storica e critica delle avanguardie e dei movimenti che avevano animato la prima metà del secolo, per consacrarne i maestri e favorire l'aggiornamento del pubblico e degli artisti italiani, mentre con segretario generale Dell'Acqua, nelle sei edizioni successive, l'obiettivo si sposta sull'arte contemporanea e, accanto ai maestri, si punta sulle nuove generazioni, che raccolgono le ceneri delle avanguardie dando vita a nuove frontiere dell'arte nella contemporaneità. È interessante notare come gli intenti di entrambi sostanzialmente coincidano con la politica che Bucarelli adotta per il suo museo: mostre, conferenze, attività didattiche volte all'aggiornamento della Galleria e del suo pubblico, alla storicizzazione del contemporaneo, comunque affiancate da uno sguardo e un sostegno costante alle novità dell'arte nazionale e internazionale. Questo approccio all'insegna della modernità si riflette inevitabilmente anche sugli acquisti che la direttrice supervisiona e realizza per la Galleria presso le Biennali di Venezia prese in esame, dal 1948 al 1968.

In qualità di dirigente, quando deve occuparsi delle questioni burocratiche e organizzative per la gestione degli acquisti volti a incrementare le collezioni della Galleria, poiché agisce con fondi del Ministero della Pubblica Istruzione deve coordinare i suggerimenti dati da un'apposita Commissione ministeriale coadiuvata da lei stessa. Da questo procedimento emergono frequenti contrasti sia con il Ministero, per i tempi lunghi della burocrazia e per la ristrettezza dei fondi che portano a dover spesso rinunciare ad acquisti rilevanti di artisti affermati, sia con il direttore dell'Ufficio vendite della Biennale, Ettore Gian Ferrari, per le modalità con cui Palma conduce le trattative direttamente con gli artisti o i loro mercanti, soprassedendo alla trattativa con l'Ufficio stesso e mettendolo in difficoltà. Bucarelli ritiene comunque indispensabile la Biennale come sguardo sulla situazione artistica europea e mondiale, considerandola una preziosa occasione per integrare e arricchire la collezione del museo tramite l'acquisto di opere internazionali di cui è ancora piuttosto sprovvista, oltre che come vetrina per artisti, giovani e non, a cui spetta, per merito e qualità, un riconoscimento a livello globale.

Se le 'Biennali di Pallucchini' sono appunto caratterizzate dalla volontà di riabilitare l'istituzione dopo la pausa dovuta alla guerra, mostrando le tendenze dell'arte contemporanea internazionale e, allo stesso tempo, ripercorrere criticamente i movimenti artistici più rilevanti dell'ultimo secolo per recuperare il ritardo culturale del pubblico italiano causato dall'autarchia fascista, la revisione 
storica di Pallucchini si attua tramite selezioni e retrospettive che fanno della Biennale un palcoscenico didattico oltre che espositivo. L'edizione del 1948 è la prima a cui Bucarelli partecipa proprio nelle veci di direttrice della Galleria e nelle negoziazioni per gli acquisti già appare evidente il divario tra la disponibilità data dal Ministero (tre milioni di lire) e i costi reali delle opere che vorrebbe ottenere: Carlo Carrà, Marc Chagall e Oskar Kokoschka rifiutano le offerte del Ministero perché decisamente inferiori rispetto alle cifre richieste. Nello specifico per Chagall, che chiedeva 2.500 dollari per Il gallo, l'offerta è di 400.000 lire; per l'opera di Carrà Mio figlio (1916), presente alla mostra nel Padiglione Italia Tre pittori italiani dal 1910 al 1920, l'offerta è di 200.000 lire a fronte delle 600.000 della richiesta ufficiale; per Energia atomica (1946) esposta nella personale dedicata a Kokoschka, l'offerta di 250.000 lire risulta del tutto inaccettabile, dato che le cifre volute dall'artista sono di gran lunga superiori. Il Ministero tuttavia riesce a concludere un buon numero di acquisti, tra i quali prevalgono ancora opere italiane, anche se già si intravede un orientamento verso le 'ultime tendenze', come dimostrano le opere di Renato Birolli, Giuseppe Santomaso, Lucio Fontana, Osvaldo Licini; ma anche verso artisti ormai affermati, come Mario Mafai e Scipione (pseudonimo di Gino Bonichi), che si distinguono per la forza innovativa e sensibilità vicina all'espressionismo. L'unica eccezione relativa a un nome internazionale, di cui è probabile suggeritrice Bucarelli, in quanto sua nota estimatrice, è quello di Henry Moore, di cui si ottiene il disegno Figura sdraiata e la scultura in bronzo Gruppo di famiglia.

Fin dall'edizione del 1948 si presentano dunque per Bucarelli una serie di problematiche che caratterizzeranno anche i suoi futuri rapporti con la manifestazione veneziana, in primis il ritardo con cui vengono avanzate le proposte d'acquisto, un dato che si riflette poi sulle trattative, nonostante la commissione per gli acquisti dello Stato venisse sempre inviata a esercitare le proprie scelte durante l'inaugurazione della Biennale, dunque orientativamente nei mesi di maggio o giugno: già in quell'anno infatti gli acquisti si concludono, con la conferma dell'ottenimento delle opere di Moore provenienti del Padiglione inglese, solo il 24 novembre.

I dipinti acquistati in quell'anno dal Ministero della Pubblica istruzione destinati alla Galleria Nazionale d'Arte Moderna di Roma, esposti nel Padiglione centrale e i cui costi fanno riferimento a quanto annotato nei registri degli acquisti della Biennale, ${ }^{4}$ sono: 
Tabella 1 Acquisti di opere italiane Acquisti destinatie alla Galleria Nazionale d'Arte Moderna di Roma, 1948

\begin{tabular}{llr}
\hline Opera & Artista & prezzo (lire) \\
\hline Lago e Barche & Angelo Del Bon & 60.000 \\
Fiori & Pio Semeghini & 100.000 \\
Uomo che legge (acquaforte) & Arnoldo Ciarocchi & 6.000 \\
Natura morta d'autunno & Carlo Levi & 80.000 \\
Il vegliardo, Avvoltoi & Mino Maccari & 45.000 \\
Testa di bambola & Mario Mafai & 100.000 \\
La sedia & Italo Valenti & 60.000 \\
Contemplazione (bronzo) & Carmelo Cappello & 40.000 \\
Natura morta in grigio & Enrico Paulucci & 50.000 \\
Interno n'1 & Giuseppe Santomaso & 60.000 \\
Gabbia e vaso di fiori & Renato Birolli & 50.000 \\
Gallo (scultura + mosaico) & Lucio Fontana & 120.000 \\
Scultura (marmo) & Marino Mazzacurati & 90.000 \\
Memorie d'oltretomba & Osvaldo Licini & 50.000 \\
Apparizioni tra le siepi (acquaforte) & Luigi Bartolini & 7.000 \\
Pensosa la luna & Atanasio Soldati & 80.000 \\
La madre (terracotta colorata) & Agenore Fabbri & 80.000 \\
Ritratto di Ungaretti & Scipione & 300.000 \\
Donne al mattino & Arnaldo Badodi & 80.000 \\
Ildrappo rosso & Alfredo Agostoni & 50.000 \\
\hline
\end{tabular}

Le opere provenienti dal Padiglione inglese sono invece:

Tabella 2 Acquisti di opere inglesi destinate alla Galleria Nazionale d'Arte Moderna di Roma, 1948

\begin{tabular}{llr}
\hline Opera & Artista & prezzo (lire) \\
\hline Figura sdraiata (disegno) & Henry Moore & 30.000 \\
Gruppo di famiglia (bronzo) & Henry Moore & 120.000 \\
\hline
\end{tabular}

Anche nel 1950 l'importo totale delle acquisizioni non deve superare i 3 milioni di lire stanziati dal Tesoro. Gli acquisti però in questa XXV Biennale, anche se inferiori per quantità, registrano un investimento maggiore su opere di artisti stranieri, e un terzo dell'intera somma totale viene addirittura investito per Cavallo, cavaliere e caseggiato (1913-14) di Umberto Boccioni.

Le trattative complessive si concludono il 6 ottobre 1950, ma subiranno una piccola modifica il 30 maggio 1951 quando Palma Bucarelli 
propone di sostituire il Ritratto di Roberto Melli con un suo altro dipinto del 1927 L'abito a scacchi. ${ }^{5}$ Tutte le opere acquistate quest'anno per la Galleria sono esposte nel Padiglione centrale, fatta eccezione per Il grido esposto nel Padiglione messicano e Coppa Chimerica presente nella sezione Scultura d'oggi. ${ }^{6}$

Tabella 3 Acquisti destinati alla Galleria Nazionale d'Arte Moderna di Roma, 1950

\begin{tabular}{llr}
\hline Opera & Artista & prezzo (lire) \\
\hline Cavallo, cavaliere e caseggiato & Umberto Boccioni & 1.000 .000 \\
Ritratto & Roberto Melli & 250.000 \\
Amalassunta & Osvaldo Licini & 100.000 \\
Coppa chimerica (bronzo) & Jean Arp & 1.000 .000 \\
Il grido & Rufino Tamayo & 1.000 .000 \\
\hline
\end{tabular}

Nel 1952 ugualmente le contrattazioni per gli acquisti incontrano varie difficoltà che Ettore Gian Ferrari, come direttore dell'Ufficio vendite della Biennale, tenta di risolvere mediando tra le esigenze del Ministero e quelle degli artisti. I maggiori problemi si riscontrano nella gestione dell'acquisto del bronzo Il giocoliere (1949) di Marino Marini, un 'pezzo forte' che fa da contrappeso a un gran numero di acquisti minori. L'artista non è disposto a cedere la sua scultura per una cifra inferiore a 1.500.000 lire, poiché fa sapere che ha già comunicato lo stesso prezzo ad altri interessati da cui aspetta una risposta. ${ }^{7}$ Inoltre Marini comunica telefonicamente a Gian Ferrari che, se l'acquisto non sarà registrato entro il primo agosto, la somma richiesta raggiungerà i 2.000.000 di lire. Ritenendo l'aumento ingiustificato, Gian Ferrari registra l'acquisto a fine luglio facendolo rientrare nei 6 milioni stanziati per questo anno dal Ministero. Impiega inoltre le 30.000 lire destinate alle incisioni di Emil Nolde, che in realtà non sono in vendita in quanto proprietà di un privato, per prendere la medaglia Il pianto del bambino di Luciano Mercante. ${ }^{8}$

5 Bucarelli propone di sostituire il Ritratto di Melli, che appartiene agli «ultimi anni della produzione dell'artista e non aggiunge nulla alla conoscenza della sua produzione, già abbastanza documentata in Galleria anche con opere recenti», con il dipinto del 1927 L'abito a scacchi comunque esposto alla Biennale. Il suggerimento viene approvato dal Ministero il seguente 12 giugno (cf. Archivio Storico GNAM, POS. 9B BIENNALI DI VENEZIA 1948-1958, b. 3, fasc. 2, Lettera di P. Bucarelli al Ministero della Pubblica Istruzione, 30 maggio 1951).

6 ASAC: Biennale 1950. Registro di vendita nr. 44.

7 ASAC, Ufficio vendite, b. 18: scambio epistolare tra E. Gian Ferrari e G. Bacchetti 28 giugno 1952; 19 luglio 1952; 29 luglio 1952.

8 ASAC, Ufficio vendite, b. 18: lettera di E. Gian Ferrari a G. Bacchetti, 23 ottobre 1952. 
Adottando questa soluzione il totale degli acquisti supera la somma prevista solo di 155.850 lire, che più di così non è riducibile poiché la Biennale non può rinunciare alla provvigione che le spetta, per non creare un pericoloso precedente. Il 7 febbraio 1953 il Ministero approva infine definitivamente gli acquisti: tra le opere, la scultura di Marini risulta comprata per 1.275.000 lire, comprensive della percentuale che spetta all'Ufficio vendite, come riportato dal direttore amministrativo della Biennale nell'atto di cessione finale del 16 marzo 1953 con il quale lo Stato conclude i suoi acquisti per 5.889.610 lire.

Le acquisizioni per la Galleria provengono per la maggior parte dal Padiglione italiano, tranne i disegni di Alfred Kubin esposti nel Padiglione austriaco, la scultura di Germaine Richier in quello francese e Gruppo che cammina di Kenneth Armitage in quello inglese; dal registro dell'Ufficio vendite risultano le opere: ${ }^{9}$

Tabella 4 Acquisti destinati alla Galleria Nazionale d'Arte Moderna di Roma, 1952

\begin{tabular}{llr}
\hline Opera & Artista & prezzo (lire) \\
\hline Gruppo che cammina (scultura) & Kenneth Armitage & 80.000 \\
Vita da cani (disegno) & Alfred Kubin & 5.000 \\
Orgia campestre (disegno) & Alfred Kubin & 35.000 \\
L'orco (scultura) & Germaine Richier & 50.000 \\
Casetta e Montmatre & Federico Zandomeneghi & 500.000 \\
Il poeta Umberto Saba & Carlo Levi & 200.000 \\
Acrobata al trapezio (scultura) & Luciano Minguzzi & 120.000 \\
Colline bolognesi (bianco/nero) & Paolo Manaresi & 10.000 \\
Natura morta in grigio & Enrico Paolucci & 50.000 \\
Guerra civile & Aligi Sassu & 300.000 \\
La ragazza con la treccia (bianco/nero) & Arnoldo Ciarocchi & 5.000 \\
Delia (bianco/nero) & Arnoldo Ciarocchi \\
Raffella (bianco/nero) & Arnoldo Ciarocchi & 5.000 \\
Paesaggio (bianco/nero) & Arnoldo Ciarocchi & 5.000 \\
Paesaggio (bianco/nero) & Arnoldo Ciarocchi & 5.000 \\
Mattino & Francesco Trombadori & 5.000 \\
Paesaggio: Strazzema & Antonio Donghi & 80.000 \\
Incisione (bianco/nero) & Mino Maccari & 100.000 \\
Donna appoggiata & Domenico Cantatore & 10.000 \\
il banchetto & Franco Gentilini & 60.000 \\
Aringhe & Gianni Vagnetti & 80.000 \\
Colazione e flauto & Fausto Pirandello & 90.000 \\
\hline & & 100.000 \\
\hline
\end{tabular}

9 ASAC: Biennale 1952. Registro di vendita nr. 47 (=46). 
Giorgia Cicalini

Palma Bucarelli e la Biennale di Venezia (1948-1968)

\begin{tabular}{llr}
\hline Opera & Artista & prezzo (lire) \\
\hline Figura in grigio & Pompilio Mandelli & 60.000 \\
\hline Gru & Mario Radice & 70.000 \\
Nudo (gesso) & Alberto Viani & 250.000 \\
Figura ${ }^{\circ}$ 3 & Ennio Morlotti & 100.000 \\
Composizione $n^{\circ}$ 32 & Mauro Reggiani & 90.000 \\
Alba & Antonio Corpora & 70.000 \\
Scultura & Berto Lardera & 200.000 \\
Conversazione (medaglia) & Nicola Rubino & 40.000 \\
La vittima (medaglia) & Paladino Orlandini & 30.000 \\
Mia figlia Franca (medaglia) & Paladino Orlandini & 30.000 \\
Lotta dicentauri (medaglia) & Otello Bertazzolo & 60.000 \\
$N^{\circ}$ 18 & Giuseppe Capogrossi & 70.000 \\
Autoritratto (scultura) & Pietro Consagra & 200.000 \\
Rammendatrici di reti & Giuseppe Migneco & 80.000 \\
Braccianti sul carro & Giuseppe Zigaina & 150.000 \\
La tagliariso (bianco/nero) & Ernesto Treccani & 30.000 \\
Immagine & Mario Davico & 50.000 \\
Graziella (bianco/nero) & Lorenzo Vespignani & 35.000 \\
Stazione di Trastevere & Lorenzo Vespignani & 35.000 \\
II limone & Bruno Cassinari & 120.000 \\
Contadino nella vigna & Renato Birolli & 150.000 \\
La seggiola e il gatto & Antonio Scordia & 70.000 \\
Il pianto del bambino (medaglia) & Luciano Mercante & 200.000 \\
Il giocoliere (scultura) & Marino Marini & 30.000 \\
\hline & & 1.500 .000 \\
\hline
\end{tabular}

Alla Biennale del 1954 Palma Bucarelli aggiorna le collezioni soprattutto sul fronte dell'arte italiana, scegliendo opere di artisti poco rappresentati in Galleria o di cui erano presenti lavori datati ai decenni precedenti. Nonostante l'inadeguatezza dei fondi ministeriali sia particolarmente evidente quando si tratta di acquisti internazionali, in questa edizione riesce ad assicurarsi anche due importanti opere di artisti di fama: Compendio della storia universale (1953) di Max Ernst e Il compianto degli amanti (1953) di Joan Mirò, pagati rispettivamente 500.000 e 1.350 .000 lire, esposte nelle personali dei relativi autori allestite nella sala 46 e 47 del Padiglione centrale. Acquisti di così alto livello saranno sempre più rari e corrisponderanno a una diminuzione del numero totale delle opere ottenute; le risorse messe a disposizione dello Stato infatti si riveleranno sempre più insufficienti. Fortunatamente, come già nelle precedenti edizioni, la direttrice riesce a concordare dei prezzi decisamente competitivi grazie alla 
sua abilità nell'interloquire direttamente con artisti e galleristi, anche se queste trattative personali non sono particolarmente gradite da Gian Ferrari, il quale si trova spesso a dover risolvere gli equivoci causati da mancate comunicazioni o incomprensioni sulla natura dei prezzi, se al netto o al lordo, in lire o in altre valute, con o senza applicazione della provvigione della Biennale.

Le opere che vanno alla Galleria dalla XXVI Biennale di Venezia sono: ${ }^{10}$

Tabella 5 Acquisti destinati alla Galleria Nazionale d'Arte Moderna di Roma, 1954

\begin{tabular}{llr}
\hline Opera & Artista & prezzo (lire) \\
\hline Toro (bronzo) & Marcello Mascherini & 500.000 \\
Chimera (bronzo) & Mirko & 585.000 \\
Bombardamento notturno (ceramica) & Leoncillo Leonardi & 350.000 \\
Agricola (bronzo) & Nino Franchina & 250.000 \\
La brughiera & Ottone Rosai & 200.000 \\
Paesaggio romano & Mario Mafai & 175.000 \\
Destinazione precisa & Alberto Magnelli & 500.000 \\
Testa di pescatore & Luigi Spazzapan & 150.000 \\
Ritmi rurali & Giuseppe Santomaso & 300.000 \\
Animismo geometrico & Enrico Prampolini & 200.000 \\
Scontro di uomini & Virgilio Guidi & 200.000 \\
Paesaggio di collina & Enrico Paolucci & 150.000 \\
Concertante ${ }^{\circ}$ 4 (incisione) & Riccardo Licata & 10.000 \\
Panchine & Bruno Caruso & 20.000 \\
Contadini (incisione) & Armando De Stefano & 30.000 \\
Hibert hise (pad. Israele) & Mordechai Ardon & 500.000 \\
Compendio della storia universale & Max Ernst & 500.000 \\
ll compianto degliamanti & Joan Mirò & 1.350 .000 \\
\hline & &
\end{tabular}

Nel 1956 l'intervento della Bucarelli è evidente negli acquisti di Ciclo della protesta $n^{\circ} 4$ (1953) di Emilio Vedova, esposta nella sala 42 dedicata all'artista nel Padiglione centrale, e della scultura Figura (1956) di Giacometti, in mostra nel Padiglione francese. La scelta delle opere da parte della Commissione risponde, come sempre, alle esigenze e alle necessità di aggiornamento delle collezioni della Galleria, andando dunque a colmare eventuali lacune presenti nel museo. Per quanto riguarda il dipinto di Vedova, Bucarelli ritiene indispensabile assicurarlo alla Galleria per incrementare la raccolta dei lavori dell'artista,

10 ASAC: Biennale 1954. Registro di vendita nr. 46 (=48). 
che consta di lavori di date antecedenti o di minore formato, rappresentandolo con «una delle migliori espressioni dell'arte drammaticamente intensa e umana di questo artista» (Bucarelli 1956, 379).

Per Figura di Giacometti, la questione è invece più complicata. È la prima opera di Giacometti ad entrare nelle raccolte della Galleria, considerata da lei una delle «più importanti e più tipiche, in cui si afferma l'intuizione di una forma e di uno spazio nuovo, stranamente suggestivo e poetico» (Bucarelli 1956, 378). L'incertezza, caratteristica che pervade Giacometti, lo condiziona anche in questa situazione; difatti, dopo un lungo carteggio non è comunque chiaro se sia infine Bucarelli a scegliere la scultura o l'artista a inviare quella che ritiene essere la più adatta. ${ }^{11} \mathrm{Il}$ bronzo arriverà effettivamente in Galleria solo il 21 febbraio 1958.

Degni di nota sono gli acquisti di altre due opere scultoree di artisti internazionali: Piccola bestia (1953) di Lynn Chadwick, vincitore del premio per la scultura e L'adolescente di Andre Arbus, prima scultura dell'artista ad entrare nelle collezioni della Galleria.

L'opportunità di acquistare opere straniere alla Biennale veneziana viene anche quest'anno limitata dalla scarsità dei fondi ministeriali, ma si presenta un ulteriore ostacolo: i Padiglioni stranieri presentano sempre più spesso artisti già famosi, la maggior parte delle opere dei quali risulta di proprietà di privati o vendibile a prezzi troppo elevati per le possibilità della Galleria, perciò, anche per questa XXVIII Biennale, nel verbale conclusivo degli acquisti del Ministero prevalgono gli italiani: ${ }^{12}$

11 Giacometti deve realizzare nel suo studio a Parigi il bronzo dai modelli in gesso esposti nel Padiglione francese per soddisfare la richiesta della direttrice, ma non ha chiaro quale sia quello prescelto e infatti le scrive di aver appena preparato otto sculture in bronzo: «ma come sapere quale Lei desidera avere? Non so se Lei pensava a una particolarmente, sono discretamente differenti una dall'altra. Se Lei non ha occasione di venire a Parigi, nei prossimi tempi potrei mandarle le fotografie che avrò prossimamente, cosa ne pensa? Mi sembra essere la sola soluzione possibile e potrà scegliere la scultura che preferisce» (Archivio Storico GNAM, POS. 9B BIENNALI DI VENEZIA 1948-1958, b. 3, fasc. 5, Lettera di A. Giacometti a P. Bucarelli, 22 aprile 1957). Nonostante le indicazioni Bucarelli (cf. Archivio Storico GNAM, POS. 9B BIENNALI DI VENEZIA 1948-1958, b. 3, fasc. 5, Lettera di P. Bucarelli ad A. Giacometti, 12 giugno 1957); la scultura tarda ad arrivare ed alla richiesta di spiegazioni da parte della Direttrice l'artista risponde:

«la prego di voler scusarmi di non aver risposto prima alla sua gentile lettera del 6 novembre ma spero che avrà ricevuto il mio telegramma e che presto riceverà anche la scultura rimessa allo speditore Lefevre - Foimet da più di una settimana. Ho fatto fondere una grande parte delle figure che erano a Venezia e le ho mandato quello che a me interessava di più anche se non è che uno studio o abbozzo come tutte le altre esposte a Venezia. Non so cosa Lei ne penserà ma sono pronto a cambiarla con un'altra se Lei lo desidera e le manderò al mio ritorno a Parigi le fotografie di queste ma almeno è un passo di fatto. Tutto questo lungo ritardo viene dal fatto che ho lasciato passare un mese prima di dare queste sculture alla fonderia (cf. Archivio Storico GNAM, POS. 9B BIENNALI DI VENEZIA 1948-1958, b. 3, fasc. 5, Lettera di A. Giacometti a P. Bucarelli, 28 novembre 1957).

12 ASAC: Biennale 1956. Registro di vendita nr. 50. 
Giorgia Cicalini

Palma Bucarelli e la Biennale di Venezia (1948-1968)

Tabella 6 Acquisti destinati alla Galleria Nazionale d'Arte Moderna di Roma, 1956

\begin{tabular}{|c|c|c|}
\hline Opera & Artista & prezzo (lire) \\
\hline Ombra bruciata & Afro & 700.000 \\
\hline Alba a Parigi & Renato Borsato & 103.000 \\
\hline Ilponte nero & Piero Martina & 206.000 \\
\hline Paesaggio di inverno & Enrico Paulucci & 103.000 \\
\hline Notturno al mare & Perizi & 103.000 \\
\hline Eglise a Etempes & Giuseppe Cesetti & 206.000 \\
\hline Sole nel cespuglio & Mattia Moreni & 400.000 \\
\hline Paesaggio grigio & Pompilio Mandelli & 103.000 \\
\hline Ciclo della protesta $n^{\circ} 4$ & Emilio Vedova & 350.000 \\
\hline Composizione $n^{\circ} 1$ & Pietro Melecchi & 103.000 \\
\hline Bestia & Gianni Dova & 206.000 \\
\hline Lume nero & De Palma & 103.000 \\
\hline Bagnante (bronzo) & Emilio Greco & 1.000 .000 \\
\hline Figura d'uomo (scultura in legno) & Marino Mazzacurati & 500.000 \\
\hline Gatto (scultura) & Enzo Assenza & 824.000 \\
\hline Centauro con ninfa (scultura) & Rubino & 412.000 \\
\hline La corte dei miracoli $n^{\circ} 1$ (disegno) & Alberto Martini & 52.500 \\
\hline La casa dell'uomo solo (acquaforte) & Arnoldo Ciarocchi & 15.450 \\
\hline Colline dopo il tramonto (disegno) & Carnevali & 30.900 \\
\hline Nudo disteso (disegno) & Carlo Mattioli & 30.900 \\
\hline Paesaggio (acquatinta) & Antonio Music & 206.900 \\
\hline Donna in poltrona (disegno) & Antonio Scordia & 41.200 \\
\hline Festa in periferia $n^{\circ} 1$ (disegno) & Anna Salvatore & 41.200 \\
\hline Studio per Madonna & Alberto Gerardi & 36.050 \\
\hline $\begin{array}{l}\text { Piccola bestia (pad. Gran Bretagna, } \\
\text { scultura in ferro) }\end{array}$ & Lynn Chadwick & 87.500 \\
\hline Figura (pad. Francia, bronzo) & Alberto Giacometti & 515.000 \\
\hline 15 febbraio & Adam Henry & 36.000 \\
\hline $\begin{array}{l}\text { Veduta di Parigi da Sacr Coeur (pad. } \\
\text { Grecia, litografia) }\end{array}$ & Galanis & 11.000 \\
\hline Venezia & Dragotescu & 51.000 \\
\hline L'adolescente (pad. Francia, bronzo) & André Arbus & 721.00 \\
\hline
\end{tabular}

La Biennale del 1958 è la prima sotto la direzione di Gian Alberto Dell'Acqua. Il nuovo segretario, che ricoprirà tale ruolo fino alla fine degli anni Sessanta, prende una strada diversa da quella intrapresa da Pallucchini, lasciando ampio spazio alle nuove generazioni di artisti. Come inviata speciale del giornale La Sera di Ro- 
ma, Bucarelli commenta il passaggio di testimone e lo svolgimento di questa XXIX Biennale difendendone la modernità e l'apertura all'arte nuova:

Ebbene dopo tutto lo sconquasso, cambiato il Segretario Generale, il Presidente e le Commissioni, questa Biennale non solo non ha mutato quel suo indirizzo verso un'apertura modernamente viva e specificatamente attuale e aggiornata quale deve avere una Mostra che si cimenta in un confronto con Paesi che son all'avanguardia dell'arte mondiale, ma anzi presenta molte tendenze "di punta" dell'arte d'oggi nel suo insieme (nonostante certe cadute dovute all'uso della Biennale di rendere omaggio ai morti recenti; pietoso e commovente uso, ma c'entrano così i buoni e i cattivi in una generale assoluzione) nell'insieme, dicevo, appare al primo sguardo più svelta e moderna di prima. Già i soliti misoneisti e interessati l'hanno chiamata, di nuovo, la "Biennale dell'astrattismo"; ma questa volta con ragione. Che cosa vuol dire tutta questa vicenda? Che l'arte astratta [...] è l'espressione propria del nostro tempo e che è insensato voler fermare una storia che si va sviluppando così naturalmente e con tanta coerenza. Una riprova di quanto sia vero sta nel fatto che questa volta, dopo aver accolto con Mostre personali di un'intera sala o con nutriti gruppi di opere di alcuni artisti di primo piano, la Biennale ha dedicato ai giovani il resto dello spazio riservato agli italiani. Se dunque la Biennale ha l'aspetto di "astratta" vuol dire che le giovani generazioni, che sono qui in preponderanza sono orientate in tal senso. Del resto ormai sarebbe meglio abbandonare il termine di "astratto" visto che tutta l'arte più o meno vi partecipa e parlare di arte moderna nelle sue diverse espressioni rappresentate dagli artisti che sono i protagonisti maggiori. ${ }^{13}$

Dai registri delle opere acquistate dal Ministero della Pubblica Istruzione per la Galleria Nazionale d'Arte Moderna di Roma durante le Biennali gestite da Dell'Acqua emerge sempre più chiaramente l'interesse a colmare le lacune presenti nelle collezioni del museo per ampliarne i confini fuori dall'ambito nazionale e verso l'attualità. È indubitabile inoltre che il carisma e le idee della Bucarelli, convinta sostenitrice dell'arte astratta, non figurativa, informale, e poi anche dell'arte cinetica, influiscono sulle scelte della commissione. La Commissione dello Stato sembra trovarsi ogni anno più in difficoltà, a causa delle esigue risorse economiche del tutto inadeguate ai prezzi del mercato internazionale; senza consi-

13 ASAC, FS, AV, b. 5/17: articolo di Palma Bucarelli, «La ventinovesima Biennale espressione viva dell'arte moderna», La Sera, 16 giugno 1958. 
derare che, per tentare di ottenere prezzi favorevoli, le trattative si dilungano notevolmente, posticipando le consegne dei verbali definitivi e mettendo a dura prova l'Ufficio Acquisti, ma anche i rapporti con gli stessi artisti e mercanti.

L'interesse della Commissione per gli acquisti nell'edizione del 1958 si concentra però esclusivamente sugli artisti internazionali: Kenzo Okada è il primo giapponese ad entrare nelle collezioni della Galleria; il grande successo del Padiglione spagnolo porta inoltre l'attenzione della Commissione ministeriale su Gesto (1957) di Eduardo Chillida e Tabula erasa (1958) di Antoni Tàpies.

Una proposta di vendita arriva anche direttamente da uno scultore italiano: Umberto Mastroianni infatti, vincitore del Premio Presidenza del Consiglio dei Ministri per uno scultore, a fronte di numerose richieste per Battaglia (1957), opera a cui Bucarelli si era mostrata interessata durante il soggiorno a Venezia, scrive al direttore dell'Ufficio delle vendite Ettore Gian Ferrari che «tra una vendita all'estero ed il collocamento presso la Galleria [...] preferisce quest'ultima, anche se ciò dovesse costituire un sensibile sacrificio economico». ${ }^{14}$ Mastroianni è disposto a dimezzare la cifra richiesta, sapendo con quanta fatica Bucarelli combatte ogni anno per impiegare dignitosamente le scarse risorse finanziarie del museo. Nonostante l'offerta dell'artista, la direttrice sembra dover comunque rifiutare per mancanza totale di disponibilità economica. Il Ministero invece infine acconsente all'acquisto, con l'unica condizione di registrarlo dopo il $1^{\circ}$ luglio 1959 per ragioni di carattere contabile.

Auspicando un aumento dei fondi la Commissione avanza le sue proposte, che solo il 18 ottobre 1958 si trasformeranno in 'acquisti fissati', esasperando Gian Ferrari, che scrive alla Bucarelli: «Mi consenta di dirLe che io non credevo più alla possibilità di registrare questo affare, senza dubbio il più laborioso ed estenuante che io abbia avuto in tanti anni di carriera: iniziato il 12 giugno si è concluso il 19 ottobre! Un record». ${ }^{15}$ Nonostante la rinuncia all'acquisto di un'opera Wols, poiché le cifre richieste per l'artista (tra i 5 e i 50 milioni di lire) superano di gran lunga le somme imposte dal Ministero, la Commissione si concentra su opere di artisti stranieri di una certa rilevanza, sulle quali si possono però applicare delle riduzioni: ${ }^{16}$

14 Archivio Storico GNAM, POS. 9B BIENNALI DI VENEZIA 1956-1960, b. 4, fasc. 1, Lettera di E. Gian Ferrari a P. Bucarelli, 17 luglio 1958.

15 Archivio Storico GNAM, POS. 9B BIENNALI DI VENEZIA 1956-1960, b. 4, fasc. 1, Lettera di E. Gian Ferrari a P. Bucarelli, 28 ottobre 1958.

16 ASAC: Biennale 1958. Registro di vendita nr. 51. 
Giorgia Cicalini

Palma Bucarelli e la Biennale di Venezia (1948-1968)

Tabella 7 Acquisti destinati alla Galleria Nazionale d'Arte Moderna di Roma, 1958

\begin{tabular}{llr}
\hline Opera & Artista & prezzo (lire) \\
\hline Lanterna & Kenzo Okada & 300.000 \\
Unità tripartita (bronzo) & Max Bill & 1.500 .000 \\
Circo trasfigurato & Mark Tobey & 1.940 .000 \\
Tabula rasa (pad. Spagna) & Antoni Tapies & 700.000 \\
Gesto (pad. Spagna, scultura in ferro) & Eduardo Chillida & 1.788 .000 \\
Nudo giacente & William Scott & 340.000 \\
Sabbia distreghe (incisione) & William Hayter & 53.000 \\
Battaglia (bronzo) & Umberto Mastroianni & 1.030 .000 \\
\hline
\end{tabular}

La Commissione per gli acquisti del Ministero per la Biennale del 1960 è composta da Palma Bucarelli, Giuli Carlo Argan, Rodolfo Pallucchini e Cesare Brandi. All'ormai consueto problema dell'inadeguatezza dei sei milioni stanziati dallo Stato rispetto alle somme richieste dal nuovo mercato internazionale si aggiungono un gran numero di opere non in vendita, o già vendute dalle Gallerie che ne sono sempre più frequentemente le proprietarie. Ciò determina l'impossibilità di comprare alcuni artisti come Hans Hartung, tra le priorità della Commissione, e Pierre Alechinsky, entrambi di proprietà della Galerie de France di Parigi, non disposta a vendere in questa occasione. Gli acquisti che il Ministero riesce a portare a termine sono comunque tutti di carattere internazionale e in linea con 'l'invasione' dell'arte informale che sembra aver caratterizzato questa XXX edizione della manifestazione veneziana.

La direttrice inoltre riesce a ricevere in dono il dipinto Tempo d'estate di Fautrier, probabilmente grazie all'intervento del mercante Sami Tarica, anche se avrebbe preferito di uno dei preziosissimi Otages per rappresentare il 'maestro dell'informale' nella sua Galleria.

Le opere acquisite risultano dunque: ${ }^{17}$

Tabella 8 Acquisti destinati alla Galleria Nazionale d'Arte Moderna di Roma, 1960

\begin{tabular}{llr}
\hline Opera & Artista & prezzo (lire) \\
\hline $\begin{array}{l}\text { Scherzo 1954 (pad. Germania) } \\
\begin{array}{l}\text { Krokadeel (pad. Gran Bretagna, } \\
\text { bronzo) }\end{array}\end{array}$ & Willi Baumeister & 2.625 .000 \\
Pittura 1959 (pad. Francia) & Camille Bryen & 1.700 .000 \\
Fioritura (pad. Giappone) & Toshimitsu Imai & 400.000 \\
Pittura (pad. Spagna) & Louis Feito & 300.000 \\
\hline
\end{tabular}

17 ASAC: Biennale 1960. Registro di vendita nr. 54. 
Per l'edizione del 1962 l'ammontare dei fondi stanziati dal Ministero rimane identico a quello delle Biennali scorse (6 milioni di lire), mentre i prezzi delle opere continuano ad aumentare.

Al momento della riunione della Commissione, le schede delle opere vendibili sono pervenute dall'ufficio della Biennale solo in parte, perciò Bucarelli viene incaricata di seguire la faccenda e di formulare in seguito, delle proposte definitive che risultano le seguenti: Arshile Gorky, Jean Paul Riopelle, Emil Schumacher, Kumi Sugai, Gullaume Corneille per quanto riguarda la pittura; Giacometti, Robert Adams, Louise Nevelson, Pablo Serrano, Shinkichi Tajiri per la scultura; Eric Heckel come incisore.

L'acquisto della scultura Grande Donna (1960) di Giacometti avviene grazie ad accordi personali presi con Bucarelli, poiché i bronzi esposti in Biennale risultavano non vendibili. Come per l'acquisto precedente di un'opera di Giacometti nel 1956, la scelta e la produzione dell'opera sono lunghe e complesse, dato il carattere particolarmente sensibile e dubbioso dell'artista. La scultura verrà effettivamente data al museo per 2.000.000 lire solo nel giugno del 1964, quando l'atto di cessione viene inviato all'ambasciata di Parigi per essere firmato da Giacometti stesso, dunque presumibilmente troppo tardi per rientrare nel bilancio del 1962. Solo il 29 novembre 1962 parte degli acquisti proposti giunge a una risoluzione: ${ }^{18}$

Tabella 9 Acquisti destinati alla Galleria Nazionale d'Arte Moderna di Roma, 1962

\begin{tabular}{llr}
\hline Opera & Artista & prezzo (lire) \\
\hline $\begin{array}{l}\text { Due forme circolari }{ }^{\circ} \text { 2 (pad. Gran } \\
\text { Bretagna, acciaio bronzato) }\end{array}$ & Robert Adams & 2.058 .822 \\
$\begin{array}{l}\text { Due linee (pad. Giappone) } \\
\text { Volta pergli uomini (pad. Spagna) }\end{array}$ & Kumi Sugai & 868.000 \\
$\begin{array}{l}\text { Franzi Coricata (pad. Germania, } \\
\text { xilografia) }\end{array}$ & Eric Heckel & 750.000 \\
\hline
\end{tabular}

L'apertura alla contemporaneità durante le Biennali guidate da Dell'Acqua viene riconfermata nel 1964 con l'innovativa rassegna Arte d'oggi nei Musei, proposta da Argan e ospitata nelle sale del Padiglione centrale. I prestatori delle opere sono diciotto tra i più importanti musei europei e d'oltreoceano, di cui tre italiani: Galleria Civica d'Arte Moderna di Torino, Galleria Nazionale d'Arte Moderna di Roma, Galleria Internazionale d'Arte Moderna di Venezia; e quindici internazionali: Moderna Museet di Stoccolma, Wallraft-Richartz Museum di Colonia, Kunsthaus di Zurigo, Museum des 20 Jahrhun-

18 ASAC: Biennale 1960. Registro di vendita nr. 60. 
derts di Vienna, Musées Royoux des Beaux Arts di Bruxelles, Hamburger Kunsthalle di Amburgo, Museu de Arte Moderna di Rio de Janeiro, Neue Staatsgalerie di Monaco, Tate Gallery di Londra, Kaiser Wilhelm Museum di Krefeld, Nationalgalerie di Berlino, Solomon R. Guggenheim Museum di New York, Musée National d’Art Moderne di Parigi, Galerja Suvremene Umjetnosti di Zagabria. Le istituzioni invitate sono tenute a esporre opere eseguite dopo il 1950, per un terzo di provenienza nazionale e per i restanti due terzi di autori internazionali, per evidenziare i circuiti internazionali del mercato e della cultura, e fornire il materiale per un'analisi della situazione attuale e reale - come scrive in catalogo lo stesso curatore Argan - attraverso «una rassegna capace di mostrare con quali criteri di valore i musei provvedono a mettere in rapporto, con finalità educativa, l'arte contemporanea con tutta la società a cui è destinata» (1964, 12). Ogni museo è libero di caratterizzarsi in base ai propri criteri e ad ogni selezione viene riservato un settore dell'Esposizione; per Bucarelli è l'occasione perfetta per esporre le sue recenti e contestate acquisizioni, e dimostrare la validità della sua linea di pensiero.

L'Esposizione viene sistemata nella grande sala Rotonda all'ingresso del Padiglione centrale e è la stessa Bucarelli a presentare in catalogo la sua Galleria. Nella presentazione (cf. Bucarelli 1964, 20-1) dopo un breve inquadramento storico, dichiara apertamente quali sono i suoi progetti e la sua politica negli acquisti, non tralasciando di puntualizzare la tanto lamentata limitatezza dei fondi, annoverando le provenienze che qui si evidenziano in un prospetto:

Tabella 10 Opere scelte da Bucarelli per rappresentare la Galleria d'Arte Moderna di Roma alla rassegna Arte d'oggi nei Musei, 1964

\begin{tabular}{llll}
\hline Opera & Artista & Anno & Acquisto in Biennale \\
\hline $\begin{array}{l}\text { Grande Sacco } \\
\text { Superficie }{ }^{\circ} \text { 335 }\end{array}$ & Alberto Burri & 1952 & \\
$\begin{array}{l}\text { Concetto spaziale - } \\
\text { attese }\end{array}$ & Giuseppe Capogrossi & 1959 & \\
$\begin{array}{l}\text { Tempi d'estate } \\
\text { Il compianto degli }\end{array}$ & Jean Fautrier & 1957 & XXX Biennale (1960) \\
$\begin{array}{l}\text { amanti } \\
\text { Lanterna }\end{array}$ & Joan Mirò & 1953 & XXVII Biennale (1954) \\
$\begin{array}{l}\text { Wood construction in } \\
\text { maroon, black and white }\end{array}$ & Kenzo Okada & 1958 & XXIX Biennale (1958) \\
$\begin{array}{l}\text { Ritmi nello spazio } \\
\text { Tabula rasa }\end{array}$ & Enrico Prampolini & 1955 & XXIX Biennale (1958) \\
$\begin{array}{l}\text { The fall of Kyperion } \\
\text { Circo trasfigurato }\end{array}$ & Antoni Tapies & 1958 & XXIX Biennale (1958) \\
Plurimo 1-lemaniaddosso & Mark Tobey & 1962 & \\
\hline
\end{tabular}




\begin{tabular}{llll}
\hline Opera & Artista & Anno & Acquisto in Biennale \\
\hline Due forme circolari ${ }^{\circ}$ 2 & Robert Adams & 1962 & XXXI Biennale (1962) \\
Le rêve passe & Fernandez Arman & 1963 & \\
Grande donna & Alberto Giacometti & 1962 & XXXI Biennale (1962) \\
Figura distesa: forma & Henri Moore & $1953-$ & \\
esterna & & 54 & \\
Sfera $^{\circ}$ 2 & Arnaldo Pomodoro & 1963 & \\
${\text { Volta pergli uomini }{ }^{\circ} \text { 51 }}_{\text {Ultime stelle }}$ & Pablo Serrrano & 1962 & XXXI Biennale (1962) \\
Orizzontale IV & Atanasio Soldati & 1950 & \\
Nudo & Francesco Somaini & 1959 & \\
\hline & Alberto Viani & 1951 & XXVIII Biennale (1956) \\
\hline
\end{tabular}

Per questa Biennale i commissari nominati dal Ministero decidono di provvedere all'acquisto di un'opera di grande importanza artistica e storica destinando quindi metà della somma disponibile per scultura di Julio Gonzalez esposta alla retrospettiva allestita nel Padiglione della Francia. La scultura in bronzo di Gonzalez Ballerina con tavolozza (1933) viene acquistata per 5.000.000 lire, riuscendo inoltre a ridurre il prezzo iniziale di ben 2 milioni. A tal proposito torna evidente il problema della trattativa diretta della Bucarelli con gli artisti/mercanti, già emerso nella passata edizione del 1962 con l'acquisto 'extra Biennale' della scultura di Giacometti. Gian Ferrari sostiene infatti che la provvigione da pagare alla Biennale spetti all'acquirente, quindi la cifra accordata con la Galerie de France risulterebbe essere di 7.470.584 lire, secondo Palma Bucarelli invece essa va presa invece dai 5 milioni già stabiliti, oltretutto erroneamente considerati in franchi dalla Galerie de France. Le trattative per definire gli acquisti giungono al termine il 18 ottobre 1964, e sono: ${ }^{19}$

Tabella 11 Acquisti destinati alla Galleria Nazionale d'Arte Moderna di Roma, 1964

\begin{tabular}{llr}
\hline Opera & Artista & prezzo (lire) \\
\hline $\begin{array}{l}\text { Convertitore dello spirito } \\
\text { (pad. Svizzera, scultura in ottone) }\end{array}$ & Zoltan Kemeny & 4.350 .000 \\
$\begin{array}{l}\text { Soluzione di continuità } \\
\text { (pad. Giappone) }\end{array}$ & Hisao Domoto & 868.000 \\
$\begin{array}{l}\text { Coelum I } \\
\text { (pad. Giappone, pannelli in legno) }\end{array}$ & Toyofoku Tomomori & 800.000 \\
Ballerina con tavolozza & Julio Gonzalez & 5.000 .000 \\
\hline
\end{tabular}

Per la XXXIII Biennale la Commissione per gli acquisti del Ministero è formata da Bucarelli, Argan e Giorgio Vigni, e si trova sempre più in

19 ASAC: Biennale 1964. Registro di vendita nr. 61. 
difficoltà nella gestione delle vendite da parte della Biennale stessa. Sono in diminuzione le opere disponibili alla vendita e la Commissione si trova sempre più spesso costretta a condurre o a far condurre all'Ufficio vendite lunghe trattative con i mercanti, a volte restii a comunicare i prezzi perché speranzosi di aumentarne le quotazioni con il successo della mostra o eventuali premi. Tali problemi si presentano, ad esempio, per l'acquisto di Vibrazione azzurra e nera (1966) di Jesus Rafael Soto indicata dalla Commissione, ma non vendibile in quanto dichiarata di proprietà privata, poi fortunatamente sostituita con il Gran muro panoramico vibrante (1966), messo in vendita all'ultimo momento dall'artista. L'opera è indice dell'interesse che Bucarelli nutre per le ricerche dell'arte cine-visuale, alla quale dedicherà quattro sale nel suo ultimo allestimento della Galleria nel 1968.

Gli acquisti del 1966 risultano dunque:

Tabella 12 Acquisti destinati alla Galleria Nazionale d'Arte Moderna di Roma, 1966

\begin{tabular}{|c|c|c|}
\hline Opera & Artista & prezzo (lire) \\
\hline Luna Nuova (pad. Gran Bretagna) & Richard Smith & 500.000 \\
\hline Quattro cerchi (pad. Giappone) & Onosato Toshinobu & 350.000 \\
\hline $\begin{array}{l}\text { Quattro incisioni colorate } \\
\text { (pad. Giappone, incisione) }\end{array}$ & Ikeda Masuo & \\
\hline $\begin{array}{l}\text { Sei incisioni colorate } \\
\text { (pad. Giappone, incisione) }\end{array}$ & Ikeda Masuo & 450.000 \\
\hline Vegetativo III & Walter Linck & 800.000 \\
\hline $\begin{array}{l}\text { Gran muro panoramico vibrante } \\
\text { (pad. Venezuela) }\end{array}$ & Rafael Soto & 2.500 .000 \\
\hline $\begin{array}{l}\text { La fuerza } \\
\text { (pad.Spagna) }\end{array}$ & Juan Genoves & 250.000 \\
\hline $\begin{array}{l}\text { Vestito d'oro } \\
\text { (pad. Jugoslavia, incisione) }\end{array}$ & Bogdan Mesko & 70.000 \\
\hline Elementi (pad. Brasile, scultura) & Sergio Camargo & 500.000 \\
\hline
\end{tabular}

Le dieci incisioni colorate a puntasecca di Masuo Ikeda, che vanno ad incrementare la raccolta di opere giapponesi già possedute dalla Galleria, sono: ${ }^{20}$

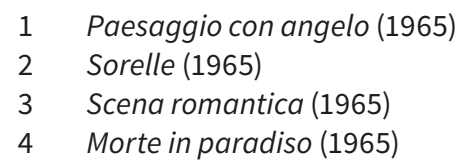

20 L'elenco delle incisioni di Masuo Ikeda è presente in Durbé 1966, 221. 
$5 \quad$ Spring and springs (1965-66)

6 Pubblicità di Cenerentola (1965-66)

7 Rosa è rosa (1965-66)

8 Sogno d'estate (1966)

9 Qualcosa I (1966)

10 Qualcosa II (1966)

Nel 1968, il clima internazionale di contestazione giovanile, e non solo, attraversa anche il settore culturale e inevitabilmente raggiunge Venezia. La Biennale è vista come un simbolo dello sfruttamento capitalistico della cultura e dell'arte, inoltre l'imponente presenza delle forze dell'ordine, schierate preventivamente dopo gli episodi della Triennale di Milano, contribuisce a creare un clima di assedio che porterà molti artisti ad aderire alla protesta. Si manifesta contro la violenza della polizia, contro lo statuto fascista della Biennale, risalente ancora al 1938, simbolo del controllo economico sull'arte da parte del governo, contro i criteri di selezione che impediscono la libera partecipazione degli artisti; contro la mercificazione delle idee e l'organizzazione burocratica delle strutture artistiche, rappresentati idealmente dai premi in denaro e dall'Ufficio vendite. La manifestazione, per quanto numericamente inferiore a molte altre ed anche allo schieramento di polizia dispiegato in tutta la città, porta alla luce i problemi strutturali della Biennale, in verità già sollevati negli anni passati da critici e commissari, e crea difficoltà negli allestimenti e all'inaugurazione (cf. Dorigo 1968, 69; Portinari 2018). Palma Bucarelli inoltre per quella edizione presenterà in catalogo Pino Pascali.

Per quanto riguarda gli acquisti del Ministero la Commissione è composta dalla Bucarelli, da Cesare Gnudi, Aldo Calò, Gino Bacchetti, Oreste Ferrari e impiega gli 8 milioni disponibili in opere esclusivamente di artisti stranieri: ${ }^{21}$

Tabella 13 Acquisti destinati alla Galleria Nazionale d'Arte Moderna di Roma, 1968

\begin{tabular}{llr}
\hline Opera & Artista & prezzo (lire) \\
\hline Lux 9, 1959 (pad. Francia, scultura) & Nicholas Schöffer & 5.000 .000 \\
Senza titolo (scultura) & John Chamberlain & 3.000 .000 \\
La casa rossa & Octav Grigorescu & 100.000 \\
(pad. Romania, disegno) & & \\
\hline
\end{tabular}

Dagli acquisti di Palma Bucarelli alle Biennali di Venezia nel ventennio 1948-68 appare dunque evidente come la Direttrice combatta le sue battaglie per l'arte astratta e non figurativa direttamente nelle

21 ASAC: Biennale 1968. Registro di vendita nrr. 66-8. 
sale del museo, motivata dall'irremovibile volontà di aggiornamento della Galleria Nazionale d'Arte Moderna di Roma sui movimenti internazionali e sull'arte del proprio tempo, presupposto per la formazione del pubblico e per elevare la qualità del museo al pari delle migliori istituzioni internazionali.

Tenendo presente che la finalità prioritaria degli acquisti ministeriali alla Biennale di Venezia è quella di ottenere opere di riconosciuto rilievo che possano rappresentare i valori fondamentali dell'arte attuale, si evince dai verbali delle varie Commissioni annuali l'intento che la Galleria è chiamata a raggiungere un livello internazionale e la conseguente esigenza di

destinare la somma ad acquisti di opere d'arte straniera, di cui la Galleria ha grande penuria. Infatti, per quanto riguarda l'arte italiana la galleria offre già un panorama se non esauriente abbastanza vasto, e in ogni modo si presentano continuamente molte occasioni di acquisti non solo in grandi esposizioni nazionali quali la Quadriennale, ma anche in mostre personali o anche presso gli artisti. Per l'arte straniera invece, la Biennale di Venezia è una delle rare occasioni di arricchire le raccolte della Galleria. ${ }^{22}$

Il numero di opere straniere sul totale degli acquisti va visibilmente aumentando da quando Palma Bucarelli entra a far parte del direttivo della Galleria: dalle due sole opere di Moore del 1948 su un totale di 22 acquisti conclusi, a cinque opere internazionali su cinque totali acquistate nel 1960. A partire dal 1960 il numero complessivo delle opere ottenute diminuisce notevolmente, ma quelle che entrano nelle collezioni sono tutte di provenienza internazionale, perciò i costi da sostenere sono più alti e non permettono le stesse quantità registrate con acquisti di opere italiane. Per colmare le lacune nel 1954 ne giungono due, una di Max Ernst e una di Joan Mirò; l'americano Tobey e gli spagnoli Tapies e Chillida vengono incamerati nel 1958; a rappresentare per la prima volta l'espressionismo tedesco è una litografia a colori di Heckel nel 1962; con Lanterna di Kenzo Okada, acquistata nel 1958, si aprono le porte all'arte contemporanea giapponese, la cui presenza nelle raccolte del museo verrà arricchita acquistando, tra il 1960 e il 1966, anche opere di Toshimitsu Imai, Kumi Sugai, Hisao Domoto, Toyofoku Tomomori, Onosato Toshinobu e dieci incisioni colorate di Ikeda Masuo; con l'acquisto di Gran muro panoramico vibrante di Rafael Soto, nel 1966, e, alla Biennale seguente, di Lux 9 di Nicholas Schöffer, Bucarelli sottolinea, infine, il suo interesse per l'arte cinetica e visuale.

22 Archivio Storico GNAM, POS. 9B BIENNALI DI VENEZIA 1956-1960, b. 4, fasc. 1, verbale della Commissione acquisti dello Stato, s.d [1958]. 
Gli acquisti di opere straniere per la Galleria Nazionale d'Arte Moderna di Roma realizzati sotto la supervisione di Palma Bucarelli dal 1948 al 1968 - con un totale di 49 opere - si riassume dunque nel seguente prospetto:

Tabella 14 Opere straniere acquistate per la Galleria Nazionale d'Arte Moderna di Roma sotto la supervisione di Palma Bucarelli dal 1948 al 1968

\begin{tabular}{|c|c|c|c|}
\hline Opera & Artista & Biennale & $\begin{array}{r}\text { prezzo } \\
\text { (lire) }\end{array}$ \\
\hline Figura sdraiata (disegno) & Henry Moore & XXIV (1948) & 30.000 \\
\hline Gruppo di famiglia & Henry Moore & XXIV (1948) & 120.000 \\
\hline Coppa chimerica (bronzo) & Jean Arp & XXV (1950) & 1.000 .000 \\
\hline Il grido & Rufino Tamayo & XXV (1950) & 1.000 .000 \\
\hline Gruppo che cammina (scultura) & Kenneth Armitage & XXVI (1952) & 80.000 \\
\hline Vita da cani (disegno) & Alfred Kubin & XXVI (1952) & 5.000 \\
\hline Orgia campestre (disegno) & Alfred Kubin & XXVI (1952) & 35.000 \\
\hline L'orco (scultura) & Germaine Richier & XXVI (1952) & 50.000 \\
\hline Hirbert Hise (pad. Israele) & Mordechai Ardon & XXVII (1954) & 500.000 \\
\hline Compendio della storia universale & Max Ernst & XXVII (1954) & 500.000 \\
\hline Il compianto degli amanti & Joan Mirò & XXVII (1954) & 1.350 .000 \\
\hline $\begin{array}{l}\text { Piccola bestia (pad. Gran } \\
\text { Bretagna, scultura in ferro) }\end{array}$ & Lynn Chadwick & XXVIII (1956) & 87.550 \\
\hline Figura (pad. Francia, bronzo) & Alberto Giacometti & XXVIII (1956) & 515.000 \\
\hline $\begin{array}{l}15 \text { febbraio (pad. Francia, } \\
\text { incisione) }\end{array}$ & Adam Henry & XXVIII (1956) & 36.000 \\
\hline $\begin{array}{l}\text { Veduta di Parigi da Sacr Coeur } \\
\text { (pad. Grecia, litografia) }\end{array}$ & Galanis & XXVIII (1956) & 11.000 \\
\hline Venezia (penna e pastello) & Dragotescu & XXVIII (1956) & 51.000 \\
\hline $\begin{array}{l}\text { L'adolescente } \\
\text { (pad. Francia, bronzo) }\end{array}$ & André Arbus & XXVIII (1956) & 721.000 \\
\hline Lanterna (pad. Giappone) & Kenzo Okada & XIX (1958) & 30.000 \\
\hline Unità tripartita (bronzo) & Max Bill & XIX (1958) & 1.500 .000 \\
\hline Circo trasfigurato & Mark Tobey & XIX (1958) & 1.940 .000 \\
\hline Tabula rasa (pad. Spagna) & Antoni Tapies & XIX (1958) & 700.000 \\
\hline $\begin{array}{l}\text { Gesto (pad. Spagna, scultura } \\
\text { in ferro) }\end{array}$ & Eduardo Chillida & XIX (1958) & 1.788 .000 \\
\hline Nudo giacente & William Scott & XIX (1958) & 340.000 \\
\hline Sabba distreghe (incisione) & William Hayter & XIX (1958) & 53.000 \\
\hline Scherzo 1954 (Pad. Germania) & Willi Baumeister & XXX (1960) & 2.625 .000 \\
\hline $\begin{array}{l}\text { Krokadeel Paolozzi (pad. Gran } \\
\text { Bretagna, bronzo) }\end{array}$ & Eduardo Paolozzi & XXX (1960) & 1.700 .000 \\
\hline Pittura 1959 (Pad. Francia) & Camille Bryen & XXX (1960) & 400.000 \\
\hline
\end{tabular}


Giorgia Cicalini

Palma Bucarelli e la Biennale di Venezia (1948-1968)

\begin{tabular}{|c|c|c|c|}
\hline Opera & Artista & Biennale & $\begin{array}{r}\text { prezzo } \\
\text { (lire) }\end{array}$ \\
\hline Fioritura (Pad. Giappone) & Toshimitsu Imai & XXX (1960) & 300.000 \\
\hline Pittura (Pad. Spagna) & Louis Feito & XXX (1960) & 665.000 \\
\hline $\begin{array}{l}\text { Due forme circolari } n^{\circ} 2 \text { (pad. } \\
\text { Gran Bretagna, acciaio bronzato) }\end{array}$ & Robert Adams & XXXI (1962) & 2.058 .822 \\
\hline Due linee (pad. Giappone) & Kumi Sugai & XXXI (1962) & 868.000 \\
\hline $\begin{array}{l}\text { Volta per gli uomini, } 51 \\
\text { (pad. Spagna, bronzo) }\end{array}$ & Pablo Serrano & XXXI (1962) & 750.000 \\
\hline $\begin{array}{l}\text { Franzi coricata } \\
\text { (pad. Germania, xilografia) }\end{array}$ & Eric Heckel & XXXI (1962) & 355.000 \\
\hline Grande Donna (bronzo) & Alberto Giacometti & XXXI (1962) & 2.000 .000 \\
\hline $\begin{array}{l}\text { Convertitore dello spirito } \\
\text { (pad. Svizzera, scultura in } \\
\text { ottone) }\end{array}$ & Zoltan Kemeny & XXXII (1964) & 4.350 .000 \\
\hline Ballerina con tavolozza (bronzo) & Julio Gonzalez & XXXII (1964) & 5.000 .000 \\
\hline $\begin{array}{l}\text { Soluzione di continuità } \\
\text { (pad. Giappone) }\end{array}$ & Hisao Domoto & XXXII (1964) & 868.000 \\
\hline $\begin{array}{l}\text { Coelum I (pad. Giappone, } \\
\text { pannelli in legno) }\end{array}$ & Toyofoku Tomomori & XXXII (1964) & 800.000 \\
\hline Luna nuova (pad. Gran Bretagna) & Richard Smith & XXXIII (1966) & 500.000 \\
\hline Quattro cerchi (pad. Giappone) & Onosato Toshinobu & XXXIII (1966) & 350.000 \\
\hline $\begin{array}{l}\text { Quattro incisioni colorate } \\
\text { (pad. Giappone, incisione) }\end{array}$ & Ikeda Masuo & XXXIII (1966) & \\
\hline $\begin{array}{l}\text { Sei incisioni colorate } \\
\text { (pad. Giappone, incisione) }\end{array}$ & Ikeda Masuo & XXXIII (1966) & 450.000 \\
\hline $\begin{array}{l}\text { Vegetativo III } \\
\text { (pad. Svizzera, scultura) }\end{array}$ & Walter Linck & XXXIII (1966) & 800.000 \\
\hline $\begin{array}{l}\text { Gran muro panoramico vibrante } \\
\text { (pad. Venezuela) }\end{array}$ & Rafael Soto & XXXIII (1966) & 2.500 .000 \\
\hline La fuerza (pad. Spagna) & Juan Genoves & XXXIII (1966) & 250.000 \\
\hline $\begin{array}{l}\text { Vestito d'oro (pad. Jugoslavia, } \\
\text { incisione) }\end{array}$ & Bogdan Mesko & XXXIII (1966) & 70.000 \\
\hline Elementi (pad. Brasile, scultura) & Sergio Camargo & XXXIII (1966) & 500.000 \\
\hline $\begin{array}{l}\text { Lux 9, } 1959 \text { (pad. Francia, } \\
\text { scultura) }\end{array}$ & Nicholas Schöffer & XXXIII (1968) & 5.000 .000 \\
\hline Senza titolo, 1965 (scultura) & John Chamberlain & XXXIII (1968) & 3.000 .000 \\
\hline $\begin{array}{l}\text { La casa rossa (pad. Romania, } \\
\text { disegno) }\end{array}$ & Octav Grigorescu & XXXIII (1968) & 100.000 \\
\hline
\end{tabular}


Per quanto riguarda le opere degli italiani, si può notare come gli acquisiti rispondano agli stessi propositi di aggiornamento del patrimonio della Galleria, ma anche di sostegno ai più dotati artisti nazionali, rendendo evidente, anche in territorio italiano, la predilezione della Bucarelli per l'arte astratta: con Cavallo, cavaliere e caseggiato (1913-14) di Boccioni, acquistato per 1.000 .000 di lire nel 1950, aggiorna la collezione sul fronte del movimento futurista; difende la qualità dell'arte non figurativa italiana nella varietà delle sue espressioni acquistando opere di Licini, Santomaso, Afro, Alberto Viani, Mirko, Vedova, Birolli, Melli, Giuseppe Capogrossi, PietroConsagra; nello stesso tempo incrementa la raccolta dei maestri del primo Novecento romano con due opere di Mafai, Scipione e Rosai; inoltre si assicura lavori di valore, come Il giocoliere di Marini nel 1952 per 1.275 .000 lire.

La partecipazione della Galleria Nazionale alla rassegna Arte d'oggi nei musei, svoltasi durante la Biennale d’Arte del 1964, è la più chiara dimostrazione del ruolo indispensabile della manifestazione per l'aggiornamento delle collezioni del museo e dell'importanza che tali acquisizioni rivestono per la Direttrice stessa: su ventuno opere da lei selezionate, ben dieci sono state infatti acquistate durante le precedenti cinque Biennali veneziane.

\section{Bibliografia}

Argan, Giulio Carlo (1964). «Arte d'oggi nei musei». Biennale 32 1964, 11-12.

Argan, Giulio Carlo (1980). Museo perché museo come. Roma: Associazione Nazionale Musei Italiani; De Luca Editore.

Bucarelli, Palma (1956). «Acquisti della Galleria Nazionale d'arte moderna». Bollettino d'arte, sr. 4, 41, 376-81

Bucarelli, Palma (1964). «Roma. Galleria Nazionale d’Arte Moderna». Biennale 32 1964, 20-1.

Camerlengo, Rita (2009). «Un museo di arte moderna non può non essere inserito in un contesto di cultura internazionale. Le acquisizioni internazionali di Palma Bucarelli (1948-72)». Margozzi 2009, 34-41.

Cantatore, Lorenzo; Zagra, Giuliana (a cura di) (2011). Palma Bucarelli a cento anni dalla nascita = Atti della giornata di studi (Roma, 25 novembre 2010). Roma: Biblioteca Nazionale Centrale.

Celant, Germano (a cura di) (1971). Catalogo della mostra di Piero Manzoni (Roma, 6 febbraio-7 marzo 1971). Presentazione di Palma Bucarelli. Roma: De Luca.

Cicalini, Giorgia (2017). Palma Bucarelli e la Biennale di Venezia (1948-68): acquisizioni, prestiti e interventi critici della Direttrice della Galleria Nazionale d'Arte Moderna di Roma [tesi di laurea]. Venezia: Università Ca' Foscari Venezia.

Dorigo, Wladimiro (1968). «La contestazione delle manifestazioni e il problema della trasformazione della Biennale». Questitalia, 125-6, agosto-settembre, 69. 
Durbé, Dario (1966). «Acquisti della Galleria Nazionale d'arte moderna». Bollettino d'arte, sr. 4, 51, 219-22.

Ferrario, Rachele (2010). Regina di quadri. Vita e passioni di Palma Bucarelli. Milano: Mondadori.

Ferrario, Rachele (2011). «L'arte nuova alla GNAM e il pensiero critico di Palma Bucarelli da Pollock a Rothko, da Pascali a Manzoni». Cantatore, Zagra 2011, 59-67.

Margozzi, Mariastella (a cura di) (2009). Palma Bucarelli: il museo come Avanguardia. Milano: Electa.

Portinari, Stefania (2018). Anni Settanta. La Biennale di Venezia. Venezia: Marsilio Editori. 


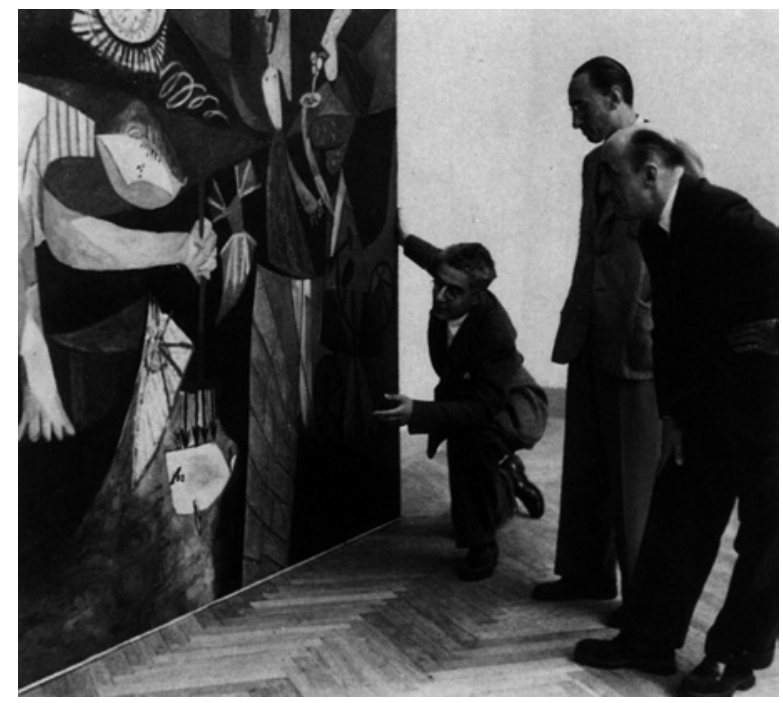

Figura 1 Umbro Apollonio tra Rodolfo Pallucchini e Giulio Baradel davanti al dipinto di Picasso, Pesca notturna ad Antibes alla Biennale di Venezia del 1948. Foto Interfoto, in Abbott, Dortch Dorazio 1994, 166 


\title{
Umbro Apollonio e l'Archivio della Biennale di Venezia (1948-1972)
}

Vittorio Pajusco

Università Ca’ Foscari Venezia, Italia

\begin{abstract}
In 1948 Rodolfo Pallucchini requested the collaboration of Umbro Apollonio to organize the 24th Venice Biennale. In 1949 the critic became the permanent curator of the Historical Archive of Contemporary Art (The Biennale Archive). First of all Apollonio organized the archival documentation of the Biennale and for this reason he thought of a new project for the library and the archive: to realize it he previously entrusted with the architect Carlo Scarpa and then with BBPR Group. After the great disorders of the 1968 edition, in 1970 Apollonio became Bienniale director. He curated with Dietrich Mahlow the special exhibition «Proposal for an experimental exhibition». On this occasion, a strong dialogue with the public was sought, focusing on issues such as art and society, art and production, analysis of seeing. The result was an exhibition holding arts from historical avant-garde to the most recent researches.
\end{abstract}

Keywords Umbro Apollonio. Biennale archive. Biennale library. Carlo Scarpa. BBPR. Domenico Varagnolo.

L'Archivio della Biennale di Venezia nasce nel 1927 grazie alla lungimirante idea del segretario generale Antonio Maraini che lo immaginava come un «centro di studi» indispensabile per i futuri storici dell'arte moderna. Il primo conservatore dei fondi della mostra veneziana è il poeta e commediografo Domenico Varagnolo che, in circa vent'anni di appassionato lavoro, mette assieme una notevole mole di documentazione eterogenea (carte, libri, fotografie), seppur con ristrette risorse finanziarie. ${ }^{1}$

1 Sulle origini dell'Archivio della Biennale si veda: Pajusco 2016a (con bibl. prec.) e su Domenico Varagnolo cf. Pajusco 2013. 
Nel primo dopoguerra la Biennale guidata dal commissario straordinario Giovanni Ponti riprende con lentezza le proprie attività, prima con la riapertura della mostra del cinema e del festival della musica, mentre per il settore principale delle arti visive bisogna attendere qualche anno e il completamento del restauro dei Padiglioni ai Giardini (Bazzoni 1962, 137).

Alla fine del 1947 Ponti nomina una giuria speciale per l'arte figurativa, composta da dieci tra i più importanti artisti e critici italiani. Queste personalità - Carlo Carrà, Felice Casorati, Giorgio Morandi, Pio Semeghini, Marino Marini, Nino Barbantini, Roberto Longhi, Rodolfo Pallucchini, Carlo Ludovico Ragghianti e Lionello Venturi - in occasione della loro prima seduta designano come segretario generale Rodolfo Pallucchini, ispettore alle Belle Arti del Comune di Venezia (Dal Canton 2001, Bandera 2011, Tomasella 2011, Poletto 2013). La XXIV Biennale di Venezia, per volontà della Commissione Ordinatrice, doveva colmare quella lacuna culturale e artistica che aveva fatto piombare l'Italia in uno stato di arretratezza rispetto al resto del mondo (Biennale 24 1948, 2a ed., XXII).

In quel frangente l'Archivio Storico della Biennale, che in tempi di guerra era stato depositato in alcuni locali del Museo Correr, ha un compito fondamentale come afferma pubblicamente Pallucchini sul Bollettino ufficiale del Ministero della Pubblica Istruzione annunciando la riapertura dell'Istituto storico, riassumendone anche la tormentata vicenda:

Il 15 marzo si è riaperto al pubblico l'Archivio Storico d'Arte contemporanea della Biennale. Quando i Tedeschi nel settembre del 1943 presero possesso di Venezia, la Biennale dovette cedere loro i locali di Ca' Giustinian e in fretta e furia i volumi e le fotografie, le cartelle con tutto il materiale riguardante gli artisti, furono posti in salvo al Museo Correr. Ora l'Archivio è stato riordinato a Ca' Giustinian e, oltre ad essere indispensabile fonte di informazioni per il funzionamento della stessa Biennale, può nuovamente assolvere il suo compito di prezioso strumento di pubblica cultura. (Pallucchini 1948, 92)

La realtà dei fatti, soprattutto per quanto riguarda le condizioni dell'Archivio, delude però le aspettative e le speranze di Pallucchini. Durante la guerra, dopo il frettoloso sgombero di Ca' Giustinian, i materiali dell'Archivio infatti erano stati appunto inscatolati e depositati al Museo Correr, mentre gli uffici amministrativi della Biennale avevano trovato alloggio nella casa dell'ex presidente Giusep- 
pe Volpi di Misurata, a palazzo Volpi sul Canal Grande. ${ }^{2}$ Sfogliando i carteggi che il segretario generale intrattiene con il conservatore Domenico Varagnolo ci si imbatte però in una serie di incomprensioni, che riguardano la situazione di precarietà dei documenti, il bilancio e le prospettive dell'Ente che si concluderanno soltanto con il pensionamento dello storico archivista nel gennaio del 1949. L'Archivio vede quindi cambiare il suo conservatore:

A succedergli fu nominato il noto critico d'arte triestino Umbro Apollonio. Scelta felice anche codesta, perché Apollonio come conoscitore di molti artisti italiani e stranieri e per l'autorità che gli deriva come scrittore d'arte poté dare nuovo impulso allo sviluppo dell'Archivio, ottenendo maggiori fondi per l'acquisto di opere di critica, riviste, pubblicazioni e monografie. (Bazzoni 1962, 123)

Umbro Apollonio si insedia ufficialmente a Ca' Giustinian il 1 aprile del 1949; il nuovo venuto è una figura totalmente diversa dal precedente direttore: critico militante attivo nel collaborare a molte riviste e giornali, ha pubblicato cataloghi e monografie d'artisti. ${ }^{3} \mathrm{Nel}$ 1948 aveva già collaborato con la Biennale nelle fasi preparatorie della XXIV Biennale di Venezia, occupandosi in particolare dell'organizzazione delle mostre personali e delle retrospettive [fig. 1], coadiuvando anche con Domenico Varagnolo alla compilazione del catalogo ufficiale, rieditato per ben tre volte dopo la prima stampa, con modifiche e precisazioni. ${ }^{4}$

Nel dicembre del 1949 Apollonio visita l'Archivio e la Biblioteca della Galleria Nazionale d'Arte Moderna di Roma e rileva le notevoli differenze con l'istituto della Biennale. Quello che emerge maggiormente è la sistemazione ottimale dei loro materiali e il numero delle persone, ben sei, che vi lavorano a tempo pieno. A Venezia il direttore aveva in quel momento a disposizione una sola segretaria, per di più a tempo parziale. Al suo ritorno dalla capitale scrive dunque subito una lettera al segretario generale per relazionare la sua trasferta, non senza lanciare qualche provocazione:

2 Giuseppe Volpi muore il 16 novembre 1947 a Roma, con la liberazione d'Italia nel 1945 i suoi averi vengono confiscati, per ulteriori dettagli e bibliografia cf. Pajusco 2015.

3 Vasta è la bibliografia sul critico triestino si veda: Salvagnini 2006, 2009, 2011. Per una biografia e bibliografia dettagliata: Dal Canton 1981, 1986. Per la parte storico-letteraria si rimanda a Rostellato 2013-14. Sulla presa di servizio di Apollonio cf. ASAC, FS, Asac 2, b. 03: lettera di U. Apollonio a G. Ponti, $1^{\circ}$ aprile 1949. Tra le sue pubblicazioni si segnala la collaborazione con le edizioni del Cavallino di Carlo Cardazzo per cui cura le monografie su Scipione (1945) e sui Disegni di Seurat (1947).

4 Le mostre personali e retrospettive di cui Apollonio si occupa direttamente sono: Arturo Martini, Massimo Campigli, Arturo Nathan, Gino Rossi; Biennale 24 1948, 4a ed., 25-8, 33-4, 38-9, 43-7. Quattro sono le edizioni del Catalogo della XXIV Biennale stampate dal 6 giugno 1948 (1a ed.) al 15 settembre 1948 (4a ed.). Biennale 24 1948, 4a ed., VI. 
Mi permetto soltanto di insistere nel voler sapere se l'archivio che dirigo deve essere, nelle intenzioni della Presidenza, un organismo vivo e attivo, sia sul piano generale della cultura che in quello più direttamente interessante l'organizzazione delle esposizioni biennali, oppure se è sufficiente che esso vivacchi, alla meglio, un po' trascuratamente. ${ }^{5}$

Apollonio si trova quindi a scontrarsi con la burocrazia di un ente che vive in funzione di una mostra che dura il tempo di una stagione, mentre per il resto dell'anno riceve scarsa attenzione e pochi incentivi.

I locali in cui è situata la biblioteca dell'Archivio necessitano di restauri e bisogna al più presto acquistare delle scaffalature per ordinare i materiali che da molto tempo si vanno accatastando negli armadi. Per una sistemazione di massima della biblioteca, tra il 1951 e il 1952 si prendono accordi con Carlo Scarpa, che in quegli anni lavorava agli allestimenti e alla costruzione di strutture stabili ai Giardini della Biennale (Lanzarini 2003; Dal Co, Mazzariol 1984, 112). Il 22 gennaio 1952 il direttore amministrativo Giovanni Piccini scrive all'architetto confermandogli l'affidamento dell'incarico e sottolineando che il progetto della biblioteca deve essere consegnato entro il 29 febbraio dello stesso anno. ${ }^{6}$ Scarpa però non risponde e a settembre il funzionario gli riscrive per verbalizzare lo scadere dell'accordo e la tacita rinuncia del progettista. ${ }^{7}$

Apollonio, accusata questa prima sconfitta, non si perde d'animo: la biblioteca deve essere sistemata, la sua credibilità di storico dell'arte potrebbe risentirne e quindi, probabilmente per antiche conoscenze triestine con Ernesto Nathan Rogers, prende contatti con i BBPR, il celebre gruppo di architetti formato da Gian Luigi Banfi, Ludovico Barbiano di Belgiojoso, Enrico Peressutti e Ernesto Nathan Rogers con studio a Milano. Il collettivo accetta l'incarico e il 22 ottobre 1952 Apollonio spedisce le planimetrie dei locali. ${ }^{8}$

Il 9 novembre Peressutti manda i disegni, le piante e le prospettive di come dovrebbe risultare la nuova biblioteca, proponendo anche una tipologia di ripiani mobili per movimentare i libri e scrive:

Ti ho lasciato l'impostazione che noi vorremmo dare al salone biblioteca. Si tratta cioè di evitare un soppalco a metà altezza con relativa scala. Gli scaffali stessi attraverso un congegno - senza funi di alcun genere - sarebbero mobili: con un minimo sforzo si può

5 ASAC, FS, Asac 2, b. 01: lettera di U. Apollonio a R. Pallucchini, 5 dicembre 1949.

6 ASAC, FS, Asac 1, b. 03: lettera di G. Piccini a C. Scarpa, 28 gennaio 1952.

7 ASAC, FS, Asac 1, b. 03: lettera di G. Piccini a C. Scarpa, 25 settembre 1952.

8 ASAC, FS, Asac 2, b. 03: lettera di U. Apollonio a G. Peresutti, 22 ottobre 1952. 
alzare o abbassare lo scaffale desiderato. E naturalmente in basso, lo spazio necessario - fisso per gli schedari e gli scaffali chiusi. ${ }^{9}$

Apollonio risponde che dal punto di vista estetico il progetto è molto gradevole ma il congegno non lo convince appieno, è preoccupato dal fatto che, come tutte le cose elettriche, dopo un po' si potrebbe logorare.$^{10}$ Peressutti manda allora altri disegni con spiegazioni dettagliate. ${ }^{11}$ Apollonio ancora non è convinto e nel dicembre del 1952 suggerisce in particolare che la forma degli scaffali debba essere allargata per contenere tutti i faldoni documentari. ${ }^{12}$ Giunti oramai all'anno successivo, Rogers si assume la responsabilità di seguire il progetto perché Peressutti deve partire per il Brasile..$^{13}$ Apollonio intanto, considerato che il piano di assetto sia ormai definito, si rivolge al presidente Giovanni Ponti per dare avvio ai finanziamenti per i lavori, ma la vicenda si protrae e dunque a marzo Rogers chiede notizie:

Caro Apollonio, che cosa succede alla nostra biblioteca?

In realtà, sono contento che tu abbia lasciato passare qualche tempo, perché siamo davvero molto occupati, ma, prima, o poi, mi piacerebbe realizzarla. Dammi qualche notizia e accogli, intanto i miei saluti più cordiali Ernest N. Rogers. ${ }^{14}$

Cui Apollonio risponde:

l'interrogativo è preoccupante anche per me. Infatti è ancora in forse la famosa questione del finanziamento per la biblioteca e quindi bisogna attendere. Spero anch'io con te che prima o poi si possa realizzarla. Di una cosa sono comunque puoi essere certo: che mi batterò per poter risolvere il problema al più presto. Con $\mathrm{i}$ migliori saluti Umbro Apollonio. ${ }^{15}$

A causa delle condizioni degli ambienti nel 1954 l'Archivio deve chiudere. Apollonio, in un messaggio rivolto al nuovo presidente, riporta tutta l'amarezza e la preoccupazione per le condizioni in cui si trovano i fondi storici della Biennale:

9 ASAC, FS, Asac 2, b. 03: lettera di G. Peresutti a U. Apollonio, 9 novembre 1952. Progetti e disegni sono conservati nel Fondo artistico dell'ASAC.

10 ASAC, FS, Asac 2, b. 03: lettera di U. Apollonio a G. Peresutti, 12 novembre 1952.

11 ASAC, FS, Asac 2, b. 03: lettera di G. Peresutti a U. Apollonio, 19 dicembre 1952.

12 ASAC, FS, Asac 2, b. 03: lettera di U. Apollonio a G. Peresutti, 27 dicembre 1952.

13 ASAC, FS, Asac 2, b. 03: lettera di E. Rogers a U. Apollonio, 20 gennaio 1953

14 ASAC, FS, Asac 2, b. 03: lettera di E. Rogers a U. Apollonio, 11 marzo 1953

15 ASAC, FS, Asac 2, b. 03: lettera di U. Apollonio a E. Rogers, 17 marzo 1953. 
Nel gennaio di quest'anno, poi, mi sono trovato costretto a proporre la chiusura dell'Archivio al pubblico a tempo indeterminato per il fatto che non ritenevo dignitoso ospitare il pubblico degli studiosi e dei frequentatori in genere in un ambiente che non offre le necessarie comodità di studio. ${ }^{16}$

Il conservatore prova a ricordare anche a Pallucchini la situazione grave in cui si trova l'istituto, scrivendogli: «Caro Pallucchini, oso sperare che l'annosa questione dell'Archivio Storico sarà presentata al prossimo Consiglio d'Amministrazione e non rimandata come più volte è avvenuto», dato che «la Direzione generale» era stata informata delle necessità della biblioteca e della proposta «per le scaffalature della nuova sala di lettura e la Detta direzione ha acconsentito all'esecuzione del lavoro». ${ }^{17}$

Il progetto però non procede, non vengono stanziati i finanziamenti ed è evidente che la biblioteca dell'Archivio non è prioritaria negli interessi all'amministrazione della Biennale.

In questi anni Umbro Apollonio, oltre a fungere da archivista, deve seguire l'attività principale dell'ente espositivo veneziano curando l'organizzazione delle mostre personali e retrospettive, italiane e straniere, e nelle vesti di rappresentante della Biennale viene spesso inviato all'estero per seguire altri eventi espositivi anche in paesi lontani, come nel caso delle prime edizioni della Biennale di San Paolo del Brasile (Durante 2011).

Nei primi anni Cinquanta Apollonio, pur oberato di impegni, riesce comunque a ritagliarsi del tempo per ricerche sull'arte, nate spesso dall'osservazione diretta delle opere presentate nelle sale dei Padiglioni ai Giardini, che confluiscono in importanti volumi strenuamente voluti da editori d’arte quali Carlo Cardazzo, Neri Pozza, Bruno Alfieri e Gino Ghiringhelli. Nel giro di pochi anni vengono pubblicate le monografie Marc Chagall nel 1949 (Alfieri editore), Pittura metafisica nel 1950 (Edizioni del Cavallino) e Pittura italiana moderna. Idea per una storia nel 1950 (Neri Pozza editore), Die Brücke nel 1952 (Alfieri editore), Marino Marini nel 1953 (Edizioni del Milione). Particolarmente significativo risulta il volume pubblicato da Neri Pozza Pittura italiana moderna [fig. 2] perché stampato volutamente in contemporanea al testo Pittura moderna in Europa di Giuseppe Marchiori (Apollonio 1950, Marchiori 1950). Marchiori e Apollonio sono colleghi e amici da molto tempo, probabilmente si sono conosciuti tra Milano e Venezia, tra la Bottega di Corrente e la Galleria del Cavallino, nel 1946 si ritrovano entrambi a Trieste per una mostra al Circolo della Cultura e delle Arti dove si raccolgono opere delle ultime due generazioni di pittori, di cui è esito il piccolo ma prezioso cata- 


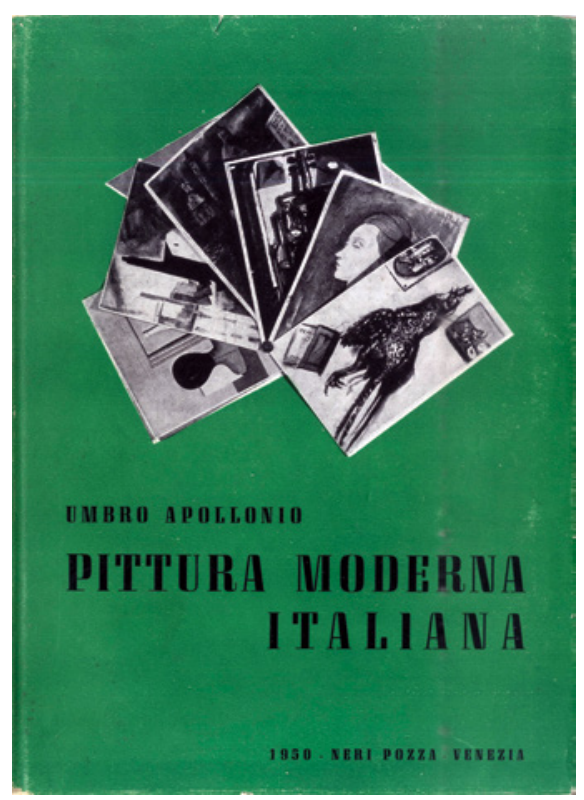

Figura 2 Umbro Apollonio. Pittura italiana moderna (sovracopertina Pittura moderna italiana). Venezia: Neri Pozza editore, 1950

logo dal titolo Pittura moderna italiana curato da Marchiori con una premessa di Apollonio (Marchiori 1946).

Quattro anni dopo Apollonio in Pittura italiana moderna. Idea per una storia si trova dunque a continuare l'itinerario tracciato dal collega mettendo assieme una serie di percorsi artistici, che spaziano da quelli di Arturo Tosi a Modigliani, a Gino Rossi, fino a Guttuso e Santomaso, senza la necessità di individuare dei movimenti o delle correnti che leghino gli artisti o che creino delle ascendenze tra i vari stili pittorici. Nell'introduzione al volume il critico triestino chiarisce bene il suo intento:

Per chi come me non ama, anzi, rimane piuttosto indifferente ai programmi, ai manifesti, alle dichiarazioni, in genere alle poetiche, la cultura figurativa si riduce a un seguito di atti ben distinti. E questo mi pare tanto meglio dimostrato in un momento, anzi, in un tempo in cui non si manifesta con evidenza, ed è quindi rintracciabile soltanto mediante qualche forzatura, un atteggiamento comune, uno sviluppo che trovi i suoi fondamenti in una polarizzazione degli interessi attorno al nucleo unico. [...] Bisogna cioè inserire così nell'arte come nella cultura la nostra facoltà di uomini vivi, capaci di uno studio che sia veramente disciplina coordinatrice e selettiva. (Apollonio 1950, 10) 
Nell'estate del 1950 viene pubblicato anche il primo numero della rivista internazionale La Biennale di Venezia che, grazie soprattutto al contributo di Apollonio, avrà una lunga durata. ${ }^{18} \mathrm{Nel}$ periodico sono accolti contributi riguardanti non solo le arti visive, ma tutte le arti. Sui primi numeri compaiono anche suoi saggi, come quello del luglio 1952 sullo scultore toscano Marino Marini, spesso presente alla Biennali di quegli anni sia nel ruolo di espositore che di commissario, che proprio nel 1952 era stato vincitore del gran premio per la scultura italiana. Il critico fa dunque trapelare la sua stima:

Dopo la delicatezza poetica di Medardo Rosso, l'esperienza ritmico architettonica di Boccioni e l'inquieta intelligenza di Martini, Marino definisce una solennità propriamente plastica, le cui cadenze gravi, asciutte, affiorate da una memoria cosciente dell'antico, non sono così devote a tale appello da restarne dominate. ${ }^{19}$

A seguito di questo articolo nel 1953 esce, per le Edizioni del Milione di Milano, la prima monografia sullo scultore toscano curata da Umbro Apollonio; l'opera, subito esaurita, viene rieditata nello stesso anno e poi ristampata nel 1958 in due edizioni, una in italiano e una in inglese.

Nel 1955 dopo la morte di Elio Zorzi, storico direttore dell'Ufficio stampa della Biennale, Umbro Apollonio vede aumentare i suoi impegni; oltre al consueto lavoro per l'organizzazione della Mostra ufficiale e la curatela del catalogo generale, gli vengono affidati anche i ruoli che deteneva il conte Zorzi, ovvero la direzione della rivista La Biennale di Venezia e la responsabilità dell'Ufficio stampa per l'Esposizione. La Biennale del 1956 sarà una edizione fortemente criticata, soprattutto dalla stampa italiana, tanto da far dire allo stesso Apollonio in uno stralcio di articolo:

Di quella critica alla quale nulla va bene, che è capace di stendere tre articoli di due e più colonne ciascuno soltanto per negare tutto, proprio tutto (tant'è vero che su 744 artisti presenti non uno ha trovato su cui soffermarsi per dirne bene), non giova tener conto. Basta leggere ciò che si scrive all'estero di questa Biennale per rendersi conto del prestigio che essa gode in tutto il mondo. ${ }^{20}$ (Apollonio 1957, 7)

18 Il primo numero della rivista La Biennale di Venezia esce il $1^{\circ}$ luglio 1950, l'ultimo numero doppio (67-8) viene editato nel dicembre 1971. Sulla storia della rivista si veda: Perosin 2015, 138-232; 2017.

19 Umbro Apollonio, «Marino», La Biennale di Venezia, 9, 1952, luglio, 35.

20 Umbro Apollonio, «La Biennale e la critica», La Biennale di Venezia, 28-9, 1957, giugno-settembre, 7 . 
Nel marzo 1957 l'editore vicentino Neri Pozza - amico di Apollonio - tuona dal settimanale di politica e letteratura Il Mondo con un titolo che non ha bisogno di spiegazioni: L'archivio storico della Biennale. Un istituto che non funziona:

La grande sala che contiene l'Archivio Storico della Biennale veneziana è chiusa al pubblico dal gennaio 1954, con un ordine del suo attuale conservatore Umbro Apollonio.

Da quella data libri, riviste, fotografie, tutto il materiale dell'Archivio si accumula e si inclina per terra, sui tavoli, negli angoli, sui bordi delle mensole, sulle sedie, in mucchi che diventano sempre più alti, polverosi e disordinati. Al punto che parlare di Archivio Storico, nelle condizioni in cui è ridotto per mancanza di spazio, è ormai improprio; com'è assurdo d'altra parte concepire la minima possibilità di ricerca e reperimento del materiale scientifico, che dovrebbe essere sempre al servizio di chi abbia la necessità di consultarlo. ${ }^{21}$

Neri Pozza porta alla luce una situazione ormai insostenibile. Conosce perfettamente l'Archivio, perché come dice lui stesso nel prosieguo dell'articolo, si è trovato «nella malaugurata necessità di ricercare dei dati» e quindi ha visto con i suoi occhi i materiali, che riesce a descrivere addirittura con numeri precisi. Parla delle riviste internazionali, dei 10.000 ritagli di stampa, della biblioteca formata da 12.000 volumi che grazie agli scambi si incrementa di 500 unità per anno e della enorme fototeca:

Le cifre parlano chiaro. Qui è raccolto un materiale prezioso per la storia dell'arte moderna e contemporanea, forse come non ne esistono in nessun altro Istituto specializzato d'Europa. E come è possibile che esso giaccia inerte e non vi sia qualcuno che provveda a trovare lo spazio sufficiente; e con lo spazio i mezzi moderni per ordinare i libri e riviste fotografie e negativi?

Prima del gennaio 1954, del giorno in cui Apollonio ordinò che l'Istituto fosse chiuso al pubblico, la situazione era di forzata coabitazione: nell'unica sala di Ca' Giustinian, dov'è il suo ufficio di conservatore, stava sia la biblioteca che la fototeca e quindi la sala di lettura: a volte, distributore del materiale bibliografico e fotografico, oltre che custode!

Neri Pozza quindi si appella agli amici Apollonio e Pallucchini, rispettivamente conservatore dell'Archivio e segretario generale del-

21 Neri Pozza, «L'archivio storico della Biennale. Un istituto che non funzione», Il Mondo, 12 marzo 1957. 
la Biennale, che però, come sa perfettamente, possono fare ben poco: chi potrebbe risolvere la faccenda è il Consiglio di Amministrazione e in particolare il presidente al quale lo scrittore chiede degli interventi d'urgenza. L'editore vicentino conclude:

Fuori dal discorso espresso per idee generali, che non serve in tempi come i nostri, va detto con la massima energia che quello di lasciar deperire Istituti come l'Archivio Storico della Biennale è una vera offesa; perché, anche stavolta, essa colpisce proprio gli studiosi insieme ai dirigenti.

Le parole di Neri Pozza raggiungono subito lo scopo, il primo di luglio di quell'anno riapre l'Archivio. ${ }^{22}$

Apollonio con la riapertura dell'Istituto riprende anche i contatti con gli architetti dello studio BBPR, che in quei mesi stavano progettando il Padiglione del Canada ai Giardini di Castello, inaugurato poi durante la Biennale d'Arte del 1958, e chiede loro altre modifiche progettuali e sollecita ulteriori preventivi dei costi dei lavori, che sembrano sempre troppo alti. ${ }^{23}$ Le condizioni dell'Archivio, se possibile, peggiorano ancora, poiché nel 1960 vengono denunciate infiltrazioni d'acqua dalle finestre della biblioteca e nel 1962 cede parte della scala d'accesso al secondo piano di Ca' Giustinian. ${ }^{24}$ Nel gennaio del 1963 Apollonio scrive una dura lettera al presidente Italo Siciliano che dichiara:

Il lavoro svolto nell'Archivio non è paragonabile nell'insieme a quello di una normale biblioteca, perché esso reperisce, riunisce e conserva oltre a libri d'arte, di musica e di teatro, anche riviste, cataloghi, fotografie, schede bio-bibliografiche, ritagli di stampa, ecc., e la raccolta di simile materiale si estende a paesi di tutti i continenti. In mie precedenti relazioni ho fornito ampi ragguagli numerici e statistici sull'entità dell'afflusso di questo materiale, nonché fatto presenti le difficili condizioni di lavoro per difetto di adeguate attrezzature e insufficienza di personale, anche in relazione ad alcune necessità di riordino dovute allo stato in cui ho trovato l'Archivio quando ne assunsi la responsabilità. Non posso che richiamarmi a quanto riferito in quelle occasioni ed alle testimonianze del Segretario Generale e del Direttore Amministrativo

22 ASAC, FS, Asac 2, b. 03: lettera di R. Pallucchini a U. Apollonio, 26 giugno 1957; lettera di U. Apollonio a R. Pallucchini, 28 giugno 1957; lettera di R. Pallucchini a U. Apollonio, 1 luglio 1957.

23 Sul Padiglione del Canada: Zevi 1958; Mulazzani 2004, 108-11; ASAC, FS, Asac 2, b. 03: lettera di U. Apollonio a E. Rogers, 24 maggio 1957.

24 ASAC, FS, Asac 1, b. 03: lettera di U. Apollonio a D. Grassi (direttore amministrativo), 5 dicembre 1960; lettera di U. Apollonio a D. Grassi, 22 marzo 1962. 
per dare un'idea del volume di attività dell'Archivio. Soltanto mantenendosi a simile livello esso può far fronte alle sue funzioni di accreditato istituto per la storia dell'arte moderna e contemporanea, quale non ha pari in Europa per consistenza patrimoniale, e rispondere alle varie richieste che da più parti pervengono. Anche eminenti critici e storici, quali il compianto prof. Venturi, il prof. Ragghianti, il Dr. Barr del Museum of Modern Art di New York, per citare solo alcuni, sono ricorsi all'Archivio per ricerche inerenti ai loro studi. [...] Confido nella Sua illuminata comprensione per le esigenze di un settore al cui contributo interno e culturale non può restare indifferente, e quindi per le mie preoccupazioni. ${ }^{25}$

Nel 1967, a quindici anni dalla creazione delle prime idee progettuali, i lavori di allestimento della biblioteca vengono finalmente assegnati alla ditta Lips Vago di Milano, che presenta la prima fattura nel febbraio $1967 .{ }^{26}$ Nel listino si parla di scaffalature, cassonature, parapetti e strutture varie ma, considerando la lentezza con cui prosegue la vicenda e il futuro incerto che si prospetta alla Biennale di quegli anni, non è dato sapere in che modo sia stato allestito lo spazio, poiché gli ultimi contatti con i BBPR risalgono ai primi anni Sessanta. ${ }^{27}$

Nel 1957 Pallucchini lascia la Segreteria Generale della Biennale, dopo aver creato alcune tra le migliori edizioni della manifestazione che, come scrive Mario Isnenghi, intendono tracciare un «progetto di storicizzazione» che passa «dalla dimensione interna - la storia dell'istituzione veneziana - a quella esterna: la storia dell'arte pregressa e rimossa, le grandi omissioni, i maestri dimenticati» (Isnenghi 1986, 467). Proprio in quell'anno si tiene a Ca' Loredan una tavola rotonda titolata Convegno di studio sulla Biennale, organizzata dal Comune di Venezia invitando i più importanti artisti e critici italiani (Atti 1957). Con la fuoriuscita di Pallucchini qualcosa sta cambiando e questo crea molte perplessità tra gli addetti ai lavori, di cui sono esemplari gli interventi di Lionello Venturi e Carlo Ludovico Ragghianti. Afferma infatti il primo:

Tutte le nazioni mandano un commissario con delle opere scelte, quelle che pensano possano far trionfare il nome delle loro nazioni. Invece l'Italia dovrebbe, per alcuni, ridursi a fare una grande sindacale. Ė necessario, e bisogna ripeterlo, diminuire enormemente il numero delle opere italiane esposte alla Biennale. (Venturi 1957, 43-4) 
Debbo constatare che all'alterazione portata all'Ente dalla presenza e dal peso diretto delle Amministrazioni centrali dello Stato, si aggiunge quella di affidare i compiti di esecuzione tecnica a un funzionario dipendente. Che cosa resta - ci si domanda - dell'autonomia dell'Ente, in tali non sane condizioni? Sempre al di fuori di ogni apprezzamento personale, quale è la differenza di posizione fra Dell'Acqua e Pallucchini? Non vi è una differenza di ordine tecnico od organizzativo, ma una differenza di sostanza. Perché? Perché il Pallucchini è un Professore universitario e pochi sanno o ricordano che quello del Professore universitario in Italia è uno stato giuridico fra i più liberi, perché i Professori universitari non prestano giuramento. Perché non prestano giuramento? Bisogna intendere bene il significato di questa distinzione, che non ha neanche la Magistratura. Il Professore Universitario non presta giuramento perché rappresenta la cultura nel suo principio, che è la libertà. (Ragghianti 1957, 89-90)

Dopo questo convegno il Governo scioglie il Consiglio Direttivo della Biennale e rinomina commissario straordinario Giovanni Ponti. Il 1957 quindi è l'anno della crisi, in cui si sente l'esigenza di una riforma dello statuto e di un cambiamento nelle modalità di ammissione delle opere degli artisti italiani che sono ancora quelle dell'epoca fascista. Così, l'ormai ex segretario generale Pallucchini, ricorda quei momenti:

Ero stanco, facevo ore impossibili: sai se ho sempre scritto di storia dell'arte, d'ogni secolo, e se ho pubblicato: anche quando ero a capo dell'Ufficio artistico del Comune, anche quando dovevo, per la Biennale, girare per gli studi, discorrere coi pittori, assistere agli arrivi delle casse, provvedere a un'infinità di cose e poi a far ordinare e appendere i quadri, con la fretta che precede una mostra d'arte moderna... Adesso mi par di potermi contentare dell'insegnamento dell'Università di Padova, dove sono succeduto al mio maestro Giuseppe Fiocco, e del mio lavoro di studioso. ${ }^{28}$

Succede a Pallucchini il soprintendente delle Gallerie di Milano Gian Alberto Dell'Acqua, che rimarrà in carica fino al 1968. Nel 1958 Umbro Apollonio, per facilitare il passaggio degli incarichi, risulta coadiutore tecnico alla Segreteria Generale e lascia definitivamente la direzione dell'ufficio stampa al giovane Wladimiro Dorigo che diviene anche un valido aiuto come redattore capo della rivista della Biennale. A partire dall'ultimo numero dell'anno 1958 il periodico presenta due facciate gialle, che il direttore impiega come una sorta di

28 Memoria riferita all'amico Aldo Camerino nell'autunno del 1957 riportata in Roddolo 2003, 45. 
editoriale, intitolandole Occasioni del tempo, su cui nel corso degli anni inserisce riflessioni sull'arte contemporanea in rapporto anche alle discipline artistiche..$^{29}$

Se gli anni Sessanta alla Biennale, da un punto di vista artistico, porteranno alla ribalta movimenti importanti del contesto internazionale come l'Informale, la Pop Art e l'Optical, il 1968 è l'anno delle contestazioni. Nei primi mesi di quell'anno, in una conversazione con il critico inglese Lawrence Alloway pubblicata nel volume The Venice Biennale from Salon to Goldfish Bowl (1968), Apollonio condivide la preoccupazione sulle sorti dell'istituzione veneziana e ammette che è necessario un forte cambiamento (Alloway 1968, 22).

Pochi giorni l'inaugurazione della mostra, il 18 giugno le proteste giovanili in piazza San Marco vengono represse con le cariche della polizia, e i giornali titolano la Biennale del manganello. La maggior parte degli artisti italiani presenti all'Esposizione, in sostegno ai manifestanti, gira le opere verso le pareti, alcuni scrivendoci dietro Biennale fascista, mentre molte delle mostre organizzate, come alcuni Padiglioni nazionali, non apriranno nemmeno. ${ }^{30}$

A conclusione di questa Biennale, nei giorni 15, 16 e 17 novembre, il Comune di Venezia propone un convegno, in cui sono presenti sia le autorità cittadine sia significativi critici e artisti, che si intitola Una nuova Biennale: contestazioni e proposte organizzato nella Sala delle Colonne di Ca' Giustinian. ${ }^{31} \mathrm{Al}$ termine dei lavori emergono alcune mozioni conclusive firmate dai critici e un documento firmato dagli artisti che inizia con una sentenza capitale: La Biennale è morta. Da molti anni non era più un centro vivo della produzione e di diffusione della cultura. ${ }^{32}$

Dopo le bufere dell'ultima edizione e le dimissioni del segretario generale Dell'Acqua, la Biennale naviga in mari turbolenti. La 35a Esposizione del 1970 è alle porte e allora si decide, caso unico nell'intera storia della Biennale, che quella edizione sarebbe stata organizzata internamente, senza un segretario generale ma soltanto con un direttore organizzativo: il conservatore dell'Archivio Umbro Apollonio. ${ }^{33}$ Le proteste del '68 avevano sortito alcuni cambiamenti radicali nell'Esposizione come l'abolizione dei premi e la rinuncia alle mostre monografiche, favorendo invece rassegne tematiche quali Ricerca e progettazione e Arte e società. ${ }^{34}$ Il direttore Umbro Apollonio

29 Alcuni di questi saggi verranno poi raccolti dallo stesso autore in Apollonio 1979.

30 Cf. Budillon Puma 1995, 171. Sulla Biennale del 1968: Portinari 2018, 47-128

31 «Una nuova biennale» 1969. Sulle proteste e il convegno si veda Pajusco 2016b.

32 «Una nuova biennale» 1969, 21.

33 Sulla Biennale degli anni Settanta e quella del 1970 in particolare: Portinari 2018, 129-68; Zanella 2017; Di Raddo 2017.

34 Sul dibattito internazionale post-Biennale 1968 si veda: Martini, Martini 2011, 46-54 
con la collaborazione di Dietrich Mahlow, già curatore della mostra di poesia concreta nel 1969, realizza la mostra speciale Proposta per una esposizione sperimentale, aperta con un mese di ritardo (Belloli, Francalanci 1969; Apollonio, Caramel, Mahlow 1970). Si cerca un dialogo con il pubblico analizzando temi quali «Arte e società», arte e produzione», «gioco e relax», «stimolazione percettiva», «analisi del vedere», da cui risulta un percorso che parte dall'avanguardia storica (Tatlin, Malevich, El Lissitky, Rodchenko, Moholy-Nagy...) arriva «fino alle ricerche più recenti» in particolare dell'arte programmata (Max Bill, Munari, Le Parc, Soto, Balocco...) (Dal Canton 1981). Nella mostra si installa anche un laboratorio di produzione manuale e meccanica usato da ventisei artisti che si avvicendano a gruppi di quattro e usano «un impianto serigrafico, un torchio litografico, una macchina per lo stampaggio sottovuoto, un torchio per l'incisione, un laboratorio fotografico, un laboratorio per la lavorazione delle materie plastiche, una macchina Rank Xerox» (Tramontin 1970, 121).

Vengono in questo modo realizzati grafiche, plurimi e piccole sculture sotto gli occhi dei visitatori che possono anche intervenire nella produzione e poi acquistare le opere, portandosi a casa un piccolo pezzo d'arte [fig. 3]. Il Fondo artistico dell'ASAC conserva moltissimo materiale di questo esperimento, composto da incisioni e stampe che rappresentano la voglia di rendere l'arte spiegabile e accessibile a tutti. ${ }^{35}$

Apollonio in quello stesso anno e per le stesse ragioni in maggio aveva organizzato il primo Convegno internazionale «Arte e didattica», le cui riflessioni sono messe a frutto all'interno della stessa Biennale del 1970, con uno spazio appositamente dedicato agli studenti. ${ }^{36}$

Attraverso questo convegno si è voluto affrontare in modo scientifico la problematica stimolante, e fin qui in genere troppo accademicamente affrontata, che sorge dall'incontro tra il mondo dell'arte e la didattica, per valutare poi le conseguenze che ne derivano sia per gli Istituti di studio, sia per le scuole di insegnamento artistico. Le relazioni di base sono state tenute da Bill, Paolo Bonaiuto, René Berger, Attilio Marcolli, Ugo La Pietra, cui hanno fatto seguito ampie discussioni da parte del pubblico presente in sala composto per la maggior parte di studiosi, artisti, studenti, insegnanti, critici e giornalisti. ${ }^{37}$

35 Alcune di queste opere sono illustrate in: Gioni 2013, 84-6 e catalogate in Piai, Scarpa Sonino 1976, 69-73.

36 Primo convegno internazionale Arte e didattica, $\mathrm{Ca}^{\prime}$ Giustinian, sala degli Specchi, 24-26 maggio 1970, gli atti delle giornate e le relazioni di base di Max Bill, Paolo Bonaiuto, Renè Berger, Attilio Marcolli e Ugo La Pietra sono contenuti nel numero monografico intitolato Arte e didattica, della rivista La Biennale di Venezia, n. 67/68, novembre 1971. 37 La Biennale di Venezia, 67-68, dicembre 1971, 86. 
Vittorio Pajusco

Umbro Apollonio e l'Archivio della Biennale di Venezia (1948-1972)

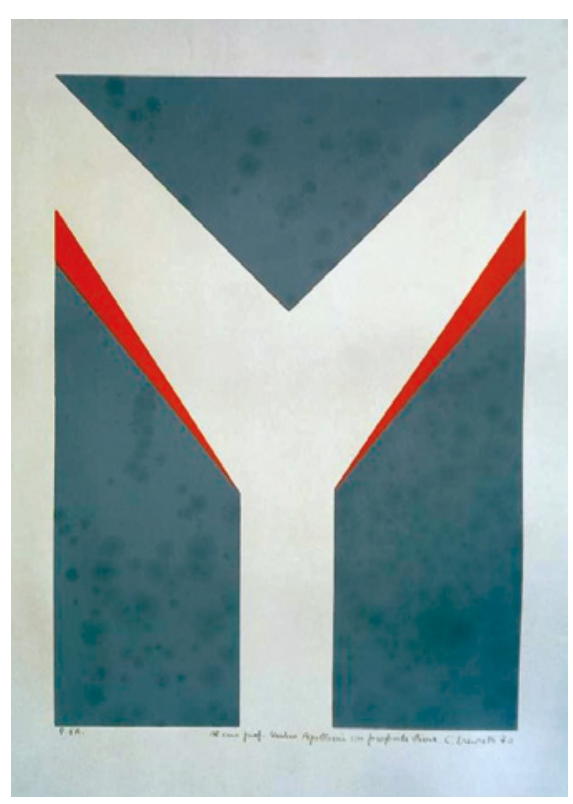

Figura 3 Carlo Lorenzetti. Struttura 1970 (grigio-rosso) Serigrafia, $50 \times 70 \mathrm{~cm}$, con dedica autografa: «Al caro prof. Umbro Apollonio con profonda stima. C. Lorenzetti'70

Collezione privata, Venezia

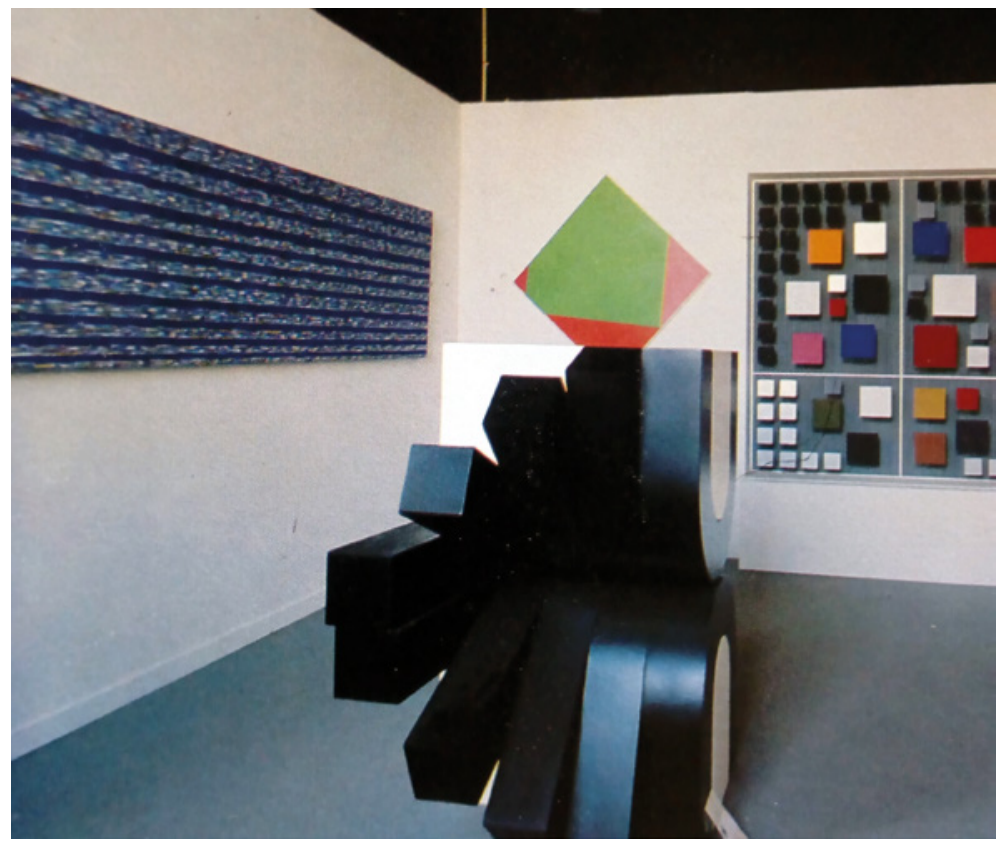

Figura 4 «Sala Apollonio» nella sede dell'ASAC a Ca' Corner della Regina, da sinistra opere di Piero Dorazio, Max Bill e Jesus Raphael Soto. Al centro una scultura di Marcello Morandin (foto in Salvagnini 1990, 67) 
Nel 1971 Mario Penelope viene nominato commissario straordinario dell'Ente Biennale e la XXXVI Biennale d'Arte mostra già significativi cambiamenti, propone infatti per la prima volta la tematica Opera e comportamento, in un'edizione ricordata anche per la liberazione delle diecimila farfalle in piazza San Marco, per il ragazzo con la sindrome di Down proposto come opera vivente da Gino de Dominicis e per la sezione Quattro progetti per Venezia che mostra gli architetti Le Corbusier, Louis Kahn, Frank Lloyd Wright e Isamu Noguchi e Carlo Scarpa. ${ }^{38}$

È proprio in quel 1972 che Apollonio, dopo ventitré anni di dure lotte e di grandi amarezze, lascia la carica di conservatore dell'Archivio della Biennale per dedicarsi a tempo pieno all'insegnamento universitario, essendo titolare della cattedra di Storia dell'arte contemporanea all'Università di Padova dal 1968 al 1979. ${ }^{39}$ Gli succede Wladimiro Dorigo, che trentenne era entrato alla Biennale in qualità di direttore dell'Ufficio Stampa e suo collaboratore alla rivista La Biennale di Venezia. Nel 1986 all'interno della nuova sede dell'ASAC a Ca' Corner della Regina, a testimonianza del riconoscimento del suo ruolo verrà allestita una sala a lui dedicata con opere d'arte donate da artisti [fig. 4] che si sono dedicati in particolare all'arte cinetica e programmata e che l'hanno conosciuto e apprezzato (Durante 2006, 19). 


\section{Bibliografia}

Abbott, Berenice; Dortch Dorazio, Virginia (1994). Peggy Guggenheim and Her Friends. Milano: Berenice.

Alloway, Lawrence (1968). The Venice Biennale from Salon to Goldfish Bowl. Greenwich: New York graphic society.

Apollonio, Umbro (1945). Scipione. Venezia: Edizioni del Cavallino.

Apollonio, Umbro (1947). Disegni di Seurat. Venezia: Edizioni del Cavallino.

Apollonio, Umbro (1950). Pittura italiana moderna. Idea per una storia. Venezia: Neri Pozza.

Apollonio, Umbro (1979). Occasioni del tempo. Riflessioni-Ipotesi. Torino: Studio Forma.

Apollonio, Umbro; Caramel, Luciano; Mahlow, Dietrich (a cura di) (1970). Ricerca e progettazione. Proposte per una esposizione sperimentale. Venezia: La Biennale di Venezia.

«Arte e didattica» (1971). La Biennale di Venezia, 67-68, dicembre.

Atti (1957). Atti del Convegno di studio sulla Biennale. Venezia: Sorteni.

Bandera, Maria Cristina (2011). «Pallucchini protagonista della Biennale». Saggi e Memorie di storia dell'arte, 35, 75-92.

Bazzoni, Romolo (1962). 60 anni della Biennale di Venezia. Venezia: Lombroso.

Budillon Puma, Pascale (1995). La Biennale di Venezia dalla guerra alla crisi 1948-1968. Bari: Palomar.

Dal Canton, Giuseppina (1981). «Umbro Apollonio: un critico militante e le sue occasioni». $13^{\circ}$ Biennale Internazionale del bronzetto piccola scultura. $\mathrm{Pa}$ dova: Come di, 8-11.

Dal Canton, Giuseppina; Dell'Acqua, Gian Alberto; Caramel, Luciano (1986). «Testimonianze per Umbro Apollonio». Archivio Storico delle Arti Contemporanee. Venezia: La Biennale.

Dal Canton, Giuseppina (2001). «Fra attiva partecipazione al "rinnovamento della cultura artistica italiana" e "collaudo della propria sensibilità": Pallucchini e l'arte contemporanea». Pilo, Giuseppe Maria (a cura di), Una vita per l'arte veneta = Atti della giornata di studio in onore e ricordo di Rodolfo Pallucchini. Monfalcone: Edizioni della Laguna, 119-29.

Dal Co, Francesco,; Mazzariol, Giuseppe (a cura di) (1984). Carlo Scarpa. Opera completa. Milano: Electa.

Di Raddo, Elena (2017). "La crisi dell'“opera”: la tecnologia entra in Biennale (le edizioni del 1970 e del 1972)». Castellani, Francesca; Charans, Eleonora (a cura di), Crocevia Biennale. Milano: Scalpendi, 215-24.

Durante, Lia (2006). «La collezione permanente della Biennale di Venezia». Durante, Lia (a cura di), Restauri. Galileo Chini e altre opere della collezione permanete. Venezia: La Biennale, 15-21.

Durante, Lia (2011). «Le mostre all'estero della Biennale di Rodolfo Pallucchini (1947-1957)». Saggi e Memorie di storia dell'arte, 35, 93-116.

Fezzi, Elda (1974). «Intervista a Umbro Apollonio». Le Arti, 7-9, luglio-agosto, 21-4.

Gioni, Massimiliano (a cura di) (2013). Amarcord. Frammenti di memoria dall'Archivio Storico della Biennale. Venezia: La Biennale.

Isnenghi, Mario (1986). «Le Biennali del secondo dopoguerra». Franzina, Emilio (a cura di), Venezia. Bari-Roma: Laterza.

Lanzarini, Orietta (2003). Carlo Scarpa. L'architetto e le arti. Venezia: Marsilio. Marchiori, Giuseppe (1946). Pittura moderna italiana. Trieste: Stampe nuove. 
Marchiori, Giuseppe (1950). Pittura moderna in Europa (Da Manet a Pignon). Venezia: Neri Pozza.

Martini, Federica; Martini, Vittoria (2011). Just Another Exhibition. Storie e politiche delle biennali. Histories and Politics of Biennials. Milano: Postmedia Srl.

Mulazzani, Marco (2004). I Padiglioni della Biennale di Venezia. Milano: Electa.

Mussa, Italo (a cura di) (1971). Aspetti della grafica europea. Venezia: Stamperia di Venezia.

Pajusco, Vittorio (2013). «Archiviare il presente. Domenico Varagnolo e l'Archivio Storico d'Arte Contemporanea». Ateneo veneto. Atti e memorie dell'Ateneo veneto: rivista mensile di scienze, lettere ed arti, 200, 12(1), 103-17.

Pajusco, Vittorio (2015). «Devozione e committenza: Giuseppe Volpi di Misurata ai Frari». Corsato, Carlo; Howard, Deborah (a cura di), Santa Maria Gloriosa dei Frari. Immagini di Devozione, Spazi della Fede. Padova: Centro studi antoniani, 199-208.

Pajusco, Vittorio (2016a). «Antonio Maraini e l'Istituto Storico d'Arte Contemporanea (1928-1944)». Saggi e Memorie di Storia dell'arte, 38, 135-51.

Pajusco, Vittorio (2016b). «Luigi Nono e la Biennale del 1968: contestazioni e proposte». Wertenson, Birgit Johanna; Storch, Christian (Hrsgg), Luigi Nono und der Osten/ Luigi Nono e i paesi dell'est. Mainz: Are Musik Verlag, 303-22.

Pallucchini, Rodolfo (1948). «Venezia - Riapertura dell'Archivio Storico d'Arte contemporanea della Biennale». Bollettino d'Arte, 1, gennaio-marzo, 92.

Perosin, Mauro (2015). Per una biblioteca di immagini del moderno. Storia, ruoli e usi della fotografia d'arte dall'archivio della Biennale veneziana (1946-1958) [tesi di dottorato]. Venezia: Università Ca' Foscari Venezia; IUAV.

Perosin, Mauro (2017). «L'uso della fotografia d'arte nella rivista "la biennale di Venezia" negli anni di Rodolfo Pallucchini: indagini e percorsi narrativi a partire da alcune fotografie». Catellani, Francesca; Charans, Eleonora (a cura di), Crocevia Biennale. Milano: Scalpendi, 117-43.

Piai, Manuela; Scarpa Sonino, Annalisa (1976). Catalogo del fondo artistico. Venezia: La Biennale.

Poesia concreta. Indirizzi concreti, visuali e fonetici (1969)= Catalogo della mostra a cura di Dietrich Mahlow, Arrigo Lora-Totino (Venezia, Ca' Giustinian, 25 settembre-10 ottobre). Venezia: La Biennale.

Poletto, Laura (2013). «La Biennale di Venezia: dai professori ai curatori». Ateneo Veneto. Atti e memorie dell'Ateneo veneto: rivista mensile di scienze, lettere ed arti, 200, 12(1), 583-97.

Portinari, Stefania (2018). Anni Settanta. La Biennale di Venezia. Venezia: Marsilio.

Ragghianti, Carlo Ludovico (1957). Atti del Convegno di studio sulla Biennale. Venezia: Sorteni, 89-90.

Roddolo, Enrica (2003). La Biennale. Arte, polemiche, scandali e storie in laguna. Venezia: Marsilio.

Rostellato, Diletta (2013-14). Scrittori triestini nell'archivio di Umbro Apollonio [tesi di laurea]. Venezia: Università Ca' Foscari Venezia.

R. Salvadori. (1972) «Quattro progetti per Venezia». Biennale 36, 1972, 17-20.

Salvagnini, Sileno (1990). «L'Archivio Storico della Biennale». Veneto ieri oggi, domani, 7, 62-7.

Salvagnini, Sileno (2006). «Da Barbantini a Marchiori. Formazioni, passioni, idiosincrasie della critica veneta d'arte della prima età del Novecento». Stringa, Nico (a cura di), Venezia '900 da Boccioni a Vedova = Catalogo della mostra (Treviso, 27 ottobre 2006-8 aprile 2007). Venezia: Marsilio, 310-29. 
Salvagnini, Sileno (2009). "La civiltà europea è crollata in un polverone, l'artista è completamente solo...”. Padova: Il Poligrafo.

Salvagnini, Sileno (2011). «Pallucchini, Marchiori, Apollonio. La critica d'arte a Venezia 1942-1947». Saggi e Memorie di storia dell'arte, 35, 35-48.

Tomasella, Giuliana (a cura di) (2011). Rodolfo Pallucchini. Scritti sull'arte contemporanea. Venezia: Fondazione Cini.

Tramontin, Giancarlo Franco (1970). «Note sui laboratori». Apollonio, Caramel, Mahlow 1970, 121.

«Una nuova biennale» (1969). «Una nuova biennale: contestazioni e proposte». La Biennale di Venezia. Rassegna delle Arti contemporanee, 64-65, gennaiogiugno, 3-21.

Venturi, Lionello (1957). Atti del Convegno distudio sulla Biennale. Venezia: Sorteni.

Zanella, Francesca (2017). «Artista vs critico architetto. La Biennale di Venezia del 1970». Castellani, Francesca; Charans, Eleonora (a cura di), Crocevia Biennale. Milano: Scalpendi, 201-13.

Zevi, Bruno (1958). «ll Padiglione BBPR ai Giardini». La Biennale di Venezia, 30, 46-8. 


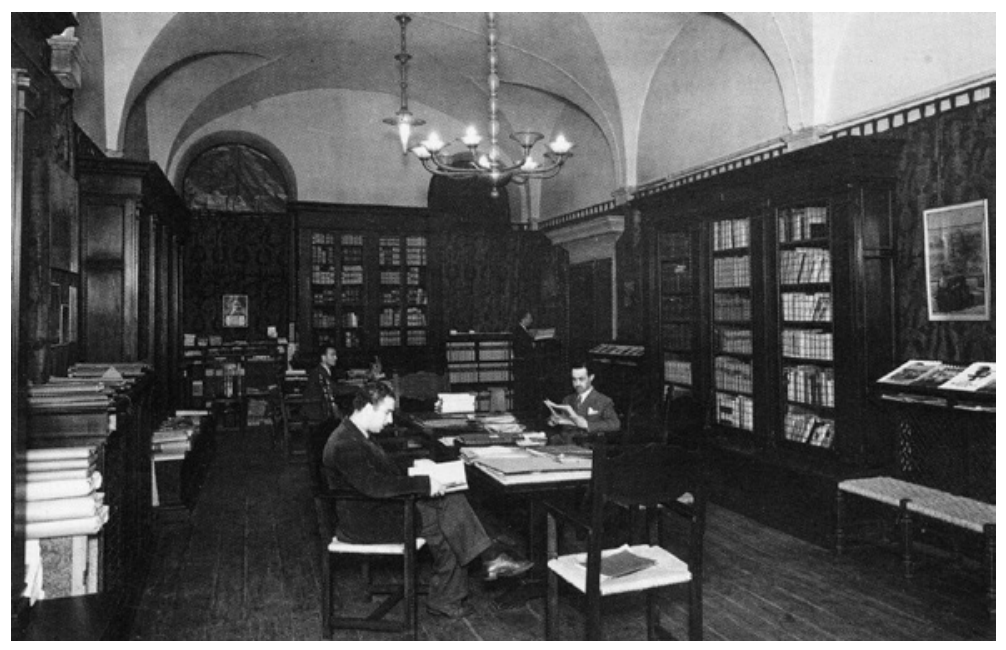

La sala di lettura dell'Archivio Storico d'Arte Contemporanea a Palazzo Ducale, 1938. Visibili Antonio Santomaso, Antonio Gnan, Angelo Tursi e Domenico Varagnolo. Courtesy Salvagnini 1990, 65 


\title{
Dell'Archivio Storico delle Arti Contemporanee «la Biblioteca n'era il principio»
}

\author{
Giorgia Marchesin
}

\begin{abstract}
The essay aims to reconstruct the history and to provide a library analysis about one of the most important libraries of contemporary art in Italy: the Library of the Venice Biennale. The library has been the founding fulcrum of what today is ASAC: Historical Archive of Contemporary Arts of the Venice Biennale. Its Iong history can be reconstructed by retracing the move of which it has been the protagonist, from the beginning in a small room on the ground floor of the Palazzo Ducale, to the current location inside the Central Pavilion of the Biennale Gardens. The heritage of books is in continuous development thanks to the Book Pavilion project and a network of exchanges between the most important artistic and cultural institutions. Today the Library heritage includes over 153,000 publications and over 3,000 periodicals. This invaluable collection, for the world of contemporary art, offers almost 23,000 volumes owned, in Italy, exclusively from the Library of the Venice Biennale.
\end{abstract}

Keywords 1928. ASAC. Library. Contemporary art. Venice Biennale.

La Venezia ottocentesca si trova ad affrontare un periodo difficile. Nel rimpianto e nella nostalgia di un passato di Serenissima ormai irripetibile, ma sulla scia di quanto andava organizzandosi oltralpe, in particolare tra Londra e Parigi, Monaco di Baviera e Vienna, nel 1887 si decise di organizzare un'importante mostra d'arte di carattere nazionale, chiamata l'Esposizione Nazionale d'Arte.

Questo saggio prende avvio da Marchesin, Giorgia, La biblioteca della Biennale di Venezia, tesi di laurea magistrale, relatore Dorit Raines; correlatore Stefania Portinari (a.a. 2015/2016). Venezia: Università Ca' Foscari Venezia. 
L'evento raccolse e espose al pubblico oltre mille opere tra pittura e scultura presso una sede temporanea, appositamente realizzata nella zona verde dei Giardini di Castello. Anche se la manifestazione alla fine non fu un grande successo incoraggiò gli intellettuali di allora a istituire un incontro d'arte in grado di inserirsi fin dall'inizio in un contesto internazionale, permanente, con cadenza biennale, inaugurando la Prima Esposizione Internazionale d'Arte della Città di Venezia il 22 aprile 1895 (cf. Donzello 1987).

Scrive Ugo Ojetti:

La prima Esposizione Internazionale d'Arte a Venezia, due anni fa, parve e fu un miracolo [...] fino allora, chi in Italia parlava o scriveva di qualche impresa artistica, era una campana che suonava a morto: triste e solitario irritava la maggioranza del pubblico affaccendata in tutt'altre faccende e non raccoglieva che i pochi fedeli memori della Morta gloriosa. Tutte quelle speranze che tendevano soltanto alle riunioni di oltre monte o di oltre mare, a Parigi o a Monaco, a Barcellona o a Vienna, a Londra o a Zurigo, ora fidano a Venezia. ${ }^{1}$

L'esigenza di documentare il presente e essere aggiornati rispetto alle tendenze storico-artistiche, ma anche di preservare i materiali legati all'organizzazione della rassegna, ha dato origine all'archivio della Biennale, costituito con il nome di Istituto Storico d'Arte Contemporanea l'8 novembre 1928 con sede a Palazzo Ducale e divenuto in seguito l'Archivio Storico d'Arte Contemporanea. ${ }^{2}$

Come afferma il suo primo conservatore, Domenico Varagnolo, «la Biblioteca n'era il principio», ne fu l'origine e rimane tutt'ora uno dei suoi fondi più rilevanti e di prestigio (Maraini 1932, 61). È infatti una delle biblioteche specializzate in arte contemporanea più significative d'Italia, è anzi la più antica ad essersi occupata di conservare volumi che ambiscono a trattare tutti gli ambiti storico-artistici del periodo su cui si estende l'esistenza della Biennale.

Il suo patrimonio librario ammonta ad oltre 151.000 volumi, con pubblicazioni legate alle discipline proprie della Biennale quali arti visive, architettura, cinema, danza, musica, teatro; tra queste, quasi 23.000 si trovano solo in questa biblioteca e in nessun altro luogo in Italia.

L'Istituto storico d'arte contemporanea nasce in un periodo di cambiamenti proficui per l’Esposizione ed è stato oggetto di vari spostamenti. Cinque sono i luoghi dove l’Archivio e la Biblioteca della Bien-

1 Ugo Ojetti, «L'arte moderna a Venezia: Esposizione mondiale del 1897», Resto del Carlino, 28 aprile 1897.

2 Sull'argomento cf. Pajusco 2013, 2016. 
nale hanno di volta in volta trovato dimora a Venezia: il primo è una alcune sale al piano terreno di Palazzo Ducale.

Sotto la gestione del segretario generale Antonio Maraini, mentre era capo dell'ufficio stampa Elio Zorzi, a Domenico Varagnolo fu assegnato l'incarico di gestire questa sezione, una compito che ricoprì fino alla sua morte, avvenuta nel 1949 (cf. De Sabbata 2006; Pajusco 2013).

Maraini ritenne che la Biennale necessitasse di disporre di un luogo adatto alla conservazione di volumi, scritti, corrispondenze, fotografie che documentassero la storia della rassegna, così che si presentasse «non più solo una esposizione, ma un centro di idee e discussioni per mezzo di congressi, un centro di pensiero e di studio per mezzo dell'Archivio e della Biblioteca» (1932, 10).

Sin dagli inizi la biblioteca - essendo uno strumento di lavoro - doveva specializzarsi in arte contemporanea, con particolare riguardo per le pubblicazioni che concernessero gli artisti internazionali partecipanti all'Esposizione veneziana, e possibilmente assicurandosi i cataloghi anche delle loro retrospettive, per completezza di informazione. Conserva inoltre sia i cataloghi di ciascuna Biennale che altri di esposizioni nazionali e internazionali.

In quella nuova collezione comparirono inoltre altre tipologie di pubblicazioni come studi, articoli, critiche d'arte concernenti la Biennale, comparsi in volumi e riviste, che oggi sono identificati come titoli analitici, recuperati e rintracciati tramite l'azione di spoglio. È su questi articoli che si formarono i più importanti e incisivi critici d'arte contemporanea del tempo tra cui Ugo Ojetti, Vittorio Pica, Margherita Sarfatti, Enrico Thovez.

Oltre all'acquisto diretto di libri e cataloghi presso librerie sia moderne che antiquarie, si ricorreva all'abbonamento a riviste specializzate; Maraini, Varagnolo e i collaboratori iniziarono poi una campagna di acquisizioni, mettendosi in contatto con gli enti culturali italiani e stranieri più influenti per intrecciare una proficua rete di scambi di materiale di interesse comune. Infine, le donazioni di volumi che potessero rientrare negli obiettivi prefissi dall'istituto erano ben accetti, se non incoraggiati. Recita infatti il Bollettino della Biennale (1, 1936):

Tornerà molto gradito l'invio dei Cataloghi, nonché di giornali, riviste ecc., riguardanti le varie mostre e gli artisti che vi partecipano. Sarà pure bene accetta qualsiasi altra pubblicazione (anche non recente) che si riferisca, in genere, all'Arte moderna e contemporanea.

Per ogni comunicazione indirizzare all'Archivio Storico d'Arte contemporanea della Biennale - (Palazzo Ducale, Venezia)». 
Il patrimonio librario della biblioteca nei primi anni contava di circa 2.000 volumi, ordinati con uno schedario alfabetico e per materia; circa 1.000 erano cataloghi di mostre. Era presente poi una notevole raccolta di riviste periodiche internazionali, conservate fin dai numeri iniziali, peculiarità che la rende una collezione molto pregiata.

Come afferma Domenico Varagnolo, l'ordinamento dei volumi era, diremmo oggi, a scaffale aperto e i libri erano catalogati in ordine alfabetico:

E tutti sono collocati bene, nelle medesime condizioni di luce e di visione senza distinzione di età, di merito, di tendenza, tutti sottoposti ad una unica legge: quella dell'alfabeto. Alla quale, pare impossibile, nessuno si ribella, benché comporti talvolta, in apparenza, delle vicinanze stridenti e dei contatti pericolosi. Ma non esistono stridori né pericoli di sorta. Anzi ne risulta un ideale simpatico affratellamento non privo di un'intima ed anche visiva armonia. (Maraini 1932, 71)

Con l'andare degli anni la raccolta libraria iniziale andò aumentando tanto da invadere alcune sale adiacenti che all'epoca erano occupate dalla biblioteca della Soprintendenza.

Negli anni Trenta cambia anche la sua denominazione: non più Istituto Storico d'Arte contemporanea, ma Archivio Storico d'Arte Contemporanea. Nel 1948 la biblioteca e l'archivio vennero trasferiti definitivamente a Ca' Giustinian, per necessità di spazio e di indipendenza. ${ }^{3}$

La Biennale del dopoguerra è guidata dal segretario generale Rodolfo Pallucchini e a dirigere l'Archivio Storico d'arte contemporanea fino al 1972 è chiamato Umbro Apollonio.

In un articolo del 1948 Pallucchini descrive la collezione della Biblioteca in quegli anni:

Oltre che custodire tutto il vasto materiale documentario riguardante la storia della Biennale, l'Archivio oggi possiede una biblioteca ricca di circa 7.000 volumi ed opuscoli, dedicati all'arte moderna italiana e straniera, una collezione di riviste italiane e straniere d'arte fra le più complete che oggi siano in Italia, una raccolta sempre più aggiornata di tutti i ritagli di stampa riguardanti gli artisti italiani, una fototeca che accoglie in cartelle riproduzioni fotografiche di opere di artisti italiani e stranieri e album con le riproduzioni delle opere più importanti esposti alle Biennali. Vari schedari consentono una facile consultazione di tutto il materiale.

3 Già dalla fine del 1942 l'Archivio si trovava a Ca' Giustinian. Nel 1943 Ca' Giustinian viene requisita dalla polizia fascista e i materiali traslocati in alcune sale del Museo Correr. Pajusco 2016, 150-1. 
Si tratta cioè ormai di una istituzione che, ben limitata e specializzata nei suoi scopi e nei suoi fini, può dirsi unica in Italia.

La Biblioteca integra la Biblioteca d'arte e di storia del $\mathrm{Mu}$ seo Correr, dedicata all'arte italiana e particolarmente veneta sino a tutto il Settecento, è uno strumento di lavoro per l'organizzazione delle Biennali, ma, soprattutto, via via che se ne vanno colmando le lacune, diviene un mezzo di studi adeguatissimo per l'arte moderna. ${ }^{4}$

Apollonio tentò di condurre un progetto di riammodernamento della biblioteca, prendendo contatti prima con Carlo Scarpa, poi lo studio BBPR e infine, dopo quindici anni di tentativi, con la ditta Lips Vago di Milano: le stanze in cui era conservata la biblioteca e gli armadi che contenevano i vari volumi avevano bisogno di ristrutturazioni e aggiornamenti, erano necessarie delle nuove scaffalature. ${ }^{5}$

In seguito la situazione conservativa del palazzo divenne precaria e sia l'Archivio che la Biblioteca necessitavano di ulteriori spazi, tanto che nel 1976, sotto la direzione del nuovo conservatore Wladimiro Dorigo, vennero trasferiti a Ca' Corner della Regina (cf. Dorigo 1975).

La Biblioteca, collocata al primo piano nobile del palazzo, al momento del trasloco conservava più di 50.000 volumi, 70.000 cataloghi e oltre 1.200 testate di periodici correnti e cessati. Per l'ordinamento e la collocazione dei volumi, a partire da questo trasferimento, vengono prese decisioni importanti, come ricorda ancora Pallucchini:

In particolare sono stati studiati i codici e le forme di segnatura e cartelli natura delle unità bibliografiche, precedentemente inesistente, sono state definite le suddivisioni per discipline e sezioni di tutto il materiale della biblioteca [...]; questo lavoro ha permesso in particolare di registrare migliaia di volumi che non figuravano ancora nel registro di ingresso, di schedare altre migliaia di volumi per la integrazione del catalogo manuale per autori con schede principali, di richiamo, di rinvio, e di spoglio, e di rifare praticamente la schedatura amministrativa - creando altresì il catalogo manuale - della sezione. ${ }^{6}$

Le sezioni o i settori disciplinari in cui sono stati suddivisi i volumi erano: arti visive, architettura, cinema, musica, teatro, mass media e periodici.

4 Rodolfo Pallucchini, «Venezia - Riapertura dell'Archivio Storico d'Arte contemporanea della Biennale», Bollettino d'Arte, gennaio-marzo, 1948.

5 Si veda il saggio di Pajusco nel presente volume.

6 Rodolfo Pallucchini, «Venezia - Riapertura dell'Archivio Storico d'Arte contemporanea della Biennale», Bollettino d'Arte, gennaio-marzo, 1948. 
Una novità apportata negli anni Settanta è un primitivo sistema di automatizzazione: furono informatizzate alcune migliaia di titoli di pubblicazioni attraverso un sistema elettronico di ricerca dell'informazione chiamato Interart-Find, creato appositamente, spingendo la Biblioteca della Biennale un passo avanti nell'impiego delle tecnologie. ${ }^{7}$

Negli anni a seguire, in concomitanza con le tensioni politiche e sociali che si erano create in Italia alla fine degli anni Settanta e con la crisi economica, a causa anche di incomprensioni tra le personalità di spicco che gestiscono l'ente, l'Archivio viene aperto e chiuso a intervalli di qualche mese. Nonostante questi ostacoli, l'attività quotidiana di catalogazione del materiale e i servizi di reference offerti al pubblico sono garantiti e vengono gestiti da Dorigo fino al 1982.

Il decennio seguente vide la Biblioteca e l'Archivio privi di una figura dirigenziale significativa fino al 1998, anno in cui venne istituita la figura del direttore di settore per le diverse sezioni della Biennale. Il ruolo direzionale dell'ASAC viene allora coperto da Giorgio Busetto dal 2004 al 2008 e dal 2009 ad oggi da Debora Rossi, nelle veci di Direttore amministrativo.

Anche a Ca' Corner della Regina si verificarono però le medesime problematiche che già si erano riscontrate in precedenza: la mancanza di fondi e i problemi strutturali resero il palazzo non idoneo alla conservazione dei materiali presenti e costrinsero la dirigenza a trasferire l'Archivio e parte dei periodici presso i depositi al Parco Scientifico e Tecnologico VEGA di Marghera nei primi anni 2000, prima nell'edificio Lybra e poi nello stabile Cygnus, dove tutt'oggi è conservata la parte dell'archivio cartaceo e fotografico dell'ASAC, abbandonando però la Biblioteca - che consisteva in circa 11.500 metri lineari di scaffalature che occupavano il primo piano nobile che rimase nello stabile fino al 2009, senza poter essere consultata con continuità. ${ }^{8}$

La consistenza della Biblioteca risalente al 2004, come si evince dall'inventario validato da Giorgio Busetto, era di circa 123.000 titoli, tra cui libri e cataloghi; tutti inventariati e il 52\% catalogati in cartaceo. ${ }^{9}$ La collezione di periodici contava 2.859 titoli di cui un terzo erano ancora in pubblicazione, tutti inventariati e catalogati. ${ }^{10}$

Lo stato di conservazione di quasi tutto il patrimonio - le monografie, i saggi, le antologie e di parte dei cataloghi - era critico, essendo stato collocato per anni a scaffale aperto, a parte certi cata-

7 Wladimiro Dorigo, «L' Archivio Storico delle Arti Contemporanee della Biennale di Venezia», Rassegna degli Archivi di Stato, 3, 1976.

8 ASAC, FS, b. 002: Carte: Carte del conservatore Giorgio Busetto, prima ricognizione su stato e necessità delle collezioni, 2008.

9 ASAC, FS, b. 001: Carte del conservatore Giorgio Busetto.

10 ASAC, FS, b. 001: Carte del conservatore Giorgio Busetto. 
loghi e pubblicazioni rare che erano conservati chiusi in armadi con vetrine e riparati da agenti esterni. I parametri relativi alle condizioni di temperatura e umidità all'interno dei locali della Biblioteca oscillavano continuamente, nessuno provvedeva alla spolveratura e alla manutenzione del materiale, nemmeno al ricambio dell'aria.

Nel 2009 la Biblioteca, secondo i voleri del presidente della Fondazione Paolo Baratta, venne finalmente trasferita in un grande spazio che si trova di fatto all'interno del Padiglione Centrale dei Giardini di Castello, un ex magazzino usato dalla Biennale anche come aula didattica per il settore Educational, di circa $1.400 \mathrm{mq}$, chiamato Ala Pastor (in onore dell'architetto Valeriano Pastor), con accesso esterno da calle Paludo per renderlo autonomo tutto l'anno. ${ }^{11}$

I lavori per un recupero funzionale di questo luogo si sono articolati in tre fasi tra il 2009 e il 2011 e il risultato finale è l'odierna Biblioteca della Biennale di Venezia, articolata su due piani comprensivi di un mezzanino e un ballatoio superiore che dà sulla grande sala conferenze open space; solo quest'ultima accoglie ben oltre $800 \mathrm{me}$ tri lineari di scaffalature. ${ }^{12}$

La nuova Biblioteca viene inaugurata il 27 agosto 2010. Come riporta un articolo del Corriere del Veneto: «Biennale di Venezia, inaugurata l'avveniristica biblioteca. La nuova biblioteca sarà la più fornita sulle tendenze artistiche degli ultimi cento anni». ${ }^{13}$

Oggi la Biblioteca della Biennale di Venezia mette a disposizione del pubblico oltre 150.000 volumi, di cui oltre un terzo catalogati nel Sistema Bibliotecario Nazionale SBN.

Il patrimonio librario si divide in volumi a scaffale aperto, i cosiddetti CONS, di cui è parte anche una sezione interamente dedicata alla Biennale: la sua storia, i cataloghi ufficiali, le partecipazioni nazionali, eventi collaterali; e volumi a consultazione controllata di cui fanno parte principalmente gli OPUS, i periodici, le tesi e volumi rari.

Esistono vari sistemi di classificazione per collocare i volumi a scaffale, tra cui il più conosciuto chiamato Dewey (CDD Classificazione Decimale Dewey), costituito da una serie di classi, identificabili attraverso determinati numeri, è utilizzato prevalentemente nelle biblioteche civiche comunali degli Stati Uniti per la molteplicità di materie trattate (cf. Della Bella 2003).

Essendo la collocazione una prerogativa specifica della biblioteca, a differenza della catalogazione del volume che è universale e uguale per tutti, l'ASAC ha deciso, anche per la sua natura specializzata, di non seguire la Dewey, ma di suddividere il materiale per discipline

11 ASAC, ASAC 2 Enti, b. 5898: Relazione descrittiva, febbraio 2009.

12 ASAC, ASAC 2 Enti, b. 5899: Relazione descrittiva, febbraio 2009.

13 Massimo Favaro, «Rinasce l'Asac, i 130mila libri nell'officina dell'arte», Corriere del Veneto, 28 agosto 2010. 
inerenti le attività della Fondazione, quindi: arte, architettura, cinema, danza, musica, teatro, fotografia. Inoltre esiste la sezione I (mass media), che ora è tutta conservata a deposito presso VEGA, perché non è più considerato un argomento veramente contemporaneo.

I volumi che sono stati collocati a scaffale aperto, trasferiti direttamente da Ca' Corner della Regina alla nuova sede una prima parte nel 2009 e il rimanente nel 2010, sono identificabili attraverso la sigla CONS (ovvero consultazione oltre le 100 pagine). Ogni segnatura attribuita al materiale a scaffale aperto della Biblioteca è composta da tre elementi: la sezione, la collocazione e la specificazione.

Gli OPUS (opuscoli sotto le 100 pagine), invece, sono posti a consultazione controllata per necessità di conservazione, data la loro delicatezza e il fatto che alcuni hanno pochissime pagine: sono stati divisi per discipline e per ogni formato è stata assegnata una lettera che ne identifica il range di centimetri. Possono essere visionati dall'utenza previa la compilazione di un modulo da consegnare al reference desk, fino ad un massimo di 5 volumi per volta e trovano collocazione nel ballatoio rialzato della grande sala conferenze inaugurata nel 2010.

Nella nuova sede della Biblioteca della Biennale ai Giardini ogni sezione dello scaffale aperto ha una sua collocazione fisica; nella prima parte inaugurata nel 2009 si trovano le pubblicazioni della Biennale, la selezione di periodici contemporanei continuamente aggiornati, le sezioni di «Arte» e di «Architettura»; nell'ala open space inaugurata nel 2010, adibita anche a sala conferenze, ci sono le sezioni di «Cinema», «Danza», «Musica», «Teatro e fotografia».

I libri negli scaffali sono collocati dal basso verso l'alto; la lettera A di ogni sezione è in basso a sinistra.

Tutti i volumi sono stati e vengono costantemente indirizzati alle varie sezioni di pertinenza, siano esse in Biblioteca, quindi strettamente inerenti l'ambito artistico contemporaneo, che in Archivio di deposito presso il VEGA, avendo questi una natura diversa, non prettamente specifica, ma comunque di valore unico, tra cui ad esempio la sezione I (mass media).

È importante sottolineare che i volumi depositati all'ASAC sono soggetti allo stesso trattamento riservato alle pubblicazioni conservate a scaffale presso la Biblioteca, vengono dunque sottoposti a catalogazione, all'antitaccheggio, a conservazione, è garantita la loro rintracciabilità e consultabilità attraverso gli Opac presenti on-line.

Il patrimonio librario viene incrementato costantemente in vari modi: attraverso l'acquisto sistematico di volumi e periodici; attraverso il progetto avviato nel 2009 La Bibliografia della Mostra o Book Pavilion per cui gli artisti e gli architetti invitati alle Esposizioni inviano e donano alla Biblioteca e all'Archivio le pubblicazioni per loro più significative relative alle opere esposte e al loro percorso artistico. L'obiettivo è creare una raccolta di volumi e cataloghi sempre ag- 
giornati. I volumi donati tramite questa iniziativa sono esposti, per la durata della singola Biennale, all'interno del Padiglione Stirling, inaugurato nel 1991 nel centro dei Giardini della Biennale (Stirling et al. 1991), in consultazione per tutta la durata della mostra, e vengono incamerati poi nella Biblioteca dell'ASAC. Un'altra forma di incremento è poi la proficua attività di dono e scambio di pubblicazioni che la Biblioteca della Biennale tiene con molte altre realtà bibliotecarie in Italia e nel mondo, che è uno dei migliori sistemi di accrescimento e è attivo sin dagli albori dell'ASAC nel primi anni '30. Le donazioni provengono principalmente da studiosi, e si tratta di tesi di laurea e di dottorato, da volumi editi da ricercatori e cataloghi di mostre di artisti che partecipano alle Esposizioni.

Gli enti italiani e stranieri con cui la Biblioteca ha sviluppato un rapporto equo e costruttivo di scambi sono moltissimi: si citano tra questi la Fondazione La Triennale di Milano; la GNAM Biblioteca e Archivio Storico Galleria Nazionale d'Arte Moderna e Contemporanea di Roma; la Biblioteca del Castello di Rivoli; la Collezione Peggy Guggenheim di Venezia; gli austriaci ESSL Museum Kunst Der Gegenwart di Klosternenburg, il MUMOK Museum Moderner Kunst Stiftung Ludwig di Vienna, la ERSTE Foundation Library di Vienna; il canadese CCA Canadian Centre Architecture di Montreal; i tedeschi Deutsche Kinemathek, lo ZKM Zentrum Kunst Und Medientechnologie: Center for Art and Media di Berlino; l'inglese Society for Theatre Research; la spagnola Fondació Joan Miro di Barcellona; gli americani Vera List Center for Art and politics - The New School, MoMA Museum of Modern Art di New York; lo svizzero CAC Centre d'Art Contemporaine di Ginevra e diversi altri.

Una fonte di prestigio per la Biblioteca della Biennale di Venezia è senz'altro il fondo periodici che raccoglie una vasta collezione di testate nazionali ed internazionali: si tratta di oltre 3.000 titoli di cui due terzi catalogati in SBN; tra cui si contano riviste di arti visive, architettura, cinema, danza, musica, teatro e una selezione di periodici interdisciplinari su fotografia, archivistica, istituzioni culturali, letterature, mass media (cf. Da Tos, Fontanin 2007).

Nel fondo è presente anche una sezione dedicata ai RARI, che all'epoca di Ca' Giustinian era collocata in uno stanzino al quinto piano del palazzo, in ordine alfabetico. Accanto a questi erano collocati i grandi formati, come i quotidiani, che fisicamente necessitavano di essere conservati stesi e non potevano essere messi a scaffale assieme agli altri: tra questi c'erano Variety ed Il Giornale dell'Arte.

La sezione dei periodici rari è costituita da 155 titoli, prevalentemente di arte visiva, oltre che di architettura, cinema, danza, teatro, mass media, musica, cultura varia e letteratura. Le caratteristiche che contraddistinguono questa tipologia di periodici e che determinano la loro rarità sono senza dubbio di tipo cronologico, tra le tante riviste ci sono le più antiche, risalenti ai primi anni dell' 800 , collezio- 
nate fin dagli albori dell'Istituto storico delle Arti Contemporanee da Varagnolo; rientrano nel fondo anche i periodici con rilegature particolari come ad esempio riviste con fogli mobili e singoli numeri o numeri speciali, magari di riviste che sono uscite per un breve periodo di tempo, con una ristretta tiratura.

Tutti, a partire dall'anno 2005, sono stati inventariati e catalogati in SBN.

Di alcune riviste poi, come Domus e Casabella, sono posseduti tutti i numeri e un centinaio di riviste correnti e in abbonamento è messo a disposizione degli utenti a scaffale aperto in Biblioteca, periodicamente sostituiti dai nuovi numeri ad ogni arrivo.

La Biblioteca fornisce inoltre all'utenza la catalogazione analitica delle riviste, cioè l'identificazione di articoli riguardanti temi inerenti alla Biennale, che porta alla realizzazione di schede di spoglio, di tutti i periodici correnti a partire dal 2010 e di alcune delle principali riviste possedute.

Tutte le riviste sono collocate in un deposito climatizzato in compatti richiudibili, in modo da garantirne la conservazione, sia mantenendo una temperatura e umidità corretta, che una protezione quasi totale dalla luce e dai raggi ultravioletti del sole. Sono suddivisi in tre formati, permettendo in questo modo di ottimizzare gli spazi: PER H, riviste da 0 a $26 \mathrm{~cm}$; PER Y, riviste da 26 a $35 \mathrm{~cm}$; PER X, riviste da $35 \mathrm{~cm}$ in su.

Un altro rapporto speciale lega la Fondazione al libro: la presenza dei libri d'arte e dei libri d'artista. Nonostante non si possano trattare come dei volumi da mettere a disposizione degli utenti in biblioteca, in quanto opere d'arte, hanno una storia e un'importanza rilevante nelle vicende della Biennale, tanto che negli anni presidenti e curatori hanno organizzare non solo mostre ma addirittura voluto un Padiglione dedicato a questo speciale tema.

La prima Mostra Internazionale del Libro d'Arte, che poi non ebbe seguito, venne inaugurata durante la $\mathrm{XX}^{\mathrm{a}}$ Biennale di Venezia nel 1936 e considerava, fra le varie definizioni date del libro d'arte, come era scritto in catalogo:

«quella che lo considera stampato con grandissima cura, su carta sceltissima, illustrato da un artista, che ne arricchisce il testo con incisioni eseguite da lui stesso su legno o su rame, o col processo dell'acquaforte, o da lui disegnate su pietra litografica. È questa l'illustrazione detta originale, il cui sviluppo la Biennale di Venezia ha voluto esporre in un sintetico quadro, il quale, se pure non completo, mostra abbastanza quel che di meglio fu fatto dagli artisti a servizio del libro in questi ultimi anni» (Mostra internazionale del libro d'arte 1936, 5) 
Questa particolare forma d'arte trovò nuova vita pochi anni dopo con la realizzazione di un vero e proprio Padiglione dedicato interamente al libro: il Padiglione del Libro d'arte. Alla fine del 1947, il collezionista, gallerista e mercante d'arte veneziano Carlo Cardazzo suggerì all'allora segretario generale della Biennale, Rodolfo Pallucchini, di realizzare uno «Stand con libreria internazionale», all'interno del Padiglione Italia.

L'iniziativa piacque a tal punto da decidere di realizzare uno 'stand esterno', con vita e personalità propria, che fungesse da "oasi di riposo intellettuale» (Assante, Bertan 2000, 32). Carlo Cardazzo affidò il progetto della realizzazione del nuovo Padiglione all'amico Carlo Scarpa, già progettista dell'allestimento della sua galleria veneziana, sia nella prima sede inaugurata nel 1942 che nella seconda del 1949. Edificato nelle vicinanze del Palazzo Centrale, il Padiglione del Libro d'arte fu concluso agli inizi dell'estate del 1950. Lo spazio venne distrutto parzialmente da un incendio nel 1984 e fu abbattuto totalmente nel 1988.

La Biblioteca della Biennale di Venezia è frequentata, secondo le statistiche eseguite annualmente dalle bibliotecarie, da un range di pubblico ristretto, essendo la sua natura altamente specializzata, prevalentemente da studenti universitari, ai quali seguono professori, dottorandi e ricercatori e, durante il periodo della mostra, da dipendenti della Biennale e delle partecipazioni nazionali e anche qualche visitatore. È comunque una biblioteca di pubblica lettura, aperta tutto l'anno e a tutti.

La Biennale di Venezia ha sentito la necessità di possedere una biblioteca sin dalla fine degli anni Venti, che fosse luogo di raccolta di volumi e garantisse riferimenti preziosi per gli studi e la testimonianza dell'arte. Questa lungimiranza oggi, nel suo continuo evolversi, la rende una delle biblioteche specializzate in arte contemporanea più fornite e importanti in Italia. 


\section{Bibliografia}

Assante, Dario; Bertan, Fiorenzo (2000). Carlo Scarpa: il Padiglione del libro alla Biennale di Venezia, La Galleria del Cavallino, 1942 e 1949. Venezia: Cavallino.

Da Tos, Valentina; Fontanin, Roberta (2004). Catalogo periodici correnti. Venezia: La Biennale.

Da Tos, Valentina; Fontanin, Roberta (2007). Il fondo periodici rari della biennale di Venezia: catalogo e spoglio. Venezia: La Biennale.

Della Bella, Marina (2003). Manuale del bibliotecario. Santarcangelo di Romagna: Maggioli

De Sabbata, Massimo (2006). Tra diplomazia e arte: le Biennali di Antonio Maraini (1928-1942). Udine: Forum.

Donzello, Giuliana (1987). Arte e collezionismo: Fradeletto e Pica primi segretari alle Biennali veneziane 1895-1926. Firenze: Firenze libri.

Dorigo, Wladimiro (1975). Archivio storico delle arti contemporanee. Storia, situazione, prospettive. Venezia: La Biennale.

Maraini, Antonio (1932). La Biennale di Venezia: storia e statistiche: con l'indice generale degli artisti espositori dal 1895 al 1932. Venezia: Ufficio stampa dell'Esposizione.

Mostra internazionale del libro d'arte (1936). Mostra internazionale del libro d'arte. Venezia: Officine grafiche $\mathrm{C}$. Ferrari.

Pajusco, Vittorio (2013). «Archiviare il presente. Domenico Varagnolo e l'Archivio Storico d'Arte Contemporanea». Ateneo veneto. Atti e memorie dell'Ateneo veneto: rivista mensile di scienze, lettere ed arti, anno CC, vol. 1, 103-17.

Pajusco, Vittorio (2016). «Antonio Maraini e l'Istituto Storico d'Arte Contemporanea (1928-1944)». Saggi e Memorie di storia dell'arte, 38-2014, 135-51.

Salvagnini, Sileno (1990). «L'Archivio Storico della Biennale». Veneto ieri oggi, domani, 7, 62-7.

Stirling, James; Wilford Michael and associates; Muirhead, Tom (1991). Padiglione del libro Electa della Biennale di Venezia. Milano: Electa. 



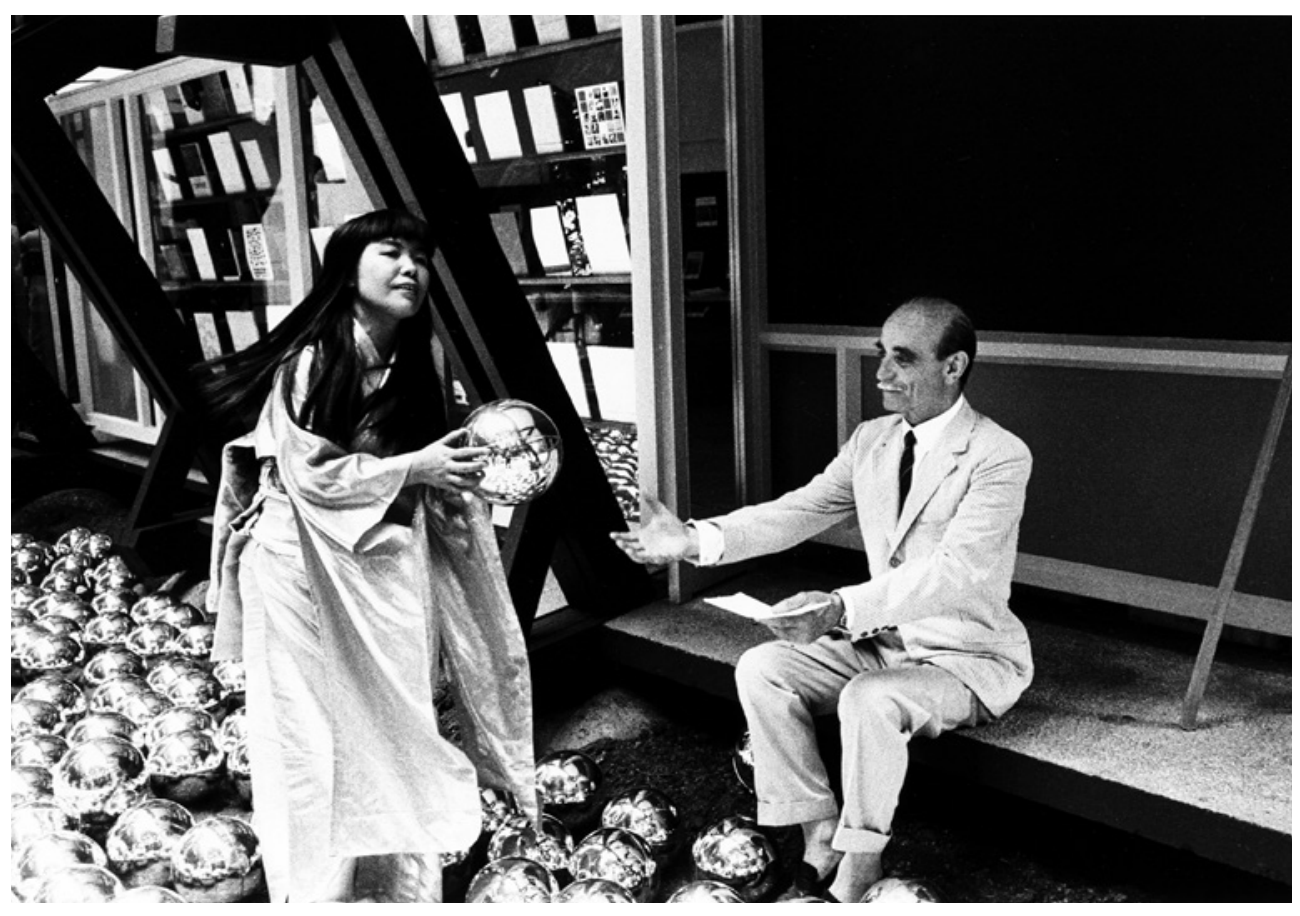

Figura 1 Venezia, Allestimento Biennale XXXIII: «Lucio Fontana», Lucio Fontana. Esterno del Padiglione del Libro; Yayoi Kusama e Lucio Fontana, giugno 1966. (c) Gianni Berengo Gardin-CISA A. Palladio 


\title{
Narcissus Garden for Sale: "one piece 2 dollars» Yayoi Kusama alla Biennale di Venezia nel 1966
}

Stefania Portinari

Università Ca' Foscari Venezia, Italia

\begin{abstract}
Things that do not exist or should not exist and 'ghetto exhibitions' mark some counterpoints on the presence of female artists and on first performance actions at Venice Biennale in the 1960s and the 1970s. Yayoi Kusama, who created Narcissus Garden without being invited in 1966; Marina Abramović and Ulay, invited for the first time in 1976 but in an external venue; and Paula Claire's action, between others, at the exhibition Materializzazioni del Linguaggio curated in 1978 by Mirella Bentivoglio, mark two crucial decades of increasingly contemporary trend at the Esposizione Internazionale d'Arte of Venice. This essay connects new relationships between Yayoi Kusama presence, art galleries in New York City, Milan, Venice and other main characters in the art system of the 1960s.
\end{abstract}

Keywords Venice Biennale. Yayoi Kusama. Lucio Fontana. Galleria del Cavallino. Padiglione del Libro. Performance. Gender art. Narcissus Garden. Japanese artists.

'Cose che non ci sono' o non ci dovrebbero essere e 'mostre ghetto' alla Biennale di Venezia tra anni Sessanta e Settanta tracciano alcuni contrappunti sulla presenza delle artiste, in omaggio anche alle ricerche provocate dalla Gender Art History che hanno cercato di marcare la 'teoria della differenza' sulla produzione artistica femminile e maschile, di cui sono state pioniere studiose della New Art History come Linda Nochlin, nel saggio «Why Have There Been No Great Women Artists?» pubblicato originariamente su ARTnews del gennaio 1971, o Lucy Lippard in From the Center: Feminist Essays on Women's Art del 1976, ma anche sulle tracce delle riflessioni di Rosalind Krauss e Judith Spector. 
Quando Nochlin pone retoricamente la «perennial question» sul perché nella storia dell'arte non possiamo annoverare grandi artiste donne, suggerisce che come per altre questioni coinvolte nel dibattito femminista si tratti di un dubbio che pare falsificare la natura della questione, indicando quasi implicitamente che sia perché le donne non sono capaci di «grandezza» (Nochlin 1988, 147). Ribadisce dunque come ovviamente l'arte non sia un'attività slegata dal contesto storico, creata da un individuo super-dotato influenzato solo dagli artisti precedenti o dal dinamismo degli eventi, ma una «situazione» che si connette sia al mercato dell'arte che alla qualità dell'opera all'interno di un contesto sociale modellato da specifiche istituzioni, in particolare dalle Accademie di Belle Arti, e dal sistema delle commissioni, come riguardi anche la concezione dell'artista come 'divino creatore' o emarginato. ${ }^{1}$ Uno dei fattori primari dell'esclusione delle donne da una certa carriera fin dall'Ottocento - rimarca - è «the question of the nude», il fatto che le donne non possano frequentare le Accademie dove posano i maschi nudi, in quanto situazione sconveniente, e siano dunque estromesse da un ambito di assimilazione tecnico-artistico e relegate ad alcune categorie di ideazione creativa considerate adatte alla loro 'indole', come scene intimiste, paesaggi o nature morte. Nel progressivo affacciarsi delle donne sulla scena del sistema dell'arte, saranno in particolare gli anni Sessanta a portare arditezza e maggiore presenza al femminile, contando numerose pioniere nel campo anche della body art, tra le quali annoveriamo Yoko Ono con Cut Piece, un'azione eseguita alla Yamaichi Concert Hall di Kyoto nel 1964 e alla Carnegie Recital Hall di New York il 21 marzo del 1965.

Alla 33a Esposizione Internazionale d'Arte di Venezia del 1966, alla metà di un decennio in cui anche alla rassegna prende slancio la partecipazione delle artiste donne, appare da 'clandestina' la giapponese Yayoi Kusama (che risiede però a New York dal 1957), compiendo una delle prime performance tenutesi alla Biennale. In quella edizione, a cui l'argentino Julio Le Parc vince il Gran Premio per la Pittura ma con due sale di opere optical e cinetiche, celebrando definitivamente la corrente che in Italia è nota anche come arte programmata (per merito di Bruno Munari e Umberto Eco che con Giorgio Soavi organizzano nel 1962 una mostra con tale titolo presso lo showroom Olivetti di Milano, poi a Venezia e a Roma), in un momento in cui i gruppi fondatori di queste ricerche già stanno dissolvendosi, Kusama installa Narcissus Garden (1966). Si tratta di mille e cinquecento sfere argentate di plastica posate sul prato antistante i tre lati del Padiglione del Libro - una struttura che si trova proprio

1 Oltre a Linda Nochlin, nel noto «Why Have There Been No Great Women Artists?», ora in Nochlin 1988, 145-78, cf. anche Hess, Nochlin 1972; Parker, Pollock 1981; Broude, Garrard 1982, 1992; Perry 1999; Portinari 2010. 
prospiciente al Padiglione Centrale - che in occasione del vernissage lei stessa, vestita con un kimono dorato dalla cintura argentata, porge in vendita al pubblico.

Il mito greco racconta di Narciso intento a riflettersi in uno specchio d'acqua e anche i globi di Kusama sono non solo riflettenti, ma anche posti l'uno accanto all'altro sull'erba dei Giardini come una superficie mobile dall'effetto cangiante. L'accumulazione stessa è un atteggiamento chiave nella poetica dell'artista, ma essi richiamano inoltre una fissazione della sua espressione creativa: i puntini che ossessionano le sue tele Infinity Net tra 1958 e 1961, le presenze-protuberanze-escrescenze morbide degli Accumulation pieces e delle Compulsive Furniture della prima metà degli anni Sessanta, i pallini obliteranti che compaiono nei lavori della serie Dots Obsession, anche come esorcismo alle crisi allucinatorie di cui soffre (Kusama 2011).

La sua presenza alla Biennale è il risultato di un soggiorno in Italia e dei contatti con Lucio Fontana, che in varie occasioni ha dimostrato molta generosità verso i colleghi più giovani. Kusama infatti ha lavorato per due mesi nello studio di lui a Milano quando, in quello stesso 1966, ha tenuto dal 26 gennaio al 9 febbraio la mostra Driving Image Show alla Galleria Naviglio 2, ovvero nelle salette interne della Galleria Il Naviglio di via Manzoni. ${ }^{2}$

In quella galleria, che lo rappresenta anche sul mercato, Lucio Fontana nel 1949 aveva realizzato l'Ambiente spaziale a luce nera e nel 1951 aveva dato sede al movimento dello Spazialismo, al tempo in cui l'attività era ancora retta dal fondatore, il veneziano Carlo Cardazzo, venuto a mancare nel 1963. È dunque con la gestione del fratello minore Renato Cardazzo che viene inaugurato uno spazio più sperimentale collegato alla galleria storica, dove Kusama allestisce un insieme composto, come recitano il poster e il piccolo catalogo-depliant della mostra, da elementi di «sex food obsession - compulsion furniture - repetitive vision - macaroni room - interminable nets - perseveration forms». Il risultato era già stato eseguito con lo stesso titolo nell'aprile del 1964 alla Richard Castellane Gallery di New York e consisteva, secondo l'affermazione dell'artista stessa, in una stanza «riempita con un simbolismo legato al sesso e al cibo» (cf. Yamamura 2009,96-8). Il pavimento era cosparso di pasta alimentare di vari

2 Carlo Cardazzo aveva fondato a Venezia la Galleria del Cavallino nel 1942 e nel 1946 aveva aperto una seconda sede a Milano, la Galleria del Naviglio. Dal novembre del 1963 ne assume la gestione il fratello minore Renato Cardazzo, che in realtà se ne occupa già dai mesi precedenti, durante la malattia di Carlo. Dal 1966 la sede di Venezia viene diretta dai figli di Carlo, Paolo e Gabriella; anche nell'estate del 1962 era stata aperta una sede chiamata Cavallino 2 a Venezia, a San Moisè 1477, per ospitare delle mostre che si tengono da giugno a ottobre; così come nell'estate del 1961 viene creata una dependance al Casinò del Lido (cf. Bianchi 2006, 92, 99; Carlo Cardazzo. Una nuova visione dell'arte 2009); nel 1966 la Cavallino 2 è aperta nella piazzetta prospiciente San Marco, a San Basso. In altre sedi sono poi le Edizioni del Cavallino e la Stamperia del Cavallino. 


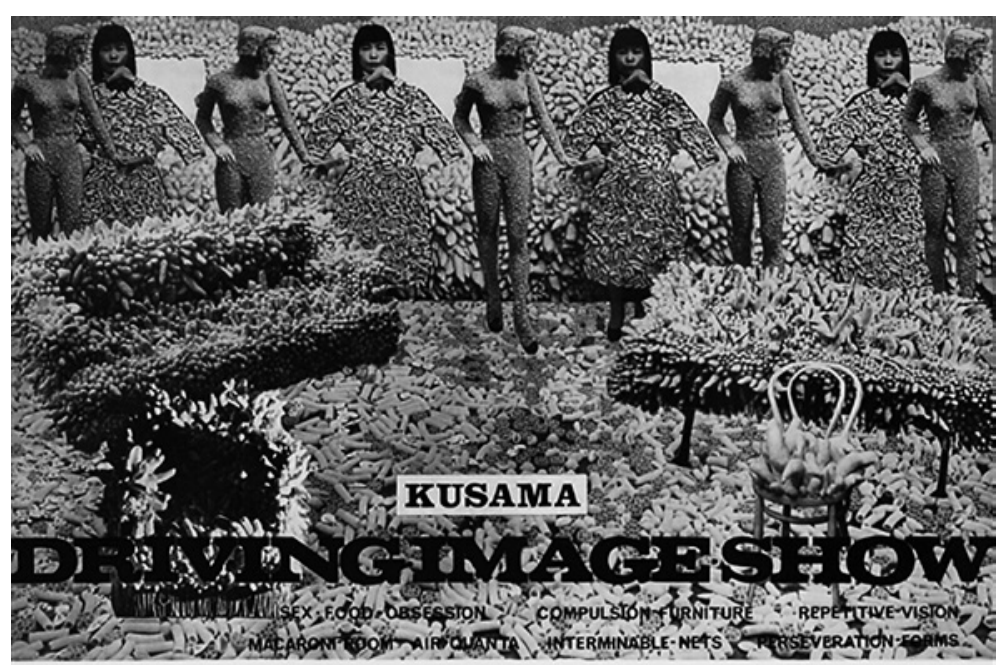

\section{NAVIGLIO 2 - GALLERIA D'ARTE - MILANO, VIA MANZONI 45 - TEL. 661538 DAL 26 GENNAIO AL 9 FEBBRAIO 1966 ORARIO: 10-12.30 - 16-20}

formati, creando un effetto che lei chiama «macaroni carpet», che le persone calpestavano facendola scricchiolare e riducendola in detriti, mentre a oggetti e mobilia erano applicate protuberanze in stoffa e decalcomanie di fiori e foglie, agglomerando altri suoi precedenti lavori del 1962-63 come Macaroni Girls (1963), dei manichini femminili ugualmente ricoperti da pezzetti di pastasciutta. La stessa idea viene riportata alla Galleria Naviglio 2: in catalogo campeggia infatti un collage piuttosto pop in cui l'effigie fotografica della stessa Kusama si alterna a nuove Macaroni Girls realizzate nello studio di Fontana (secondo quanto mostrano anche delle foto custodite alla Fondazione Lucio Fontana di Milano), attorno alle quali fanno carosello delle sedie, una panca, una poltrona e un divano ricoperti di prominenze morbide, tra un all over di pasta.

In catalogo è riportata inoltre una «Intervista fatta da Gordon Brown per la Radio W.A.B.C.», che l'executive director di Art Voices aveva registrato ancora nel luglio 1964. L'artista vi racconta come i Net Paintings fossero «very large canvases without composition - without beginning, end, or center. The entire canvas would be occupied by monocromatic net. This endless repetition caused a kind of dizzy, empty, hypnotic feeling» (Yayoi Kusama 1966) e come quella 
sensazione avesse iniziato a espandersi. Afferma infatti: «I came under the spell of repetition and aggregation. My nets grew beyond myself and beyond the canvases I was covering with them. They began to cover walls, the ceiling, and finally the whole universe. I was always standing at the center of the obsession over the passionate accretion and repetition inside of me». ${ }^{3}$ Dopo le Aggregation-Sculpture del 1962 dunque aveva già avuto l'idea di un ambiente ( I planned an environment based on the idea of aggregation», dichiara), che diventa quello mostrato alla Castellane Gallery. «Driving images» è il nome dato alle presenze che mette in scena: sono i visitatori che le riferiscono che hanno su di loro una «irresistible force» e pure lei guardandole si sente come se stesse guidando su un'autostrada o su un nastro trasportatore senza fine, continuando a bere centinaia di tazze di caffè o mangiando centinaia di metri di «macaroni» (cf. anche Kusama 1966). Da quelle sensazioni deriva anche l'idea di Narcissus Garden; l'interesse per il coinvolgimento del pubblico ha un ruolo significativo fin da allora, precisa infatti: «I am always deeply interested in the background of problems involved in the relationships of people and society. My artistic expression always grow from the aggregation of these».

A quella intervista si affianca un breve testo di Herbert Read del 13 marzo 1964 che la definisce alla ricerca di una «super reality»; mentre quando la mostra Driving Image Show dalla Galleria Naviglio 2 viene trasferita dal 29 aprile alla Galerie M.E. Thelen di Essen in Germania è accompagnata da uno scritto di Udo Kultermann, in cui già si anticipa che l'artista sarà alla Biennale.

Kusama alla Biennale rappresenta solo se stessa: non è in una mostra speciale, né in un padiglione nazionale, tanto meno in quello del Giappone, esistente fin dal 1952 e che nel 1966 ospita Toshinobu Onosato, Masuo Ikeda, Morio Shinoda e Ay-O, alias l'artista fluxus Takao Iijima, e che anzi ospiterà una personale di Yayoi Kusama solo nel 1993, con la 45a Esposizione Internazionale d'Arte intitolata Punti Cardinali dell'Arte curata da Achille Bonito Oliva.

Nell'autobiografia edita nel 2011 l'artista asserisce ambiguamente di aver ricevuto il «permesso» dal «bureau» della rassegna (Kusama 2011, 54), ma in realtà non le viene recapitato un invito e la sua comparsa irregolare è dovuta all'autorità esercitata da Renato Cardazzo che gestisce il Padiglione del Libro progettato nel 1950 da Carlo Scarpa proprio su mandato del fratello Carlo Cardazzo (cf. Lanzarini 2003; Duboÿ 2016; Carlo Scarpa. Disegni di Carlo Scarpa 2000), essendo quel luogo una sorta di bookshop della Biennale dove vengono vendute edizioni d’arte e cartelle create in primis dalle Gallerie del Cavallino e del Naviglio, oltre che allestite piccole mostre dedicate 
ai libri d'arte: la presenza di Kusama, anche nel senso proprio di collocazione spaziale, è collegata a quella struttura. ${ }^{4}$ Tra le mostre che la Galleria del Cavallino tiene a Venezia in quella estate se ne annovera tra l'altro proprio una di Fontana (dal 7 al 29 luglio) e una intitolata Modern Art of Japan, allestita dal 15 giugno al 15 luglio allo spazio Cavallino 2, sempre in piazza San Marco ma nella sala di San Basso, che espone tredici artisti giapponesi (Nobuya Abe, Shusaku Arakawa, Kumiko Imanaka, Masuo Ikeda, Josaku Maeda, Tomio Miki, Hiroshi Okada, Toshinobu Onosato, Yoshishige Saito, Yoshio Sekine, Morio Shinoda, Jiro Takamatsu, Tomonory Toyofuku). Se inoltre durante i primi mesi dell'anno si erano tenute piccole personali di Shu Takahashi e Juuko Ikewada, seguite dopo la Biennale da quella di Ichiro Haryu, in quel novero d'anni nel 1965 c'erano state altre personali di Katsumi Natai, Yukihisa Isobe e Nobuya Abe, nel 1963 quelle di Hisachika Takahashi e Susumu Shingu. Questo interesse si inserisce in una crescente attenzione verso l'arte contemporanea giapponese che già si era posta, legata ancora a Gutai e all'informale in connessione con le correnti americane e europee, da mostre come Arte Nuova. Esposizione Internazionale di Pittura e Scultura che era stata organizzata nel 1959 a Palazzo Granieri a Torino, curata da Angelo Dragone ma emanata da un comitato in cui erano il gallerista Luciano Pistoi, Coichi Tominaga e Michel Tapié (che dal 1956 si è stabilito in quella città e che aveva una attività di mediatore col Giappone instaurata fin dall'anno precedente), e dalle collaborazioni col Centro Internazionale delle Arti e del Costume di Palazzo Grassi, che nel 1960 aveva invitato a Dalla Natura all'Arte anche Sofu Teshigahara, artista e fondatore della Scuola di ikebana Sogetsu di Tokyo. Dunque la presenza di Kusama presso il Padiglione del Libro con una installazione (che però si trasforma in azione) poteva passare come un complemento all'attività dei Cardazzo considerato che, oltre alle due gallerie con le rispettive dependances, gestiscono le Edizioni del Cavallino e la Stamperia del Cavallino. Se pure nei documenti inerenti al Padiglione del Libro conservati all'archivio storico della Biennale non sia menzionata Yayoi Kusama, risulta comunque come da Milano il 10 maggio 1966 Renato Cardazzo avesse risposto a una lettera del 2 maggio di Deuglesse Grassi, direttore amministrativo dell'ente, confermando come anche quell'anno avrebbero organizzato «come consuetudine [...] la mostra del Libro d'Arte al Padiglione del Libro in occasione della Biennale», mettendosi a disposizione per sti-

4 Il Padiglione del Libro era una piccola struttura, dalla pianta a forma di freccia, divisa all'interno in due ambienti, uno a scopo espositivo (nel 1966 nella mappa in catalogo la struttura viene infatti nominata come Mostra Internazionale del Libro d'Arte) e l'altro per la vendita. È stato distrutto da un incendio nel maggio del 1984 e definitivamente demolito nel 1988; ne ha preso il posto il Bookshop Pavilion for the Venice Biennale, creato dalla Stirling Wilford and Associates di James Stirling nel 1991. 
pulare l'atto di concessione della struttura, che viene data in affitto all'impreditore. ${ }^{5}$ Il gallerista ed editore diviene poi l'epicentro a sua volta delle richieste di altri interlocutori, come le Edition du Griffon di Neuchatel o Allemandi di Torino, per poter vendere le loro edizioni. Prova del suo ruolo e della libertà che lo contraddistingue è anche l'istituzione di un premio di 500.000 lire dedicato alla memoria di Carlo Cardazzo da assegnare tra i premi ufficiali che, come già era avvenuto due anni prima, Renato offre alla presidenza della Biennale. ${ }^{6}$

Il ruolo di Renato Cardazzo e di Lucio Fontana, che a quella Biennale ha assegnata una sala allestita da Scarpa come fosse un sacello di monoliti bianchi che reggono suoi cinque Concetti spaziali. Attesa e che vince il premio da due milioni di lire dato dal Comune di Venezia per un pittore italiano, sono rivelati come un indizio cruciale anche dalle foto scattate da Gianni Berengo Gardin: Fontana siede sugli scalini del Padiglione del Libro, guardando Kusama che fa volteggiare le sfere argentate. ${ }^{7}$ E stato lui tra l'altro a finanziare la produzione dei globi, mettendo Kusama in contatto con la ditta Italvalmet di Levane, in provincia di Arezzo, e prestando il corrispettivo di circa 600 dollari per la realizzazione (Tatehata 2009, 25). I due si erano conosciuti probabilmente l'anno prima, nel 1965, all'esposizione collettiva ZERO/Nul. Negentienhonderd vijf en zestig allo Stedelijk Museum di Amsterdam, e successivamente avevano esposto entrambi alla collettiva itinerante Zero Avanguardia che, curata da Nanda Vigo, era iniziata nello stesso anno proprio dallo studio di Fontana a Milano e aveva toccato in maggio anche la Galleria del Cavallino, dove viene accompagnata da un cataloghino con testo di Gillo Dorfles (Zero Avantgarde 1965; Galleria del Cavallino. Mostre 1966 1966).

5 ASAC, FS, AV, b. 146: Padiglione del Libro. Lettera di R. Cardazzo a D. Grassi, 10 maggio 1966.

6 ASAC, FS, AV, b. 146: Padiglione del Libro. Lettera di R. Cardazzo a M. Marcazzan, 11 marzo 1966: «in occasione della 33a Biennale di Venezia vorremmo affidare, come due anni fa, alla Giuria Internazionale della Biennale stessa un premio di L. 500.000 dedicato a Carlo Cardazzo. Noi speriamo che la Presidenza voglia accettare questo premio che per noi è oramai una consuetudine».

7 Fontana aveva esposto alla Biennale nel 1930, nel 1954 (diciotto opere nel Padiglione Italiano), nel 1958 (quaranta opere, allestite già da Carlo Scarpa). Nel 1966 l'allestimento consiste in una sorta di «involucro ovale a tutta altezza» ricoperto in tela bianca, "chiuso in alto da un velario a teli - che racchiude cinque espositori a forma di C. Ciascuno di essi è destinato a contenere - come una piccola quinta teatrale - uno dei Concetti spaziali» (Lanzarini 2003, 205-9). Cf. Duboÿ 2016, 175-9: alla sala si accede tramite due porte anch'esse strutturate in forma elissoidale, poste entrambe sulla medesima parete. Il progetto inizialmente doveva essere un effetto di labirinto, contenente una decina di opere, secondo un progetto di Fontana stesso, poi aveva preso il sopravvento la necessità di mostrare davvero le opere e il fatto che la sala fosse più piccola del previsto, anche se l'artista in un'intervista di Carla Lonzi nel 1967 riferisce che avrebbe persino preferito presentare solo «una sala come ambiente, come pensiero puro», «una forma tutta ovale», come un «uovo». 
Kusama, tra la mostra al Naviglio 2 e la Biennale, ha allestito nel marzo del 1966 Kusama's Peep Show. Endless Love Show alla Castellane Gallery di New York, consistente in una struttura in legno esagonale ricoperta di specchi, posti anche su soffitto e pavimento, su cui si riflettono flash di luci colorate emesse da file di lampadine che si accendono e spengono in sequenza random, attivate da uno strumento elettrico, che gli spettatori possono esperire da due aperture poste all'altezza del viso, affacciandosi e vedendo riflessa - apparentemente all'infinito - la propria immagine. Un sound di sottofondo con musica rock e adesivi che promettono «Love Forever» punteggiano inoltre l'environment. Se già l'artista aveva impiegato superfici riflettenti alla mostra Floor Show del 1965 sempre alla Castellane Gallery, ricoprendo le pareti di specchi e la pavimentazione con pannelli a cui erano fissate fitte protuberanze in stoffa bianca a grandi pallini rossi, il concetto di visione riflessa torna nelle sfere argentate della Biennale che rispecchiano addirittura tutto il mondo esterno, visitatori compresi (Yamamura 2009, 104). E un'osservazione che segnala fin da subito il critico Pierre Restany su Domus, scrivendo come le persone si avvicinino all'installazione per esaminare le sfere, vedendo i loro volti deformati e riflessi all'infinito (Restany 1966, 37). La stessa Kusama ribadisce al critico Gordon Brown, che si reca successivamente nello studio di lei a Manhattan per una intervista che pubblica sulla rivista italiana D'Ars Agency:

Le mie 1500 sfere riflettono l'erba verde del giardino, esprimendo simbolicamente l'unione dell'uomo con la natura. La gente si ferma come ipnotizzata da quei 1500 riflessi del Sole nel cielo e dell'ambiente circostanze in movimento. Nelle biglie i riflessi riflettono i riflessi. Vedere l'immagine del proprio viso moltiplicata all'infinito colpisce la sensibilità di quelli che si fermano a guardare: è come se essi intuissero che non vi sono limiti alla capacità dell'uomo di proiettare se stesso nello spazio. (Brown 1966)

Quest'ultima dichiarazione è molto vicina agli intenti dello Spazialismo e anche per quanto concerne i materiali delle «biglie» Kusama afferma che gli artisti devono oramai impiegare $\mathrm{i}$ «nuovi ritrovati della chimica e dell'industria», dunque esse sono di plastica, anzi «i materiali tradizionali dovrebbero essere banditi, specialmente se sono costosi come il marmo e il bronzo, le cui spese di trasporto, ad esempio, vengono pagate a più riprese: dallo scultore, dal venditore $\mathrm{e}$ dal pubblico». L'idea di usare i materiali industriali, anche per le loro stesse proprietà di colore e consistenza, è annettibile alle sperimentazioni dell'arte programmata, pensando a come è cambiata la poetica di Kusama dagli imbottiti cuciti da lei stessa e dall'uso della pasta 
alimentare impiegati fino a pochi mesi prima. ${ }^{8}$ Per questo motivo secondo lei occorre un mutamento nelle pratiche dell'artista, che non va inteso come una limitazione dei mezzi scelti e continua spiegando:

il mio "Giardino di Narciso" è un esempio di piena libertà artistica. In passato si usavano pennelli, colori, scalpelli. Oggi l'opera d'arte è frutto esclusivamente della sensibilità dell'artista. Non si è più ostacolati dalle difficoltà del mestiere. Oggi l'artista non deve far altro che avere l'idea dell'oggetto e rivolgersi all'industria per grosse ordinazioni. All'arte si offrono nuove possibilità. Non si crea più per pochi privilegiati ma per la massa. Invece di attingere soggetti dalla società, cosa sempre dannosa, l'artista diventa parte integrante della vita economica vendendo i suoi lavori a prezzi accessibili a tutti. La gente può fare acquisti d'arte facilmente, come al supermercato o in merceria. (Brown 1966)

Trova inoltre molto «lusinghiero» che le persone giochino con le sfere, che sono molto leggere e dunque «ognuno può portarsele a casa da solo e costruirsi la propria scultura. L'uso continuo dell'identica forma e della stessa misura garantisce l'unità artistica in questi lavori fatti in casa» (Brown 1966), quasi insomma un anticipo di arte relazionale.

Al vernissage della Biennale Kusama indossa lo stesso kimono dai colori metallici con il quale è ritratta proprio all'inaugurazione della mostra allo Stedelijk Museum (cf. Sullivan 2015; Yoshimoto 2005, 4577), e si fa fotografare successivamente tra le sfere vestita con body, calze e scarpe rossi, come nelle installazioni alla Castellane Gallery: la messa in scena della sua figura, della sua 'esoticità' o estraneità, di una componente di spettacolo, come in occasione delle successive performance newyorkesi, è dunque parte attiva dell'immaginario che intende costruire.

Tra le sfere poste l'una accanto all'altra sul prato, sul versante più in vista, spicca un cartello con la scritta «Narcissus Garden. Kusama», mentre davanti all'ingresso un altro promette "Your Narcissium [sic] for Sale. One piece \$ 2. Narcisizzati. Al pezzo L. 1200», tanto che il giornale romano La Discussione, fraintendendo il messaggio, ne pubblica una foto con didascalia indicando che lei stia presentando le sue sfere di plastica per «un giardino di narcisi». ${ }^{9}$

8 Cf. Alfred Carl, «Call Her Dotty», Sunday News, August 13 1967, 31: ricorda come la pratica nasca dai gesti compiuti dall'artista da bambina, disegnando pallini, e come le protuberanze imbottite in stoffa che sono servite a comporre le sue sculture precedenti, apposte anche su arredi in quelle che chiama le Compulsion Furniture, fossero state eseguite da lei con la macchina da cucire, e come in Driving Image Show fosse presente un appendiabiti che regge vestiti da donna tempestati di «macaroni».

9 La discussione, 26 giugno 1966. 
Le foto scattate a Kusama, sia quelle che si premura di far realizzare che quelle che vengono pubblicate su riviste come L'Europeo o L'Espresso, la mostrano anche intenta a interloquire con le signore eleganti, reggendo una sfera o facendone volteggiare in aria più d'una, tra la sorpresa dei visitatori. ${ }^{10}$ L'atto di venderle e di presentarsi nelle forme di una apparizione all'esterno dei padiglioni si conforma dunque come un happening di cui il pubblico diviene complice, come quando nel 1960 Piero Manzoni alla sua Galleria Azimut nella Consumazione dinamica del pubblico divorare l'arte aveva venduto le uova sode con l'impronta del pollice o nel 1962 aveva ceduto al prezzo simbolico del valore dell'oro al grammo le scatolette di Merda d'artista. La Biennale di Venezia come istituzione però gestisce fino al 1972 un Ufficio vendite interno, tramite il quale devono passare tutti gli acquisti effettuati alla rassegna e le cui procedure sono chiaramente indicate nello statuto dell'ente e sui cataloghi (cf. Portinari 2018), che ha sede tra l'altro proprio in una stanzina nel Padiglione Centrale lì di fronte e fino al 1968 è seguito dal noto gallerista milanese Ettore Gian Ferrari, dunque l'azione dell'artista viene subito bloccata.

A Gordon Brown in quel 1966 Kusama rivela che comunque quello della Biennale rappresenta per lei un «lavoro pilota» e che la prossima volta che esporrà le sfere intende davvero venderle a due dollari l'una, ma ribadisce che non è una speculazione commerciale, che ha inteso lavorare sulla percezione e rendere possibile a tutti il possesso di un'opera d'arte. Quarant'anni dopo, ricordando nuovamente come per lei fosse stato importante il gesto di vendere le mirror balls in tale luogo, aggiunge che nella sua intenzione era come se avesse venduto «hot dogs o gelato», che non trovava nulla di sbagliato in quello che stava facendo, dato che le sue erano opere di pop art - suggerendo dunque implicitamente, e ambiguamente, di considerarle alla stregua di una merce - e che c'erano file di persone pronte ad acquistarle. ${ }^{11}$ Se effettivamente la pop art nel 1966 è una corrente già molto affermata, oggetto di un symposium al MoMA fin nel dicembre del 1962, quella dichiarazione si lega a un periodo tardo, già di grande notorietà per l'artista, mentre la conformazione reale di Narcissus Garden, in primis il suo aspetto composto da sfere luccicanti vicine a tutta una serie di altre realizzazioni presenti proprio a quella Biennale, e le frequentazioni dell'artista in quel momento inducono a collocare ben altrove quella sua traiettoria, più vicina semmai alla contestazione del mercato dell'arte che, con altri modi, andavano

10 Cf. anche Irene Brin, «Milledue cadauna», L’Europeo, 23 giugno 1966.

11 Tatehata 2000, 19: «what was most important about Narcissus Garden was my action of selling the mirror balls on site, as if I were selling hot dogs or ice cream». Germano Celant, «Yayoi Kusama», Interview, June 2005, 80: «What's wrong? This is Pop art. There were lines of people waiting to buy». 
compiendo proprio i gruppi dediti all'arte optical e cinetica con l'idea anche di multiplo o, tanto più, a tutto quell'underground newyorkese che, dagli ultimissimi anni Cinquanta, inizia a coltivare l'arte performativa e anticipa quella che sarà l'environmental art. ${ }^{12}$

Malgrado ne sia impedita la vendita, i globi restano in esposizione per tutta la durata della Biennale. Il Gazzettino riporta però che a causa del sole la «patina lucente» che li riveste va virando verso «il colore del caffelatte» e che l'artista stessa « disperata» ha raccomandato via lettera alle commesse del Padiglione del Libro di girare «i palloncini più volte al giorno». ${ }^{13}$

La giornalista Ornella Ripa del settimanale Novella nota che quella Biennale «é il trionfo delle palline e degli specchi»: le offre «la giapponese», ci sono "palline lucenti» sulla scultura La Grande Madre (1966) di Alberto Viani, «pallone» su quella dello svizzero Walter Linck, i pois del giapponese Ay-O, i dischi di Toshinobu Onosato, le sculture a sferette dell'austriaco Curt Stenvert, l'israeliano Buky Schwartz ha posto una pallina rossa su una molla semirigida fissata davanti a una striscia di alluminio concava e il pubblico è invitato a colpirla, molte sono poi quelle presenti da Le Parc. ${ }^{14}$ Il premiato Julio Le Parc infatti, che fa parte del Group de Recherche d'Art Visuel e occupa due sale del Padiglione Centrale con quarantadue opere, ${ }^{15}$ impiega la forma sferica in una versione delle Palle vibratili del 1963 e in una del 1966, in Palla su una molla (1963-65), due Giochi di una palla (1964), Gioco con una palla da ping pong e Gioco con due palle da ping pong (1965) o una Tavola da gioco con venti palle da ping pong (1965); ha soprattutto un vivissimo rapporto con lo spettatore

12 Nel 1972 la stessa Biennale, dopo le contestazioni del 1968 e in coincidenza con un cambiamento del suo stesso pubblico, che annovera più giovani, per la richiesta di maggiore 'democraticità' e pure a seguito del rinnovamento dello statuto nel 1973, organizzerà una sezione nella mostra principale con un Laboratorio di stampa che offre molti medium, dall'incisione alla xerocopia alla stampa su plexiglass. A questo lavorano alternandosi degli artisti invitati per creare dei multipli, che vengono sia impiegati per mostrare ai visitatori come operano, pensando utopicamente di coinvolgerli in come si svolge il processo creativo (anche provando direttamente la realizzazione), sia venduti dall'Ufficio vendite della Biennale (che in quell'anno esiste per l'ultima volta) per cifre irrisorie, e i cui guadagni vengono impiegati per sostenere in parte le spese di ospitalità degli artisti stessi (un'altra parte dei fondi viene dal reimpiego della cifra usualmente destinata ai premi, aboliti dopo l'edizione del 1968) (cf. Portinari 2018). Una delle opere prodotte ad esempio è Souvenir (1972) di Ugo La Pietra, rilasciato accompagnato da un certificato-opera emesso dall'artista.

13 «Uno dei fori di Ay-O chiuso per consiglio medico», Il Gazzettino, 5 luglio 1966.

14 Ornella Ripa, «Vi piace l’arte d’oggi?», Novella, 31 luglio 1966, 8-9.

15 Biennale 33 1966, 121-2. A quella Biennale nel Padiglione Centrale, oltre agli artisti italiani, sono anche le rappresentanze nazionali di altri Paesi senza padiglione, oltre all'Argentina rappresentata da Le Parc, come Bolivia, Cuba, Ecuador, India, Iran, Perù, Sud Africa, vi si affiancano anche le retrospettive dedicate a Umberto Boccioni, Giorgio Morandi e la mostra Aspetti del primo astrattismo italiano. Milano-Como 1930-1940. 
del quale incentiva una partecipazione percettiva attiva, ma molti altri artisti in quella edizione corteggiano il contatto con il pubblico, in una dimensione persino ludica.

Il Giappone, che come nazione è alla sua decima partecipazione alla Biennale, essendo stato presente alla seconda del 1897, nel 1924 e poi regolarmente dal 1952, presenta tra i quattro artisti esposti AyO, che come Kusama viene da New York, dove lui vive da otto anni, e che lavora da tempo sullo spettro dell'arcobaleno. Allestisce infatti l'opera Rainbow Environment 3, definita in catalogo Arcobaleno e camera tattile di Venezia (1966), un ambiente composto da elementi chiamati Paesaggio (Veduta della stanza), Tenda, Scansia e tavola e Muro (Arcobaleno animato), che ha un grande successo per la sua singolare interattività. Le persone possono insinuare un dito nelle fessure tonde della camera tattile, attraverso una membrana di gomma che stringe la falange, e dentro si possono percepire delle sensazioni inusitate, dei «misteri soavi e sconcertanti» dati da sostanze come borotalco, sabbia, pelo, un guanto di gomma, pulsantini che provocano musiche e suoni di campanello, o provare delle sorprese rischiando di farsi male, come capita a un visitatore punto da uno strano ago, persino sentire «le delissie» (le delizie), come dichiara l'inserviente che compie le pulizie nel padiglione a Milena Milani, che è scrittrice e artista e era stata la compagna di Carlo Cardazzo. ${ }^{16}$ In un articolo intitolato Una biennale tutta sexy, lei che nel 1964 aveva avuto il suo romanzo Una ragazza di nome Giulio incriminato per oltraggio al pudore, annuncia che aspetta al varco il patriarca di Venezia, le signorine di buona famiglia, persino il vice procuratore generale della Repubblica che ha incriminato la sua pubblicazione per sentire cosa dicono di questa edizione della rassegna. Ha percepito infatti la vernice «più gioiosa e sconvolgente che la storia della Biennale ricordi, in un'atmosfera elettrica, ma anche piena di umanità» e riporta come artisti, critici e belle donne con le gambe nude, pittori e attrici abbiano «bivaccato» fino a tarda notte in piazza san Marco: «tutta la Biennale è giovane». Persino l'esperienza tattile procurata da Ay-O le pare una «rivoluzione erotica» rappresentata con ironia. Un occhiello dell'articolo è dedicato poi espressamente alla Kusama, visto che Milani accompagna un brigadiere ad acquistare quella che chiama una sua boule, che l'uomo intende portare come omaggio al capo della polizia, e nota come i riflessi abbiano sulle sfere un «effetto decorativo notevole» e la gente dica che per guardarle «ci vogliono gli occhiali da sole».

16 Milena Milani, «Una biennale tutta sexy», $A B C, 3$ luglio 1966, 12-13: l'articolo è pubblicato sul numero di luglio della rivista, ma è scritto in giugno in occasione del vernissage della Biennale. Sui materiali contenuti nella camera tattile cf. anche Ornella Ripa, «Donne e mostri alla Biennale», Novella, 31 luglio 1966, 8-13. 
Nel Padiglione Centrale la sala 50, che ha un grande rilievo essendo la prima alla destra dell'ingresso, è assegnata a Bruno Munari che presenta un ambiente composto da quattro Polariscop (1966) a luce polarizzata come variazioni di un unico «oggetto» - spiega nella presentazione Filiberto Menna - in cui la «funzionalità della macchina si accompagna alla gratuità del gioco e alla libertà del contemplare». Sono dunque «macchine per contemplare» che rappresentano una «piena espressione dei principi dell'arte programmata», strutture cinetiche in continua trasformazione e sperimentazione visiva, oggetti con una loro autonomia estetica (Menna 1966), così come altre due sue aurorali Macchine inutili (1933-34) sono nelle sale 7-11 alla mostra Aspetti del primo astrattismo italiano. Milano-Como 1930 1940. Persino Michelangelo Pistoletto in sala 53 presenta un'opera specchiante intitolata Biennale 1966 (1966) che diventa simbolica del concetto di spettatore, raffigurando sulla carta velina incollata sullo specchio un ragazzino nell'atto di guardare, posto dietro a un riflettore da palcoscenico.

La stessa Ornella Ripa si domanda anche quante siano le donne artiste presenti a quella Biennale, su 220 espositori di 37 Paesi, e va a chiederlo a Gian Ferrari che risponde «anche troppe», mentre gli organizzatori dicono che non lo sanno: lei le conta dal catalogo e constata che sono 14. Dunque in realtà sono $14+1$, dato che Kusama non è annoverata da nessuna parte mentre - sottolinea - è la più fotografata e apprezzata da tutti, «non invitata alla Biennale», e che però sta «in quel pezzo di giardino fuori concorso che una galleria di Venezia ha allestito vicino all'ingresso». ${ }^{17}$

Quando il segretario generale della Biennale, Gian Antonio Dell'Acqua, aveva scritto nell'introduzione in catalogo che per ragioni di carattere tecnico e organizzativo la fisionomia della rassegna si definisce nel suo complesso

come convergenza di scelte indipendenti, soltanto al momento della 'vernice' o nei giorni immediatamente precedenti. Soltanto allora, infatti, è possibile rendersi conto in concreto, nel confronto reciproco, del peso delle singole persone e del rilievo che questo o quell'orientamento o tendenza vengono ad assumere nel quadro d'insieme dell'esposizione. Qui sta l'affascinante incertezza, fino all'apertura dei cancelli ai critici e al pubblico, di ogni Biennale. (Dell'Acqua 1966),

presagiva proprio situazioni come questa: quando un artista o una situazione non prevista diventano invece il centro dell'attenzione. Questo capiterà negli anni successivi ad esempio con la performance di 
Gino de Dominicis nel 1972 o con la prima presenza ufficiale di performance (dopo il fallimento dell'organizzazione della sezione Pérsona a cura di Achille Bonito Oliva nel 1972) che si tiene nel luglio del 1976 nella sezione intitolata Attivo, programmata da Tommaso Trini per la rassegna "Attualità internazionali '72-'76», nella sede decentrata degli ex Cantieri navali della Giudecca (che mostra azioni, tra gli altri, di Vettor Pisani, Giuseppe Chiari, Michelangelo Pistoletto, e Marina Abramović e Ulay, che presentano Relation in space)..$^{18}$ Allora saranno trascorsi dieci anni da Narcissus Garden e solo nel 1978, ancora in una sede decentrata, i Magazzini del Sale, si terrà una mostra di sole donne con ottanta artiste di diciotto nazioni, dedicata alla poesia visiva e alla scrittura visuale, con una sezione di performance che si estende anche all'esterno, grazie a Materializzazioni del Linguaggio curata da Mirella Bentivoglio. ${ }^{19}$

In quello stesso 1966 in cui Kusama compare da 'irregolare' ai Giardini invece, al di fuori di ogni connessione con la Biennale e ugualmente all'aperto, avviene una singolare performance a mezzanotte, il Venice Gondola Happening di Charlotte Moorman e Nam June Paik. Avendo annunciato tramite i giornali locali che il 5 luglio alle ore 23 avrebbero tenuto un'azione sul Canal Grande di fronte a un bar di Rialto frequentato dagli artisti, la sera dell'evento i tavolini sono occupatissimi e gli astanti «tutti schierati sulle due spallie-

18 Accanto alla mostra è stato approntato uno spazio per gli interventi degli artisti che realizzano performance o azioni di teatro e danza. Per il catalogo Abramović invia le foto di Art Must Be Beutiful, Artist Must Be Beautiful (1975), ma il 16 luglio alle 23 lei e Ulay mettono in atto Relation in space, in cui per 58 minuti, partendo dai due estremi opposti del luogo, si scontrano nudi sempre con più forza, ripresi da una videocamera fissa il cui filmato viene proiettato simultaneamente da una tv posta di fronte al pubblico: cf. Portinari 2018, 292; ASAC, FS, AV, b. 242: Lettera di M. Abramović a T. Trini, 3 maggio 1976 (l'artista aveva accettato con entusiasmo all'invito, scrive: «I went mad with joy»); Achille Bonito Oliva, «Quel pasticciaccio brutto della Giudecca», Corriere della Sera, 18 luglio 1976. Si tratta della prima partecipazione dell'artista alla Biennale, che in precedenza in Italia aveva compiuto azioni a Contemporanea, curata da Bonito Oliva e Graziella Lonardi Buontempo nel parcheggio sotterraneo di villa Borghese a Roma, ricreando Rhythm 10 (1973) presentato per la prima volta al Festival di Edinburgo, Rhythm 4 alla galleria Il Diagramma di Luciano Inga Pin a Milano nel 1974 e Rhythm 0 allo Studio Morra di Napoli nel 1975.

19 Materializzazione del linguaggio. La donna tra parola e immagine si tiene in quello che è chiamato Spazio Aperto, dal 20 settembre al 15 ottobre 1978, dunque praticamente sullo scorcio finale della Biennale; le cinque performance che si tengono il 21 e 22 ottobre sono soprattutto azioni di letture di poesia fonetica, spiccano in particolare quelle del soprano Joan Logue che esegue Concerto per voce sola: Stripsody (1966), di cui è autrice Cathy Berberian, e Paula Claire che con Codestones of Venice crea un'azione basata in parte sull'improvvisazione in 9 lingue su 3 ponti lungo le Zattere, coinvolgendo il pubblico e in parte dedicata al disastro ambientale di Seveso: cf. Portinari 2018. È Bentivoglio che parla esplicitamente di 'mostre ghetto' indicando le mostre 'al femminile' che lei stessa ha organizzato anche in precedenza, ma precisa come esse siano necessarie, in quel momento, a dare risalto alle loro ricerche. 
re del Ponte». ${ }^{20}$ Si dice che i due sarebbero arrivati in gondola, che lei avrebbe suonato il violoncello e che si sarebbe messa in bikini, che il pittore giapponese Ay-O - che come loro è parte del movimento fluxus - avrebbe fatto «qualcosa di straordinario». Egli si trova infatti sul ponte, ma all'orario stabilito non accade nulla, mentre vicino al bar sono posti «un giradischi, un registratore con grande altoparlante e un riflettore». Verso mezzanotte compare finalmente la gondola promessa, che nel frattempo ha solcato i rii e il canale con a bordo un altro musicista: dall'altoparlante escono rintocchi di campane, un riflettore inquadra Moormann che suona sul violoncello una partitura di John Cage, una rivisitazione di 26'1.1499 for a Sing Player, e delle variazioni su tema di Saint-Saens, mentre l'altoparlante a quel punto suona musica jazz e una canzone di Fred Bongusto. Paik, che è alla sua prima performance italiana, impiega uno strumento orientale con sonagli, poi si toglie la camicia e a torso nudo si intromette sotto una corda del violoncello di lei, che la suona. Dopodiché lei si tuffa in acqua e la ripescano «con molta fatica». Issata sulla gondola, suona il Largo di Haendel, poi ancora la composizione di Cage, mentre Paik inizia «a travasare l'acqua» del Canal Grande e riprendono la via del canale.

Anche Kusama procede verso una pratica maggiormente performativa e dall'interagire con le persone passa a dipingerle, ancora all'aperto. L'esperienza della Biennale ha potenziato la sua audacia. L'anno successivo infatti - scrive il Sunday News del 13 agosto 1967 - «proclama Love Forever grazie al permesso del Parks Department di New York»: chi va in Washington Square, a Tompkins Square o a Central Park lei «lo punteggia di» pallini, intitolando appunto Love Forever l'azione. Quando si presenta, inizia a dipingere su rotoli di carta, poi esegue dei cerchiolini bianchi su dei cavalli e infine 'pittura' la gente (aveva già messo in atto una sessione di questa body painting in giugno, partecipando a una open air exhibition, «Artsy» a Woodstock), che presta gambe, braccia e torace. Da questa esperienza ha iniziato a definire tali sessioni Body Festivals e ha composto un Manifesto (forse memore dei vari manifesti degli Spazialisti dell'amico Lucio Fontana) in cui protesta contro la guerra e reclama che è stata dimenticata la bellezza del corpo, che è invece tutto ciò che possediamo veramente e afferma: «we all need pleasure in our forever now. Our painted bodies reflect and will continue to hold the psychedelic colors. We are but lonely spirits of light in

20 L.M., «Lettere da Venezia. Decadenza dell“'happening'», Giornale del Mattino, 10 luglio 1966. Cf. anche «A mezzanotte sotto i riflettori. L'happening a Rialto si è concluso con un bagno», Il Gazzettino, 19 giugno 1966; "Su gondola 'happening' musica ultramoderna», Oggi a Venezia, 18 luglio 1966; Jeanne Molli, «Beauty of Tediousness Performed. Joan of Arc of New Music Leads Happening in Rome», New York Herald Tribune (edizione di Parigi), July 51966. 
our trip through the happening of life». ${ }^{21}$ Il quotidiano segnala come avesse già tenuto l'anno precedente una «outdoor exhibit in Venice» con The Garden of Narcissus, in cui aveva iniziato a vendere le millecinquecento sfere argentate a due dollari l'una: «the balls reflected like mirrors», proprio come era composto di specchi, sei pareti più soffitto e pavimento, Kusama's Peep Show. Endless Love Show (1966) che nel 1967 si evolve nella versione The Infinity Mirror, che è in vendita per 1.500 dollari. ${ }^{22}$

\section{Bibliografia}

Bianchi, Giovanni (2006). Un cavallino come logo. Venezia: Edizioni del Cavallino. Broude, Norma; Garrard, Mary D. (1982). Feminism and Art History. Questioning the Litany. New York: Harper and Row.

Broude, Norma; Garrard, Mary D. (eds) (1992). The Expanding Discourse. Feminism and Art History. New York: Icon Harper Collins.

Brown, Gordon (1966). «Yayoi Kusama». D’Ars Agency, 7(1-2), 140.

Carlo Cardazzo. Una nuova visione dell'arte (2009). Carlo Cardazzo. Una nuova visione dell'arte = Catalogo della mostra a cura di Luca Massimo Barbero (Venezia, Collezione Peggy Guggenheim, 1 novembre 2008-9 febbraio 2009). Milano: Electa.

Carlo Scarpa. Disegni di Carlo Scarpa (2000). Carlo Scarpa. Disegni di Carlo Scarpa per la Biennale di Venezia. Architetture e progetti, 1948-1968= Catalogo della mostra (Venezia, Padiglione Venezia, 2002; Roma, MAXXI 2002). Roma: Gangemi.

Dell'Acqua, Antonio (1966). «Introduzione». Biennale 33 1966, 29.

Duboÿ, Philippe (2016). Carlo Scarpa. L'arte di esporre. Milano: Johan \& Levi.

Galleria del Cavallino. Mostre 1966 (1966). Galleria del Cavallino. Mostre 1966. Venezia: Edizioni del Cavallino.

Hess, Thomas B.; Nochlin, Linda (1972). Woman as Sex Object. Studies in Erotic Art. 1730-1970. London: Allen Lane

Menna, Filiberto (1966). «Bruno Munari». Biennale 33 1966, 87-8.

Nochlin, Linda (1988). Women, Art, and Power. New York: Harper and Row.

Parker, Rozsika; Pollock, Griselda (1981). Old Mistresses: Women, Art and Ideology. London: Routledge and Kegan Paul.

Perry, Gill (ed.) (1999). Gender Art. New Haven; London: Yale University Press. Kusama, Yayoi (2011). Infinity Net. The Autobiography of Yayoi Kusama. Transl. by Ralph McCarthy. London: Tate Publishing.

Kusama, Yayoi (1966). «Autografi: Yayoi Kusama». D’Ars, 5, 82.

Lanzarini, Orietta (2003). «Gli anni '60. Le Biennali dal 1962 al 1966». Carlo Scarpa. L'architetto e le arti. Gli anni della Biennale di Venezia 1948-1972. Venezia: Marsilio Editori, 189-210.

21 Alfred Carl, «Call Her Dotty», Sunday News, August 131967.

22 Alfred Carl, «Call Her Dotty», Sunday News, August 13 1967: «The Garden of Narcissus: For an outdoor exhibit in Venice she bought 1,500 silvers balls from a plastics manifacturer and spread them on a lawn. The balls reflected like mirrors. So many visitors wanted one, she started selling them at two bucks each». 
Portinari, Stefania (2010). «Cherchez la femme: indagine sul potere dello sguardo». Il pittore e la modella. Da Canova a Picasso = Catalogo della mostra a cura di Nico Stringa (Treviso, Casa dei Carraresi, novembre 2010-marzo. 2011). Treviso: Canova, 107-22.

Portinari, Stefania (2017). «Materializzazioni del linguaggio alla Biennale di Venezia». Caldura, R. (a cura di), Verbovisioni. Una regione di segni in movimento. Pordenone: Mimesis, 38-60.

Portinari, Stefania (2018). Anni Settanta. La Biennale di Venezia. Venezia: Marsilio.

Restany, Pierre (1966). «La Biennale vista da Pierre Restany. L’Homo Ludens contro l'Homo Faber». Domus, 441, agosto, 37.

Sullivan, Marin (2015). «Reflective Acts and Mirrored Images. Yayoi Kusama's Narcissus Garden». History of Photography, 39(4), 405-23.

Tatehata, Akira (2000). «In Conversation with Yayoi Kusama». Hoptman, Laura; Tatehata, Akira; Kelterman, Udo, Yayoi Kusama. London: Phaidon, 19-27.

Tatehata, Akira (2009). «Reconsidering Yayoi Kusama». Yayoi Kusama: I Want to Live Forever. Milano: Federico Motta, 20-55.

Yamamura, Midori (2009). «Re-Viewing Kusama, 1950-1975: Biography of Things». Yayoi Kusama. Mirrored Years = Catalogo della mostra a cura di Franck Gautherot, Jaap Guldemond, Seungduk Kim (Rotterdam, Museum Boijmans Van Beuningen, 23 August-19 October 2008; Sydney, Museum of Contemporary Art, 5 February-8 June 2009; Wellington, City Gallery, 4 September-29 November 2009). Rotterdam: Museum Boijmans Van Beuningen, 66-113.

Yayoi Kusama (1966). Yayoi Kusama = Catalogo della mostra (Milano, Galleria Naviglio 2, 26 gennaio-9 febbraio 1966). Galleria del Naviglio: Milano.

Yoshimoto, Midori (2005). Into Performance. Japanese Women Artis in New York. New Brunswick: Rutgers University Press.

Zero Avantgarde (1965). Zero Avantgarde = Catalogo della mostra (Venezia, Galleria del Cavallino, 4-14 maggio 1965). Venezia: Edizioni del Cavallino. 


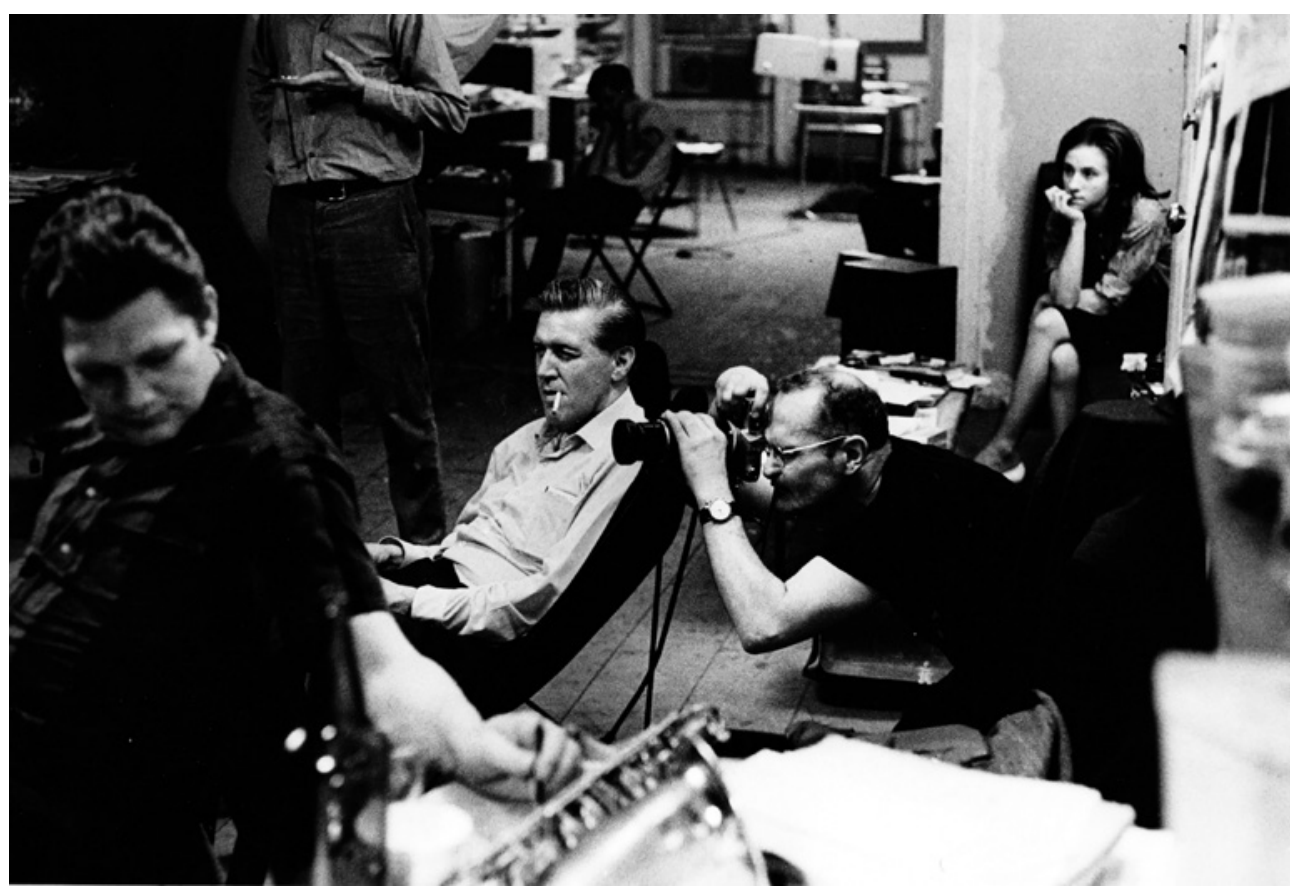

Figura 1 Paolo Monti, William Eugene Smith, Romeo Martinez e Stan Getz nello studio di Gene Smith negli Stati Uniti, 1961. Courtesy Archivio Romeo Martinez 


\title{
Il gioco di nessuno La forma biennale tra Venezia e Parigi nella fotografia del dopoguerra fino agli anni Ottanta
}

\section{Francesca Dolzani}

\begin{abstract}
The purpose of this article is to start mapping the trajectories of artistic dispositives, exhibiting formats and reciprocal cultural influences across Europe since its economic and political foundation in the 1950s, and up until the 1980s. Lines are drawn between Venice and Paris, two of the most visually identified cities on the continent, to explore the contribution given by photography and Biennales towards a European self-representation.
\end{abstract}

Keywords Biennale Photo-Cinéma. Biennale della Fotografia Venezia. Venezia '79. Mois de la Photo.

Nel clima di tensione internazionale dei primi anni Cinquanta e con il delinearsi di una politica dei blocchi si moltiplicano gli appelli al dialogo da parte degli intellettuali. Nel 1946, dalla constatazione che per la costruzione di una pace duratura non fossero sufficienti accordi politici ed economici, era nata a Parigi l'Organizzazione delle Nazioni Unite per l'Educazione, la Scienza e la Cultura (UNESCO), nel cui atto costitutivo si legge che «l'ignoranza dei reciproci modi di vivere è stata la causa, nella storia dell'umanità, della diffidenza e della sfiducia tra i popoli, differenze che hanno troppo spesso portato alla guerra». In una fase in cui lo slancio del pensiero federalista europeo si affie-

Questo saggio ha origine dalla partecipazione al convegno Storie della Biennale di Venezia, a cura di Nico Stringa e Stefania Portinari (Venezia, Università Ca' Foscari Venezia, Ca' Dolfin, 6-7 dicembre 2016). 
voliva a causa della profonda crisi economica e politica del dopoguerra, il Congresso americano, dopo l'annuncio della dottrina Truman nel 1947, approvava una risoluzione in cui dichiarava di sostenere la creazione degli Stati Uniti d'Europa e le iniziative per l'integrazione. L'atto che sancisce il primo passo concreto in questa direzione è la creazione della Comunità europea del Carbone e dell'Acciaio, proposta dal ministro degli Esteri francese Robert Schuman nel maggio 1950. L'ispirazione funzionalista di questo primo passo verso l'integrazione trova un completamento ideale in iniziative culturali che incoraggiano un sentire europeo comune (cf. Mammarella, Cacace 2018).

A distanza di pochi giorni si riunisce a Venezia l'assemblea costitutiva della Société Européenne de Culture, fondata da Umberto Campagnolo su ispirazione delle Rencontres Internationales che si erano svolte a Ginevra nel 1946 per discutere dell'esprit européen (Esposito 2016, 199). Accanto al filosofo italiano, intellettuali e studiosi provenienti da orizzonti e Paesi diversi: Julien Benda che aveva firmato nel 1933 un Discours à la nation européenne, Thomas Mann, autore dell'Avertissement à l'Europe nel 1937, Julian Huxley, primo direttore dell'UNESCO e ancora Federico Chabod, Ignazio Silone, Diego Valeri, Giuseppe Ungaretti, André Breton, Marc Chagall, Louis Jouvet e Henri Matisse che riceve nello stesso anno il Gran Premio per la pittura all'Esposizione Internazionale d'Arte, per menzionare solo alcuni dei presenti. La Société de Culture si appoggia, come prevede il suo statuto, all'Ente autonomo La Biennale. Dopo l'interruzione della guerra, la prima Esposizione d'Arte si è tenuta nel 1948: Rodolfo Pallucchini, il segretario generale, aveva organizzato una mostra sugli Impressionisti con Roberto Longhi e invitato numerosi artisti attivi sulla scena francese contemporanea.

I primi anni Cinquanta sono caratterizzati da vivaci scambi culturali tra Parigi e Venezia. L'editrice italo-francese Daria Lapauze Guarnati - che fin dai primi anni Quaranta collaborava con Gio Ponti, Curzio Malaparte e con lo stesso Pallucchini - nel 1953 pubblica in un volume fotografico intitolato Immagini di Venezia le fotografie di Ferruccio Leiss, precedute da un'introduzione di Jean Cocteau e Filippo de Pisis (cf. Zannier 1979, 2005). La rivista fotografica svizzera Camera parla di una vera e propria «scuola veneziana» di fotografia, grazie all'opera di Ferruccio Leiss, Gino Bolognini e dei più giovani Fulvio Roiter, Paolo Monti, Gianni Berengo Gardin, Toni del Tin, membri del circolo fotografico La Gondola, attivo nella ricerca di un confronto con la fotografia francese, attraverso esposizioni organizzate dal 1954 con il circolo parigino Les 30×40 (Monti 1980). Fulvio Roiter pubblica per la Guilde du Livre di Losanna Venise à fleurs d'eau nel 1954 e l'anno successivo Ombrie Terre de Saint François, che gli vale un Premio Nadar a Parigi nel 1956.

La fotografia gode in questi anni di un'industria in forte espansione e di un riconoscimento sempre maggiore grazie alla stampa e 
all'editoria. Con la ripresa dell'attività industriale si inaugurano in Europa fiere e grandi esposizioni, prendendo a modello quelle organizzate dal MoMA di New York fin dagli anni Trenta: in Germania Photokina nel 1950 e le esposizioni curate da Otto Steinert; in Svizzera la grande mostra fotografica di Lucerna del 1952 e la fiera Photo-Ciné a Losanna nel 1953. Nel 1955 anche Parigi inaugura una sua fiera-esposizione internazionale, che chiama Biennale de la Photo e du Cinéma. Jean Adhémar, conservatore del Cabinet des Estampes della Bibliothèque Nationale, nel presentarla evoca l'Esposizione Universale del 1867. Il Grand Palais ospita nel maggio 1955 materiali fotografici e immagini provenienti da quaranta Paesi e un grande numero di esposizioni e seminari vengono organizzati nei musei della capitale francese. «La Biennale di Parigi celebrerà gli immensi progressi tecnologici raggiunti dopo la guerra, e il ruolo essenziale dell'immagine nella sua forma fotografica, trasformando le nostre vite e l'idea stessa dell'umanità. Ė opportuno ricordare le parole del pittore Alfred Stevens: 'L'invenzione della fotografia ha operato nell'arte una rivoluzione pari a quella dell'invenzione della ferrovia per l'industria'» (Adhémar 1955). Nel fornire una lista degli autori presenti, la direzione evidenzia come lo scopo fosse quello di fornire una panoramica delle possibilità offerte dalla fotografia, grazie alle ultime novità tecnologiche presenti al Grand Palais; l'assenza di un catalogo della manifestazione viene giustificata con la volontà di dare al visitatore una visione d'insieme e non «le opere classificate per autore» («Album officiel» 1955). La rivista PhotoMonde dedica alla Biennale di Parigi, nel mese di maggio, un album al quale Paul Sonthonnax, responsabile della sezione artistica e culturale della manifestazione, contribuisce illustrando i criteri di selezione e allestimento. Accanto ai fotoamatori il comitato di selezione ha scelto dei fotografi professionisti, le cui immagini sono state raggruppate per temi, categorie o per serie (Sonthonnax 1955). Fanno parte della rassegna le esposizioni fotografiche curate dall'ONU, dall'UNESCO, da Subjektive Fotografie, e dalla FIAP (Fédération Internationale d'Art Photographique), che riunisce, accanto a quelle della Société Française de Photographie, immagini provenienti dalla Royal Photographic Society of Great Britain e dalla Photographic Society of America. Tra i partecipanti anche i membri dell'agenzia Magnum: da Robert Capa a David Seymour, da Ernst Haas a Henri Cartier-Bresson, che in questo stesso anno pubblica un libro intitolato Les Européens. I fotografi più noti del momento sono presenti, tuttavia, non nella loro qualità di autori.

In collaborazione con l'UNESCO, la Biennale de la Photo et du Cinéma organizza gli Incontri internazionali sul ruolo dell'immagine nella civiltà contemporanea, dove sei commissioni presiedute da esperti internazionali affrontano tematiche legate all'immagine fissa e in movimento. Un gruppo di studio coordinato da Vasco Ronchi, 
direttore dell'Istituto di Ottica di Firenze, analizza gli aspetti psicofisiologici delle immagini fotografiche e cinematografiche. Un altro studia gli effetti sociologici e di propaganda nell'utilizzo della fotografia e del cinema ed è presieduto da Edward Steichen, direttore del dipartimento di fotografia del MoMA di New York dal 1947. Originario del Lussemburgo ma cresciuto negli Stati Uniti, aveva trascorso molto tempo in Europa, a Parigi in particolare, fin dai primi anni del Novecento. La sua conoscenza dell'arte e della fotografia dai due lati dell'oceano gli aveva permesso di svolgere un'interessante opera di mediazione culturale tra i due continenti. Il piano Marshall, accanto all'obiettivo del risanamento finanziario dell'Europa, aspirava anche alla diffusione delle idee del giovane capitalismo americano. Gli scambi culturali promossi dal MoMA e dal Dipartimento di Stato sono strumenti diplomatici privilegiati. Nel caso del MoMA, l'attenzione che dedica alla fotografia e al cinema diventano la cifra della modernità del museo rispetto alle istituzioni europee, dove queste discipline ancora non sono ammesse (Barrère 2007). Nel 1955 il museo americano presenta al Musée national d'art moderne di Parigi un'esposizione intitolata 50 ans d'art aux États-Unis, nella quale accanto all'Espressionismo astratto, manifestazione propriamente 'americana' in ambito artistico, Steichen cura una sezione fotografica, in cui nella scelta delle opere rinuncia «alla funzione di testimone e di registrazione dell'immagine, per celebrare esclusivamente le possibilità creative ed espressive del mezzo» (Barrère 2007, 47). Nello stesso 1955, Edward Steichen è anche il curatore della celebre, quanto controversa, mostra The Family of Man inaugurata a New York, e che negli anni successivi circolerà in buona parte del globo, con le sue 503 immagini scattate da 273 fotografi di 68 Paesi, selezionate tra 2 milioni di fotografie spedite al museo nell'arco di tre anni (Edward Steichen, 2007) (Back, Schmidt-Lisenhoff 2004). La formula sintetica con cui si riassume la natura di questa esposizione faraonica è eloquente, con lo straordinario numero dei Paesi partecipanti ha l'ambizione di esprimere un punto di vista globale. Tra il 1952 e il 1956 il MoMA invia all'estero trenta esposizioni, che a loro volta danno vita a circa settanta mostre presentate in Europa e Asia, inclusa Venezia con l'Esposizione Internazionale d'Arte, grazie a un finanziamento di 625.000 dollari del Rockefeller Brothers Fund. ${ }^{1}$ Le immagini selezionate per la mostra The Family of Man parlano della grande famiglia umana, attraverso momenti e situazioni che il curatore vorrebbe universali. L'allestimento cinematografico nella varietà dei formati e la grande qualità dei materiali fotografici giustificano il successo planetario riscosso dall'esposizione, sebbene non siano mancate le

1 International Activities of The Museum of Modern Art 1952-1956, RdH Papers, VI.71 MoMA Archives, New York, in Barrère 207, 60. 


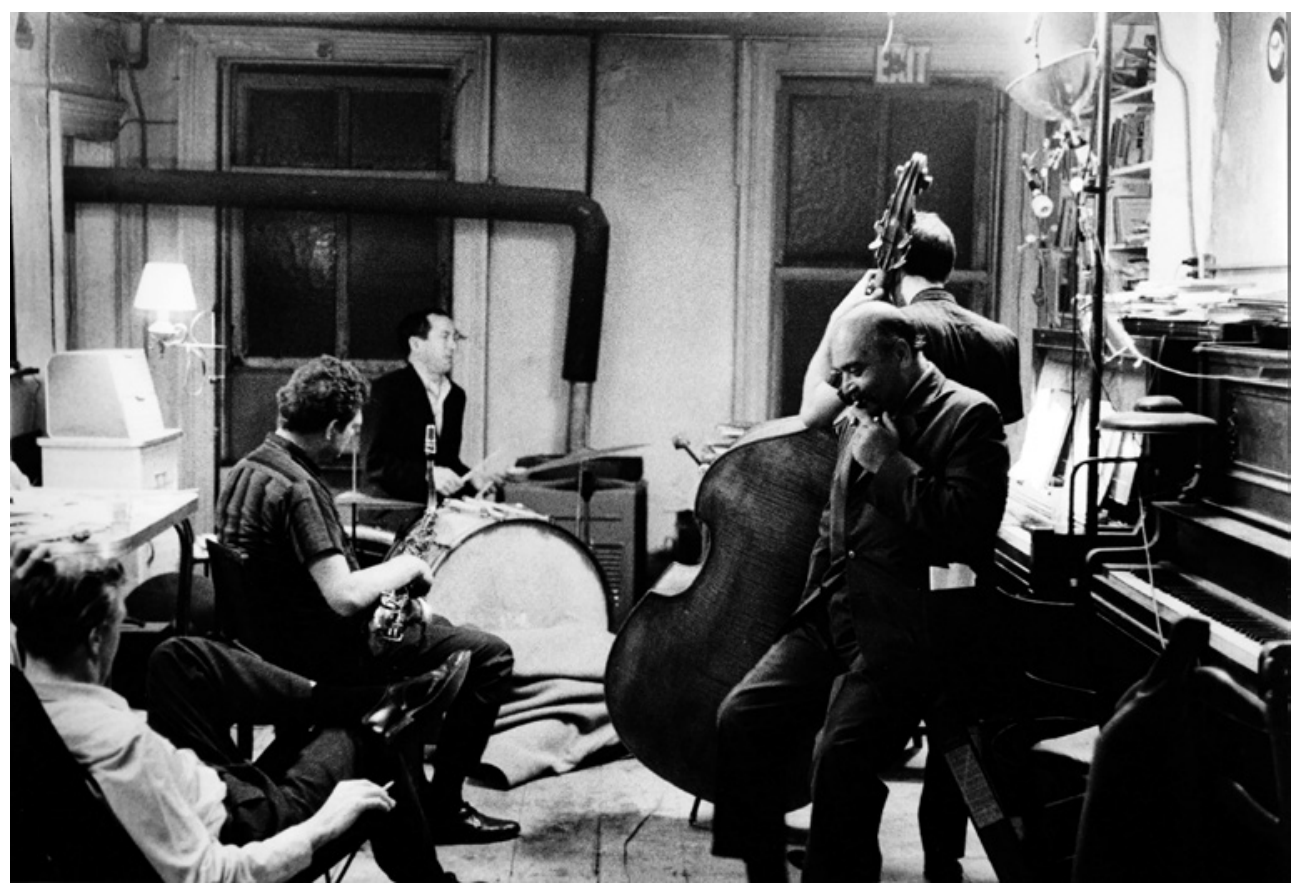

Figura 2 Paolo Monti, William Eugene Smith, Romeo Martinez e Stan Getz nello studio di Gene Smith negli Stati Uniti, 1961. Courtesy Archivio Romeo Martinez

critiche. Celebre è quella di Roland Barthes, espressione di un più generale sentimento anti-americano da parte francese in questo periodo, che rifiuta la mitizzazione astratta delle vicende umane, nascita e morte, guerra e pace estrapolati dal loro contesto storico, e ancor più l'idea di una idillica comunità umana (cf. Barthes 1970). Ciononostante, la dimensione popolare della fotografia riesce a comunicare il volto 'democratico' dell'America, restituendo l'immagine di un Paese libero, da contrapporre all'orbita comunista, in una fase politicamente delicata, dopo il naufragio del Trattato della Comunità europea di difesa, fortemente voluto dagli Stati Uniti (cf. Mammarella, Cacace 2018). Per Romeo Martinez, direttore della rivista svizzera Camera, che risiede a Parigi e ha partecipato agli incontri organizzati dalla Biennale de la Photo et du Cinéma e dall'UNESCO, questa esposizione pone la questione del ruolo del curatore e della sua responsabilità verso il pubblico (Martinez 1955).

Alla Biennale di Parigi accanto a Mario De Biasi, Ferruccio Ferroni, Bruno Cot, Arrigo Orsi, Giulio Parmiani, Antonio Persico, Mario Finocchiaro, per menzionare solo i più noti, hanno esposto i loro lavori anche i fotografi della 'scuola veneziana' Fulvio Roiter, Paolo 
Monti e Toni del Tin, che nel 1957, con la collaborazione di Camera e la direzione artistica del suo direttore Romeo Martinez, contribuiscono alla creazione di una Biennale Internazionale della Fotografia a Venezia. Il Comune partecipa all'organizzazione della manifestazione, con l'Ente provinciale del Turismo, il circolo fotografico La Gondola e il CCF di Luigi Crocenzi. Con l'Esposizione Internazionale d'Arte e la Mostra del Cinema, che hanno ormai raggiunto prestigio e notorietà internazionali, Venezia sembra essere il luogo ideale per ospitare una rassegna che aspira alla legittimazione culturale della fotografia, o più precisamente dei suoi autori. Proprio questo elemento definisce la singolarità del progetto rispetto ad altri di impostazione simile. Se, infatti, le mostre cui si è fatto riferimento trattavano di immagini fotografiche secondo tematiche specifiche o messaggi politici e sociali, in cui quindi le fotografie servivano a illustrare un'idea del curatore o dell'ente organizzatore, la Biennale della Fotografia di Venezia si concentra sugli autori e sui contesti professionali, la carta stampata in particolare, nei quali gli autori trovano espressione. Un ulteriore elemento di innovazione risiede nella modalità di selezione a invito, marcando una differenza fondamentale rispetto alla pratica espositiva della fotografia amatoriale, caratterizzata dal libero invio di materiali successivamente selezionati da una commissione, il criterio utilizzato anche da Edward Steichen per la mostra The Family of Man. Nel regolamento della Biennale Internazionale della fotografia di Venezia si specifica che "parteciperanno solo i fotografi invitati dal Comitato di organizzazione», che «la manifestazione ha un carattere esclusivamente artistico e culturale», prendendo quindi le distanze dalle rassegne contemporanee che invece associavano alla fotografia anche l'aspetto industriale dei materiali. Si preannuncia la possibilità di acquistare alcune fotografie a scopi non commerciali, nel tentativo di incoraggiare un collezionismo fotografico pressoché assente in Europa. Attraverso la Biennale di Fotografia Martinez aspira a modificare la percezione della fotografia, riconoscendo il suo valore di arte plastica, con «diritto di cittadinanza [...] accanto alla pittura, alla scultura, alla musica e al cinema, nel calendario regolare di queste manifestazioni veneziane» (Martinez 1957).

La prima Biennale fotografica si tiene nella primavera del 1957 nell'Ala Napoleonica del Museo Correr e in parte a Ca' Giustinian e presenta la fotografia europea, gli aspetti più rappresentativi del reportage fotografico di Magnum e della rivista Vogue, straordinaria illustrazione della tecnica e dell'estetica della fotografia di moda contemporanea. Il mondo della fotografia internazionale arriva a Venezia per i giorni dell'inaugurazione. La prima edizione esplora il campo della fotografia europeo, due mesi dopo la firma dei Trattati di Roma, che sanciscono la nascita della Comunità economica europea. Nel 1959 è la volta della fotografia extra-europea, giapponese e americana e nel 1961 Martinez organizza per la terza Biennale una 
grande retrospettiva su Robert Capa. Ogni edizione approfondisce il lavoro di singoli autori attraverso l'allestimento di mostre personali: vi si celebrano Man Ray, Albert Renger-Patzsch, Arnold Newman, e il 1963 segna l'inizio della riscoperta di André Kertész, caduto in un lungo oblio dopo il trasferimento da Parigi negli Stati Uniti. L'esperienza delle Biennali della Fotografia si conclude nel 1965: la grande alluvione che devasta Firenze e il centro Italia nel 1966 colpisce anche Venezia e il Comune, dopo mesi di incertezza, decide di annullare la Biennale fotografica del 1967 (cf. Dolzani 2013).

La fine degli anni Sessanta e gli anni Settanta rappresentano il momento di piena assimilazione del linguaggio fotografico nei circuiti dell'arte contemporanea. Numerose pratiche artistiche ricorrono al mezzo fotografico, che inizia a essere sempre più presente nelle esposizioni d'arte, e uno dei primi esempi è la mostra alla Kusthalle di Berna del 1969, When Attitudes Become Form, curata da Harald Szeemann. Alla stessa Esposizione Internazionale d'Arte di Venezia il Padiglione degli Stati Uniti allestisce nel 1972 una mostra di Diane Arbus e Franco Vaccari presenta l'Esposizione in tempo reale n.4: lascia su queste pareti una traccia fotografica del tuo passaggio. Se negli Stati Uniti l'ingresso della fotografia nei musei risale agli anni Trenta, in Europa il processo di istituzionalizzazione inizia negli anni Settanta.

In Francia nel 1970 hanno inizio i Rencontres de la Photographie di Arles, prendendo a modello i festival estivi di teatro. Dal 1975 il festival inizia a ricevere sovvenzioni dallo Stato, che rapidamente passano da 40.000 franchi iniziali a 1 milione nel 1980. Il processo di istituzionalizzazione della manifestazione si compie definitivamente nel 1979, quando il ministro della cultura Jean-Philippe Lecat, interviene alla prima serata pubblica con una conferenza stampa. Secondo Jean-Luc Monterosso, la tendenza dei musei ad assimilare la fotografia alle arti plastiche, relegherebbe il medium al rango di arte minore, da cui la necessità di concepire delle istituzioni specifiche per la fotografia (Morel 2006, 130). L'associazione Paris Audiovisuel viene creata nel 1978 con il supporto del Comune di Parigi, dopo la realizzazione del Centro Pompidou, e nel 1996 confluisce nella Maison Européenne de la Photo, il primo museo interamente dedicato alla fotografia a Parigi, di cui Jean-Luc Monterosso è stato direttore.

In Italia sono l'Istituto Centrale per il Catalogo e la Documentazione, nel quale confluisce nel 1975 il Gabinetto Fotografico Nazionale risalente al 1895, e l'Istituto Nazionale per la Grafica le istituzioni preposte alla conservazione del patrimonio fotografico, sebbene in questa fase ancora poco interessate alla creazione contemporanea. A Venezia l'apertura del presidente della Biennale Carlo Ripa di Meana alla fotografia non è seguita da una progettualità a lungo termine. Luigi Carluccio, che aveva curato nel 1973 a Torino la mostra Combattimento per un'immagine con Daniela Palazzoli, dopo la no- 
mina a curatore del settore Arti Visive organizza nel 1978 una mostra fotografica dal titolo L'immagine provocata, come sezione parallela della 38. Esposizione Internazionale d'Arte. È invece il Comune di Venezia a impegnarsi l'anno successivo nell'organizzazione di una delle manifestazioni più significative - e come tale oggetto di molte critiche - mai dedicate alla fotografia in Italia: Venezia '79 - La Fotografia. Definita una «grande festa della fotografia», l'idea era nata dal direttore dell'International Center of Photography, Cornell Capa, che aveva visitato la città in occasione della retrospettiva dedicata al fratello Robert alla Biennale della Fotografia del 1961. In collaborazione con il Comune e l'UNESCO, Capa organizza una rassegna di proporzioni inedite: da giugno a settembre 25 esposizioni fotografiche si accompagnano a seminari e workshop con celebri esponenti della fotografia internazionale. Cornell Capa con l'ICP si occupa della direzione artistica dell'evento e delle mostre internazionali. Un comitato scientifico italiano, nel quale figurano Daniela Palazzoli e Italo Zannier, è incaricato invece delle esposizioni che hanno come oggetto la fotografia italiana. Le critiche rivolte alla manifestazione riguardano la forte presenza della fotografia americana, a scapito di quella europea e italiana in particolare, tuttavia non si può trascurare l'attenzione che Venezia '79 ha catalizzato intorno alla fotografia, stimolando progetti editoriali ed espositivi, nell'anno in cui a Modena si tiene anche il convegno La fotografia come bene culturale, che per la prima volta pone la questione della tutela, della conservazione e dello studio della fotografia.

Come recita il comunicato stampa, questa iniziativa si inserisce in una 'linea di politica culturale' del Comune di Venezia, che ha dato avvio a un tentativo di valorizzazione e riscoperta della fotografia organizzando delle mostre a Palazzo Fortuny, che negli anni successivi avranno un seguito grazie al Centro di Documentazione con sede nel museo, che fino alla fine degli anni Novanta organizzerà circa settanta mostre fotografiche. Le voci critiche in ambito italiano sono riprese dalla recensione di Hervé Guibert su Le Monde, che scrive: «Non c'è la Biennale quest'anno, ma Venezia festeggia la fotografia tutta l'estate, sontuosamente, con molto respiro, mezzi e intelligenza». La spesa per l'organizzazione dell'evento si aggira tra i $700 \mathrm{mi}$ lioni di lire e il miliardo, e le polemiche non mancano, in particolare, scrive Guibert, sono assenti le riviste fotografiche italiane, che non hanno apprezzato che Venezia '79 fosse organizzato dagli americani. E prosegue: «Questo evento, che è senza dubbio la più fenomenale celebrazione che la fotografia abbia mai conosciuto, non compete né fa impallidire i Rencontres internationales d'Arles, perché è un episodio puntuale, non potrà ripetersi, potrà al limite essere trasportato, e adattato, in altre città» (Guibert 1999, 132-6).

L'anno successivo, nel 1980, a Parigi si inaugura il Mois de la Photo. Jacques Chirac, allora sindaco della città, annuncia che la capita- 
le francese sarà nel mese di novembre anche la capitale della fotografia mondiale e si riferisce alla manifestazione come a una «festa dell'immagine». Il Mois de la Photo è organizzato dall'associazione Paris Audiovisuel, e dalla Direzione dell'Assessorato alla cultura della città di Parigi. Nel consiglio artistico quasi tutti i presenti hanno partecipato anche a Venezia '79: Robert Delpire, Agathe Gaillard, Pierre Gassmann, Romeo Martinez e Roger Therond. Alcune esposizioni di Venezia '79 sono riproposte a Parigi nel 1980, e così avverrà per altre esposizioni negli anni successivi. Jean-Luc Monterosso, delegato generale della manifestazione, presenta nell'«Introduzione» al catalogo il progetto, che avrà una cadenza biennale, e proclama che

Paris Audiovisuel si impegna a ingaggiare da oggi un combattimento permanente che ha come obiettivo quello di riportare la nostra capitale al ruolo di primo piano che occupava all'inizio del secolo. Il settore privato e quello semi-pubblico non hanno fatto mancare la loro collaborazione attiva al sostegno di questa idea guida, ciascuno persuaso che l'industria, allo stesso titolo della creazione fotografica, non è necessariamente condannata a restare al traino degli Stati Uniti. Su questo punto, il Mois de la Photo avrà anche il valore di un test per il futuro di questa scommessa: dipenderà dalla risposta popolare, in effetti, il ruolo che Parigi potrà svolgere nei prossimi vent'anni nell'ambito della fotografia, espressione viva della nostra cultura quotidiana.

Con la vittoria di François Mitterrand alle elezioni presidenziali francesi del 1981, si assiste, con le parole dello storico Pascal Ory, «alle professioni di fede ultra-culturaliste» delle principali personalità del nuovo governo, per il quale «il socialismo è innanzitutto un progetto culturale» (Ory 1984, in Morel 2006, 39). Jack Lang, ministro della cultura, ha come obiettivo l'integrazione nel campo culturale di discipline precedentemente poco considerate, tra le quali la fotografia, e la creazione di nuovi spazi di legittimazione. Il ministero incoraggia inoltre la collaborazione tra settori della cultura e dell'economia, e la fotografia, come fa notare Gaëlle Morel, rappresenta il «medium esemplare per questo progetto politico» (39).

Il Mois de la Photo, a cadenza biennale, è considerato un'esperienza originale che ha creato un vero e proprio fenomeno di emulazione nel mondo intero e questa manifestazione si colloca in una chiara linea di continuità rispetto a Venezia '79 - La Fotografia. Nel corso degli anni nel catalogo ufficiale si fa riferimento al Mois de la Photo con l'appellativo informale di 'biennale', fino alla formulazione impiegata dal presidente di Paris Audiovisuel e assessore alla cultura del Comune di Parigi Françoise de Panafieu nel 1990, che nel salutare la sesta edizione chiama la manifestazione «Biennale Internazionale». L'ormai trentennale manifestazione parigina, cui si è associata dal 
novembre 1997 Paris Photo - una delle più importanti fiere internazionali per il mercato delle gallerie - hanno fatto di Parigi, proprio come gli organizzatori auspicavano, una tappa obbligata per il mondo fotografico internazionale.

L'ambiziosa densità di Venezia '79 aveva individuato un punto di equilibrio tra le esigenze del mondo fotografico, da una parte, e della politica dall'altra. Come era accaduto nel 1895 per l'arte e nel 1932 per il cinema, anche per la fotografia Venezia ha saputo produrre, con le Biennali della Fotografia prima e Venezia '79 poi, un modello che rispondeva in maniera adeguata alle esigenze di visibilità e legittimazione del campo, un dispositivo che ancora oggi non ha esaurito la sua utilità. Nella modalità antagonistica e ricorsiva che è propria della molteplicità europea, il dialogo continuo e lo scambio di idee fanno «il gioco di nessuno, che è poi il vantaggio di tutti» (Bobbio [1955] 2005, XLIII).

\section{Bibliografia}

Adhémar, Jean (1955). «Présentation de la Biennale de la photo et du cinéma de Paris». Camera, 34(5), mai, 198.

«Album officiel» (1955). «Album officiel Biennale Photo-Cinéma». Photo-Monde, mai, 91.

Back, Jean; Schmidt-Lisenhoff, Viktoria (2004). The Family of Man 1955-2001. Humanismus and Postmodernism: A reappraisal of the Photo Exhibition by Edward Steichen. Marburg: Jonas Verlag.

Barrère, Laetitia (2007). «Influence culturelle? Les usages diplomatiques de la photographie américaine en France durant la guerre froide». Études Photographiques, 21, décembre, 45-61.

Barthes, Roland (1970). Mythologies. Paris: Editions du Seuil.

Bobbio, Norberto [1955] (2005). Politica e cultura. Torino: Einaudi.

Dolzani, Francesca (2013). Camera 1953/1964: gli anni di Romeo Martinez. Venezia: Edizioni MUVE.

Edward Steichen (2007). Edward Steichen. Une épopée photographique. Paris: Editions du Jeu de Paume.

Esposito, Roberto (2016). Da Fuori. Una filosofia per l'Europa. Torino: Einaudi.

Guibert, Hervé (1999). La Photo, inéluctablement. Paris: Gallimard.

Leiss, Ferruccio (1953). Immagini di Venezia. Milano: Daria Guarnati.

Mammarella, G.; Cacace P. (2018). Storia e politica dell'Unione europea. RomaBari: Laterza.

Marra, Claudio (1999). Fotografia e pittura nel Novecento. Una storia "senza combattimento". Milano: Bruno Mondadori.

Martinez, Romeo (1955). «Le message d'une exposition». Camera, 7(34), juillet, 343.

Martinez, Romeo (a cura di) (1957). I Mostra Internazionale Biennale della Fotografia. Venezia: Edizioni Biennale.

Fotografica, 1957. Testi introduttivi di Paolo Monti e Romeo Martinez

Mois de la Photographie. Parigi. Cataloghi generali delle rassegne 1980-19821984-1990. 
Francesca Dolzani La forma biennale tra Venezia e Parigi nella fotografia del dopoguerra fino agli anni Ottanta

Monti, Paolo (1980). 30 anni di fotografia a Venezia. Il Circolo Fotografico La Gondola 1948-78. Venezia: Marsilio.

Morel, Gaëlle (2006). Le photoreportage d'auteur. L'institution culturelle de la photographie en France depuis les années 70. Parigi: CRNS Editions.

Ory, Pascal (1984). «La politique du ministère Jack Lang: un premier bilan». The French Review, 56(1), October, 77-83.

Sonthonnax, Paul (1955). «L'image - Problèmes d'aujourd'hui et demain». Photo-Monde, mai, 81-6.

Steichen, Edward (ed.) (1955). The Family of Man = Exhibition catalogue. New York: The Museum of Modern Art.

Valtorta, Roberta (2008). Il pensiero dei fotografi. Un percorso nella storia della fotografia dalle origini a oggi. Milano: Bruno Mondadori.

Zannier, Italo (1979). Ferruccio Leiss Fotografo a Venezia. Milano: Electa.

Zannier, Italo (a cura di) (2005). Fotografia a Venezia nel Dopoguerra, da Ferruccio Leiss al circolo La Gondola. Firenze: Alinari. 


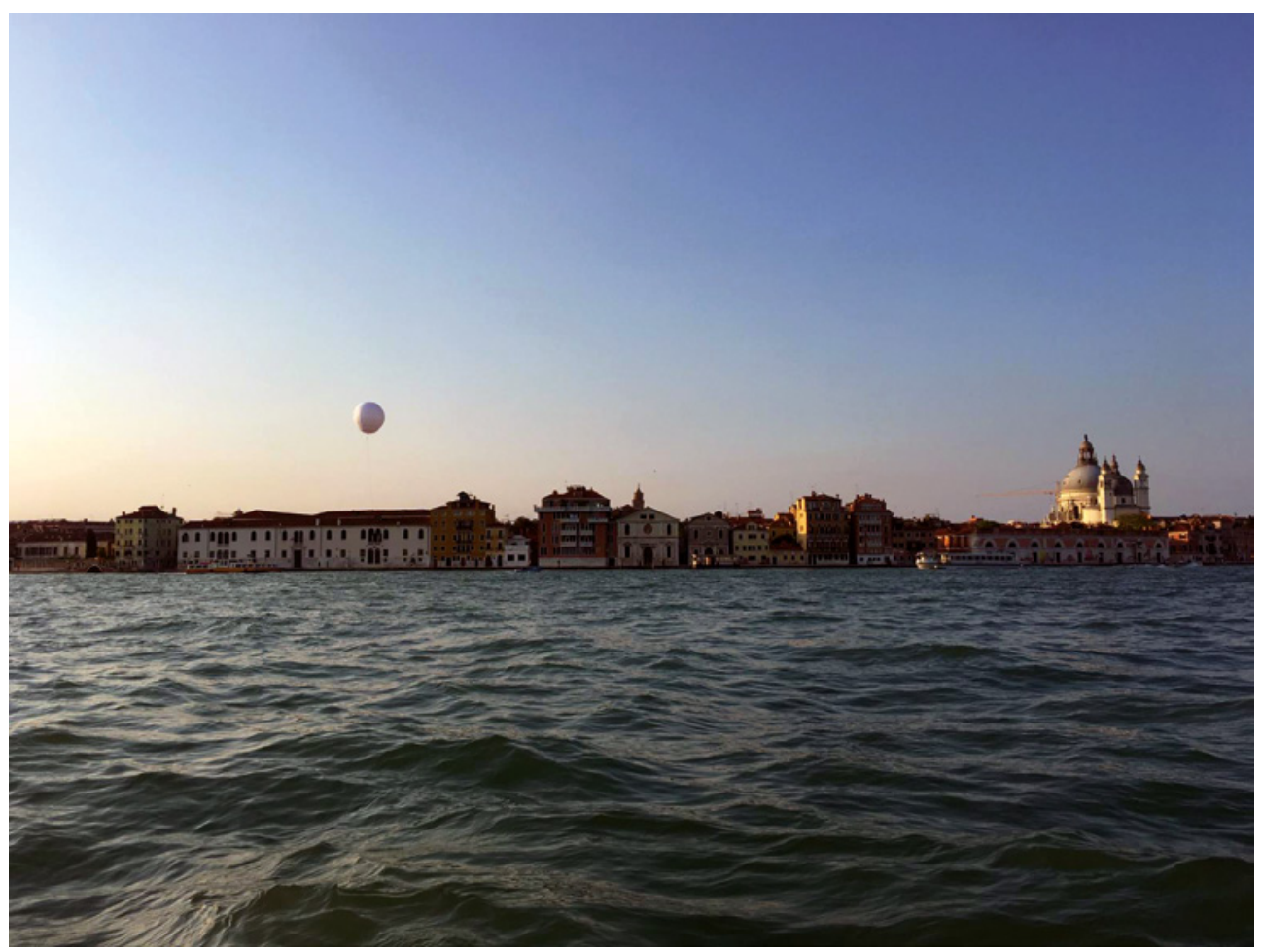

Venezia, veduta delle Zattere con i Magazzini del Sale, dove si trovava la prima sede dello spazio Aperto, nel 1980 


\title{
Jeff Koons alla XLIV Esposizione Internazionale d'Arte La Biennale di Venezia (1990)
}

Sabina Laura De Stefano

\begin{abstract}
In 1990, the thirty-five-year-old Jeff Koons (York, Pennsylvania, USA, 1955) officially debuted in Italy as participant artist of the 44th edition of Venice Biennale. He was invited to take part of the section called Aperto, which was dedicated to those young artists who had never exhibited at the Biennale. At that time Koons was not so famous and decided to present a disrespectful complex of works created in collaboration with the famous pornstar Cicciolina. Koons' choice grants him the attention of critics and press. Despite the critical rejections, Koons' participation at Venice Biennale and the interest of the media contributed to launch him on a successful international career.
\end{abstract}

Keywords Jeff Koons. Biennale 1990. Aperto'90. Scandal. Sculpture.

Jeff Koons (York, Pennsylvania, USA, 1955) è uno dei più celebri artisti contemporanei e, tra i viventi, uno dei più quotati: tanto che nel 2013 una delle sue opere più note, l'imponente scultura in acciaio inox Balloon Dog (Orange) (1994-2000), che evoca un palloncino a forma di cane, è stata battuta all'asta alla cifra record di 58,4 milioni di dollari. Al pari di una star cinematografica hollywoodiana, Koons si trova sovente al centro dell'attenzione mediatica internazionale e non è raro che le testate giornalistiche più importanti e le riviste specializzate in arte gli dedichino articoli a commento di suoi progetti più o meno scandalosi o kitsch o riportando le diatribe legali di cui si vede protagonista a causa delle accuse di plagio che gli sono state più volte mosse nel cor-

Il presente contributo prende avvio da: De Stefano, Sabina (2017). Jeff Koons e i rapporti con l'Italia: dalla Biennale di Venezia del 1990 a «Jeff Koons in Florence» (2015-2016) [tesi di laurea magistrale]. Relatore Stefania Portinari; correlatore Nico Stringa, a.a. 2016/2017. Venezia: Università Ca' Foscari Venezia.

Storie dell'arte contemporanea 4 | Atlante delle Biennali 1

ISSN 2704-9973

ISBN [ebook] 978-88-6969-366-3 | ISBN [print] 978-88-6969-367-0

Edizioni 
so degli anni. ${ }^{1}$ Koons è considerato un artista controverso e il dibattito intorno al personaggio è costantemente tenuto vivo. È anche uno dei creativi che rappresenta al meglio quello 'stile di vita americano' di cui il filosofo francese Jean Baudrillard tratta nel suo libro Amérique (1986), che analizza i valori della classe media statunitense impegnata a rincorrere l'autopromozione e a porre come punto focale della propria esistenza i beni di consumo che spesso idolatra e trasforma in feticci (Baudrillard 1986, 52-53). Koons con la sua arte esplicitamente neo-pop - una corrente artistica sorta proprio negli Stati Uniti nella seconda metà degli anni Ottanta e diffusasi in seguito in Europa e Italia (cf. Terraroli 2009, 344; Foster 1996, 115-120; Pancotto 2010, 96) - si rivolge in primis proprio al gusto statunitense, realizzando un vasto corpus di opere spesso ritenuto dal pubblico e dalla critica espressamente kitsch. Nonostante la vastità della produzione artistica di Koons e la sua longeva attività, questo testo si sofferma su un momento circoscritto della sua carriera: la partecipazione alla Biennale d'Arte di Venezia del 1990, che rappresenta il suo esordio artistico italiano. Nello specifico l'artista statunitense prende parte alla XLIV Esposizione Internazionale d'Arte La Biennale di Venezia invitato a far parte di una sezione della rassegna denominata Aperto, che è stata creata per la per la prima volta nella sede esterna dei Magazzini del Sale alle Zattere in occasione dell'edizione della Biennale del 1980, introdotta da Bonito Oliva e Harald Szeemann per dedicare uno spazio di rilievo ad artisti giovani e esordienti che non abbiano mai preso parte ad altre edizioni della rassegna e che verrà riproposta fino al 1993.

Aperto '90 si pone l'intento di esporre i lavori di artisti emergenti under 35 e presenta opere di Koons inedite e provocatorie tratte dalla serie Made in Heaven (1989-91), realizzate servendosi della collaborazione della sua compagna di allora, la pornostar ungherese Ilona Staller, in arte Cicciolina, che al tempo era molto nota, soprattutto in Italia: proprio la grande attenzione mediatica che si è sviluppata intorno alla sua presenza ha concorso a lanciare la carriera di Koons a livello internazionale (cf. «Aperto '90», Biennale 44 1990, 253-349). L'artista statunitense prenderà nuovamente parte alla Biennale d'Arte solo in un'altra occasione: la seconda e per ora ultima partecipazione è infatti il suo intervento alla XLVII edizione del 1997, invitato

1 Cf. Ruth La Ferla, «Art, and Handbags, for the People», The New York Times, 23 July 2014; Simone Cosimi, «Snapchat si dà all'arte: Jeff Koons in realtà aumentata», La Repubblica, 3 ottobre 2017; Mariacristina Ferraioli, «Jeff Koons firma per Louis Vuitton una collezione di borse ispirata a Monet», Artribune, 30 ottobre 2017. Koons è stato anche talora accusato di plagio, cf. Benjamin Sutton, «Jeff Koons Accused of Plagiarism in Paris, Again», Hyperallergic, 29 December 2014; Anonymous, «Jeff Koons sued for appropriating 1980s gin ad in art work sold for millions», The Guardian, 15 December 2015; Henry Samuel, «Jeff Koons accused of plagiarising iconic French advertising campaign for multi-million-puond sculpture», The Telegraph, 24 September 2018. 
da Germano Celant alla rassegna Futuro, Presente, Passato: 19671997, un'occasione in cui espone due opere della serie allora inedita Celebration (1994-2006). La presenza di Koons a quella Biennale però delude le attese di chi si aspettava una reiterazione del grande successo di sette anni prima (cf. De Stefano 2017, 70-9). Anche se la Biennale va naturalmente considerata in un contesto internazionale e i suoi riverberi giungono oltre l'ambito nazionale, queste sue due partecipazioni possono comunque essere considerate particolarmente interessanti anche nel novero delle sue mostre in Italia.

Nel 1992 Jeff Koons è uno dei protagonisti dell'epocale mostra collettiva itinerante «Post Human» - allestita al Fae Musée Contemporain Pully di Losanna, al Deste Foundation for Contemporary Art di Atene, al Deichtorhallen Hamburg di Amburgo e al Castello di Rivoli - a cui viene invitato dal curatore statunitense Jeffrey Deitch per esporre alcune opere della serie Made in Heaven con la quale si era fatto conoscere proprio in occasione della Biennale del 1990 (cf. Deitch 1992). Nel 2003 Achille Bonito Oliva è l'ideatore della prima mostra antologica di Koons in Italia tenutasi presso il Museo Archeologico di Napoli, che ospita una ricca selezione di opere dalla sua produzione dal 1981 al 2002 (cf. Codognato 2003). Koons in seguito intraprende un proficuo rapporto con la Fondazione Pinault di Venezia, tanto che dal 2006 al 2013 la sua presenza diviene pervasiva grazie a questo sodalizio. Il magnate francese François Pinault, che intende condividere con il pubblico una parte della sua vasta collezione d'arte contemporanea, riserva infatti a Koons un posto d'onore nell'organizzazione di esposizioni collettive ospitate dalle due sue sedi veneziane (cf. Gingeras 2006; Gingeras Bankowsky 2006; Gingeras 2009; Bourgeois 2011). Dal 24 settembre 2015 al 15 gennaio 2016 si è tenuto un ulteriore intervento artistico di Jeff Koons in Italia, a cura di Sergio Risaliti: Jeff Koons in Florence, in occasione del quale l'artista espone nel centro storico del capoluogo toscano due sculture che corteggiano un gusto molto kitsch: Pluto and Proserpina (201013) in acciaio inox con cromatura color oro in Piazza della Signoria e Gazing Ball (Barberini Faun) (2013) nella Sala dei Gigli in Palazzo Vecchio, che hanno creato un intenso dibattito (cf. Risaliti 2015).

Il successo di Koons in Italia è dunque indelebilmente legato a quel suo intervento alla XLIV Esposizione Internazionale d'Arte di Venezia, che rappresenta l'inizio di un rapporto molto particolare. Quella Biennale, che apre il 23 maggio 1990 con i tre giorni di vernice dedicati alla stampa, alla critica e ad altri 'addetti ai lavori' che anticipano l'inaugurazione ufficiale alla presenza del presidente del Consiglio Giulio Andreotti tenutasi il 27 maggio, ha come curatore Giovanni Carandente che intitola l'esposizione Dimensione Futuro.

Lo storico dell'arte, direttore del settore arti visive della Biennale dal 1988, intende rappresentare lo spirito di cambiamento e la propensione verso il futuro che a suo avviso caratterizza l'inizio dell'ul- 
timo decennio del XX secolo, come scrive in catalogo (Carandente $1990,16)$, sottolineando in particolare il rapporto che gli artisti mettono in atto con lo spazio:

L'artista e lo spazio [...] è l'altra possibile definizione che si può affidare a questa Biennale, come essa è venuta configurandosi, non solo in Italia, ma anche negli altri paesi del mondo, man mano che gli artisti prendevano contatto con lo spazio a loro assegnato. (Carandente 1990,16$)$

La strategia curatoriale di Carandente è la presentazione delle tendenze più attuali dell'arte contemporanea e intende dunque invitare giovani artisti operanti sulla scena internazionale, tralasciando le mostre retrospettive o tematiche che nel corso degli anni hanno caratterizzato le edizioni della manifestazione veneziana. ${ }^{2}$ Una delle più importanti sezioni della XLIV edizione della Biennale è considerata allora proprio Aperto '90: lo spazio assume un ruolo di interprete speciale dello spirito della Biennale, essendo la rassegna dedicata agli artisti emergenti.

Grande importanza riveste anche la rassegna «Ambiente Berlin», curata da Jörn Merkert, Ursula Prinz, Hermann Raum, Werner Schmalenbach e Carandente stesso, che riunisce e espone opere di artisti non solo di nazionalità tedesca per i quali Berlino sia stato un luogo vitale per le proprie creazioni artistiche, documentando la vivace situazione culturale della capitale tedesca (cf. Carandente 1990, 61), tanto più alla luce degli avvenimenti seguiti alla caduta del muro di Berlino, avvenuta il 9 novembre del 1989. Un'altra rassegna significativa della Biennale del 1990 è quella in omaggio allo scultore basco Eduardo Chillida (1924-2002), curata da Carandente allestendo a Ca' Pesaro le opere dell'artista che nel 1958 aveva ricevuto il Leone d'oro per la scultura (cf. Carandente 1990, 91). Per quanto riguarda la rappresentanza italiana, il Padiglione Italia è curato da Laura Cherubini, Flaminio Gualdoni e Lea Vergine e riceve un diffuso riscontro positivo in quanto ritenuto in grado di illustrare in modo diversificato il panorama artistico del paese sul finire degli anni Ottanta. In assonanza con lo spirito della Biennale, la maggior parte degli artisti scelti sono molto giovani come ad esempio: Davide Benati (1949), Nicola De Maria (1954), Giuseppe Gallo (1954), Alberto Garutti (1948), Franco Guerzoni (1948) e Marco Tirelli (1956), e si presentano con opere recenti caratterizzate dal pluralismo dei linguaggi e delle tecniche (cf. Cherubini, Gualdoni, Vergine 1990, 23-41). Per Luciano Caramel è proprio la rappresentanza italiana quella più viva alla Bien-

2 Pier Francesco Listri, «Il parere di Giovanni Carandente, responsabile delle arti figurative», Il Tempo, 21 marzo 1990. 
nale, grazie alla presenza di artisti impegnati, anche se con maggiore libertà e anticonvenzionalità, con le forme tradizionali della pittura e della scultura. ${ }^{3}$ I loro lavori colpiscono positivamente anche la giuria, infatti è Giovanni Anselmo (1934) ad aggiudicarsi il Leone d'oro per la pittura (cf. «La giuria», Biennale 44 1990, 9). Per gli altri Padiglioni nazionali, stampa e critica muovono un giudizio piuttosto unanime. Il Padiglione che riscontra il maggior successo, fin dal primo giorno identificato come il più promettente, è quello degli Stati Uniti, che risulta vincitore del Leone d'oro per la Miglior Partecipazione Nazionale (cf. «La giuria», Biennale 44 1990, 9). ${ }^{4}$ A rappresentanza degli Usa è stata scelta Jenny Holzer (1950), allora giovane artista americana di tendenze neo-concettuali (cf. Auping 1990, 212-17). Grande attenzione viene dimostrata anche nei confronti del Padiglione della Repubblica Federale di Germania dove vengono esposte foto realizzate dai coniugi Becker (Bernhard, 1931-2007 e Hilla, 1934-2015), vincitori del Leone d'oro per la scultura grazie alla plasticità dei loro scatti, ${ }^{5}$ e l'imponente l'installazione dell'artista Reinhard Mucha (1950), ricca di associazioni e simbolismi (cf. Klaus Bussmann 1990, 200-5). Il Padiglione inglese è interamente occupato dalla mostra monografica di Anish Kapoor (1954), che realizza installazioni in pietra arenaria, ardesia o fibra di vetro e che riceve il Premio Duemila come miglior artista esordiente (cf. Thomas McEvilley 1990, 156). Considerando la XLIV Biennale nel suo insieme, si può verificare come Carandente sia intenzionato a rispecchiare nella manifestazione la complessità della ricerca artistica del periodo presentando una varietà di opere molto ampia e eterogenea.

Già dai primi giorni di vernice giungono comunque anche le critiche. ${ }^{6}$ Alcuni articoli sono concordi nell'asserire che la Biennale non sia riuscita a mantenere le premesse, secondo le quali sarebbe dovuta essere un'esposizione internazionale «giovane, provocatoria, ricca

3 Luciano Caramel, «Ecco la Biennale '90 pochi quadri e molti audiovisivi», Il Giornale, 26 maggio 1990 .

4 Cf. la rassegna stampa del 1990 presso l'ASAC, tra cui in particolare: Virginia Baradel, «Alieni d'una terra morta», La Nuova Venezia, 24 maggio 1990; Luciano Caramel, «Ecco la Biennale '90 pochi quadri e molti audiovisivi», Il Giornale, 26 maggio 1990; Nello Forti Grazzini, «La Biennale del Revival», L’Unità, 26 maggio 1990; Manlio Onorato, «Biennale: inultile, scandalosa, lottizzata, caotica, però...», Nuova Vicenza, 6 giugno 1990. Sul Padiglione USA cf. «La giuria», Biennale 44 1990, 9.

5 Cf. Nello Forti Grazzini, «La Biennale del Revival», L’Unità, 26 maggio 1990; Andrew Renton, «La Biennale di Venezia», Flash Art Italia, 157, 1990, 117-18

6 Cf. Franco Miracco, «Il villaggio dell'arte che vive di frammenti più che di sostanza», La Nuova Venezia, 24 maggio 1990; Nello Forti Grazzini, «La Biennale del revival», L'Unità, 26 maggio 1990; Claudio Spadoni, «E l'arte finì al muro», Il Resto del Carlino, 26 maggio 1990; Luciano Ferraro, «Biennale, commedia dell'arte», La Nuova Ve nezia, 28 maggio 1990; Enrico Crispolti, «Clima di omologazione celebrata», La Gazzetta delle Arti, 5/6, estate 1990. 
di spunti, originale», ${ }^{7}$ come afferma il gallerista Lucio Amelio, ma a distanza di tempo si può constatare come quella Biennale fosse proprio lo specchio dell'arte postmoderna dell'epoca, che spesso guardava al passato facendo uso di revivalismi e di linguaggi eterogenei.

La Biennale chiude il 30 settembre con un risultato comunque decisamente poco soddisfacente, registrando «solo» centomila visitatori paganti, una soglia davvero bassa che la Biennale raggiunge in poche altre occasioni. ${ }^{8}$ Jeff Koons diventa però il personaggio più discusso di questa edizione. Aperto '90 occupa l'intero spazio alle Corderie dell'Arsenale, un luogo tradizionalmente deputato alla rassegna fin dall'edizione di Aperto '86, mettendo a punto un allestimento di notevoli dimensioni che per struttura si rifà a quello delle fiere d'arte, con la presenza di stand tutti di identica estensione. I curatori che si sono susseguiti nel corso delle diverse edizioni di Aperto si sono sempre prodigati per creare una mostra che potesse rappresentare le tendenze più innovative e internazionali, come ha sottolinea Renato Barilli (Barilli 1990, 255), che nel 1990 è uno dei curatori della sezione assieme a Bernard Blisténe, Stuart Morgan, Wenzel Jacob e Linda Shearer. Questa edizione è particolarmente significativa anche per l'introduzione di un nuovo fattore discriminante per la scelta degli artisti: per la prima volta in cinque edizioni viene, infatti, indicato un limite di età per poter partecipare alla rassegna, fissato a trentacinque anni. La volontà di Carandente a questo proposito è di garantire la presenza all'interno della Biennale di uno spazio davvero dedicato interamente ad «una selezione quanto mai varia e qualificata dei nuovi, giovani talenti» (Caradente 1990, 17). Nonostante il desiderio di privilegiare «artisti alla loro prima apparizione internazionale» si lascia ampio spazio anche ad artisti già conosciuti e «divulgati dai media» (Carandente 1990, 17), soprattutto nei loro rispettivi paesi, una decisione che non resta incolume dalle polemiche, come nel caso di Koons e di altri artisti statunitensi selezionati dalla curatrice Linda Shearer che, sul finire degli anni Ottanta, sono molto noti negli Stati Uniti ma decisamente meno in Italia e a livello internazionale. ${ }^{9}$

Linda Shearer giustifica la presenza di nomi famosi, riferendosi nello specifico a Koons e Ashley Bickerton, sostenendo che sono stati scelti perché la loro indagine artistica si sta orientando verso nuo-

7 Lucio Amelio, «La Biennale di Venezia 1990», Domus, luglio-agosto 1990.

8 Fabio Marangoni, «Centomila visitatori alla Biennale di Venezia», Il Gazzettino, 1 ottobre 1990

9 Cf. Anonimo, «Come gli americani tentato il bis alla biennale», Il Giornale dell'Arte, 1990, marzo; Giancarlo Politi, «Dialogo dei massimi sistemi», Flash Art Italia, 157, 1990, 172. In particolare sono meno noti gli artisti Ashley Bickerton (1959) e Cady Noland (1956). 
ve strade inedite e stimolanti. ${ }^{10}$ Anche Barilli replica alle polemiche e afferma che la decisione di non escludere dalla manifestazione Koons e altri artisti non definibili strettamente come emergenti è dipeso dal fatto che nel 1990 questi artisti sono ancora i punti cardine dell'universo artistico contemporaneo e in loro è individuabile l'espressione del gusto corrente..$^{11} \mathrm{Il}$ curatore, inoltre, si sofferma su quali siano le tendenze riscontrabili all'interno di Aperto '90, che in questa edizione è caratterizzato dalla notevole presenza di fotografie, video, strutture minimali, con la netta prevalenza di installazioni, come si può evincere dalle riproduzioni presenti nel catalogo generale della Biennale.

Secondo quanto affermato da Barilli, ad Aperto si possono individuare da un lato artisti che utilizzano mezzi espressivi «duri» come l'indagine fotografica e le scritte concettuali, dando manifestazione a esperienze artistiche che restano legate alle pratiche «fredde» del neoconcettualismo, ${ }^{12}$ dall'altro lato è presente un indirizzo che non deve essere sottovalutato: è una tendenza kitsch con cui è necessario confrontarsi senza cercare di evitarla a prescindere, che dà luogo ad opere che non hanno timore di risultare eccessive e ridondanti (Barilli 1990, 255), di cui sono autori in particolare Jeff Koons, il gruppo di creativi Plumcake - composto dagli artisti italiani Gianni Cella (1953), Romolo Pallotta (1954) e Claudio Ragni (1955) -, l'israeliano Izhar Patkin (1955), il belga Wim Delvoye (1965). Per lui l'apoteosi di questo orientamento è proprio il lavoro di Koons, che fa luce sull'importanza di «fare i conti con i depositi immani di kitsch accumulati nella nostra società» (Barilli 1990, 255).

All'interno della rassegna Aperto è quindi possibile identificare una radicata eterogeneità di espressioni linguistiche, per le quali Barilli propone una definizione applicabile alla maggior parte dei giovani che espongono alle Corderie: il Barocco Freddo. Questa espressione, che sembra nascere dall'unione di due contrari, viene spiegata dal commissario nel catalogo generale della Biennale:

Il Barocco è l'arte dell'eccesso, della complicazione, dell'abbondanza, e pertanto sembrerebbe doversi al connotato del 'caldo', all'esuberanza d'animo e di sentimenti, di un'organicità portata in qualche modo a dialogare con la natura. Invece il fatto di caratte-

10 Anonimo, «Linda Shearer: una storia di eroi e antieroi», Il Giornale dell'Arte, marzo 1990.

11 Renato Barilli, «Un novanta aperto ai giovani», Arte e Dossier, 47, giugno 1990.

12 Tra questi artisti troviamo gli statunitensi Stephen Prina (1954), Lorna Simpson (1960), Annette Lemieux (1957) e l'olandese Mirjam de Zeeuw (1959). Il concetto espresso da Barilli è valido anche per il gruppo di artisti italiani della cosiddetta Scuola di Piombino: Salvatore Falci (1950), Pino Modica (1952), Cesare Pietroiusti (1955) e Sandro Fontana (1955). 
rizzarlo con il 'freddo' significa che oggi questa operazione di accumulo per eccesso di deve condurre su elementi e oggetti forniti dal panorama urbano, artificiale, tecnologico della nostra attuale scena mondiale. [...] questa operazione di assemblaggio [...] invade di necessità la terza dimensione dell'ambiente. (Barilli 1990, 256)

Come accade sempre con i momenti più significativi della storia della Biennale, la rassegna riceve molte critiche: da chi disapprova la viscerale necessità di novità ricercata da questa edizione della Biennale e da Aperto in particolare a chi considera l'ambiente un «luna park dei giovani» o un «museo degli orrori», il cui emblema è proprio Jeff Koons. ${ }^{13}$

Nel dialogo tra l'editore Giancarlo Politi e la curatrice Helena Kontova pubblicato su «Flash Art», quest'ultima contesta il meccanismo attraverso cui vengono scelti gli artisti di Aperto, affermando che vi è un serio problema derivato dai compromessi che i curatori devono affrontare per arrivare alla rosa di artisti selezionati. ${ }^{14}$ Secondo la giornalista Maria Torrente, invece, Aperto '90 segue lo stesso modus operandi della Biennale, dove gli artisti peccano di mancanza di autenticità affidandosi al meccanismo dei rimandi, al ready-made e a un neoconcettualismo manieristico privo di sostanza. ${ }^{15}$ Tra questa profusione di giudizi negativi spicca invece il commento meno ostile di Enrico Crispolti che, in riferimento alla rassegna dei giovani, afferma che è individuabile una tendenza degli artisti partecipanti a elaborare opere basandosi sulla ricerca, dando luogo a proposte nate dalla volontà «di cogliere situazioni di attualità linguistica». ${ }^{16}$ Nonostante il suo parere, nel complesso la maggior parte degli interventi boccia però la rassegna, mettendo fortemente in dubbio la sua validità ed efficacia. Ė stata comunque Linda Shearer a proporre agli altri commissari di Aperto e a Carandente l'inserimento di Koons nella rosa degli artisti da invitare alla rassegna. Dai documenti presenti presso l'Archivio Storico della Biennale (ASAC), si apprende che la partecipazione dell'artista viene fin da subito sostenuta da tutti. ${ }^{17}$ Nello scambio di corrispondenza tra Shearer e Carandente si viene a sapere che Koons ha accettato con entusiasmo l'invito; tramite fax Shearer inoltre comunica a Carandente la volontà dell'artista di presentarsi con

13 Angela Maria Caracciolo Aricò, «E c'è anche Cicciolina scolpita da un americano», Il Tempo, 27 maggio 1990. Maria Campitelli, «Il Luna Park dei giovani», Il Piccolo, 26 maggio 1990.

14 Giancarlo Politi, «Dialogo dei massimi sistemi», Flash Art Italia, 157, estate 1990, 173

15 Maria Torrente, «Le trasformazioni dell'arte e la Biennale», L'umanità, 12 luglio 1990.

16 Enrico Crispolti, «Clima di omologazione celebrata», La Gazzetta delle Arti, 5-6, estate 1990.

17 ASAC, FS, AV, b. 497: «Verbali sezioni», 17-18 novembre 1989. 
opere inedite a cui aveva cominciato a lavorare dal $1989 .{ }^{18}$ Lo statunitense viene inizialmente contattato dalla curatrice per prendere parte all'Esposizione Internazionale d'Arte fin dal dicembre del 1989. Koons ha oramai un decennio di attività alle spalle, ma è un periodo in cui il suo modus operandi subisce una profonda trasformazione e passa dal rappresentare oggetti e immaginari ben riconoscibili dalla middle class statunitense a incentrare i propri lavori su se stesso. Il momento in cui lui in persona diventa protagonista delle sue opere, tramite la realizzazione dei lavori inclusi nella serie Made in Heaven (1989-91), è altresì quello in cui comincia la sua ascesa internazionale. Koons partecipa alla Biennale rappresentato della prestigiosa galleria newyorkese di Ileana Sonnabend; il legame con lei, che lo sostiene fin dal principio della sua carriera, ha costituito una sorta di garanzia sulla validità del suo operato. La preminenza della galleria gli ha permesso di prendere parte alla Biennale con un progetto irriverente e per certi versi coraggioso, che ha contribuito a cambiare il corso della sua carriera. Il complesso di opere presentate fa infatti parte della «scandalosa» serie Made in Heaven. Gran parte di queste sono imponenti dipinti a olio i cui protagonisti sono Koons e Ilona Staller, che diventerà la moglie dell'artista, ritratti in pose molto sensuali. L'interesse della stampa si accende fin da quando, nel mese di marzo del 1990, si diffonde la notizia che un artista americano si sarebbe presentato con opere che avrebbero avuto come soggetto Cicciolina e lui stesso in pose simil-pornografiche. Due testate riportano addirittura la notizia, rivelatasi in seguito un equivoco, dell'intenzione di Koons di presentarsi con un film pornografico con loro due come protagonisti. ${ }^{19} \mathrm{Il}$ malinteso viene presto chiarito in via ufficiale da Carandente in persona, il quale esclude la possibilità che Koons esponga una simile produzione..$^{20}$ Nonostante la smentita, l'attesa nei confronti della vena scandalistica di Koons resta alta. Le aspettative comunque non vengono deluse infatti, nonostante l'assenza del discusso filmato erotico, le opere con cui Koons si presenta alla Biennale non passano inosservate: si tratta di tre dipinti a olio creati a partire da fotografie alterate con l'ausilio della manipolazione digitale e una scultura. Per l'ideazione degli scatti si ispira ai servizi fotografici comunque ideati nell'ambito dell'industria dell'erotismo. Le imponenti tele, Ilona on Top (Rosa Background) (1990), Ilona with Finger Between Legs (Blue Background) (1990) e Silver Shoes (1990), realizzate proprio in vista

18 ASAC, FS, AV, b. 502: «Aperto 90», «Fascicolo nominativo di Jeff Koons».

19 Cf. Anonimo, «Cicciolina a luci rosse in mostra alla Biennale», La Repubblica, 7 marzo 1990; Virginia Baradel, «E l'artista sceglie il porno. Cicciolina attrice alla Biennale», La Nuova Venezia, 7 marzo 1990

20 Enrico Tantucci, «Cicciolina alla Biennale ma solo in scultura», La Nuova Venezia, 14 maggio 1990. 
della partecipazione alla Biennale, raffigurano lui e la sua musa in pose plastiche di reale richiamo pornografico. I dipinti vengono allestiti in modo da accentuare il centro focale della sala: una scultura in legno policromo raffigurante Koons e Cicciolina a dimensioni naturali in atteggiamenti inequivocabili, accompagnati da un serpente a squame verdi e dorate e adagiati su una base rocciosa decorata da fiori rosa e azzurri. Né le altre opere dell'installazione, né la scultura intitolata Jeff \& Ilona. Made in Heaven, nonostante la nudità esibita e l'ispirazione all'immaginario pornografico, raffigurano un atto sessuale vero e proprio. L'intera composizione, come Koons ha dichiarato in un'intervista rilasciata a Andrew Renton prima dell'inizio della Biennale, richiama il peccato originale di Adamo ed Eva e la cacciata dal paradiso terrestre. ${ }^{21}$ L'artista aggiunge, in riferimento alle opere destinate a essere esposte ad Aperto, che la sua aspirazione è quella di commisurarsi anche a livello filosofico con un tipo di arte che è in grado di entrare in relazione con i visitatori, illustrando loro la possibilità di raggiungere i propri desideri. Secondo il teorico e critico di arte contemporanea Marco Senaldi, l'artista statunitense implementa il suo proposito «filantropico» sfruttando se stesso come «icona mediale» e trasformando Cicciolina, incarnazione del suo più grande desiderio, in arte, «usandola» come un ready-made vivente (Senaldi 2003, 212). La scelta di imperniare le opere su quella figura farebbe dunque parte di un piano di azione ben studiato dettato dalla consapevolezza che la Staller sia un personaggio molto noto e dibattuto in Italia. Per il critico, l'intento di Koons è quello di sfruttare tutto ciò a proprio vantaggio assicurandosi una pubblicità facile ed efficace incentrando su di sé l'interesse mediatico (Senaldi 2003, 212). Tale interessamento subisce in effetti un forte incremento già poche ore dopo l'inaugurazione della Biennale. Secondo Vittorio Sgarbi si crea un fenomeno mediatico non a causa della presenza di un vero scandalo ma piuttosto perché

la possibilità di giudicare è favorita dall'indiscutibile evidenza del linguaggio. [...] Cicciolina serve a far parlare la stampa, i giornalisti pettegoli, e insieme contribuisce a scandalizzare quei benpensanti che fossero ormai assuefatti alle trovate dell'avanguardia, e quindi sul punto di non scandalizzarsi più. ${ }^{22}$

Koons sdogana il cattivo gusto portando alla sua rivalutazione, diventa il maestro dell'espressione artistica kitsch che è una vera e propria tendenza quell'anno all'interno di Aperto e più in generale di tutta la

21 Andrew Renton, «Jeff Koons. Sono in competizione con il Vaticano: ho già le mani nell'eternità», Flash Art Italia, 157, 1990, giugno-luglio, 108.

22 Vittorio Sgarbi, «Venice Biennale», Vogue Uomo, luglio-agosto 1990. 
Biennale «Dimensione Futuro». La critica non nasconde la propria ostilità nei confronti delle sue opere, mettendone in dubbio il valore artistico; contrariato rispetto alla sua presenza in mostra è però anche Flaminio Gualdoni, uno dei tre curatori del Padiglione italiano, il cui pensiero viene riportato nell'articolo di Claudio Altarocca:

Troppo facile farsi pubblicità mettendosi con Cicciolina, Koons fa solo del Kitsch, [...] dell'arte senza tensione morale. Koons ha capito il circuito arte-media e lo sfrutta cinicamente. ${ }^{23}$

Durante i primi giorni della vernice subito la maggior parte delle testate giornalistiche italiane che si occupano della Biennale lanciano commenti più o meno ostili nei suoi confronti. Il lavoro di Koons viene etichettato da Enzo Di Martino come «porn-art» e lui si guadagna l'appellativo di «gran maestro del massimo cattivo gusto» dalla giornalista Natalia Aspesi, che afferma con malizia che quella partecipazione alla Biennale è benvista da Carandente soprattutto per la grande risonanza che l'artista ha sui media, fungendo quindi da ottimo stratagemma pubblicitario per attirare l'attenzione sulla manifestazione veneziana. ${ }^{24}$ Duccio Trombadori su «Epoca» afferma che le opere destano molta ilarità tra i visitatori attirati alle Corderie dell'Arsenale per «ammirare» l'installazione tanto chiacchierata. ${ }^{25} \mathrm{Il}$ giornalista Paolo Rizzi su «Il Gazzettino», dal canto suo, è molto infastidito dalla presenza di Koons, tanto da definire la scultura la «cosa più orripilante» della Biennale, mentre anche per l'articolista de «Il Messaggero» Massimo Di Forti l'artista statunitense è l'emblema dell'eccesso e della provocazione e ha come fine ultimo solo la notorietà; condivide questa posizione anche Piergiorgio Dragone, che ne biasima il sensazionalismo e la mancanza di buon gusto. ${ }^{26}$ Tra questi vi è anche chi, come la giornalista Caracciolo Aricò, sostiene che Koons abbia fallito nel suo tentativo di scandalizzare, provocando nel pubblico solo scetticismo. ${ }^{27}$

I commenti nei confronti dell'operato artistico di Koons sono dunque eterogenei, tuttavia è evidente che gran parte dei recensori si

23 Claudio Altarocca, "Cicciolina nuda alla Biennale», La Stampa, 24 maggio 1990.

24 Enzo Di Martino, «La pantera ruggisce alla Biennale», Il Gazzettino, 24 maggio 1990. Natalia Aspesi, «Questa elettrica biennale Sesso \& Soldi», La Repubblica, 26 maggio 1990

25 Duccio Trombadori, «Biennale cronache di povera arte», Epoca, 13 giugno 1990, 66-76.

26 Paolo Rizzi, «Il bello non abita più qui», Il Gazzettino, 25 maggio 1990. Massimo Di Forti, «Barocco Freddo, anzi freddissimo», Il Messaggero, 26 maggio 1990. Piergiorgio Dragone, «Alle Corderie non tutti pezzi da novanta», Il Giornale, 3 giugno 1990.

27 Anna Maria Caracciolo Aricò, «E c'è anche Cicciolina scolpita da un Americano», Il Tempo, 27 maggio 1990. 
trova d'accordo nell'affermare quanto le sue opere siano l'emblema del cattivo gusto. L'attenzione della stampa nei confronti della Biennale dopo la prima settimana di apertura inizia a scemare e gli articoli che continuano ad occuparsene si trovano per lo più all'interno di riviste d'arte. Queste ultime ugualmente dedicano sempre meno spazio a Koons e alle sue opere. Nonostante questa tendenza generale, Duccio Trombadori in un articolo del 13 giugno apparso nel settimanale Epoca dedica all'artista alcune righe all'interno della sua critica della Biennale: lo descrive come un personaggio che incarna perfettamente i dettami dello yuppismo americano e che nella creazione delle proprie opere adopera

il kitsch o il gusto del pessimo gusto [...] l'estetismo e l'imitazione del barocco esaltato del genere 'beata Ludovica Albertoni' in formato New Age approdando ad un prodotto ultra pubblicitario della società post-industriale. ${ }^{28}$

Un'affinità con una cultura visuale che si rifà alla religione è individuata anche dal vicedirettore di «Il Giornale dell'Arte», Franco Fanelli, il quale sostiene che la scultura policroma ricordi le «salme di cera che nelle chiese contengono le reliquie dei santi». ${ }^{29}$ Anche Carlo Montanaro insiste sull'immaginario religioso a cui Koons sembra ispirarsi e asserisce addirittura che con la sua installazione schernisca i corredi diffusi nella religiosità popolare con il risultato di far scaturire dalla sua creazione un sensazionalismo banale e «blasfemo». ${ }^{30}$ Con l'inizio di settembre, ultimo mese di apertura della Biennale, l'attenzione nei confronti di Koons sembra essere totalmente assopita. L'intera manifestazione passa in secondo piano rispetto alla Mostra Internazionale d'Arte Cinematografica , in programma dal 4 al 15 settembre. Tuttavia, il 3 settembre si riaccendono i riflettori sull'artista a causa di un evento imprevisto: le principali testate riportano che le tre tele esposte alle Corderie sono state sfregiate ad opera di un giovane, il quale, secondo quanto riportato dai testimoni, senza indugio ha estratto un coltello e ha tagliato i quadri dello «scandalo» causando lacerazioni lunghe anche un metro e mezzo. ${ }^{31}$ L'autore del gesto, che ha causato un danno in termini monetari stimato in circa 600.000 dollari, ${ }^{32}$ non è stato individuato

28 Duccio Trombadori, «Biennale cronache di povera arte», Epoca, 13 giugno 1990, 66-76.

29 Franco Fanelli, «La Biennale di Venezia», Il Giornale dell'Arte, 1990, maggio.

30 Carlo Montanaro, «Se la scultura si annida in una fotografia», La Nuova Venezia, 29 maggio 1990.

31 Anonimo, «'Sfregiate' le tele dello scandalo», Il Corriere della Sera, 3 settembre 1990.

32 ASAC, FS, AV, b. 512: «Rapporti Codess», «corrispondenza relativa a danni opere». 
e resta un'incognita anche la motivazione che lo ha portato a compiere questo atto increscioso. L'ipotesi avanzata è che possa essere

una sorta di 'condanna' che il giovane a suo modo ha voluto infliggere a opere che offendevano il suo senso morale, oltre che estetico. ${ }^{33}$

Nonostante la gravità del fatto accaduto, quest'ultimo non ha fatto che aumentare l'attenzione mediatica nei confronti di Koons e delle sue opere, garantendo ulteriore visibilità all'artista.

La serie Made in Heaven, di cui sono parte le opere esposte alla Biennale, riscontra molto successo, tanto da essere ampliata e integrata da nuovi dipinti e sculture e divenire protagonista di quattro mostre omonime allestite tra la fine del 1991 e l'inizio del 1992 alla Sonnabend Gallery di New York, alla Galerie Max Hetzler di Colonia, alla Galerie Lehmann di Losanna, alla Gramo Fine Art di Anversa e al Christophe Van de Weghe di Bruxelles. Nel 1992 la consacrazione di Koons diventa definitiva con l'organizzazione della sua prima mostra monografica retrospettiva «Jeff Koons: Retrospective», allestita in contemporanea al San Francisco Museum of Modern Art di San Francisco e allo Stedelijk Museum di Amsterdam. L'attenzione mediatica ricevuta alla Biennale ha dunque prodotto l'effetto auspicato: l'ascesa artistica internazionale. La molteplicità di giudizi negativi rivolti a Koons non ha scalfito la sua credibilità, ma al contrario ha contribuito ad accrescere la curiosità e l'interesse nei suoi confronti, dando prova di come anche nel sistema dell'arte valga la frase pronunciata da Lord Henry nel romanzo di Oscar Wilde The Picture of Dorian Gray «There is only one thing in the world worse than being talked about, and that is not being talked about» (Wilde [1890] 1985, 24). 


\section{Bibliografia}

Auping, Micheal (1990). "Stati Uniti d’America». Biennale 44 1990, 212-17. Barilli, Renato (1990). «Verso un Barocco Freddo». Biennale 44 1990, 255-6 Baudrillard, Jean (1986). Amerérique. Paris: Editions Grasset \& Fasquelle.

Bourgeois, Caroline (a cura di) (2011). Elogio del Dubbio = Catalogo della mostra (Venezia, Punta della Dogana, 10 aprile 2011-31 dicembre 2012). Milano: Electa.

Bussmann, Klaus (1990). «Repubblica Federale di Germania». Biennale 44 1990, 200-5.

Carandente, Giovanni (1990). «Dimensione Futuro. L'artista e lo spazio». Biennale 44 1990, 15-17.

Carandente, Giovanni (1990). «Novembre a Berlino». Biennale 44 1990, 61.

Carandente, Giovanni (1990). «Omaggio a Eduardo Chillida». Biennale 44 1990, 91. Cherubini, Laura (1990). «Le divergenze dell'arte». Biennale 44 1990, 23-6.

Codognato, Mario (a cura di) (2003). JeffKoons: Napoli Museo Archeologico Nazionale = Catalogo della mostra (Napoli, Museo Archeologico Nazionale, 9 giugno-15 settembre 2003). Napoli: Electa.

De Stefano, Sabina Laura (2017). Jeff Koons e i rapporti con l'Italia: dalla Biennale di Venezia del 1990 a «Jeff Koons in Florence» (2015-2016) [tesi di laurea]. Venezia: Università Ca' Foscari Venezia.

Deitch, Jeffrey (a cura di) (1992). Post human: FAE Musée d'Art Contemporain, Pully Lausanne: Castello di Rivoli Museo d'Arte Contemporanea, Rivoli: Deste Art Foundation for Contemporary Art, Athens: Deichtorhallen Hamburg, Hamburg= Catalogo della mostra (FAE Musée d'Art Contemporain, Pully Lausanne; Castello di Rivoli Museo d'Arte Contemporanea, Rivoli; Deste Art Foundation for Contemporary Art, Athens; Deichtorhallen Hamburg, Hamburg, giugno 1992-maggio 1993). Amsterdam: Idea Books European distribution.

Foster, Hal (1996). The Return of The Real: Art and Theory at the End of the Century. Cambridge (MA): The MIT Press. Trad. it.: Carneglia, Barbara, /l ritorno del reale: l'avanguardia alla fine del Novecento. Milano: Postmedia, 2006.

Gingeras, Alison M. (a cura di) (2006). La collezione François Pinault: Una selezione Post-Pop = Catalogo della mostra (Venezia, Palazzo Grassi, 12 novembre 2006-11 marzo 2007). Milano: Skira.

Gingeras, Alison M.; Bankowsky, Jack (2006). Where Are We Going? Opere scelte dalla Collezione Francois Pinault = Catalogo della mostra (Venezia, Palazzo Grassi, 29 aprile-1 ottobre 2006 ). Milano: Skira.

Gingeras, Alison M. (a cura di) (2009). Mapping the Studio: artisti dalla collezione François Pinault = Catalogo della mostra (Venezia, Palazzo Grassi, Punta della Dogana, 6 giugno 2009-10 aprile 2011). Milano: Electa.

Gualdoni, Flaminio (1990). «In margine». Biennale 44 1990, 37-40.

McEvilley, Thomas (1990). «Gran Bretagna». Biennale 44 1990, 156.

Pancotto, Pier Paolo (2010). Arte contemporanea: dal minimalismo alle ultime tendenze. Roma: Carocci, 96.

Risaliti, Sergio (a cura di) (2015). Jeff Koons in Florence = Catalogo della mostra (Firenze, Palazzo Vecchio, Piazza della Signoria, 24 settembre 2015-15 gennaio 2016). Firenze: Forma.

Senaldi, Marco (2003). Enjoy! il godimento estetico. Roma: Meltelmi editore.

Terraroli, Valerio (a cura di) (2009). L'arte del XX secolo Neoavanguardie, postmoderno e arte globale (1969-1999). Milano: Skira.

Vergine, Lea (1990). «Echi del Gamelan, dopo Debussy». Biennale 44 1990, 41-58. Wilde, Oscar [1890] (1985). The Picture of Dorian Gray. London: Penguin Books. 

BorDER ARt Workshop/TAller de Arte Fronterizo

$(619) 575.9293$ or $(619) 5751830$ - (619) 4631907

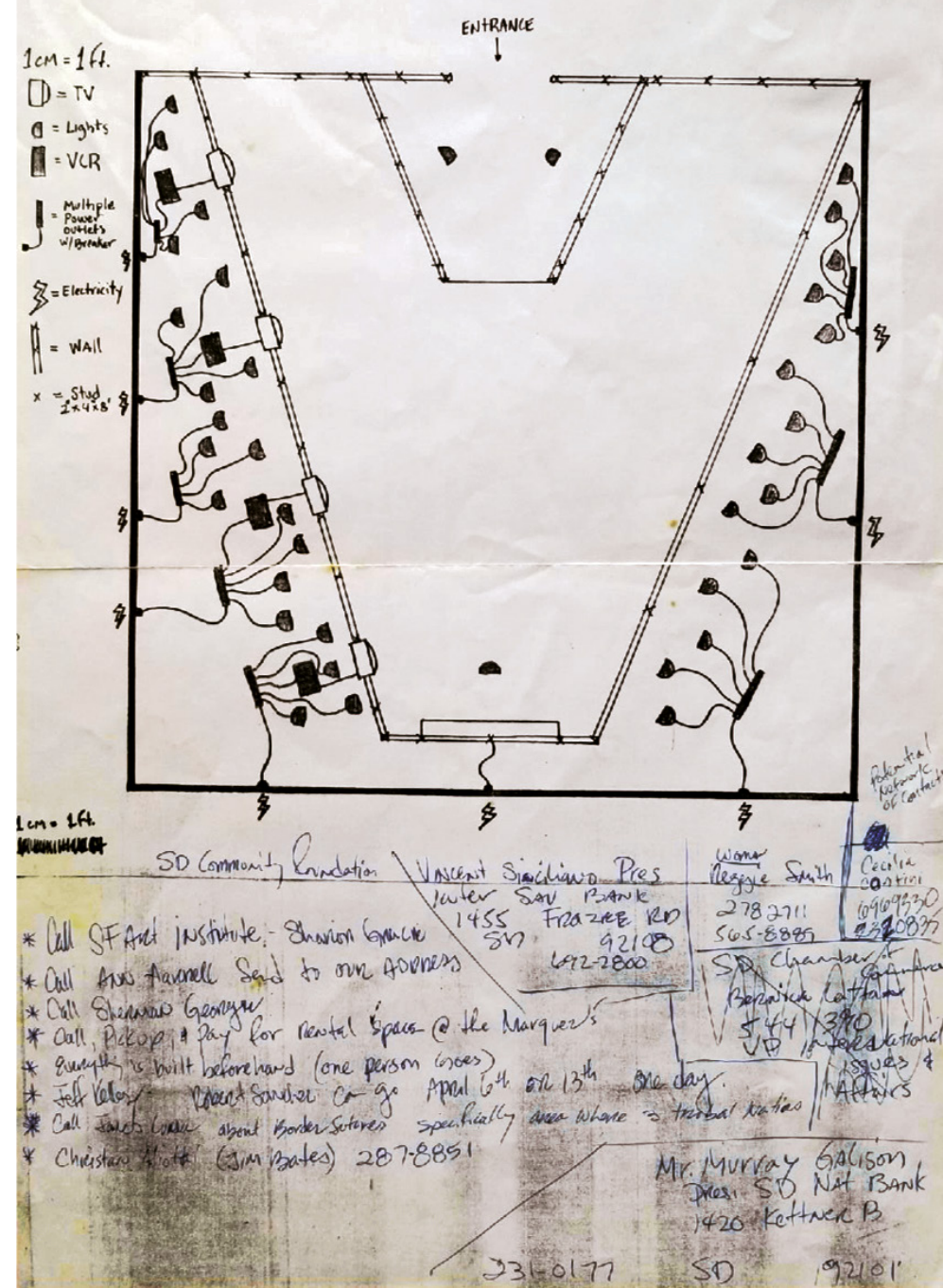

Border Art Workshop/Tallér de Arte Fronterizo (BAW/TAF), Pianta dell'installazione Colón Colonizado - Tutto è Mio - ¿De Quién?, 1990, Venezia, La Biennale di Venezia. Coutesy dell'artista Richard Alexander Lou 


\title{
"What kind of dirt do you need?» Da San Diego e Tijuana a Venezia: Border Art oltre i confini
}

Andrea Masala

\begin{abstract}
Only one year after the fall of the Berlin Wall, in 1990, another border could be observed at the Aperto section of the Venice Biennale. It was the work Colón Colonizado - Tutto è Mio - ¿De Quién? by the Border Art Workshop/Tallér de Arte Fronterizo (BAW/ TAF), a binational collective of artists from the San Diego-Tijuana border region. The project corresponds to the first landing of Border Art overseas, but also to the dissolution of the BAW/TAF. The work is therefore subject and conductor of the essay. Its site-specificity aspects are deepened in relationship to the Biennale's exhibition context. The analysis of this case study will present the Venice Biennial as a key moment and a stage for the transformation of the border space from geographical-territorial to mental and conceptual.
\end{abstract}

Keywords Border. Space. Border Art. BAW/TAF. Site-specificity.

Quando siamo messi con le spalle al muro, possiamo provare un senso di oppressione o claustrofobia; se ci voltiamo verso di esso, invece, può nascere in noi un'esplosione di creatività. ${ }^{1}$ I confini nazionali, siano essi marcati o no da muri e barriere, hanno il medesimo effetto sugli artisti, che riescono a trasformare l'angoscia dell'occlusione in arte e bellezza. Allo stesso modo, voltare le spalle a un problema e fingere che non esista non fa che incrementarlo, mentre guardarlo

Questo saggio prende avvio da Masala, Andrea (2019). T/HERE. Borders Art a San Diego e Tijuana (1970-2005) [tesi di laurea magistrale]. Relatore Giuseppe Barbieri; correlatore Cristina Baldacci. a.a. 2018/2019. Venezia: Università Ca' Foscari Venezia.

1 La riflessione è ispirata a Casey, Watkins 2014.

Storie dell'arte contemporanea 4 | Atlante delle Biennali 1

ISSN 2704-9973

ISBN [ebook] 978-88-6969-366-3 | ISBN [print] 978-88-6969-367-0

(a)ioni 
dritto in faccia offre l'opportunità di superarlo e renderlo un punto forte della nostra identità e della nostra storia. Questo fu lo spirito che mosse l'attività del Border Art Workshop/Tallér de Arte Fronterizo (BAW/TAF), una collettiva bi-nazionale e attivista di artisti impegnati nell'affrontare il tema del confine (border) in un particolare contesto: il tratto del lungo confine tra Stati Uniti e Messico che attraversa il complesso urbano di San Diego e Tijuana. Nel 1990, il BAW/TAF ${ }^{2}$ ebbe modo di portare questo confine all'interno della sezione Aperto della XLIV Biennale di Venezia Dimensione Futuro. La loro opera, Colón Colonizado - Tutto è Mio - ¿De Quién?, ebbe modo di far superare alla cosiddetta Border Art statunitense e messicana i confini territoriali entro i quali si era espressa fino a quel momento. In altre parole, questo progetto corrisponde al primo esempio oltreoceano di un genere artistico sviluppatosi e manifestatosi appositamente sul confine e lungo i territori ad esso adiacenti.

La partecipazione del BAW/TAF con questa installazione non sarà solamente l'argomento di questo intervento, ma anche il suo filo conduttore. Intendo, infatti, avanzarne una lettura in chiave di site-specificity, ma anche adottare questo caso di studio come pretesto per riportare l'attenzione sul particolare rapporto tra artisti e confini e sulle difficoltà dell'adattare un simile spazio geo-politico ad altri contesti.

Era il 1990 e, solamente un anno prima, il confine che aveva diviso il mondo in due, il Muro di Berlino, era caduto lasciando spazio all'idea di libertà e globalizzazione, incarnate dalla sensazione di uno spazio riacquistato e nella consapevolezza di essere alla fine di un lungo secolo contraddistinto da divisioni e muri politici (GREPPI 2019). Alla Biennale di Venezia il nuovo spazio post-Berlino echeggiava nella mostra Ambiente Berlin all'interno del Padiglione Italia, dove Emilio Vedova esponeva Absurdes Berliner Tagebuch e Hans Ticha la sua tela Il Muro. A conferma della fine dell'incomunicabilità dei due blocchi mondiali stava l'opera dello statunitense Rauschenberg assieme a un gruppo di giovani artisti dell'ex Unione Sovietica (Di Martino 2003, 78). Un'epoca era finita e un'altra prendeva il suo posto, come dimostra l'attenzione verso il futuro spiegata dall'allora direttore del settore arti visive, Giovanni Caradente, come un occhio di riguardo per gli artisti emergenti:

Il titolo Biennale '90, Dimensione Futuro voleva essere semplicemente benaugurante, dato che vi era internazionalmente privilegiata la selezione di artisti più giovani rispetto alla passata Biennale. [...] "L'artista e lo spazio" [...] è l'altra possibile definizione

2 Gli artisti del BAW/TAF che collaborarono al progetto per la Biennale furono: Yareli Arizmendi, Carmela Castrejon, Berta Jottar, Richard Alexander Lou, Robert Sanchez, Michael Schnorr. 
che si può affidare a questa Biennale, come essa è venuta configurandosi, non solo in Italia, ma anche negli altri paesi del mondo, a mano a mano che gli artisti prendevano contatto con lo spazio loro assegnato. (Caradente 1990, 16)

In questa generale ripresa di fiato a seguito della lunga immersione nel secolo degli 'ismi' e delle grandi fazioni mondiali, la presenza in Biennale di un gruppo artistico incentrato sul tema del confine sembrava riportare le coscienze davanti alla realtà. Nonostante ciò, la partecipazione del BAW/TAF rimaneva perfettamente contestualizzata e giustificata. La riflessione sullo spazio, su cui insisteva Caradente, era stata, infatti, centrale all'attività del gruppo, già dalla sua fondazione, precedente di soli sei anni. Lo si può leggere chiaramente in una prima dichiarazione d'intenti, che afferma quanto segue in toni da manifesto avanguardistico:

The BAW/TAF proposes the planning and production of the first border opera defining our region from the perspective of people who live and work in our binational borderland. The San Diego/Tijuana region, Los Angeles, Seoul and Tokyo are the spokes of the Pacific Rim. We feel that our cross-cultural binational neighborhood is more than ready for a complex multilayered-layered mixed media product with people who understand the beauty and problems of living in the most trafficked international border crossing in the world. ${ }^{3}$

Si comprende bene che l'intera poetica artistica del BAW/TAF era stata sviluppata a partire proprio da una riflessione sullo spazio, declinata, in questo caso, in base alla delicatezza e alle peculiarità di un luogo che merita di essere introdotto prima di un'analisi dell'opera in Biennale.

L'area geo-politica e socio-culturale sviluppatasi lungo le due città frontaliere di San Diego e Tijuana è ancora oggi teatro di una forte cultura ibrida, in cui linguaggi messicani si mescolano a pratiche statunitensi influenzandosi a vicenda (Anzaldúa 1987, 3). Questo conferma in primis quanto espresso in apertura, ovvero che un confine non impedisce affatto gli scambi culturali, ma funziona da stimolo creativo e da punto di incontro. Inoltre, questo aspetto è prova ulteriore della simbiotica e intensa connessione tra cultura e spazio in questa particolare area. Un sito diviso da un confine risulta, infatti, pregno di una marcata intensità semantica. Essa è la conseguenza del principale scopo di un border, ovvero, quello di delimitare, definire e di-

3 San Diego (CA), University of California in San Diego, Geisel Library, Special Collections \& Archives, Michael Schnorr Collection of Border Art Workshop/Tallér de Arte Fronterizo Records, 1978-2008, MSS 0760, Box 1, Folder 10, Group Statement. 
stinguere ciò che sta 'da questa parte' da ciò che sta 'da quella parte'. Un confine nazionale spesso presuppone che al di là di esso vi sia un 'altrove', in cui si parla un'altra lingua, dove abita un'etnia diversa e dove si portano avanti differenti culture e tradizioni. In sostanza lo spazio diventa doppio e si fa carico di diversi significati e accezioni che costantemente influenzano e rimodellano la vita delle persone in base alla loro posizione rispetto alla linea frontaliera.

Sebbene il BAW/TAF avesse già affrontato queste tematiche in una serie di performance e installazioni eseguite sulla borderline stessa, il progetto per la Biennale fu particolarmente difficoltoso da realizzare. Come si sarebbe potuta rendere la medesima intensità dello spazio di frontiera all'interno di un contesto geografico, culturale e espositivo così lontano da esso com'era, di fatto, la Biennale di Venezia?

Per rispondere al quesito e entrare nel merito della questione si rende necessario fare delle premesse e delle precisazioni, che torneranno utili al lettore per recepire meglio il linguaggio dell'opera Colón Colonizado - Tutto è Mio - ¿De Quién?.

Innanzitutto occorre chiamare le cose con il proprio nome: il termine border si riferisce a ogni tipo di confine esistente, geografico o mentale che sia. È quindi possibile riferirsi in termini di border a questioni relative al genere, all'identità, all'orientamento sessuale, all'etnia e alla classe sociale. Ci troviamo dinnanzi a un border ogniqualvolta vi sia una divisione tra ciò che è consueto e ciò che è diverso, tra il consentito e il proibito, tra il comune e il diverso, tra il dentro e il fuori, tra noi e loro. In sostanza i confini stanno contemporaneamente ovunque e da nessuna parte, poiché ne esistono infinite tipologie che costantemente instauriamo e oltrepassiamo. Di conseguenza la Border Art finisce per riflettere questo caleidoscopico ventaglio di applicazioni tramite l'adozione di un'insieme di linguaggi multimediali che presentano le due principali accezioni di border - territoriale e mentale - come interconnesse e spesso interdipendenti tra loro. Anche per questa ragione, lo status quaestionis è andato nel tempo focalizzandosi sempre più sugli effetti meno tangibili e più sociali del border, fino ad abbattere i confini disciplinari dei border studies, dei gender studies, dei de-colonial studies a favore di approcci transdiciplinari che, di volta in volta, sottolineano forza e debolezza di questo concetto (Zaccaria 2004, 8).

L'accezione che maggiormente interessa questo scritto, ovvero la separazione tra due territori o due nazioni, rimane quella più immediata e tangibile di questo sfaccettato e poliedrico concetto. Non a caso, l'etichetta Border Art venne a galla per la prima volta proprio in relazione all'operato del BAW/TAF (Chávez 1993, 5) che - come si è precisato - era focalizzato sul comprendere e divulgare le controverse dinamiche geo-politiche e socio-culturali della border region.

Prima dell'operato del BAW/TAF era stata la Chicana/o Art ad occuparsi di questi problemi, sebbene con un'accezione e con degli in- 
tenti differenti. Quest'ultima si era protratta durante i tormentati e movimentati anni '60 e '70 del Novecento, gli anni derivati dal precedente rifiuto di Rosa Parks a cedere il suo posto sull'autobus a un uomo bianco. Durante quel periodo di lotte per i diritti civili e per l'uguaglianza tra le minoranze e tra i sessi, lo spirito rivoluzionario non era venuto a mancare tra le minoranze messicane degli USA, i cui membri sono meglio noti come chicanas/os. Esse avevano trovato nell'arte, specialmente nel muralismo (cf. Barnett 1984) e nella grafica, il principale strumento per la promozione delle proprie idee e per l'avanzamento delle loro lotte civili. Le due tecniche artistiche avevano permesso loro di lamentare le difficoltà derivate dalla propria condizione minoritaria, come la subalternità lavorativa e le varie ingiustizie spesso subite dal razzismo comune nella società angloamericana dominante. Il tema dell'immigrazione era il collante tra tutti questi problemi, nonché quello più connesso al border.

Questo consolida ulteriormente il rapporto di interdipendenza e consequenzialità tra border fisico e mentale. Infatti, questi problemi avevano avuto origine dalla creazione della linea territoriale, tracciata sulle mappe nel 1848 e stabilita sulla carta tramite il trattato Guadalupe Hidalgo. Ancora oggi essa costituisce il lungo percorso del confine tra il primo e il terzo mondo, tra Stati Uniti e Messico. Ad animare il Movimiento Chicano accorreva il grido «we did not cross the border, the border crossed us», perfetta testimonianza della diaspora (Davalos 2001, 21) a cui il popolo messicano venne destinato da questa linea. In sintesi, il confine aveva tagliato in due il Nuovo Mondo collegando idealmente l'Oceano Atlantico a quello Pacifico e frammentando in molteplici border mentali le coscienze delle popolazioni che abitavano a cavallo di questa linea.

La parola workshop come denominativo del BAW/TAF restituisce l'idea di un laboratorio impegnato a comprendere ciò che avviene in questa zona, piuttosto che il nome di un gruppo di artisti. Non a caso, alcuni fondatori del BAW/TAF provenivano dalla precedente esperienza chicana, che si era affievolita verso la fine degli anni '70. Ad essi apparteneva Victor Ochoa, il quale mi racconta durante un'intervista: ${ }^{4}$

I am one of the original founders of the Centro Cultural. [...] We had different disciplines and artists: poets, dancers... we had all the different disciplines, etc. So we were going to a different direction and - you know - I think in 1984 we received a grant. [...] I was a director there at the Centro, and we decided what kind of a project we wanted to do..., and we came up with this thing on the border. So, then, what we did was starting to invite artists to dialogue about doing some project about the border as a group of artists. 
Assieme a Ochoa si radunarono attorno al Centro Cultural de La Raza (un edificio espositivo del Balboa Park di San Diego, sede del gruppo), gli artisti Michael Schnorr, David Avalos, Sara-Jo Berman, Isaac Artenstein, Guillermo Gómez-Peña e Jude Ederhart. Tutti vantavano trascorsi, linguaggi artistici, idee, genere, provenienza e cultura differenti: alcuni di loro, infatti, erano statunitensi, altri chicanos, altri ancora messicani.

In base a quanto affermato finora, non sarebbe una forzatura intendere questo aspetto come una prima influenza da parte dello spazio sulla border art. La provenienza degli artisti da luoghi differenti causò, infatti, la convergenza di molteplici linguaggi, di diversi panieri tematici, di più medaglioni iconografici. Ogni artista rappresentava quasi un'enclave del proprio Paese, del quale portava con sè i tratti, la lingua e le principali caratteristiche. Per questo motivo, il periodo di attività del BAW/TAF viene identificato come la fase della 'multiculturalità' della Border Art (Berelowitz 2003, 143-81). L'eterogeneità etnico-culturale del gruppo vide, inoltre, il numero di artisti partecipanti variare di volta in volta. Le differenze interne si esprimevano anche in termini di genere: tra i sette membri fondatori, solamente due (Jude Eberhardt e Sara Jo Berman) erano donne. Le due artiste, sposate rispettivamente con Isaac Artenstein e Guillermo Gómez-Peña, si ritrovarono spesso a ricoprire un ruolo marginale nei progetti e nelle decisioni nel gruppo. Per questo motivo, nel 1988, decisero di separarsi dal gruppo e di fondare con altre artiste un corrispettivo femminile del BAW/TAF, da loro denominato Las Comadres (Sánchez 2007, 158-201).

Le differenze proprie del gruppo finirono per complicare ulteriormente la difficoltà implicita nel parlare di border a Venezia. Infatti, oltre che ricreare una dimensione spaziale, il gruppo dovette amalgamare più approcci, tecniche e background culturali in un'opera, che sarebbe dovuta risultare come un intervento coeso e unitario. Da qui nacque l'articolazione linguistica di Colón Colonizado - Tutto è Mio - ¿De Quién?.

Come si può evincere dal disegno progettuale, l'installazione consisteva in una stanza di sei metri di altezza, sei di larghezza e sei di profondità. Dall'esterno la pianta risultava quadrata ma all'interno essa aveva una forma triangolare dettata dalla presenza di due muri progressivamente convergenti. La parete di fondo e il soffitto erano oscurate da un rivestimento in plastica nera, che faceva scomparire lo spazio esterno a favore di una dimensione spaziale 'altra'. Le pareti laterali incrementavano questa sensazione: a sinistra erano presenti delle fotografie e a destra dei murales a carboncino entrambi raffiguranti il paesaggio della zona frontaliera. Nella parete decorata con i murales erano incastonati quattro schermi che proiettavano degli estratti di alcune precedenti performance del gruppo riguardanti tematiche come le atrocità della conquista e della colo- 
nizzazione europea del Nuovo Mondo, l'invasione di Panama e l'abbattimento del Muro di Berlino.

Lo spettatore era invitato ad accedere in questa stanza attraverso una porta sovrastata da un'immagine di Cristoforo Colombo. Una volta all'interno, l'esperienza provata era multisensoriale, oltre che riflessiva. Il visitatore poteva infatti interagire con l'installazione camminandovi dentro e misurando con i suoi passi lo spazio circostante. La convergenza delle pareti interne in un unico punto, se da un lato rendeva questa stanza opprimente, dall'altro, invece, come fosse un corridoio prospettico del Brunelleschi in tre dimensioni, permetteva di sentirsi protagonisti del paesaggio riprodotto ai lati. La vista e l'udito venivano stimolati ulteriormente dalle immagini del border riprodotte alle pareti e dai suoni delle proiezioni trasmesse negli schermi; il tatto, invece, veniva chiamato in causa attraverso i piedi.

Quest'ultimo aspetto è solo apparentemente insignificante, dal momento che, come mi conferma l'artista Richard Alexander Lou in un'intervista, ${ }^{5}$ in fase progettuale gli autori chiesero agli uffici della Biennale, tra le altre cose, del materiale 'sporco' da inserire all'interno della stanza. La risposta della Biennale recita il titolo che ho scelto di dare a questo testo: «What kind of dirt do you need? In anycase no smelling dirt». ${ }^{6}$ Questa semplice frase racchiude in maniera emblematica il limite principale costituito dal dover eseguire un'opera per un museo e non sul sito esatto in cui i due paesi si incontrano. In altre parole, la domanda «che tipo di sporco vi serve?» è ciò che meglio testimonia quest'opera come una riproduzione del confine e non come un intervento sul sito originale. Il BAW/TAF aveva chiesto un tipo di sporcizia che ricordasse la terra del deserto, in modo tale da dare al visitatore la sensazione del suolo sotto le scarpe e per restituire il suono del crepitio della terra al passaggio su di essa. A pensarci bene, lo spazio è una cosa che si può percepire pienamente quando si ha la possibilità di muoversi dentro di esso, di attraversarlo e di sperimentarne le dimensioni. La terra, dunque, avrebbe restituito la concretezza dello spazio geografico, della dimensione tangibile e concreta del deserto tagliato in due da una linea immaginaria. In quest'ottica, chi percepiva meglio il confine era il migrante illegale senza documenti, undocumented negli USA, che tentava (e questo accade ancora oggi) di perseguire il suo sogno americano viaggiando di nascosto nel deserto quando il cielo era nero come il soffitto di questa stanza.

5 Intervista di Andrea Masala a Richard Alexander Lou, 17 dicembre 2018, San Diego (CA).

6 San Diego (CA), University of California in San Diego, Geisel Library, Special Collections \& Archives, Michael Schnorr Collection of Border Art Workshop/Tallér de Arte Fronterizo Records, MSS 760, Box 13, Folder 8, The New Museum exhibit notes and correspondence, 1990. 
L'importanza di questo materiale può essere meglio compresa tramite il suo accostamento spontaneo con gli insegnamenti di Robert Smithson, artista pioniere del concetto di site-specificity. Ad esempio, la sua celebre opera Spiral Jetty del 1970 consisteva in un'enorme spirale di rocce dentro il Great Salt Lake in Utah. La sua peculiarità era il suo essere in costante dialogo con il lago, che ne influenza ancora adesso continuamente colore, visibilità, forma e, dunque, percezione da parte dello spettatore. L'opera è dunque a diretto contatto con le condizioni atmosferiche, geologiche e ideografiche del luogo e vuole far riflettere esattamente sulle caratteristiche di questo determinato paesaggio. Se Spiral Jetty rappresentava un chiaro esempio di opera site-specific in un contesto naturale, i vari non-sites dell'autore avevano accezioni differenti. Esse erano sempre delle opere sitespecific, ma, in questo caso, consistevano nella riproduzione di un sito all'interno di un contesto museale. Tuttavia, non bisogna intenderle come copie esatte e dettagliate di paesaggi, ma sculture sintetiche, concettuali e simboliche, caratterizzate dall'unione di specchi, materiali organici, terra, sassi raccolti in precedenza dall'autore durante i suoi viaggi lungo paesaggi naturali. Scopo di questi lavori era stimolare una dialettica tra spazio interno e esterno o, meglio ancora, delle riflessioni su tempo, sito, entropia, materia, vista, natura e cultura. In poche parole, la lezione di Smithson riguardava la possibilità di creare opere site-specific anche fuori dal sito e i suoi nonsites confermavano dunque che ciò era possibile grazie al ricorso a una dimensione concettuale.

Se trasponiamo questo ragionamento alla stanza realizzata in Biennale dal BAW/TAF, risulta più semplice capire come il gruppo fosse riuscito a ricreare il border in un contesto differente, come quello del white cube. La Biennale era infatti un contesto espositivo dalle caratteristiche più simili all'arido e asettico spazio museale moderno e contemporaneo evidenziato dalla canonica riflessione di O'Doherty (1976). Sebbene non completamente ascrivibile al biancore e alla trasparenza del white cube, l'assenza di appigli culturali o paesaggistici, rendeva, infatti, il compito ancora più complicato. Alla maniera di Smithson, pertanto, il BAW/TAF non si limitò a riprodurre un paesaggio in maniera canonica, ma a restituirne una dimensione che unisse tratti tangibili e concettuali. La loro stanza funse da rappresentazione non soltanto di un confine geografico, ma dell'intero sfaccettato concetto di border, precedentemente introdotto in base alle sue differenti accezioni. Oltre che restituire la sensazione totalizzante di trovarsi in un altro paesaggio, l'opera del BAW/TAF stimolava la riflessione grazie a un processo di ricostruzione delegato allo spettatore. Ne è prova principale la presenza della foto di Cristoforo Colombo al di sopra dell'ingresso. Se letta in relazione al titolo dell'installazione, si palesa chiaro il messaggio secondo il quale sia necessario passare sotto la colonizzazione di Colombo per com- 
prendere il luogo in cui si sta accedendo. Linee di frontiera artificiali e arbitrarie come quella qui presa in considerazione sono tipiche di un territorio post-coloniale, così come lo sono i vari border mentali a esse conseguenti e profondamente radicati nelle controversie del melting-pot statunitense. In sostanza, se a Spiral Jetty era necessaria l'azione della natura per essere considerata site-specific, all'intervento del BAW/TAF in Biennale serviva il fattore umano e sociale come nesso tra opera e paesaggio. Il border, infatti, è un teatro quotidiano per movimenti e dinamiche sociali, così come il lago è oggetto di continui mutamenti atmosferici e idrografici.

La nostra analisi sta facendo emergere sempre più la volontà del BAW/TAF di raggiungere una dimensione concettuale della Border Art tramite l'adozione delle potenzialità comunicative offerte dallo spazio e dal sito, anche quando in realtà ci si trovava ad agire lontano da esso. A questo proposito l'artista Michael Schnorr spiega:

In our specific case "site" has always two sides: it has been determined by nationalistic sparring, has a layered as well as ruptured component, and comes with an established mass media presence. These geo-political realities place the phenomenon of border-assite in a context other than that of conventional site specific "locations". Accumulated evidence concerning the border-site is thrown into chaos when one realizes that if seen from only one side, the site eliminates its other side. Within the racist and ethnocentric form of this chaos (of the "other") there are four primary models by which the border site might be defined and engaged. To construct tangible, participatory relationships among artists and audience on the site, as well as to redefine a place that is not politically neutral, is the true potential of working with the border-as-site. (Schnorr 1988, 42)

La dichiarazione suggerisce che il BAW/TAF abbia sviluppato la sua riflessione sul border lungo due piani principali: quello reale e quello della percezione che si ha di questa realtà. Sostenendo che, se visto da un solo lato, il confine elimina l'altra parte, Schnorr non fa riferimento solamente alla caratteristica implicita ai muri e alle barriere $^{7}$ di bloccare lo sguardo. Egli allude, piuttosto, alla necessità di un approccio alla zona che vada oltre quello dei media, della cultura dominante o di quella minoritaria. Queste finivano sempre per lasciare un'ombra su un aspetto della verità, analizzandone solo una componente, esattamente come fa un muro sul terreno. La necessità espressa dal BAW/TAF era pertanto quella di smettere di ragionare nei termini di uno sguardo 'da questa' e 'da quella parte' e comincia-

7 Occorre precisare, tuttavia, che ai tempi in cui agiva il BAW/TAF, non vi era ancora un muro divisorio tra San Diego e Tijuana, ma solamente una recinzione di filo spinato. 
re ad adottare un'ottica più evoluta. Questa avrebbe dovuto considerare ogni singolo aspetto e ogni singola prospettiva del border in un complesso culturale dai tratti globali che fosse orientato anche verso le similitudini con altri confini critici del mondo.

Tutto ciò giustifica anche la presenza degli estratti delle performance proiettati alle pareti. Il loro scopo era di mostrare gli aspetti sociali, umani e psicologici di quel particolare spazio e di aiutare la riflessione del visitatore sul tema. A loro volta, queste facevano parte di una serie composta da sette performance mensili, intitolata Destination L.A., eseguita tra il 1989 e il 1990 al Soccer Field, una spianata tra il Canyon Zapata di San Diego e il quartiere Colonia Libertad di Tijuana. Ai tempi il sito si riempiva quotidianamente di migranti, che aspettavano insieme il tramonto per tentare di entrare negli USA di notte. Gli artisti erano soliti cominciare gli eventi disegnando un enorme piano da gioco nel terreno (generalmente un monopoli o una scacchiera) e, successivamente, con l'aiuto di vari oggetti (croci di legno, bare o riproduzioni di monumenti di demarcazione territoriale in polistirolo), mettevano in scena una serie di passaggi trans-frontalieri dapprima da nord verso sud e poi in direzione contraria (Kelley 1990, 24-5). Questo restituisce l'idea che lo spazio si percepisca al meglio in base ai movimenti che esso consente e, nel caso del confine, ciò assume ancora più intensità. Il BAW/TAF presentava il confine come un luogo possibile solamente in base ai passaggi da esso permessi o ostacolati. L'immagine era quella di luogo d'attesa, di passaggio, o meglio, come di quello che il celebre antropologo francese Marc Augé avrebbe definito un 'non-luogo' (Augé 1992). Il rapporto tra la collettiva e questo sito era dunque quello di un paesaggio fisico che era soprattutto teatro di pratiche sociali, culturali e artistiche, in cui processi di territorializzazione e deterritorializzazione partecipano ogni giorno a un gioco intricato (Malagamba-Ansotegui 2001, 72).

L'intervento in Biennale dunque, grazie alla sua accezione sensoriale, esperenziale e concettuale, risulta essere una sintesi non solo della situazione frontaliera, ma anche dei vari approcci dei singoli artisti del BAW/TAF allo spazio. Si potrebbero circoscrivere le loro opere con la chiave di lettura della site-specificity in quella che vorrei proporre come una 'doppia via'. Questa può, altrimenti, essere intesa come la predominanza di due tendenze principali nell'adozione del sito. Una di esse è la 'tendenza all'accentramento' e l'altra, invece, è quella 'all'espansione'. In altri termini, la prima corrisponde alle performance site-specific eseguite esattamente sulla linea di confine, come Destination L.A., mentre la seconda equivale alle installazioni lontane da essa, tra cui troviamo appunto l'intervento per Aperto. Se si riflette ulteriormente su questo dato alla luce di quanto esposto finora, si vede confermato da parte del BAW/TAF il medesimo approccio di Smithson al site-specific. Dunque, con la loro insistenza sul sito e sul concetto di site-specificity, gli artisti del BAW/TAF ridefinirono 
il confine: solamente dopo aver assunto uno status di entità solida e fisica e aver assunto il ruolo di un partecipante alla produzione artistica, questo potè diventare successivamente portatile e metaforico per essere presentato a un pubblico più vasto (Sheren 2005, 25).

Questa trasformazione del confine fu lateralmente proprio il motivo che portò alla rottura del gruppo. Il problema era proprio la destinazione che la Border Art avrebbe dovuto raggiungere, qualora con destinazione si intenda il contesto espositivo e istituzionale, piuttosto che il contesto geografico.

Si pensi agli spazi della Biennale di Venezia: sebbene il sistema dei Padiglioni possa ricordare, di fatto, quello di una riproposizione ideale e virtuale dei confini nazionali, la circolazione di persone al loro interno non viene affatto bloccata ma, al contrario, incoraggiata. La Biennale Arte è infatti un enorme contesto culturale e artistico dove, una volta ogni due anni, converge l'intera comunità artistica mondiale (e non) che riflette nell'acqua della laguna veneziana cultura, storia e pensiero contemporaneo. In questo immenso calderone artistico non mancano grandi firme dell'arte contemporanea, come Jeff Koons nella stessa sezione Aperto in cui esponeva il BAW/ TAF. Fu esattamente l'accostamento a grandi firme e a grandi istituzioni a generare dissidi interni al gruppo, che si sarebbero addizionati alla già descritta eterogeneità interna che lo contraddistingueva.

In realtà il gruppo avrebbe continuato a lavorare ancora, anche se con un numero di partecipanti ridotto ai minimi termini. La data convenzionale per indicarne lo scioglimento equivale, tuttavia, a quella della loro partecipazione alla Biennale di Venezia. Responsabile di ciò non fu propriamente la mostra veneziana, ma una più generale attitudine da parte dei border artists a collaborare con istituzioni simili ad essa. Nel 1989, infatti, il Museum of Contemporary Art of San Diego si era rivolto al National Endowment for the Arts (NEA), agenzia federale statunitense per la promozione degli artisti, al fine di intraprendere un progetto di tre anni a carattere bi-nazionale, che sarebbe sfociato nel 1993 nella mostra La Frontera/The Border Art About the Mexico/United States Border Experience (Berelowitz 2003, 168). Il ricorso a un'istituzione tipica del mondo dell'arte statunitense, gestita ai tempi principalmente da persone di etnia anglos, portò l'artista del BAW/TAF Guillermo Gómez-Peña a lamentare una specie di 'svendita della Border Art'. In un articolo del 1991 intitolato Death on the Border: An Eulogy to Border Art egli affermò:

A movement that began as an attempt to dismantle Anglo-Saxon patriarchal authority end up being appropriated, controlled, promoted and presented by Anglo-Saxon patriarchs... The border as metaphor has become hollow. Border aesthetics have been gentrified and border culture as a utopian model for dialogue is temporarily bankrupt. (Gómez-Peña 1991) 
Se si riconsidera nuovamente lo sviluppo temporale della Border Art, già il suo distacco dalla Chicano Art presenta una tendenza da parte di questo genere artistico a espandersi verso contesti geografici progressivamente più lontani, ma soprattutto a raggiungere un pubblico sempre più vasto. La polemica di Gómez-Peña conferma questa attitudine. Separatasi da un'arte minoritaria, la Border Art, agli albori degli anni ' 80 , aveva ammesso tra i suoi addetti anche personalità estranee alle poetiche chicane, esattamente come Gómez-Peña, che era in realtà originario di Città del Messico o Michael Schnorr, un ebreo statunitense nato a Honolulu e trasferitosi a San Diego. ${ }^{8}$ La Border Art, in sintesi, stava via via assumendo un carattere globale anche nei suoi partecipanti, oltre che nei suoi destinatari. A ulteriore conferma di ciò, si sarebbe susseguito tra San Diego e Tijuana dal 1992 al 2005 il festival d'arte contemporanea InSite Specific: questo avrebbe permesso la convergenza di artisti provenienti da tutto il mondo sul confine e avrebbe consentito a questi di trattare anche tematiche relative al border, a prescindere dalla loro provenienza. Questa equivale alla terza fase della Border Art, quella che la Berelowitz definisce, per l'appunto, quella della 'globalizzazione' (Berelowitz 2003, 172) e che vede annullata la polemica su chi sia autorizzato o meno a trattare le tematiche frontaliere e su quali siano le istituzioni escluse o preposte alla promozione di un tale genere artistico.

Dovendo descrivere in sintesi e graficamente l'espansione degli attori della Border Art, si può dire che corrisponde a un cammino che assume i contorni e i tratti di un'enorme parabola, i cui vertici - al contrario dei muri di Colón Colonizado - Tutto è Mio - ¿De Quién? - proseguono allargandosi a simboleggiare una crescita e un progressivo allargamento.

Resta dunque da comprendere quale significato e quale ruolo abbia giocato la Biennale all'interno di questa crescita. Non dev'essere infatti casuale la coincidenza tra la fine dell'esperienza del BAW/ TAF e la sua incoronazione a gruppo artistico internazionale ottenuta in questa circostanza. La Biennale assume, a questo punto, un ruolo chiave, per comprendere il quale basta osservare la situazione attorno alla stanza buia del BAW/TAF. Oltre a Koons, infatti, esposero accanto ad essa artisti come Lorna Simpson e il Gran Fury, attenti a quelle tematiche, da me descritte in precedenza come border mentali: la sessualità, i pregiudizi sull'AIDS, il razzismo nei confronti donne nere americane... In sostanza, la sezione Aperto '90 agì da arena politica per una serie di problemi che in alcuni luoghi erano senz'altro più sentiti di altri, ma che, in fondo, erano condivisi a livello in-

8 San Diego (CA), University of California in San Diego (UCSD), Geisel Library, Special Collections \& Archives, InSite Archive, MSS 707, BOX 208, Folder 17, Michael Schnorr's curriculum. 
ternazionale. Questo venne perfettamente espresso dalle parole del curatore della rappresentanza statunitense di Aperto:

Osservando il lavoro degli artisti statunitensi possiamo delineare due fenomeni: la continua espansione dei parametri artistici in termini formali, così come di contenuto e tematica, e, forse in maniera più esplicita, la politicizzazione dell'arte e la preoccupazione per le problematiche sociali negli anni ottanta. La loro opera esemplifica l'ulteriore integrazione fra arte e politica, istanze sociali, economia e religione, un'arte che riesce a travalicare le frontiere del mondo artistico, in netta opposizione con la rimozione formalista dell'arte dal mondo "reale". (Shaerer 1990, 269)

Il comune denominatore tra gli artisti americani poteva essere individuato nella multimedialità e nella tendenza ad affrontare più tematiche allo stesso momento. Colón Colonizado - Tutto è Mio - ¿De Quién? fu esemplare in questo senso, ma più importante ancora fu la tensione politico/attivista che accomunò l'opera agli altri lavori. La politica, intesa come azione su problemi comuni, venne rispecchiata anche nella scelta degli artisti invitati ad Aperto: vi esponevano, infatti, centoquattro artisti, trentuno dei quali divisi in sette gruppi. La condivisione del processo artistico, dunque, annullava completamente il dogmatico rapporto tripartito autore-opera-spettatore. L'annullamento del singolo creatore andava allora a favore di un'opera riassuntiva che racchiudeva, oltre che più tematiche, anche più autori, approcci, punti di vista e linguaggi. Ancora una volta, la partecipazione del BAW/TAF risulta coerente, soprattutto se la si inserisce nella 'prospettiva internazionale' evidenziata da Shaerer (1990, 269-72). Era questa la propensione ad affrontare problemi comuni a più nazioni e a più persone, in altri termini: problemi politici.

Trattare temi politici spinge sempre a suscitare polemiche; farlo alla Biennale di Venezia significa lanciare benzina nel bracere di opinioni, critiche e discussioni che ogni due anni, dalla fondazione della mostra, brucia puntualmente in laguna. Il Grand Fury aveva infatti accostato un fallo all'immagine di Papa Giovanni Paolo II, Damien Hirst aveva sezionato una vacca e l'aveva immersa nella formaldeide suscitando le ire degli animalisti e degli ambientalisti. Più di tutti, però, fu Koons a suscitare scandalo con la scultura che ritraeva lui e la celebre sex symbol Cicciolina in esplicite pose sessuali. Il decennio si apriva pertanto con una serie di strascichi di poetiche e di problematiche che avevano alimentato gli anni Ottanta, ma adottava una nuova arte, militante e impegnata nel sociale.

La Biennale funzionò da perfetto teatro per mostrare il cambiamento, non soltanto dei parametri della Border Art, del rapporto artista-spazio e artista-confine, ma, soprattutto, del periodo politico che si presentava come il fotofinish cronologico del Vecchio Millennio. 
In estrema sintesi, nell'economia di questo lavoro si è avuto modo di osservare il modo in cui lo spazio 'terzo' della Biennale permise allo spazio binario del confine USA-Messico di diventare uno 'spazio mentale'. Per giungere a Venezia il border dovette essere dapprima de-costruito, poi ricostruito e infine reso applicabile a ogni contesto. Questo processo rispecchia l'espansione in ogni campo dell'esperienza umana del concetto di border e presenta la Biennale di Venezia del 1990 come un piccolo tassello che consentì, almeno alla Border Art, di seguire la medesima espansione a macchia d'olio. Infine, il BAW/TAF confermò che il problema dei confini non era affatto finito con Berlino, ma era ancora presente e pressante oltreoceano o, se non altro, che l'unico modo di non sentirsi oppressi da questi confini è sempre quello di affrontarli, di superarli, di voltarsi verso essi come faremmo davanti a un muro alle nostre spalle.

\section{Bibliografia}

Anzaldúa, Gloria (1987). Borderlands. La Frontera. The New Mestiza. San Francisco (CA): Aunt Lute Books.

Augé, Marc (1992). Non-lieux. Introduction à une anthropologie de la surmodernité. Paris: Seuil.

Barnett, Alan W. (1984). Community Murals. The People's Art. Philadelphia (PA): The Art Alliance Press.

Berelowitz, Jo-Anne (2003). «Border Art Since 1965». Dear, Michael; Leclerc, Gustavo (eds), Postborder City. Cultural Spaces of Bajalta California. New York: Routledge, 143-81.

Casey, Edward S.; Watkins, Mary (2014). Up against the Wall. Re-Imagining the U.S.-Mexico Border. Austin (TX): University of Texas Press.

Chávez, Patricio (1993). «Multi-Correct Politically Cultural». Chávez, Patricio; Grynsztejn, Madeleine; Kanjo, Kathryn (eds), La Frontera/The Border. Art About the Mexico/United States Experience. San Diego (CA): Centro Cultural de la Raza; Museum of Contemporary Art San Diego.

Davalos, Karen Mary (2001). Exhibiting mestizaje: Mexican (American) Museums in the Diaspora. Albuquerque (NM): University of New Mexico Press.

Di Martino, Enzo (2003). Storia della Biennale di Venezia 1895-2003: arti visive, architettura, cinema, danza, musica, teatro. Venezia: Papiro arte.

Gómez-Peña, Guillermo (1991). «Death on the Border: An Eulogy to Border Art». High Performance, 55, 16(3).

Greppi, Carlo (2019). L'età dei muri. Breve storia del nostro tempo. Milano: Feltrinelli Editore.

Kelley, Jeff (1990). «Border Art». Art Forum, March, 23-4.

Malagamba-Ansotegui, Amelia (2001). Tracing Symbolic Spaces in Border Art: de Este y del Otro Lado. Austin (TX): The University of Texas at Austin.

O'Doherty, Brian (1976). Inside the White Cube. The ideology of the Gallery Space. Santa Monica (CA): The Lapis Press.

Sánchez, Rita (2007). «Chicanas in the arts, 1970-1995: with Personal Reflections». Griswold del Castillo, Richard (ed.), Chicano San Diego. Cultural Space and the Struggle for Justice. Tucson (AZ): The University of Arizona Press. 
Andrea Masala «What kind of dirt do you need?» Da San Diego e Tijuana a Venezia: Border Art oltre i confini

Sheren Ila, Nicole (2005). Portable Borders/Mythical Sites: Performance Art and Politics on the US-Frontera, 1968-present. Cambridge (MA): Massachussets Institute of Technology.

Schnorr, Michael (1988). «Site Redefinition». The Border Art Workshop (BAW/ TAF) 1984-1989, A documentation of 5 years of interdisciplinary art projects dealing with U.S.-Mexico border issues (a binational perspective) - Tallér de Arte Fronterizo (BAW/TAF) 1984-1989, documentación de 5 años de proyectos de arte interdisciplinario sobre asuntos de la frontera de Estados Unidos con México (una perspectiva binacional). San Diego (CA): Border Art Workshop/ Tallér de Arte Fronterizo, 1988.

Zaccaria, Paola (2004). «Border Crossing». Cultural Studies. Dizionario degli studi culturali, 1-11. 


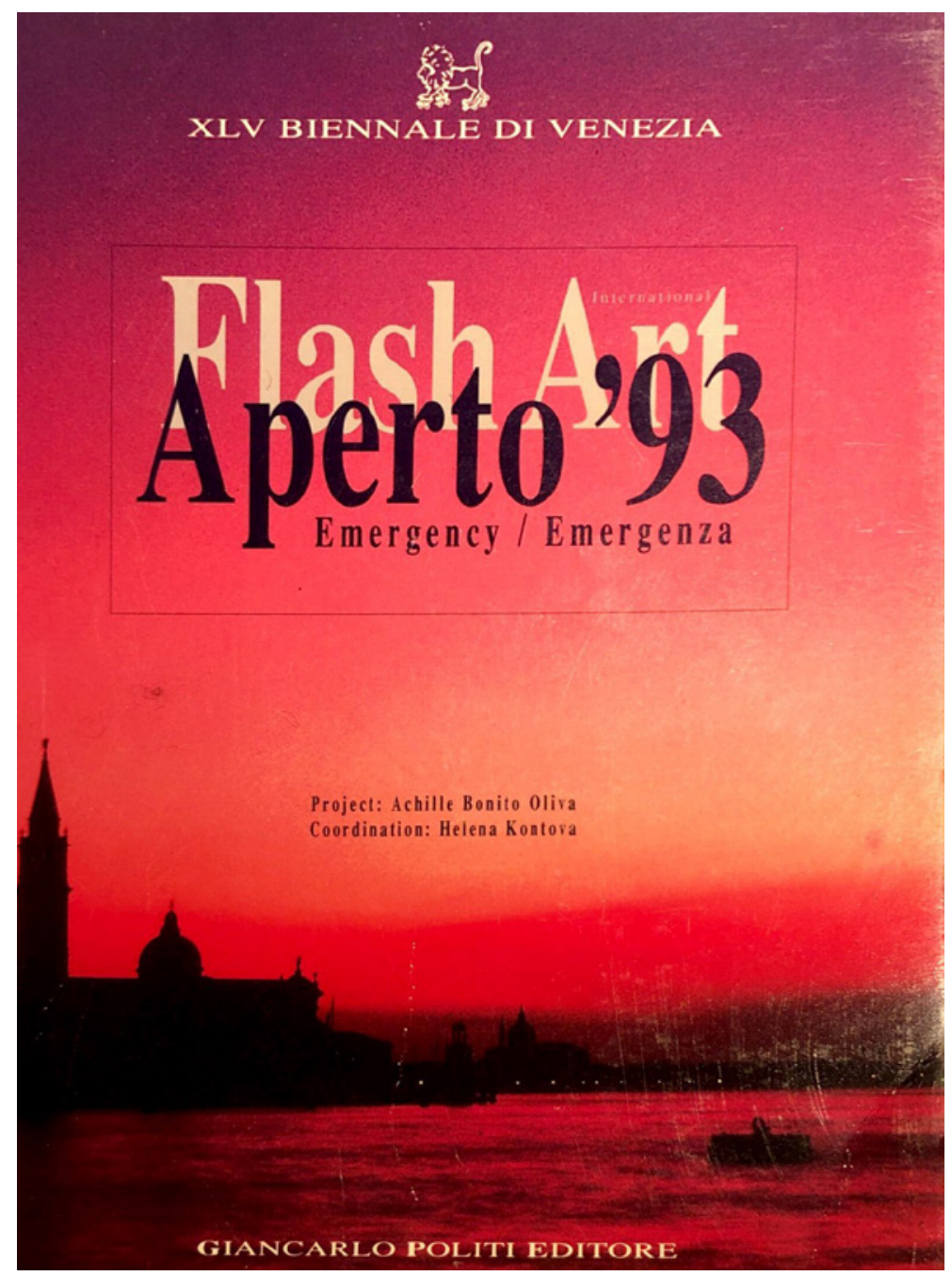

Flash Art. Aperto '93. Emergency/ Emergenza (1993). Courtesy Flash Art Editore 
«All We Are Saying Is Give Pizza Chance» L'effetto YBA e l'irruzione di una nuova generazione alle Biennali degli anni Novanta del Novecento

\author{
Diego Mantoan \\ Università Ca' Foscari Venezia, Italia
}

\begin{abstract}
The chapter addresses the rise of the Young British Artists (YBA) in their home country and their subsequent international spread during the 1990s as seen in connection with the Venice Biennale occurring between 1993 and 2003. Particularly, the author argues that the Aperto '93 section devoted to young artists at the Arsenale for the 45th Biennale was an unexpected starting point for the YBAs on the global stage and their first Venetian appraisal convinced home institutions such as the British Council to finance a large program of international exhibitions for this new artistic generation. Throughout the 1990s the Biennale thus became a major stage for the rising YBAs, which in turn deeply influenced the artistic language and installation practices shown at the Venetian exhibition. In conclusion, the chapter intends to present the 1990s at the Biennale as a decade of progressive struggle between different artistic generations - of both artists and curators - that climaxed with the 50th Biennale in 2003 and eventually shaped the new face of the Venetian exhibitions for the new millennium.
\end{abstract}

Keywords Young British Artists. Aperto '93. General Release. Achille Bonito Oliva. Francesco Bonami.

Sommario 1 Rivendicazioni generazionali alla soglia del nuovo millennio. - 2 La giovane Albione ribolle. - 3 Nuove sponde istituzionali in patria. - 4 Aperto '93 o il primo approdo internazionale . - 5 Giovani artisti britannici sull'altare di Venezia. - 6 La Biennale come specchio d'un avvicendamento generazionale.

Storie dell'arte contemporanea 4 | Atlante delle Biennali 1

ISSN 2704-9973

ISBN [ebook] 978-88-6969-366-3 | ISBN [print] 978-88-6969-367-0 


\section{Rivendicazioni generazionali alla soglia del nuovo millennio}

Vi è un curioso aneddoto lagunare su Sarah Lucas, fra gli astri della generazione di artisti britannici che negli anni Novanta vennero definiti young British artists, da cui il celebre acronimo YBA. Esattamente vent'anni prima di quel 2015, anno in cui il Padiglione britannico alla Biennale di Venezia scelse infine di dedicarle una mostra personale, l'artista fu già presente alla kermesse internazionale, ma soltanto come accompagnatrice del nutrito gruppo di coetanei e amici presenti nella compagine giovanile del suo paese per la mostra intitolata General Release del 1995. Tra questi figuravano vecchi compagni di studi al Goldsmiths come il pittore Gary Hume con cui la Lucas aveva intessuto una relazione lunga e autodistruttiva. L'episodio, riferito nelle sue memorie da uno dei curatori della mostra, Gregor Muir, riguarda una serata di euforia e alcol protrattasi fino a tarda notte e conclusa nelle calli ormai deserte del sestiere di San Marco. Alla Lucas parve il palcoscenico perfetto per intonare una nota canzone pacifista di John Lennon, storpiando la parola 'peace'. Finì per urlare a squarciagola: «All we are saying is give pizza chance!» (Muir 2011, 127). ${ }^{1}$ Forse ispirata dalle stereotipate specialità italiane, inconsciamente prese a modello la pizza, un alimento fortemente regionale assurto a simbolo di una internazionalità pervasiva. Un grido liberatorio, con cui la giovane artista esprimeva goliardicamente per la sua intera generazione il desiderio di essere riconosciuti a livello globale per la propria tipicità, sognava insomma che conquistassero il mondo dell'arte con la loro giovane e cruda Britishness.

Pur trattandosi di un simpatico aneddoto, questa presenza in laguna non fu affatto isolata, bensì la conseguenza di un progressivo avvicendamento fra generazioni di artisti e curatori sulla scena dell'arte internazionale - nonché persino del progressivo mutamento di sensibilità artistiche e più in generale del modo di intendere l'arte contemporanea. Un po' per caso e un po' per spudorata pianificazione, infatti, l'appuntamento biennale di Venezia si trasformò fra il 1993 e il 2003 - nonostante la delicata fase di trasformazione sul piano aziendale e istituzionale - in una perfetta cassa di risonanza per queste nuove aspirazioni e rivendicazioni generazionali, con diversi esponenti della nuova arte britannica a distinguersi fra i protagonisti. A dare il via quasi involontariamente a questa stagione fu l'ultima edizione della sezione Aperto nel 1993, voluta da Achille Bonito Oliva alle Corderie dell'Arsenale proprio per dare spazio alle giovani promesse dei vari paesi partecipanti alla sua Biennale. Fu in quell'oc-

1 La sonorità del cantato dava l'impressione di rendere la pronuncia «give pizza [a] chance», evocando Give Peace a Chance (1969). 
casione che dalla Gran Bretagna giunse un drappello di ventenni capitanato proprio da un Damien Hirst fresco del suo debutto allo ICA londinese ${ }^{2}$ e del provocatorio squalo in formalina alla Saatchi Gallery. ${ }^{3}$ In verità fu una partenza assai contestata dalla critica, che tuttavia convinse il British Council a investire massicciamente sugli YBA nelle successive edizioni della Biennale e, perfino, a perseguire poi una sistematica politica di diffusione con mostre collettive in numerose istituzioni museali nel continente europeo (Mantoan 2015, 30822). I frutti di questa tattica a Venezia furono l'acclamata collettiva General Release nell'anno del centenario della Biennale alla Scuola di San Pasquale, nonché due anni dopo la vittoria del premio come miglior giovane artista allo scozzese Douglas Gordon, infine la presenza di un enorme pillolario di Hirst quale pezzo centrale della 50esima Esposizione Internazionale nel 2003.

In questa sede si intende ripercorrere la strategia, più o meno consapevole, messa in campo per il consolidamento della reputazione internazionale dei giovani artisti britannici, facendo perno sulla Biennale. Pertanto, in questa disamina si passerà in rassegna il percorso che condusse gli YBA a irrompere sulla scena mondiale grazie alla Biennale, dapprima quasi inavvertitamente, ma poi fornendo un modello di propagazione delle innovazioni artistiche sfruttato dal British Council a partire dal 1995. Nel contempo, questa vicenda offre l'occasione per osservare come alla Biennale - nell'arco di un decennio compreso fra le edizioni di Bonito Oliva (1993) e Jean Clair (1995) fino alla cinquantesima di Francesco Bonami (2003), passando per le due rassegne firmate da Harald Szeemann - si sia consumato un progressivo stravolgimento delle priorità artistiche a livello globale. Tale cambiamento traghettò le arti visive dal ripiegamento degli anni Ottanta sul neoespressionismo alla proliferazione di nuovi media e alla prassi fra il neo-concettuale e il neo-pop, portando con sé un avvicendamento generazionale che coinvolse sia gli artisti che i curatori. Facendo ricorso a un ampio spettro di materiali e fonti, fra cui cataloghi, recensioni e documenti d'archivio, si vuole quindi illuminare una vicenda assai articolata e sinora poco approfondita, quella della graduale ribalta internazionale degli YBA che si rafforzò alla kermesse veneziana, e al tempo stesso far emergere come la Biennale negli anni Novanta del Novecento abbia funto da termometro della situazione globale registrando l'avvicendamento generazionale e forse addirittura la progressiva emersione di una nuova sensibilità artistica e curatoriale.

2 Il riferimento è alla mostra Internal Affairs all'Institute of Contemporary Arts di Londra nel 1993.

3 Il riferimento è all'opera The Impossibility of Death in the Mind of Someone Living (1992) presentata alla mostra collettiva Young British Artists 1 presso la Saatchi Gallery di Londra nel 1992. 


\section{La giovane Albione ribolle}

Prima d'addentrarsi nello studio del processo d'espansione nel mondo dell'arte che caratterizzò la Gran Bretagna negli anni Novanta, è d'uopo mettere in luce gli avvenimenti salienti che condussero un intero sistema-paese a scommettere su una nuova generazione artistica quale portatrice di una diversa immagine della Britishness, assai più cool e attraente di quella ingessata e noiosa a cui la perfida Albione era stata consegnata dall'austero periodo di Margaret Thatcher. Non può che stupire come nel giro di un decennio il Regno Unito fosse passato dall'impersonare una 'vecchia zia' - memorabile in tal senso è il pubblico ludibrio cui viene consegnata l'indole britannica nella celebre commedia A Fish Called Wanda (1988) - alla trasmutazione in un'autentica fucina delle ultime tendenze globali nei campi più disparati, a partire da quelli artistici e musicali. Invero, persino a una superficiale disamina storica si può notare come all'indomani della recessione iniziata con lo scoppio della bolla giapponese nel 1989 la Gran Bretagna avesse poi tentato il proprio rilancio economico a livello mondiale - più per opportunità che per strategia - appoggiandosi sempre più alle arti come fattore d'attrazione e di rinnovamento della propria immagine internazionale: per certi versi, il sensazionalismo originato dagli YBA in patria fu presto sfruttato globalmente per conferire alla nazione una nuova immagine, innovativa e alla moda (Stallabrass 2006a, 197-203). Per di più, fu uno stratagemma che negli anni Novanta accomunò indistintamente i governi conservatori e quelli laburisti: sia John Major che Toni Blair diedero grande importanza alle arti visive, moltiplicando gli eventi artistici e allocando ingenti fondi per la loro circolazione internazionale. All'apice di questa parabola, nel 1997, il ministro della cultura Virginia Bottomley giunse a esprimere la sua ferma convinzione che l'arte britannica fosse la più stimolante e innovativa al mondo. ${ }^{4}$ Senza dubbio si tratta di un'affermazione tendenziosa e grossolana, eppure indicativa della fede che l'establishment britannico di quel decennio ripose nelle arti - fra cui confluivano a pari merito gli YBA, la moda, la musica pop e la cinematografia - quale fondamentale asset per il rafforzamento dell'immagine del paese: attraverso la massiccia esportazione artistica si sarebbe così contribuito a ridefinire la Britishness in un tal modo da favorire non solo il trionfo sui mercati artistici, bensì il più generale rilancio economico del paese (Stallabrass 2006a, 198). La generazione dei giovani artisti britannici funse pertanto da propulsore di questo periodo, ma si trovò per certi versi al posto giusto nel momento giusto, dopo essersi fatta le ossa per anni fra gallerie autogestite sparse per il regno e mostre improvvisate in capannoni abbandonati dei

4 George Walden, «Leave your weapons at the door. Democracy, State modernism and the official embrace of the arts», London Review of Books, 26 September 1997, 10-11. 
Docklands della capitale inglese. ${ }^{5}$ Invero, l'avvio di questa rivalsa generazionale può essere convenientemente collocato nell'estate del 1988, quando un nutrito gruppo di studenti del Goldsmiths College di Londra guidato da Damien Hirst, Abigail Lane ed Angus Fairhurst si ritrovò a organizzare tre esposizioni consecutive intitolate Freeze nell'edificio svuotato - e rimesso a nuovo dagli stessi giovani rampanti fra cui Sarah Lucas, Tracy Emin, Simon Patterson e Gary Hume - della London Port Authority presso gli Surrey Docks (Cooper 2012, 24). Da subito quest'evento si configurò come modello organizzativo, più che artistico, da replicare ad libitum al fine d'esporsi precocemente ed efficacemente agli occhi dell'establishment londinese. ${ }^{6}$ Seguirono infatti altre imprese degli stessi protagonisti, in primis l'operazione Building One del 1990 alla Peak Freans Factory di Bermondsey, ossia le tre mostre Modern Medicine, Gambler e Market curate sempre da Hirst assieme a Carl Freedman e Billee Sellman, le quali attirarono l'attenzione definitiva del magnate e collezionista Charles Saatchi, nonché del futuro co-fondatore della rivista Frieze Matthew Slotover (Serota 2011, 93). A lasciare entrambi stupefatti fu in particolare l'installazione $A$ Thousand Years (1991) di Hirst, una doppia vetrina con mosche vive e testa di vacca in decomposizione. Vi furono però anche insuccessi, quali l'East Country Yard Show organizzato da Sarah Lucas ed Henry Bond nello stesso anno a poca distanza negli South Docks. ${ }^{7}$ In retrospettiva, i cosiddetti warehouse show nel periodo compreso fra il 1988 e il 1990 furono certamente strumentali all'emersione e alla successiva legittimazione degli YBA, ma l'efficacia di questo modo di porsi non era affatto scontata in partenza (Craig-Martin 2012, 39). Nonostante gli alti e bassi, gli eventi in spazi alternativi di quegli anni - specie quelli originati dal gruppo originario del Goldsmiths College - finirono per catturare l'attenzione di critici ed esperti, fra cui Sacha Craddock (The Guardian), Sarah Kent (Time Out), Andrew Renton (Blitz), Kate Bush (Artscribe), David Batchelor e Adrian Searle (Art Monthly). Un esempio autorevole dell'accettazione che andavano maturando questi giovani artisti fu l'intervento di Andrew Graham-Dixon sulle pagine de The Independent nel luglio 1990, in cui lodò alquanto lo spirito di iniziativa e la competenza in fatto di allestimento dimostrata da Hirst e sodali:

Goldsmiths graduates are unembarrassed about promoting themselves and their work: some of the most striking exhibitions in London over the past few months - The East Country Yard Show, or Gambler, both staged in Docklands - have been independently organised

5 Per una disamina più completa di avvenimenti e protagonisti dell'ondata giovanile nel Regno Unito a cavallo del 1990 si rimanda a Mantoan 2017.

6 Matthew Collings, «The Hirst effect», Telegraph Magazine, 21 September 1996.

7 David Lillington, «The East Country Yard Show», Time Out, 18 June 1990. 
and funded by Goldsmiths graduates as showcases for their work. This has given them a reputation for pushiness, yet it should also be said that in terms of ambition, attention to display and sheer bravado there has been little to match such shows in the country's established contemporary art institutions. They were far superior, for instance, to any of the contemporary art shows that have been staged by the Liverpool Tate in its own multi-million-pound dockland site. ${ }^{8}$

La narrazione più diffusa riguardo all'ascesa degli YBA ha spesso teso a incentrare il racconto sugli avvenimenti nella capitale inglese, sorvolando sul pur vivace fermento in altri centri artistici del Regno Unito. Siano essi memorie dettagliate di alcuni giovani curatori dell'epoca come Gregor Muir (2011), Matthew Collings (1997) e James Roberts (1995), oppure studi accademici anche assai critici quale il fondamentale contributo di Julian Stallabrass (2006a), nei testi di riferimento sul periodo la piazza londinese la fa sempre da padrona, minimizzando invece le energie sprigionate nella vorticosa proliferazione di gallerie alternative o autogestite in tutta l'isola. A differenza dei warehouse show nei Docklands infatti, realizzati come eventi unici per attrarre nell'immediato l'attenzione della stampa e dell'establishment artistico, nei primi anni Novanta sorgevano cosiddetti artist-run spaces, spazi gestiti da giovani artisti in numerose città britanniche e intesi quali luoghi per sviluppare un discorso artistico più articolato e duraturo, utile per connettere la realtà artistica locale con analoghe esperienze nazionali ed estere (Mantoan 2017, 74-5). Fra le gallerie autogestite di maggior rilievo si contavano all'epoca Transmission di Glasgow, Collective e New 57 di Edimburgo, Catalyst Arts a Belfast, Locus+ a Newcastle upon Tyne e The International 3 di Manchester, mentre nella stessa Londra proliferavano City Racing, Clove nei pressi di Butler's Wharf, Cubitt a King's Cross, Infanta of Castile, Matt's, Milch Gallery a Bloomsbury e infine Nosepaint (poi chiamata Beaconsfield). Questa situazione di fermento giovanile non può essere sottovalutata, bensì rappresenta il brodo di coltura dell'intera generazione YBA nei primi anni Novanta, poiché numerosi odierni protagonisti della scena artistica mondiale si muovevano proprio nelle esposizioni realizzate da amici e sodali in questi spazi autogestiti - basti pensare alle prime mostre personali concesse ad artiste quali Sarah Lucas, Gillian Wearing e Fiona Banner negli spazi di City Racing (Burgess et al. 2002, 1), oppure alle numerose iniziative pilotate dalla Transmission di Glasgow in cui figuravano futuri vincitori del Turner Prize come Douglas Gordon, Martin Boyce e Martin Creed (Lowndes 2010, 117-39; Richardson 2011, 133-56). Proprio la piccola galleria autogesti-

8 Andrew Graham-Dixon, «The Midas Touch? Graduates of Goldsmiths School of Art dominate the current British art scene», The Independent, 31 July 1990. 
ta nella città scozzese avviò a partire dal 1989 nella nuova sede sulla King Street un percorso di avvicinamento al mondo dell'arte internazionale attraverso la creazione di un gruppo giovanile artisticamente coerente e umanamente coeso $^{9}$ che attrasse presto l'attenzione di mentori d'eccezione quali Lawrence Weiner, Alan Johnston e Thomas Lawson (Mantoan 2015, 159). L'antagonismo fra Londra e Glasgow fu presto notato dai critici più attenti e già nel 1991, sulle pagine della neonata rivista Frieze, venne dedicato un lungo servizio a una mostra collettiva auto-organizzata nell'abbandonato Seamen's Institute in Scozia, dal titolo Windfall 1991, che venne celebrata quale modello alternativo alla prassi dei warehouse show londinesi e sintomo di un'effervescenza giovanile geograficamente assai più vasta della sola capitale inglese (Slotover 1991, 40-1). La testimonianza di Andrew Cross delle James Hockey Galleries suonava in proposito come un elogio esplicito e al tempo stesso come riconoscimento di una compagine artistica che si andava articolando già agli inizi degli anni Novanta:

What I liked about the Windfall project is that although the work individually may not have been the best pieces by each artist, the whole event had a freshness, a relaxed attitude which I found very positive. Dare I say it, in some of the most recent initiatives in London there has been an over-emphasis on presentation. Which there wasn't in this case. [...] Up there, there was a greater willingness to all work together. ${ }^{10}$

\section{$3 \quad$ Nuove sponde istituzionali in patria}

Sempre nel 1991, l'inclusione di tre artisti ventenni fra i quattro finalisti del Turner Prize, appena riformato dal nuovo direttore della Tate Nicholas Serota, fu il segnale che oltre alla critica pure le grandi istituzioni del paese stavano puntando il radar verso questa generazione emergente (Morgan 1991, 5). Rachel Whiteread, Ian Davenport e Fiona Rae ${ }^{11}$ non avevano certo chance di vittoria contro il ben più ac-

9 Quell'anno il testimone nel comitato direttivo passò a un gruppo di amici appena diplomati presso l'Environmental Art Department della Glasgow School of Art, fra cui figuravano Dave Allen, Christine Borland, Billy Clark, Douglas Gordon e Craig Richardson, mentre più tardi furono coinvolti anche Martin Boyce, Katrina Brown, Roderick Buchanan ed Elsie Mitchell.

10 Andrew Cross in Slotover 1991, 41.

11 Si noti che Ian Davenport e Fiona Rae furono fra gli studenti del Goldsmiths che parteciparono nel 1988 a Freeze e furono ivi notati, nonché immediatamente rappresentati da importanti galleristi londinesi. Inoltre Fiona Rae era stata presente alla Biennale del 1990 nella sezione Aperto, pertanto fu di fatto la prima esponente degli YBA a partecipare alla kermesse veneziana. 
clamato Anish Kapoor, appena di ritorno dalla sua personale al Padiglione britannico alla Biennale del 1990, ma la loro presenza rendeva plastica e inequivocabile la direzione che avrebbe preso il Regno Unito in quel decennio, a cui proprio il rinnovato format televisivo del Turner Prize contribuì in maniera fondamentale (Stallabrass 2006a, 1845). Invero, già nel 1992 Damien Hirst figurò fra i finalisti, grazie allo scalpore suscitato dal suo squalo in formalina realizzato per la prima mostra alla Saatchi Gallery intitolata Young British Artists $1,{ }^{12}$ mentre nel 1993 alla Whiteread riuscì il colpo grosso alla Tate sull'onda delle controversie per la sua scultura pubblica di quell'anno intitolata House (Muir 2011, 44-5). Fatta eccezione per il successo di Antony Gormley nel 1994, tutte le successive edizioni del Turner negli anni Novanta furono dominate da artisti riconducibili agli YBA consacrando il progressivo monopolio di attenzione artistica e mediatica di questa generazione.$^{13} \mathrm{Il}$ risultato fu inoltre una drastica riduzione dell'età anagrafica degli artisti finalisti e un autentico exploit di presenze di pubblico alla Tate, nonché sul medio periodo la massiccia esportazione a livello globale degli YBA (Cooper 2012, 153). ${ }^{14} \mathrm{~A}$ dare il via a questa rapida ascesa furono dunque diversi eventi nel corso del 1993, quando potè in effetti dirsi conclusa la fase germinale degli YBA, ormai stabilmente inseriti sotto ai riflettori del sistema dell'arte nazionale.

Invero, prima del trionfo della Whiteread a fine 1993, i mesi precedenti erano stati costellati da eventi che indicavano la progressiva e fondamentale legittimazione di numerosi giovani protagonisti da parte del mercato artistico britannico. Fra i segnali più evidenti si debbono annoverare le mosse di tre rilevanti soggetti della piazza londinese: lo spregiudicato magnate Charles Saatchi, il decano dei galleristi

12 La numerazione nel titolo della mostra presumeva ve ne sarebbero state altre, invero ne fu organizzata una all'anno fino alla controversa e iper-popolare mostra collettiva Sensation del 1997 alla Royal Academy.

13 I vincitori furono Damien Hirst nel 1995, Douglas Gordon nel 1996, Gillian Wearing nel 1997, Chris Ofili nel 1998 e Steve McQueen in 1999. Inoltre, fra il 1996 e il 1999 tutti i finalisti erano riconducibili alla nuova generazione artistica, fra cui: nel 1996 Douglas Gordon, Craigie Horsfield, Gary Hume e Simon Patterson; nel 1997 Christine Borland, Angela Bulloch, Cornelia Parker e Gillian Wearing; nel 1998 Tacita Dean, Cathy de Monchaux, Chris Ofili e Sam Taylor-Wood; nel 1999 Tracey Emin, Steve McQueen, Steven Pippin, Jane e Louise Wilson.

14 Si propongono in merito i dati ufficiali:

Età media dei finalisti del Turner Prize (Edizione: $\Delta$ età) 1984: 44; 1985: 46; 1986: 43; 1987: 39; 1988:45; 1989: 50; 1991: 30; 1992: 37; 1993: 37; 1994: 38; 1995: 36; 1996: 35; 1997: 35; 1998: 33.

Presenza di pubblico alla Tate Gallery di Londra (anno: visitatori) 1980: 1.330.937; 1981: 885.168; 1982: 1.219.102; 1983: 1.270.925; 1984: 1.265.605; 1985: 980.105; 1986: 1.137.070; 1987: 1.725.084; 1988: 1.581.467; 1989: 1.234.281; 1990: 1.562.431; 1991 : 1.816.421; 1992: 1.575.637; 1993: 1.760.091; 1994: 2.226.399; 1995: 1.769.662; 1996: 2.203.001; 1997: 1.757.735.

Fonte: dati dal sito ufficiale www.tate.co.org rielaborati dall'autore 
londinesi Nicholas Logsdail e il neofita di successo Jay Jopling. In primis, nel 1993 vi fu la seconda mostra interamente dedicata agli Young British Artists alla Saatchi Gallery, la quale indicava la serietà delle nuove intenzioni e ambizioni manifestate dal collezionista, anche per risalire la china dopo il necessario smantellamento della sua precedente raccolta di opere. ${ }^{15}$ Nel corso delle cinque mostre organizzate fra il 1992 e il 1996 nei suoi spazi a Saint John's Wood Saatchi fu capace di articolare una strategia di massiccia sovraesposizione mediatica dei suoi giovani protetti come mezzo per lanciarli rapidamente nell'olimpo dell'arte, riuscendo inoltre a conferire il nome a un'intera generazione artistica, ben oltre ai singoli autori che crebbero sotto la sua egida (Muir 2011, 121-3). ${ }^{16}$ La definizione Young British Artists - non ancora l'acronimo YBA, tuttavia, che entrò in uso soltanto dal 1995 - si estese a macchia d'olio inglobando una serie di artisti e raggruppamenti assai eterogenei e presenti su tutto il territorio del Regno Unito, fra cui la compagine di Glasgow che, nonostante si sentisse in contrapposizione con Londra, salì definitivamente agli onori della cronaca nazionale con la presentazione della video-installazione 24 Hour Psycho di Douglas Gordon a maggio del 1993 presso la Tramway Gallery della città scozzese (Lowndes 2010, 174).

Ad accorgersi di questa ampia compagine giovanile giunse lo stesso anno pure il rispettato e potente gallerista Nicolas Logsdail, patron di Lisson Gallery, dove il 17 luglio si inaugurò Wonderful Life che costituì la prima estensiva collettiva degli YBA in un'autentica istituzione del mercato artistico londinese, andando così a rafforzare la loro reputazione anche nei confronti di altri attori pubblici e privati, come collezionisti e musei. ${ }^{17}$ Scorrendo l'elenco degli artisti partecipanti, tuttavia, si può notare come Logsdail avesse optato per una linea legata alle indagini concettuali e processuali a lui molto care, nonché contraddistinta da una raffinatezza di fondo, evitando cioè lo scalpore a tutti i costi che caratterizzava invece le scelte della Saatchi Gallery (Muir 2011, 125). Pur non presentando opere nuove né radical-

15 A questo proposito è utile ricordare che il 1991 era stato l'annus horribilis di Charles Saatchi, poiché la sua agenzia di comunicazione aveva perso 64 milioni di sterline e lo colpì anche il divorzio dalla prima moglie Doris Lockhart, costringendolo a liquidare tramite Sotheby's buona parte della collezione di opere che aveva accumulato nel corso degli anni Ottanta, tanto che la scelta di optare per artisti autoctoni e giovani va in parte letta come soluzione di ripresa a basso costo (Stallabrass 2006a, 206-7).

16 Nel 1992 Saatchi presentò opere di Rachel Whiteread, John Greenwood, Alex Landrum, Langlands \& Bell e Damien Hirst, mentre nel febbraio 1993 fu il momento di Rose Finn-Kalcey, Sarah Lucas, Marc Quinn e Mark Wallinger. L'anno successivo espose lavori di Simon Callery, Simon English e Jenny Saville, quindi nell'aprile del 1995 mise in mostra John Frankland, Marcus Harvey, Brad Lochore, Marcus Taylor e Gavin Turk (Rose et al. 1995, 80-102).

17 La mostra si tenne dal 17 luglio al 16 ottobre del 1993 presso la sede di Lisson Gallery a Londra. 
mente innovative, vi esposero futuri finalisti o vincitori del Turner Prize quali Christine Borland, Martin Creed, Liam Gillick, Douglas Gordon, Damien Hirst, Gary Hume e Simon Patterson, creando così un'originale miscela fra protagonisti della scena londinese e di quella di Glasgow (Lowndes 2010, 174). ${ }^{18}$ Un ulteriore aspetto di rilievo fu poi la scelta del curatore, il giovane critico e giornalista di Frieze James Roberts, il quale era coetaneo degli YBA e da sempre loro grande sostenitore, oltre che fautore di quella che definiva una svolta pop giovanile nell'arte britannica, tanto da difendere a spada tratta pure le provocazioni dei fratelli Dinos e Jake Chapman (Roberts 1996, 32). Prima con la nascita della rivista Frieze, poi con la promozione di Roberts a curatore per una galleria riconosciuta, la compagine giovanile andava così arricchendosi di sostenitori e nuovi entranti nei diversi ruoli del sistema dell'arte britannico (Stallabrass 2006a, 122).

Di lì a breve fu il momento della comparsa sulla scena londinese di un nuovo e ambizioso gallerista, Jay Jopling, il quale iniziò sempre nel 1993 la sua rapida ascesa con uno spazio pensato per promuovere proprio i giovani protagonisti degli YBA, tanto da offrire a Hirst, Hume, Emin e altri le loro prime mostre personali, nonché affiancandovi un indefesso lavorio mediatico: il White Cube divenne presto la galleria distintiva degli anni Novanta in Gran Bretagna e venne a trovarsi nell'epicentro mondiale dell'onda di propagazione giovanile originata dagli YBA (Muir 2011, 75). La raffigurazione plastica della svolta avvenuta nel mercato artistico britannico la si ebbe sempre quell'anno alla fiera di Colonia, dove parteciparono un numero senza precedenti di vecchie e nuove gallerie londinesi: ben dieci furono gli esercizi storici presenti nella sezione principale - fra cui Victoria Miro, Saatchi Gallery e Lisson Gallery con opere di esponenti degli YBA - mentre le quattro gallerie emergenti e dedite interamente alla nuova generazione - ossia Interim Art, White Cube, Cabinet Gallery e Karsten Schubert - si presentarono nella sezione aggiunta UnFair (Muir 2011, 88). Per di più, nel cuore del Padiglione centrale Charles Saatchi portò il meglio della sua nuova collezione presentandola con l'inequivocabile titolo Junge Britische Kunst aus der Sammlung Saatchi, ivi inclusi lo squalo in formalina di Hirst e Ghost della Whiteread, ossia il calco in gesso d'un salotto vittoriano. Qualora ci fosse stato bisogno di conferme del fatto che il mercato artistico britannico si fosse ormai schierato alle spalle degli emergenti YBA, la fiera di Colonia del 1993 fugò ogni dubbio e preparò il campo all'imminente invasione globale di questa nuova generazione.

18 A questi si aggiungevano Terrence Bond, Roderick Buchanan, Adam Chodzko, Graham Fagen, Thomas Gidley, Marysia Lewandowska, Jason Martin. 


\section{Aperto '93 o il primo approdo internazionale}

A ben guardare, il fermento giovanile e quella generale aria di rinnovamento che caratterizzavano i primi anni Novanta si era manifestato già alla documenta del 1992, per la quale il curatore Jan Hoet aveva stabilito un'apertura senza precedenti a nuovi media e linguaggi (Schwarze 2012, 144). Fra tutti spiccava senza dubbio il ricorso a grandi videoinstallazioni, come la disturbante camera di proiezione realizzata per Anthro/Socio (1992) di Bruce Nauman, in cui la voce assordante e la testa rotante del performer ripreso dall'artista texano aggredivano fisicamente lo spettatore (Bruggen 2002, 66). A gran merito di questa edizione di documenta andò l'inclusione di produzioni video di tipo pseudo-narrativo ben più fresche e visionarie rispetto alle sperimentazioni tecnologiche dei pionieri della video arte. In quest'ultimo senso colpiva l'opera di un giovanissimo Matthew Barney, una sorta di epopea onirica dal titolo OTTOshaft che seguiva l'allenamento volutamente massacrante del campione di football Jim Otto per raggiungere una personalissima hybris, il cui stile di ripresa pareva una reminiscenza estetizzante della cultura giovanile formatasi sui videoclip musicali (Bonami 2009, 79-82). Nonostante lo iato generazionale fosse ben presente nella rassegna di Kassel, esso era diluito e ben amalgamato fra le sale del Fridericianum e le altre sedi espositive.

L'anno seguente, con la realizzazione di Aperto '93 alle Corderie dell'Arsenale, a Venezia si colse invece l'occasione per presentare in maniera esclusiva e organica la compagine giovanile che pareva far breccia a livello internazionale. Più che il risultato di un'operazione pianificata, Aperto '93 fu innanzitutto il frutto di una brillante intuizione da parte di Achille Bonito Oliva, il quale voleva rinverdire per l'ultima volta questa sezione esterna alla mostra principale offrendo un palcoscenico alle generazioni emergenti. D'altra parte la mostra emerse anche grazie a una parziale sottovalutazione della spinta propulsiva che molti giovani artisti stavano profondendo: non ci si aspettava insomma nulla di eclatante, almeno non se lo aspettava chi non conosceva il fermento generazionale che stavano vivendo città come New York e Londra (Kontova 1995, 61). Infine, la vera chiave del successo di questa sezione, per molti versi anticipatrice delle nuove tendenze generali e di specifici artisti emergenti, fu l'assetto multi-curatoriale che essa stessa si diede (Obrist 2017). Aperto era stata lanciata dallo stesso Bonito Oliva oltre dieci anni prima, nel 1980, intendendola come compartimento sperimentale della Biennale, del tutto libero e slegato dalle tendenze più consolidate e ingessate del mondo dell'arte (Vecco 2002, 33). Sotto la direzione della XLV edizione dell'Esposizione Internazionale di Bonito Oliva, nel 1993 per l'appunto, la sezione fu dedicata ad artisti sotto ai quarant'anni. Il motivo può forse essere ricercato proprio nell'ipotesi che Bonito Oliva, 
una volta diventato direttore della sezione principale, ritenesse la sua Biennale comunque già la continuazione del lavoro di ricerca da lui profuso per Aperto negli anni precedenti. Di conseguenza, nei suoi intenti la rimanente sezione Aperto '93 non poteva che distinguersi in maniera sostanziale dalla mostra principale, pertanto la divisione generazionale poteva apparire la misura di differenziazione più immediata e logica. Per certi versi era anche la suddivisione meno insidiosa per il curatore della Biennale, poiché ipoteticamente i giovani artisti di Aperto '93 avrebbero avuto minore possibilità di mettere in ombra i colleghi più acclamati della sezione principale. Si può presumere, insomma, che la soluzione paresse ideale poiché era la meno concorrenziale fra Aperto e la sezione principale, così da non caricare i giovani artisti di troppe aspettative, nonché al tempo stesso per evitare di mescolare autori di età e livelli di carriera disomogenei. Che le cose stessero così è possibile desumerlo dal disappunto con cui Bonito Oliva accolse poi la notizia inaspettata che la giuria internazionale avesse scelto di conferire un premio a un artista proposto da un giovane Francesco Bonami nella sezione Aperto '93: si trattava nella fattispecie di Matthew Barney, assente il giorno della premiazione, ma il cui riconoscimento non venne fatto ritirare a Bonami su interdizione, pare, dello stesso Bonito Oliva. ${ }^{19}$

Aperto '93 si dimostrò un banco di prova internazionale per la generazione di artisti e curatori emergenti, i quali risultarono favoriti da una sottovalutazione iniziale da parte dell'establishment artistico più maturo, tanto da amplificare l'effetto novità che osservatori più giovani non trovarono invece affatto sorprendente (Verzotti 1993, 104). L'ingrediente segreto di quest'esperienza fu invero la scelta iniziale di affidare la curatela di Aperto '93 alla direttrice di Flash Art Helena Kontova, dunque a una critica e scrittrice - e non a una curatrice - che ritenne opportuno coinvolgere un gran numero di colleghi per offrire una rappresentazione fedele della molteplicità di sfaccettature nel mondo artistico globalizzato dell'epoca (Obrist 2017). L'idea di fondo era dunque che la complessità del mondo contemporaneo e della sua moltitudine di voci non potesse essere rappresentata attraverso l'espressione di un curatore unico, la cui visione veniva impressa o perfino imposta sugli altri: non pare un caso che dieci anni più tardi, nel 2003, questa stessa filosofia venisse applicata da Bonami alla sua 50esima Esposizione Internazionale col ricorso alla collaborazione con tredici altri curatori. In un'intervista rilasciata a Hans Ulrich Obrist, Kontova ricorre alla metafora della rivista per illustrare la struttura conferita ad Aperto '93:

19 A questo proposito si confronti il resoconto parziale fornito dallo stesso Francesco Bonami in Curator: Autobiografia di un mestiere misterioso (2014). 
deve trattarsi di qualcosa di più simile al numero di una rivista. Il numero di una rivista è una piattaforma polifonica. Allo stesso modo "Aperto '93" offriva una prospettiva plurale e un angolo di mondo nella sua autenticità. Non era più un solo curatore o un team curatoriale a confrontarsi sul concetto della mostra e su chi invitare a parteciparvi, ma si lasciava a tanti curatori la libertà di rappresentare ciascuno la propria idea. Nella sua dinamica curatoriale, la mostra incarnava il concetto deleuziano di rizoma, come opposizione alla 'teologia' del centro e dell'uno occidentale. ${ }^{20}$

Proprio il principio della rivista d'arte guidò Kontova, assieme al patron di Flash Art Giancarlo Politi, a selezionare curatori che non erano affatto tali, ossia non curatori puri, bensì esperti impegnati a diverso titolo nel commentare, organizzare o fare arte. ${ }^{21}$ Scelsero pertanto personalità di varia estrazione professionale quali redattori di riviste (Francesco Bonami e Matthew Slotover) e critici (Benjamin Weil, Nicolas Bourriaud, Robert Nickas e Berta Sichel), nonché un artista (Thomas Locher) e persino un gallerista (Jeffrey Deitch). A dire il vero, la critica non colse tanto la novità di questa polifonia curatoriale di per se stessa, bensì rimase colpita dai suoi effetti sia per la scelta degli artisti partecipanti, sia per la fluidità con cui vennero riempiti gli spazi delle Corderie (Verzotti 1993, 106). Scorrendo l'elenco degli artisti partecipanti, si possono trovare già quasi tutti i futuri protagonisti del mondo dell'arte internazionale: oltre a Matthew Barney erano presenti per la prima volta in una grande mostra internazionale come la Biennale Damien Hirst, Maurizio Cattelan, Rudolf Stingel, Gabriel Orozco, Rirkrit Tiravanija, Pipilotti Rist, John Currin, Andrea Zittel, Félix González-Torres, Renée Green, Carsten Höller, Sylvie Fleury e molti altri ancora, con una notevole presenza femminile (cf. Bonito Oliva, Kontova 1993). Le opere più acclamate e controverse di questa rassegna furono senza dubbio la mucca con vitello dimezzati in formalina di Hirst, che nel 1995 gli valsero la vittoria al Turner Prize, ${ }^{22}$ oppure la tavola calda allestita da Tiravanija

20 Kontova in Obrist 2017.

21 Il principio della rivista d'arte guidò a tal punto la curatela della mostra che il catalogo risultò infine in un numero speciale di Flash Art, un fatto che creò gravi problemi con l'editore del catalogo principale, il quale avendo l'esclusiva ottenne in un primo momento il sequestro della rivista (Obrist 2017).

22 Il riferimento è all'opera Mother and Child (Divided) del 1993, poi esposta alla finale del Turner Prize nel 1995, in cui Hirst risultò vincitore battendo l'altra favorita Mona Hatoum. A Venezia l'opera fu contestata aspramente degli animalisti per l'utilizzo di una mucca e un vitello segati poi a metà per essere posti in quattro teche riempite di formalina. 
memore delle sue opere relazionali nelle gallerie newyorkesi, ${ }^{23}$ nonché lo spazio pubblicitario venduto da Cattelan in sostituzione della sua opera ${ }^{24}$ e il formicaio a forma di bandiera sovietica di Yukinori Yanagi ${ }^{25}$ (Obrist 2017). In generale le opere presentate lungo gli spazi delle Corderie apparvero fresche e mai banali, seppur talvolta ancora fragili dal punto di vista formale, tanto che Giorgio Verzotti non lesinò complimenti sulle pagine di Artforum promuovendo senza indugi l'intera sezione e ribattezzandola inequivocabilmente «The better Biennale» (Verzotti 1993, 105).

In questa Biennale migliore si fece notare la massiccia presenza di giovani artisti britannici grazie alle scelte operate da Matthew Slotover e Benjamin Weill, il primo fondatore della rivista Frieze e il secondo una delle sue penne di punta, entrambi apertamente schierati a favore della generazione emergente dalla stagione dei warehouse show. Assieme invitarono ben otto artisti ascrivibili agli YBA: Christine Borland, Henry Bond, Angela Bulloch, Mat Collishaw, Damien Hirst, Simon Patterson, Julie Roberts e Georgina Starr (Rose at al. 1995, 88). La compagine di artisti britannici era dunque dominata da londinesi, di cui molti provenienti dal gruppo originario del Goldsmiths College. La vera novità erano invece due artiste scozzesi, Borland e Roberts, le quali provenivano entrambe dalla galleria autogestita Transmission di Glasgow. Invero, nell'opinione di Slotover che aveva seguito le vicende attorno alla città scozzese fin dai primordi nel 1991, Glasgow era l'unico luogo al di fuori di Londra che avesse dato vita a un fermento giovanile capace di catturare l'attenzione internazionale, pertanto la città andava necessariamente rappresentata ad Aperto '93 (Lowndes 2010, 179). Borland, che quattro anni dopo sarebbe figurata fra i finalisti del Turner Prize nel 1997, presentò a Venezia un'opera molto apprezzata, intitolata A Lifetime of Love (1993) e composta da una pila di coperte di lana cucite all'uncinetto, le quali intendevano tematizzare la strisciante sopraffazione sessista che si cela dietro alle attività casalinghe o tradizionalmente attribuite alle donne (Lowndes 2010, 187). L'inclusione delle due artiste scozzesi fu il prodromo di una costante associazione delle compagini giovanili di Londra e Glasgow su cui il British

23 Il riferimento è alla performance realizzata nel 1990 alla Paula Allen Gallery e poi nel 1992 per la 303 Gallery a Manhattan, in cui Tiravanija realizzò una specie di tavola calda dove cucinava per amici, visitatori, curiosi e li invitava poi a tessere relazioni attorno al tavolo della mensa.

24 Provocatoriamente Cattelan scelse infatti di vendere il proprio spazio alle Corderie a una nota ditta di cosmetici, la quale lo utilizzò come spazio pubblicitario, dunque per apporvi cartelloni di richiamo commerciale.

25 Il riferimento è all'opera The World Flag Ant Farm Project (1990) che fu altrettanto contestato dagli animalisti a Venezia per via dell'utilizzo di formiche vive, le quali tuttavia chiuse nella teca alla fine morivano per inedia. 
Council negli anni a venire costruì una politica di diffusione internazionale della nuova arte britannica, consentendo alla capitale inglese di tornare al centro del mondo dell'arte, mentre la città scozzese divenne all'improvviso - per rimanerlo tuttora - un nuovo punto di riferimento nella geografia artistica contemporanea (Richardson 2011, 156). Ad ogni modo, il nutrito gruppo di giovani artisti britannici fu indubbiamente decisivo per il successo di Aperto '93, specie in considerazione della capacità di queste ragazze e questi ragazzi nell'utilizzo di edifici industriali abbandonati per trasformarli in contenitori d'arte. In fin dei conti, le antiche Corderie dell'Arsenale non erano tanto dissimili dai capannoni caduti in disuso nelle periferie urbane e che si erano dimostrati luoghi ideali per realizzarvi sofisticati warehouse show.

\section{$5 \quad$ Giovani artisti britannici sull'altare di Venezia}

Il notevole interesse, per non dire clamore, suscitato dai giovani britannici ospitati alla Biennale per la sezione Aperto '93 funse da banco di prova internazionale che questa nuova generazione superò brillantemente, tanto da convincere finalmente in patria anche importanti istituzioni pubbliche come il British Council ad aprire una linea di credito in favore degli Young British Artists. Il British Council infatti, in vista del successivo appuntamento veneziano che coincideva col centenario dalla fondazione della Biennale, scelse finalmente di affidarsi a curatori appartenenti alla stessa coorte di Hirst e sodali, a dimostrazione del fatto che spesso le nuove ondate generazionali nell'arte portano a emergere assieme agli artisti anche una vasta serie di attori fra cui critici, giornalisti, galleristi e altri ancora (Vettese 2007, 22). Nonostante la nuova generazione non condividesse un autentico manifesto artistico, bensì si evidenziassero anche notevoli discrepanze come quelle fra Londra e Glasgow, nel corso dei primi anni Novanta gli Young British Artists operarono come una fazione abbastanza omogenea: si potrebbero perfino considerare una cordata, per cui chi faceva un passo avanti creando un varco nell'establishment - indifferentemente se artista, curatore o critico - provava poi a portare dentro coetanei e amici (Stallabrass 2006a, 4). All'indomani del 1993, pertanto, si fecero strada non soltanto gli artisti fin qui citati, ma anche un numero crescente di giovani curatori, fra i quali ricorrevano i nomi di Liam Gillick, Gregor Muir, Graham Fagen e James Roberts, la cui reputazione crebbe rapidamente fino a poter collaborare ancora giovanissimi con la Tate e il British Council (Muir 2011, 125). Già nel 1994 furono convocati da queste istituzioni per realizzare i due principali avvenimenti artistici finanziati con fondi pubblici per il 1995, ossia la mostra itinerante The British Art Show in patria, nonché la trasferta veneziana per la partecipazione britannica alla Biennale. 
Ai fini del presente capitolo interessa soltanto il secondo di questi due eventi, poiché la Biennale del centenario offriva un palcoscenico internazionale senza pari. Oltretutto, con l'avvicendamento alla guida della rassegna veneziana fra Achille Bonito Oliva e Jean Clair, si creò una situazione che giocò a favore delle mire espansionistiche del British Council. Invero, il curatore francese appuntato per la 46. Esposizione Internazionale abolì senza indugio la sezione Aperto, poiché l'ultima esperienza si era dimostrata troppo distante dal suo modo - abbastanza tradizionalista - d'intendere l'arte contemporanea (Vecco 2002, 34). Nonostante questa abolizione, o forse persino rafforzato nelle proprie convinzioni da quanto avvenuto, il British Council progettò di portare in blocco a Venezia un nutrito gruppo di giovani artisti britannici affittando all'uopo una intera sede esterna, la Scuola di San Pasquale a San Francesco della Vigna. In questo modo, la Gran Bretagna si sarebbe presentata al centenario della Biennale con ben due sedi: lo storico Padiglione sulla sommità dei Giardini di Castello, in cui presentare un artista affermato, il pittore Leon Kossoff, e un Padiglione esterno a due passi dall'Arsenale in cui concentrare il meglio della generazione emergente di artisti under-quaranta (Muir 2011, 122). Questa scelta fu propiziata dalla nuova direttrice per la sezione arti visive del British Council, Ann Gallagher, poiché fu lei a convocare nel 1994 due giovani critici e curatori legati agli Young British Artists per progettare la mostra alla Scuola di San Pasquale (Rose 1995, 7). Si trattava di Gregor Muir e James Roberts, entrambi celebri firme di Frieze, i quali approfittarono dell'occasione offerta per rivendicare una supposta straordinarietà della nuova generazione di artisti britannici. Per di più, nelle sue memorie Muir attribuisce la nascita dell'acronimo YBA proprio alla primissima delle riunioni preparatorie alla mostra veneziana, poi intitolata General Release: Young British Artists (1995): ${ }^{26}$

It was during this meeting, towards the end of 1994, that we stopped saying 'young British artists' and started saying 'YBA'. In truth, we'd simply given up stumbling through the unabridged version and 'YBA' made for easy shorthand, but this was the first time I'd ever heard the term used in regular discussion. [...] YBA was not the result of the 'Young British Artists' shows taking place at the Saatchi Gallery. Instead, the term referred to an all-encompassing phenomenon that included a number of different artists from a variety of backgrounds. (Muir 2011, 122-3)

I due curatori invitarono a esporre ben nove artisti più tre coppie, tutti neofiti alla Biennale, nonostante si fossero già distinti a livello inter- 
nazionale negli anni precedenti. Si trattava nell'ordine di Fiona Banner, Adam Chodzko, Ceal Floyer, Tom Gidley, Douglas Gordon, Gary Hume, Jaki Irvine, Elizabeth Wright e Cerith Wyn Evans, più la coppia formata dai fratelli Dinos e Jake Chapman, il duo composto da Matthew Dalziel e Louise Scullion, infine le gemelle Jane e Louise Wilson. Oltre la metà degli invitati aveva partecipato due anni addietro alla mostra Wonderful Life presso Lisson Gallery, curata proprio da Roberts. Il centro di gravità della delegazione britannica era sempre collocato nella capitale inglese, con dodici su quindici artisti formatisi in una delle art school londinesi, ma soltanto sette di questi erano diplomati al Goldsmiths, un chiaro segnale che il gruppo originario di Freeze stava ormai perdendo la sua egemonia sugli YBA. I soli tre artisti non londinesi fra i selezionati - ossia Gordon, Dalziel e Scullion - erano ancora una volta di Glasgow e tutti loro avevano frequentato la locale art school, a conferma del notevole fermento alternativo che si era creato nel milieu artistico della città scozzese (Muir 2011, 129-30). Fra le opere presentate si ricordano specialmente le pitture realizzate con vernici lucenti fra l'astratto e il rappresentativo di Gary Hume, per certi versi analoghe ai suoi precedenti Door Paintings. Hume era da poco tornato alla ribalta grazie al supporto del White Cube che gli permise di gettarsi alle spalle il fallimento del rapporto col suo primo gallerista Karsten Schubert risalente ancora ai tempi di Freeze (Muir 2011, 80-1). Si fece però anche notare una ben più articolata video installazione dal titolo Hysterical (1994-95) di Douglas Gordon, il quale utilizzò due schermi leggermente inclinati e posti fra loro ad angolo ottuso, sui quali scorrevano estratti di filmati clinici di inizio Novecento: queste bobine ritrovate e manipolate dall'artista scozzese mostravano due medici che assistevano un donna apparentemente affetta da una crisi di isteria, ma i fotogrammi sui due schermi erano sfasati temporalmente oltre che specchiati fra loro, così da creare un profondo senso di disagio nello spettatore (Brown 2004, 47-8). Pare curioso, ma per nulla casuale, che proprio questi due artisti si sarebbero fronteggiati l'anno dopo con opere molto simili alla mostra finale per il Turner Prize del 1996 alla Tate, dove lo scozzese la spuntò come primo video artista ad aggiudicarsi il premio contro tutti i pronostici che davano invece per certa una vittoria del pittore londinese. ${ }^{27}$

Oltre a dimostrarsi profetica, General Release fu per i suoi curatori anche l'occasione per giungere a una prima storicizzazione del fenomeno degli YBA, poiché inclusero nel catalogo una dettagliata cronologia che restituiva tutti gli avvenimenti salienti nell'ascesa di questa nuova generazione (Rose at alia 1995, 72-103). I saggi in catalogo offrivano invece una narrazione tendenzialmente eroica e a tratti per-

27 Richard Dorment, "Artists find a new frame of reference», The Daily Telegraph, 29 novembre 1996. 
sino esagerata circa la forza autonoma e la capacità imprenditoriale con cui gli YBA erano riusciti a farsi rapidamente strada (Roberts $1995,49)$. Al netto delle iperboli, la mostra veneziana e il volume d'accompagnamento si dimostrarono una prima rilevante quanto omogenea rappresentazione degli YBA ad opera di due autentici insider, Muir e Roberts, che la esposero e narrarono alla platea internazionale assisa alla Biennale del centenario. Infine, va enfatizzato un aspetto che si rivelò centrale nella marcia trionfale degli YBA fuori dalla madre patria: General Release fu la prima di una vasta serie di mostre collettive organizzate e finanziate direttamente dal British Council (Muir 2011, 132). Invero, all'indomani della chiusura della 46. Esposizione Internazionale, la mostra fu spostata in blocco dalla Scuola di San Pasquale ad altre sedi italiane ed estere, fra cui si annovera una mostra a cura di Mario Codognato dal titolo inequivocabile Artisti britannici a Roma (22 maggio-29 giugno 1996). ${ }^{28}$ Altre collettive realizzate o sponsorizzate dal British Council sull'onda dell'esperienza veneziana furono inoltre nel 1995 Arte inglese d'oggi alla Galleria Civica di Modena, quindi nel 1996 The Scottish Autumn al Museum Ludwig di Colonia e al Kortars Muvesti Museu di Budapest, poi Full House - Young British Art al Kunstmuseum di Wolfsburg e Swan-off al Konstmuseum di Uppsala, infine nel 1998 Emotion: Young British and American Art from the Sammlung Goetz alle Deichtorhallen di Amburgo. Questa sistematica proliferazione della giovane arte britannica promossa dal British Council si dimostrò efficace e pervasiva, tanto da operare perfino una concorrenza a tratti sleale nei confronti di altre nazioni europee: a tal proposito lo studioso Friedrich Meschede fece notare già all'epoca come secondo i suoi calcoli nel solo biennio 1997-98 il British Council avesse promosso su suolo tedesco ben 70 progetti e dodici collettive che avevano coinvolto 153 artisti britannici, mentre la Germania nello stesso periodo aveva finanziato con fondi statali le iniziative nel Regno Unito di soli 10 artisti tedeschi (Meschede 1998, 175). Anche secondo uno studioso britannico assai critico degli YBA come Julian Stallabrass, l'intervento del British Council fu davvero imponente in quel periodo e del tutto senza precedenti per il Regno Unito, oltre che particolarmente efficace sfruttando l'egemonia globale della lingua inglese e i canali internazionali a questa riservati in virtù della predominanza commerciale degli Stati Uniti d'America (Stallabrass 2006a, 196).

Che il British Council facesse ormai sul serio con gli YBA lo si capì nuovamente alla Biennale di Venezia per l'edizione del 1997, quando il proprio Padiglione nazionale fu affidato per la prima volta a una gio-

28 Nel catalogo stesso della mostra il curatore fa riferimento al fatto che l'iniziativa deriva direttamente dalla tappa veneziana per la Biennale del centenario (cf. Codognato 1996). 
vane artista britannica, Rachel Whiteread, già salita agli onori della cronaca per la sua vittoria al Turner Prize nel 1993. ${ }^{29}$ Nella sezione principale della mostra, invece, si distinse a sorpresa il vincitore in carica del Turner Prize, ossia il trentenne scozzese Douglas Gordon, il quale a Venezia venne insignito del riconoscimento per il miglior giovane artista presente alla Biennale grazie a un'installazione ambientale intitolata 30 Seconds Text (1996): si trattava di una stanzetta buia con le pareti dipinte di nero e una lampadina al centro, la quale si accendeva e spegneva ogni trenta secondi per disvelare sulla parete un breve testo che raccontava i macabri esperimenti di un dottore francese di inizio Novecento, il Dottor Baurieux di Montpellier, che interrogava le teste dei decapitati fino a che spiravano definitivamente dopo all'incirca mezzo minuto (Brown 2004, 37). A questo proposito è importante sottolineare come l'opera di Gordon fosse la rielaborazione di un intervento site specific sviluppato proprio per una precedente mostra sponsorizzata dal British Council, ossia Swanoff (1996) nel Castello di Uppsala, e poi presentato lo stesso anno in forma rinnovata anche al Musée d'art moderne de la Ville of Paris in occasione della collettiva life/live curata dal giovanissimo Hans Ulrich Obrist (Haase 1999, 144-5). Nelle due edizioni successive della Biennale, curate da Harald Szeemann, la sezione principale non riservò particolari onori agli YBA, mentre il Padiglione britannico persistette nella stessa direzione portando nel 1999 il pittore Gary Hume e poi nel 2001 Mark Wallinger curato dalla stessa Ann Gallagher. Si dovette attendere il 2003 per la definitiva consacrazione di questa generazione, in virtù della nomina di Francesco Bonami a direttore della 50esima Esposizione Internazionale d'Arte, il quale scelse come pezzo centrale al Padiglione Italia dei Giardini proprio un immenso armadietto di pillole di Damien Hirst (Bonami, Frisa 2003, 34-5).

\section{La Biennale come specchio d'un avvicendamento generazionale}

Che il mondo dell'arte sia diviso in fazioni è cosa ben nota, seppur si tenda nella narrazione storico artistica a circostanziare le contrapposizioni per evidenziare uno sviluppo dettato invece da una presunta necessità che procede fra continuità e discontinuità (Vettese 2007, 21-2). Nella sua ricostruzione della repentina emersione degli YBA, invece, Julian Stallabrass enfatizza proprio lo scontro fra generazioni che caratterizzò gli anni Novanta del Novecento e condusse al de-

29 Cf. «British pavilions at the Venice Biennale - in pictures», The Guardian, 15 May 2012, https://www.theguardian.com/artanddesign/gallery/2012/may/15/venicebiennale-british-pavilions-pictures (2019-09-12). 
finitivo strappo fra il periodo precedente dominato dal neo-espressionismo e un presente progressivamente conquistato da tendenze neo-pop e neo-concettuali (Stallabrass 2006a, 190-1). Nella presente trattazione si è tentato di dimostrare come questo strappo vada forse inquadrato meglio quale passaggio progressivo o per tappe, ossia una transizione che si protrasse almeno per un decennio e che permise a giovani artisti - in particolare britannici - di affermarsi a livello internazionale con una considerevole rapidità. A tal fine, tuttavia, si rese indispensabile il concerto di altri soggetti oltre agli artisti, dunque l'apporto di quanti fra galleristi, critici, curatori e collezionisti - sia vecchi che nuovi - scommisero sulla nuova generazione.

Lungo il corso di questo repentino sviluppo l'appuntamento veneziano dell'Esposizione Internazionale - grazie alla sua cadenza biennale rispetto alla quinquennalità di documenta - si dimostrò particolarmente adeguato a registrare il cambiamento artistico in atto. Per di più la Biennale - talvolta per caso, altre volte in maniera più programmatica - si rivelò una tappa necessaria per la graduale affermazione degli YBA e, più in generale, di un'intera coorte di giovani artisti, critici e curatori. La stessa Biennale, come riportato nei paragrafi precedenti, si trasformò persino in una sorta di campo di battaglia fra diverse generazioni, nonché fra modi molto differenti di concepire l'arte contemporanea stessa. A questo proposito basti considerare la distanza fra le posizioni di due curatori centrali nelle vicende veneziane durante il periodo considerato, ossia da una parte Jean Clair che ebbe a organizzare la mostra del centenario nel 1995 e dall'altra Francesco Bonami che guidò il drappello di colleghi responsabili della 50esima Esposizione Internazionale nel $2003 .{ }^{30}$ L'abolizione da parte di Clair della sezione Aperto non deve infatti sorprendere, se si considerano la sua definizione di arte, ancora saldamente incentrata sulle categorie tecniche tradizionali, e le sue affermazioni circa il fine ultimo della stessa, romanticamente votato a una rappresentazione armonica della nostra idea del mondo, dei suoi abitanti e divinità (Clair 2005, 74). Nei suoi scritti il curatore francese si scaglia pertanto contro quella cultura angloamericana colpevole a suo avviso d'aver prodotto un eccesso d'attenzione sull'artista, anziché sull'opera, e causato uno stravolgimento nel ruolo dei musei, intesi ormai come soggetti attivi nella speculazione artistica, invece di essere ricettori e custodi dell'arte universale (Clair 2011, 25). Una simile visione fa a pugni con le convinzioni espresse da Bonami, il quale intende al contrario l'arte quale espressione cruciale sul mondo nel tentativo di comprenderlo, tanto che il suo scopo ultimo si ridurrebbe alle idee convogliate dall'artista attraverso la propria opera (Bonami 2009, 5-7). L'artista verrebbe allora identificato in colui 
(o colei) che è stato capace di trasformare per necessità interiore la propria vita in arte realizzando opere inevitabili (Bonami 2010, 5).

Al netto delle semplificazioni o imprecisioni nella visione teorica di entrambi i curatori, la distanza siderale fra le convinzioni di Clair e di Bonami rende plasticamente l'idea del capovolgimento che si consumò sul palcoscenico internazionale della Biennale negli anni Novanta del Novecento. La kermesse veneziana si tramutò così nello specchio di un avvicendamento generazionale, sia di artisti che di curatori. Un nuovo modo di intendere l'arte, nonché di presentarla al pubblico, si fece largo fra i Padiglioni dei Giardini e nei nuovi spazi dell'Arsenale. Quest'ultimi in particolare si dimostrarono ideali per accogliere la vera grande novità artistica del periodo, ossia la proliferazione di installazioni ambientali quale nuovo genere o categoria artistica (Stallabrass 2006b, 16). A questo proposito, gli YBA avevano saputo cavalcare precocemente il filone emergente producendo ancora giovanissimi opere installative dal forte impatto quali $A$ Thousand Years di Damien Hirst, House di Rachel Whiteread oppure 24 Hour Psycho di Douglas Gordon. Il critico del New York Times Michael Kimmelmann sottolineò in maniera pregnante la pervasività del nuovo genere e il suo impatto sul mondo dell'arte di fine Novecento:

Installation art is in the late 90's roughly what Abstract Expressionism was by the late 50's. I suspect the term itself will eventually come to seem too vague to be of lasting value. [...] Having exhausted their value, catchwords or phrases may then be abandoned. [...] But unlike Pop, installation art is not even a style or movement; it is a strategy, a way of working. ${ }^{31}$

Non vi è dubbio, altresì, che la scalata internazionale degli YBA sia passata per Venezia, anzi che sia stata sancita dalla Biennale nella misura in cui rafforzò il British Council nella decisione di avviare una massiccia campagna di diffusione di questa nuova generazione. ${ }^{32} \mathrm{Al}$ tempo stesso, giovani artisti britannici come i summenzionati Hirst, Whiteread e Gordon hanno contribuito in maniera decisiva a cambiare l'arte esposta in Biennale, nonché il modo stesso di presentarla. Si può affermare, dunque, che la singolare richiesta canterina di Sarah Lucas ricordata in avvio di questo saggio sia stata alfine esau-

31 Michael Kimmelmann, «Installation art moves on, moves in», New York Times, 9 agosto 1998.

32 A questo proposito si noti che il British Council giunse infine a promuovere a Venezia dal 2003 attraverso il proprio braccio operativo scozzese anche un Padiglione dedicato alla Scozia e separato da quello britannico, così da poter rappresentare anche la crescente compagine artistica originata anzitutto dalla Glasgow School of Art e presentando a Venezia vincitori del Turner Prize non inglesi come Martin Boyce o Duncan Campbell, https://scotlandandvenice.com/years/ (2019-09-12). 
dita: alla pizza - ossia a questa nuova generazione e al suo modo di intendere l'arte - fu data una chance. A lei nello specifico accadde nel 2003 nella sezione principale assieme a Damien Hirst, evento poi celebrato nel 2004 alla Tate con la vituperata mostra In-A-Gadda-Da-Vida assieme a Hirst stesso e ad Angus Fairhurst, in occasione della quale Lucas presentò proprio un'opera intitolata All We are Saying is Give Pizza Chance (2003) già presentata alla Biennale, ossia un missile rivestito di volantini di pizza da asporto. ${ }^{33}$ Per approdare da sola al Padiglione britannico della Biennale dovette invece attendere fino al 2015. Nel frattempo la consacrazione mondiale degli YBA, e più in generale della loro generazione, era già cosa fatta: fondamentale fu dunque negli anni Novanta il ruolo della Biennale di Venezia, sulla quale questi giovani artisti britannici lasciarono di converso un segno profondo.

\section{Bibliografia}

Bonami, Francesco (2009). Lo potevo fare anch'io: Perché l'arte contemporanea è davvero arte. Milano: Mondadori.

Bonami, Francesco (2010). Si crede Picasso. Come distinguere un vero artista contemporaneo da uno che non lo è. Milano: Mondadori.

Bonami, Francesco (2014). Curator: Autobiografia di un mestiere misterioso. Venezia: Marsilio.

Bonito Oliva, Achille; Kontova, Helena (a cura di) (1993). Aperto '93 Emergency/ Emergenza = Catalogo della mostra. Milano: Giancarlo Politi Editore.

Brown, Katrina (2004). DG: Douglas Gordon. London: Tate Publishing.

Bruggen, Coosje van (2002). «Sounddance». Morgan, Robert C. (ed.), Bruce Nauman. Baltimore: Johns Hopkins University Press.

Burgess, John; Coventry, Keith; Hale, Matt; Noble, Paul; Owen, Peter (2002). City Racing: The Life and Times of an Artist-Run Gallery. London: Black Dog Publishing.

Clair, Jean (2005). De Immundo. Milano: Abscondita.

Clair, Jean (2011). L'hiver de la culture. Paris: Café Voltaire-Flammarion.

Codognato, Mario (1996). Artisti Britannici a Roma = Catalogo della mostra (Galleria Bonomo, Roma, 1996). Torino: Umberto Allemandi.

Collings, Matthew (1997). Blimey! From Bohemia to Britpop: the London Artworld from Francis Bacon to Damien Hirst. London: 21 Publishing Ltd.

Cooper, Jeremy (2012). Growing up: the Young British Artists at 50. Munich: Prestel Publishing.

Craig-Martin, Martin (2012). «Damian Hirst: The Early Years». Gallagher, Anna (ed.), Damien Hirst = Exhibition catalogue (London, 2012). London: Tate Publishing, 38-9.

33 Laura Cumming, "Oh do look, a calf with six legs», The Guardian, 7 March 2004, https://www.theguardian.com/artanddesign/2004/mar/07/art.tatebritain (2019. 09-12). 
Haase, Armin (1999). "On the other side of the mirror». Gordon, Douglas (ed.), Déjà-vu: questions \& answers. Volume 2. 1997-1998. Paris: MARC Musée d'art moderne de la ville de Paris, 127-48.

Kontova, Helena (1995). «Uno, cento, mille Aperto: due anni dopo». Flash art, 192, 61-3.

Lowndes, Sarah (2010). Social Sculpture: The Rise of the Glasgow Art Scene. Glasgow: Lutah Press.

Mantoan, Diego (2015). The Road To Parnassus: Artist Strategies in Contemporary Art. Wilmington: Vernon Press.

Mantoan, Diego (2017). «Diverging Collectives: Artist-Run Spaces versus Warehouse Shows. Different models of art production and cooperation among young British artists». RE_BUS, 8.

Morgan, Stuart (1991). «The Turner Prize». Frieze, 1, September, 4-12.

Muir, Gregor (2011). Lucky Kunst. The Rise and Fall of Young British Art. London: Artum Press.

Obrist, Hans Ulrich (2017). «In primo piano: The Better Biennale». Flash Art, 23 ottobre. URL https://flash---art.it/article/the-better-biennale/.

Richardson, Craig (2011). Scottish Art since 1960: Historical Reflections and Contemporary Overviews. Farnham: Ashgate Publishing.

Roberts, James (1995). «Never had it so good...». Rose, Andrea; Muir, Gregor; Roberts, James (eds), General Release: Young British Artists at Scuola di San Pasquale $=$ Exhibition catalogue (Venezia, 1995). London: The British Council, 48-71.

Rose, Andrea; Muir, Gregor; Roberts, James (eds) (1995). General Release: Young British Artists at Scuola di San Pasquale = Exhibition catalogue (Venezia, 1995). Londra: The British Council.

Rose, Andrea (1995). «Foreword». Rose, Muir, Roberts, 6-7.

Schwarze, Dirk (2012). Meilensteine: Die Documenta 1 -13. Berlin, Verlag B\&S.

Serota, Nicholas (2011). «Nicholas Serota interviews Damien Hirst». Gallagher, Anna (a cura di) (2012), Damien Hirst = catalogo della mostra (London, 2012). Londra: Tate Publishing, 91-9.

Slotover, Matthew (1991). «Windfall: Northern Lights». Frieze, 1, September, 36-41.

Stallabrass, Julian (2006a). High Art Lite. the Rise and Fall of Young British Art. London; New York: Verso.

Stallabrass, Julian (2006b). Contemporary Art. A Very Short Introduction. Oxford; New York: Oxford University Press.

Vecco, Marilena (2002). La Biennale di Venezia - Documenta di Kassel: Esposizione, vendita, pubblicizzazione dell'arte contemporanea. Milano: Franco Angeli.

Verzotti, Giorgio (1993). «Aperto 93: the better Biennale». Artforum International, 2, 104-6.

Vettese, Angela (2007). Ma questo è un quadro? Il valore nell'arte contemporanea. Roma: Carocci. 


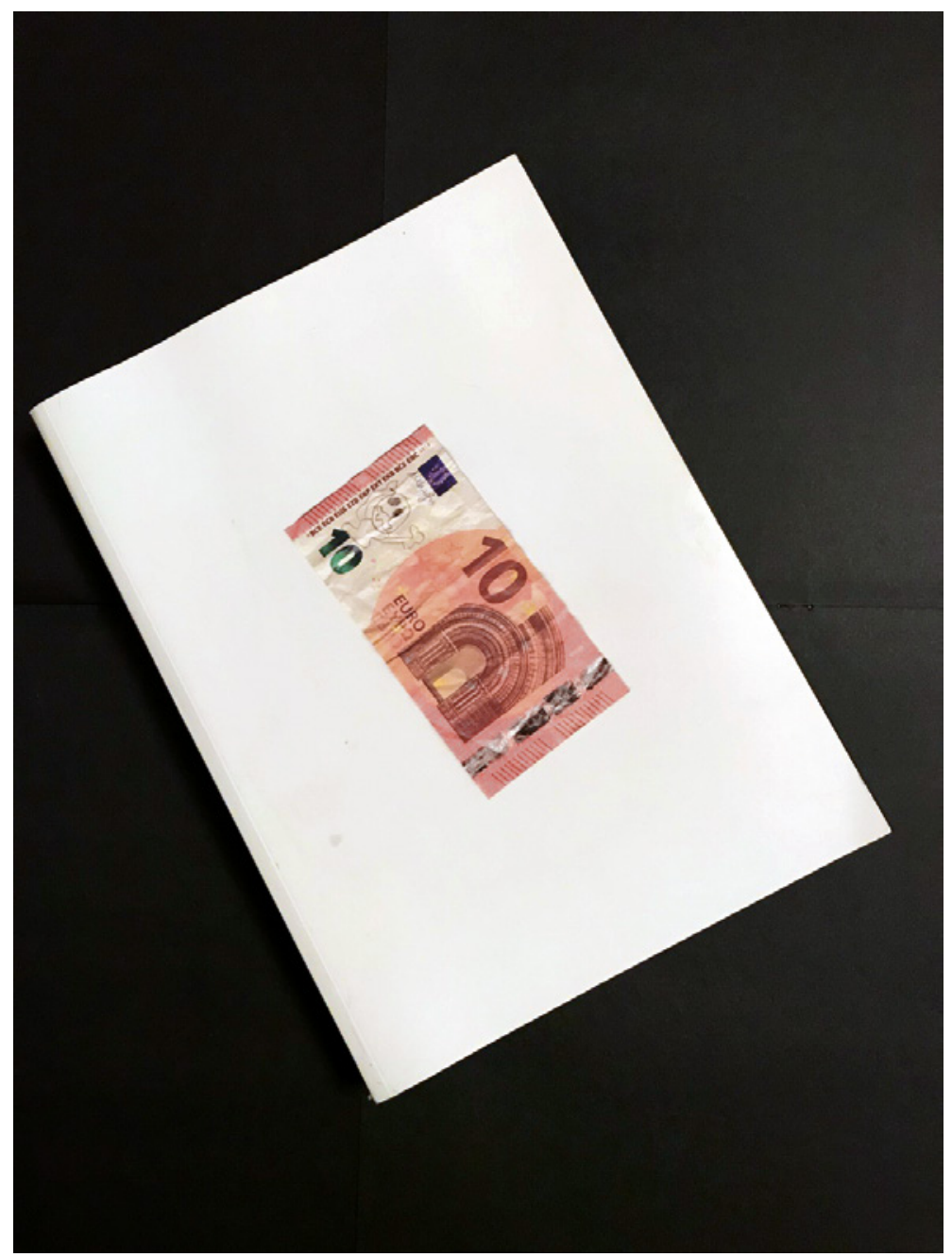

Marco Fusinato, From the Horde to the Bee (Edizioni Colibrì, 2015). volume componente l'installazione esposta alla Biennale del 2015.

Courtesy Edizioni Colibri 


\title{
Arte relazionale alla Biennale di Venezia dal 1999 al 2017
}

Francesca Amadi

\begin{abstract}
This essay traces the presence of Relational Art at Venice Biennale, considering ten editions from 1999 to 2017, immediately following the publication of Esthétique relationelle by the critic Nicolas Bourriaud in 1998 and covers the most recent experiences. Analysing the past two decades, a variety of approaches relations are revealed, from the theme of human to the growing interest for involving people in artistic actions. Performances, installations, multimedia projects, workshops involve a heterogeneous public.
\end{abstract}

Keywords Venice Biennale. Relational Art. Rirkrit Tiravanija.

La Biennale di Venezia dagli anni Novanta ospita interessanti proposte di artisti che lavorano nell'ambito dell'arte relazionale e propongono spazi d'aggregazione che consentono di sviluppare liberamente connessioni umane dal carattere aleatorio.

La prima opera di arte relazionale viene presentata alla Biennale nel 1993 durante l'edizione Punti cardinali dell'arte a cura di Achille Bonito Oliva. Untitled (1271) (1993) di Rirkrit Tiravanija viene esposta nella sezione Aperto '93 che raccoglie le proposte dei giovani artisti alle Corderie dell'Arsenale. È un'installazione che consiste in una canoa da cui viene fornita ai visitatori acqua calda per preparare tazze di noodles, i classici spaghetti cinesi, che si possono consumare in uno spazio adiacente allestito con sedie e tavoli. Tiravanija vuole costruire un ponte ideale tra Oriente e Occidente ricollegandosi all'esperienza di Marco Polo e all'anno in cui partì per la Cina. L'artista

Questo saggio prende avvio da Amadi, Francesca (2017). Arte relazionale alla Biennale di Venezia dal 1999 al 2017 [tesi di laurea magistrale]. Relatore Nico Stringa; correlatore Stefania Portinari. a.a. 2016/2017. Venezia: Università Ca’ Foscari Venezia.

Storie dell'arte contemporanea 4 | Atlante delle Biennali 1

ISSN 2704-9973

ISBN [ebook] 978-88-6969-366-3 | ISBN [print] 978-88-6969-367-0

Edizioni 
propone la pratica conviviale, come aveva già fatto a New York con Untitled 1992 (Free), come microutopia democratica per favorire il dialogo tra culture (Obrist 2010, 12).

Grazie alla pubblicazione di Nicolas Bourriaud Esthétique relationnelle del 1998 questa nuova corrente artistica gode di un riconoscimento effettivo, come testimonia la presenza crescente di opere di questo genere nelle edizioni successive della rassegna.

Nel 1999 alla Biennale intitolata dAPERTutto curata da Harald Szeemann (che riprende nel titolo la citazione dello spazio Aperto, inventato nel 1980 con Achille Bonito Oliva), viene presentato il Progetto Oreste, nato nel 1997, i cui membri si definiscono un insieme variabile di persone che condividono spazi per idee, invenzioni e progetti. Essi non vogliono essere definiti gruppo, collettivo o associazione e le loro opere si basano sulla collaborazione e la relazione con gli altri attraverso laboratori, residenze estive, meeting, convegni e pagine web (Biennale 48 1999, 344). Alla Biennale, all'interno del Palazzo delle Esposizioni nella sede dei Giardini, il gruppo Oreste propone un vasto numero di attività e un presidio permanente nel cuore della mostra progettata da Bonito Oliva, per suscitare il confronto e la conversazione tra i membri del progetto e con il pubblico (cf. Norese 2000).

Ai Giardini, vicino al Padiglione degli Stati Uniti, Tiravanija progetta The First Thai Pavilion in Venice (1999), un podio che viene utilizzato come padiglione dagli artisti thailandesi, che non trovano posto in alcuna struttura. L'opera viene realizzata proprio ai Giardini quale sede storica delle rappresentanze nazionali, in cui i Padiglioni, edificati per la maggior parte prima della Seconda guerra mondiale da Paesi che già godevano di un ruolo riconosciuto, fungono quasi da ambasciate. Il progetto di un meta-padiglione aperto a vari interventi artistici vuole conferire alla Thailandia uno status diplomatico, come se si trattasse di una rappresentanza nazionale. Significative sono le dimensioni dell'opera che equivalgono a quelle della camera da letto del curatore dell'esposizione: l'artista sovrappone la dimensione privata al tema pubblico (Biennale 48 1999, 162).

Alle Corderie dell'Arsenale il pubblico viene coinvolto dall'opera dell'artista Chen Zhen Jue Chang-Fifty Strokes Each (1998), un'installazione con decine di sedie, letti e oggetti di recupero, rivestiti con pelli animali a formare un enorme strumento musicale a percussioni che ricorda quello tradizionale cinese, il bianzhong, che durante il vernissage viene suonato da un gruppo di monaci tibetani e messo poi a disposizione del pubblico come pratica per affrontare l'angoscia e la rabbia quotidiane liberando le proprie emozioni attraverso la musica. L'arte per Chen Zhen attraverso la pratica della transexperience diviene terapia, come una medicina, e contribuisce al raggiungimento dell'armonia risolvendo i conflitti (Hanru 2003, 23-36). Gli objets trouvés secondo questa pratica, dopo essere divenuti rifiuti nella società dei consumi, vengono purificati nell'opera d'arte e tro- 
vano una nuova vita e un nuovo senso nel flusso dinamico di energie che compongono l'armonia universale (Zhen 2003, 152-87). L'opera ha un intento politico: esposta per la prima volta nel 1998 a Tel Aviv, era stata progettata per portare l'attenzione suoi conflitti in corso in Medio Oriente e viene riproposta a Venezia per trattare delle tensioni politiche in Kosovo (Biennale 48 1999, 98).

Alla Biennale nel 2001 Platea dell'umanità, la seconda edizione curata da Harald Szeemann, l'artista Santiago Sierra presenta 133 Persons Paid to Have their Hair Dyed Blond (2001); l'azione si svolge all'Arsenale per poi trasferirsi nella città. L'artista invita centotrentatré venditori ambulanti che operano in città a lasciarsi tingere i capelli di biondo in una sala all'interno della mostra, in cambio di un salario. L'operazione pone in luce la loro presenza e sollecita riflessioni sul loro stato di emarginazione: l'opera è incentrata sul rapporto tra i venditori ambulanti e il tessuto sociale urbano composto da cittadini, pendolari e turisti (Biennale 49 2001, 208). Sierra critica il sistema capitalista e riflette sulla condizione dei lavoratori sottopagati che non godono dell'assistenza sociale e sul tema dell'emarginazione in contrasto con il sistema dell'arte, impiegando per le sue opere persone ai margini della società, retribuendole il minimo sindacale e trattandole come materia artistica, suscitando disagio e perplessità negli spettatori. ${ }^{1}$

All'Arsenale Michael Schmitz presenta Heli Global Art Tour (2001), un progetto umanitario che prevede un tour 'artistico' del globo in elicottero, finalizzato a raccogliere fondi per costruire un istituto per l'infanzia in Sud Africa. L'itinerario prevede soste presso musei, istituzioni culturali e artisti. Il progetto vuole essere un momento di scambio e incontro tra culture e religioni con un invito aperto al pubblico (Biennale 49 2001, 300).

Alla 50a Biennale del 2003 viene nominato direttore Francesco Bonami, che sceglie di farsi affiancare da undici curatori da lui scelti tra critici e artisti, che si occupano di diverse sezioni tematiche, una di queste è Stazione Utopia curata da Molly Nesbit, Hans Ulrich Obrist e Rirkrit Tiravanija. Il progetto sviluppa il concetto di stazione come luogo di passaggio, di scambio, di incontri e di riflessione. All'interno degli spazi di Stazione Utopia vengono esposte opere, hanno luogo performance, si tengono presentazioni di libri e dibattiti; tutto si basa sul coinvolgimento del pubblico e sull'opportunità di creare relazioni tra i visitatori all'interno dell'ambiente. Questa 'stazione' diventa un luogo di condivisione ideale per riflettere sul senso della parola utopia, è un punto di arrivo da percorsi differenti, di sosta o transito e di partenza in una nuova direzione. Vi partecipano più di sessanta artisti con il coinvolgimento di intellettuali, scrittori, architetti, filosofi (Biennale 50 2003, 319-419). 
Nel Padiglione olandese, che raccoglie le opere di cinque artisti, Jeanne van Heeswijk espone l'installazione Draw a Line (2003): l'artista riflette su come grazie alla competizione le competenze possano essere incrementate. L'opera consiste in un gioco tradizionale olandese che affronta il tema della territorialità ed ha a che fare con la negoziazione di spazi tra giocatori. L'installazione prevede la partecipazione del pubblico e si collega ad un progetto più ampio The Future from the Sidelines in corso nella cittadina olandese di Gorinchem, incentrato sul tema del gioco sportivo inteso come opportunità di miglioramento sia per il singolo individuo che per la collettività (We Are the World 2003, 34-9).

Santiago Sierra lavora invece sull'esclusione con l'opera Muro cerrando un espacio (2003) che consiste di un muro in mattoni costruito a sessantacinque centimetri dal portone d'ingresso, all'interno del Padiglione, in modo da impedire l'accesso all'area espositiva ma da rendere comunque visitabili il foyer, il bagno e un piccolo magazzino. L'accesso al Padiglione è concesso - solo dal retro - esclusivamente ai possessori di passaporto spagnolo e vietato a tutte le altre nazionalità. L'opera è uno spartiacque che impedisce la comunicazione tra i cittadini spagnoli che hanno avuto il permesso di accedervi e gli esclusi e permette di evidenziare riflessioni sui temi di nazionalità e identità facendo subire al pubblico l'effetto di esclusione sofferto dai migranti ai quali viene negato il permesso di soggiorno (Martínez 2003, 146).

Alla Biennale del 2005 L'esperienza dell'arte. Sempre un po' più lontano curata da María de Corral e Rosa Martínez troviamo Olaf Nicolai con l'opera Welcome to the Tears of St. Lawrence (2005). Si tratta di una convocazione, l'invito l'invito è effettuato tramite il catalogo, con manifesti, con volantini e per e-mail, a partecipare allo spettacolo delle stelle cadenti la notte di San Lorenzo ovunque ci si trovi. Si tratta di un'opera che ha origine nello spazio espositivo per poi ampliarsi al di fuori di esso grazie ai visitatori che vi prendono parte e formano una comunità. L'opera non assume una dimensione spaziale o materiale definita ma si espande a ovunque le stelle siano visibili: in tal caso il pubblico diviene parte dell'opera (Olaf Nicolai 2006, 46). Nicolai vuole porre attenzione sulla natura, invitando i visitatori a prendervi parte. ${ }^{2}$

Nel Padiglione tedesco espone Tino Sehgal, che tramite performance tratta aspetti dell'incontro umano. Organizzando azioni all'interno di spazi espositivi rende i visitatori parte dell'opera e presenta a quella Biennale This is so Contemporary (2005) in cui gli interpreti, danzando, cantano «This is so contemporary, contemporary, contemporary». Le opere di Sehgal si caratterizzano per l'immateriali- 
tà; l'unica componente materiale è la presenza fisica dei performer. L'artista giunge alla scelta radicale di non effettuare né concedere foto e riprese delle sue performance e desidera che l'esperienza della fruizione dell'opera venga trasmessa solo oralmente dal pubblico: la performance dunque si basa su una dimensione esperienziale piuttosto che cognitiva. La fruizione dell'opera non dovrebbe essere guidata da ulteriori apparati quali il catalogo o la guida ma esclusivamente dall'opera stessa. ${ }^{3}$

Nel 2007 ha luogo l'edizione Pensa con i sensi, senti con la mente curata da Robert Storr che accoglie nel Padiglione degli Stati Uniti opere di Felix Gonzalez-Torres considerato uno dei primi artisti relazionali (Bourriaud 2010, 51-64). Per Gonzalez-Torres la relazione tra il visitatore e l'opera è fondamentale per completarne il senso, il ruolo dello spettatore è attivo nel contribuire a dare forma all'opera con il suo intervento (cf. Felix Gonzalez-Torres 1997). L'esposizione a cura di Nancy Spector riprende un progetto espositivo del 1995 ed include le opere "Untitled" (America) (1994), "Untitled" (Natural History) (1990), "Untitled" (Memorial Day Weekend) (1989), "Untitled" (Veterans Day Sale) (1989), "Untitled" (Repubblican Years) (1992), "Untitled" (Public Opinion) (1991), "Untitled" (Leaves of Grass) (1993), "Untitled" (Strange Bird) (1993). "Untitled" (Public Opinion) consiste in un tappeto di caramelle argentate, che possono essere raccolte e consumate dai visitatori e che vengono quotidianamente aggiunte dagli addetti in modo che la forma dell'opera si modifichi costantemente in relazione al comportamento del pubblico.

Il collettivo Mourrinho è composto da ex meninos de rua che collaborano con la fotografa Paula Trope. Il gruppo propone alla Biennale una copia di un modellino che raffigura un villaggio brasiliano. Si tratta di un quartiere in miniatura della periferia di Rio de Janeiro costruito con materiali di recupero provenienti dalle favelas, con l'intento di porsi in polemica con la pianificazione urbanistica attuata in Sud America. L'installazione si pone come una realtà in cui accadono eventi positivi ma anche negativi come può capitare nelle periferie in cui vige la criminalità e si può considerare arte relazionale per la modalità 'collettiva' con cui è stata composta e perché il pubblico avrebbe potuto idealmente manometterlo, una volta in esposizione a Venezia (Biennale 52 2007, 346).

All'esposizione Fare Mondi, curata da Daniel Birnbaum, Miranda July espone Eleven Heavy Things (2009) nel Giardino delle Vergini all'Arsenale. L'opera si compone di una serie di undici sculture a forma di piedistallo o di lastra sulle quali sono apposte delle iscrizioni che invitano i visitatori a interagire. Il pubblico è invitato a provare le emozioni suggerite nell'iscrizione attraverso un intervento che

Michel Gauthier, «Tino Sehgal Keeps Us Talking», Art Press, 313, 2005, 44. 
affronta ironicamente i temi della colpa e dell'incomunicabilità. ${ }^{4}$ Le opere sono Lace Shape, Double Pink Shape, Three Hole Tablet, Pedestals for Guilty Ones, Finger Tablet, Burberry Shape, Two Faced Tablet, Pedestal for Strangers, Pedestal for a Daughter. L'iscrizione sull'opera Pedestal for Strangers recita «We don't know each other we're just hugging for the picture» e invita i visitatori che non si conoscono a salire insieme sul piedistallo abbracciandosi per una foto. I tre podi dell'opera Pedestals for Guilty Ones con le iscrizioni «The guilty one, The guiltier one, The guiltiest one» suggeriscono ai visitatori che vi salgono di assumere lo stato d'animo relativo alla colpevolezza. Miranda July lavora dunque sull'emotività; il titolo dell'opera Eleven Heavy Things si riferisce all'aspetto materiale dei supporti e a quello immateriale delle emozioni (Biennale 53 2009, 90).

Alla stessa rassegna Xu Tan ai Giardini presenta il progetto Keywords School (2008), si tratta di una scuola all'aperto, in cui si generano discussioni e dialoghi tra partecipanti a partire da alcune parole chiave. L'idea è quella di lasciare libera la creatività del pubblico a partire da un indizio, la parola chiave, che permetta di sviluppare una discussione: non è un'indagine svolta con il singolo ma un lavoro di gruppo al quale tutti i partecipanti apportano materiale. Si tratta di un laboratorio temporaneo sul linguaggio, una scuola dalla quale apprendere grazie all'artista stesso che studia i dialoghi generati dal pubblico. Il progetto nasce dall'interesse dell'artista per l'arte concettuale in quanto i concetti sono parole che rappresentano processi mentali, Xu Tan è interessato alla relazione tra concetto ed emozione (Biennale 53 2009, 170).

Durante l'edizione Fare Mondi gli artisti Massimo Bartolini, Tobias Rehberger, Rirkrit Tiravanija vengono chiamati a progettare ai Giardini spazi per i visitatori dal carattere relazionale. Massimo Bartolini progetta la Sala F adibita a spazio Educational, uno spazio che si plasma a seconda delle necessità con una scalinata che può ospitare persone sedute e che estendendosi diviene un tavolo per attività manuali (Biennale 53 2009, 10). Tobias Rehberger progetta il bar caffetteria in connubio tra arte e design, una grande installazione che con la sua decorazione a forme geometriche rende frammentaria la percezione e contribuisce ad una lieve sensazione di disorientamento nei visitatori. ${ }^{5}$ Rirkrit Tiravanija progetta il bookshop come spazio di relazione concentrandosi sull'aspetto di scambio culturale tra i visitatori piuttosto che sull'aspetto commerciale del luogo. L'ambiente è provvisto di una serie di sgabelli dove i visitatori possono accomodarsi, consultare libri e conversare (Biennale 53 2009, 156).

4 Maria Angela Piga, «Miranda July», L'uomo Vogue maggio-giugno, 2009.

5 Michele Fossi, «Making Worlds. Tobias Rehberger», L'Uomo Vogue, maggio-giugno, 2009. 
Nell'edizione del 2011 Illuminazioni, curata da Bice Curiger, Dora García espone al Padiglione spagnolo Lo Inadecuado (2011). Si tratta di un programma di performance, incontri, interviste e dibattiti tra gli artisti che si compiono attraverso vari eventi (cf. García 2011). Lo spazio del Padiglione diviene un palco teatrale sul quale agiscono visitatori e performer. ${ }^{6}$ L'artista è interessata alla reazione del pubblico; lo spazio all'interno del Padiglione non mostra opere ma contiene oggetti e archivi in relazione alle performance. Per tutta la durata dell'esposizione il Padiglione ospita l'opera Istant Narrative (2008) che consiste in un computer attraverso il quale a turno un osservatore può descrivere la situazione che lo circonda all'interno del Padiglione. Il racconto viene proiettato su uno schermo visibile a tutti i visitatori rendendo il pubblico protagonista inconsapevole della narrazione. ${ }^{7}$

Norma Jeane presenta l'opera Who's Afraid of Free Expression? un grande cubo di plastilina dei colori della bandiera egiziana. Il pubblico è invitato a manipolarlo a piacimento liberando la propria creatività. Il titolo dell'opera affronta il tema della libertà di espressione, è un omaggio a un manifesto esposto durante le proteste di Piazza Tahrir al Cairo durante la Primavera araba e che a sua volta riprende il titolo della commedia inglese di Edward Albee Who's Afraid of Virginia Woolf. L'opera d'arte fa riferimento ai divieti ancora vigenti in molti paesi in merito alla libertà di espressione considerata un diritto fondamentale e allo sfruttamento da parte dei paesi europei di risorse provenienti dai paesi africani. Al termine della Biennale, grazie alla partecipazione dei visitatori, dal cubo scultura minimalista nella sala si è ottenuta una scultura collettiva (Biennale 54 2011, 212).

Il Padiglione danese Speech Matters curato da Katerina Gregos, espone sculture, disegni, incisioni e anche installazioni ideate per luoghi pubblici legate a tematiche sociali e politiche oltre che sul tema della libertà di parola. Ospita opere di diciotto artisti provenienti da dieci paesi diversi tra cui Thomas Kilpper che propone un'installazione, Pavillon for Revolutionary Free Speech (2011), consistente in una struttura esterna, che si colloca direttamente nel giardino e costruita con materiali di reimpiego provenienti dalla Biennale architettura dell'anno precedente. Il para-Padiglione di Kilpper costituisce un'area in cui rifugiarsi fermarsi e socializzare e ospita lo Speaker's Corner una terrazza in legno dove hanno luogo performance e letture e che può essere fruita dai visitatori che desiderano condividere messaggi tramite un grande megafono di metallo. L'opera è una scultura sociale, uno spazio politico, un luogo per la libera espressione in cui riflettere sul linguaggio dei discorsi pubblici (Biennale 54 2011, 70).

6 Zbyszek Sypniewski, «Padiglione Spagnolo. Dora García - L'inadeguato/ Lo Inadecuado/ The Inadequate», Cura.magazine, 6 luglio 2011.

7 «La 'inadecuada' Dora García», Yo dona, 1 de junio de 2011. 
Una volta terminata la Biennale, l'artista utilizza poi i 140 metri quadrati di legno che costituiscono l'installazione come matrice per incisioni per la mostra Incisioni veneziane che si tiene l'anno successivo a Reggio Emilia presso Dispari\&dispari Project. Le opere sono stampe su carta e su stoffa e riguardano ancora una volta i temi della libertà di espressione, la censura e l'esclusione sociale al fine di promuovere una maggior consapevolezza riguardo alla questione dell'integrazione dei migranti e dei diritti umani.

Anche il colombiano Nicolás Paris, che occupa la Sala F nel Padiglione centrale ai Giardini con il suo progetto Classroom: Partial exercises (2011), prevede incontri con il pubblico, laboratori di disegno, un archivio di disegni e materiale pedagogico. Si tratta di un processo di arricchimento continuo grazie all'apporto dei visitatori che contribuiscono ad incrementare l'archivio con le loro opere grafiche (Biennale 54 2011, 242).

Il Padiglione tedesco ospita opere di Christoph Schlingensief, mancato nel 2010: Plan B è il progetto portato avanti dopo la sua scomparsa e diviene una dedica retrospettiva, una riflessione sulla sua arte, la sua vita e le sue opere. Tra le opere esposte, A Church of Fear vs the Alien Within (2008) affronta il tema personale della malattia dell'artista e allo stesso tempo costituisce una riflessione sui temi di vita, morte e spiritualità. Nell'ambiente espositivo viene ricostruita la chiesa di Oberhausen in Germania dove Schlingensief aveva svolto servizio come chierichetto da giovane e dove si sono tenuti i funerali alla sua morte. Il corpo dell'artista, in questa chiesa ricreata, prende il posto dell'eucarestia e vi sono materiali quali foto e video che documentano la malattia e, al posto degli arredi liturgici, elementi che ricordano la sua vita e la sua carriera. Si tratta di un oratorio Fluxus che si presta a rappresentazioni teatrali, visioni cinematografiche e che consente l'esperienza collettiva di emozioni quali paura, angoscia e insicurezza (Cornish 2012). All'interno vengono proiettati film prodotti dall'artista, mentre in un'altra ala del Padiglione è esposto il suo progetto Remdoogo, realizzato in Burkina Faso, e vengono proiettate alcune scene dell'opera teatrale Via Intolleranza II (2010) la quale si ispira ad Intolleranza 1960 di Luigi Nono (Gaensheimer 2011). A Schlingensief viene riconosciuto il premio del Leone d'Oro.

Marinella Senatore nel Padiglione centrale ai Giardini presenta il progetto Estman Radio Drama (2011) che coinvolge cinquecento persone della cittadina veneziana di Marghera. Il lavoro parte da delle ricerche sociologiche che coinvolgono un gruppo di operai della zona industriale di Porto Marghera e le loro famiglie e si estendono agli studenti delle università veneziane Ca' Foscari e IUAV oltre che della Scottish Accademy of Music and Drama di Glasgow. Il titolo dell'opera Estman Radio Drama si riferisce al teatro mobile del nord della Spagna che negli anni Cinquanta era una fonte di svago per gli operai. Senatore prevede la scrittura collettiva del Drama da parte del- 
la comunità locale e viene trasmessa da una postazione in mostra e attraverso canali radio locali e nazionali. L'opera riflette sulle condizioni lavorative degli operai nel polo industriale negli anni Settanta attraverso lo studio dell'Archivio operaio «Augusto Finzi» e le rapporta a quelle contemporanee, ai problemi occupazionali nell'attualità (Biennale 54 2011, 262).

Alla Biennale del 2013 Il Palazzo Enciclopedico, curata da Massimiliano Gioni, Tino Sehgal presenta nel Padiglione centrale ai Giardini la performance Senza titolo (2013) interpretata da una coppia o un gruppo di interpreti di età diversa che danzano e cantano una nenia, seduti o inginocchiati sul pavimento, in un rituale di trasmissione orale tra generazioni. Gli interpreti si muovono a contatto con il pavimento e in raccoglimento, ascoltando a turno l'altro; i gesti sono eseguiti in base alle sensazioni che si trasmettono reciprocamente. Alla base dell'opera vi è l'idea di un sapere che viene trasmesso in modo ciclico, all'infinito, attraverso le differenti età dei partecipanti. La performance si basa sull'entrare in relazione continua l'uno con l'altro. ${ }^{8}$ I temi toccati riguardano l'istintività del corpo, l'intimità sentimentale ed il racconto orale. ${ }^{9}$ L'azione dei performer che si raccolgono in ginocchio o seduti ad accogliere il discorso altrui vuole evidenziare un comportamento in disuso nella società contemporanea. L'atteggiamento che assumono è anche quello di prendersi cura l'uno dell'altro e di apertura alla comprensione reciproca, un comportamento che dovrebbe comunicarsi anche al pubblico. L'opera rappresenta un rituale sociale in cui la memoria collettiva viene trasmessa tra gli interpreti e il pubblico presente (Il Palazzo Enciclopedico 2013, 163). A Sehgal viene conferito il Leone d'Oro per l'eccellenza e la portata innovativa del suo lavoro.

In quella stessa Biennale si tiene all'isola della Giudecca un evento collaterale dell'artista Dora García, The Joycean Society (2013), consistente nella proiezione di un film che ha come soggetto la lettura corale di Finnegans Wake di James Joyce presso un circolo letterario di Zurigo. Nello spazio espositivo troviamo un tavolo con tre opere di Joyce - Ulysses, Finnegans Wake e Dubliners - e le annotazioni dell'artista, una lavagna con diagrammi e disegni e il video proiettato sulla parete..$^{10}$ L'oggetto dell'opera non è tanto il racconto di Joyce quanto l'esperienza di lettura nel gruppo che affronta temi moderni come il discorso onirico e schizofrenico e antichi, riprendendo la lettura corale ad alta voce diffusa in ambienti sacri e prima dell'av-

8 Marta Calcagno Baldini, «I vincitori e le opere imperdibili di questa 55. Biennale d'Arte a Venezia», Touring Club Italiano blog, 1 giugno 2013.

9 Chiara Cartuccia, «Tino Sehgal, o del gesto», Doppiozero, 14 giugno 2013.

10 Juliette Soulez, «Venice Report: Dora García Finds Utopia in Finnegan's Wake, Bloiun Artinfo, 18 July 2013. 
vento della stampa. García è affascinata dall'esperienza dei circoli di lettura in quanto perseguono progetti di comprensione collettiva attraverso la condivisione di idee, esperienze di vita, interessi ed emozioni con la finalità di formare un sapere comune, costituendo la comunità The Joycean Society. Si tratta di un poema ciclico senza fine che può essere letto all'infinito e che accompagna i lettori per la vita; la circolarità della lettura suggerisce un'interpretazione non gerarchica dell'opera ed il discorso appare come un fluire di parole ma con una struttura precisa che ricorda quella del discorso schizofrenico (García 2013a).

L'edizione del 2015 All The World's Futures è curata da Okwui Enwezor, l'artista Marco Fusinato alle Corderie dell'Arsenale presenta l'opera From the Horde to the Bee (2015) un'installazione composta da pile di libri posizionati lungo il bordo di un tavolo: si tratta di copie della omonima pubblicazione che raccoglie documenti provenienti dall'Archivio Primo Moroni di Milano. Il libro composto dall'artista si configura come un archivio portatile di retorica radicale nel quale tutti i documenti sono stati scansionati nello stesso formato (cf. Fusinato 2015). I volumi si possono acquistare in cambio di un'offerta di dieci euro per sostenere l'Archivio; l'artista non vuole che alcun controllo venga esercitato sulle transazioni e l'esposizione dell'atto di compravendita vuole evidenziare il ruolo del pubblico nel sistema economico: i visitatori non sono semplici acquirenti ma fanno attivamente parte dell'opera (Biennale 56 2015, 571).

Ana Gallardo nel Giardino delle Vergini all'Arsenale espone l'opera El Pedimento (2009-15), frutto di un progetto con il carcere femminile di Venezia. Si tratta di un luogo in cui plasmare delle statuine d'argilla con finalità propiziatoria secondo una pratica ispirata a un rito messicano della comunità di Oxaca. Presso un santuario dedicato alla Vergine Maria i pellegrini infatti creano delle figurette che vengono poi lasciate all'aperto e che con la pioggia e le intemperie vengono distrutte, tornando alla terra dalla quale provengono e portando a compimento il desiderio espresso da chi le ha modellate. I visitatori dell'esposizione sono invitati ad entrare in questa stanza scura, collocata nel giardino e riempita di terreno, a riflettere sulla fertilità del suolo e sul rapporto tra la vita e la morte. Essi possono plasmare la propria statuina propiziatoria (All the World's Futures. Guida Breve 2015, 326).

Dora García alle Corderie dell'Arsenale prosegue il lavoro sul linguaggio e la comunicazione iniziato con le opere degli anni precedenti e presenta la performance The Sinthome Score (2014-15). La performance è generata dalla lettura della traduzione inglese non ufficiale del seminario di Jaques Lacan numero XXIII Le Sinthome (1975-76), e prevede l'interpretazione del testo tramite la danza. La coreografia è suddivisa in dieci movimenti ognuno dei quali corrisponde a una delle dieci lezioni del seminario (cf. García 2013b). L'opera è anima- 
ta da due interpreti ma è aperta alla partecipazione del pubblico che può interpretare la lettura con il movimento (All the World's Futures. Guida Breve 2015, 179).

Ivana Müller nel padiglione centrale ai Giardini presenta la performance We Are Still Watching (2012) in collaborazione con Andrea Bozic, David Weber-Krebs e Jonas Rutgeerts. L'opera viene messa in scena dal pubblico e prende forma in base ai suoi comportamenti. I visitatori seduti nell'Arena leggono un copione ad alta voce e ognuno recita un ruolo all'interno di una comunità. Il tema è la partecipazione politica attiva e la delega di rappresentanza. L'opera è una sorta di provino in cui ognuno legge un testo per la prima volta con il copione in mano. La performance permette ai partecipanti di formare una micro comunità temporanea per la durata della recita, di circa un'ora, per un totale di dieci episodi più epilogo e ha un carattere aggregativo (All the World's Futures. Guida Breve 2015, 130).

Olaf Nicolai lavora con media differenti e progetta performance sonore che si basano sull'esecuzione di melodie con la voce. A quella Biennale nel Padiglione Centrale ai Giardini presenta l'opera Non consumiamo... (to Luigi Nono) (2015) che si ispira all'opera del compositore Luigi Nono incisa su nastro magnetico Un volto del mare / Non consumiamo Marx (1969) in cui erano presenti le registrazioni audio dei manifestanti del Sessantotto alla Biennale unite con il suono delle onde del mare e letture tratte dall'opera di Cesare Pavese. Nicolai invita nell'Arena otto performer a reagire con il canto alla lettura del testo Il Capitale di Karl Marx. Il loro canto viene registrato con uno smartphone e diffuso in mostra attraverso degli zaini attrezzati con altoparlanti portatili che sono messi a disposizione dei visitatori che vogliano partecipare propagando il suono della performance (All the World's Futures. Guida Breve 2015, 133).

Adrian Piper all'Arsenale espone The Probable Trust Registry (2013), che risulterà vincitrice del Leone d'Oro, un'installazione in cui un addetto, posto dietro a un desk, fornisce ai visitatori un documento da firmare che attesta la loro volontà di iscriversi al «registro della fiducia». Chi compie questo atto dichiara di assumersi una responsabilità morale verso se stesso e gli altri. I documenti raccolti includono clausole come «I will always mean what I say» ed entrano a far parte del APRA, Adrian Piper Research Archive Foundation a Berlino. L'opera vuole ricostruire la fiducia nel contratto sociale e creare una micro comunità basata sulla sincerità reciproca e la pratica di empatia per un approccio altruista al prossimo (All the World's Futures. Guida Breve 2015, 101).

Rirkrit Tiravanija nella sede dell'Arsenale espone l'opera Untitled $2015(14,086)$ Si tratta di un'installazione di mattoni crudi prodotti proprio in situ da appositi addetti e il numero legato al titolo dell'opera fa riferimento ai pezzi necessari a costruire una piccola casa famigliare in Cina. L'idea dell'opera nasce infatti in Cina, a Pechino, 
nel 2004 osservando le strade con mattoni ammassati utilizzati per ricostruire le case dopo un terremoto, ma su quelli creati all'interno della Biennale viene inciso in cinese il motto situazionista «Ne travaillez jamais» che vuole sensibilizzare il pubblico sulle condizioni di vita e lavoro degli operai. Il pubblico inoltre può portarne a casa degli esemplari in cambio di una donazione di dieci euro all'associazione senza scopo di lucro ONG ISCOS, Istituto Sindacale per la Cooperazione allo Sviluppo, che si occupa di diritti dei lavoratori in ambito internazionale (All the World's Futures. Guida Breve 2015, 72).

L'edizione del 2017 Viva Arte Viva viene curata da Christine Macel, Olafur Eliasson prosegue il progetto Green Light-An Artistic Workshop (2016) iniziato l'anno precedente a Vienna presso la galleria Thyessen-Bornemisza Art Contemporary. L'artista invita rifugiati, migranti, studenti e pubblico ad assemblare lampade poliedriche che vengono raccolte nello spazio espositivo del Padiglione centrale ai Giardini e formano una collezione collettiva risultante dalla ricerca e dalla sperimentazione dei partecipanti che si relazionano nella costruzione e possono essere utilizzate singolarmente o in costruzioni più complesse date dall'assemblaggio di più moduli (Biennale 57 2017, 74). Il laboratorio è un ambiente favorevole allo scambio e alle relazioni ed è in particolare rivolto a rifugiati e migranti affinché si possano relazionare con la cultura ospitante attraverso un approccio creativo in uno spazio sociale. I migranti che partecipano al progetto sono volontari che per due mesi si recano quotidianamente alla Biennale e sono stati reclutati attraverso le organizzazioni che gestiscono i centri per i richiedenti asilo. Essi possono beneficiare di un corso di lingua italiana e di colloqui con legali che offrono suggerimenti per affrontare il percorso burocratico di integrazione, dato che Eliasson considera la partecipazione del pubblico in arte una componente fondamentale e sostiene l'importanza di un atteggiamento critico di valutazione da parte del pubblico impegnato nell'opera (cf. Obrist 2008).

Lee Mingwei presenta due opere, la prima all'Arsenale The Mending Project (2009) è un'installazione che si compone di un lungo tavolo, due sedie e rotoli di filo di tessuto. L'artista attende seduto al tavolo un visitatore alla volta; i visitatori portano abiti da rammendare e l'artista esegue il rammendo con del filo colorato. Durante l'operazione di cucito tra il visitatore e l'artista si instaura un rapporto che è simbolicamente rappresentato dal filo di colore evidente lasciato attaccato alle cuciture. A differenza delle usuali operazioni di sartoria l'artista non vuole nascondere il danno ma simboleggiare con una cucitura che il tempo trascorso nell'operazione ha portato all'instaurazione di una relazione (Biennale 57 2017, 194). Ai Giardini Lee Mingwei presenta la performance When Beauty Visits (2017) sul tema del dono della bellezza. Una performer invita un visitatore a turno tra il pubblico a passare del tempo in contemplazione della bellez- 
za nel piccolo ambito-giardino ideato da Carlo Scarpa all'interno del Padiglione Centrale. Dopo aver trascorso il tempo del raccoglimento meditativo al visitatore viene consegnata in dono una busta con l'invito ad aprirla al prossimo «incontro con la bellezza» ovunque esso avvenga. All'interno della busta si trova la testimonianza di un'altra persona, scritta in precedenza, al suo incontro con la bellezza: il progetto era iniziato infatti già un anno prima dell'apertura dell'Esposizione con la 'raccolta' di episodi incorsi a conoscenti, amici, parenti, colleghi e sconosciuti. Quest'opera stimola la riflessione su come la bellezza viene percepita, condivisa e ricordata offrendo una modalità di esperienza che può essere ripetuta in altri luoghi (Biennale 57 2017, 194). L'artista definisce le sue opere 'creature viventi' poiché si accrescono nel tempo grazie alla relazione con il pubblico.

Ernesto Neto nella mostra all'Arsenale presenta Um Sagrado Lugar (2017) una struttura di poliammide ispirata al gazebo di rete $\mathrm{Cu}$ pixawa in uso da parte degli indios Huni Kuin in Amazzonia per le loro cerimonie sociali: è appesa al soffitto e scende fino a terra accogliendo al suo interno i visitatori che possono accomodarsi su cuscini disposti sul pavimento, respirare gli odori delle spezie che segnano l'ambiente e suonare o ascoltare musica prodotta con gli strumenti messi a disposizione. Neto crea un ambiente multisensoriale che favorisce l'aggregazione rifacendosi al modello indigeno in cui hanno luogo rituali volti a ristabilire le connessioni tra l'uomo e la natura e ad allontanare le energie negative. L'opera vuole evidenziare come nel mondo contemporaneo si sia perduta la sacralità della natura e fa parte di un progetto più ampio che prevede la divulgazione della cultura autoctona della foresta amazzonica (Biennale 57 2017, 352).

Alla cinquantasettesima Biennale la Tunisia presenta il progetto collettivo The Absence of Paths (2017) sul tema delle migrazioni, con una piattaforma on-line intesa come luogo di dialogo sul tema dove addetti del settore e persone comuni possono inserire materiali audio, audiovisivi e testuali da condividere con la comunità digitale e tre postazioni collocate invece a Venezia per l'emissione di uno speciale documento di viaggio che si propone di abbattere le frontiere (Biennale 57 2017, 164).

Le opere di arte relazionale presentate alla Biennale condividono dunque l'orizzonte comune delle relazioni con il pubblico e ciò avviene attraverso differenti approcci: il primo consiste nell'organizzazione di laboratori e la conseguente produzione di materiale, si tratta di opere in cui le componenti immateriali si fondono con quelle materiali e in cui vengono creati risultati differenti: nel caso del Progetto Oreste una pubblicazione, per Keyword School di Xu Tan uno studio, per Classroom di Nicolas Paris un archivio, per Green LightAn artistic Workshop di Olafur Eliasson delle lampade ecosostenibili. Altri artisti adottano il linguaggio della performance: Chen Zhen, Tino Sehgal, Dora García, Olaf Nicolai, Ivana Müller e Lee Mingwei. 
Le opere di Michael Schmitz e Christoph Schlingensief rientrano in più articolati progetti umanitari. Altre opere si caratterizzano per l'approccio ludico: The Future from the Sidelines di Jeanne van Heeswijk, Eleaven Heavy Things di Miranda July e Who's Afraid of Free Expression? di Norma Jean. Altre ancora si focalizzano sull'aspetto economico delle relazioni esponendo i meccanismi della compravendita in mostra: From the Horde to the Bee di Marco Fusinato e Untiteld 2015 (14086 unfired) di Rirkrit Tiravanija. Altri artisti propongono la creazione di una comunità provvisoria alla quale ogni visitatore può partecipare; si tratta di opere come Stazione Utopia, The Probable Trust Registry e The Absence of Paths. L'opera Estman Radio Drama di Marinella Senatore si può collocare nell'ambito dell'arte pubblica collettiva mentre Santiago Sierra realizza opere che evidenziano il tema delle relazioni attraverso la loro negazione. Sono ispirate alla spiritualità di culture differenti le opere Jue Chang-Fitfty Strokes Each di Chen Zhen, El pedimiento di Ana Gallardo e Um Sagrado Lugar di Ernesto Neto. L'aspetto comune a tutte è però l'esperienza guidata dalla percezione: la percezione del sé muta e matura in relazione all'ambiente e la conoscenza del sé si modifica in relazione agli altri e all'esperienza vissuta.

\section{Bibliografia}

All the World's Futures. Guida Breve (2015). Venezia: Marsilio.

Bourriaud, Nicolas [1998] (2010). Estetica relazionale. Trad. di Marco Enrico Giacomelli. Milano: Postmedia.

Cornish, Matt (2012) «Art is Magic/It Cannot Succeed. Cristoph Schlingensief Via Intoleranza II». The Drama Review. A Journal on Performance Studies, 56, 191-7.

Felix Gonzalez-Torres (1997). Felix Gonzalez-Torres: Museum moderner Kunst Stiftung Ludwig Wien, 12. September 1997-1. November 1998=Catalogo della mostra (itinerante 12 settembre 1997-1 novembre 1998). Ostfildern-Ruit: Cantz Verlag.

Fusinato, Marco (2015). From the Horde to the Bee. Milano: Colibrì Edizioni.

Hanru, Hou (2003). «Chen Zhen, an Extraordinary Adventure into the Realm of Sinergy». Rosenberg, David; Xu, Min (eds), Chen Zhen, Invocation of Washing Fire. Prato: Gli Ori, 22-36.

Gaensheimer, Susan (ed.) (2011). Cristoph Schlingensief. German Pavillon 2011. 54th International Art Exhibition La Biennale di Venezia. Berlin: Srenberg Press.

García, Dora (a cura di) (2011). L'Inadeguato. Lo Inadecuado. The Inadequate. Mad Marginal Cahier 2. Berlin: Sternberg Press.

García, Dora (2013a). The Joycean Society. Milano: Silvana Editoriale.

García, Dora (2013b). The Sinthome Score: The Seminar of Jacques Lacan, book 23. Joyce and the Sinthome. S.I.: s.n.

Il Palazzo Enciclopedico. Guida breve (2013). Venezia: Marsilio.

Martínez, Rosa (ed.) (2003). Santiago Sierra. Pabellón de España. 50a Bienal de Venecia. Spanish Pavillon. 50th Venice Biennale. Madrid: Ministerio de Asunto Esteriores. 
Norese, Giancarlo (a cura di) (2000). Oreste alla Biennale. Milano: Charta.

Obrist, Hans Ulrich (2008). Olafur Eliasson. Köln: Walther König.

Obrist, Hans Ulrich (2010). Rirkrit Tiravanija. Köln: Walther König.

Olaf Nicolai (2006) = Olaf Nicolai 2003-2006 = Catalogo della mostra (Leipzig, Galerie EIGEN+ART, 29 aprile-26 agosto 2006). Nürnberg: Verlag für moderne Kunst.

Speech Matters (2011). Milano: Mousse.

We Are the World (2003). We Are the World. Biennale di Venezia. Dutch Pavillon 2003. Amsterdam: Artimo.

Zhen, Chen (2003). «Transexperiences. A Conversation between Chen Zhen and Zhu Xian». Rosenberg, David; Xu, Min (eds), Chen Zhen, Invocation of Washing Fire. Prato: Gli Ori, 152-87. 


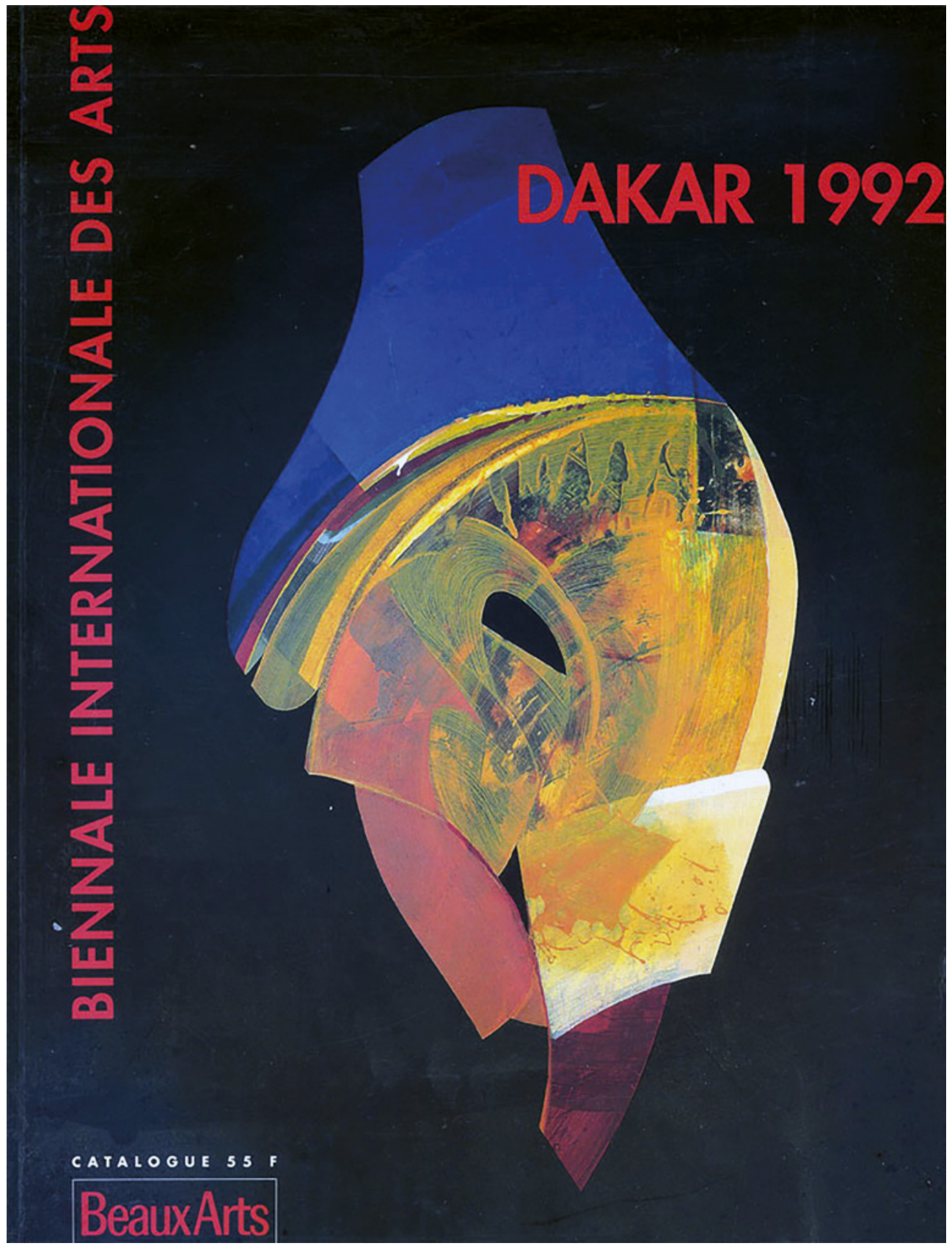

Copertina del catalogo della Biennale Internationale des Arts di Dakar del 1992 


\title{
Dak'Art. Biennale de l'Arte Africain Contemporain
}

\author{
Chiara Pattaro
}

\begin{abstract}
Dak'Art, the Dakar Biennale of Art was devised in Senegal in 1989 as the Biennale de Dakar des Arts et des Lettres but - after an edition committed only to literatures, the Biennale des Lettres in 1990 - was achieved in 1992 as the Biennale Internationale des Arts de Dakar. Its creation is influenced by Leopold Sedar Senghor's ideas: between 1960 and 1980, he was the first President of Independent Senegal and was always a passionate promoter of African art and culture. Under his government the nation developed a flourishing cultural context that will later lead to the birth of Dak'Art which, from its second edition in 1992, is exclusively dedicated to visual arts. This essay describes history, management and organization of its editions, until 2014, analysing some artists and artworks as case histories. The study identifies therefore the characteristics of a Biennale that is purposefully Pan-African, committed to giving visibility and prominence to African contemporary art, which is still poorly represented in the international art system.
\end{abstract}

Keywords Dak'Art. Senegal. Art Biennial. Contemporary African Art. Leopold Sedar Senghor.

La critica Caroline Amber Jones sostiene che le biennali d'arte ereditano alcuni dei loro principi e caratteri dalle Grandi Esposizioni ottocentesche: il concetto di universalità, il bisogno di produrre e diffondere conoscenza, la volontà di migliorare il mercato, di implementare il turismo, come pure di 'abbellire' le città ospitanti con nuove infrastrutture e infine per presentare e diffondere nuove opere di carattere internazionale (Van Hal, Øvstebø, Filipovic 2010,69), ma in realtà agli esordi si ispirano al modello del Salon.

Questo saggio trae origine anche dalle ricerche compiute in occasione della tesi di laurea magistrale Pattaro, Chiara (2016). La Biennale di Dakar: "Dak'Art 2014", un caso di studio [laurea magistrale]. Venezia: Università Ca' Foscari Venezia. Relatore Stefania Portinari, correlatore Nico Stringa, a.a. 2015/2016 e dalle ricerche compiute negli archivi delle istituzioni di Dakar nel 2014.

Storie dell'arte contemporanea 4 | Atlante delle Biennali 1

ISSN 2704-9973

ISBN [ebook] 978-88-6969-366-3 | ISBN [print] 978-88-6969-367-0 
Le biennali sono luoghi in cui gli artisti possono rappresentare la loro realtà all'interno di un contesto globalizzato, con la speranza di entrare nel mercato mondiale (F. Martini, V. Martini 2011, 13). Per l'artista e curatore austriaco Peter Weibel (2015, 2-4) la globalizzazione può ancora essere intesa come la diffusione dei valori occidentali su scala globale, se si propongono alle biennali soprattutto opere europee o si ammettono quelle che rispecchiano questi canoni artistici. Weibel crede profondamente nel format biennale come luogo di conoscenza e crescita animato da uno spirito di resilienza. Le biennali infatti possono richiamare un grande pubblico e dare visibilità agli artisti e alle nazioni ospitanti, alimentandone la notorietà e il prestigio. Queste esposizioni possono inoltre promuovere e scoprire nuove correnti d'arte e, se animate da un forte spirito democratico, anche quelle delle periferie, che sono ancora poco rappresentate dalle gallerie, dai musei, dalle fiere internazionali e nelle aste.

Negli anni Novanta il fenomeno delle Biennali diventa 'virale' e se ne contano un'impressionante numero, sempre in crescita: ognuna di esse cerca una propria individualità per risaltare rispetto alle altre. La crescita culturale e artistica di questi avvenimenti si avrà però solo se sapranno svilupparsi e passare da una globalizzazione dettata e guidata dal commercio, a una globalizzazione in cui l'opera d'arte riflette e completa il mondo.

La città sede di questi eventi, in cui si riscoprono edifici o ne vengono costruiti di nuovi, così come le infrastrutture che garantiscono l'afflusso di visitatori, acquisiscono un carattere cosmopolita proprio grazie al pubblico e agli espositori internazionali: essa diviene quindi un luogo di conoscenza, investito da un ruolo specificatamente pedagogico come la scoperta del mondo che vi prenderà parte, senza dimenticare i benefici economici che un così importante evento può garantire, che comporta al contempo anche il rischio dell'assunzione del ruolo di gentrification e di promozione urbana.

La Biennale di Venezia è l'archetipo da cui tutte le altre traggono ispirazione. Anche Alfredo Sigolo analizza il fenomeno della diffusione del modello biennale parlando di «biennalizzazione»: nell'articolo Biennali invisibili, obiettivi sensibili ${ }^{1}$ evidenzia come sia indispensabile la periodicità dell'evento per mantenere l'arte contemporanea attuale, evitando la «musealizzazione» che la renderebbe già parte della storia. Per «biennalizzazione» si intende quindi il fenomeno di globalizzazione che pone l'arte, anche locale, su di una piattaforma internazionale, ovvero a una conoscenza più ampia possibile, in grado di soddisfare il bisogno primordiale di scoperta dell'essere umano. Gli Stati promotori sostengono le Biennali per diffondere e pubblicizzare la loro real- 
tà locale, ma soprattutto per collegarla a un sistema internazionale. Il bisogno di 'scoperta' di nuove ricerche artistiche viene appagato non solo dalla mostra in sé, ma anche dalla possibilità di spostarsi e relazionarsi. Le biennali africane e orientali sono quelle che - all'interno di questo discorso sull'altro' e sul 'diverso' - meglio soddisfano questo bisogno, soprattutto da parte del ricettore occidentale.

La separazione tra ideali positivi e motivi capitalisti risulta sottile, in questo processo di attuazione e 'invenzione' di nuove biennali: si tratta di una contraddizione intrinseca che appartiene anche alle altre precedenti biennali come quella di San Paolo e di Kassel e ancora più presente nella realtà del 'mondo globalizzato', ma al contempo sottolinea il bisogno del Sud del mondo di sentirsi valorizzato al pari dei Paesi occidentali. Nel concepire una rassegna che mostri diversità di modi e espressioni si contribuisce comunque a un processo di conoscenza degli altri, che si auspica porti a rispettarli e apprezzarli, sostenendo così non solo la cultura, ma anche una profonda interazione. Dak'Art viene vissuta dalla comunità artistica e intellettuale senegalese proprio come un luogo di ricerca e sperimentazione comune per creare nuovi modelli artistici e conoscere nuovi modi d'espressione, si prefigge di valorizzare le creazioni di artisti emergenti, principalmente del continente africano, per proporre un'arte che sappia interpretare e influenzare il presente.

La nascita e la storia della Biennale di Dakar è strettamente legata al rinnovo della società e della politica dell'Africa, avvenuto durante il XX secolo, ovvero con la fine formale del colonialismo. Achille Bonito Oliva descrive così questa evoluzione:

L'Africa per molto tempo è stata un'enorme discarica di veleni coloniali e postcoloniali. I primi, per durata storica, avevano prodotto un'inevitabile globalizzazione politica e dipendenza economica dalle potenze occidentali. I secondi, frutto dell'abbandono al loro destino da parte dei paesi colonizzatori, nei decenni scorsi (e forse tuttora) hanno sviluppato ineluttabili forme di tribalizzazione. Tra globalizzazione e tribalizzazione, alla fine del travagliato Ventesimo secolo, l'Africa presenta le sue credenziali, istituzionali e artistiche, per poter esprimere una propria identità culturale. ${ }^{2}$

La Biennale di Dakar è la continuazione del processo di diffusione della cultura africana intrapreso con il Festival Mondial des Arts Nègres del 1966 voluto da Leopold Sedar Senghor, uno dei rappresentanti del movimento culturale, politico e letterario che prende il nome di nègritude, eletto nel 1960 primo presidente della Repubblica del Senegal.

2 Achille Bonito Oliva, «Le identità dell'Africa a Dak'Art 98». Africa e Mediterraneo, giugno 1999. 
La négritude è la presa di coscienza, da parte dell'africano, del valore della propria differenza culturale e sociale e in tale processo si esalta la produzione letteraria e artistica precoloniale di un'Africa tradizionale concepita in modo idilliaco e ideale.

Abdou Diouf, l'ex primo ministro di Senghor che sale al potere nel 1981 e vi rimane fino al 2000, ha cercato di proseguire l'azione del suo predecessore e di prendersi merito di grandi azioni nel campo delle arti, ma non sono poche invece le critiche che ha ricevuto per quanto sia stato invece poco percepito il suo patrocinio artistico e per come abbia anzi profondamente strumentalizzato i suoi interventi per la sua ascesa politica. Ė durante il suo mandato che, nel 1985, un gruppo di artisti si unisce nell'Association Nationale des Artistes Plasticiens du Sénégal (ANAPS) e fonda a Dakar il primo Salon national des artistes plasticiens, un'esposizione di scultura senegalese che verrà in seguito organizzata in alternanza alla Biennale di Dakar.

Nel 1989 Diouf progetta la Biennale de Dakar des Arts et des Lettres come un evento che alterni un'edizione dedicata alla letteratura a una all'arte. L'annuncio dell'istituzione della Biennale avviene nell'ottobre del 1989, durante una conferenza su L'écrivain et les drois de l'homme organizzata dall'Association des Écrivains du Sénégal (fondata nel 1973 da Senghor per sostenere gli scrittori senegalesi) e il Centre d'Animation et d'échanges culturels, un centro che promuove iniziative culturali. Nel 1990 si inaugura così la Biennale des Lettres, dedicata esclusivamente alla letteratura: sono presentate aree culturali e creazioni letterarie provenienti dall'intera Africa. Senghor è invitato come spettatore onorario. Nello stesso anno gli artisti senegalesi, tramite l'ANAPS, chiedono espressamente al presidente Diouf una manifestazione di risonanza internazionale, che permetta di esporre e far conoscere l'arte senegalese: la loro proposta è di istituire una Biennale in Africa dedicata solo all'arte contemporanea.

La seconda edizione della Biennale si tiene nel 1992 ed è dedicata alle arti visive: la Biennale Internationale des Arts de Dakar, intitolata Arts e Regards croisés sur l'Afrique. Vi partecipano artisti provenienti da varie aree divisi per nazionalità, prendendo a modello la Biennale di Venezia e i suoi Padiglioni nazionali.

Il segretario generale è il poeta Amadou Lamine Sall, che aveva ricoperto lo stesso ruolo alla Biennale precedente, dedicata solo alla letteratura, e vengono riconfermati anche molti altri membri dell'organizzazione. A Sall vengono affiancati tre assistenti: lo storico dell'arte Christian Tonani, Ismaïla Diouf, responsabile della Galerie Nationale d'Art di Dakar, e lo storico Mamadou Diouf; sono inoltre istituiti un Comitato Tecnico per la gestione organizzativa dell'evento e una Giuria Internazionale per la selezione e premiazione delle opere esposte.

Questa seconda edizione subisce pesanti critiche perché viene considerata particolarmente strumentalizzata dal potere politico, se- 
condo quanto riportato anche da studiosi come il critico d'arte ivoriano Yacouba Konaté (2009) o dalla specialista di arte africana contemporanea Iolanda Pensa (2006): il presidente Diouf la promuove per ottenere l'appoggio degli intellettuali senegalesi nelle imminenti elezioni. In effetti la selezione degli artisti partecipanti è affidata alle ambasciate internazionali presenti in Senegal, alle istituzioni culturali straniere, alle organizzazioni internazionali e ai ministeri, utilizzando quindi una rete legata principalmente al governo, arricchita solo in parte da poche conoscenze dirette dei curatori.

Questa però è anche l'unica edizione in cui siano ammessi artisti internazionali, di qualsiasi nazionalità. Gli artisti senegalesi però sono numerosi, tra cui gli scultori Ousmane Sow, con opere in bronzo, e Moustapha Dimé, che propone lavori composti in legno e materiale riciclato.

I problemi tecnici e finanziari si dimostrano rilevanti: come riporta Pensa (2006), l'evento lascia un debito corrispondente a 55.307,15 euro, saldato dal budget della Biennale successiva.

Nel giugno del 1993 il Ministero della Cultura organizza una settimana di incontri per analizzare e valutare quanto offerto dalla Biennale, per rispondere alle domande e alle critiche mosse a queste prime due edizioni. In seguito, nell'ottobre dello stesso anno, il ministro della Cultura Coura Ba Thiam annuncia la decisione di dedicare la Biennale esclusivamente alla promozione dell'arte contemporanea africana e di istituire un segretario generale e un Comitato Scientifico per assicurare una gestione più controllata e imparziale. Il segretario generale deve occuparsi della realizzazione tecnica, ovvero definire il piano delle attività, nominare i commissari del Comitato d'Esecuzione e del Comitato Tecnico, coordinare gli interventi, e della gestione finanziaria, cioè elaborare il budget e amministrare i conti. Il Comitato Scientifico invece è l'organo incaricato di indicare obiettivi, strategie e criteri di valutazione della Biennale; i suoi componenti, personalità senegalesi del mondo dell'arte e della cultura nominate dal Ministero della Cultura, eleggono i membri del Comitato Internazionale di Selezione e di Giuria e identificano i professionisti da invitare. Il Comitato Scientifico è anche responsabile delle valutazioni finali nell'assegnazione dei premi. Il presidente della Biennale di Dakar, che deve essere obbligatoriamente un critico d'arte, e il segretario generale costituiscono parte del Comitato di Selezione e di Giuria. ${ }^{3}$

La Biennale del 1996, che prende il nome di Biennale de l'Art Africain Contemporain, si svolge tra il 7 e il 14 maggio e rispecchia questa struttura organizzativa, escludendo gli artisti occidentali per valorizzare l'arte contemporanea dell'Africa; gli artisti dell'Africa Occi-

3 Thomas Fillitz, «The Biennial of Dakar and South-South Circulations», ARTL@S, 2016, 57-69. 
dentale inoltre sono in numero dominante. Questo Panafricanismo attribuisce una particolare specificità all'evento, distinguendolo da altre Biennali nate negli ultimi anni.

L'intellettuale senegalese Rémi Sagna, molto vicino al presidente Senghor, è nominato segretario generale. Sagna, dopo aver conseguito una laurea in Geografia presso l'Université Cheikh Anta Diop di Dakar, si trova a coprire dei ruoli significativi in Senegal, come direttore di alcune biblioteche pubbliche e funzionario per l'Organization Internationale de la Francophonie (OIF), un ente che promuove la cultura e i diritti umani in tutti i territori di lingua francese. Yacouba Konaté riporta nel suo saggio La Biennale de Dakar. Pour une esthétique de la création africaine contemporaine-têtê à avec têtê Adorno (2009) un discorso che Sagna tiene nella sede francese dell'associazione Afrique en créations per presentare il progetto di questa Biennale. In quell'occasione, secondo Konaté $(2009,30)$, non chiese fondi, ma un sostanziale sostegno nella pubblicizzazione dell'evento in Europa. Sagna è determinato a dimostrare che in Africa esistono persone competenti, oneste e capaci di concepire, creare e gestire un grande evento, senza l'appoggio economico e intellettuale dell'Occidente. La Biennale per lui è un simbolo di rivalsa del popolo africano che sottolinea la propria indipendenza e il proprio valore. Tuttavia è bene ricordare che se l'organizzazione rimane sostanzialmente africana, la maggior parte dei finanziatori dell'evento sono ancor oggi europei.

Dak'Art richiama comunque l'interesse di un pubblico internazionale, pertanto è considerata una grande opportunità sia per gli artisti che per gli specialisti del settore. Allo stesso modo l'impostazione panafricana risveglia l'interesse dell'intero continente, ciò significa che agli artisti senegalesi, considerati al pari degli altri, sono dedicati spazi espositivi più limitati rispetto alle Biennali precedenti.

La mostra comprende il Programma Ufficiale, costituito dall'Esposizione Internazionale con opere africane contemporanee, i Saloni del Design e della Creatività Tessile e mostre individuali; l'esposizione non è più divisa in Padiglioni nazionali, ma segue un allestimento dettato da criteri estetici. Vengono organizzati anche dibattiti, proiettati film, creati laboratori d'arte per i ragazzi delle scuole. Purtroppo per quanto concerne le prime edizioni di questa rassegna mancano informazioni dettagliate e fotografie che ci diano conto della gestione degli spazi e delle opere degli artisti realmente presenti, perché non esistono documenti e materiali ufficiali attendibili. In alcune tabelle riassuntive riportate da Konaté (2009), che non inserisce per esempio il Salone della Creatività Tessile, sono trascritti i nomi dei dodici artisti partecipanti al Salone del Design, di cui sei sono senegalesi, e è riportato solamente che l'ivoriano Vincent Amian Niamien vince, con l'opera Souami, il Prix de la Créativité.

Secondo quanto indicato dallo stesso Konaté $(2009,56)$, gli artisti che adottano una ricerca più contemporanea creano scandalo per- 
ché ritenuti troppo innovativi rispetto alla tradizione. Tra questi riporta il camerunense Pascal Marthine Tayou, che partecipa anche alla Biennale di Venezia del 2005 e del 2009. La sua opera, Exotisme tribal, propone un'installazione con bambole trafitte da frecce, come feticci di stregoni, unite ironicamente a escrementi e preservativi. Appare evidente così l'enorme differenza tra artisti davvero contemporanei e quelli invece più conservatori.

Nelle edizioni successive si richiede che gli artisti, per essere ammessi alla selezione, debbano essere in possesso del passaporto di uno dei Paesi del continente africano e compilare il modulo di candidatura presente nel sito ufficiale. La scelta di presentare solo l'arte africana contemporanea intende dare alla Dak'Art una specificità che la contraddistingua. Gli artisti sono scelti dal Comitato Internazionale di Selezione e di Giuria, nominato dal Comitato Scientifico senegalese, che esamina i dossier di presentazione inviati dagli artisti stessi e costituiti da sei opere ciascuno, poste su cd o in diapositive. Fino al 2000 questi dossier rimangono anonimi durante la fase di selezione, per mantenere l'imparzialità nella scelta delle opere. Considerato che chiunque può candidarsi, la Biennale viene intesa come una grande opportunità. I curatori e i critici invitati a effettuare la scelta dei partecipanti vengono incoraggiati a impiegare comunque 'criteri internazionali', assicurando la qualità delle opere secondo una nuova idea di arte africana contemporanea.

Dal 1998 la Biennale è strutturata in due sezioni: un programma ufficiale, chiamato In, e un programma laterale, formato da iniziative indipendenti, chiamato Manifestations d'environnement, che - dalla quarta edizione del 2000 - prende il nome di Off.

L'esposizione ufficiale si sviluppa solitamente in tre parti: un'Esposizione Internazionale, dove espongono artisti africani contemporanei, un Salone del Design (non presente in tutte le edizioni), che in alcune occasioni comprende il salone della Creatività Tessile e le Esposizioni Individuali, ovvero delle retrospettive che focalizzano l'attenzione su alcuni protagonisti dell'arte contemporanea del Paese.

Col tempo, al programma ufficiale sono associate altre iniziative come il Padiglione degli Artisti Senegalesi, i laboratori per le scuole, i concerti, la mostra-vendita di opere, le conferenze, le sfilate di moda e altri eventi chiamati «animazioni».

L'edizione della Dak'art del 1998 si è svolta tra il 24 e il 30 aprile con Rémi Sagna confermato nel ruolo di segretario generale. L'italiano Achille Bonito Oliva è stato nominato presidente della Commissione Selezione e Premiazione degli Artisti (cf. 1999). Le Manifestations d'environnement hanno dato come esito ventinove siti espositivi distribuiti nella città, anche presso centri culturali internazionali, per coinvolgere il più possibile le persone. È stato istituito anche il Marché des Arts Plastiques Africaines (MAPA) presso la Casa della Cultura Douta Seck a Dakar, dove era possibile comprare delle opere d'arte. 
A quell'edizione sono presenti anche i cosiddetti «artisti della diaspora», cioè personalità di origine africana ma formate in Europa o negli Stati Uniti e oramai residenti all'estero, spesso percepiti come più 'vicini' all'occidente, per i mezzi utilizzati o per le tematiche affrontate. Tra questi vanno considerati i camerunensi Goddy Leye, con opere concettuali, e Barthélémy Toguo, fotografo, scultore e pittore autore di dipinti astratti, che diffondono stili e mezzi espressivi considerati molto innovativi in quel contesto, in un connubio di video, fotografie e installazioni. Entrambi si sono stabiliti in Europa (Leye in Olanda e Inghilterra; Toguo in Francia e Germania), per poi tornare a vivere in madrepatria fondando studi d'arte.

Yacouba Konaté, che in quell'edizione cura il Salon de la Jeune Création Ivoirienne, racconta di come l'artista tradizionale Mohamede Diabaté, profondamente colpito dalle opere presentate all'Esposizione Internazionale, decida di non usare più la sabbia e la scorza di cocco per le proprie sculture, elaborando invece una nuova forma espressiva con disegni su carta dal gesto immediato e semplice $(2009,31)$. La manifestazione quindi risulta fondamentale nel relazionare artisti sconosciuti e/o tradizionali con realtà nuove e stimolanti, capaci di trasformare la loro arte.

Tra le opere esposte sono particolarmente interessanti quelle del ghanese Seth Kane Kawei, che ha partecipato alla significativa mostra Magiciens de la Terre tenutasi al Centre Georges Pompidou nel 1989 e anche in occasione della Africa Explores: 20th Century African Art, esposizione itinerante iniziata dal Center for African Art e dal New Museum for Contemporary Art di New York nel 1991. Le sue bare a forma di automobili di prestigiosi marchi occidentali sottolineano provocatoriamente come anche la morte e il funerale affermino lo status del defunto.

I nigeriani e i sudafricani propongono opere soprattutto sui temi della resistenza e della segregazione razziale, criticando la politica e il sistema sociale. Tra questi il nigeriano Aniedi Okon Akpan - che ugualmente ha esposto a Magiciens de la Terre, nella sezione Africa Remix, e alla Biennale di Venezia del 2001 - usa la scultura per creare massicce opere in cemento che ritraggono africani in smoking, cravattino e bastone.

Nel 2000 le elezioni del governo si tengono proprio pochi mesi prima dell'apertura della manifestazione e viene eletto presidente $\mathrm{Ab}$ doulaye Wade. Il governo conferma il suo sostegno all'evento, che ormai ha raggiunto un certo prestigio che ne assicura l'esistenza, e la Biennale si svolge regolarmente, prendendo ufficialmente il nome di Dak'Art ed estendendosi per la prima volta per un mese intero, dal 5 maggio al 5 giugno.

Rémi Sagna è riconfermato segretario generale per la terza volta e gli artisti vengono scelti ancora tramite dossier anonimi dal Comitato Internazionale di Selezione, composto da dieci membri di cui cinque 
europei, mentre per le Esposizioni Individuali sono nominati sei commissari, due dei quali europei, che propongono direttamente gli artisti.

L'esposizione In è allestita al museo dell'IFAN; le Esposizioni Individuali al Village des Arts, nei pressi dell'aeroporto Leopold Sédar Sénghor e alla Galerie Nationale d'Art; il Salon International du Design africain et de la Créativité textile nello spazio VEMA nel porto dell'isola di Gorée; il MAPA presso la casa della cultura Douta Seck e il Salon de la Jeune Création plastique Sénégalaise al centro culturale Blaise Senghor di Dakar.

Iolanda Pensa (2006) sottolinea che questa edizione della Biennale è inaugurata per la prima volta dal presidente del Senegal accanto a Mamadou Diop Decroix, ministro della Cultura e della Comunicazione. ${ }^{4}$ Durante la cerimonia sono assegnati i premi e Wade descrive le riforme in atto per promuovere le attività culturali del Paese. Nel programma Off si segnala la mostra Alimentation d'Art, curata da Peter Wollenweber e Abdoulaye Guissé dell'organizzazione tedesca Querformat Art Support: essa propone l'opera del senegalese Mansour Ciss, che ha allestito sue opere e prodotti alimentari all'interno di un negozietto di quartiere, la Boutique d'Alimentation.

Sebbene in catalogo siano riportati i nomi degli artisti in ordine alfabetico, con l'indicazione del Paese d'origine, le immagini delle opere e alcune informazioni biografiche, i testi e i documenti su questa edizione sono pochi e spesso riportano dati approssimativi o tra loro contrastanti.

Alla Dak'Art 2002, che si svolge tra il 10 maggio e il 10 giugno, viene organizzato un programma parallelo che prende il nome di $\mathrm{Da}$ kartoff, seguito per la prima volta da un curatore: l'italiano Mauro Petroni, proprietario dell'atelier di ceramica Almadies di Dakar. L'esposizione si compone di novantanove mostre disseminate nei quartieri della città e viene creato un logo da impiegare in un'apposita segnaletica che, assieme al posizionamento di bandiere, identifica le varie esposizioni.

Le Esposizioni Individuali sono suddivise nelle sezioni Afrique, curata dalla critica senegalese N'Gone Fall; Diaspora, curata ugualmente da un senegalese, Ery Camara, e Monde curata dall'italiano Bruno Corà che propone tre artisti europei: il greco Jannis Kounellis, lo spagnolo Jaume Plensa e il viennese Franz West. È molto difficile reperire informazioni affidabili su questa edizione e persino il suo catalogo, ma Konaté riporta che quest'ultima sezione è molto criticata dai più integralisti, come il senegalese Iba Ndiaye Diadji, professore di Estetica all’Università Cheikh Anta Diop di Dakar, in particolare per la scelta di esporre Kounellis. Ndiaye accusa l'artista e il suo curatore di essere poco attenti alla realtà africana, proponendo opere 
che paiono alla gran parte dei visitatori dei semplici oggetti, come il Sans-titre consistente in sacchi di iuta contenenti cereali. Si sottolinea così ancora una volta il divario fra l'Africa e l'Occidente.

Dopo quell'esperienza un'ipotesi molto accreditata è quella di sostituire l'edizione successiva con la ripresa del Festival Mondial Des Arts Nègres, che sarà effettivamente riproposto, ma con un suo corso a parte, dal 2010. Dak'Art viene invece confermata e ha luogo dal 5 maggio al 5 giugno 2004 e il numero di visitatori internazionali continua ad aumentare rispetto alle precedenti edizioni.

Le scelte del comitato di selezione prediligono giovani artisti che impiegano modalità artistiche innovative. La sezione Afrique, dedicata alle Esposizioni Individuali, è curata da Yacouba Konaté; la sezione Diaspora curata dal critico brasiliano Ivo Mesquita e quella Monde dallo svizzero-londinese Hans Ulrich Obrist, che presenta dodici artisti internazionali. Sappiamo poco però dell'allestimento della mostra, ancora una volta per una mancanza di documentazione: nelle esposizioni si associano opere più contemporanee (nelle sezioni Diaspora e Monde con artisti provenienti da vari Paesi) ad altre di soli artisti africani che vivono e operano nel continente (nella sezione Afrique), innovativi rispetto a un'arte tradizionale, ma che continuano a usare gli stessi mezzi espressivi. Si intende comunque che l'edizione della Biennale va verso un tentativo di apertura, in cui si mette in atto un contrasto positivo tra le tre esposizioni, riconoscendone la diversità e l'unicità.

Il critico statunitense Okwui Enwezor, in occasione di una delle tavole rotonde che vengono organizzate durante questa Dak'Art, disapprova fortemente questa partizione delle mostre individuali. Sottolinea come questo tipo di suddivisione non permetta di confrontare liberamente le opere e i diversi background degli esecutori. A tal proposito evidenzia come molti degli artisti che vivono in Africa si siano formati in Europa o in America e che quelli con doppia nazionalità si sentano a proprio agio sia nel paese d'origine che d'accoglienza. L'organizzazione sembra troppo preoccupata di mettere in evidenza l'appartenenza o l'origine africana, mentre gli stessi specialisti (come lo stesso Enwezor, di origine nigeriana) si percepiscono sempre più parte di un contesto globalizzato che non pone troppe suddivisioni. Secondo l'antropologo francese Jean-Loup Amselle $(2004,177)$ invece questa Biennale diventa il «crocevia dei percorsi che conducono dal panafricanismo alla globalizzazione» per il legame tra un'arte popolare tradizionale africana, che doveva essere rinnovata, e un'arte occidentalizzata, che da questo incontro poteva essere rivitalizzata.

La Biennale del 2004 è segnata anche da una numerosa presenza di artisti dal Maghreb e dall'Egitto: tra questi gli egiziani Khaled Hafez, che abbina quadri e video relazionando i mezzi della tradizione con quelli della modernità, e Amal El Kenawy che con la sua 
installazione descrive il matrimonio come una prigione del cuore e dei sentimenti. Tra i premiati è il franco-congolese Michèle Magema che con l'opera La porte (2002), caratterizzata da tre video, mette in relazione il ruolo della donna durante la dittatura di Mobutu Sese Seko, nella Repubblica Democratica del Congo, la tratta degli schiavi e il campo nazista di Dachau.

Il Padiglione del Design propone un atelier-laboratorio condotto dall'algerino Mohamed Yahyaoui detto Yamo, dal camerunense Jules Bertrand Wokam, dai maliani Madeleine Bombote e Cheikh Diallo, dove la maggior parte degli oggetti esposti sono prodotti in loco.

Il programma Off ha ancora come commissario Mauro Petroni ed è caratterizzato da centotrenta esposizioni collocate in siti diversi della città, di cui però non rimane menzione, non essendo stati segnalati nel catalogo ufficiale. L'esigenza, ancora una volta, è quella di sensibilizzare la popolazione all'arte contemporanea: con la collaborazione dell'École de Beaux Arts di Dakar viene formata un'equipe di guide, sviluppato un piano educativo da proporre alle scuole e proiettati dei video sulla Biennale nei quartieri della città. Vengono persino organizzati dei concerti serali accompagnati da proiezioni delle opere dell'edizione ritenute più significative.

Nel 2005 Dakar è colpita da una terribile alluvione, una catastrofe che distrugge interi quartieri, tanto che molte case sono dichiarate inagibili e 250.000 persone perdono ogni cosa. Sorgono dunque forti inevitabili polemiche sul finanziamento da assegnare a Dak'Art, a fronte di una tale emergenza umanitaria, ma l'edizione del 2006 è confermata e ha luogo tra il 5 maggio e il 5 giugno.

Nel settembre del 2005 Safiatou Ndiaye Diop, ministro della Cultura e del Patrimonio Storico chiede a Konaté di assumere il ruolo di direttore artistico: questo ruolo compare dunque per la prima volta. L'idea che regge quell'edizione è l'intento di promuovere una Biennale panafricana dedicata all'arte contemporanea e pensata per un pubblico africano, che coinvolga tutti gli artisti di origine africana del mondo. I commissari cercano di promuovere opere che trattino di temi legati a queste istanze e che possano aiutare il continente a promuovere la propria identità.

Le opere esposte sono pensate per entrare in relazione con lo spettatore. La sociologa e critica d'arte francese Nathalie Heinich sostiene che parte del budget della Biennale debba essere speso per sensibilizzare il 'popolo', non solo per le scuole, tanto che nel laboratorio d'arte digitale proposto per l'edizione, dal nome Dak'Art Lab, l'artista britannico Keith Piper gestisce uno stage per la creazione di video dedicati alla popolazione di Dakar, con l'obiettivo di coinvolgere gli abitanti della capitale che visitano poi la Biennale anche per riconoscersi nelle riprese. I filmati sono inoltre diffusi in televisione e proiettati in diversi punti della città. Con la stessa intenzione agisce l'afroamericano William Popel, che propone un'installazione fo- 
tografica da realizzarsi nelle vie di Dakar, dove lui fotografa i passanti o fa realizzare loro delle fotografie. I protagonisti diventano dunque la gente comune, incontrata per strada, che poi si spera possa visitare l'esposizione per ritrovarsi fotografata o riconoscersi come autrice delle immagini.

Konaté nomina sei commissari aggiunti i quali, oltre a vagliare i dossier di candidatura presentati dagli artisti, propongono loro stessi possibili partecipanti; si tratta di sei collaboratori responsabili ciascuno di una zona geografica. I curatori, oltre a selezionare le candidature libere inviate da artisti con passaporto africano, votano collettivamente sui partecipanti proposti da loro stessi.

I dossier presentati dagli artisti della diaspora sono più professionali rispetto a quelli dei connazionali e le loro opere hanno uno sguardo più critico e lucido verso la propria cultura d'origine. L'esposizione almeno però non suddivide i partecipanti in base alla loro provenienza, riducendo la barriera che da sempre divide l'esposizione Internazionale da quelle Individuali, e cerca un equilibrio tra i linguaggi tradizionali di scultura e pittura e quelli considerati 'nuovi', come video, installazioni, performance. Gli artisti della diaspora vengono considerati importanti per accelerare la ricezione in patria delle nuove ricerche e Dak'Art si rivela sempre un'ottima occasione di scambio, pur con le inevitabili critiche, come quelle dirette dal pittore senegalese Djibathen Sambou che ritiene alcune opere troppo «occidentalizzate» e reputa gli artisti che le propongono troppo disinibiti, travestiti di libertà, segno di una sorta di «anarchia mentale». Il giudizio negativo a volte colpisce anche i materiali utilizzati, che giudica «immondizia di discarica» (Amselle 2007, 70).

Un quarto delle opere esposte sono di giovani artisti, un altro quarto di artisti ritenuti tra i fondatori dell'arte africana contemporanea, e i due quarti rimanenti sono opere di artisti affermati. Anche se i temi sono diversi (ecologia, urbanizzazione, politica, spiritualità e identità), costante è la ricerca di un dialogo: lo stile astratto affianca quello figurativo e l'arte popolare incrocia quella concettuale.

Anche in questa edizione gli allievi dell'École de Beaux Arts di Dakar sono impegnati come guide didattiche. Il Padiglione del Design presenta mobilio, lampade e oggetti di altro tipo; alcuni sono prodotti negli atelier organizzati durante l'apertura e possono essere acquistati durante la Biennale.

Si cerca anche di portare le esposizioni in spazi più centrali: il Centre International des Conférences et Echanges du Sénégal (CI$\mathrm{CES}$ ), che è uno dei luoghi espositivi storici, è sentito come troppo periferico. Il cuore dell'evento è trasferito all'ex museo dell'Istitut Français d'Afrique Noire, l'INFAN: al piano terra sono organizzate le esposizioni, nel prato anteriore è costruito un Padiglione con una struttura mobile per ampliare la proposta espositiva. Nei pressi sono organizzate altre mostre, alla Maison de la culture quella di Dou- 
ta Seck, altre alla Chambre de Commerce, alla Crypte de la Cathédrale e all'Espace Culturel Vema.

Se per la prima volta viene previsto un premio per il programma Off, tra quelle esposizioni risulta particolarmente significativa l'iniziativa Exit Tour (2006), dell'artista e intellettuale camerunense Goddy Leyes di Douala, sostenuta da Art Bakery, un'organizzazione che intende promuovere l'arte contemporanea camerunense. L'opera consiste in un itinerario attraverso la città di Dakar nei luoghi simbolo dell'arte, durante il quale è possibile incontrare artisti e istituzioni.

Fino a questa edizione i membri del Comitato di Selezione sono numerosi, talora anche più di dieci; la metà di essi è di origine africana e gli altri vengono dall'Europa o dal Nord America. Thomas Fillitz, professore di antropologia culturale e sociale presso l'Università di Vienna, spiega così la scelta di portare a Dak'Art professionisti occidentali d'arte contemporanea:

It was a strategy of the Biennale to ensure an international quality of the selected artworks. Above all, it was a conscious act for raising the visibility of Dak'Art within the global culture of biennials. (Fillit 2016, 62)

Dopo il 2006 i membri del Comitato di Selezione vanno progressivamente diminuendo, dal 2010 sono quattro al massimo e devono essere specialisti d'arte di cittadinanza africana.

L'edizione del 2008 - su cui è ugualmente molto difficile ottenere informazioni - si svolge dal 9 maggio al 9 giugno con il titolo Afrique: miroir?. Come commissario generale nominato è Maguèye Kassé, professoressa di studi germanici e sociologici presso il Dipartimento di Lingue e Civiltà Germaniche dell'Université Cheikh Anta Diop. L'organizzazione appare fragile e su 317 dossier sono scelti solo 35 artisti, di cui 10 senegalesi, e 13 designer. Due sono gli artisti invitati, entrambi senegalesi: Iba Ndiaye e la pittrice Amina Ndiaye Leclerc.

Con la Dak'Art del 2010 si celebra il ventesimo anniversario della manifestazione. La Biennale viene gestita in autonomia, con molte difficoltà organizzative, senza il contributo dell'Unione Europea, che fino ad allora aveva sempre coperto più della metà del budget. Iolanda Pensa scrive su Domus (2011) un reportage sull'esposizione In, composta da una sezione Internazionale, una Retrospettiva e una di Artisti Invitati; ma le informazioni sull'organizzazione e quelle che si possono ricavare dal catalogo sono davvero poche, tra cui il fatto che il premio Off non è riconfermato, ma vengono allestite 150 mostre in vari siti e viene, per la prima volta, anche coinvolta la città di Saint-Louis.

I curatori dell'edizione del 2012, che ha luogo dall'11 maggio al 10 giugno, sono Christine Eyene, critica d'arte di origine camerunense che vive in Inghilterra, Nadira Laggoune, curatrice e critica di origine algerina, e il curatore sudafricano Riason Naidoo. 
Nell'esposizione In sono presenti 42 artisti provenienti da 21 Paesi africani scelti tra 329 dossier presentati. All'atelier de Joe Ouakam in Place du Souvenir sono organizzate due mostre intitolate Hommages, dedicate agli artisti senegalesi Papa Ibra Tall e Issa Samb detto Joe Ouakam. Alla Maison de la culture 'Douta Seck' di Dakar è presente per la prima volta una sezione dedicata tutta al femminile: la mostra Créativité de femmes in cui espongono dieci artiste donne e le esposizioni Off si estendono su circa 150 siti, mentre la sezione istituita a Saint-Louis è in crescita con altre 40 occasioni espositive.

L'undicesima edizione della Biennale, promossa dal Ministère de la Culture et du Patrimoine del Senegal, si svolge tra il 9 maggio e l'8 giugno 2014. Nel 2013 il ministro della Cultura Abdoul Aziz Mbaye nomina segretario generale il senegalese Babacar Mbaye Diop, tra i maggiori specialisti d'arte dell'Africa subsahariana classica e contemporanea e della diaspora. Mbaye è un grande sostenitore della Biennale, convinto che contribuisca a creare in Senegal una rete di esperti, mercanti, critici, collezionisti, galleristi e conservatori, indispensabili intermediari tra gli artisti e gli acquirenti, connazionali o internazionali.

Per sostenere l'organizzazione della Dak'Art è nominato un Comité d'Orientation che, con il segretario generale, gestisce l'intera manifestazione decidendo il personale dei diversi apparati direttivi e i contenuti della Biennale. La presidentessa di questa commissione è Thérèse Turpin Diatta, responsabile della Kemboury, una delle principali galleria d'arte di Dakar.

Il ministro Mbaye sente il bisogno di dimostrare l'indipendenza non solo economica ma anche culturale e sociale dell'Africa e allo stesso tempo vede come minacciosa la forte presenza dello Stato all'interno dell'apparato organizzativo e teme che l'evento diventi, come d'altronde già successo in passato, un mezzo di propaganda politica piuttosto che un luogo di promozione artistica (2014). ${ }^{5}$ La Biennale, secondo lui, deve puntare alla completa autonomia con partner esclusivamente africani. Questo forte bisogno di autonomia sottolinea come non ci si senta ancora liberati dall'aver subito una dominazione.

Questa Dak'Art ha come titolo Produire le commun, per dimostrare comunque la volontà di unire le diversità del mondo, ma soprattutto di cercare gli ideali comuni e condivisi per la crescita dell'intera umanità. Produrre il comune è un impegno politico perché crea un luogo di scambio pubblico che, grazie all'arte, permette di comunicare, quindi costruire relazioni, senza divisioni e distinzioni tra esseri umani. L'idea è quella di produrre uno spazio comune in cui gli artisti contemporanei possano inserire le loro opere nell'oggi per aiu-

5 Antonella De Gregorio, «L'arte africana si mostra al mondo. A Dak'Art 2014 tutta la creatività del Continente. Tra gli artisti presenti, il Picasso d'Africa, che partecipa anche al progetto Imago Mundi, di Benetton», Corriere della Sera, 22 maggio 2014. 
tarci a interpretare la realtà che ci circonda. Proprio per questo gli artisti non sono divisi all'interno delle esposizioni per nazionalità o Paesi d'origine ma sono collocati negli stessi spazi.

L'edizione è costituita da diverse esposizioni situate in luoghi e città differenti. Comprende infatti una mostra internazionale principale, intitolata In; l'esposizione Les artistes invités con gli artisti invitati direttamente dai commissari e dedicata alla diversité culturelle; il Salon de la sculpture africaine contemporaine; l'esposizione Anonymous, Dak'Art au Campus; le Expositions Hommage e le mostre collaterali appartenenti al programma Off. Durante la settimana d'apertura vengono anche organizzate una serie di conferenze e dibattiti sulle arti visive, ma anche concerti, laboratori e dibattiti in vari luoghi della città e promossi direttamente dall'organizzazione o delle nazioni rappresentate. Questi incontri mirano essenzialmente a formare una nuova classe di intellettuali esperti d'arte africana contemporanea e a incoraggiare la collaborazione tra specialisti del settore provenienti dai diversi continenti.

L'esposizione In, e gran parte delle conferenze e dei concerti, sono proposti al Village della Biennale, la sede principale della manifestazione, situata in un nuovo edificio industriale più integrato e vicino alla città. Il sito è composto da tre edifici grandi e spaziosi, ideali per ospitare qualsiasi tipo di realizzazione artistica. Gli altri luoghi impiegati per l'esposizione sono il Musée Théodore Monod de l'IFAN, che ospita le opere degli artisti invitati dai commissari, il Jardins du Musée Théodore Monod de l'IFAN che ospita l'esposizione delle sculture africane, la Place du Souvenir Africain che accoglie l'esposizione omaggio a Mbaye Diop e Mamadou Diakhaté, la Galerie Nationale per l'esposizione omaggio di Moustapha Dimé e il Campus de l'Université Cheikh Anta Diop (UCAD) per l'esposizione Art Vert. Nella Place du Souvenir Africain, situata a ovest di Dakar, direttamente sull'oceano, è allestito un grande palco per i concerti e gli eventi organizzati durante le serate e sono presenti gli unici due Padiglioni nazionali della Biennale, quelli del Marocco e dell'Algeria.

Per partecipare alle manifestazioni Off invece è sufficiente individuare uno spazio espositivo, un budget per coprire i propri costi e inviare l'apposito modulo alla sede organizzativa. Non viene effettuata una selezione e l'ammissione è assicurata. I formulari presentati servono per raccogliere gli iscritti nella guida della Biennale e fornire ai partecipanti una bandiera con la scritta Off da esibire davanti al luogo d'esposizione.

Le opere più significative nella sezione In sono quelle della sudafricana Candice Breitz e dell'angolano Kiluanji Kia Henda. Breitz in particolare presenta Extra (2011), una installazione video accompagnata da fotografie in cui si mostra lei stessa o particolari parti del suo corpo nudo che compaiono innestati in modo surreale nelle scene di una vera serie televisiva intitolata Generations, una delle fic- 
tion più celebri e amate in Sudafrica, ambientata nel periodo postapartheid e recitata da soli attori neri. La sua presenza straniante, sia perché fuori luogo e con un effetto collage o comunque perché il suo essere bianca risalta in modo provocante e irrispettoso, la rende appariscente, come un'intrusa ironica ma costante.

Kia Henda presenta invece una critica agli aiuti umanitari e militari elargiti dall'Occidente al continente africano, in pieno mood post-coloniale. Trasforma ironicamente la sigla ONG in ORGASM, Organization of African States for Mellowness, usandola come titolo del collage fotografico O.R.G.A.S.M. (As God Wants and Devil Likes It) (2011) che denuncia come secondo lui l'aiuto caritatevole verso l'Africa serva a sostenere l'economia occidentale. L'artista vuole rivelare gli abusi di potere, anche sessuali, e il razzismo che si nascondono dietro le istituzioni della cooperazione internazionale che rendono la carità un business, facendone propaganda politica. Questo lavoro propone satiricamente la creazione di una presunta «Organizzazione africana» istituita a sostegno dell'Occidente, mostrando le fotografie dei grandi leader europei che si africanizzano con acconciature voluminose e appariscenti, ritratti con una bandiera identica a quella europea ma con al centro l'immagine dell'Africa e il logo di ORGASM sullo sfondo, in cui si alternano immagini provocatorie dell'artista, ripreso come una Madonna con una corona di stelle, dei suoi amici e immagini delle diverse parti di un corpo africano nudo.

Se pure è complesso e spesso irrealizzabile reperire materiale archivistico sulla Dak'Art - tanto che a oggi l'istituzione manca di un proprio archivio e spesso i cataloghi dell'evento si possono acquistare solo durante la prima settimana di vernice - e molto raro sia ad ora l'apporto bibliografico su di essa, la Biennale di Dakar risulta davvero un luogo anche simbolico, in cui la molteplice identità africana trova per ora una voce, in cui avviene uno scambio culturale e sociale e viene vissuta dalla comunità artistica e intellettuale senegalese come un luogo di ricerca e sperimentazione comune, per creare nuovi modelli artistici e conoscere modi d'espressione, per proporre un'arte in grado di rinnovare e influenzare il presente. 


\section{Bibliografia}

Dak'Art 2004 (2004). Dak'Art 2004: 6ème Biennale de l'art africain Contemporain = Catalogue de l'exposition (Dakar, 7 mai-7 juin). Dakar: Saii.

Dak'Art 2014 (2014). Dak'Art 2014: 11e Biennale de l'art africain Contemporain = Catalogue de l'exposition (Dakar, 9 mai-8 juin). Dakar: La Rochette.

Altshuler, Bruce (ed.) (2013). Biennials and Beyond Exhibitions That Made Art History 1962-2002. London: Phaidon.

Amselle, Jean-Loup (2007). L'arte africana contemporanea. Torino: Bollati Boringhieri.

Bargna, Ivan (2000). Arte Africana. Milano: Jaca Book.

Bonito Oliva, Achille (1999). «Le identità dell'Africa a Dak'Art 98». Africa e Mediterraneo, 28-9(2-3), giugno, 17-18.

Buggenhagen, Beth (2016). «Dak'Art 11th Biennale of Contemporary African Art». Africa Arts, 49, February.

Busca, Joëlle (2002). L'arte contemporanea africana. Torino: L'Harmattan Italia. Busca, Joëlle (1999). «Uno sguardo dislocato». Africa e mediterraneo, 28-9, febbraio.

Cafuri, Roberta (2005). L'arte della migrazione. Memorie africane tra diaspora, arte e musei. Torino: Trauben.

Castellaneta, Carlo (1961). Sédar Senghor. Milano: Nuova accademia editrice.

Eulisse, Eriberto (a cura di) (2003). Afriche, diaspore, ibridi. Il concettualismo come strategia dell'arte africana contemporanea. Bologna: Aiep Editore.

Filipovic, Elena; Øvstebø, Solveig; Van Hal, Marieke (eds) (2010). The Biennial Reader. An Anthology on Large-scale Perennial Exhibitions of Contemporary Art. Germany: Ed. Bergen Kunsthall.

Fillit, Thomas (2016). «The Biennial of Dakar and South-South Circulations». ARTL@S, 36, May, 83-93.

Konaté, Yacouba (2009). La Biennale de Dakar. Pour une esthétique de la création africaine contemporaine-têtê à avec têtê Adorno. Paris: L'Harmattan.

Hinderliter, Beth (2015). «Producing the Common, Dak'Art 2014». NKA Journal of Contemporary African Art, 36.

Martin, Jean-Hubert (éd.) (1989). Magiciens de la terre = Catalogue de l'exposition (Paris, Centre Pompidou, 18 mai-14 août 1989). Paris: Éditions du Centre Pompidou.

Martini, Federica; Martini, Vittoria (2011). Just Another Exhibition. Storie e politiche delle biennali. Milano: Postmedia.

Okeke Agulu, Chika (2014). «Dak’Art 2014. Various venues, Dakar, Senegal». Artforum, October.

Pensa, lolanda (2006). "Les biennales et la géographie: Les biennales de Venise, du Caire et de Dakar». Créations artistiques contemporaines en pays d'Islam: des arts en tension. Paris: Kimé Editions.

Pensa, Iolanda (2011). "Dak'Art 2010: prospettive e retrospettive». Domus, 3 febbraio. URL https://www.domusweb.it/it/arte/2011/02/03/dakart-2010-prospettive-e retrospettive.html.

Sophie, Perryer (ed.) (2004). 10 Years 100 Artists, Art in a Democratic South Africa. Cape Town: Bell-Roberts Publishing.

Van Dyk Van Niekerk, Barend (1970). The African Image (Négritude) in the Work of Léopold Sédar Senghor. Cape Town: Balkema Publishers.

Weibel, Peter (2015). «Introduction». Biennals: Prospect and Perispectives. International Conference at ZKM / Center for Art and Media Karlsruhe (Germany, 27 February-1 March 2014). Germany: Center for Art and Media Karlsruhe. 

Mapping pavilions 


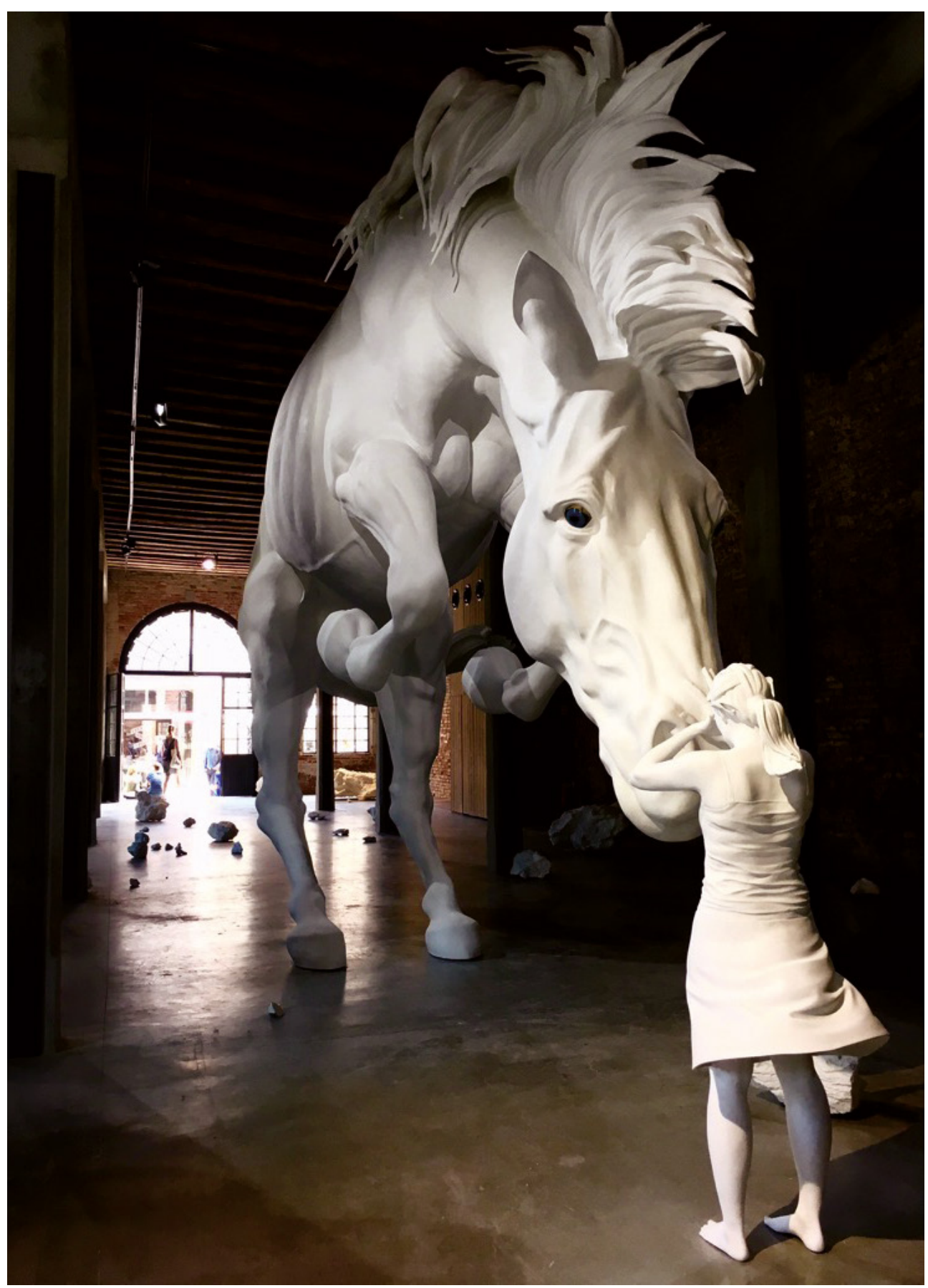

Figura 1 Claudia Fontes, El problema del caballo, La Biennale di Venezia 2017. Courtesy Claudia Fontes 


\title{
L'Argentina alla Biennale d'Arte di Venezia
}

\author{
Paola Natalia Pepa
}

\begin{abstract}
The presence of Argentinian art at Venice Biennale was hosted for the first time in 1901, and then in 1903 and 1922. Argentina, not owning a building at the Giardini venue, showed its artists in the Central Pavilion, in collective or solo exhibitions, since when in 1972 the Argentinian artists took part at the newly founded Latin American Pavilion. In 2011 an arrangement was set to give it a space - finally the Argentina Pavilion - in the Sale d'Armi at the Arsenale. Other important presences were for instance, Lucio Fontana, Nicolás Garcia Uriburu, Oscar Bony and León Ferrari, who was awarded as Best Artist in Biennale during 2007. In conclusion, a comparison is carried out between some significant examples in the lagoon event, up to the most recent participants of last years.
\end{abstract}

Keywords Argentina. Venice Biennale. Arsenale. South America. Latin America.

Il rapporto fra Argentina e Italia in campo artistico è sempre stato significativo: dall'Ottocento al contemporaneo anche l'insegnamento degli italiani presso le istituzioni accademiche e le scuole d'arte del Paese ha costituito una presenza importante.

L'Argentina di inizio secolo è quella della Prima Indipendenza (1816), in cui le ampie distese delle pampas, la vita e le tradizioni dei gauchos rappresentano un mito affascinante e di forte ispirazione per gli intellettuali europei dell'epoca, che vedono diffondersi anche in queste 'nuove' terre l'urbanizzazione e numerosi distretti carboniferi e siderurgici (Sartor 2009, 17-19). Non

Questo saggio trae origine dalle ricerche compiute in occasione della tesi di laurea magistrale Arte argentina: dall'indipendenza al contemporaneo con suggestioni europee. Quattro artisti contemporanei nella Biennale di Venezia. Relatore Nico Stringa, correlatore Stefania Portinari Venezia: Università Ca' Foscari Venezia, a.a. 2015/2016 e dalla partecipazione al convegno Storie della Biennale di Biennale, a cura di Nico Stringa e Stefania Portinari (Venezia, Università Ca' Foscari Venezia, Ca' Dolfin, 6-7 dicembre 2016).

Storie dell'arte contemporanea 4 | Atlante delle Biennali 1

ISSN 2704-9973

ISBN [ebook] 978-88-6969-366-3 | ISBN [print] 978-88-6969-367-0 
si può scindere questa riflessione da un'ulteriore premessa: una stratificazione sociale e culturale dovuta agli anni delle conquiste ha interessato l'intero continente centro e sud americano, ma il fatto che l'Argentina sia stata colonizzata dalla Spagna ha ovviamente posto le basi per alcune caratteristiche etniche, sociali, culturali e di conseguenza artistiche che in seguito all'Indipendenza verranno vissute come una memoria collettiva contrastante. Da un lato è una storia di sottomissione, soprusi e massacri da cui si può evincere una struttura culturale comune tipica dell'America Latina; dall'altro emerge una volontà di allontanarsi da una conversione forzata per ritrovare un'identità individuale che trasmetta le reali origini di questi popoli (Basoalto 2008, 55-65). Nella rivendicazione di un'indipendenza globale - e non solo territoriale - l'arte assume un ruolo fondamentale, è concepita come la voce di uno spirito collettivo che brama la propria libertà. Ne è consapevole Enrico Crispolti che a tal proposito in Arte argentina dalla indipendenza ad oggi. 1810-1987 scrive:

il raggiungimento di una propria identità essendo non soltanto la maggiore soddisfazione esistenziale per chi faccia arte, e che proprio anzi nel fare arte trova l'occasione per raggiungere più agevolmente rispetto ad altri uomini tale necessario quanto disagevole traguardo d'identità. (Crispolti 1987, 11)

Mentre il Sud e il Centro America vivevano le conseguenze di una multiculturalità che ora non era più imposta da altri, gli intellettuali europei salpavano numerosi verso il Nuovo Mondo, motivati da spedizioni scientifiche o semplice curiosità, lasciando memorie su carta o su tela delle loro esperienze. Alcuni artisti si stabilivano definitivamente aprendo atelier o insegnando nelle scuole, portando la tradizione del modello europeo e delle Accademie di Belle Arti: è il caso di moltissimi italiani, come quello del pittore Ignazio Manzoni, che aprì uno studio a Buenos Aires e seppe coniugare i generi in voga nel momento (pittura storica, religiosa e nature morte) con i costumi argentini (Ribera 1987, 14). Fra i suoi allievi si ricorda Cándido Lopez, protagonista di uno slancio artistico di modernità che si avvicina ad Henri Rousseau, dovuto anche ad esperienze militari che deciderà di raccontare in una cinquantina di vedute sulla vita nei campi di battaglia e negli accampamenti (Sartor 2009, 17-23, 49-50).

Gli italiani furono molto presenti come professori anche durante la nascita delle prime scuole di Belle Arti: nel 1877 la Sociedad Estímulo Bellas Artes, l'anno dopo la Escuela Nacional de Bellas Artes, nel 1895 il Museo Nacional de Bellas Artes sancivano la confluenza di una forte componente di provenienza italiana in Argentina. Agli studenti inoltre, grazie ad appositi premi, veniva offerta la possibilità di viaggiare in Europa e essere testimoni della modernità artistica, contribuendo allo scambio di canoni e alla contaminazione della di- 
sciplina. Gli allievi che si formavano con questi maestri erano fautori di una nuova arte sudamericana: Carlos Morel ad esempio studiò con il maestro italiano Pietro Caccianiga, dedicandosi a pittura e litografia e ritraendo aspetti rilevanti della società argentina, assieme a elementi folcloristici, quali gauchos o militari, ritratti della borghesia, corse di cavalli, cacce agli struzzi, rodei... (Sartor 2009, 17-30).

Afferma a tal proposito Ernesto Sábato:

gli intellettuali che guidarono la nazione dell'ultimo trentennio del diciannovesimo secolo [...] popolarono le nostre terre di immigrati da quell'Europa che costituiva il paradigma della filosofia, della scienza e della tecnica. [...] Ne consegue che quella che oggi possiamo chiamare la Nuova Argentina è stata fatta con sangue e spirito europei. (Sábato 1987, 6-7)

L'Argentina più d'ogni altra nazione latino-americana saprà assorbire con entusiasmo la pratica artistica europea, italiana in primer orden, anche in parte per l'assenza di arte indigena in questo Paese. Questa terra così generosa fu la patria di artisti portatori di una nuova visione di matrice italiana, con un certo qual raffinato aggiornamento in campo artistico, sebbene legato ancora a una tradizione ottocentesca e seppur mantenendo una pluralità di linguaggio: dal paesaggismo all'impressionismo al puntinismo (con autori come Walter de Navazio), dai Macchiaioli al post-impressionismo al simbolismo (con Faustino Brughetti), dalla vitalità della scuola fiorentina (come nel caso di Benito Quinquela Martín) al divisionismo italiano (in Pio Collivadino) (Sartor 2009, 63-4). Faustino Brughetti ad esempio si trasferì in Italia nel 1896, rimanendo affascinato dalla «luce e la vita» di questa nazione, entrando in contatto con intellettuali quali Mario Puccini e Giacomo Balla, che lo definì «un artista dal dolore umanizzato» (Brughetti 1946, 11-14).

Nascono forti connessioni da entrambe le sponde, un laccio invisibile che traccia una parentela artistica indissolubile.

La presenza argentina alla Biennale d'arte di Venezia inizia nel 1901 con l'artista Pio Collivadino, come evidenzia lo stesso catalogo della rassegna:

nacque nel '69 a Buenos Aires nell'Argentina e dimora a Roma, dove compì i suoi studi presso l'Accademia di Belle Arti. È la prima volta che espone, ed il suo lavoro è una incoraggiante promessa. (Biennale 4 1901, 50)

Il suo dipinto Vita onesta (1901) fu acquisito dalla Galleria Marangoni di Udine e è attualmente ancora parte della collezione del Museo d'Arte Moderna e Contemporanea di quella città.

Nel 1901 l'Argentina e il Brasile erano considerati i Paesi più significativi dell'America Latina per quanto concerneva il campo dell'edu- 
cazione e della cultura: la partecipazione alla Biennale era dunque il primo sintomo di un riconoscimento considerevole (cf. Biennale 48 1999, 2). Un'altra partecipazione nel 1903 vede il ritorno di Pio Collivadino con Ora di Pranzo (1903) nella Sala K (Biennale 5 1903, 67).

Nonostante l'Argentina non possedesse ancora un proprio Padiglione come altri Paesi, il panorama artistico argentino veniva sorvegliato assiduamente: da ricordare un evento rilevante nel 1922, quando nella sala 20 fu allestita una «Mostra degli Artisti Argentini» composta da venticinque dipinti di Antonio Alice (Formiche umane), Jorge Bermudez (Ritratto di signora), Francesco Bernareggi (Sole d'aprile), Italo Botti (Tranquillità del mezzogiorno), Alessandro Bustillo (Autoritratto), Guillermo Butler Fray (Serenità), Ceferino Carnacini (Pomeriggio piovoso), Emanuele G. Castilla (Piazza di Anacapri), Emilio Centurion (Misia Mariquita), Tito Cittadini (Primo verde), Pio Collivadino (Elevatori di grano), Luis Adolfo Cordiviola (Capra), Cupertino Del Campo (Il giardino degli aranci), Fernando Fader (Fine d'Inverno), Rodolfo Franco (Primi giorni d'Inverno), Alfredo Guido (Ritratto del Pittore Vena), Hector Nava (Il carrettiere), Americo Panozzi (Chiaro di Luna), Adan Luis Pedemonte (Peri cordovesi), Octavio Pinto (Giardino del Heredero), Enrique Prins (Giardino), Cesáreo Bernaldo De Quirós (El embrujador), Carlos Pablo Ripamonte (Dominio), Valentin Thibon de Libian (Camerino), Angel Domingo Vena (Biche); e cinque sculture di Alberto Lagos (Ansietà), Gonzalo Leguizamon Pondal (Hyalis), Agustin Riganelli (Maschera Faunesca e Il poeta Bufano), César Antonio Sforza (Bambina) (Biennale 13 1922, 74-6).

Negli anni Trenta è di particolare rilevanza la presenza di Lucio Fontana, un esempio perfetto dello scambio artistico fra Argentina e Italia: nato a Rosario in Argentina da genitori italiani, alternerà soggiorni nell'una e nell'altra patria, in una continua ricerca di sperimentazione artistica. Nel 1930 Fontana partecipa alla XVII Biennale di Venezia, presentando i due bronzi Eva (1928) nella sala 15 e Vittoria fascista (1929) nel Giardino (Biennale 17 1930, 73, 148).

Dopo vari anni di assenza dalla kermesse veneziana, l'Argentina ritorna nel 1950 con Ernesto Scotti, scelto dal commissario di allora Riccardo Nino García Moritán, e le cui ventinove tele sono considerate di una «purezza di aggressiva semplicità» (Biennale 25 1950, 261-2).

Nel 1952 in sala XXXIII vengono esposti quindici dipinti e sette sculture dei più importanti artisti argentini del momento, fra cui Juan Del Prete con Astrazione (1932), di origini italiane e considerato il primo artista latinoamericano a uscire dall'ambito figurativo con tendenze astratte; Raul Soldi - che studierà all'Accademia di Brera a Milano e esporrà molte volte in Italia - con la sua opera Sarita (1947); Miguel Carlos Victorica, che durante un viaggio a Venezia scoprì il fascino della pittura del Cinquecento, in particolare quella di Giorgione, Tiziano, Tintoretto e Veronese, la cui ispirazione influenza il suo dipinto El collar de Venecia (1914); Pablo Curatella Manes, noto per essere 
stato uno dei primi scultori in Argentina ad adottare nuove istanze plastiche moderne, da cui la sua opera El pajaro (1952) (Biennale 26 1952, 185-9). ${ }^{1}$

Durante l'edizione del 1956, Miguel Ocampo, commissario dell'allora Padiglione Argentina, sottolinea una consapevolezza ormai nazionale e necessaria dei legami artistici fra i due Paesi, seppur con una multiforme espressione di cui fanno uso gli artisti del momento.

Potrà darsi che più tardi [...] si darà la possibilità di scoprire i caratteri specifici di una espressione artistica nazionale, e tutto ciò sarà allora un frutto che verrà ad aggiungersi, poichè - come del resto è nell'ordine logico - l'artista non dirige mai di sua volontà la propria ricerca artistica nell'ambito di uno stile, ma soltanto in quello della propria espressione. (Biennale 28 1956, 309)

La Sala LXV ospita i dipinti di Manuel Alvarez, Victor Chab, Santiago Cogorno, Armando A. Coppola, Ernesto Farina, José Antonio Fernández Muro, Leonidas Gambartes, Jadwiga Alicia Giangrande, Sarah Grilo, Oscar Herrero Miranda, Alfredo Hlito, Victor Magariños, Francisco Maranca, Miguel Ocampo, Rafael Onetto, Ana M. Payró, Leopoldo Pedro Presas, Raul Russo, Ideal Sanchez, Luís Seoane, Clorindo Testa, Carlos Torrallardona, Carlos Enrique Uriarte, Leonor Vassena. Gli scultori presenti sono: José Alonso, Libero Badii, Martín Blaszko, Carlisky, Noemi Gerstein, Gyula Kosice (Biennale 28 1956, 311-15).

Fra le altre edizioni, nella XXIX del 1958 Lucio Fontana avrà assegnata una sala personale in cui presenta, in una mostra antologica di ben ventisette opere, le sue più recenti produzioni: Gessi, Barocchi, Inchiostri e le sculture spaziali, realizzate fra il 1931 e il 1958. (Biennale 29 1958, 21-2). Lo presenta così Guido Ballo nel catalogo di quell’anno:

Estrosa urgenza di rompere ogni schema precostituito, felicità di sperimentare, bisogno di avventurarsi oltre: sempre con un candido, rinnovato stupore poetico che riscatta nell'animo la prestigiosa abilità delle mani. È questo Lucio Fontana. (Biennale 29 1958, 19)

Nella Sala LI di quell'anno, il commissario del Padiglione Argentina Miguel Ocampo sceglierà come rappresentanti Juan Batle Planas, Juan Del Prete e Raquel Forner (Biennale 29 1958, 172-3).

Nel 1966, in occasione della XXXIII edizione, Lucio Fontana è premiato con il Premio Comune di Venezia riservato a un pittore italia-

1 Gli artisti presenti oltre a quelli citati sono: Roberto Azzoni, Eugenio Daneri, Cesareo Bernaldo de Quiros, Roberto Rossi, Lino Eneas Spilimbergo, Francisco Vidal per la sezione pittura; per la scultura gli artisti Carlos de La Carcova, José Fioravanti, Vicente Puig, Antonio Sassone, Antonio Sibellino, Ernesto Soto Avendaño. 
no. Il suo nome è accanto ad artisti quali Alberto Burri, Michelangelo Pistoletto, Renato Guttuso, Agostino Bonalumi, nella sezione Artisti Italiani Invitati: la Sala XXVI a lui dedicata è il risultato di un allestimento ideato da Carlo Scarpa, in cui l'artista considera «la pittura - la tradizionale pittura da cavalletto - o la tradizionale statuamonumento - come alcunché di decisamente tramontato o in via di tramontare» e rende la sua volontà artistica

a dimostrare che lo spazio è anche dietro e intorno e non solo davanti al dipinto, che il dipinto deve essere un "tutto spaziale", una configurazione di forze, di momenti, di processi, piuttosto che una struttura chiusa ed opaca, immobile e definitiva. (Biennale 33 1966, 43)

L'Argentina quell'anno poteva vantare un'altra presenza significativa con Julio Le Parc, collocato nelle sale LXVII e LXVIII. Fondatore del Groupe de Recherche d'Art Visuel (GRAV), le sue creazioni cinetico-ottiche aspiravano ad un contatto con l'osservatore e ad una sua partecipazione attiva. A rafforzare l'importanza di tale presenza, Le Parc verrà premiato con il riconoscimento del Gran Premio (Biennale 33 1966, 121-2).

Gli anni Sessanta per il continente sudamericano sono particolarmente fervidi, sia dal punto di vista culturale che per il dibattito politico l'arte diviene spesso mezzo di espressione di dramma sociale e oppressione, come nel caso dell'Argentina (Marzona 2006, 24). Dal 1930 il Paese infatti è vittima di un'alternanza di colpi di stato e di governi militari, che sarebbe perdurata fino agli anni Ottanta. Dal 1966 un golpe porta al potere le truppe militari e solo presidenti appoggiati dall'esercito; solo nel 1973, dopo dieci anni, in Argentina si svolgono finalmente le elezioni generali con la vittoria del comunista Juan Domingo Perón, che muore però l'anno dopo. Gli succedette la moglie Isabel Martínez de Perón, a causa della quale ci furono pesanti repressioni degli oppositori politici. Nel 1974 un golpe militare filostatunitense rovesciava la presidentessa in favore del generale Jorge Rafael Videla, dando luogo ad una pagina nera nella storia della nazione: atti illeciti di terrorismo di stato perpetrati dal governo militare anticomunista causeranno la morte di più di 40.000 persone, i cosiddetti desaparecidos (Di Tella, 79-104). In questo clima di proibizionismo e censura gli artisti scelgono palchi internazionali per rivelare la loro voce (Carpani, Di Genova 1966).

Delle presenze degli artisti argentini alla Biennale negli anni Sessanta è dunque particolarmente emblematica l'azione performativa di Nicolás Garcia Uriburu nel 1968, che decide di evadere i confini fisici della Biennale colorando di verde fluorescente le acque del Canal Grande, come testimonia infatti il critico Pierre Restany: 
nel 1968, nella Biennale di Venezia, in pieno disordine contestatario, la poesia recuperò tutti i propri diritti per alcune ore: con un liquido biologicamente innocuo e utilizzato dalle Marine in tutto il mondo per identificare le imbarcazioni nell'acqua, Uriburu aveva colorato di un verde elettrico e fluorescente le acque del Canal Grande. La corrente verde della metamorfosi aveva dissipato per alcuni istanti gli spessi miasmi demagogici della giungla dei Giardini. Uriburu era riuscito in un golpe maestro, una splendida dimostrazione di igiene morale dell'arte. (Restany 2001)

Dal 1972 la presenza argentina è stata inclusa nel Padiglione dell'America Latina, che si trovava nello spazio dei Giardini di Sant’Elena: in quella data infatti la Biennale di Venezia invita l'Istituto Italo-Latino Americano (IILA) a formare quel Padiglione, per includervi gli artisti di Perù, Cuba e Cile. Per l'occasione Federico Brook, artista argentino che dal 1956 risiede a Roma, viene nominato commissario del Padiglione e segretario culturale. Da allora l'Argentina si è vista rappresentare, oltre che da eventuali singole partecipazioni di artisti volute nella mostra generale dai curatori delle Biennali, anche all'interno del Padiglione dell'America Latina (cf. IILA 2009, 9).

Gli anni Ottanta vedono l'Argentina in grave difficoltà economica ma nel 1983, dopo la caduta della dura dittatura che dal 1976 ha represso con ferocia ogni libertà, avviene almeno il ripristino della democrazia (1983). La Transavanguardia italiana di Achille Bonito Oliva e il generale ritorno alla pittura nel sistema dell'arte internazionale vengono recepiti con clamore e si assiste a una evoluzione nei soggetti e nei canoni anche nell'arte argentina. La pittura diventa il supporto privilegiato con cui descrivere il dolore per il recente passato e la sperenza per l'inizio di una nuova era (Glusberg 1986, 122). L’anno seguente alla liberazione, nel 1984, l'Argentina viene rappresentata da Antonio Seguí con una serie di dieci dipinti che raffigurano la tumultuosa vita urbana, in maniera caricaturale e umoristica (Biennale 41 1984, 82).

Gli anni Novanta vedranno un gran numero di artisti argentini riconosciuti ufficialmente dal panorama internazionale. Persistono le tematiche socio-politiche: terminata la dittatura i governi di destra e di sinistra che si sono succeduti hanno lasciato debiti incolmabili che tutt’oggi gravano sulla nazione e non permettono una risalita economica. Molti artisti continuano a denunciare questa situazione attraverso l'arte, come Oscar Ruben Bony, presente alla Biennale del 1999 con l'opera Broadcasting News, che - come gran parte della sua produzione dagli anni Novanta in poi - consiste in un polittico fotografico composto da cinque parti, attraversato da spari di pistola inferti dall'artista stesso, in riferimento alle tematiche di violenza e morte. Bony era noto al pubblico della Biennale già per la performance El limite, compiuta nel 1995, in cui una gondola trasportava una bara e una donna dipinta di bianco che allattava un bambino (Alonso 2011, 20-1). 
Un'altra artista significativa che alla Biennale ha dimostrato di voler trascendere le percezioni reali e virtuali è stata Marcela Cernadas, alla 50esima edizione del 2003 curata di Francesco Bonami e Daniel Birnbaum, incentrata sulla dinamica evocativa dell'opera d'arte con il titolo Ritardi e Rivoluzioni. Cernadas, come già altri prima di lei - Nicolás Garcia Uriburu e Oscar Bony per esempio - nell'opera Il gioco del mondo desidera superare i confini fisici della Biennale ampliando la portata della ricezione e trasmissione artistica all'intera città di Venezia. Ispirandosi al romanzo Rayuela (1963) dello scrittore Julio Cortázar, al di fuori dei Giardini della Biennale viene concepita un'installazione site specific realizzata con lastre di legno e resina bianca specchiante che fanno da base a numeri e lettere in acciaio inossidabile, mentre una seconda parte dell'opera prevede una serie di performance eseguite da un gruppo di partecipanti che hanno disegnato 'mondi' tramite gessetti colorati perché, come scrive l'autore

Il gioco del mondo si fa con una pietruzza che si deve spingere con la punta del piede. Ingredienti: un marciapiedi, una pietruzza, una scarpa e un bel disegno con il gesso, preferibilmente colorato. In alto è il Cielo, sotto la Terra. (Cortázar 1963)

Come ultimo spunto di riflessione, nelle università veneziane si tenevano delle letture di brani tratti dal romanzo di Cortázar.

L'opera-itinerario, come è definita dall'artista, diventa un'operagioco a cui tutti possono accedere tramite 'mappe' distribuite alla Biennale; la Cernadas è riuscita così nell'obiettivo di eliminare le distanze fra osservatore e opera d'arte, coinvolgendolo attivamente in un vero e proprio gioco, seguendo le regole e ottenendo il premio finale. Ogni partecipante ha ricevuto, alla fine di ogni episodio, una perla/biglia di vetro di Murano a testimonianza della sua partecipazione come coautore dell'opera. Solo chi ha completato l'intera collezione di perle però ha potuto ricevere il premio finale e visitare l'Osservatorio Meteorologico del Seminario Patriarcale della Basilica della Salute, godendo di una veduta privilegiata della città e della Laguna di Venezia.

Tra le presenze argentine alla Biennale spicca anche quella di Carolina Raquel Antich, da tempo residente a Venezia, che è stata finalista del Premio per la Giovane Arte Italiana nel Padiglione Venezia nel 2005, in occasione della 51. Biennale. Per l'occasione ha esposto cinque dipinti: Galvani, I Malandrini, Noia, $1 \times$ 7, I figli di Robert Kennedy e una animazione, Tamburino di 3', insieme a Manfredi Beninati, Lara Favaretto e Loris Cecchini. Le opere di Raquel Antich prendono forma da pennellate docili e delicati colori pastello. I protagonisti, uno o più bambini, sembrano esistere in un mondo distante dal nostro, forse memori di un ricordo o di una esperienza vissuta. Carolina Raquel Antich rappresenta i suoi personaggi attraverso 
Paola Natalia Pepa

L'Argentina alla Biennale d'Arte di Venezia

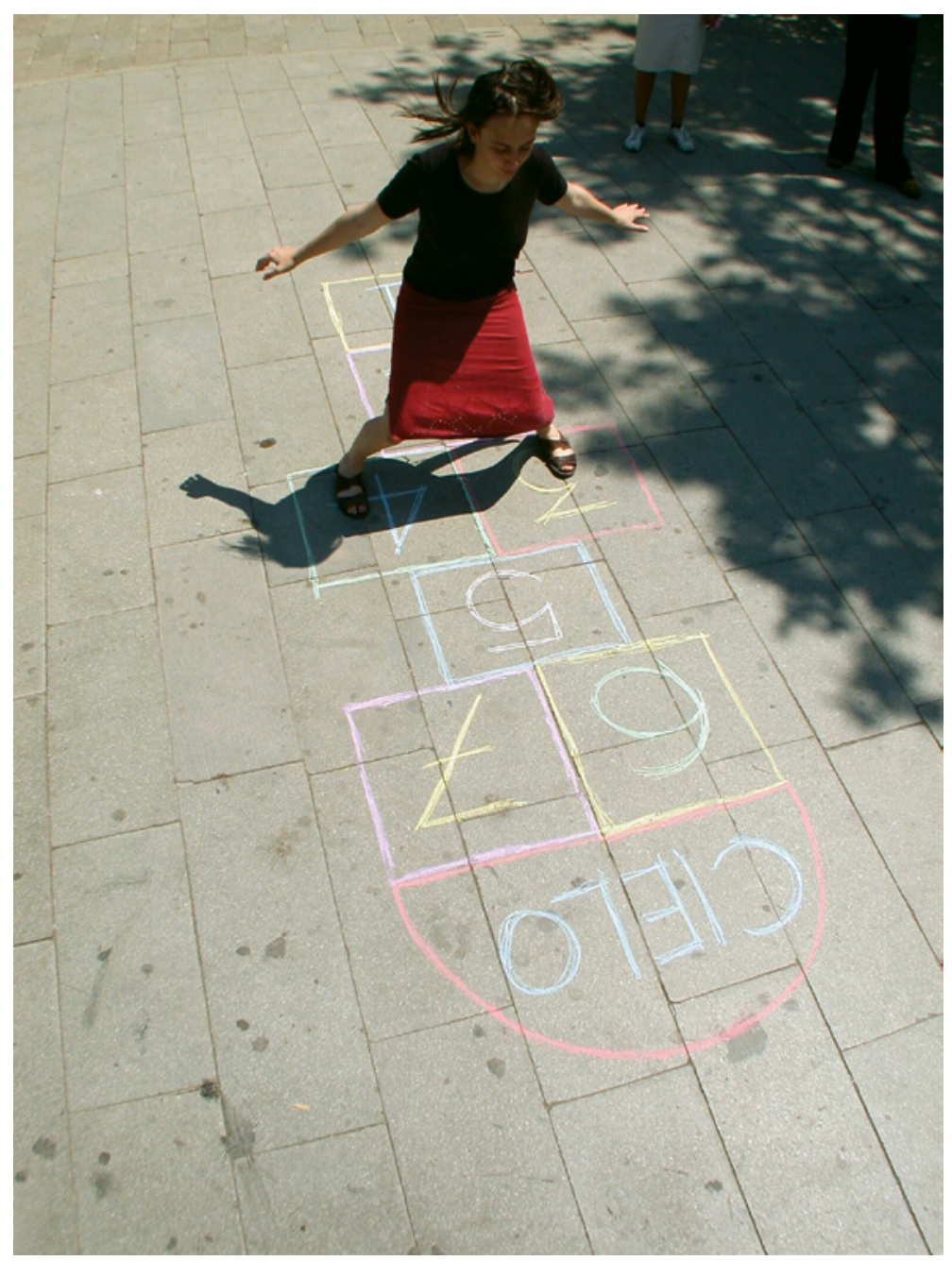

Figura 2 Marcela Cernadas, Il gioco del mondo (2017), performance alla Biennale di Venezia 2017

(foto: Michele Lamanna) 


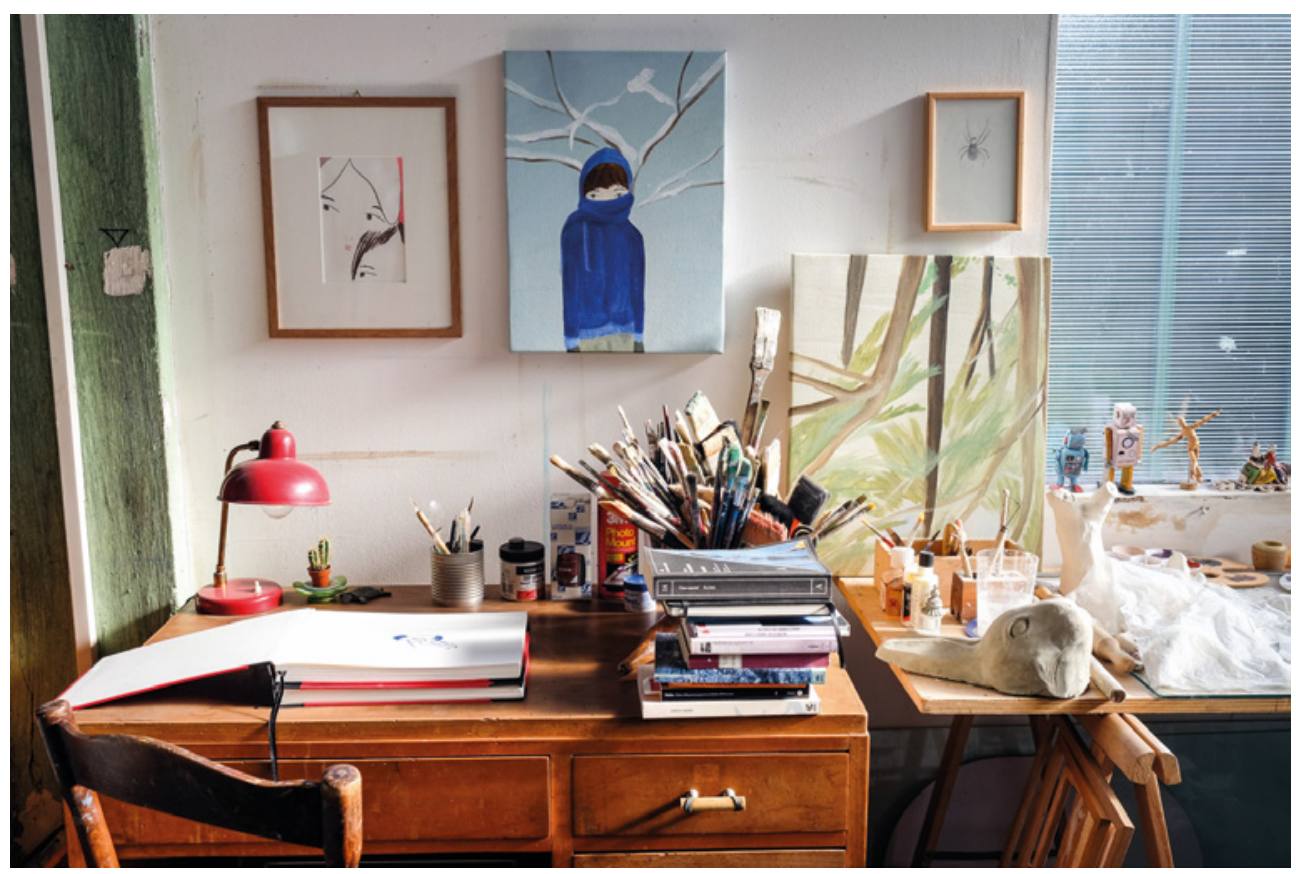

Figure 3 Studio di Carolina Raquel Antich, Venezia 2019 (foto: Augusto Maurandi)

un utilizzo sapiente della linea: con raffinata bravura include nel segno l'essenziale di una posa, l'abitudine di un atteggiamento, la semplicità mai ostentata di un contegno. I suoi soggetti appartengono a una stirpe eletta che abita luoghi chimerici da calpestare e in cui scomparire, mantenendo intatto lo stupore e la malinconia, in disegni di intenso lirismo (Paltenghi Malacrida 2015, s.p.; Colombo, Morano 2005, 38-9; Portinari 2010).

In tema di riconoscimenti importanti occorre citare León Ferrari, artista concettuale e attivista politico. Radicale figura dell'avanguardia di Buenos Aires dagli anni Sessanta, fu subito riconosciuto per l'impronta provocatoria: alla Biennale del 2007 è stata esposta un'opera significativa che l'artista aveva creato nel 1965 per l'Istituto Di Tella a Buenos Aires, ma per la quale fu ferocemente attaccato dalla critica e dalla stampa, un episodio che portò all'impossibilità di esporla perché il curatore della mostra e critico Jorge Romero Brest temeva di offendere la sensibilità religiosa di un Paese fortemente cattolico (Alonso 2011, 23-4). L'opera La civilización occidental y Cristiana consiste in un Cristo posto nella posa della croce in- 
chiodato però a un aereo militare statunitense di quelli impiegati al tempo nella guerra in Vietnam: un'opera definita da Andrea Giunta un'«immagine-manifesto» che combina icone di guerra e religione in maniera provocatoria. Con l'intento di scagliarsi contro l'ipocrisia dei valori cristiani, Ferrari aveva creato un confronto/scontro fra la cronaca, la storia e la storia dell'arte (Giunta 2006, 376).

Nel 2007 l'artista, oramai ottantaseienne, vince il Leone d'Oro per la forza comunicativa con cui riecheggia l'oltraggio e il terrore della storia presente e passata (Biennale 52 2007, 94). Le sue parole chiariscono più d'ogni altro ciò che ha mosso la sua pratica artistica:

Tutto ciò che chiedo all'arte è che mi aiuti a dire quello che penso con la maggior chiarezza possibile, a inventare i segni visivi che mi permettano di condannare le barbarie dell'Occidente nel modo più efficace. (Ferrari 2007)

Nel 2011, a dimostrazione della presa d'atto della necessità di possedere un luogo rappresentativo, viene siglato un accordo che assegna per ventidue anni uno spazio, chiamato Padiglione Argentina, nelle Sale d'Armi dell'Arsenale veneziano. La nazione in quell'anno espone inoltre a Ca' Giustinian la mostra Memoria y libertad en el arte argentino del siglo $X X$, che intende porsi come una testimonianza che rende omaggio alla Biennale di Venezia e alla partecipazione dell'Argentina a questa manifestazione (Alonso 2011, 5-6). Esauriente è l'affermazione di Magdalena Faillace nel prologo del catalogo:

l'apertura alla modernità, alla creatività e all'innovazione, il ruolo dell'immigrazione italiana nella costruzione dell'industria nazionale argentina e il permanente dialogo d'interscambio transoceanico tra artisti ed intellettuali, è la base dell'antica amicizia tra Italia e Argentina. (Alonso 2011, prologo)

La situazione storico-politica di quel Paese è spesso evocata nelle opere dei suoi artisti, come nel 2013 quando Nicola Costantino, un'artista accolta dalla critica in maniera spesso controversa a causa della forte componente provocatoria delle sue creazioni, ha presentato Rapsodia Inconclusa che consta di quattro installazioni tramite cui viene evocata l'eroina politica Eva Perón, detta Evita, sposa del presidente Juan Domingo Perón e morta a soli 33 anni. Eva. Los sueños è una videoinstallazione in cui Costantino, travestita nei panni da Evita, si aggira come una figura spettrale all'interno di una abitazione. Eva. El espejo propone la vista di quella che potrebbe essere la camera da letto della protagonista, che osserviamo riflessa negli specchi. Eva. La fuerza è una scultura in ferro che si muove tramite un motore e rappresenta il busto di Eva Perón, ricordando una diceria popolare che raccontava come poco prima di morire, volendo sa- 
lutare il popolo ma sentendosi mancare nelle forze, si fosse fatta costruire una macchinario per essere sorretta. L'ultima installazione, Eva. La lluvia, è un tavolo chirurgico su cui è posto un cumulo di gocce di ghiaccio che sciogliendosi evocano una pioggia di lacrime, simbolo della disperazione di molti argentini dopo la scomparsa di Evita.

L'opera nasce con l'intento di celebrare quella figura in forma romantica, oltrepassando lo stereotipo storico-politico in favore di quello umano ed emotivo (Biennale 55 2013, 12-13). L'artista però ha subito un'interferenza dall'intervento del governo argentino: la presidentessa Cristina Kirchner l'ha costretta a modificare il titolo originale in «Eva-Argentina. Una metáfora contemporánea». L'idea di liberare l'immagine di Evita da un ruolo veicolato anche con eccessi dai mass media è stata usata come mezzo di propaganda governativa, diventando il simbolo di uno dei più grandi problemi della nazione: un proibizionismo politico che non lascia via d'uscita.

Un'altra esperienza importante è stata quella presentata alla 57. Biennale di Venezia del 2017 da Claudia Fontes, applaudita da critica e pubblico, configurandosi come una delle opere che più ha lasciato il segno. El problema del caballo, posta nelle Sali d'Armi dell'Arsenale, è un'installazione colossale composta da tre sculture: un cavallo di cinque metri d'altezza, una ragazza e un ragazzo a grandezza naturale, e quattrocento rocce di polvere di marmo, le cui ombre si stagliavano sulla parete, disegnando la parvenza di un cavallo. La scena - che lasciava esterrefatti i visitatori - si ispirava alle icone culturali del XIX secolo, in particolare al dipinto La vuelta del malón (1892) di Ángel della Valle, considerato fondamentale per l'identità culturale argentina, da cui erano stati estrapolati i due personaggi secondari (la donna e il cavallo), rendendoli qui invece i veri protagonisti. L'opera evocava la conquista dell'America, mettendo in discussione il ruolo dell'eroe in favore invece dell'animale (Biennale 57 2017).

L'edizione del 2019 ha visto la novità di una selezione condotta in base a un bando pubblico emanato dalla Cansillería Argentina che ha determinato i protagonisti del Padiglione Argentina alla 58. Biennale d'Arte di Venezia. Per la prima volta nella storia della Nazione e della sua partecipazione alla manifestazione, la Dirección de Asuntos Culturales del Ministerio de Relaciones Exteriores y Culto ha indetto un concorso con lo scopo di «dare trasparenza e carattere federale alla convocazione»le cui proposte curatoriali sono state vagliate da una commissione. La giuria che ha selezionato il progetto vincente era presieduta dall'Ambasciatore Sergio Baur e composta dal Direttore del Museo Nacional de Bellas Artes, Andrés Duprat, Laura Malosetti Costa dell'Academia Nacional Bellas Artes, l'artista Jorge Macchi e dal Consejo Asesor Ad Honorem de la Dirección de Asuntos Culturales (Teresa Anchorena, Eleonora Jaureguiberry, Adriana Rosenberg, Mauro Herlitzka, Esteban Tedesco). 


\section{Bibliografia}

Alonso, Rodrigo (a cura di) (2011). Memoria y libertad en el arte argentino del siglo XX = Catalogo della mostra (Venezia, Ca' Giustinian, 3 giugno-27 novembre 2011). Buenos Aires: Ministerios de relaciones exteriores, comercio internacional y culto.

Basoalto, Manuel (2008). «Colores para una poética de America Latina». Malacchini, Mariano (ed.), Ojo Latino. La mirada de un continente. Milano: Skira.

Brughetti, Romualdo (1946). Veinte expresiones de arte humanista. Buenos Aires: Imprenta Lopez.

Carpani, Di Genova (1966). Ricardo Carpani. Roma: Due mondi galleria d'arte internazionale.

Colombo, Paolo; Pignatti Morano, Monica (a cura di) (2005). Premio per la giovane arte italiana 2004-2005. Manfredi Beninati, Lara Favaretto, Loris Cecchini, Carolina Raquel Antich = Catalogo della mostra (Venezia, Padiglione Venezia Biennale d'Arte, 2005). Venezia: Mondadori Electa.

Crispolti, Enrico (1987). «ll posto dell'Argentina». Malacchini 1987.

Ribera, Adolfo Luis (1987). «Primo Periodo (1810-1910)». Malacchini 1987, 14.

Sábato, Ernesto (1987). «Introduzione». Malacchini 1987, 6-7.

Dorfles, Gillo (1966). «Lucio Fontana». Biennale 331966.

Di Tella, Andrés (1999). «ll posto dell'Argentina». Devoto, Fernando; Madero Marta (eds), La Argentina entre multitudes y soledades. De los años treinta a la actualidad. 3 vols. Buenos Aires: Taurus.

Istituto Italo-Latino Americano (2009). Mundus novus: Arte contemporaneo de América Latina = Catalogo della mostra (Venezia, giugno-novembre 2009). Roma: Istituto Latino Americano.

Giunta, Andrea (ed.) (2006). Leon Ferrari: retrospectiva. Obras 1954-2006 = Catalogo della mostra (Buenos Aires, Centro Cultural Recoleta, novembre 2004-gennaio 2005; São Pauo, Pinacoteca do Estado, ottobre 2006-novembre 2006). São Paulo: Cosac Naify.

Glusberg, Jorge (1986). Art in Argentina. Milano: Giancarlo Politi.

Malacchini, Mariano (a cura di) (1987). Arte argentina dalla indipendenza adoggi: 1810-1987 = Catalogo della mostra. Roma: IILA.

Marzona, Daniel (2006). Arte concettuale. S.l.: Taschen.

Paltenghi Malacrida, Barbara (a cura di) (2015). Controcorrente = Catalogo della mostra (Lugano, Galleria Doppia V, 2015). S.I.: Galleria Doppia V.

Portinari, Stefania (a cura di) (2010). Carolina Raquel Antich. Capricci = Catalogo della mostra (AB23, Vicenza). Vicenza: Comune di Vicenza.

Restany, Pierre (2001). Uriburu - Utopia del Sur. S.l.: Fundación Nicolás García Uriburu.

Sartor, Mario (2003). Arte Latinoamericana contemporanea: dal 1825 ai giorni nostri. Milano: Jaka Book. 



\title{
La Spagna alle prime Biennali veneziane del secondo dopoguerra La ricezione della stampa
}

Romina Viggiano

Universidad Complutense de Madrid, España

\begin{abstract}
This essay aims to analyse the Spanish Pavilion at Venice Biennale in the years from 1948 to 1956. Rosalía Torrent calls this chronological arc años de travesía: from 1948 precisely because within the Biennales under Francoism (1938-76) it marks the passage to the commissioner of Luis González Robles, who materializes and exports a 'modern' image of Spain that justifies on a political level the tacit acceptance of the regime among the Western powers in defence of the 'red danger'. The period shows the weakness of liberal hopes and the exploitation of art by the dictatorship.
\end{abstract}

Keywords Spanish Pavillion. Venice Biennale. Pablo Picasso.

La fine della Seconda guerra mondiale rappresenta uno dei momenti più complessi per la sopravivenza del regime totalitario imposto alla Spagna dalla vittoria dello schieramento nazionalista nella guerra civile del 1936-39. Dopo la sconfitta delle dittature nazifasciste le pretese del generale Francisco Franco risultano ostili rispetto all'atteggiamento democratico che si diffonde in Europa. Tra gli effetti del nuovo contesto storico-politico, va rimarcata l'assenza della Spagna alla prima Biennale veneziana del secondo dopoguerra, mentre la nazione era stata una delle più assidue partecipanti fin dalla creazione della rassegna. Di conseguenza nel 1948 l'edificio del gover-

Questo saggio prende avvio da: Viggiano, Romina (in corso di stesura). La cuestión española en el debate internacional: el arte y la cultura de España en las revistas italianas 1945-1951 [tesi di dottorato]. Relatori: María Dolores Jiménez-Blanco (Universidad Complutense de Madrid) e Massimo Bignardi (Università degli Studi di Siena). 
no spagnolo ai Giardini di Castello rimane chiuso, sebbene per alcuni visitatori della manifestazione internazionale la sua anima resti implicitamente presente nella mostra del malaghegno Pablo Picasso, simbolico esponente di tutti gli esiliati di Spagna, che si tiene nel Padiglione Centrale. ${ }^{1}$

Questo saggio si propone di analizzare le adesioni spagnole alla Biennale di Venezia negli anni dal 1948 al 1956. Rosalía Torrent (1997) chiama quest’arco cronologico Años de travesía: 1948-1956 proprio perché queste Biennali, durante il franchismo (1938-76), segnano il passaggio, scandito da tante tribolazioni, al commissariato di Luis González Robles che materializza ed esporta un'immagine 'moderna' della Spagna atta a giustificare sul piano politico la tacita accettazione del regime tra le potenze occidentali, in difesa dal 'pericolo rosso' dell'avanzata comunista.

Quel periodo manifesta la debolezza delle speranze liberali e la strumentalizzazione dell'arte da parte della dittatura. Al fine di rivelare il processo da una prospettiva il più possibile obiettiva, oltre ai cataloghi pubblicati per la rassegna di Venezia dal comitato dei Beni Culturali spagnolo, è stato dato rilievo alle informazioni sulle presenze artistiche esposte reperite sulla stampa italiana del tempo. Il confronto tra queste recensioni contribuisce a ricostruire le dinamiche della politica artistica spagnola e la loro ricaduta nella ricezione del nostro Paese.

Il lasso di tempo preso in esame coincide con la nomina a segretario generale di Rodolfo Pallucchini che fin dalla ripresa della rassegna veneziana adotta una visione storicistica dell'arte organizzando, nelle prime cinque Biennali del dopoguerra e con l'impegno dei curatori dei Padiglioni stranieri, retrospettive e personali dei principali rappresentanti delle avanguardie europee, con la finalità di informare, studiare, sistematizzare e riconoscere ufficialmente l'importanza dei movimenti più innovativi degli ultimi cinquant’anni.

Una delle lacune più vistose da colmare era la presenza di Pablo Picasso: come sapeva bene la commissione speciale per le arti figurative, dopo che il suo quadro La famiglia dei saltimbanchi fu fatto ritirare dalla Biennale nel 1905, l'artista aveva rifiutato gli inviti successivi. Le trattative non risultavano semplici ma in Italia spirava un vento di speranza e di fiducia ben espresso da un articolo apparso nel gennaio del 1948 sulla rivista Emporium che così informa i lettori: «Nelle sezioni straniere sono previste mostre personali di Maillol, Laurens, Braque, Rouault, Moore, Kokoschka e forse Picasso». ${ }^{2}$

Anche il Bollettino d'Arte del Ministero della Pubblica Istruzione comunica l'imminente ripresa della Mostra Internazionale d'Arte e

1 Sulla vicenda cf. Rodriguez 1992, 1993; Rodriguez, Richter 1985.

2 U.V., «Venezia», Emporium, 637, gennaio 1948, 32. 
segnala alcune esposizioni di artisti internazionali enfatizzando la presenza della collezione di Peggy Guggenheim da New York, «forse tra le più importanti al mondo di arte astratta, cubista e surrealista». ${ }^{3}$ All'inizio della primavera la stampa italiana pubblica fotografie del maestro spagnolo in compagnia del segretario Rodolfo Pallucchini e del direttore dell'ufficio stampa dell'ente Elio Zorzi e comunica il risultato positivo della negoziazione avvenuta in Costa Azzurra. ${ }^{4}$ Anche Emporium riporta che:

Invitato dalla Presidenza della Biennale Pablo Picasso ha accettato volentieri di partecipare alla XXIV Biennale, inviando una scelta di dipinti del periodo 1940-1947 e alcune ceramiche da lui eseguite in questi ultimi mesi a Vallauris. Picasso ha promesso anche di recarsi a Venezia durante l'apertura della Biennale. È la prima volta che il celebre pittore spagnolo espone alla mostra veneziana. ${ }^{5}$

L'ottimismo verrà presto ridimensionato perchè arriveranno a Venezia solamente ventidue dipinti per di più prestati da collezionisti privati francesi e svizzeri, ai quali si aggiungeranno le sei opere - tra quelle del periodo cubista e le acqueforti Sogni e Menzogne di Franco (1937) - esposte nel Padiglione della Grecia che ospitava la raccolta di Peggy Guggenheim (Biennale 24 1948, 190-1).

La stampa italiana nella maggior parte dei numeri speciali dedicati alla Biennale, forse per ovviare al fatto che l'evento risultasse più ridotto di quanto si fosse sperato, invece di descrivere l'allestimento della piccola ma influente esposizione della sala L nel Padiglione Centrale, puntava sulla attesa partecipazione di Picasso per inquadrare l'artista al fine di suscitare la polemica tra ammiratori e detrattori del suo 'multilinguaggio versus camaleontismo', che spesso era una ripercussione della più ampia contrapposizione tra realismo e astrattismo, tra accademici e progressisti o, nel clima iper ideologizzato dell'epoca, un contraccolpo di natura politica (Picasso 1937-1953 1998).

A favore del conclamato creatore si era schierato lo studioso Bruno Maier evidenziando come, al di là del virtuosismo e delle trasformazioni stilistiche, le opere dell'andaluso fossero inconfondibili perchè mantenevano un carattere specifico: per lui Picasso è il riflesso del tempo attuale, un «proteo multiforme» dell'arte e il fondamento unitario di

3 «Manifestazioni artistiche», Bollettino d'Arte, sr. 4, 33(1), gennaio-marzo 1948, 93

4 Vernice, 3(22-3), aprile-maggio 1948, 26.

5 Attilio Podestà, «Picasso e il Museo Guggenheim alla Biennale», Emporium, 640, aprile 1948, 76; il direttore della rivista è tra i pochi critici che nel numero speciale creato per la Biennale gli dedicherà un breve articolo, tra i più obiettivi: Attilio Podestà, «Picasso», Emporium, 643-4, luglio-agosto 1948, 35-9. 
tutta la sua produzione è la sua personalità. ${ }^{6}$ Questa lettura si diffonde e, qualche tempo dopo, su L'Immagine, Carlo Levi comprova che

Per quanti sforzi si facciano, Picasso resta ormai una figura simbolica: un simbolo gigantesco che copre con la sua lunga ombra i primi cinquant'anni di questo secolo. E, come tutti i simboli, si può interpretare in mille modi diversi e anche opposti. ${ }^{7}$

Sull'altro versante, tra i più accaniti oppositori dell'arte contemporanea, il settimanale Il Mondo di Mario Pannunzio fin dal titolo di Riccardo Bacchelli Picasso despota e tiranno chiarisce la sua posizione: ${ }^{8}$ le sue creazioni sono il «successo di una moda» e finiscono per «isterilire ed uccidere l'arte» e, come scrive Piero Dorazio, sono tra le colpevoli di quella «frattura che oggi esiste fra pubblico e arte». ${ }^{9}$

Nel clima che vorrebbe essere internazionale ma che spesso si chiude in un contesto provinciale e conservatore, gli attacchi a Picasso scaturiscono da un'accusa indiretta ai cosiddetti 'pittori picassiani' - giovani antifascisti dapprima agglutinati nella rivista milanese Corrente e, nel primo dopoguerra, nel raggruppamento del Fronte Nuovo delle Arti -, anch'essi esposti alla medesima Biennale del '48, proprio in uno spazio adiacente a quello di Picasso.

Un esempio di questo approccio ostile sono gli articoli di Orio Vergani ne L'Illustrazione Italiana, in cui per spronare ad un'arte autoctona indipendente così sottolinea:

Sembra che da una quarantina d'anni a questa parte agli italiani sia difficile essere italiani, e italiani del nostro tempo. Nel primo decennio del secolo scorso si era o spagnoli zuloagheggianti o tedeschi di Monaco, o viennesi alla Klimt..$^{10}$

La visita ai Giardini non lo rassicurerà e nel numero riservato alla Biennale commenta deluso come le nuove generazioni sembrino dei meccanismi «fermi o in ritardo», legati alle rivoluzioni del passato, e non «orologi in anticipo sul pubblico». Nella stessa pubblicazione esplicita la presa di posizione del settimanale o, almeno, il poco interesse al contesto artistico cosmopolita: la sostanziosa recensione è infatti illustrata con molte riproduzioni di opere internazionali - tra cui Il Gatto (1939) di Picasso e La nascita dei desideri liquidi (1932) di Dalí - ma il testo è riservato alla storia dell'evento e alle parteci- 
pazioni italiane. Nella conclusione giustifica la sua scelta: approfondire la sessione straniera sarebbe equivalso a "scrivere una specie di sommario di storia dell'arte moderna» e avrebbe avuto un valore meramente informativo e non un giudizio critico. ${ }^{11}$

Un resoconto ufficiale sulla Biennale del '48 viene pubblicato nel Bollettino d'Arte dallo storico Giorgio Castelfranco che, se da una parte declama con entusiasmo i risultati conseguiti dalla rassegna che:

ha esposto 3144 opere, delle quali 1698 italiane e 1446 straniere. Gli ingressi sono stati 216.471 in 125 giorni, dal 6 giugno al 10 ottobre, con una media di 1730 visitatori al giorno. Le opere vendute sino al giorno della chiusura erano 344 per l'importo di L. 22.458.000

dall'altra osserva, in parte giustificando la Commissione organizzativa, che i quadri della mostra di Picasso come quelli di Braque (Gran Premio per un pittore straniero) - e a differenza del percorso espositivo su Marc Chagall - non erano stati valorizzati, e questo era accaduto:

soprattutto per Picasso, molto noto, ma, in fondo, poco conosciuto in Italia. Sembra che incertezze sulla sua stessa partecipazione abbian resa un po' limitata la sua mostra; e poi Picasso è quanto mai malagevole a esporsi data la diversità impressionante tra gruppo e gruppo dei suoi dipinti e l'incredibile dispersione delle opere dal Periode bleu al Periode Antique. ${ }^{12}$

In definitiva la mostra dedicata a Pablo Picasso alla prima Biennale del dopoguerra sarà interessante per una sua prima effettiva partecipazione, ma nessuno gli darà un peso per il suo 'essere spagnolo in esilio' o farà riferimento alla situazione politica iberica. Cesare Brandi ad esempio supera la questione sostenendo che come Giambologna è considerato uno scultore italiano anche Picasso ha ormai acquisito la 'nazionalità artistica' francese..$^{13} \mathrm{O}$ altri, come Giulio Carlo Argan nella presentazione della Collezione di Peggy Guggheneim, eludono la presa di posizione affermando che:

11 Orio Vergani, «La XXIV biennale di Venezia», L'Illustrazione Italiana, 18-25 luglio 1948.

12 Giorgio Castelfranco, «La XXIV Biennale Internazionale d'Arte di Venezia», Bolletino d'Arte, 3, luglio-settembre 1948, 277-83.

13 Cesare Brandi, «La pittura contemporanea francese alla biennale», L'Immagine, 2(9-10), agosto-dicembre 1948, 607-10. 
in artisti perfetti come Picasso o Klee l'astrazione non è più mera ideologia, ma vale in quanto la si raggiunge attraverso un processo interiore, cioè attraverso il superamento graduale e controllato della nozione storica o naturalistica. (Biennale 24 1948, 334-5)

Non si può non notare che in uno dei periodi più ideologizzati dell'occidente, quando in molti propugnavano la 'libertà della cultura per migliorare la società', l'arte sia interpretata indipendentemente dal suo essere nella storia. Anche l'introduzione redatta in catalogo da Renato Guttuso, in qualità di membro della Giuria di accettazione dell'Ente, non contestualizza storicamente l'arte di Picasso. Nel suo «Saluto» ribadisce la necessità morale di una connessione con le opere picassiane perchè rappresentano una «fiducia non astratta, non culturale, ma umana di lotta e di speranza» (Biennale 24 1948, 190) - difendendo così le accuse fatte in realtà al Fronte Nuovo delle Arti - ma astraendole ad idea universale. La contraddizione è forse ancora più evidente se si confronta con la politica culturale in atto nel regime franchista: permettere la produzione di creazioni moderne in sintonia con i movimenti europei ma deviarne il significato più profondo, cioè quell'urgenza che nonostante la censura aveva spinto l'arte a rinnovarsi.

Nei primi anni del secondo dopoguerra la dittatura mette in pratica diversi tentativi per servirsi della cultura in generale e dell'arte in particolare come strumenti diplomatici atti a potenziare un'immagine oltre i confini che migliorasse la propria condizione politico-economica durante l'isolamento internazionale. Dapprima questo accorgimento muove il proprio interesse verso i paesi iberoamericani, fomentando la 'politica dell'ispanità' e quindi una propaganda per unire alla Spagna i paesi 'originati dalla sua conquista'. Con l'Argentina di Juan Domingo Perón la Spagna instaura il legame più risolutivo e il suo riflesso è la Exposición de arte español contemporáneo a Buenos Aires nel 1947: che risulta la prova del regime per mostrare un'arte moderna all'estero interpretata attraverso la continuità dei caratteri spirituali, culturali e religiosi 'degli spagnoli'. Successivamente, legandosi agli avvenimenti mondiali, l'attenzione è spostata verso l'Europa e l'attualità' artistica della Spagna diviene la prova tangibile della supposta 'democrazia organica' del suo governo (Cabañas Bravo 1996, 147).

Il processo di polarizzazione ideologica innescato dalla Guerra Fredda aveva fatto acquistare al territorio spagnolo un gran valore strategico nel sistema di difesa voluto dagli Stati Uniti, per questo motivo verso la fine del decennio diminuisce e infine è revocata la condanna decisa dai membri delle Nazioni Unite nel 1946 (Tusell 1989, 131-9). I governi occidentali antepongono la loro stabilità capitalista ai concetti etici di cui si erano fatti portavoce, tuttavia era necessario convincere l'opinione pubblica: a questo scopo nei program- 
mi di politica estera, insieme all'aspetto economico e militare, diventa fondamentale la diplomazia culturale (Gómez-Escalonilla 1992, 2-4).

All'inizio del 1950 il quindicinale romano Alfabeto di arti scienze e lettere annuncia per la nuova Biennale la continuazione del lavoro di conoscenza e divulgazione dell'arte d'avanguardia promosso nell'edizione precedente con la pianificazione delle mostre dedicate ai Fauves, ai Futuristi e ai Cubisti. ${ }^{14}$ Anche la stampa periodica italiana riprende le interpretazioni di due anni prima, infatti sul mensile diretto da Roberto Longhi, Paragone, una breve riflessione dell'allievo Francesco Arcangeli puntualizza rispetto al reportage di Alfredo Mezio, pubblicato su Il Mondo, che $^{15}$

i più intensi Boccioni battono Léger cubista e Juan Gris; in quanto a Carrà sapevamo già che i suoi capolavori dell'11 e del '12 avrebbero retto in pieno alla vicinanza di Braque e di Picasso. ${ }^{16}$

Sorvolando sulle polemiche comparative, l'istruttivo articolo di Giorgio Castelfranco annota che, nonostante l'accurata selezione e l'allestimento delle opere, il ritardo delle mostre retrospettive - rispetto al ritmo incalzante con cui si erano originate le avanguardie - le ha sottoposte ad una lettura distorta: spesso i primi movimenti del '900 sono descritti come delle scelte prefissate per giungere all'arte astratta. Questa evoluzione non riflette le posizioni originarie, antitetiche e vitali dell'inizio del secolo, verificabili nel Picasso esposto alla Sala V del Padiglione Centrale.

Per chiarire il suo discorso l'autore traccia le differenze tra i quadri picassiani - la progressione tra quelli vicini a Cézanne e e quelli ispirati all'arte africana, i postcubisti e le ultime pitture dove la figurazione va scomparendo - fornendo al lettore del Bollettino d'Arte la fotografia della parete con sette delle tredici pitture presenti in Quattro Maestri del Cubismo. ${ }^{17}$

L'informata rassegna mensile Spazio diretta dall'architetto Luigi Moretti pubblica una nota comunicando che nell'appena inaugurato Padiglione del Libro, costruito su disegno di Carlo Scarpa nei Giardini della Biennale, il gallerista Carlo Cardazzo ha organizzato per quell'agosto una mostra dedicata all'editoria spagnola. ${ }^{18}$ Il breve messaggio attesta una delle prime attività della diplomazia bila-

14 «Tre mostre alla Biennale», Alfabeto quindicinnale di arti scienze e lettere, 15-31 gennaio 1950 .

15 Alfredo Mezio, «Posta di Venezia», Il Mondo, 1 luglio 1950.

16 Francesco Arcangeli, «Appunti», Paragone, 1(7), luglio 1950, 62.

17 Giorgio Castelfranco, «La XXV Biennale di Venezia», Bollettino d'Arte, sr. 4, 35(3), luglio-settembre 1950, 279-80.

18 «Edizioni. Il libro d’arte a Venezia», Spazio, 1(2), agosto 1950, 73. 
terale, messa in atto anche in campo artistico e ufficialmente riattivata in Europa, tra la metà del 1949 e la fine dell'anno successivo, con l'ammissione dei rappresentanti spagnoli negli organismi internazionali e il ritorno degli ambasciatori a Madrid. I legami tra Spagna e Italia comunque si erano intensificati già dal 1947, quando riprende l'attività l'Accademia Spagnola di Belle Arti a Roma proprio con una mostra libraria. ${ }^{19}$ Un'altra prova è il gran numero di «Pubblicazioni entrate in Biblioteca» nell'Archivio dell'istituzione veneziana, soprattutto cataloghi di mostre tenute negli anni immediatamente precedenti nelle gallerie di Barcellona e Madrid ma anche di riviste come Archivio Español de Arte o Indice. ${ }^{20}$

In occasione della XXV Edizione della Biennale Internazionale d'Arte, l'Illustrazione italiana registra l'adesione di nuovi Paesi o il loro ritorno:

Alcune nazioni partecipano per la prima volta: il Messico, il Brasile, l'Irlanda, la Columbia. La Spagna, la Jugoslavia, la Svezia, l'Egitto, sono tornate solo ora, dopo l'intervallo della guerra. ${ }^{21}$

Negli anni d'intesa col fascismo, il falangismo iberico non si era interessato particolarmente alla promozione culturale, accontentandosi di sbandierare alle manifestazioni straniere artisti compromessi, illustratori della causa nazionalista. Nel 1950, alla riapertura del Padiglione spagnolo, l'apparato critico-artistico perfettamente controllato dallo Stato predispone una mostra in sintonia con gli interventi anteriori, proponendo un'arte accademica, o comunque rigorosamente figurativa, che si distingue per i suoi valori di fedeltà alla tradizione e al cattolicesimo.

L'organizzazione fu affidata allo scultore Enrique Pérez Comendador, un habitué dell'evento vicino ai curatori dei precedenti allestimenti, interessato a presentare opere stilisticamente affini alle proprie o portavoci dei valori plastici del secolo XIX. A lui si affiancò una Commissione governativa con a capo il direttore delle Relazioni Culturali Carlos Cañal che seppe differenziare il progetto senza renderlo troppo dispendioso per le, ancora provate, finanze nazionali durante l'autarchia. All'eclettica mostra, risultante da questa combinazione, saranno esposti tra gli altri i pittori Mariano Fortuny y Marsal e Mariano Fortuny y Madrazo, la personale del 'tenebrista' José Gutiérrez Solana e un seguito di numerosi artisti con poche opere che spaziavano dal conservatorismo alla prima avanguardia, tra i quali lo scultore Pablo Gargallo e i paesaggisti della Scuola di Madrid. 
Il tentativo non riuscì a nascondere il ritardo della proposta rispetto al contesto plasmato dagli aggiornamenti di Pallucchini, e per questo è ripetutamente stroncato dai giornalisti italiani. In un minuzioso articolo dedicato alla Biennale Attilio Podestà commenta brevemente che «nel Padiglione spagnolo l'interesse è ancora accentrato su Solana $»^{22}$ mentre Mario Balocco sulla rivista AZ, Arte Oggi afferma che, escluso Gutierrez Solana, il padiglione «avrebbe figurato molto meglio in un'esposizione di cinquant'anni fa», ${ }^{23}$ anche Orio Vergani - sempre con un fondo polemico - concede il primato all'artista definendolo il

più potente della pittura spagnola contemporanea dopo Picasso e Salvador Dalí che ormai è difficile considerare ancora pittori iberici. $^{24}$

I dipinti di José Guitérrez Solana - macabri, barocchi e di «amara ironia» - sono dunque avallati come «gli unici di valore» per il Nuovo Caffè. La Rassegna di libera polemica artistico-letteraria è tra i pochi ad offrire un discorso approfondito sulla mostra decisa dalla Spagna e ne evidenzia la dissonanza temporale: «Gli amanti dell'Ottocento troveranno nella retrospettiva dei Madrazo e dei Fortuny largo campo per il loro entusiasmo». ${ }^{25}$

Nelly Inghilleri, ugualmente, critica tutte le 'inconfondibili personalità' dell'arte iberica (Cañal 1950, 5-6) con delle rigorose sentenze: nella ritrattistica di Federico e Raimondo de Madrazo «l'eleganza decorativa spesso si sostituisce alla profondità», in Mariano Fortuny «la passiva imitazione» goyesca è troppo evidente e, addirittura, suo figlio «non portò nessun contributo d'originalità in una pittura per se stessa "di scuola"». Diorama cita inoltre la presenza, all'interno del Padiglione, del quadro di Salvador Dalí Donna alla finestra (1925) e, nella sala dei cubisti, degli «spagnoli d'origine e francesi d'elezione» Gris e Picasso. ${ }^{26}$

Irene Brin, in quegli anni anche redattrice di articoli sulla vita mondana italiana, ribadisce la rilevanza di Dalí come artista sperimentatore, protetto della sua galleria e inoltre tra gli ospiti di riguardo «che si aspettano, o che già si incontrano in istrada». Diver-

22 Attilio Podestà, «La partecipazione straniera», Emporium, 670, ottobre 1950, 161

23 Mario Balocco, « Padiglioni stranieri », AZ, Arte Oggi, 2(8), giugno-luglio 1950, 3.

24 Orio Vergani, «Panorama della XXV Biennale di Venezia», L'Illustrazione Italiana, 9 luglio 1950.

25 Nelly Inghilleri, «Diorama della XXV Biennale», Il Nuovo Caffè. La Rassegna di libera polemica artistico-letteraria, 2(4), luglio-agosto 1950, 14-21.

26 Nelly Inghilleri, «Diorama della XXV Biennale», Il Nuovo Caffè, 2(5), settembreottobre 1950, 7-13. 
samente dalla Inghillery, che scrive per Nuovo Caffè, secondo la quale quell'unico dipinto presente alla Biennale del '50 era un «documento per la storia dell'attività dell'artista, considerato il gran maestro del surrealismo», la gallerista ritiene che quella pittura:

fa dubitare del suo istinto, se non della sua fortuna [infatti] la scialba ragazza turchinaccia poteva precedere una serie infinita di smorte fruttiere ${ }^{27}$

Di tutt’altro pensiero è Arturo Lancellotti, forse il solo ad esprimere un'opinione esaltante del Padiglione spagnolo:

Certamente uno dei più belli, per noi che, pur non essendo chiusi alle nuove tendenze quando sono appena accettabili, amiamo la grande arte del passato ${ }^{28}$

Il critico della rivista Brutium si riallccia alle parole del direttore artistico Cañal e ne sostiene le scelte. Nella sua opinione, data la promiscuità dei modelli dell'arte spagnola,

è naturale che anche oggi essa non può completamente staccarsi dalla sua tradizione, così ha fatto benissimo a darci, innanzi tutto, una retrospettiva di Fortuny padre e figlio e di Solana.

Nelle due fitte colonne della Rassegna artistica calabrese, come in un testo alla Diderot, si articolano le descrizioni di quasi tutte le opere mostratate nel Padiglione della Spagna, delle quali il cronista apprezza le qualità o l'onestà', a volte a dispetto del valore pittorico (4-5).

Da questa breve panoramica emerge che gli italiani non seppero evidenziare sulla loro stampa - cosa evidente invece dalla prospettiva storica attuale - l'inizio del processo di dissimulazione e l'utilizzo dell'arte spagnola da parte del Governo. Nel 1950 diversi piccoli accorgimenti stabiliscono le fondamenta dell'appropiazione degli artisti d'avanguardia. Il più evidente riguarda Juan Gris, esposto nella retrospettiva del cubismo coordinata dalla direzione veneziana, però 'obbligatoriamente' presente nella collettiva spagnola attraverso un ritratto eseguito nel periodo parigino (1907) da Daniel Vázquez Díaz e ricordato nel catalogo come 'spagnolo', precisando che il suo «vero cognome era González». Ma il debole tentativo di avvicinamento si intuisce anche nei titoli di altre opere: il Ritratto di Miguel de Unamuno seduto nel suo studio e il Cenacolo del Pombo di Guitierrez Solana; due opere dello scultore avan- 
guardista Pablo Gargallo tra cui un Ritratto di Picasso in pietra (1913). In questo modo personaggi scomodi al regime - soprattutto di quella che viene chiamata Generazione del '98 - venivano indirettamente 'riabilitati'.

Un'altra strategia del franchismo era utilizzare i preconcetti internazionali, e italiani in particolare, sulla nozione di 'spagnolo' e su ciò che si conosceva della Spagna per farsi accettare, trasformando la cultura in ponte tra le culture, con un dispositivo diplomatico.

Nel periodo tra la XXV e la XXVI Biennale d'Arte di Venezia il contesto spagnolo è marcato da cambiamenti fondamentali per il riconoscimento dell'arte astratta da parte dello Stato. Nel 1951 tra i ministri più influenti della sesta amministrazione del generale Franco occupa un posto d'onore Joaquín Ruiz-Giménez, un uomo cattolico di prestigio internazionale - che era stato ambasciatore nella Santa Sede - e direttore dell'Instituto de Cultura Hispánica. L'ente responsabile della valorizzazione dei valori culturali ispanici in ottobre inaugura a Madrid la I Biennale d'Arte Ispanoamericana: l'iniziativa è la prima divulgazione massiva delle esperienze d'avanguardia nello stesso territorio spagnolo. La Fiera Letteraria pubblica la traduzione di un saggio di Eugenio D'Ors sulla manifestazione:

Quello che importa al nostro tema è stabilire che anche in Spagna l'attuarsi pubblico degli interessi dell'arte è giunto al suo colmo. L'atmosfera che circonda la benvenuta Biennale ispanica lo dimostra. Un decennio ha preparato questa fioritura sociale ${ }^{29}$

L'evento segna il vertice di un processo progettato per la costruzione del racconto di «una Spagna libera, aperta alla modernità e di una cultura depoliticizzata» (Cabañas Bravo 1995, 13-19): la celebrazione di una propria grande rassegna d'arte contemporanea era un ennesimo artificio per realizzare l'apertura internazionale a livello politico, e il carattere artistico era una facciata.

La simulazione funzionava a svantaggio della genuinità dei movimenti di rinnovamento artistico nati durante l'autarchia, come dimostra il 'trasferimento' a Madrid degli incontri annuali della Escuela de Altamira. Le riunioni che dal 1948 convocano intellettuali e artisti nazionali ed internazionali nella città cantabrica, con la III Settimana, sono così ufficializzate. Tra i promotori c'era anche lo storico dell'arte Enrique Lafuente Ferrari, incaricato nel 1952 di pianificare la selezione da presentare alla manifestazione lagunare.

Il Bollettino dell'Archivio Storico di Venezia nel comunicato stampa informa sull'adesione di Svizzera, Polonia e Spagna; a proposito di

29 Eugenio d'Ors (trad. Francesco Tentori), «La Biennale Iberoamericana», La Fie ra Letteraria, 20 luglio 1952. 
quest'ultima, si annota con fiducia che il commissario sarà Enrique Lafuente Ferrari e che «tra i primi contatti sembra che [questo Padiglione] sarà tra i più importanti». ${ }^{30} \mathrm{Gli}$ italiani del settore conoscevano il critico perchè, seguendo le orme del filosofo Eugenio D’Ors, aveva presentato delle conferenze anche nel nostro Paese. ${ }^{31}$

Nel 1952 la Biennale aveva incentivato e coordinato insieme al governo spagnolo una retrospettiva delle opere di Francisco Goya: una personale che come quella di Picasso si aspettava da tempo, giacchè nel 1946 era caduta la ricorrenza dei duecento anni dalla nascita dell'artista e la stampa italiana vi aveva dedicato vari approfondimenti. Le cronache artistiche quindi, trattando del Padiglione spagnolo, si concentrano soprattutto sulla mostra del saragozziano sottolineando che, nonostante i pochi quadri - sette arrivati dal territorio iberico - «è pur sempre una mostra di dipinti di Goya e sconosciuti in Italia». ${ }^{32}$ L'artista è identificato come uno dei maestri antesignani delle 'rivoluzioni' dell'arte moderna e dimostra la continuità del discorso curatoriale del segretario generale Pallucchini oltre che il legame con le precedenti retrospettive degli inglesi Turner e Constable (e poi del francese Courbet). Giorgio Castelfranco nel suo consueto resoconto sulla Biennale ricorda come

il grande pittore spagnuolo [...] abbia sentito già all'età dei primi capelli grigi tutto il travaglio politico del secolo come una immensa folle tragedia ${ }^{33}$

Il testo ricalca il modello della storiografia ufficiale e riassume le nozioni diffuse sul pittore:

complesso di pateticità ed impulsività illogica sulla base di una corposità naturalistica intuitiva ed inabolibile, è peculiariamente romantica, è il grande contributo della Spagna al Romanticismo nascente.

Il 'ritratto romanzato' che ne risulta dimostra le idee precostituite esistenti in Italia su Goya e sulla cultura spagnola, infatti lo storico dell'arte sottolinea:

la grande forza di Goya precorritore stilistico consistette propriamente nell'aver fatto dell'abbreviatura, che era nei suoi contempo-

30 «Bollettino dell'ASAC», La Biennale di Venezia, 3(8), aprile 1952, 51.

31 «L'arte a Roma», La Fiera Letteraria, 29 maggio 1947.

32 Benjamin Storey, «Goya», Emporium, 691-2, luglio-agosto 1952, 3.

33 Giorgio Castelfranco, «La XXVI Biennale di Venezia», Bollettino d'Arte, sr. 4, 37(3), luglio-settembre 1952, 279. 
ranei gioiosa, rapidità di resa di un contenuto edonistico, un mezzo di resa realistico-patetica, linea d'urto, quasi direi, tra lui e il dramma che gli appare improvviso (280).

La pittura di Goya trasforma il modello della colorata e pagana festa andalusa nel paradigma dello spagnolo austero, composto da elementi drammatici e della tradizione religiosa.

Nel Padiglione era comunque presente, per la prima volta, un'antologia di pittura e scultura attuali. La presentazione bilingue di Lafuente Ferrari notifica con dedizione qual era il desiderio della Commissione:

ha voluto, dentro i limiti possibili, presentare un complesso scelto con un criterio ampio e liberale, il solo che possa riflettere con alcuna esattitudine la complessità dell'arte spagnola e la varietà delle sue tendenze. Nessuna di essa è stata esclusa a priori dal Comitato, il quale ha creduto di servire in questo modo, senza divieti nè passioni, la vitalità artistica di un paese che può aggiungere ogni giorno alla sua grande tradizione certe apportazioni e varianti che, in definitiva verranno giudicate dalla storia come tanti altri arricchimenti. (Lafuente Ferrari 1952, 22)

L'arte contemporanea è riscattata attaverso il suo legame con la tradizione; nonostante tutto era evidente un aggiornamento rispetto alle precedenti edizioni: erano presenti meno artisti e rappresentati da più opere, a nomi conosciuti si aggiungevano creatori innovativi della nuova generazione come José Caballero, Manuel Capdevilla, José Guinovart, Ramon Rogen e Antoni Tàpies. Attilio Podestà delineando «Le sezioni straniere» della Spagna ricorda:

un gruppo di artisti che sono stati posti in primo piano all'esposizione dell'arte ispano-americana celebrata a Madrid nei mesi dell'autunno e dell'inverno scorsi: una trentina di artisti, fra cui Beniamino Palencia e Daniele Vasquez Diaz, vincitori dei grandi premi per la pittura, e Giovanni Rebull, vincitore del gran premio per la scultura ${ }^{34}$

La stampa italiana non si sofferma sui giovani artisti spagnoli. La selezione di Lafuente Ferrari rappresenta un assaggio di quelli che saranno i 'vincitori' della Biennale '58 ma non riesce a depurare il Padiglione dall'eclettismo e favorisce la completa legittimazione del regime franchista tra le potenze mondiali. Gli esiti dell'arte spagnola sono infatti paralleli ai 'successi' del suo governo; all'ufficiale ritorno dei diplomatici seguiranno: da una parte la firma del Concordato

34 Attilio Podestà, «Le sezioni straniere», Emporium, 691-2, luglio-agosto 1952, 87. 
con il Vaticano che lo riabilita sul piano religioso (1951) e, dall'altra, la firma degli accordi di cooperazione economica e militare tra i generali Francisco Franco e Dwight Eisenhower - poco dopo sugellati dalla visita del presidente statunitense in territorio spagnolo - che riconoscono la 'democrazia organica' sul piano politico (1953).

Nelle edizioni XXVII e XXVIII della Biennale, l'incarico di mostrare l'arte della Spagna contemporanea è affidato al direttore dell'Accademia Spagnola di Belle Arti di Roma Juan de Contreras y López de Ayala, più conosciuto come il Marchese di Lozoya. A lui si affiancheranno storici dell'arte fautori dei discorsi critici di premessa nei cataloghi e quindi influenti per la costruzione di una ideologia della 'modernità spagnola', che rivalutava la cultura prima scomoda al regime, fino a reinterpretare le opere di Pablo Picasso (de la Nuez 2014, 189-212).

Seguendo la tendenza delle Biennali precedenti, la manifestazione del 1954 portava all'attenzione l'arte surrealista: nel Padiglione centrale, insieme alle mostre individuali di Max Ernst e Jean Arp, una sala era riservata a Joan Miró. La mostra presentava una trentina di quadri che ripercorrevano la sua carriera artistica dal 1918 al 1953. Il governo spagnolo (con intermediario il gallerista parigino Aimé Maeght) aveva convinto Miró a esporre una ventina di litografie e acqueforti nel Padiglione spagnolo, grazie alle quali si era aggiudicato il Gran Premio per la Grafica.

L'artista catalano attira l'attenzione di molte riviste. Garibaldo Marussi gli riconosce che

ha saputo fondere il capriccio spagnolesco al dettato automatico, dentro ad un fastoso e rutilante giuoco di immagini: caleidoscopio per adulti, sollievo e giuoco nello stesso tempo. Davvero, quella di Miró è una delle sale fra quelle dei cosiddetti pittori fantastici che più delle altre si salva e che dimostra quanto molta pittura recente debba a questo prestigiatore insuperabile ${ }^{35}$

Di questo 'astrattismo fantastico' Gillo Dorfles esalterà la «grafia» e il «ritmo» di Mirò, indicando però la sua distanza dal surrealismo:

C'è una vigile ricerca del risultato artistico, dell'attualità del mezzo espressivo impiegato. Non c'è quadro, litografia, scarabocchio di Miró che non sia equilibrato, intonato, ritmizzato, cromatizzato, sapientemente, con eleganza e finezza suprema. ${ }^{36}$

35 Garibaldo Marussi, «La XXVII Biennale veneziana», La Fiera Letteraria, 27 giugno 1954.

36 Gillo Dorfles, «Grafia e ritmo di Mirò, dolcezza e veemenza di Arp: profilo di due maestri dell'astrattismo fantastico», La Fiera Letteraria, 18 luglio 1954. 
Il prestigio gli è conferito dalla rivista La Biennale di Venezia che gli destina la copertina del numero dedicato alla rassegna; all'interno una recente litografia è corollario di un articolo di Raymond Queneau. Lo scrittore esalta la dignità di Miró, che «non vuole essere mescolato» con gli altri, e la sua vicinanza alla poesia. Lo distacca dall'interpretazione dell'infantilismo diffusa da Breton o dal grafismo suggerito da Michel Leiris, sostenendo che

come ogni pittura di valore, essa vuol rappresentare (e non ridurre) su una superficie piana un mondo a cinque dimensioni: le quattro dimensioni dello spazio-tempo e una quinta (o la prima) che è la dimensione particolare ad ogni pittore. ${ }^{37}$

A conferma della stima riposta nell'artista catalano lo Stato italiano acquista Il compianto degli amanti (olio su tela, cm $47 \times 39$, 1953), il primo dipinto di Miró ad entrare nella sue collezioni. La direttrice della Galleria Nazionale di Roma, Palma Bucarelli, riprende le parole del critico Charles Estienne in catalogo e commenta:

Era la sola opera in vendita della bella sala con cui la Biennale ha celebrato quest'anno il famoso pittore, ma non la meno interessante, anzi una delle più fini e poetiche creazioni dell'artista [...] che si annovera tra le più nuove e alte espressioni dell'arte moderna, in assoluta libertà creatrice, per il bisogno di esprimere un mondo fantastico di forme e colori, di apparenza quasi fanciullesca, fiabesca e gioiosa. ${ }^{38}$

Quasi da contrappeso a Mirò nel Padiglione spagnolo erano esposti una ventina di acquerelli di Salvador Dalí. Negli anni Cinquanta l'eccentrico artista e la sua nuova pittura mistico-nucleare sono spesso protagonisti dei rotocalchi, e le recenti tavole per illustrare la Divina Commedia avevano già turbato il pubblico nella mostra personale della capitale..$^{39}$

Oltre ai due, la commissione spagnola mantiene la consuetudine di presentare una retrospettiva, questa volta dedicata al barcellonese Isidro Nonell. L'importanza del pittore modernista si riflette nel catalogo in cui le riproduzioni delle opere a pagina intera accompagnano il lungo testo scritto dal critico José Camon Aznar. La sua pittura postimpressionista non passa inosservata alla stampa italiana,

37 Raymond Queneau, «Joan Miró», La Biennale di Venezia, 19-20, aprile-giugno 1954, 39-41.

38 Palma Bucarelli, «Acquisti alla XXVII Biennale veneziana», Il Bollettino d'Arte, sr. 4, 39, dicembre 1954, 379-80.

39 «Una grande inchiesta: Che ne pensate di Salvador Dalí», La Fiera Letteraria, 30 maggio 1954. 
Giorgio Castelfranco commenta il «graditissimo incontro con Isidoro Nonell» e la piacevole scoperta dei suoi quadri..$^{40}$

L'«ampia rassegna dei più caratteristici pittori delle nuove tendenze dell'arte spagnola» (Marchese di Lozoya, 1954, 378) comprendeva una trentina di artisti viventi. Tra i vari nomi ricordiamo il neocubista Daniel Vazquez Diaz, il paesaggista della Escuela de Madrid Ortega Muñez - quell'anno premiato alla II Biennale Ispanoamericana tenutasi all'Avana - e Antoni Tàpies con opere del periodo «magicista» (Camón Aznar 1954, 17).

La recensione più ottimista su questo Padiglione è quella di Mario Portalupi su La Fiera Letteraria, che giudica la mostra una «interessante documentazione su una civiltà pittorica poco conosciuta» e sostiene che le centosessanta opere esposte

non bastano davvero a illuminarci sulla esatta essenza e consistenza del mondo artistico della Spagna d'oggi. Semmai, quel diorama commentando, riassumendone aspetti e limiti, tendenze e invocazioni, facilita al visitatore accorto il giudizio, e col giudizio il formarsi d'uno schermo mentale entro cui approssimativamente vedere come e a che punto stiano laggiù, oggi, le cose dell'arte..$^{41}$

In questo articolo l'autore è tra i primi a sostenere l'annessione dell'arte spagnola ai traguardi dell'arte contemporanea «è in ogni parte del globo». L'idea è chiarificata dal sottotitolo «Realismo nel Padiglione spagnolo. Accanto agli artisti di chiare tendenze "sociali" non mancano naturalmente gli astrattisti», e scrive:

ai lati un realismo spesso popolare e sociale al quale fa riscontro un astrattismo 'immaginoso' e funambolesco; al centro, diciamo, un generico lirismo che solo in certi e infrequenti casi si alimenta, ma svogliato, alle non più turgide mammelle della tradizione. ${ }^{42}$

Il concetto ricorda le diatribe italiane degli ultimi tempi e, in realtà, si dissocia dalla presentazione ufficiale, nella quale si cercava di dare valore al carattere realista degli artisti spagnoli: «l'attecchimento alla realtà più elementare della terra o degli uomini gli impediscono di lasciarsi andare alle astrazioni di tipo mentale che sono l'indice di modernità di altre scuole» (Camón Aznar 1954, 11).

40 Giorgio Castelfranco, «La XXVII Biennale», Bolletino d'Arte, sr. 4, 39(4), ottobredicembre 1954, 352.

41 Mario Portalupi, «Realismo nel Padiglione spagnolo», La Fiera Letteraria, 9(29), 18 luglio 1954, 6.

42 Mario Portalupi, «Realismo nel Padiglione spagnolo», La Fiera Letteraria, 9(29), 18 luglio 1954, 6. 
Il Bollettino d'Arte infatti osserva che questo Padiglione si trova «fuori dalla zona di combattimento» della Biennale di quell'anno «segnata dalle tre direttrici neo-realismo, astrattismo, surrealismo». ${ }^{43} \mathrm{Ri}$ prendendo l'idea di Camón Aznar, l'unità della selezione da lui presentata si riscontra: «non nell'impostazione né nelle soluzioni estetiche, ma sì nel clima di modernità che la avvolge» (Camón Aznar 1954, 11)

In definitiva, nella panoramica internazionale della Biennale, la Spagna propone una mostra molto diversificata con la predisposizione per i soggetti figurativi. La diplomazia culturale del franchismo si è appropriata completamente dell'arte d'avanguardia seppur mantenendo il 'realismo' della tradizione, in questo momento caratteristica essenziale per definire la 'sua modernità'. In Spagna però, come abbiamo notato, dal 1951 gli artisti astratti non erano più censurati e anzi venivano incoraggiati, con l'appoggio del Congresso Internazionale a Santander e del progetto di un museo dedicato all'arte contemporanea alle Canarie.

Nell'anno di pausa della Biennale in Spagna si verificano altri episodi di vivacità culturale segnalati dal successo della terza e ultima Biennale Ispanoamericana di Arte a Barcellona. La versatilità di stili è mantenuta ma il gran numero di premi, artisti e opere di qualità sottolineano l'integrazione delle proposte iberiche con la modernità artistica europea e il consolidamento del riconoscimento degli artisti nazionali nel sistema mondiale. La rispettabilità dell'evento barcellonese è aumentato dalla mostra El arte moderno en los Estados Unidos. Selección de las colecciones del Museum of Modern Art, prima presentazione ufficiale dell'arte nordamericana in territorio spagnolo, segno della vicinanza a questo circuito che coincide, sempre nel 1955, con l'ammmissione della Spagna a membro dell'ONU.

La Biennale di Venezia del 1956 ospita un numero eccezionale di esposizioni e di artisti - il mercante Daniel-Henry Kanhweiler presenta la grande retrospettiva di Juan Gris $-{ }^{44}$ con la partecipazione di trentaquattro nazioni, tra le quali la Russia, paese assente dalla rassegna da ventotto anni. ${ }^{45}$ L'arte realista sovietica è al centro del mirino, espressione della società e del comunismo, che viene intesa come incompatibile con quella americana, espressione dell'individuo e del capitalismo ma anche segno di innovazione e di libertà.

La proposta spagnola risulta ancora poliedrica e ambigua: sono allestite le personali del pittore ottocentesco Juan de Echevarría e delle sperimentazioni metalliche dello scultore Pablo Gargallo accompagnate da una rassegna di artisti contemporanei. Assieme a quadri figurativi per la prima volta il Padiglione mostra delle opere astratte,

43 Giorgio Castelfranco, «La XXVII Biennale», Bollettino d'Arte, sr. 4, 39(4), ottobredicembre 1954, 352.

44 «Cronache: Venezia», Emporium, 734, febbraio 1956, 76.

45 «Cronache: Venezia», Emporium, 736, aprile 1956, 175. 
confermando la conformità alle scelte dell'arte contemporanea internazionale. Nella presentazione però è mantenuto il modello degli anni anteriori e l'arte contemporanea è giustificata dalla sua tradizione. Il Marchese di Lozoya, nuovamente commissario, insiste sull'assenza di scuole e sulla presenza nel tempo di una successione di creatori isolati ma accomunati dal «carattere collettivo» dello «spagnolo»:

La Spagna è l'antitesi dell'Accademia ed è l'unico paese che seppe interrompere la tirannia neoclassica della fine del settecento con l'apparizione inattesa di Goya. L'anarchia iberica provoca la reazione di ogni artista di fronte all'ambiente. (Biennale 28 1956, 470-1)

Gli artisti a-formali - Tàpies ma anche José Tharrats, Antonio Saura, Rafael Canogar, Manuel Millares, Luis Feito, Manuel Mampaso Bueno, Enrique Planasdurá e Ángel Ferran in scultura - approdano a Venezia presentati da Luis Felipe Vivanco, un intellettuale vicino alle avanguardie, prima e dopo la guerra civile, che in catalogo ripercorre le esperienze artistiche spagnole degli ultimi anni evidenziando il rinnovamento dell'arte religiosa e le mostre promosse dall'Academia Breve de Crítica de Arte di Eugenio D’Ors o dalle Biennali Ispanoamericane. Vivanco reputa la 'nuova arte' uno stile spirituale in cui l'artista segue la sua «necessità interiore», come i suoi antenati quando disegnarono i bisonti nella caverna di Altamira. L'interpretazione consente di giustificare il tradizionale realismo della pittura spagnola anche nelle composizioni non figurative. L'architetto chiarisce che, con questo concetto:

non si allude a nessuna realtà naturale ma a tutta l'operazione formale dell'immaginazione. L'immaginazione risolve le forme che l'intelligenza non può risolvere. Quanta più forza d'immaginazione ha il pittore, più reale come creatura dell'arte avrà la sua opera. (Artistas espanoles en la 28. Bienal 1956, 24-5)

L'impronta «spagnola» parte dalla preistoria e giunge all'informale, passando per le opere di El Greco, Velázquez, Zurbarán, Goya unendo Palencia, Zabaleta, Solana e infine includendo quelle di Picasso e Miró. La struttura a catena della cultura nazionale annulla la dimensione storica ed esistenziale originaria, neutralizzando la carica politica e ideologica di un'arte spesso nata in contrapposizione al regime.

La stampa italiana documenta le esperienze informali spagnole e spesso pubblica i 'muri' di Antoni Tápies che, durante la permanenza a Parigi, era stato inserito tra i materici europei promossi dalla geografia dell'Art Autre di Michel Tapié. ${ }^{46}$ Dipinto (1955) è riprodotto tra le foto

46 Enrico Crispolti sottolinea l'importanza del critico francese: «I primi tentativi di ampia e comprensiva recensione, non soltanto europea, datano al '51 con la mostra or- 
con le sculture di Gargallo nel numero speciale de La Biennale di Venezia («Le Nazioni») ${ }^{47}$ e in Arti Visive, Marco Balzarro lo descrive come:

Il muro che avanza. Ma il grande segno, il cerchio ovale e la croce non liberano dallo sgomento. Così le firme dei gitani sugli intonaci abbandonati non comunicano che il sonno ipnotico del muro. La materia affaticata respinge la traccia umana in un grigiore che pare l'estremo, spettrale calco del naturalismo. ${ }^{48}$

Su Aut Aut la composizione del rappresentante della Spagna è accompagnata dalle opere informali di Fritz Winter per la Germania e di Marc Mendelson per il Belgio, alludendo a quella «normalizzazione» dell'arte descritta da Gillo Dorfles che nel biennio sucessivo toccherà il suo apice con la progettazione del nuovo segretario Gian Alberto dell'Acqua. ${ }^{49}$

A Venezia, nel 1958, la corrente dell'informale è molto presente, come una tendenza sovranazionale: se una chiave di lettura la voleva indicare come tipica dei Paesi in cui vigeva la libertà di espressione, come gli Stati Uniti, in realtà era evidente che la nuova pittura si era diffusa anche in nazioni in cui non vi era un governo democratico come in Yugoslavia e in Spagna. L'ordinato discorso curatoriale di Luis González Robles ottiene inoltre il premio per il migliore Padiglione.

Il percorso tracciato attorno alle prime Biennali dopo l'interruzione della Seconda guerra mondiale ha illustrato il destreggiarsi del franchismo per uscire dall'isolamento che era stato imposto al Paese dal 1946. L'esportazione di una cultura che intende mostrare modelli moderni e la ripresa di stereotipi esistenti nel contesto internazionale gradualmente migliorano la ricezione dell'arte spagnola sulla stampa italiana. Il prestigio che guadagna col tempo mitiga l'allontanamento, relegando in secondo piano la vera realtà del Paese, approvando implicitamente il processo di apertura della politica del regime.

Nel giro di un decennio dunque il sistema dell'arte spagnolo si è depurato dagli elementi fascisti concentrandosi su quelli religiosi e tradizionali (1950); si è collocato in Europa tramite la sua «spagnolizzazione» che gli dona una percezione più affabile (1952); ha subito una assimilazione attraverso un discorso teorico-retorico che coniu-

ganizzata da Michel Tapié a Parigi Véhemences Confrontées, e nel '52 con il volumetto Un art autre [...]», per tutti gli anni Cinquanta organizzerà esposizioni collettive: in Spagna la mostra Otro Arte alla Galleria Gaspar (1957) è anticipata dalla pubblicazione del suo saggio "Esthétique en devenir», nell'ultimo numero della rivista di Barcellona Dau al Set (9[54], dicembre 1956). Per un approfondimento cf. Crispolti 1970, 12

47 La Biennale di Venezia, 28-9, giugno-settembre 1956, 55.

48 Marco Balzarro, «Pittori», Arti Visive, sr. 2, 5(5), 1956, 22.

49 Gillo Dorfles, «La macchia e il gesto alla XXIX Biennale», Aut Aut, 17 settembre 1958, 284-9. 
ga l'arte moderna con la propria tradizione e spiritualità, che sono dei valori franchisti (1954). Parte di questo processo è anche l'accettazione dell'astrattismo, che viene giustificata e diventa intermediaria per la promozione all'estero di un'aspetto moderno (1954) che culmina con l'assunzione dell'Informale ad arte ufficiale (1956-58).

La produzione artistica esposta alla Biennale di Venezia è dunque coinvolta in un discorso ideologico che mostra di assecondare la libertà artistica degli artisti affinché la diplomazia culturale del regime si riprenda un posto tra le potenze europee per la lotta contro l'espandersi del comunismo sovietico.

\section{Bibliografia}

Cabañas Bravo, Miguel (1996). Política artística del franquismo: el hito de la Bienal Hispanoamericana de Arte. Madrid: Consejo Superior de Investigaciones Científicas.

Cabañas Bravo, Miguel (2017). Imaginarios en conflicto: lo español en los siglos XIX y XX. Madrid: Consejo Superior de Investigaciones Científicas.

Cabañas Bravo, Miguel; López Yarto Elizalde, Amelia; Rincón García, Wifredo (2008). Arte, poder y sociedad en la España de los siglos XV a XX.

Camon Aznar, José (ed.) (1954). Artistas españoles en la XXVII exposición bienal internacional de arte de Venecia = Catalogo della partecipazione spagnola alla XXVII Esposizione Internazionale d'Arte (Venezia, 19 giugno-17 ottobre 1954). Madrid: Altamira.

Camon Aznar, José; Lafuente Ferrari, Enrique; Marques de Lozoya; Vivanco, Luis Felipe (eds) (1956). España en la XXVIII Bienal de Venecia=Catalogo della partecipazione spagnola alla XVIII Esposizione Internazionale d'Arte (Venezia, 16 giugno-21 ottobre 1956). Madrid: Magerit.

Cañal, Carlos; Marques de Lozoya (eds) (1950). 25. Exposición Bienal de arte en Venecia: pabellón espanol = Catalogo della partecipazione spagnola alla XXV Esposizione Internazionale d'Arte (Venezia, 8 giugno-15 ottobre 1950). Madrid: dirección general de relaciones culturales.

Crispolti, Enrico (1970). Pittura d'avanguardia nel dopoguerra in Europa. Milano: Fratelli Fabbri Editori.

Delgado Gómez-Escalonilla, Lorenzo (1992). Imperio de papel: acción cultural y política exterior durante el primer franquismo. Madrid: Consejo Superior de Investigaciones Científicas.

Di Martino, Enzo (2003). Storia della Biennale di Venezia 1985-2003. Torino: Papiro Arte.

Díaz Sánchez, Julían (2000). El triunfo del informalismo. Las consideraciones de la pintura abstracta en la época de Franco. Madrid: Metáforas del Movimiento Moderno.

Díaz Sánchez, Julían (2013). La idea del arte abstracto en la España de Franco. Madrid: Cátedra.

Donaggio, Adriano (1988). La Biennale di Venezia, un secolo distoria. Firenze: Giunti.

Fagiolo dell'Arco, Maurizio; Terenzi, Claudia (a cura di) (2002). Roma 1948-1959: arte, cronaca e cultura dal neorealismo alla dolce vita = Catalogo della mostra (Roma, Palazzo delle Esposizioni, 30 gennaio-27 maggio 2002). Milano: Skira. 
Fuentes Vega, Alicia (2011). «Franquismo y exportación cultural. El papel de "lo español" en el apadrinamiento de la vanguardia» en del Val Moreno, Gloria; Fuentes Lázaro, Sara (eds), «Extra: Saberes artísticos bajo signo y designios del 'Urbinate'», vol. extraordinario de Anales de Historia del Arte, noviembre, 183-96.

Fuentes Vega, Alicia (2011). «Franquismo y exportación cultural. El caso de la Exposición de Arte Español en Buenos Aires 1947». Iberoamericana. América Latina-España-Portugal, 11(44), 25-46.

González Robles, Luis (ed.) (1958). España en la XXIX Bienal de Venecia = Catalogo della partecipazione spagnola alla XXIX Esposizione Internazionale d'Arte (Venezia, 14 giugno-19 ottobre 1958). Madrid: Valera.

Jiménez-Blanco, María Dolores et al. (eds) (2016). Campo cerrado: artey poder en la posguerra española: 1939-1953 = Catalogo della mostra (Madrid, Museo Nacional Centro de Arte Reina Sofía, 27 abril-26 septiembre, 2016). Madrid: MNCARS.

Lafuente Ferrari, Enrique (ed.) (1952). Catalogo del Pabellón Español en la exposición Bienal de Venecia = Catalogo della partecipazione spagnola alla XXVI Esposizione Internazionale d'Arte (Venezia, 14 giugno-19 ottobre 1952). Madrid: Hauser y Menet.

Llorente Hernández, Ángel (1995). Arte e Ideología en el franquismo (1936-1951). Madrid: Visor.

Mantura, Bruno; Mattirolo, Anna; Villari, Anna (a cura di) (1998). Picasso 19371953. Gli anni dell'apogeo in Italia = Catalogo della mostra (Roma, Galleria nazionale d'Arte Moderna, 12 dicembre-15 marzo 1998). Torino, Allemandi.

Marí, Antoni; Mercadé, Albert (eds) (2014). La modernitat cauta. 1942-1963. Resistència, resignació, restauració. Barcelona: Angle Editorial.

Marzo, Jorge Luis (2007). Arte moderno y Franquismo. Los orígenes conservadores de la vanguardia y de la política artística en España. Girona: Fundació Espais d'Art Contemporani.

Marzo, Jorge Luis (2010). La memoria administrada. El barroco y lo hispano. Madrid-Buenos Aires: Katz.

Negri, Antonello; Pirovano, Carlo (1993). Esperienze tendenze e proposte del dopoguerra. Il Novecento: 1945-1990. Milano: Electa.

de la Nuez Santana, José Luis (2014). «Picasso en el laberinto de la crítica española de los cincuenta». Marí, Mercadé 2014, 189-212.

Rodriguez, Jean François; Richter, Mario (1985). Picasso a la Biennale de Venise (1905-1948): sur deux lettres de Picasso a Ardengo Soffici. Venezia: Istituto Veneto di Scienze, Lettere e Arti.

Rodriguez, Jean François (1992). Soffici, Paresce, de Pisis, Tozzi intermediari di cultura tra la Francia e l'Italia: Ia sala Picasso alla Biennale (1926-1932). Venezia: Istituto Veneto di Scienze, Lettere e Arti.

Rodriguez, Jean François (1993). Picasso alla Biennale (1905-1948). Soffici, Paresce, de Pisis, Tozzi intermediari di cultura tra la Francia e l'Italia. Padova: Cleup.

Torrent Escalpé, Rosalía (1997). España en la Bienal de Venecia 1895-1997. Castelló: Col·leció Universitària.

Tudelilla Laguardia, Chus (2014). Mathias Goeritz: recuerdos de España, 19401953. Zaragoza: Prensas de la Universidad de Zaragoza.

Tusell Gómez, Javier (1989). La España de Franco: el poder, la oposición y la política exterior durante el franquismo. Madrid: Historia 16.

Tusell García, Genoveva (2003). «La internacionalización del arte abstracto español: el intercambio de exposiciones con los Estados Unidos (1950-1964)». Espacio, tiempo y forma, sr. 7, 16, 223-32. 


\section{BIENNAL DE VENĖCIA}

AVANTGUARDA ARTISTICA I REALITAT

SOCIAL A L'ESTAT ESPANYOL 1936-1976

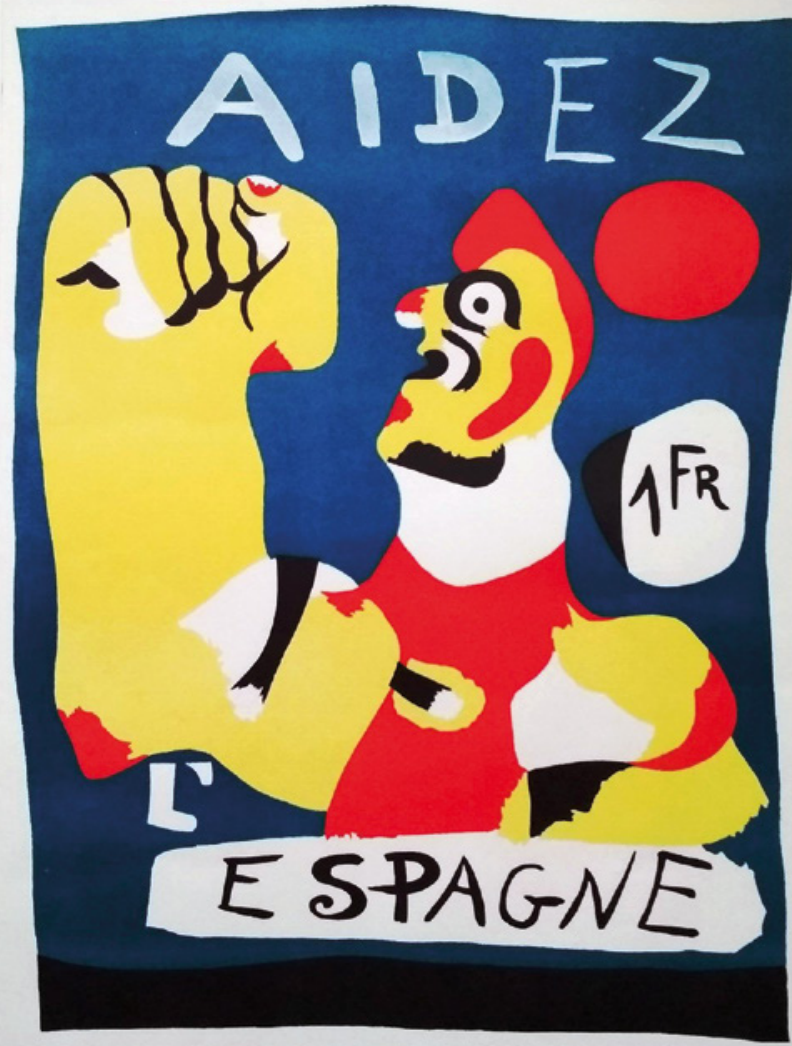

\section{FUNDACIÓ JOAN MIRÓ}

18 desembre 1976 - 13 febrer 1977

Parc de Montjuic

Barcelona

Manifesto dell'esposizione España, Vanguardia artística y realidad social. 1936-1976, trasferita alla Fundació Joan Miró di Barcellona nel dicembre 1976-febbraio 1977 dopo la Biennale di Venezia del 1976 


\title{
Breve storia di una liberazione La Spagna alla Biennale di Venezia dal 1979 al 1999
}

Giulia Crespi

Archivio Emilio Isgrò, Milano, Italia

\begin{abstract}
The essay offers a specific recollection of the participation of Spain at the Venice Biennale since 1976 to 1999. The starting date has a particular relevance both for historical and artistic reasons. 1976 coincides in fact with a democratic beginning for the Country, which has just witnessed Franco's death. This meant the end of a long period of isolation and the recovery from years of repression and dictatorship. Through that time, artistically, Spain was not left behind, thanks to the strength of many artists who kept contact with other countries, always up to date on what was new. However, they had been forced to choose between being artist of the regime or stay hidden in an interior exile. With the Biennale edition of 1976, the special project, promoted by the institution and two of the most renowned art critics at the time, Valeriano Bozal and Tomàs Llorens, called España, Vanguardia artistica y realidad social. 1936-1976, tried to draw a critical and historical view on the Spanish artistic languages consumed and silenced by censorship. Through the 80 s and the 90s Spain has experienced a renewed awareness of its internationally artistic role and that has reflected on the choices made for Venice Biennale. Although seeing the evolution of Spanish art in the last decades through the Biennale is limited and incomplete, it has an undeniable interest and relevance worth being investigated.
\end{abstract}

Keywords Spanish Pavillion. Biennale. Venice. Seventies. 
Non è possibile ripercorrere la storia della partecipazione della Spagna alla Biennale di Venezia degli ultimi quarant’anni, senza prendere in considerazione un anno fondamentale, il 1976, che non solo coincide storicamente con l'elezione di Adolfo Suárez González, primo ministro democraticamente scelto dopo la morte del Caudillo Franco, ma anche con la fine di un periodo di isolamento culturale determinante nello sviluppo artistico della nazione. Vedere la storia dell'arte spagnola degli ultimi quarant’anni attraverso lo specchio della manifestazione veneziana permette di riflettere su quali artisti il Paese abbia ritenuto degni rappresentati di un determinato momento culturale.

Durante gli anni fascisti, lo stesso Franco non impedisce mai la partecipazione della Spagna alla Biennale, anzi sceglie di utilizzarla a proprio favore, diffondendo, attraverso gli artisti invitati, l'idea menzognera di un paese libero e aperto. Il generale aveva capito l'importanza che poteva avere l'arte e, soprattutto, l'importanza di utilizzare quell'arte alla Biennale e, quindi, a livello mondiale.

Dopo la morte di Francisco Franco, il 20 novembre del 1975 l'arte dominante è certamente quella concettuale, interessata alla ricerca sul linguaggio, sulla forma e sull'uso di nuove tecnologie. Nella nuova libertà ritrovata, gli artisti spagnoli si aprono a linguaggi nuovi ma che, curiosamente, perdono un elemento vitale del loro sviluppo: un nemico contro cui combattere, aspetto fino ad allora caratteristico.

Segnale importante e che desta grande scalpore a livello internazionale, è il progetto di rottura pensato per la Biennale del 1976 dai critici Valeriano Bozal e Tomás Llorens, che vuole mette in scena il vero percorso della produzione artistica dei precedenti anni di regime, includendo anche chi era stato costretto a fuggire all'estero o a chiudersi in un esilio interiore. Dopo la decisione di chiudere ufficialmente il Padiglione spagnolo dei Giardini, una commissione ufficiale, incaricata dall'allora presidente della Biennale Carlo Ripa di Meana, allestisce la mostra, all'interno del Padiglione Centrale, intitolata España, Vanguardia artística y realidad social. 1936-1976, con l'obiettivo di analizzare le relazioni tra l'avanguardia e le realtà sociali che avevano caratterizzato la Spagna durante gli anni del franchismo. Si configura così un evento internazionale di dibattito democratico che ben si inserisce nel nuovo indirizzo intrapreso dalla Biennale, nel pieno clima di rinnovamento italiano degli anni Settan-

Questo saggio trae origine da: Crespi, Giulia (2012). La Spagna alla Biennale di Venezia dal 1976 al 2009 [tesi di laurea magistrale]. Relatore Nico Stringa; correlatore Stefania Portinari, a.a. 2011/2012. Venezia: Università Ca' Foscari Venezia; e dalla partecipazione al convegno Storie della Biennale di Venezia, a cura di Nico Stringa e Stefania Portinari (Venezia, Università Ca' Foscari Venezia, Ca' Dolfin, 6-7 dicembre 2016). 
ta, che voleva porsi in prima linea per la denuncia di ideologie fasciste e di limitazioni alle libertà perpetrate ancora da alcuni regimi. ${ }^{1}$

Nonostante il Ministerio de Asuntos Exteriores avesse inizialmente riconfermato Luis Gonzáles Robles come commissario incaricato dell'organizzazione della Biennale del 1976 programmando l'abituale esposizione all'interno del Padiglione spagnolo con gli artisti Modest Cuixart, Luis Feito López e l'Equipo Realidad, scelti per far conoscere il punto sull'arte contemporanea spagnola, inizia a delinearsi l'ipotesi di promuovere una mostra di carattere marcatamente antifascista e ci si interroga sull'appoggio che questa idea avrebbe potuto ottenere in Spagna. Racconta Ripa di Meana, ricordando i momenti antecedenti all'apertura dell'edizione del 1976:

Noi prendemmo i contatti con chi era in Italia. Stabile in Italia fu per molti anni Rafael Alberti con sua moglie. Intorno a lui c'era un gruppo di esuli spagnoli che aveva sempre tenuto uno sguardo molto ampio su quello che succedeva in Spagna e tra questi spiccava un giovane pittore che faceva spola tra Francia e Italia: Eduardo Arroyo. Amico di molti artisti italiani del tempo e di tutta la pittura politica italiana di Guttuso etc., aveva un suo peso. Il retroscena politico di tutti questi esuli era a maggioranza comunista. I comunisti spagnoli avevano avuto un ruolo leggendario nella guerra civile incoraggiati dalle retrovie sovietiche durante il periodo della dittatura, e avevano il vantaggio della tradizione organizzativa leninista che le altre forze politiche non avevano. In più, i comunisti, vantavano una preponderanza nelle brigate internazionali. Questo partito guidato da Santiago Carrillo, che stava a Parigi, aveva contatti anche con un figlio del popolo, autodidatta, molto geniale Marcelino Camacho, alla guida delle Comisiones Obreras. Avevamo anche dei contatti con una forza ancora indistinta che poi sarà quella dell'attuale primo ministro Mariano Rajoy, che negli auspici avrebbe voluto essere la democrazia cristiana: il Partido Popular. ${ }^{2}$

L'arresto di Arroyo, tornato in Spagna clandestinamente, all'aeroporto di Valencia, dove si era diretto per organizzare una mostra di artisti spagnoli in concomitanza con l'edizione della Biennale, è il primo fatto eclatante che fa ripensare all'invito ufficiale alla Spagna. È la Biennale stessa a levare un forte grido di protesta e a denuncia-

1 Si veda in particolare il progetto speciale dedicato al Cile nel 1974, a favore della libertà del Paese sudamericano dopo il colpo di stato del generale Pinochet, e l'edizione del 1977, conosciuta come 'Biennale del dissenso', dedicata all'arte sovietica.

2 Carlo Ripa di Meana a colloquio con Giulia Crespi in La Spagna alla Biennale di Venezia dal 1976 al 2009 [tesi di laurea magistrale], 40-1. 
re il caso, così come aveva in precedenza fatto anche per l'arresto di Alfonso Sastre e Genoveva Forest e altri sei intellettuali spagnoli.

I propositi di Ripa di Meana in quei mesi si indirizzano così all'organizzazione e direzione di un'edizione nuova della Biennale che risponda alla «necessità di far fronte alle gravi costrizioni che hanno luogo in varie parti del mondo dell'arte, della cultura e della libertà» (Carlo Ripa di Meana in Torrent 2003, 67).

España, Vanguardia artística y realidad social. 1936-1976 porta con sé diverse polemiche sia prima sia dopo l'inaugurazione. Il Padiglione nazionale viene lasciato chiuso e tutta l'attenzione si sposta sul Padiglione centrale. In accordo con l'idea generale, la mostra doveva seguire tre principi fondamentali: doveva avere carattere storico, occupandosi del periodo dal 1936 al 1976; doveva essere discorsiva, quindi articolata secondo diverse unità che creassero un discorso narrativo; e infine doveva essere analitica, ovvero concepita in modo che lo spettatore capisse l'esposizione anche attraverso l'analisi delle relazioni intercorse allora tra i fenomeni artistici e il contesto storico complessivo, politico, economico, sociale e culturale, della dittatura franchista.

Il nostro progetto voleva fare una teoria dell'arte contemporanea in Spagna sotto Franco. Il punto di partenza era: c'è un regime dittatoriale per quarant'anni e, nonostante la repressione, è certo che ci sono movimenti d'avanguardia. La questione era determinare che carattere storico avevano questi movimenti d'avanguardia, dove posizionarli. Credo che l'argomento proposto fosse molto semplice: l'avanguardia è un progetto che ha avuto sempre una vocazione di trasformazione sociale e l'avanguardia artistica ha, in una maniera o nell'altra, una vocazione di trasformazione politica. E quindi: come si può accettare che quest’avanguardia sia nata durante un momento immobilista e dittatoriale? La risposta è stata: perché l'idea di novità è tollerata dal Regime fino al punto concreto in cui si produce l'assorbimento e l'integrazione dell'avanguardia stessa, dopo di che nasce una nuova ondata di avanguardia che vuole rompere con quel livello fino a che anche questo torna ad integrarsi, e così via. Alla fine succede che l'idea di avanguardia evapora. (Tomás Llorens in Torrent 2003, 153) ${ }^{3}$

3 Enriqueta Antolín intervista Tomás Lorens (Torrent 2003).

EA Non c'è mai stata una polemica così grande rispetto alla partecipazione spagnola in una Biennale. Come definirebbe oggi quella esposizione della quale fu il principale responsabile?

TL Un grande insuccesso ... Per quanto riguarda me e tutti i collaboratori fu un grande impegno intellettuale, però fu mal compreso sia in Spagna che fuori. In questo senso dico che fu un insuccesso. 
A partire da questo momento, che concretamente segna una linea tra il prima e il dopo nella presenza spagnola a Venezia, è interessante vedere quali sono state le scelte curatoriali e quali sono stati gli artisti che hanno avuto il compito di aver rappresentato il Paese all'estero.

Sul finire degli anni Settanta e nei primi anni Ottanta i commissari che si sono susseguiti nella direzione del Padiglione spagnolo hanno scelto di presentare giovani artisti sulla scia del rilancio della società; Ferrán García Sevilla, Miquel Navarro, Cristina Iglesias, José María Sicilia, Eduardo Arranz Bravo, José Luis Pascual e Nacho Criado sono alcuni degli artisti che espongono e che oggi rappresentano un successo di critica a livello nazionale e internazionale e che hanno vissuto con la Biennale di Venezia un momento di affermazione artistica

Non bisogna caratterizzare il nostro momento con una o un'altra ideologia, con uno o un altro stile, ma abbiamo selezionato personalità, artisti che si rappresentano da soli e che rappresentano, non un gruppo determinato, ma un momento sociale. (Gonzáles Robles 1982, 5)

Nel 1984 il commissario Luis Gonzales Robles sceglie di dedicare l'intero Padiglione a Antoni Clavé, dando vita a una interessante retrospettiva a partire dagli anni Trenta. La sua carriera era iniziata come autore di incisioni, illustrazioni pubblicitarie e cartellonistica, ma anche come costumista. Spinto poi, a seguito della Guerra Civile, ad abbandonare la Spagna e a trasferirsi in Francia, entra in contatto con artisti del calibro di Picasso, che producono su di lui un cambio radicale di stile. Si avvicina quindi al cubismo e all'espressionismo che lo aiutano a maturare uno stile personale, caratteristico di tutta la sua carriera. Infatti, già a partire dagli anni Cinquanta, Clavé si libera dalle influenze esterne e si indirizza alla creazione di opere che sono diretta materializzazione delle sue idee, che si rivelano solo nell'atto della realizzazione. Da ciò deriva il suo rispetto per la materia bruta 0 , meglio, per le forme brute che la materia può acquisire accidentalmente: pieghe, deterioramenti, strappi. La grande innovazione è però l'introduzione del tempo nelle sue opere.

Il riutilizzo di materiali e oggetti che entrano a fare parte delle sue opere non ha l'intento di riabilitare gli scarti e la volgarità, ma piuttosto ambiscono a rispettare il peso del tempo sullo spazio del quadro e della scultura. (Daix 1984, 7)

Nel 1986 il nuovo commissario Francisco Calvo Serraller ${ }^{4}$ si concentra sulla rivalutazione dei precedenti dieci anni di arte spagno-

4 Francisco Calvo Serraller è uno storico, critico d'arte, saggista e docente presso l'Universidad Complutense di Madrid. 
la. Una decade, secondo lui, «segnata dalla fretta e dal desiderio più incontenibile di omologazione internazionale, con tutto ciò che un atteggiamento del genere implica, sia di negativo che di positivo» (Calvo Serraller 1986, 330). Serraller aggiunge che nelle ultime quattro edizioni della Biennale «a parte la sempre polemica discriminazione dei nomi, si è quasi sempre verificato un divorzio tra ciò che si intendeva nei circoli specializzati del paese come arte più rappresentativa del momento e ciò che si presentava ufficialmente nel Padiglione» (Calvo Serraller 1986, 330). La sua scelta ricade su artisti che già avevano attirato l'attenzione della critica nazionale e internazionale e che, soprattutto, sembrano slegarsi dall'eredità del franchismo, di cui conservano un ricordo solo nei racconti di infanzia. L'opera di Ferrán García Sevilla, Miquel Navarro, Cristina Iglesias e José Maria Sicilia rappresenta un nuovo traguardo nello sviluppo artistico del Paese.

Non bisogna dimenticare che gli anni Ottanta sono anni cruciali nel rinnovamento della Spagna; contemporaneamente alla diffusione del mercato dell'arte, con la nascita di importanti gallerie tra Madrid e Barcellona e di una fiera internazionale come ARCO Madrid (1982), e contemporaneamente alla nascita di un vero museo di arte contemporanea, il Centro de Arte Reina Sofia di Madrid (1986), crescono anche le libertà espressive ed una nuova consapevolezza di poter occupare un ruolo importante nel panorama mondiale.

A Venezia, ancora negli anni Ottanta, si gioca anche il predominio dell'interesse della critica per la scultura a discapito della pittura: Juan Muñoz, Cristina Iglesias e Susana Solano rinnovano la scultura tradizionale e si contrappongono alle esperienze pittoriche di Ferrán García Sevilla, José Maria Sicilia e Miquel Barcelò, che nel 1982 era stato l'unico rappresentante spagnolo a Documenta VII di Kassel. Le esposizioni di Oteiza nel 1988 e Chillida, a Ca' Pesaro, nel 1990, sanciscono invece il definitivo riconoscimento internazionale dei due maestri baschi.

Le premesse teoriche dell'opera di Oteiza si possono rintracciare nell'apprezzamento per certe culture primitive e nella visione contemporanea del mondo. Studiando la mitologia e le forme artistiche della cultura sudamericana in Spagna, Oteiza le riutilizza per comprendere le relazioni della metafisica dell'uomo con l'universo. La sua indagine e, quindi, la sua estetica si basano sull'idea che l'intera attività cosmica ubbidisce a delle regole cicliche, all'interno delle quali si collocano i processi dinamici di movimento, crescita e cambiamento. L'artista moderno deve dunque riuscire a creare un vocabolario formale per mezzo del quale lo spazio esterno e lo spazio interno riescano a fondersi, dando vita ad espressioni con significato universale e spirituale (Rowell 1988).

Eduardo Chillida, già premiato con il Premio Internazionale di Scultura del Comune di Venezia nel 1958 in occasione della XXIX 
Biennale, ritorna a Venezia con una selezione di trenta sculture datate tra il 1951 e il 1990 e così scrive per lui Giovanni Carandente.

Formalmente, la scultura di Chillida, esprime la stasi, l'imperturbabilità dell'assoluto, evita il dettaglio superfluo, carpisce i rapporti segreti delle forme e il ritmo che si istituisce tra gli elementi, crea la dialettica dei pieni e dei vuoti, del peso e della gravitazione, indaga sulle unità originarie che compongono una struttura (Carandente 1990, 15)

L'opera scultorea di Chillida si è caratterizzata per forte intimismo e poeticità, legati alla terra d'origine, e per la sorprendente molteplicità di materiali utilizzati. Con il legno, il granito, il cemento (riprendendo la lezione di Le Corbusier), l'acciaio, ma soprattutto il ferro, l'artista basco crea forme che non solamente occupano uno spazio, ma lo penetrano, grazie al sensuale alternarsi di curve e segmenti retti che creano dinamicità, senza mai sconfinare in un vero e proprio movimento (cf. Fullaondo 1976). Le sue opere sono il risultato di un calcolo preciso, quasi matematico delle strutture, derivato dalla sua formazione di architetto, prematuramente abbandonata, e dalla profonda ammirazione per la misura geometrica di Piero della Francesca e per la perfezione della forma racchiusa nella sezione aurea.

Attraverso il confronto con le altre realtà artistiche internazionali sullo sfondo della Biennale, la Spagna è riuscita velocemente ad uscire da anni di isolamento a cui il regime l'aveva costretta, grazie anche alla grande risonanza che questa manifestazione da sempre porta con sé. Permettere al pubblico di conoscere la situazione artistica di un Paese che, nonostante la censura, non ha mai smesso di essere all'avanguardia, è stato uno degli obiettivi maggiormente perseguiti. Così, le grandi mostre organizzate in patria e l'opera di fondazioni pubbliche e private hanno fatto della cultura uno strumento di trasformazione e di ammodernamento, parallelamente all'intensificarsi della ricerca critica degli esperti.

Sempre in occasione della Biennale di Venezia si è celebrato un modello espositivo destinato ad avere fortuna; quello di accostare un artista consacrato ad uno più giovane e meno conosciuto. È il caso delle esposizioni congiunte di Susana Solano con Jorge Oteiza (1988), di Antoni Tàpies con Cristina Iglesias (1993), di Joan Brossa con Carmen Calvo (1997), che hanno permesso un diretto confronto tra generazioni e punti di vista diversi, ma compatibili.

Probabilmente gli esiti più interessanti dell'arte spagnola sono passati per le sale del Padiglione spagnolo o nel Padiglione Centrale della Biennale anche durante tutti gli anni Novanta; Antoni Miralda (1990), Andreu Alfaro (1995), Manolo Valdés e Esther Ferrer (1999) hanno consacrato definitivamente la loro presenza nel panorama artistico internazionale. 
Il tema dell'opera di Miralda nel 1990 sono le 'nozze' tra due monumentali statue: la Statua della Libertà a New York e la Statua di Colombo a Barcellona. Si tratta di un progetto in divenire che ha trovato una conclusione solo nel 1992, in occasione delle celebrazioni del quinto centenario dello sbarco di Colombo nel Nuovo Mondo. Honeymoon continua un percorso, iniziato sul finire degli anni Settanta da Miralda, interessato a indagare la «permanenza del rito e della cerimonia nella società attuale, il tutto visto sotto il suo aspetto umoristico e inteso come una festa che riunisce le genti in pace e allegria» (Biennale 44 1990, 208). Per il centenario della Statua della Libertà nel 1986, con l'intenzione di riscoprire i riti e le celebrazioni del matrimonio occidentale, l'artista inizia a concepire questo «viaggio di nozze» tra le due statue, concluso con il matrimonio vero e proprio solo dopo sette anni. Rovesciando il concetto di viaggio di nozze, che normalmente avviene solo a seguito delle effettive nozze, Miralda programma un viaggio attraverso differenti Paesi per consentire a tutti i popoli di esserne partecipi.

Nel 1993 Achille Bonito Oliva dedica la XLV Biennale di Venezia, per la quale è stato nominato direttore del settore arti visive ai «Punti cardinali dell'arte», immaginando una esposizione «transnazionale e interdisciplinare, che darà all'Europa un segnale di conciliazione in questi tempi di estrema divisione e di crisi economica, morale e politica». ${ }^{5}$

La Spagna dedica l'intero Padiglione a Antoni Tàpies e Cristina Iglesias, progetto pensato da Aurora García, critica d'arte e storica che, a partire dagli anni Ottanta, si era dedicata alla curatela indipendente. La ricerca di interdisplinarietà, che Bonito Oliva aveva incoraggiato, è ben rappresentata dai due artisti spagnoli che con le opere esposte si collocano in un campo che supera la mera scultura e pittura per farsi portatori di un nuovo linguaggio artistico.

Sia Tàpies che Iglesias realizzano opere espressamente pensate per il Padiglione spagnolo che, quindi, non sono solamente traslazione di opere preesistenti, ma nascono e si concludono in relazione allo spazio a loro concesso. La convivenza tra le loro creazioni risulta riuscita, suscitando riflessioni su una poetica che si nutre di silenzio, dando vita a luoghi che portano con loro connotazioni quasi mistiche e spirituali.

Il titolo dell'opera di Tàpies, che gli vale anche il Leone d'Oro per la pittura, è esplicativo: Rinzen, che dal giapponese si traduce 'improvviso risveglio", porta con sé la connotazione semantica 'zen' e ciò che crea è uno spazio che induce alla contemplazione e alla riflessione: «Rinzen è una idea che viene dal buddismo, dove il pensie-

5 Achille Bonito Oliva in Peru Egurbide, «Los cuatros puntos cardinales del arte, lema de la Bienal de Venecia», El País, 26 de mayo de 1993. 
ro della illuminazione o risveglio improvviso è abituale». ${ }^{6}$ Le diciannove opere di Cristina Iglesias creano «ambienti fisici in cui i binomi come il reale e l'illusorio, il possibile e l'impossibile, l'evidente e l'occulto, sono privi di delimitazioni precise» (Garcìa 1993, 182). L'artista originaria di San Sebastian produce delle immagini che, come quelle di Tàpies, inducono ad una riflessione sul rapporto tra mondo esterno e mondo interno, sensazione accentuata dall'uso degli specchi. Invita lo spettatore a circolare intorno sue proprie opere e a rimanerne sorpreso, trasportato da un territorio all'altro in un costante rimando al profondo rapporto tra uomo e natura, in cui l'utilizzo di vari materiali e diversi generi diventa elemento costante.

Per l'edizione del centenario della Biennale il Padiglione spagnolo inaugura esponendo le opere di Andreu Alfaro e Eduardo Arroyo, scelte dal commissario Fernando Huici. A differenza dell'anno precedente, in cui le opere di Tàpies e Iglesias, nonostante le ovvie diversità, dialogavano perfettamente e in un certo senso si completavano vicendevolmente, in questa edizione i due artisti appaiono fortemente contrapposti. Se gli esordi di Arroyo lo avvicinano ai movimenti neofigurativi della Parigi degli anni Sessanta e alla pop art, grazie a «l'appropriazione e manipolazione degli stereotipi culturali di ordine assai diverso, che gli permettono la costruzione di un discorso mordace e appassionato, di forte senso critico» (Huici 1995, 186), le opere di Alfaro sono, invece, in forte connessione con la geometrizzazione delle forme e dello spazio di eredità costruttivista, e sembrano anticipare le posizioni minimaliste degli anni Settanta (cf. Llorens, Todolí 1991).

Per comprendere meglio la partecipazione spagnola nel 1997, partiamo da una definizione che il poeta-artista Joan Brossa dà si se stesso e della sua arte:

Io non sono né pittore, né scultore, né nessun'altra cosa. C'è una definizione della poesia visiva che dice che cerca una nuova dimensione tra il visivo e la semantica, senza cadere né nell'una né nell'altra cosa. Si tratta di operare un cambio di codice, di supporto, per poter esprimere qualcosa che si può scrivere. Con gli oggetti si possono creare metafore. E mi pare che questo è ciò che ho raggiunto molte volte. Però è difficile, perché, se ti lasci andare, puoi perdere l'equilibrio. ${ }^{7}$

La commissaria Victoria Combalía, scelta per curare il Padiglione spagnolo alla Biennale di quell'anno, utilizza la formula, ormai diven-

6 Antoni Tàpies, «La Bienal concede el León de Oro de pintura a Tàpies», El País, 14 de junio de 1993.

7 Joan Brossa in Peru Egurbide, «El Balón con peineta de Brossa, imagen de España en la Bienal de Venecia», El País, 14 de junio de 1997. 
tata consuetudine, di accostare tra di loro due artisti appartenenti a generazioni differenti: l'ormai riconosciuto talento di Joan Brossa viene affiancato alla emergente valenciana Carmen Calvo. Entrambi gli artisti scelti fanno dell'annullamento delle frontiere tra le varie discipline artistiche l'elemento fondante della propria carriera. Per Brossa il confine tra poesia e poesia visiva, così come la produzione di poesia e oggetto viene totalmente superato, mentre Carmen Calvo sfrutta la sottile linea che distingue la pittura dalla scultura per farne il perno delle proprie opere. Come scrive per loro José Maria Parreno, «hanno costruito un linguaggio poetico supportato dall'oggetto, e questo è una lingua più universale dell'Esperanto e che va oltre la parola per coniugare associazioni, per pensare agli opposti e dare forma a intuizioni nelle quali lo spettatore si riconosce». ${ }^{8}$

Nel 1999 la Biennale di Venezia viene affidata a Harald Szeemann che, con il titolo dAPERTutto, organizza una edizione all'insegna del superamento di ogni distinzione tra artisti affermati e artisti giovani. Nel 1980 Szeemann era stato il promotore insieme a Bonito Oliva di Aperto, sezione dedicata alla promozione dei nuovi talenti emergenti, ma nel 1995 Jean Clair aveva preferito abolirla. Ora, 1999, l'intera Biennale diviene 'aperta' e si annullano i confini. Gli spazi espositivi aumentano notevolmente, creando in tale modo una nuova relazione con l'utilizzo di spazi inconsueti. L'edizione di Szeemann è un vero successo, tanto da dare l'impressione di costituire una nuova Biennale (cf. Ricci 2010).

Un'altra innovazione apportata dal curatore svizzero è la maggiore presenza di arte proveniente dall'oriente, con una grande quantità di artisti cinesi. Come scrive lui stesso:

«Siamo saturi di storia europea e nordamericana che, con questa guerra assurda, a mio dire è arrivata alla fine. Non è che voglio oppormi alle culture o dare un tocco caotico alla Biennale. Voglio solamente ampliarla e offrire una maggiore apertura. ${ }^{9}$

L'anno precedente, inoltre, l'Ente Biennale aveva subito una nuova modifica. Con l'approvazione del decreto legislativo del 23 gennaio 1998 veniva trasformato in persona giuridica privata assumendo la denominazione di Società di cultura La Biennale di Venezia. Al suo interno venivano delineati sei settori di attività: architettura, arti visive, cinema, teatro, musica e danza.

Nel 1999 il Padiglione spagnolo, curato da David Pérez, professore e saggista, propone le opere di due artisti che hanno avuto un

8 José Maria Parreno, «Brossa \& Calvo: el objeto español», $A B C$ de las artes, 13 de junio de 1997.

9 Harald Szeemann in Carmen del Val, «Estamos saturados de arte occidental», El País, 8 de junio de 1999. 
ruolo molto importante negli orientamenti artistici del paese nel corso dei precedenti decenni: Manolo Valdés e Esther Ferrer. Entrambi iniziano la propria carriera artistica all'interno, rispettivamente, di Equipo Cronica e Zaj; due gruppi apparsi in Spagna nel 1964, nati dalla necessità di «sovvertire i codici e i concetti artistici del momento» (Pérez 1999, 168).

Questa tendenza ha avuto successivamente forti ripercussioni sulla nascita di movimenti concettuali agli inizi degli anni Settanta, come il Grup da Traball e anche su manifestazioni come gli 'incontri di Pamplona' del 1972. Con la mostra Biennale, come scrive David Pérez, «non si propone un dialogo tra generazioni, una opera complementare o un dialogo artistico. È un esempio di una determinata situazione dell'arte spagnola che si colloca nel segno di una rottura artistica, degli anni Sessanta e Settanta, con l'arte accademica del franchismo e con le basi della modernità». ${ }^{10}$

La rivalutazione del passato e il passo verso la modernità è ciò che ha maggiormente caratterizzato queste due decadi di presenza spagnola a Venezia. Dal Duemila si prediligeranno invece artisti affermati e linguaggi sperimentali, segno di una nuova radicata consapevolezza che ha ridato al Paese una voce protagonista nel dibattito artistico internazionale.

\section{Bibliografia}

Carandente, Giovanni (1990). Eduardo Chillida = Catalogo della mostra (Venezia, Ca' Pesaro). Milano: Fabbri.

Daix, Pierre (1984). Antoni Clavé: Pabellón de España. Bienal de Venecia. Madrid: Programa español de acción cultural en el exterior.

Fullaondo, Juan Daniel (ed.) (1976). Oteiza y Chillida en la moderna historiografia del arte. Bilbao: La Gran Enciclopedia Vasca.

Gonzáles Robles, Luis (1982). España en la Bienal de Venecia '82. Madrid: Dirección General de Relaciones Culturales.

Llorens, Tomás; Todolí, Vicente (eds) (1991). Alfaro = Catálogo de la exposición (Valencia, 2 de octubre-9 de diciembre de 1991). Valencia: Institut Valenciá d'Art Modern.

Oteiza, Jorge; Solano, Susana (eds) (1988). De varia commensuracion = Catálogo de la exposición (Venezia, 43ma Biennale di Venezia, Padiglione della Spagna). Madrid: Ministerio de Asuntos Exteriores, Ministerio de cultura.

Ricci, Clarissa et al. (ed.) (2010). Starting From Venice. Milano: et. al. edizioni. Rowell, Margit (1988). «Una modernidad atemporal». Oteiza, Solano 1988.

Torrent, Rosalía (2003). Un siglo de arte español en el exterior: España en la Bienal de Venecia, 1895-2003. Madrid: Ministerio de Asuntos Exteriores, Dirección General de Relaciones Culturales y Científicas; Turner.

10 David Pérez, «El pabellón español participa del «dapertutto» de la Bienal de Venecia», El País, 28 de mayo de 1999. 


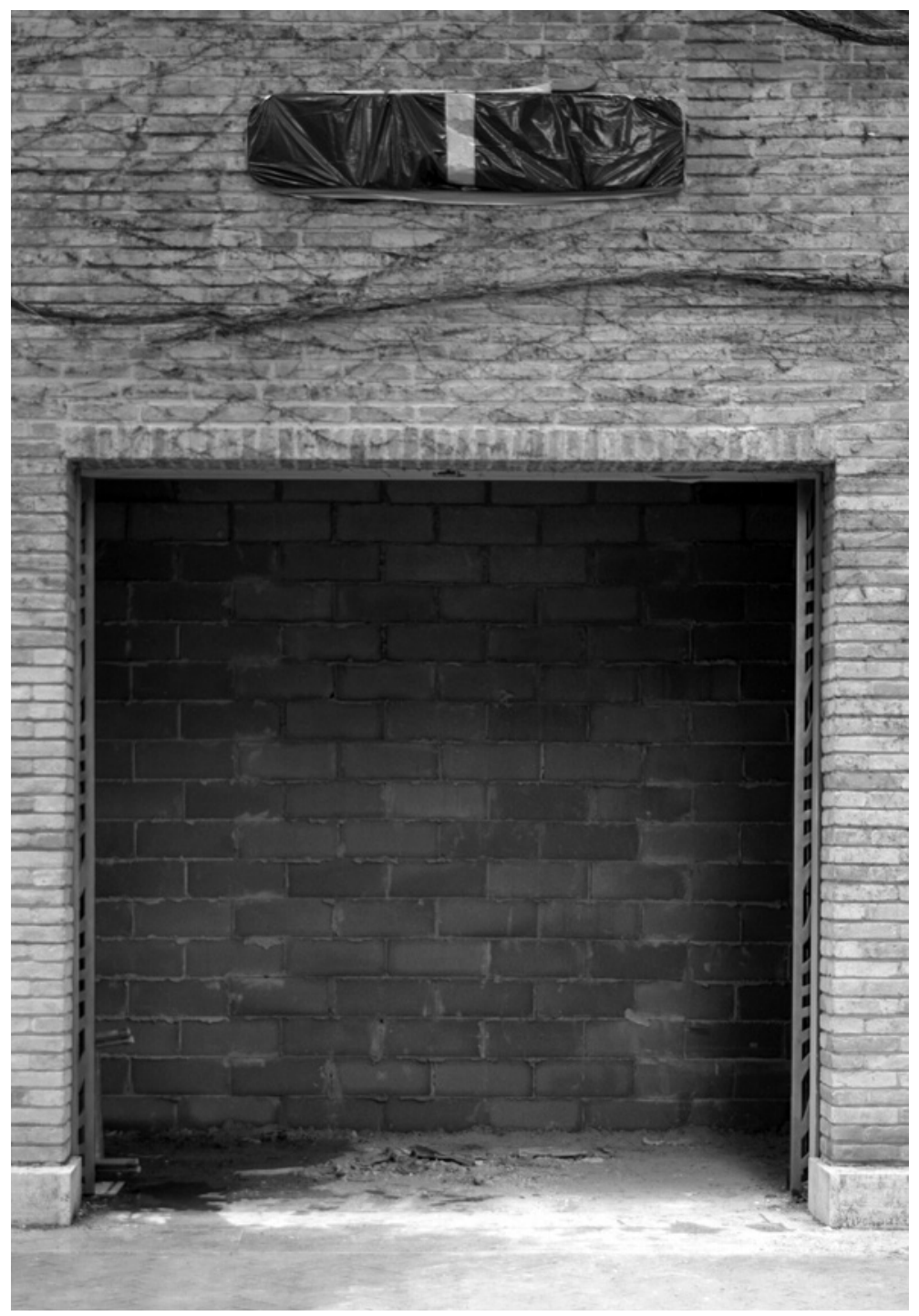

Figura 1 Santiago Sierra, Palabra tapada, 2003. Padiglione spagnolo, 50esima Esposizione Internazionale, Venezia, Italia.

(c) Santiago Sierra. Courtesy dell'artista 


\title{
Padiglioni che denunciano, riflettono e astraggono Un'analisi critica e trasversale della partecipazione spagnola alla Biennale di Venezia (2003-2011)
}

\section{Anita Orzes}

Universitat de Barcelona, Espanya

\begin{abstract}
Studying the national participations at the Venice Biennale means studying part of the spine of the exhibition as the creation of national pavilions is generated at a time when the Biennale was still defining its structure. Through an in-depth analysis of national participations it is possible to study the foreign cultural policy of the various countries, the evolution of a supposedly national art and the local-global contradictions of the contemporary art system. This essay aims to present and analyze some of the projects exhibited in the Spanish pavilion in the first editions of 21 century to demonstrate its utility to discuss the obsolescence or topicality of the national pavilions in Venice and also to treat the 'biennial phenomenon', its apparent unstoppability and the trend of the 'discursive biennials'.
\end{abstract}

Keywords Spanish Pavilion. Venice Biennale. On Translation. National pavilions. Discursive biennials.

Sommario 1 Perché studiare le partecipazioni nazionali alla Biennale di Venezia? 2 La partecipazione spagnola alla Biennale di Venezia: lo stato dell'arte. - 3 Il Padiglione nazionale come (s)oggetto della riflessione e della denuncia. - 4 L'(in)adeguatezza delle discursive biennials. -5 Conclusione. 


\section{Perché studiare le partecipazioni nazionali alla Biennale di Venezia?}

Studiare le partecipazioni nazionali alla Biennale di Venezia significa studiare parte della colonna vertebrale della mostra. Infatti, l'idea di creare architetture dove i Paesi partecipanti possano esporre i propri rappresentanti si genera contemporaneamente al definirsi dei contorni della struttura della Biennale stessa, materializzandosi fisicamente già nel 1907 con il primo Padiglione nazionale, quello del Belgio. ${ }^{1}$ Inoltre, questa struttura continua ad essere vigente, a differenza di altri biennali che, nonostante abbiamo preso Venezia come modello di riferimento, l'hanno ormai abbandonata. ${ }^{2}$

Fino alla data odierna gli studi sulla struttura della Biennale di Venezia e, conseguentemente, sulle partecipazioni nazionali sono esigui. Se si escludono alcuni progetti artistici come On Translation: I Giardini (2005) e Altavoces (2005), essi si riducono a studi datati (cf. Alloway 1968) o ampliati cronologicamente ma non aggiornati (cf. Di Martino 1995, Di Martino 2013) e con tesi di laurea triennale, magistrale o di dottorato.

Eppure, lo studio delle partecipazioni nazionali, sia dei Paesi che possiedono un Padiglione dentro i Giardini, sia di quelli che, privi di esso, vi partecipano in Padiglioni satelliti (ossia in spazi di varia natura come istituti di cultura, palazzi o chiese concessi per un limitato periodo di tempo, e disseminati per l'urbe veneziana e le isole adiacenti), permettono di svolgere una complessa ed articolata analisi della realtà socio-politica, culturale-economica ed identitaria-nazionale. I Giardini di Castello sono stati, e continuano ad essere, un vero e proprio punto di precipitazione delle tensioni geo-politiche a livello mondiale, basta ricordare l'arrivo di Israele con il proprio Padiglione nel 1952, dopo aver partecipato per la prima volta nel 1950 (in uno spazio concessogli nel Palazzo dell’Esposizione), successiva-

Questo saggio ha origine dalla partecipazione al convegno Storie della Biennale di Venezia, a cura di Nico Stringa e Stefania Portinari (Venezia, Università Ca' Foscari Venezia, Ca' Dolfin, 6-7 dicembre 2016).

1 L'origine risale alla Seconda edizione della Biennale (1897), nella quale si nota che il carattere internazionale comportava che gli artisti italiani non avessero spazio sufficiente per presentare i propri lavori nel Palazzo dell'Esposizione, l'unico spazio espositivo in quel momento loro accessibile. Nel 1907, di fronte all'esigenza di offrire una superficie maggiore, Antonio Fradeletto, segretario generale della Biennale, propose di collocare le opere degli artisti stranieri nei Padiglioni nazionali che si sarebbero costruiti nel recinto dei Giardini.

2 Un esempio è la Biennale di San Paolo (1951) che negli anni Ottanta inizia a riflettere sulla validità della struttura in partecipazioni nazionali, a favore invece di sezioni tematiche. Ciò sino al 2006, con Como viver junto (27a Biennale di San Paolo, 7 ottobre-17 dicembre 2006), quando si abbandonando le rappresentazioni nazionali. 
mente alla proclamazione dello Stato di Israele nel 1948; o la partecipazione della Germania con il Padiglione della Repubblica Democratica Tedesca e della Repubblica Federale di Germania durante la Guerra Fredda. Al giorno d'oggi queste tensioni rivelabili, per esempio, ai Giardini con il Padiglione coreano, dove partecipa solo la Corea del Sud, si sono estese a tutta la città di Venezia e sono visibili nelle cartografie tracciate dai Padiglioni satelliti. Ne sono esempio la partecipazione della Catalogna o la reiterata assenza di Porto Rico, colonia degli Stati Uniti.

Attraverso un'approfondita analisi delle partecipazioni nazionali è quindi possibile studiare la politica culturale estera dei Paesi partecipanti, l'evoluzione di un'arte presumibilmente nazionale e le contraddizioni locale-globale del sistema artistico contemporaneo. Altrettanto interessante è esaminare quali artisti si inviano a Venezia e quali ad altre biennali occidentali e del Sud Globale dato che corrispondono all'immagine che si vuole dare delle pratiche artistiche del proprio Paese nei diversi contesti esteri.

Data una piattaforma internazionale quale la Biennale di Venezia, che possiede più di cento anni di storia, e le cui partecipazioni nazionali sono numerose eppure in parte discontinue, non è facile portare a termine un'analisi esaustiva. È però possibile individuare casi di studio paradigmatici in quei Paesi abituati a partecipare alla Biennale di Venezia, che approfittano da sempre della struttura della manifestazione per godere di una maggiore visibilità e i cui progetti permettono di arricchire concettualmente la Biennale stessa, per svolgere a partire dall'analisi di questi casi paradigmatici - anche considerazioni più generali. Il caso scelto è quello della Spagna. Il presente saggio si prefigge quindi di presentare ed analizzare alcuni dei progetti esposti nel Padiglione spagnolo della Biennale di Venezia nelle prime edizioni del Duemila, e dimostrarne l'utilità per discutere la questione generale dei Padiglioni nazionali e per trattare il fenomeno biennale e la sua apparente inerzia. Per una corretta contestualizzazione, prima di soffermarsi sulla partecipazione di Santiago Sierra (Palabra tapada e Muro cerrando un espacio, 2003), Antoni Muntadas (On Translation: I Giardini, 2005) e Dora García (Lo inadecuado, 2011), si ritiene opportuno realizzare una breve digressione sulla partecipazione della Spagna e lo stato dell'arte degli studi ad essa relativi. Questa digressione si ripropone di fornire una cornice storica nella quale inserire l'analisi di questi tre progetti artistici. 


\section{La partecipazione spagnola alla Biennale di Venezia: lo stato dell'arte}

La Spagna è un Paese assiduo alla Biennale di Venezia: oltre a parteciparvi fin dalla sua Prima edizione, è tra i primi a costruire un Padiglione nazionale (1922). L'attività espositiva del Padiglione spagnolo è un oggetto di studio paradigmatico. Infatti, l'analisi critica della sua traiettoria dopo più di un secolo di partecipazione pressoché costante permette di eviscerare la sua politica culturale estera, individuando nel franchismo uno spartiacque che segna un prima, un durante e un dopo. ${ }^{3}$ Così, si può assistere all'omologazione ai canoni globali di artisti isolati dal contesto internazionale per anni, oltre ad operare un confronto critico tra gli artisti inviati a rappresentare la Spagna in diverse biennali nello stesso arco cronologico. ${ }^{4}$

La complessità di una ricerca di questo tipo deve confrontarsi con la scarsezza delle ricerche realizzate fino al momento. L'unico libro dedicato alla partecipazione spagnola alla Biennale di Venezia è Un siglo de arte español en el exterior. España en la Bienal de Venecia 1895-2003 (2003) di Rosalía Torrent Esclapés. ${ }^{5}$ Quasi un decennio prima della pubblicazione di questo libro, nel 1988, Torrent Esclapés discuteva la sua tesi di dottorato, dal titolo La Bienal de Venecia. Datos para su historia. La participación española: el hito del 76 presso l'Universitat de Valéncia. Ricerche accademiche più recenti, corrispondenti a tesi di laurea magistrale, sono state realizzate presso l'Università Ca' Foscari Venezia: La Spagna alla Biennale di Venezia dal 1976 al 2009 di Giulia Crespi (a.a. 2011-12), Il Padiglione spagnolo alla Biennale di Venezia: un'analisi critica dell'attività espositiva dal 2001 al 2013 dell'autrice di questo saggio (a.a. 2013-14) e Videoarte e arti performative: il caso del Padiglione spagnolo alla Biennale di Venezia dal 2001 al 2017 di Eleonora Varotto (a.a. 2017-18).

Nel momento in cui si sta scrivendo questo saggio sta avendo luogo presso l'Institut Valencià d'Art Modern (IVAM) la mostra España. Vanguardia artística y realidad social. 1936-1976, ${ }^{6}$ dedicata dell'omonima mostra curata da Valeriano Bozal e Tomás Llorens nel Palazzo dell'Esposizione durante la 37a edizione della Biennale di Venezia.

3 Gli unici anni in cui non partecipa alla mostra sono dal 1942 al 1950 nel 1974. Nel 1976 il Padiglione rimane chiuso ma si organizza la mostra España. Vanguardia artística y realidad social: 1936-1976.

4 Interessanti casi di studio sono il Padiglione della Spagna all'Esposizione Internazionale di Parigi del 1937 in contrapposizione a quello nella Biennale di Venezia del 1938 o, negli anni Cinquanta, la partecipazione spagnola alle Biennali di Venezia ed alle Biennali Ispanoamericane.

5 Nel 1997 Rosalía Torrent Esclapés pubblicò il libro España en la Bienal de Venecia 18951997. L'edizione aggiornata al 2003 è stata finanziata dal Ministero degli Affari Esteri.

6 Dal 13 settembre 2018 al 13 gennaio 2019. 
In questa edizione, che appartiene alle «biennali solidali» promosse durante la presidenza di Carlo Ripa di Meana, ${ }^{7}$ la Spagna non venne invitata all'Esposizione e si promosse quel progetto curatoriale ${ }^{8}$ il cui obiettivo era dimostrare come per molti anni la Biennale era stata lo scenario di una Spagna che aveva mostrato una realtà distorta, dove l'avanguardismo spagnolo era stato modellato dall'interno, diventando uno strumento di propaganda politica a favore del regime franchista. La decisione di Carlo Ripa di Meana, il dibattito e le contestazioni che si generarono al riguardo in Spagna (e anche in Italia), la chiusura del Padiglione nazionale e la mostra stessa sono un altro esempio di come le tensioni politiche si riflettano, grazie alla struttura della Biennale, nei Giardini di Castello.

Per quanto riguarda lo studio della politica culturale post-dittatura, adottata dal Governo spagnolo, si cita l'articolo Política cultural del gobierno español en el exterior (2000-2004) (marzo 2005), il progetto di ricerca Arte contemporaneo español en el exterior (Beirak 2013) e la sezione La (re)construcción de la institución de arte in Art/nsición, Tra/nsición (Albarrán 2018). In queste ricerche viene offerta un'analisi della politica culturale e degli organismi statali creati per la diffusione dell'arte spagnola all'estero in contrapposizione a quelli per lo sviluppo di una rete stabile all'interno del paese ed il loro 'traballare' con i cambi di governo, si esaminano i presupposti, si ribadisce (indicazione alla quale soggiace una denuncia) che non è il Ministero di Cultura (quando esiste e non viene inglobato ad altri ministeri) ad occuparsi della partecipazione spagnola alle mostre internazionali, bensì il Ministero degli Affari Esteri. ${ }^{9}$

A dispetto dell'interesse di questi studi, continua ad essere assente uno studio minuzioso della politica culturale estera adottata nelle biennali internazionali ed è, conseguentemente, mancante quella relativa al Padiglione spagnolo. Lacuna che si è cercata di colmare con la tesi di laurea magistrale Il Padiglione spagnolo alla Biennale di Venezia: un'analisi critica dell'attività espositiva dal 2001 al 2013 che si configura come una proposta per progredire nella rilettura della qualità della presenza spagnola alla Biennale di Venezia nelle prime edizioni del Duemila.

7 Il 20 marzo 1974 si costituisce il nuovo Consiglio della Direzione della Biennale e si nomina presidente Carlo Ripa di Meana, che voleva denunciare la situazione dei paesi sottomessi ad un sistema dittatoriale. La Biennale del 1974 è stata dedicata al Cile.

8 Si articolava in due grandi blocchi ed analizzava e 'correggeva' l'immagine dell'avanguardia spagnola che si era mostrata alla Biennale. Il primo blocco studiava tanto gli avvenimenti artistici che avvennero in piena guerra civile quanto quelli relativi agli artisti in esilio; il secondo tentava un'analisi cronologica-visiva dell'arte spagnola post-bellica.

9 Il Ministero degli Affari Esteri è l'organismo che dal 1950 si occupa della coordinazione della partecipazione spagnola alla Biennale di Venezia. 


\section{Il Padiglione nazionale come (s)oggetto della riflessione e della denuncia}

Il carattere internazionale della Biennale di Venezia e la sua struttura in partecipazioni nazionali, albergate in «ambasciate simboliche» (Medina 2003, 232) dei Paesi, la trasforma in un luogo in cui si può mettere in discussione, contemporaneamente, la globalizzazione ed il nazionalismo (cf. Basualdo 1999, s.p.). L'intervento di Santiago Sierra nel Padiglione nazionale, nella 50esima Biennale, ne è un esempio. Le tre parti in cui si articola si inseriscono nelle direttrici abituali del suo lavoro: l'ostruzione (Muro cerrando un espacio), la provocazione linguistica (Palara tapada) e la tematica del lavoro come castigo (Mujer con capirote sentada de cara a la pared). In quest'occasione si analizzeranno le prime due, senza dimenticarsi di sottolineare che, nonostante ogni parte esista indipendentemente, la coesistenza ne amplifica la forza.

In Palabra tapada [fig. 1] Santiago Sierra ha coperto la parola Spagna, collocata all'entrata del Padiglione, usando della plastica nera e del nastro adesivo. Il suo carattere effimero obbliga gli addetti alla manutenzione delle installazioni a 'ristrutturarla' costantemente. Il messaggio contenuto in Muro cerrando un espacio [fig. 2] riafferma l'identità dello spazio e le sue connotazioni storiche e simboliche. Quest'ultimo consiste nella costruzione di un muro di mattoni, dal suolo al tetto, parallelo alla porta d'ingresso, che impedisce di varcarne la soglia. L'accesso all'edificio è bloccato e la porta, lasciata aperta, aumenta la forza visiva del muro. Lo spettatore vi si può solo avvicinare, scontrarsi con l'ostacolo fisico, e percorrere lo spazio con la vista, fino a dove il campo visivo glielo permette. Vicino al muro c'è un cartello, in castigliano, che indica che l'entrata si trova nella parte posteriore della costruzione e che vi può accedere solo chi possiede la nazionalità spagnola, presentando prima un documento identificativo.

Muro cerrando un espacio si riferisce «alla restrizione dell'accesso rappresentata dai confini e dai limiti (in)visibili che collocano le persone in differenti territori geografici, sociali e ideologici. Il muro polarizza gli spettatori della Biennale su entrambi i lati di uno scenario ipotetico [...] e mostra che, nonostante le apparenze, i confini non sono stati aboliti ma consolidati» (Martínez 2003, 24). Portando a termine questo progetto in uno dei Padiglioni nazionali della Biennale di Venezia, Santiago Sierra ha convertito questa ambasciata artistica simbolica in una extraterritorialità, un Padiglione esclusivamente per spagnoli, il cui retro si trasforma in un ufficio di revisione migratoria. Ha enfatizzato, attraverso la strumentalizzazione linguistica e l'ostruzione fisica, quella che giudicava essere la politica migratoria del governo spagnolo di fronte ai flussi migratori provenienti dal Nord Africa e dall'America Latina. 


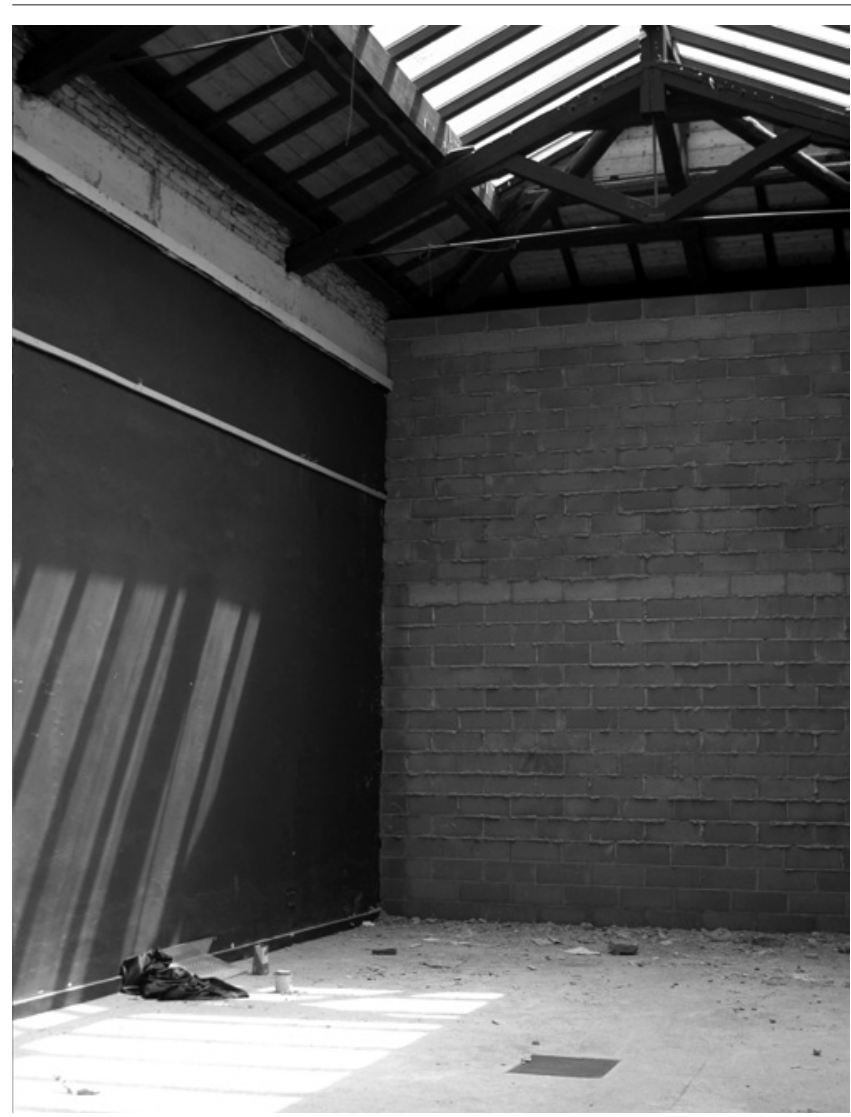

Figura 2 Santiago Sierra, Muro cerrando un espacio, 2003. Padiglione spagnolo, 50esima Esposizione Internazionale, Venezia, Italia. (c) Santiago Sierra. Courtesy dell'artista

La forza del lavoro risiede nella capacità di Santiago Sierra di mettere in relazione gli avvenimenti (la politica migratoria) con il luogo (il Padiglione nazionale come ambasciata simbolica del paese) e di mettere in discussione, conseguentemente e contemporaneamente, la globalizzazione ed il nazionalismo nella struttura nazionalista della Biennale di Venezia, che si pone come una mostra internazionale. Quest'opera è tutt'ora di grande attualità se si considera la politica migratoria che stanno intraprendendo gli Stati Uniti verso i paesi dell'America Latina, il desiderio di innalzare frontiere fisiche o il comportamento di alcuni Paesi dell’Unione Europea di fronte alla crisi migratoria. Nonostante la lettura politica fosse importante nel momento in cui è stata presentata alla Biennale, e nonostante sia molto attuale, in quest'occasione ci si vuole soffermare sul ruolo che ha giocato l'architettura che ha ospitato il progetto di Santiago Sier- 
ra e su come i Padiglioni nazionali sono «uno degli alibi estetici per la persistenza dello stato nazione», un anacronismo a cui si ricorre per «rinazionalizzare la produzione artistica» (Medina 2003, 232).

Per approfondire questi aspetti si utilizzerà On Translation: I Giardini, ${ }^{10}$ progetto con cui Antoni Muntadas partecipa alla 51. edizione della Biennale di Venezia, costruendo una metafora riflessiva dei Giardini di Castello.

Il punto di partenza è la struttura della Biennale che, nel 1976, anno della sua prima partecipazione alla mostra con España. Vanguardia artística y realidad social: 1936-1976, ne ha catturato l'attenzione. Già in quest'occasione Muntadas identifica i Giardini come un «giardino amabile», una «micro-città dove la storia è visibile nei dettagli della sua pianificazione» (Muntadas 2005, 140): l'organizzazione territoriale corrisponde alla realtà geo-politica ed economica della prima metà del XX secolo (principalmente), mentre gli ordini e gli stili architettonici dei Padiglioni riflettono il governo dei singoli Paesi e l'epoca in cui furono innalzati. ${ }^{11}$

Secondo Antoni Muntadas, la struttura e i modi in cui i Giardini accolgono e presentano l'arte non sono adatti all'età contemporanea e l'idea del Padiglione come rappresentazione di un Paese è obsoleta. La tesi si articola su tre linee: la storia della Biennale di Venezia, la struttura topografica dei Giardini di Castello e l'esistenza stessa dei Padiglioni nazionali.

L'analisi dell'articolarsi delle ultime decadi dimostra che la manifestazione ha luogo non solo nei Giardini di Castello e nell'Arsenale ma coinvolge tutta l'urbe veneziana. Vi sono gli storici Padiglioni nazionali (Belgio, Spagna, Francia, Germania e Stati Uniti) all'interno del recinto dei Giardini ed altri che, per la saturazione dello stesso, si disperdono nella città lagunare.

Il fenomeno dell'abbondante presenza dei Padiglioni satelliti ha una causa ed una conseguenza. Da una parte, lo storico modello imposto dalla Biennale sembra affermare che «chi non ha un Padiglione nella Biennale non è un paese» (Muntadas 2005, 276), trasformandosi, in questo modo, in un dispositivo di legittimazione dell'identità nazionale. ${ }^{12}$ Conseguentemente, tutti i Paesi che non lo possiedono, se vogliono partecipare dignitosamente all'evento, dovranno trova-

10 On Translation raccoglie una serie di progetti iniziati nel 1995. Non è un lavoro finito e si arricchisce con il tempo. Sono progetti complementari che danno continuità ad ampie tematiche: la comunicazione, la cultura del nostro tempo ed il ruolo dell'arte nella società contemporanea.

11 Il Padiglione spagnolo è stato costruito nel 1922. La sua facciata è stata rimodellata nel 1938 e nel 1952.

12 Muntadas, 2005, 292-4. La preoccupazione degli Stati di possedere un Padiglione nazionale, pur lontano dal nucleo centrale, invece di concentrare l'attenzione principale sulla qualità della propria esposizione, dimostra che il modello imposto da Ve- 
re una collocazione negli spazi disponibili (istituti di cultura, antichi palazzi, chiostri o chiese) ed investire molto tempo, forze e denaro (in pubblicità) per attrarre le persone e, soprattutto, per informarle della propria esistenza.

Con il suo progetto, Muntadas vuole marcare una distanza rispetto ai problemi della rappresentazione e della nazionalità. La strategia consiste nell'organizzare il Padiglione spagnolo secondo la stessa struttura della Biennale, in modo che racchiuda una serie di sedi internazionali, da Bogotá (On Translation: El aplauso) alla Corea del Sud (On Translation: Listening) e New York (On Translation: The Bank), riproducendo così la conformazione di un parco tematico. La struttura architettonica del Padiglione agevola l'organizzazione in due spazi: il nucleo centrale, la prima sala visitata dallo spettatore, in cui colloca On Translation: Stand By e On Translation: I Giardini, opere che si collegano direttamente alla critica evidenziata. Nelle sale adiacenti, ${ }^{13}$ si espongono tutti quei lavori che sottolineano l'internazionalità del Padiglione, a sinistra On Translation: On View, On Translation: Warning, On Translation: The Interview, On Translation: The Internet Project; a destra, On Translation: Listening, On Translation: El aplauso e, nella sala perpendicolare al nucleo centrale, On Translation: La mesa de negociación II, On Translation: The Bookstore e On Translation: The Bank (cf. Bonet 2005, 386-429).

In On Translation: Stand By, l'artista vuole mostrare il primo contatto che le persone instaurano con gli spazi culturali o religiosi, gli uffici di migrazione e gli aeroporti: una lunga attesa prima di poter accedere al luogo desiderato; caratterizzata iconograficamente da larghe file che costituiscono il contatto visivo iniziale con i diversi scenari. La situazione di attesa è stata ricreata in On Translation: I Giardini [fig. 3] dove il nucleo centrale si trasforma in un vestibolo che evoca le sale di aspetto e di transito degli aeroporti con le sue file di panchine, monitor e schermi pubblicitari. Una struttura rettangolare attraversa diagonalmente lo spazio e assume la funzione di un database: da un lato, raccoglie le fotografie, antiche ed attuali, dei Padiglioni dei Paesi partecipanti ed offre informazioni sulla loro storia; dall'altro, presenta una lista con i nomi dei Paesi esclusi dalla 51. Biennale di Venezia.

Per mezzo dell'installazione, Muntadas trasforma il Padiglione in una complessa metafora di quello che reputa essere, al giorno d'oggi, la Biennale: una struttura gerarchica che vede i paesi storicamente e politicamente importanti in una posizione privilegiata, che conservano

nezia trasforma il Padiglione nel principale protagonista mentre gli artisti e la loro arte rimangono in secondo piano.

13 Dato che i progetti presentati alla Biennale non corrispondono sempre alla versione originale della serie On Translation, in quanto soggetti a modifiche necessarie per la mostra, si consiglia lo studio accurato della versione originale per confrontarla con quella presentata nel 2005. Cf. Mercader 2000 e Alonso 2002. 


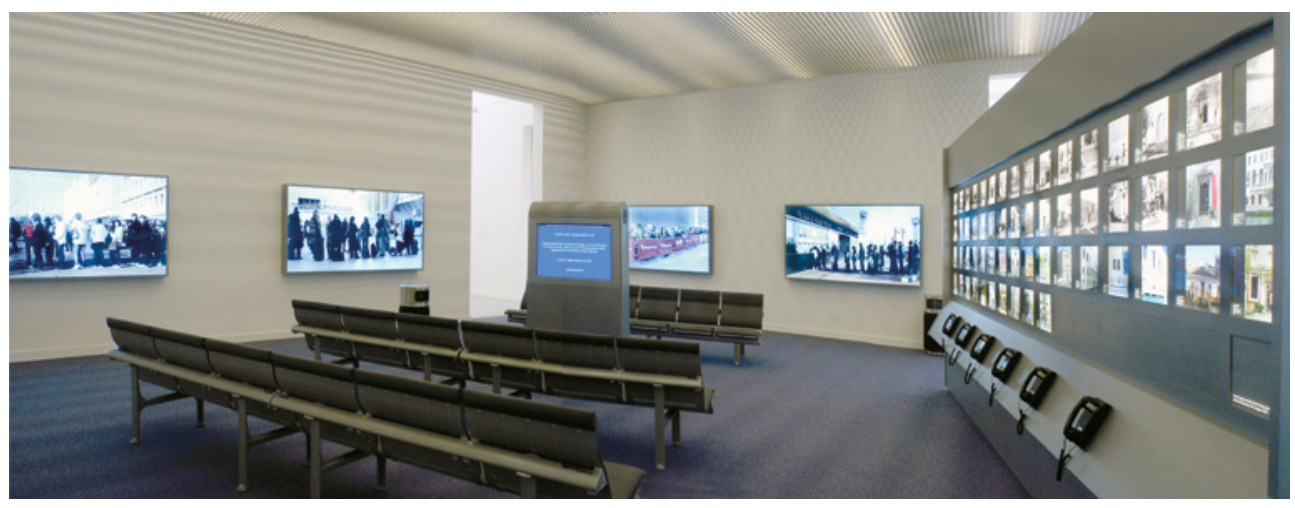

Figura 3 Antoni Muntadas, On Translation: / Giardini, 2005 Padiglione spagnolo, 51esima Esposizione Internazionale d'Arte, Venezia, Italia. (c) Muntadas. Courtesy dell'artista. Fotografo: Claudio Franzini

senza la necessità di legittimarla, e i Paesi storicamente poveri, che si trovano in una posizione svantaggiata e lontani dai flussi delle persone che vanno a visitare l'esposizione. In aggiunta, la struttura dei Padiglioni dimostra l'esistenza di una gerarchia tanto tra i partecipanti, coloro che possiedono un Padiglione dentro l'ambito recinto e coloro che l'hanno fuori, quanto con i Paesi che non vi prendono parte. Il gap geopolitico tra i paesi poveri e i ricchi è evidente: quelli che sono dotati di un Padiglione nei Giardini rappresentano il 24\% della popolazione mondiale e producono l'83\% del PIL globale; quelli che si collocano fuori corrispondono al 34\% e contribuiscono al suddetto PIL con il 9\%. Per concludere, gli Stati che non vi partecipano rappresentano il $42 \%$ e contribuiscono solo con l' $8 \%$ del PIL all'economia mondiale..$^{14}$

Il progetto di Muntadas si inserisce nel dibattito internazionale sull'obsolescenza della struttura della Biennale di Venezia (cf. Alloway 1968, 12-29; Filipovic 2005, 63-84), secondo il quale i Padiglioni nazionali sono un'eredità del diciannovesimo secolo ed è urgente abbandonarla, modificarla o aggiornarla. Una voce dissonante è quella di Caroline A. Jones che sostiene che la «componente del Padiglione» (Jones 2010, 83) sia molto utile per il suo ruolo politico e concettuale. Si condivide questa visione e si ritiene che, sebbene la struttura della Biennale di Venezia rifletta il modello ideologico con cui si interpretavano le nazionalità alla fine del XIX secolo, essa sia (e sia stata) un utile strumento da un punto di vista geo-politico, economico e identitario-nazionale.

14 Le statistiche appartengono a Altavoces, opera di sound art con cui Santiago Sierra partecipa alla 51. Biennale di Venezia. Il contenuto della registrazione è disponibile nella pagina web dell'artista: https://www.santiago-sierra.com/200502_1024.php (2018-11-07). 
Si reputa inoltre che il motivo per cui le critiche sono così aspre e poco costruttive, dal momento che si indica che la struttura è obsoleta e che dev'essere abbandonata, modificata o aggiornata con urgenza ma non si suggeriscono nuovi approcci, si deve a una conoscenza superficiale della sua storia, a una disinformazione rispetto alle (tras)formazioni topografiche che hanno avuto luogo nei Giardini di Castello tra la costruzione del primo e dell'ultimo Padiglione, rispettivamente nel 1907 e nel $1995 .^{15}$

Al giorno d'oggi esistono solo due pubblicazioni concernenti la storia dei Padiglioni nazionali a Venezia: I Padiglioni della Biennale di Venezia (Mulazzani 1988, 2004) ${ }^{16}$ e On Translation: I Giardini (Muntadas 2005). La prima è una pubblicazione di carattere compilativo: la breve introduzione sulla storia dei Giardini di Castello è seguita da schede concernenti i Padiglioni nazionali con informazioni relative al momento in cui se ne è proposta la costruzione alla Biennale, ${ }^{17}$ dettagli del progetto architettonico ed una documentazione visiva (piantine e fotografie). La seconda è il catalogo del progetto presentato nel Padiglione spagnolo ed è attualmente il testo più aggiornato e completo. Contiene un saggio sulla storia dei Giardini di Maria Vittoria Martini, le piantine di varie edizioni che permettono visualizzare come si è formato il nucleo originale della mostra ed un importante corpus fotografico dei Padiglioni nazionali.

In entrambi i testi si spiega qual è stata la genesi dei Padiglioni nazionali, le modalità per edificarli e la formazione progressiva del nucleo originale della Biennale. È totalmente assente la ricostruzione delle vicende di quei Paesi che in un momento determinato hanno disposto di un Padiglione nel recinto della Biennale per poi cederlo ad un altro o di quei Paesi a cui è stato offerto il terreno per la costruzione dell'architettura nazionale ma che hanno declinato l'offerta. La ricerca e (ri)costruzione di queste storie eclissate è di grande utilità per riaffermare come la struttura della Biennale di Venezia sia un utile strumento per svolgere una complessa ed articolata analisi della realtà geo-politica, culturale-economica ed identitaria-nazionale.

15 Gli anni in cui i Paesi hanno costruito e/o occupato i rispettivi Padiglioni nei Giardini di Castello sono: 1907 Belgio, 1909 Germania, Ungheria e Gran Bretagna, 1912 Francia e Olanda, 1922 Spagna, 1930 Stati Uniti, 1932 Danimarca e Svizzera, 1934 Austria, 1956 Giappone e Finlandia, 1958 Canada, 1964 Brasile, 1988 Australia e 1995 Corea del Sud.

16 L'edizione ampliata del 2004 include il Padiglione del libro Electa e quello della Corea del Sud. Si può ricordare anche lo studio di Romanelli, Giandomenico (1976). Ottant'anni di architettura e allestimenti alla Biennale di Venezia. Venezia: Archivio storico delle arti contemporanee.

17 Per ottenere la concessione del terreno, il Paese doveva presentare un progetto e ottenere l'approvazione del comune. Una volta ottenuto il consenso, il Paese poteva decidere di costruire da solo il Padiglione, occupandosi delle spese e nominando un architetto, o affidarne il compito al Comune di Venezia e farsi carico dell'edificio posteriormente. 
Al giorno d'oggi, dato che i Giardini di Castello godono di una conformazione definita e non sono soggetti a cambiamenti importanti, l'oggetto di studio dovrebbe essere le partecipazioni nazionali dei Padiglioni satelliti. Esse, infatti, non sono costanti, ma corrispondono a determinate politiche culturali, riflettendo l'ordine geo-politico ed economico mondiale (come dimostra Altavoces di Santiago Sierra), e non rispecchiano quindi solo la vecchia Europa e le vecchie potenze mondiali, come è invece il caso dei Giardini. Inoltre, il loro aspetto nomadico, diluito nella città di Venezia, e i loro tentativi di avvicinarsi ai Giardini, o entrare nell'Arsenale, oggi il secondo nucleo principale della Biennale, dimostra che non è sufficiente partecipare alla Biennale di Venezia con un Padiglione nazionale, il cui potere simbolico è ancora molto forte, ma che è necessario ubicarsi nelle immediate vicinanze dei Giardini [fig. 4] come se, con questo gesto, si volesse autolegittimarsi, dimostrando di 'essere all'altezza'.

Un esempio lo costituisce la partecipazione del Messico alla 56. Biennale di Venezia. Gli artisti Tania Candiani e Luis Felipe Ortega presentano Possessing Nature, una ricerca storica che sfocia in una proposta concettuale, la quale si vincola con la memoria idrica di due città anfibie, Città del Messico e Venezia, e che riflette il viaggio fatto dal Messico per arrivare all'Arsenale. L'opera si configura come una scultura d'acciaio che nasconde un dispositivo idraulico il quale aspira l'acqua dai canali veneziani, la fa transitare attraverso un canale interno alla scultura, la riversa in uno specchio d'acqua, per poi restituirla alla laguna. Nello specchio d'acqua si proietta un video che, alternando immagini del passato e del presente, mette in relazione la Città del Messico e Venezia (cf. Ponce-López 2015, 61-79). È importante segnalare che la forma della scultura corrisponde all'unione delle architetture che hanno ospitato il Messico dal 2007 (anno in cui ritorna a partecipare alla Biennale con un Padiglione nazionale dopo vent'anni di assenza $)^{18}$ al 2015. ${ }^{19}$ Possessing Nature è una testimonianza visiva dell'arduo viaggio intrapreso dal Padiglione messicano per avvicinarsi, progressivamente, ai Giardini di Castello ed entrare nell'Arsenale. ${ }^{20}$

Un altro esempio è la Spagna, Stato plurinazionale che in varie occasioni è ricorso alla struttura della Biennale di Venezia per affermare la sua plurinazionalità. In quest'occasione si riporteranno due esempi: il primo relativo a Euskadi (Paese Basco) ed il secondo relativo alla Catalogna.

18 Il Messico ha partecipato alla Biennale di Venezia nel 1950, 1952, 1958, 1968 ai Giardini di Castello e nel 1986 nell'Arsenale. Il saggio di Carolina Ruiz Nieto, nel presente volume, ricostruisce le prime partecipazioni del Messico ed indica l'esistenza di una storia ricordata e di una eclissata.

19 Palazzo Soranzo Van Axel (2007), Palazzo Rota Ivancich (2009 e 2011) e Chiesa di San Lorenzo (2013).

20 La concessione dello spazio avrà luogo fino al 2024. 


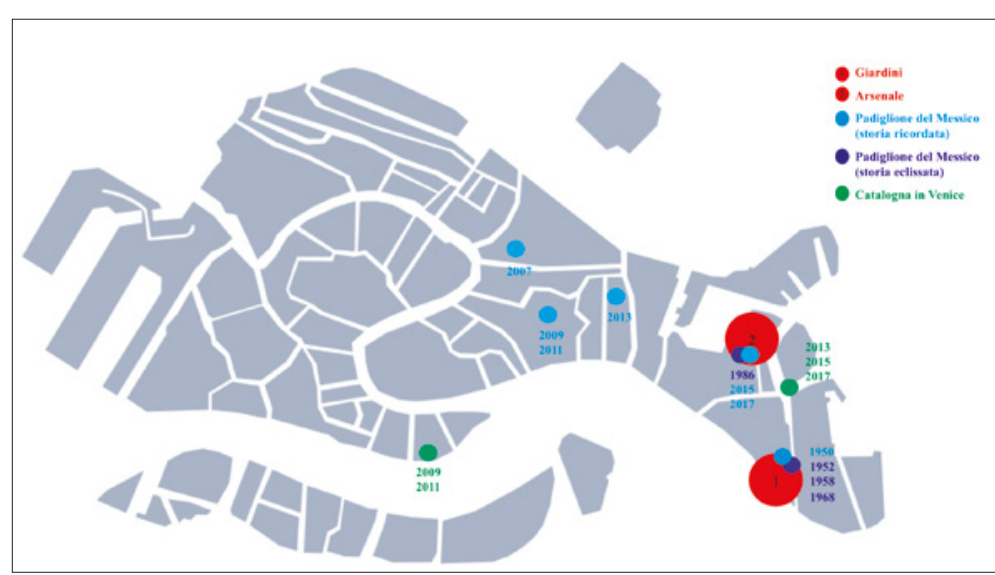

Figura 4 Ricostruzione della partecipazione del Messico (1950-2017) e della Catalogna (2009-2017) alla Biennale di Venezia (ricostruzione dell'Autrice)

Nel 1976, gli scultori baschi Jorge Oteiza ed Eduardo Chillida, invitati a partecipare alla mostra España. Vanguardia artística y realidad social: 1936-1976, rifiutano l'offerta ritenendo che le loro opere non si potessero concepire fuori dal contesto del popolo basco, reclamando così un proprio padiglione. ${ }^{21}$ Alla fine Euskadi ha abbandonato qualsiasi progetto espositivo e l'ha sostituito con una solitaria Ikurriña situata in uno spazio concesso dagli artisti italiani nel proprio Padiglione. Ciononostante, fuori dai Giardini sono stati prodotti atti che volevano ricordare l'identità del popolo basco (cf. Torrent 2013, 292-4).

Nel 2009 alla Biennale di Venezia si poteva visitare Miquel Barceló nel Padiglione nazionale, La comunitat inconfesable ai Magazzini del Sale, partecipazione catalana promossa dall'Istituto Ramon Lull, ed il Pabellón de la Urgencia. La sociedad del miedo nell'isolotto dell'Arsenale, promosso dalla regione di Murcia. La Spagna partecipava quindi con tre progetti, la consueta partecipazione nazionale nell'omonima sezione e due progetti nella sezione degli Eventi Collaterali. ${ }^{22}$ Se Pabellón de la Urgencia. La sociedad del miedo è un

21 Jesús Ceberio, «El País Vasco estará presente en la Bienal de Venecia», El País, 3 agosto 1976.

22 La Biennale di Venezia si divide in tre sezioni: l'esposizione del curatore, le partecipazioni nazionali e gli eventi collaterali. Le modalità di partecipazione sono differenti in ogni sezione. In linea generale, nel caso degli Eventi Collaterali bisogna inviare un progetto che deve risultare utile complemento alla Mostra e deve ricevere l'approvazione del curatore, il vaglio degli uffici della Biennale per l'aspetto organizzativo e, successivamente, deve essere sottoposto al Consiglio di Amministrazione della Biennale per l'ammissione definitiva. 
evento collaterale sporadico, promosso da istituzioni spagnole, come è stato il caso di Ofelias y Ulises. En torno al arte contemporáneo español $^{23}$ nel 2001, la partecipazione catalana corrisponde ad una rivendicazione identitaria e politica che si avvale del potere simbolico dei Padiglioni nazionali a Venezia. Tutt'altro che innocentemente, ci si riferisce a questa partecipazione con la denominazione popolare di Padiglione catalano ${ }^{24}$ e non con quella ufficiale che è «Catalogna in Venice ». Il Padiglione catalano partecipa, inoltre, al progressivo avvicinamento di molti Padiglioni satelliti ai due nuclei centrali della Biennale: se nelle 53. e 54. edizioni è ospitato nei Magazzini del Sale alle Zattere, dalla 55. si ubica in uno spazio nei Cantieri Navali di Castello, a pochi metri dall'Arsenale ed a pochi passi dai Giardini.

Per concludere, si ricorda come la curatela di Carlos Basualdo di Bruce Nauman: Topological Gardens, per la partecipazione degli Stati Uniti alla 53. Biennale di Venezia, ha fatto della struttura della Biennale di Venezia il suo punto di forza e non una debolezza. Il punto di partenza è stato interrogarsi se un'esposizione, grazie alla sua struttura, può aiutare il visitatore a stabilire una relazione tanto con le opere quanto con il contesto in cui si espongono. Nel caso specifico di una mostra che si prefigge di rappresentare un Paese, esponendo un'arte locale-globale in un'architettura nazionale, la sfida consiste nel riconoscere questa impossibilità ed integrarla, in modo produttivo, ad un evento il cui sistema arte-Padiglione-nazione ha creato sia gerarchie tra i Paesi partecipanti sia aree di maggiore o minore interesse e fruibilità (dentro e fuori o 'nei pressi' dei Giardini di Castello e l'Arsenale). Il curatore ha quindi deciso di articolare la mostra in tre spazi espositivi, tra il centro e la periferia: il Padiglione nazionale ai Giardini di Castello, Ca' Foscari Esposizioni ed il chiostro dell’Università IUAV nella sede dei Tolentini. Le tre sedi sono una manifestazione visibile della struttura della Biennale e, per mezzo delle sequenze concettuali (Heads and Hands, Sound and Space e Fountains and Neons), si vuole mostrare la logica topologica della produzione di Bruce Nauman e permettere al pubblico di avvicinarsi all'opera dell'artista americano mediante la sua esperienza nella città e viceversa (cf. Basualdo 2009, 188-92).

23 Ha avuto luogo agli Antichi Granai della Giudecca. Nel Padiglione nazionale si esponeva Viaje a Venecia, mostra curata da Estrella de Diego.

24 José Oliva, «Cataluña tendrá pabellón propio en la Bienal de Venecia de 2009», El Mundo, 15 octubre 2007; Anónimo, «El jurado del pabellón catalán en Venecia amaga con dimitir», El País, 31 de julio de 2009; Anónimo, «Cataluña por libre en Venecia», La Razón, 25 de marzo de 2009. 


\section{L'(in)adeguatezza delle discursive biennials}

Dora García è un'artista che nutre alcuni dubbi su quello che, al giorno d'oggi, è la Biennale di Venezia e quello che significa rappresentarvi una nazione. Reputa che «un artista è inadeguato per rappresentare un Paese, [e] un Paese non è adeguatamente rappresentato da un artista» (García 2011, 207), considerazione a cui si aggiunge il fatto che, nel momento della nomina, non viveva in Spagna da più di 20 anni. Conseguentemente, usa la sua sensazione di 'inadeguatezza', di difficoltà e scomodità, come materiale di lavoro, dando vita a Lo inadecuado, una performance che si prolunga durante tutti i sei mesi della 54. Biennale, che è il culmine di progetti affini realizzati anteriormente, con cui mette in discussione i parametri che caratterizzano una mostra.

Dora García trasforma il Padiglione in un laboratorio di idee ed esperienze. Al suo interno si svolgono le prime due parti del progetto, mentre la terza prende forma nella realtà virtuale di internet. Nell'edificio spagnolo non sono presenti né opere d'arte tangibili né indicazioni precise rispetto alle performance che avranno luogo. Le creazioni d'arte sono sostituite da «strumenti d'appoggio», «contenitori concettuali» (Del Rio 2003, 43), per eventi e performance che prenderanno forma durante la Biennale. Il pubblico che entra nell'edificio e visita le sale trova cartelli, bacheche, tavole, immagini e testi che non hanno, apparentemente, alcuna relazione tra di loro. Può sentirsi smarrito, non riuscendo a rintracciare un filo conduttore del discorso e interrogandosi, senza esito, su quale sia l'inizio e quale la fine, dato che non esiste né un inizio né una fine prestabilita. Gli «strumenti d'appoggio» si 'attivano', diventano oggetti utili, durante le performance, i discorsi e gli incontri ai quali partecipano attori ed esperti di arte contemporanea. Ogni giorno 'si attiva' una parte de Lo inadecuado per 'assopirsi' poco dopo e lasciare, l'indomani, spazio ad un'altra azione.

La seconda parte dell'intervento è Instant Narrative, una performance continua, una «dattilografia di eventi» (Brea 2008, 176). Consiste in un personaggio, seduto sulla piattaforma della sala centrale che scrive quello che vede e sente nel Padiglione. Gli scritti si proiettano su uno schermo nel quale si 'imbatte' lo spettatore in un momento determinato della visita. Egli può riconoscersi o meno nelle frasi proiettate, nello stesso modo in cui può aver catturato l'attenzione o essere passato inosservato alla persona che scrive al computer. Se egli si riconosce, Dora García ha raggiunto l'obiettivo desiderato: mettere a confronto due livelli di percezione, quella che una persona ha di se stessa e quella che hanno gli altri. Si stabilisce così una frontiera tra la realtà e la finzione, tra la presunta spontaneità e l'imposto, un distacco radicale tra oggettività e soggettività, per porre in discussione il grado di verità presente nella realtà. 
La terza parte di questo laboratorio di idee ed esperienze si materializza nel blog Contribute to The Inadequate: un diario collettivo in cui si può consultare un riassunto dettagliato di quello che succede e dove qualsiasi persona può intervenire per commentare le performance, partecipare virtualmente ai workshops o semplicemente raccontare la propria esperienza nel Padiglione.

Lo inadecuado è privo di un copione predefinito nei minimi dettagli. Si vuole intervenire in situazioni concrete e in un tempo reale, si definiscono quindi linee guida generiche ma mutanti che non permettono di prestabilire né la 'durata' del prodotto artistico, né l'interazione del pubblico. Ciò corrisponde con le linee di ricerca e di sperimentazione di Dora García: come lei stessa afferma, nel momento in cui ha accettato di rappresentare la Spagna alla Biennale di Venezia, ha accettato l'incarico di presentare il suo modo di lavorare ${ }^{25}$ nel Padiglione nazionale e non di essere la rappresentante dell'arte spagnola del momento.

La sua traiettoria artistica è scandita da importanti e significative partecipazioni a biennali internazionali come Sydney (2008), Istanbul (2009) e San Paolo (2010). Nei progetti presentati in questi eventi l'artista ha focalizzato la sua attenzione sulla marginalità dettata dall'esclusione, ${ }^{26}$ mentre a Venezia si concentra sull'inadeguatezza dell'inclusione e pone l'accento sull'analisi della condizione dell'artista in queste manifestazioni, sulla sua condizione di inadeguatezza, e usa tale sensazione come materiale di lavoro, creando un progetto assolutamente adeguato grazie al quale ci si interroga, implicitamente, sulla maggiore o minore adeguatezza del contesto in cui lo si realizza: la Biennale di Venezia in particolare e le altre biennali in generale. Di conseguenza, suscita, coscientemente o meno, una riflessione sull'adeguatezza delle biennali e del loro formato nell'epoca contemporanea, in un momento in cui le biennali stesse si sono rese auto-riflessive.

Dopo i primi cinquant'anni di attività della Biennale di Venezia, si incomincia ad assistere alla proliferazione delle biennali nei centri e ai margini del sistema dell'arte. ${ }^{27}$ Attualmente si contano tra due-

25 Dichiarazione dell'artista alla presentazione del progetto che ha avuto luogo nel Círculo de Bellas Artes de Madrid il 5 maggio 2011.

26 Si fa riferimento a The Deviant Majority: From Basaglia to Brazil (Biennale di San Paolo, 2010) e The Beggar's Opera (Münster Sculpture Projects, 2007).

27 La maggior parte della bibliografia pubblicata fino al momento indica che la proliferazione delle biennali d'arte contemporanea inizia negli anni Ottanta, conseguentemente alla Prima edizione della Biennale dell'Avana (1984). Questo dato, più che una verità assoluta, corrisponde alla storicizzazione eurocentrica del 'fenomeno biennale' Uno studio recente (Green, Garden 2016, 81-108) indica come vi sia stata una 'seconda ondata' di biennalizzazione nel Sud Globale tra gli anni Cinquanta e Ottanta (Biennale di Alessandria, 1955; Biennale di Arte Coltejer, 1968 e Biennale di San Juan, 1970). Studi in atto, e non ancora pubblicati, dell'autrice di questo saggio, suggeriscono l'esistenza di multipli centri di cultura, anteriori alla Biennale dell'Avana, in America Latina. 
cento e trecento biennali nel mondo, includendo sia quelle che hanno avuto una o poche edizioni, quelle che dopo un periodo di pausa hanno provato o stanno cercando di imporsi come evento artistico nella propria area geografia, sia quelle la cui longevità le ha rese le più conosciute, studiate ed attuali modelli di riferimento.

Dagli anni Cinquanta alla seconda metà degli anni Novanta, le biennali si sono affermate nel sistema dell'arte contemporaneo perché offrivano una nuova modalità di produzione, esposizione, circolazione e ricezione dell'arte e portavano, di conseguenza, un nuovo formato, sperimentando, influenzandosi reciprocamente e analizzando le proprie capacità e le rispettive zone d'influenza. Tra la seconda metà degli anni Novanta e la prima decade del Duemila si assiste alla cristallizzazione e fossilizzazione di questo 'nuovo' sistema dell'arte e all'eccessivo protagonismo dell'evento in sé, a discapito delle pratiche artistiche, tanto che si arriva ad affermare che «la biennale è il soggetto e l'arte è l'oggetto» (Paasche 2010, 20). Di fronte a queste presumibilmente ipotetiche ma sempre più tangibili e evidenti conseguenze, sorgono vari interrogativi: qual è il futuro delle manifestazioni? Sono un modello esaurito o hanno ancora qualcosa da offrire alla contemporaneità? La biennalizzazione dell'arte e la sua arte biennalista è un fatto irreversibile? Per ultima la domanda più logica, provocatoria, apparentemente scontata ma estremamente necessaria per un fenomeno che da tempo è globale, ma è ormai più omogeneo che eterogeneo: cos'è una biennale?

In un momento in cui le biennali hanno incominciato a ridefinire e legittimare il loro modello, non deve sorprendere la lista stilata da José Roca, in cui egli indica cosa debba essere una biennale puntualizzando ciò da cui deve allontanarsi: una biennale non è un museo, una biennale non è Documenta, una biennale non è né una mostra né una fiera d'arte e neanche una scuola d'arte e, per concludere, una biennale non deve essere solo una biennale (cf. Roca 2011, 18-25). Sebbene Massimiliano Gioni, nel saggio intitolato In Defense of Biennials (cf. Gioni 2013, 171-7), garantisca che una biennale non è né un modello né un formato, quanto uno strumento versatile che si può usare per ottenere i risultati più eterogenei, ogni biennale ha una propria forma e una propria struttura, seppure molte facciano riferimento, almeno in partenza, allo stesso modello. Se così non fosse, non si spiegherebbe l'attuale tendenza delle discursive biennials (Ferguson, Hoegsberg 2010, 361), vale a dire, l'analisi della propria storia e traiettoria per ridefinire la propria identità o lo studio minuzioso del fenomeno biennale prima di incominciare a definire il proprio modello. Em vivo contato (San Paolo, 2008) e To Biennial or Not Biennial? (Bergen 2009) sono un esempio di questa nuova tendenza.

Em vivo contato è il progetto curato da Ivo Mesquita e Ana Paula Cohen nella 28esima Biennale di San Paolo. Per mezzo dell'auto- 
analisi (la storia e traiettoria della Biennale di San Paolo a livello globale e nel contesto latinoamericano) si auspica di gettare le basi per rigenerare il proprio modello e di far scaturire una lettura critica e riflessiva sul biennalismo (cf. Barriendos, Spricigo 2009, 16-27). Per quanto concerne il primo punto, si lavora con l'Archivio Wanda Svevo, ${ }^{28}$ archivio storico che, prima dell'edizione in esame, conteneva solo documenti sulla storia della Biennale di San Paolo e del Museo de Arte Moderno ${ }^{29}$ e volumi di arte contemporanea. Visto il carattere riflessivo della 28esima Biennale di San Paolo si decide di incrementarne i fondi, per convertirla in un Centro di Documentazione sulle biennali ${ }^{30}$ ed invitare gli artisti partecipanti alla Biennale a sviluppare un progetto partendo da quanto conservato nell'archivio e trasformarlo in uno spazio di riflessione (cf. Mesquita, Cohen 2008, 22). Per quanto riguarda il secondo punto, sono stati organizzati una serie di incontri in cui si analizza la storia, il ruolo e il futuro della Biennale di San Paolo (The Bienal de São Paulo and the Brazilian artistic milieu: memory and projection e Backstage), il modello espositivo adottato da quest'ultima e da altre biennali per identificare tipi e categorie e studiarne l'evoluzione negli anni (Biennials, biennials, biennials...) ed il futuro delle pratiche artistiche contemporanee (History as a flexible matter: artistic practies and new system of reading) (cf. Mesquita, Cohen 2008, s.p.). Gli assi di queste conferenze (storia, pratica artistica e futuro) sono stati i medesimi attraverso i quali si articola il simposio To Biennial or Not to Biennial? ${ }^{31}$

Nel 2007 il comune di Bergen manifesta il desiderio di istituire una biennale d'arte contemporanea in Norvegia. Dopo aver consultato i professionisti della Bergen Kunsthall, si decide di sostituire la prima edizione con un simposio, in cui critici, artisti, curatori e storici dell'arte avrebbero analizzato, esposto e riflettuto sui temi 'topici' delle biennali e le problematiche che le caratterizzano. L'obiettivo è che nella nuova esposizione internazionale conviva sia un interessante ed innovatore contesto artistico sia il coraggio di parlare ed affrontare i problemi che emergono quando gli obiettivi della politica si scontrano con la libertà artistica. L'equilibrio possibile tra questi fattori sarebbe dovuto emergere dalla discussione.

28 Em vivo contato si è articolato in tre parti: l'agora, le plan libre e l'archivio Wanda Svevo.

29 Il Museo de Arte Moderno (MAM) è l'istituzione che promosse la Biennale di San Paolo dal 1948 al 1962, anno in cui si fondó la Fundação Bienal de São Paulo.

30 In occasione del simposio To Biennial or Not To Biennial?, l'Archivio Wanda Svevo è stato portato a Bergen ed esposto nella Bergen Kunsthall dal 17 settembre al 18 ottobre 2009 .

31 Il simposio ha luogo dal 18 al 20 settembre 2009. 
In soli tre giorni si ricorda il passato (dalle prime biennali alla proliferazione del formato); si analizzano le pratiche artistiche (i 'tipi' di biennali, la loro funzione, il ruolo del curatore e la tendenza ad adottare il modello della discursive biennial) e si conclude chiedendosi se, in un momento in cui la post-modernità è arrivata alla fine e una nuova era, la altermodernity, è stata dichiarata, queste esposizioni possano essere o no rilevanti. I relatori sottolineano che uno dei modelli emergenti si caratterizza per possedere un'approssimazione discorsiva (cf. Niemojewski 2010, 88-103; Ferguson, Hoegsberg 2010, 360-75), la quale stimola un re-thinking sul compromesso tra l'arte e la città sede dell'evento e la manifestazione artistica diviene una piattaforma che favorisce l'incontro ed il dialogo.

Tanto l'esperienza della 28esima Biennale di San Paolo quanto il simposio a Bergen sono chiari esempi delle discursive biennials, in molti casi caratterizzate da una forte autoreferenzialità che vincola le biennali al passato e le converte in prigioniere del presente. Solo quando si avrà accettato e processato l'esistenza di un 'vecchio stile' nella storia delle biennali, da cui distanziarsi mantenendolo però sempre presente, si potranno incominciare a liberare le biennali dalla prigione di 'contemporaneità antica' in cui molte si trovano attualmente. Fino a quando ciò non avviene, non bisogna sorprendersi se nei circoli d'arte ci si chieda: «Did Biennials Change Art?», affermando implicitamente che «la Biennale è il soggetto e l'arte l'oggetto» (Paasche 2010, 19-20), invece di interrogarsi su come l'arte possa cambiare una biennale o su come si possa cambiare una biennale utilizzando l'arte.

\section{Conclusione}

Il presente saggio è stato scritto con il proposito di presentare e analizzare alcuni dei progetti esposti nel Padiglione spagnolo nelle prime edizioni del Duemila e dimostrarne l'utilità per discutere l'obsolescenza o l'attualità delle partecipazioni nazionali alla Biennale di Venezia e per trattare brevemente la tendenza delle discursive biennials. Da una parte, si è cercato di fare chiarezza sulla potenzialità e contemporaneità di una struttura che è generalmente giudicata obsoleta a causa di letture superficiali e della disinformazione derivata da studi approssimativi e datati. On Translation: I Giardini ha permesso di dimostrare che la struttura della Biennale di Venezia è un utile strumento da un punto di vista geo-politico, economico ed identitario-nazionale tanto che è stata enunciata la mossa politica e culturale della Catalogna e la palpabile denuncia di Santiago Sierra. Dall'altra, si è fatto riferimento alla tendenza discorsiva di alcune biennali e come la prolungata auto-referenzialità possa derivare in 
studi e congressi ridondanti piuttosto che generare modelli più consoni per le realtà locale e la specificità temporale.

Invece di soffermarsi su quesiti la cui ridondanza stessa li ha resi retorici (La biennalizzazione dell'arte e la sua arte biennalista è un fatto irreversibile? Qual è la prima biennale d'arte contemporanea?) si considera opportuno allontanarsi dalla prospettiva eurocentrica e genealogica, quindi lineare, e studiare il fenomeno biennale da una pluralità di luoghi e tempi. Nel caso specifico di Venezia, si considera di grande utilità continuare con la ricerca iniziata da Muntadas, avendo essa dimostrato la potenzialità della struttura della Biennale. Difatti, siccome i Giardini godono ormai di una conformazione definita e non sono soggetti a cambiamenti importanti, l'oggetto di studio dovrebbero essere le partecipazioni nazionali dei Padiglioni satelliti. Esse infatti non sono pre-esistenti, riflettono il mutare dell'ordine geo-politico mondiale e, soprattutto, corrispondono a determinate politiche culturali. Inoltre, a livello globale, sono sempre determinate politiche culturali quelle che decidono di impiantare il modello biennale nella propria area geografica, con l'ambizione di incrementare la visibilità artistica (ma non solo) della città, della regione e del Paese. Si vuole quindi concludere con una domanda che connette la struttura nazionale della Biennale di Venezia internazionale con l'altrettanto internazionale fenomeno biennale: è possibile che il fenomeno biennale si rifletta nella Biennale di Venezia attraverso le partecipazioni nazionali?

\section{Bibliografia}

Albarrán, Juan (ed.) (2018). Art/nsición, Tran/nsición. Madrid: Brumania. Alonso, Rodrigo (2002). Muntadas con/texto. Buenos Aires: Ediciones Simurg. Alloway, Laurence (1968). The Venice Biennale 1985-1968. From Salon to Goldfish Bowl. Greenwich: New York Graphic Society.

Baravalle, Marco; Casavecchia, Barbar; Daneri, Anna; De Bellis, Vincenzo; Fabbris, Eva; Graziani, Stefano; Pietroiusti, Cesare; Roccasalva, Bruna (2011). «Pensieri intorno a un tavolo». García, Dora (ed.), Mad Marginal Cahier \#2: L'inadeguato/Lo inadecuado/The Inadequate = Catalogo della mostra (Venezia, Biennale di Venezia, Padiglione spagnolo, 4 giugno-27 novembre 2011). Berlin: Sternberg Press, 206-2019.

Barreiro López, Paula (2018). «Vanguardía artística y realidad social: una batalla por el significado del arte moderno». Albarrán, Juan (ed.), Art/nsición, Tra/nsición. Madrid: Brumania, 517-44.

Barriendos, Joaquín; Spricigo, Vinicius (2009). «Horror Vacui: Crítica institucional y suspensión (temporal) del sistema internacional del arte. Una conversación con Ivo Mesquita sobre la 28a Bienal de Sao Paulo». Ramona: revista de artes visuales», 95, 16-27.

Basualdo, Carlos (ed.) (2009). Bruce Nauman: Topological Garden = Exhibition Catalogue (Biennale, La Biennale di Venezia, Padiglione degli Stati Uniti, 7 giugno-22 novembre 2009). Philadelfia: Philadelphia Museum of Art. 
Bazzoni, Romolo (1962). 60 anni della Biennale di Venezia. Venezia: Lombroso. Bianchi, Mario (2006). I miei esordi saggististici. Padova: Il Glifo.

Bonet, Eugeni (2003). «Pabellón On Translation / On Translation Pavilion». Muntadas 2005, 383-429.

Bozal, Valeriano; Llorens, Tomás (eds). (1976) España. Vanguardia artística y realidad social: 1936-1976 = Catálogo de la exposición (Venezia, La Biennale di Venezia, 18 luglio-10 ottobre 1976). Barcellona: Gustavo Gili.

Brea, José Luis (2008). «Dora García». Olivares, Rosa (ed.), 100 artistas españoles. Madrid: EXIT publicaciones, 176-9.

Crespi, Giulia (2012). La Spagna alla Biennale di Venezia dal 1976 al 2009 [tesi di laurea magistrale]. Venezia: Università Ca' Foscari Venezia.

Del Rio, Victor (2003). «Dora García». Cimal. Arte Internacional, 56, 42-3.

Di Martino, Enzo (1995). La Biennale di Venezia 1895-1995. Milano: Mondadori.

Di Martino, Enzo (2013). La Biennale di Venezia 1895-2013. Venezia: Papiro Arte.

Di Martino, Enzo; Rizzi, Paolo (1982). Storia della Biennale 1895-1982. Milano: Electa.

Ferguson, Bruce; Hoegsberg, Milena (2010). «Talking and Thinking about Biennials: The Potential of the Discursive». Filipovic, Van Hal, Ovstebo 2010, 360-75.

Filipovic, Elena (2005). «The Global White Cube». Filipovic, Elena; Vanderlinden, Barbara (eds), The Manifesta decade: Debates on Contemporary Art Exhibitions and Biennials in Post-wall Europe. Cambridge (MA): MIT Press, 63-84.

Filipovic, Elena; Van Hal, Marieke; Ovstebo, Solveig (eds) (2010). The Biennial Reader. Ostfildern: Hatje Cantz Verlag.

Gioni, Massimiliano (2013). «In Defense of Biennials». Dumbadze, Alexander; Hudson, Suzanne (eds), Contemporary Art: 1989 to the Present. Oxford: Wiley-Blackwell, 171-7.

Green, Charles; Gardner, Anthony (2016). Biennials, Triennials, and Documenta. The Exhibitions that Created Contemporary Art. Hoboken: Wiley.

Jasso, Karla (ed.) (2015). Tania Candiani and Luis Felipe Ortega. Possessing Nature $=$ Catálogo de la exposición (Venezia, Biennale di Venezia, Padiglione del Messico, 9 maggio-22 novebre 2015). Ciudad de México: INBA.

Jones, Caroline (2010). «Biennial Culture: A Longer History». Filipovic, Van Hal, Ovstebo 2010, 66-87.

Lagnado, Lisette; Pedrosa, Adriano (2006). 27a. Bienal de São Paulo: Como Viver Junto = Catálogo da exposição (São Paulo, Bienal de São Paulo, 7 ottobre-17 dicembre 2006). São Paulo: Fundação Bienal.

Martínez, Rosa (2003). «La mercancía y la muerte». Martínez 2003, 14-25.

Martínez, Rosa (a cura di) (2003). Santiago Sierra = Catálogo de la exposición (Venezia, Esposizione Internazionale d'Arte, Padiglione spagnolo, 15 giugno-3 novembre 2003). Madrid: Dirección General de Relaciones Culturales y Científicas.

Martini, Martia Vittoria (2005). «Breve historia de I Giardini». Muntadas 2003, 205-33.

Marzo, Jorge Luis (2005), « Política cultural del gobierno español en el exterior (2000-2004)». Desacuerdos, 2, 57-121.

Medina, Cuauhtémoc (2003). «Aduana/customs». Martinés 2003, 214-51.

Mercader, Antoni (a cura di) (2000). MUNDATAS: Proyectos/Projects. Madrid: Fundación Telefónica.

Mesquita, Ivo; Cohen, Ana Paula (eds) (2008). Em Vivo contacto / In living contact $=$ Catalogo da exposição / Exhibition Catalogue (San Paolo, 18a Bien- 
nale di San Paolo, 26 ottobre-6 dicembre 2008). São Paulo: Fundação Bienal de São Paulo.

Mulazzani, Marco [1988] (2004). I Padiglioni della Biennale di Venezia. 2a ed. Milano: Electa.

Muntadas, Antoni (2005) (ed.). On translation: $i$ Giardini = Catálogo de la exposición (Venezia, Biennalde di Venezia, Padiglione spagnolo, 12 de junio-6 de noviembre 2005). Madrid: Dirección General de Relaciones Culturales y Científicas.

Niemojewski, Rafal (2010). «Venice or Havana: A Polemic on The Genesis of the Contemporary Biennial». Filipovic, Van Hal, Ovstebo 2010, 88-103.

Ofelias y Ulises (2001). Ofelias y Ulises. En torno al arte contemporáneo español = Catalogo della mostra (Venezia, Giudecca, Antichi Granai, 10 giugno-4 novembre 2001). Madrid: Ministerio de Asuntos Exteriores.

Orzes, Anita (2014). Il Padiglione spagnolo alla Biennale di Venezia: un'analisi critica dell'attività espositiva dal 2001 al 2013 [tesi di laurea magistrale]. Venezia: Università Ca' Foscari Venezia.

Paasche, Marit (2010). «The Cage of the Contemporary. On "Late Style" and Biennials». Filipovic, Van Hal, Ovstebo 2010, 19-22.

Ponce-López, Roberto (2015). «Lo que la laguna nos dejó. Cambio en el espacio urbano de México y Venecia». Jasso 2015, 61-79.

Roca, José (2011). «[duo]decálogo». Roca, José (ed.), Bienal do Mercosur. Ensaios de geopoética = Catálogo da exposição (Porto Alegre, Biennale del Mercosur, 10 setiembre-15 noviembre 2011). Porto Alegre: Fundação Bienal do Mercosul, 18-25.

Rubira, Sergio (ed.) (2018). Caso de Estudio. España. Vanguardia Artística y realidad social. 1936-1976 = Catálogo de la exposición (Valencia, IVAM, 13 setiembre 2018-13 enero 2019). Valencia: Institut Valencia d'Art Modern.

Spricigo, Vinicius (2011). Modos de rapresentaçao da Bienal de São Paulo. A passagem do internacionalismo artístico à globalização cultural. São Paulo: Hedra.

Stella, Alessandro (1913). Cronistoria della Esposizione Internazionale d'arte della città di Venezia 1895-1912. Venezia: Fabbris di S.

Torrent Esclapés, Rosalía (1988). La Bienal de Venecia. Datos para su historia. La participación española: el hito del 76 [tesi di dottorato]. Valencia: Universitat de Valencia.

Torrent Esclapés, Rosalia (1997). España en la Bienal de Venecia (1895-1997). Castellón: Servicio de Publicaciones de la Diputación.

Torrent Esclapés, Rosalia (2003). Un siglo de arte español en el exterior: España en la Bienal de Venecia 1895-2003. Madrid: Ministerio de Asuntos Exteriores.

Varotto, Eleonora (2018). Videoarte e arti performative: il caso del Padiglione spagnolo alla Biennale di Venezia dal 2001 al 2017 [tesi di laurea magistrale]. Venezia: Università Ca' Foscari Venezia.

Wigley, Marc (2005). «Conversación entre Antoni Munatdas y Mark Wigley, Nueva York / A conversation Between Antoni Muntadas and Mark Wigley, New York». On Translation: I Giardini (51st Venice Biennale). Madrid: Spanish Ministry of Foreign Affairs and Cooperation, 269-322. 



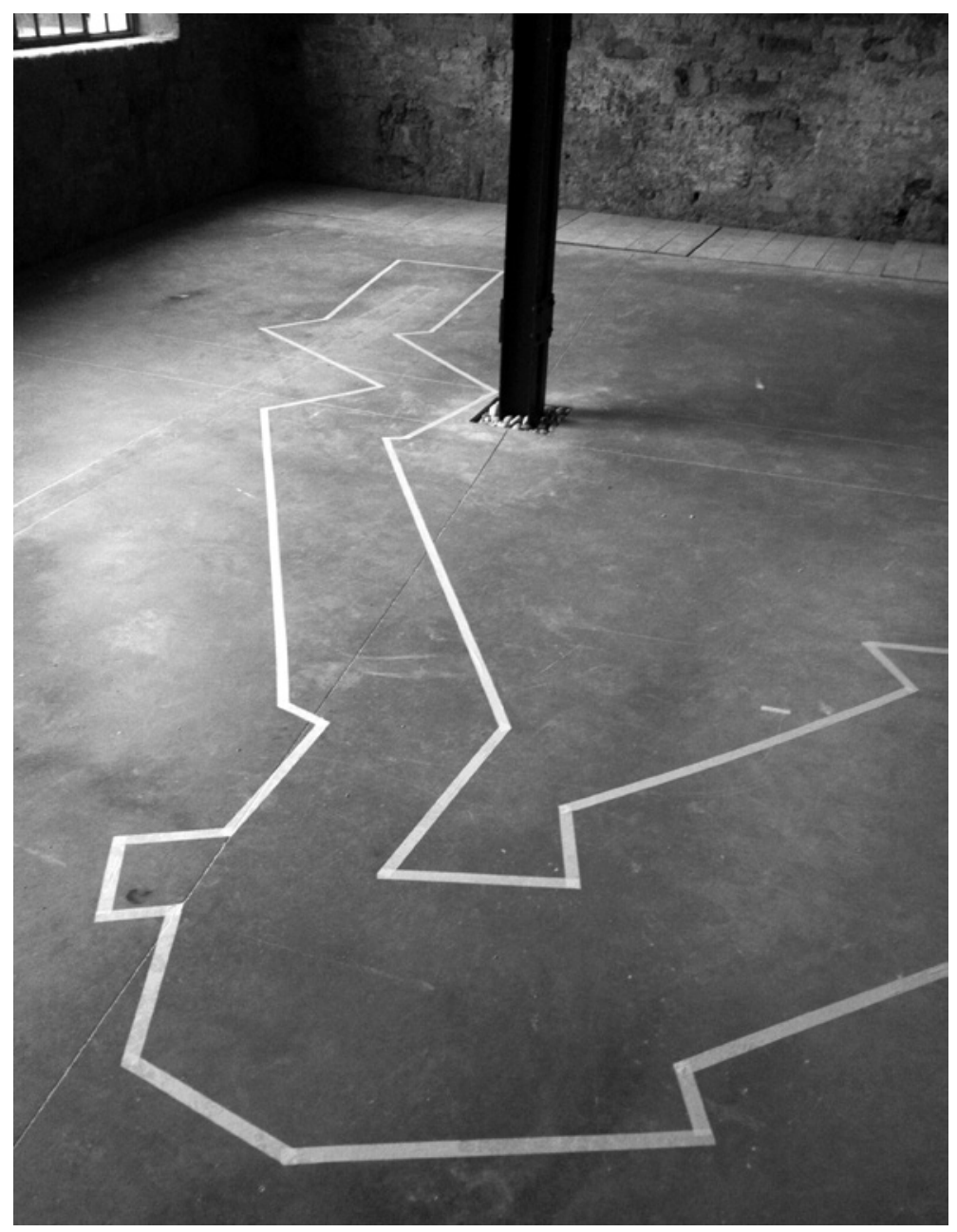

Figure 1 Tania Candiani and Luis Felipe Ortega, Possessing Nature, Pavilion of Mexico: Arsenale. Venice Biennale 2015 (press kit) 


\title{
Retelling the History of the Mexico Pavilion at La Biennale di Venezia
}

Carolina Nieto Ruiz

University of Washington, USA

\begin{abstract}
This chapter presents a revised history of the Mexico Pavilions in La Biennale di Venezia. Official discourse on Mexican Pavilions published in the twenty-first century contain significant inconsistencies and omissions about Mexico's twentieth century participations. These inaccuracies have been repeatedly published in the press, effectively rewriting history. I argue that a more complete historical narrative is necessary for a richer understanding of Mexico's contribution to the international art scene. First, I examine historical inconsistencies in exhibition publications from 2007-17. Next, I construct a narrative history of the Mexico Pavilions in three stages, in accordance with their aesthetics and contexts of production: post-revolutionary (1950, 1952, 1958, 1968), rupture (1986), and transnational (2007, 2009, 2011, 2013, 2017, 2019). Finally, I offer some concluding remarks and suggestions for further research.
\end{abstract}

Keywords Mexico Pavilion. La Biennale di Venezia. Historical revision. Twentieth century Mexican Art. Twenty-first century Mexican Art.

Summary 1 Introduction. - 2 Inconsistencies in the History of the Mexico Pavilion. - 3 Post-Revolutionary Nationalistic Mexico Pavilions: 1950, 1952, 1958, 196. - 4 The Rupture Pavilion: 1986. - 5 The Transnational Pavilions: 2007, 2009, 2011, 2013, 2015, 2017. -6 Concluding Thoughts.

\section{Introduction}

La Biennale di Venezia is the oldest and most important international art exhibition in the world. Since the first Biennale in 1895, it has become both a world-renowned international art institution and a critical tool for cultural diplomacy. The first national pavilion, belonging to Belgium, appeared in 1907 
(cf. Ventimiglia 1996). National pavilions have grown in number ever since, increasing to 29 in 1995, and to 70 in 2015.

The scholar Edgardo Bermejo Mora (2015) claims that when a nation presents a pavilion at La Biennale, it demonstrates economic stability and a strong interest in developing its artistic and cultural production. The process requires economic resources, tough negotiations, international logistics, promoting the event at home and abroad, and mediating the voices of many different actors without losing sight of the diplomatic importance of the event. For those involved, a national pavilion is a tremendous source of pride. In the case of Mexico, narratives of pride in the national pavilions are somewhat undermined by inconsistencies in the historical record of $\mathrm{Bi}$ ennale participation. Starting in 2007, official documentation of the Mexico Pavilions began to omit almost all of the twentieth century pavilions. This may be due to a lack of effort to collect, track, or verify this history. Instead, Mexican art historians have addressed individual pavilions as part of their work on specific artists, curators, or movements. ${ }^{1}$ In this chapter, I offer a more complete history of Mexican participation in international art exhibitions by shedding light on the repeated omissions of the Mexico Pavilions from official histories. First, I will examine historical inconsistencies in exhibition publications from 2007-17. Next, I will construct a narrative history of the Mexico Pavilions in three stages, in accordance with their aesthetics and contexts of production: post-revolutionary (1950, 1952, 1958, 1968), rupture (1986), and transnational (2007, 2009, 2011, 2013, 2017, 2019). Finally, I will offer some concluding remarks and suggestions for further research [tab. 1].

I gathered evidence for this work through archival research at the Archivio Storico delle arte contemporanee di Venezia in June, 2015; at Biblioteca de las Artes in Mexico City in May, 2016; using the online database of the International Center for the Arts of the Americas, created by the Museum of Fine Arts in Houston; and by reviewing the online digital archives and published materials of Mexico's National Institute of Fine Arts (INBA).

I want to thank my colleague K.C. Lynch from the University of Washington for her enormous help revising and editing the manuscript of this essay. In addition, the funding for this research project was provided by the Mexico's Consejo Nacional de Ciencia y Tecnología in 2015. This essays was created for the conference Storie della Biennale di Venezia, curated by Nico Stringa and Stefania Portinari (Venice, Ca' Foscari University, Ca’ Dolfin, 6-7 December 2016).

1 See: Carlos A. Molina (2013, 2014) and Alejandro Ugalde (2014) for the Mexico Pavilions from 1950 to 1968, García Gomez (2009) for the Mexico Pavilion in 1986 and Edgardo Bermejo Mora (2015) for the Pavilion of 2009. 


\section{Inconsistencies in the History of the Mexico Pavilion}

Founded in 1947, INBA has produced all of the Mexico Pavilions to date, and has been responsible for the selection of its contributors (Molina 2014). As Martínez Martínez (2008) states, in 1988 the INBA became part of the National Council for Art and Culture (CONACULTA), so catalogues from the Mexico Pavilion contain joint press releases from INBA and CONACULTA, including remarks from the directors of both institutions on how Mexico is honoured to be included in La Biennale. In spite of national pride, the published history of the Mexico Pavilions is incomplete. Mexico's presence at twentieth century Biennali has been largely omitted from twenty-first century INBA publications.

Mexico Pavilions have been featured at every Biennale since 2007. The same text, written by Mexico's curatorship team, appears in both the Mexico Pavilion catalogue for 2007 and in Mexico's section of the catalogue of the 52. Esposizione Internazionale d'Arte:

Mexico's first presence at the Venice Biennale was over 50 years ago at its 25th edition. Fernando Gamboa, a leading promoter of Mexican art and commissioner of the Mexico Pavilion, presented the works of Orozco, Rivera, Siqueiros and Tamayo, giving rise to what European critics called 'The revelation of Mexican painting' [...] The 52th International Art Exhibition of the Venice Biennale is a great opportunity for Mexico to reopen an institutional door for its contemporary art to the international forum. Achieving an active of Mexican artists on the world stage is a shared desire of Mexican society and its national cultural institutions. (Biennale 52 2007, 88)

The language of "first presence", juxtaposed with "opportunity... to reopen", implies that there was only one Mexico Pavilion prior to 2007 - effectively omitting five pavilions from the historical narrative. Mexican newspapers referencing these texts reproduced the error. For example, the cultural section of La Jornada published an article that states:

After more than 50 years, Mexico will officially participate for the second time at the Venice Biennale. ${ }^{2}$

The inaccuracy is even more pronounced in the official press bulletin for the 2007 Mexico Pavilion, which claims that it is Mexico's "first

2 Carlos Paul, "México vuelve a la Bienal de Venecia tras una ausencia de más de 50 años", La Jornada, 6 de junio de 2007. 
official national representation". ${ }^{3}$ This same inaccuracy occurred in 2009, where there were no specific mentions of earlier pavilions in the Mexico Pavilion catalogues, nor in La Biennale's catalogues of earlier national participations. However, the press bulletin for the 2009 Mexico Pavilion states:

Mexico presents its second official national representation at the 53rd International Art Exhibition, La Biennale de Venezia. ${ }^{4}$

This implies that the INBA is not considering any of the Mexico Pavilions of the twentieth century as an official national representation.

In the 2011 Mexico pavilion catalogue, an essay by Gastón Ramirez Feltrin states:

La primera aparición de México en la Bienal de Venecia se remonta a 1914 ... Lamentablemente, debido a las contradicciones y conflictos por los que pasaba el país, el arte mexicano perdió su presencia en Venecia por muchos años.

Durante la década de 1950 México participó en tres ocasiones, proyectando el arte mexicano en el ámbito internacional. Y en un largo paréntesis de casi medio siglo nuestro país participó cinco veces más; la últimas tres como invitado de las exposiciones del Instituto Italo-Latinoamericano. Es hasta el año 2007 que México cuenta finalmente con un pabellón nacional. (Ramirez Feltrin $2011,109)^{5}$

Despite his apparent concern over the 'lost presence' of Mexican art, Ramirez Feltrin completely omits the Mexico Pavilions of 1968 and 1986, and diminishes the Mexico Pavilions of the 1950s by referring to them only as "participations", comparable with the works of Mexican artists presented in collective international exhibitions within La Biennale. Additionally, Dario Ventimiglia recalls Mexico's participation in the 1914 Biennale, but not as a pavillion (1996, 76-7). Archival research corroborates this: there is no evidence or mention of a

3 ASAC, Mexican Pavilion at the 52nd International Art Exhibition, La Biennale di Venezia, June 2007.

4 ASAC, FS, AV: Biennale 2009, Mexican Pavilion.

5 "The first participation of Mexico in the Venice Biennale goes back to $1914 \ldots$ Unfortunately, due to the contradictions and conflicts that the country would go through, Mexican art lost its presence in Venice for many years. During the 1950s Mexico participated on three occasions... And in a long absence of almost half a century our country participated five times more; the last three as a guest of the exhibitions of the Italo-Latin American Institute. It is not until 2007 that Mexico finally has a national pavilion" (here and after: translations by the Author). 
Mexico Pavilion in any of the catalogues of the Esposizione Internazionale d'Arte della Città di Venezia before 1950. In 2013, INBA Director María Cristina García Cepeda states in the foreword for the Mexico Pavilion exhibition catalogue:

Mexican artists who have participated in the Biennale include figures of the stature of Diego Rivera, José Clemente Orozco, David Alfaro Siqueiros, Rufino Tamayo, Leonora Carrington, Fernando Leal Audirac, and Gabriel Orozco. Since setting up its own pavilion in 2007, Mexico has presented artists and works of great vitality. (García Cepeda 2013, 105)

García Cepeda omits the Mexico Pavilions from 1950-1986 by distinctively mentioning Mexican artists who participated as part of the Mexico Pavilion and that have presented in collective international exhibitions within La Biennale instinctively.

In 2014, INBA Director Maria Cristina García Cepeda signed a contract to place the Mexico Pavilion in the Arsenale. Mexico can make use of it to exhibit diverse cultural manifestations without having to invest in restoration and paying for maintenance, assembly, damage insurance, and other third parties expenses. ${ }^{6}$

In 2014, García Cepeda expressed pride because, "for the first time" the Mexico Pavilion would have a spot in one of the main venues, the Arsenale, and would keep it for the following two decades. ${ }^{7}$ In an interview for the newspaper La Jornada she said:

Me parece fundamental que México esté representado dentro del circuito de la Bienal. ${ }^{8}$

This new opportunity to occupy a privileged space was reiterated not only to mass media and specialized journals, but also within the 2015 exhibition itself. García Cepeda states in the foreword of the catalogue of the Mexico Pavilion of 2015:

Within this new space within the official circuit of the Biennale, we have confidence in increasing the visibility of the Mexican Pavilion. (García Cepeda 2015, 37)

6 Sonia Ávila, "México firma convenio por 20 años en la Bienal de Venecia”, Excélsior, 2 de junio de 2014.

7 Alejandra Ortiz, "México tiene un lugar en el circuito de la Bienal de Venecia por 20 años", La Jornada, 8 de junio de 2014.

8 "It seems fundamental for me that Mexico is represented within the main circuit of La Biennale". María Cristina Cepeda García entrevistada en Alejandra Ortiz, "México tiene un lugar en el circuito de la Bienal de Venecia por 20 años", La Jornada, 8 de junio de 2014 
In addition an important part of the artwork presented in 2015 was a cartographic retrospective of Mexico Pavilion sites, but only those from 2007-15 were included. Both the official discourse and the exhibition itself omitted all five twentieth century pavilions, four of which took place in The Giardini, the other main venue of La Biennale. In other words, the 2015 Mexico Pavilion was the first in the twenty-first century to be located in one of the two main areas (I Giardini and the Arsenale), but all of the twentieth century Mexico Pavilions shared that same honor.

The same omissions have been repeated in discourse around the 2017 Mexico Pavilion. In the Mexico Pavilion catalogue, INBA Director Lidia Camacho characterizes the pavilion as a commemoration of the first decade of Mexico's participation in La Biennale:

In 2017 Mexico celebrates the tenth anniversary with its own pavilion at La Biennale di Venezia. It was in 2007 that Rafael Lozano-Hemmer opened the doors of this venue to the arts community from Mexico, which gave him the opportunity to represent our country, through his outstanding artistic project to a broad audience. (Camacho 2017, 10)

The omissions have even been reinforced on the official webpage of the Mexico Pavilion, created by INBA and CONACULTA (https://bienaldevenecia.mx/es/), which includes only the Mexico Pavilions from 2007 to the present.

Knowing the importance of having a national pavilion in La Biennale, and the pride it brings to the state's cultural institutions, it is hard to believe that the individuals involved made these omissions and discrepancies on purpose. The more likely explanation is a lack of academic and institutional effort to verify the history. When scholars cite sources from 2007 onward, they are repeating the mistakes of their predecessors, reifying an inaccurate narrative. A more comprehensive history is needed. In the following sections, I bring together evidence from archival research to construct a historical narrative that includes all the Mexico Pavilions, organized in three phases that align with the context of their production.

\section{Post-Revolutionary Nationalistic Mexico Pavilions: 1950, 1952, 1958, 1968}

In the first four Mexico Pavilions presented in La Biennale di Venezia, the curatorial decisions and the aesthetic characteristics of the selected artworks were coherent, cohesive, and in accordance with the Mexican state's post-revolutionary nationalistic discourse, which was reinforced both internally and internationally. The aesthetic that came from this ideology became part of the imaginary of Mexican 
art that is still present in many visual products. The post-revolutionary nationalistic discourse of this period draws on multiple threads from Mexico's history. From 1884-1911, general Porfirio Diaz Mori served seven terms as President of Mexico, developing infrastructure, bringing in foreign capital, and keeping the peace through a dictatorship that benefited the bourgeoisie while increasing economic disparity between urban and rural populations. He also supported the production of eurocentric art, especially works inspired by the French (Tenorio-Trillo 1998). The collapse of the Diaz regime led to the Mexican Revolution in 1910, which, along with the 1917 Constitution, was seen as the victorious indigenist sequel to the War of Independence (1810-1821). Together, these events liberated Mexico from imperialism and its vestiges - first from Spain, then from the imperialist countries that invaded Mexico in the nineteenth century, and finally, from the French-inspired Porfirian ideals. Consequently, Mexico in the early twentieth century was seen as a direct descendant of the Revolution and its ideals: embracing Mexico's pre-Hispanic history, racial legacy, and rural and indigenous tradition - while eschewing European aesthetics (Aguilar Camín, Meyer 1993).

In the first half of the twentieth century, the state used this postrevolutionary discourse to try to unify the country by appealing to Mexican pride. In 1947, the Mexican state founded INBA, which particularly supported arts and exhibitions aligned with this discourse, letting them flourish (Luna Arroyo 1962). The post-revolutionary ideology was widely spread in cities through public art, especially murals. This period is known as Renacimiento Mexicano (Mexican Renaissance). Diego Rivera (1886-1957), José Clemente Orozco (1883-1949), David Alfaro Siqueiros (1896-1974), and Rufino Tamayo (1899-1991) were the leading artists (Ugalde 2014). The movement focused on artistic media that the government supported: paintings, and wood and linoleum engravings. Artists used traditional academic techniques, but their subjects were liberated from Eurocentric cannons, and instead used idealized images of pre-Hispanic history, rural peasant folklore, and popular historical images that spoke to the independent national Mexican spirit.

The images created during the Mexican Renaissance were bricks in the construction of the historic-aesthetic of Mexico. At the same time, these images integrated concepts and forms from the international avant-garde, thereby renewing the links between Mexican and western art. This movement became the seed of the Escuela Mexicana (Mexican School), which reiterated the same post-revolutionary subjects through the nuances of social realism until the end of the 1960s.

The first four Mexico Pavilions were presented within this political-artistic context, with artists and curators encouraged by postrevolutionary ideology. The pavilions were made during the political-economic bonanza called the 'Mexican Miracle' (1945-1970). They 
included artworks from the two preferred mediums, engraving and painting (Martínez Martínez 2008). The works and artists chosen were related to the aesthetic movement of the Mexican Renaissance and the Mexican School. Just two curators were responsible for choosing, assembling, and making presentation text for the art in all four pavilions: Fernando Gamboa (1950, 1952, 1968) and Miguel Salas Anzures (1956). Both were high officials of the newly formed INBA. In 1950, the first Mexico Pavilion debuted at La Biennale in an assigned space in The Giardini. It presented an exhibition curated by Fernando Gamboa, then Assistant Director of INBA. One of the most important twentieth century museographers in the history of Mexican art, Gamboa made great strides in presenting national art as a trans-historic continuum that linked pre-Hispanic art, Colonial art from 1500s1800 s, the nationalistic art of the 1800s, and post-revolutionary art. The result is an aesthetic discourse that constitutes a nationalist and indigenist image of Mexico, and has been reiterated in different venues inside and outside Mexico to this day (Molina 2013).

For the first Mexico Pavilion, Gamboa selected the best wellknown artists of the Mexican Renaissance, who also started the Mexican School. These artists were committed to the state's post-revolutionary ideology, giving them access to important mural commissions from the government. The exhibition held works by the four great Mexican muralists: Diego Rivera, José Clemente Orozco, David Alfaro Siqueiros, and Rufino Tamayo (Biennale 25 1950). As with all his curatorial work, Gamboa's exhibition also portrayed the indigenist face of Mexico by representing pre-Hispanic myths and scenes of indigenous and rural life - all with the decolonised, figurative, and colourful character of the aesthetics of the Mexican School. This exhibition was a success for Mexico and the visibility of Mexican Art worldwide. ${ }^{9}$

In 1952, Mexico was once again invited to participate in La Biennale with a pavilion at The Giardini, with Gamboa curating for the second time. He presented a collection of 134 engravings by twentyfive visual artists: Ignacio Aguirre, Carlos Alvarado Lang, Luis Arenal, Avelardo Ávila, Alberto Beltrán, Angel Bracho, Federico Cantú, Fernando Castro Pacheco, José Chavez Morado, Francesco Dosamantes, Jesús Escobedo, Arturo García Bustos, Andreina Gómez, Franco Gómez Lazaro, Manuel Manilla, Leopoldo Méndez, Francisco Mora, Isidoro Ocampo, José Clemente Orozco, Paolo O’Higgins, José Guadalupe Posadas, Everardo Ramírez, Diego Rivera, David Alfaro Siqueiros, and Alfredo Zalce.

Manuel Manilla and José Guadalupe Posadas were the earliest engravers of the group. They were from the same workshop, and gained wide recognition for their representations of death through cartoons

9 Anonymous, "México triunfa en Venecia", Tiempo: Semanario de La Vida Y La Verdad. Ciudad de México, junio de 1950. 
of skulls in a festive mood. Posadas also did political cartoons criticizing the Porfirio Diaz government and the gap between the privileged and disadvantaged peoples. Manilla died in 1895 and Posadas in 1913 - both before the end of the Revolution - but their work inspired many Mexican artists who were engaged with post-revolutionary discourse (Crespo de la Serna 1952).

The third Mexico Pavilion, in 1958, was curated by Miguel Salas Anzures, head of INBA's visual arts department. It was located inside the central palace of La Biennale. Salas Anzures presented a collective exhibition with 18 paintings by artists described as "social realists;" disciples of Rivera, Orozco, and Siqueiros; and heirs of Mexican muralism. These artists were: Raúl Anguiano, Jorge Gonzáles Camarena, Ricardo Martínez, Guillermo Meza, Carlos Orozco Romero, and Manuel Rodríguez Lozano (Biennale 29 1958).

The fourth Mexico Pavilion took place ten years later, in 1968, the same year in which the Olympics Games took place in Mexico City. Located in the central palace of La Biennale, the Mexico Pavilion was once again curated by Fernando Gamboa. It was a retrospective exhibition of Rufino Tamayo that included fifty paintings (Biennale 34 1968). According to Alejandro Uribe (2014), Tamayo's work during the 1920's, like the work of other abstract artists, did not receive support from state agencies that preferred works in the social realist style. Instead, Tamayo found economic support in the United States. The first exhibition of Tamayo's work in the United States was held at the Weyhe Gallery, New York, in 1926. His striking use of colour and the mystical way he represented Mexican folklore were key for his international success - and made the Mexican government take notice. In 1932, he received his first of many mural commissions in Mexico, to paint the National School of Music in Mexico City. He became the fourth great muralist, after Orozco, Rivera, and Siqueiros. In 1936 Tamayo moved to New York, and throughout the late thirties and early forties, the Valentine Gallery, New York, gave him shows. In 1948, Tamayo's first retrospective took place at the Instituto de Bellas Artes, Mexico City. The greatness Mexico finally saw in his work was later seen by the world at La Biennale in 1968.

\section{The Rupture Pavilion: 1986}

La Ruptura (The Rupture) was an artistic movement in Mexico that started in the 1950s. It included artists from different disciplines who were not favored by the state because their work was not aligned with the post-revolutionary nationalistic artistic enterprise. The movement was not formally recognized until 1988, after an art exhibition presented in the Museum Carrillo Gil called Ruptura 1952-1965 recuperated visual works from artists who criticized, challenged, at- 
tacked, and distanced themselves from the nationalist hegemonic discourse supported by the main figures of the Mexican School and its followers (Del Conde 2014).

The members of the Rupture movement did not have a cohesive aesthetic style, and they were supported by private organizations and the art market. These artists appreciated the work of the muralists, especially the adventurous art of Rufino Tamayo, as well as new developments in the European and American Art scenes. They were critical of the bureaucratic dictatorship and the 'official art' that was supported and exhibited nationally and internationally by members of the Mexican School that mimicked the themes and style of the muralists. The artists of the Rupture movement argued that even when the government patronage made life and artistic work easier, the cost of limiting the development of personal creativity and style was too high (Del Conde 1979).In 1970 the economic bubble in Mexico broke, and post-revolutionary ideology lost its power. Artists from the Rupture movement who had been excluded from the institutional circuit in Mexico started appearing in official venues This was especially true for those who found recognition and better markets for their artworks outside Mexico.

Throughout the 1970s, the Mexico Pavilion was absent from La Biennale. It reappeared in 1986, the same year that the World Cup was held in Mexico, thereby affording Mexico another opportunity for increased visibility in the international arena. The curator Sara Bolaño was in charge of presenting the Mexico Pavilion in the Arsenale. This was the first time Mexico presented artists that were not attached to the Mexican School. The exhibition included 10 engravings and one painting by Manuel Felguerez, and 10 paintings by Raymundo Sesma. As part of the Rupture movement, neither artist was supported by the state in their early careers; their work was too 'abstract' and therefore unable to convey the post-revolutionary ideology relied on so heavily by the social realists. However, they found good markets for their work in Europe, particularly in France and Italy. When the Mexican government later declared itself to be open to art outside of the Mexican School, Felguerez, and Sesma got support and recognition inside Mexico (García Gomez 2009).

In the catalogue of the XLII Esposizione Internazionale La Biennale di Venezia, Bolaño includes text written by 1990 Nobel Laureate Octavio Paz as the foreword of the Mexico Pavilion. ${ }^{10}$ Paz states that:

Il nuovo muralismo di Felguérez ha rotto con la tradizione della scuola messicana... Gli anni di consolidamento del regime nato dalla rivoluzione messicana (1930-1945) furono anche quelli della gra-

10 This essay was published for the first time in the catalogue of the exhibition Espacio Multiple [Multiple Space] at the Museo de Arte Moderno in Mexico City in 1973 (1-7). 
duale divisione delle correnti universali nella sfera dell'arte e della letteratura. Alla fine di questo periodo il paese tornò a chiudersi in sé stesso ed il movimento artistico e poetico, originariamente fecondo, degenerò in un nazionalismo accademico, non meno asfissiante e sterile dell'europeismo di Porfirio Diaz. I primi a ribellarsi furono i poeti e, quasi immediatamente, seguirono i romanzieri ed i pittori. Tra il 1950 ed il 1960 la generazione di Felguérez... inizió un lavoro di pulizia estetica e mentale. (Paz in Biennale 42 1986) ${ }^{11}$

This text is representative of the commonalities of the Rupture movement: criticizing the constraints of the hegemonic Mexican School and praising the work of Felguerez as being able to break away from it. After the four first pavilions that proudly supported and reinforced the aesthetic of the social realism reproduced by the Mexican School, the Mexico Pavilion of 1986 presented the result of its decline as dominant style.

\section{The Transnational Pavilions: 2007, 2009, 2011, 2013, 2015, 2017}

After another long absence of 20 years, the Mexico Pavilion reappeared in 2007. Since then, the Mexico Pavilion has been present at each consecutive Biennale. These are the 'transnational' pavilions because they share common characteristics with what Nestor García Canclini (2014, 21) calls the globalized art system of the twenty-first century.

While the twentieth century Mexico Pavilions attempted to represent a national Mexican artistic identity, the twenty-first century translational pavilions have worked to create cross-border relations and multicultural alliances. This change is also reflected in the presentation texts. Exhibitions prior to 2007 describe a Mexican cultural essence. From 2007 on, supporting texts explain how the exhibitions contribute to the global art system, highlighting concepts that relate to social and cultural concerns across nations.

The exhibitions in the twentieth century were curated by INBA officials. In the twenty-first century, INBA has had a more managerial role, organizing a contest with an external jury. Curators submit pro-

11 "Felguérez's muralism broke with the tradition of the Mexican School... A gradual division of the universal movements in the art and literature spheres occured in the years when the political regime that was born from the Mexican Revolution was consolidating (1930-1945). At the end of this period [Mexico] closed itself again and the artistic and poetic movement... degenerated into an academic nationalism, none less suffocating and sterile than Porfirio Diaz Europeism. The first who rebelled were the poets followed almost immediately by the novelists and the painters. Between 1950 and 1960, Felguerez's generation... started an aesthetic and mental cleaning". 
posals, and the winning project becomes the Mexico Pavilion for La Biennale. This effectively shifts power away from the Mexican state and towards the curators, who can work with artists to bring their transnational concerns and identity to the exhibition.

The artists and curators chosen for the transnational Mexico Pavilions have lived, worked, and presented their art in different nations. This is manifested in the way they fluidly incorporate codes from different cultures, rework their local traditions, insert them significantly into a transnational exchange, and sell their work in cities around the world. Additionally, transnational art enhances what Arthur Danto (2009) calls post-historic art, in which painting is no longer the main platform as it was in the first half of the twentieth century. Instead, transdisciplinary practices prevail; it is no longer necessary for the artist to work solely in one medium. While all the twentieth century Mexico Pavilions presented painting and engraving, all the twenty-first century exhibitions have embraced multimedia approaches, including installation, interactive art, performance, object art, video, photography, and sound art.

In 2007, officials from La Biennale and INBA agreed that the Mexico Pavilion would be held in the gothic Soranzo Van Axel Palace, one of the venues outside of the main circuit of La Biennale. The pavilion presented the exhibition Algunas cosas pasan más veces que todo el tiempo (Some things happen more times than all the time). Curated by Priamo Lozada and Bárbara Perea, the show included six art installations created by the Mexican artist Rafael Lozano-Hemmer.

Lozano-Hemmer is a transnational artist: he has lived, studied, worked, and presented his work in different countries. Raised in Mexico, he studied physics and chemistry at Concordia University in Montreal, Canada, where he has lived since 1990. In his career as a visual artist, he explores science and technology through interactive installations; kinetic sculptures; and public space interventions using video projections, sound, photographs, internet connections, and sensors. Perhaps the highlight of his works in the 2007 Mexico Pavilion, the installation Almacén de Corazonadas (Pulse Room) consists of a hundred light bulbs that turn on and off according to the heartbeat of different spectators as they touched two sensors. ${ }^{12}$ The exhibition aimed to generate an embodied experience around

the paradoxical phenomenon found in certain scientific fields, such as quantum physics, where prediction and uncertainty models have proven the existence of behaviours that happen more often than one hundred percent of the time. (Lozada, Perea 2007)

12 Germaine Gómez Haro, "México en la Bienal de Venecia”, La Jornada Semanal, 2 de septiembre de 2007. 
Instead of any attempt of portray a national identity, the curators and the artist presented a universal concept pulled from physics.

The 2009 Mexico Pavilion held an exhibition titled ¿De qué otra cosa podríamos hablar? (What else could we talk about?), with work of the Mexican artist Teresa Margolles. The show was curated by Cuahutemoc Medina and situated in the Renaissance building of the Rota Ivancich Palace, a space built in the 16th century, located near to St. Mark's Square and out of the main circuit of La Biennale. Margolles' work comments on the thousands of deaths that occured in Mexico in relation to the war against narcotraffic, which began in 2007 under President Felipe Calderón. ${ }^{13}$ In the installation Lemas (Mottos), a set of sheets is completely stained with blood collected from the floor of a 2008 violent crime scene is embroidered with phrases related to drug-violence in golden thread. In the performance Limpieza (Cleaning), relatives of people who were murdered in drug trafficking conflicts mop the floor of the exhibition space with a mixture of water and fluids from the morgue. Margolles and Medina requested that the exhibition space not be cleaned, so the surrounding decay became part of the artworks, adding to the spectator's sense exposure to repulsion, hatred, and pain. ${ }^{14}$ In the catalogue for La Biennale 2009, Medina frames these artworks with a transnational appeal:

Margolles' work carefully balances the fear of contamination, the social need for political awareness, to a crisis which is yet another of the faces of globalization. The works presented at the Mexican Pavilion are subtle chronicle of the effect of a devilish international economy: the vicious circle of prohibition, addiction, accumulation, poverty, hatred, and repression that transmogrifies the transgressive pleasures and puritan obsession of the North into the South as Hell. (Biennale 53 2009, 90)

Here Medina presents the exhibition as a political piece, criticising the socio-economic inequalities that negatively affect nations around the world. It connects the Mexico Pavilion with broader problems occurring in the transnational scene.

13 There was a controversy around this exhibition. As it was a direct critique to the President decision, and portrayed a negative image of Mexico, the Secretary of Foreign Affairs (SRE) attempted to stop the INBA to produce it. The SRE even cancelling funds had been destined to support the pavilion was going to had. The decision of the jury and cultural public opinion, which generally leans towards a liberal-progressive position, won the battle, the exhibition was presented, portraying an idea of Mexican State as open to critic and freedom of speech (Villanueva Rivas 2014).

14 Alejandra Ortiz Castañeda, “Por la violencia, 'México es un país que llora': Teresa Margolles", La Jornada, 11 de junio de 2009. 
In 2011, Melanie Smith was selected to exhibit her work for the Mexico Pavilion at the Rota Ivancich Palace. She is an intercultural border-crossing artist: she is British, has lived in Mexico since 1989, and became a naturalized Mexican citizen in 2006. The exhibition titled Cuadro Rojo, Imposible Rosa (Red Square, Impossible Pink), included an installation, expanded paintings, and a short version of three of Smith's videos: Azteca Stadium: malleable prowess (2010), Xilitla (2010), and Bulk (2011). The curator of the exhibition, Jose Luis Barrios (2011), situates Smith's videos in discourse on the globally fractured concept of modernity. He states that Smith's analyzes the way in which modernities are placed outside the hegemonic power and how they are interpreted outside this space that we consider modernity (Barrios 2011, 15-21).

In other works for the pavilion, Smith incorporated disaggregated images from Latin American social symbols, in order to question the political condition of modernization by creating the kind of disjointed narratives that post-colonized nations share.

The 2013 Mexico Pavilion exhibition was presented in the former San Lorenzo Church and curated by Itala Schmelz. Under the title Cordiox, it consisted of one piece from the artist Ariel Guzik, whose work has been shown internationally. Guzik is a musician, scientist, and visual artist who has dedicated three decades of research to creating devices that can detect sound waves imperceptible to the human ear and translate them into audible sound. His goal has been to be able to hear and communicate with plants, and animals, with a special focus on marine life. Cordiox is a complex, four-meter tall machine that uses a quartz system sensitive to electromagnetic fields and converts those fields into sound waves. The acoustics of the church made the space function as a resonance box into which the spectators could penetrate and be surrounded by the unrecognizable sound. ${ }^{15}$ The Mexico Pavilion catalogue of 2013 states that Guzik's work:

poses a science without pragmatic ends which explores the living planet... it fosters and enriches a debate that is currently on the agenda worldwide. As the development of our species has become a serious threat to nature, contemporary individuals have detached themselves, through urbanization, from their natural surroundings. In the face of this situation, Guzik invites us to reestablish a dialogue with the planet. (Schmelz 2013, 111)

The unrepeatable and uncomparable experience of hearing and moving through the sound of the building was the strength of the piece. As this quote shows, the piece also appealed to global concerns as the other transnational exhibitions do. 
The 2015 Mexico Pavilion also presented a one-piece exhibition titled Possessing Nature. It was curated by Karla Jasso and created jointly by the Mexican neo-conceptual artists Tania Candiani and Luis Felipe Ortega, who have worked and shown in a number of countries around the globe. Both artists work with installations, machines, interventions, and videos regarding the relation between artefacts, language, culture, and space. For the Mexico Pavilion, their work aimed to show different relations between Venice and Mexico City as "amphibian" lake cities (Jasso 2015). The installation pulls associations with drainage and lagoons into conversation with the emplaced history of Mexico Pavilions. It consists of a three-meter high metallic structure which looks like a serpentine wall in an irregular and angled zig-zag, and works as a hydraulic artefact. Water brought from Venice's lagoon circulates over the wall in a narrow canal; the running water fills the space with its sound. At one end of the structure, a contained pool of water lays on the floor, making a screen. Black and white photographs from Mexico City and Venice are projected onto the surface, overlapping to enhance the similarities between the two cities. The path of the wall over the floor traces a cartographic retrospective of the places where the Mexico Pavilion has been presented from 2007-15. In La Biennale, Possessing Nature provided a critical view of power dynamics in the history of urbanism, which is shared globally in westernized cities.

In 2017, the Mexico Pavilion presented the exhibition La vida en los pliegues (The Life in the Folds) by the artist Carlos Amorales, curated by Pablo León de la Barra. Amorales' work combines the imaginaries of contemporary subculture, traditional crafts, popular culture, and conceptual art. Carlos Amorales lives in Mexico, studied in Amsterdam in the Rijkskasacademies Van Beeldende Kunsten (1992-1995) and in the Gerrit Rietveld Academie (1996-1997), and has completed artistic residencies in France and the United States (León de la Barra 2017). The exhibition consisted of a set of artefacts related to a code created by Amorales. Each letter of the alphabet corresponds to the abstract shape of a black ocarina, a small wind instrument, and the sound it produces. The ocarinas are displayed over white tables as texts, and the tables are surrounded by a set of images and a video called The cursed village. The video uses black paper figures and shadow theatre to tell a story of a family of immigrants who were lynched. The characters 'speak' the code of the ocarinas' sound. The video has subtitles for the dialogue that is displayed using the visual code of the abstract shapes. In the wall text, Amorales explains:

I believe we are in a time when it is crucial to discuss freedom of thought if we want... understanding of equality and justice. I've research the way in which writing is encrypted... as a strategy to preserve contents that would be silenced if they were in legible 
Carolina Nieto Ruiz

Retelling the History of the Mexico Pavilion at La Biennale di Venezia

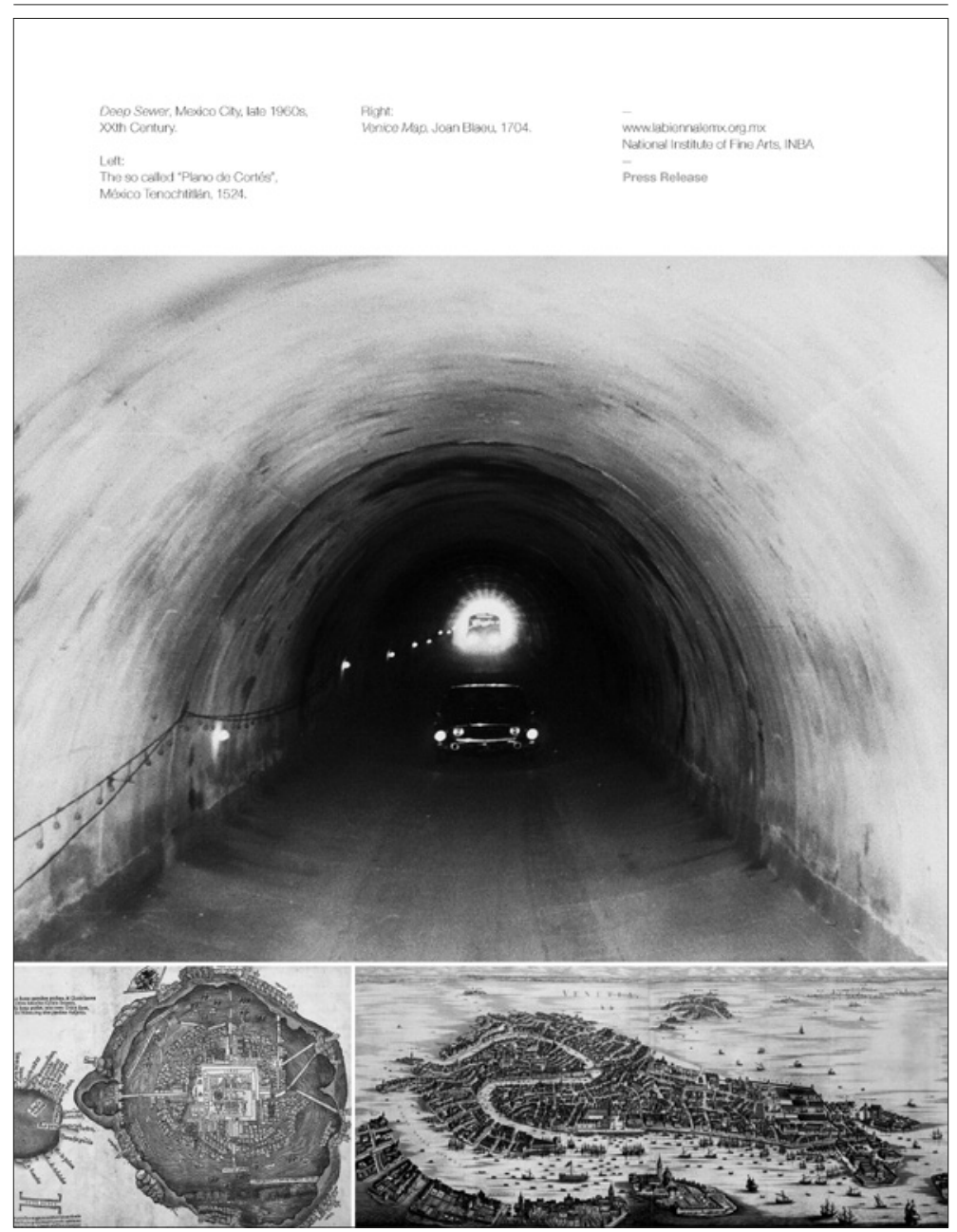

Figure 2 Tania Candiani and Luis Felipe Ortega, Possessing Nature, Pavilion of Mexico at the 56th International Art Exhibition of La Biennale di Venezia, 2015 (press kit) 
form... In this piece, images of migration and lynching are metaphors of a generalized crisis that we need to discuss. On the one hand migration is the consequence of current economic policies... On the other murders... carried out by citizens and private militias... where people administer justice subjectively. ${ }^{16}$

Amorales presents her work as able to speak on and critique global economic disparities and incoherences. It is a relational piece that is tied to a wider argument about global social practices.

\section{Concluding Thoughts}

In this chapter, I have attempted to do a revision to the history of the Mexico Pavilion in La Biennale di Venezia. I did this by first, supplying evidence that discrepancies and omissions about the history of the Mexico Pavilion have been published and reiterated since 2007 up to today; then, by presenting the results of an investigation that clarify this history, through a narrative that groups the Mexico Pavilions according to the commonalities of their exhibitions, and situates each group within the Mexican Art context.

Mexico Pavilions fall into three categories: post-revolutionary nationalist (1950, 1952, 1958, 1968), rupture (1986), and transnational (2007-17). The first group is characterized by the inclusion of artists from the Mexican School who were supported by the Mexican state. As part of the state's ideological enterprise, artworks embraced the aesthetics of social realism to create a unified mexican identity - one that celebrated the ideals of the 1910 Revolution and a common preHispanic ethnicity. The second group included abstract, bidimensional works by artists who rejected the hegemony of the Mexican School and could not be nationally recognized until the decline of the post-revolutionary state. The third group of Pavilions is shaped by consecutive appearances in La Biennale, each presenting exhibitions less interested in presenting a national art identity. The artists in these pavilions are already part of the transnational art circuit, and use non traditional media to discuss concepts that speak to concerns about the globalized world.

This chapter presents a stable ground for future research about the Mexico Pavilions. I hope that this work will help recognize the ef-

16 This quote was taken from the text wall of the exhibition La vida en los pliegues [Life in the folds] curated by Pablo León de la Barra with the artwork of Carlos Amorales, and presented in the Mexico Pavilion at La Biennale di Venezia (2017). The text was signed by the artist. 
forts involved in the exhibitions of the twentieth century that have been omitted reiteratively in the twenty-first century. These omissions inadvertently diminish the strength and appreciation that Mexican Art has had in the international scene. Moreover, we cannot truly understand the evolution of themes, aesthetic values, and artistic movements that have represented Mexican Art to the world if we do not acknowledge the complete historical record of the Mexico Pavilions in La Biennale di Venezia. 
Carolina Nieto Ruiz

Retelling the History of the Mexico Pavilion at La Biennale di Venezia

Table 1 Schematic representation of the chronology of the presence of the Mexico Pavilion in the Esposizione Internazionale D'Arte la Biennale di Venezia. It includes for each pavilion its year, location, curator, exhibition title, and artist that were presented.

\begin{tabular}{|c|c|c|c|c|c|}
\hline & Year & Location & Curator & Exhibition Title & Artists \\
\hline \multirow{4}{*}{$\begin{array}{l}\text { Post- } \\
\text { revolutionary } \\
\text { nationalistic } \\
\text { pavilion }\end{array}$} & 1950 & I Giardini & $\begin{array}{l}\text { Fernando } \\
\text { Gamboa }\end{array}$ & - & $\begin{array}{l}\text { Diego Rivera, José Clemente Orozco, } \\
\text { Rufino Tamayo }\end{array}$ \\
\hline & 1952 & I Giardini & $\begin{array}{l}\text { Fernando } \\
\text { Gamboa }\end{array}$ & - & $\begin{array}{l}\text { Ignacio Aguirre, Carlos Alvarado Lang, } \\
\text { Luis Arenal, Avelardo Ávila, Alberto } \\
\text { Beltrán, Angel Bracho, Federico Cantú, } \\
\text { Fernando Castro Pacheco, José Chavez } \\
\text { Morado, Francisco Dosamantes, Jesús } \\
\text { Escobedo, Arturo García Bustos, Andreina } \\
\text { Gómez, Franco Gómez Lazaro, Manuel } \\
\text { Manilla, Leopoldo Méndez, Francisco } \\
\text { Mora, Isidoro Ocampo, José Clemente } \\
\text { Orozco, Paolo O'Higgins, José Guadalupe } \\
\text { Posadas, Everardo Ramírez, Diego Rivera, } \\
\text { David Alfaro Siqueiros, and Alfredo Zalce }\end{array}$ \\
\hline & 1958 & $\begin{array}{l}\text { Padiglione } \\
\text { Centrale } \\
\text { La Biennale, } \\
\text { i Giardini }\end{array}$ & $\begin{array}{l}\text { Miguel Salas } \\
\text { Anzures }\end{array}$ & - & $\begin{array}{l}\text { Raúl Anguiano, Jorge Gonzáles } \\
\text { Camarena, Ricardo Martínez, Guillermo } \\
\text { Meza, Carlos Orozco Romero, and Manuel } \\
\text { Rodríguez Lozano }\end{array}$ \\
\hline & 1968 & $\begin{array}{l}\text { Padiglione } \\
\text { Centrale } \\
\text { La Biennale, } \\
\text { i Giardini }\end{array}$ & $\begin{array}{l}\text { Fernando } \\
\text { Gamboa }\end{array}$ & - & Rufino Tamayo \\
\hline $\begin{array}{l}\text { The Rupture } \\
\text { Pavilion }\end{array}$ & 1986 & Arsenale & Sara Bolaño & - & Manuel Felguerez, Raymundo Sesma \\
\hline \multirow{6}{*}{$\begin{array}{l}\text { Transnational } \\
\text { Pavilions }\end{array}$} & 2007 & $\begin{array}{l}\text { Palazzo } \\
\text { Soranzo Van } \\
\text { Axel }\end{array}$ & $\begin{array}{l}\text { Priamo } \\
\text { Lozada and } \\
\text { Barbara Perea }\end{array}$ & $\begin{array}{l}\text { Algunas cosas pasan } \\
\text { más veces que todo el } \\
\text { tiempo. [Some Things } \\
\text { Happen More Often } \\
\text { Than All of the Time] }\end{array}$ & Rafael Lozano-Hemmer \\
\hline & 2009 & $\begin{array}{l}\text { Palazzo } \\
\text { Rota } \\
\text { Ivancich }\end{array}$ & $\begin{array}{l}\text { Cuauhtémoc } \\
\text { Medina }\end{array}$ & $\begin{array}{l}\text { ¿De qué otra cosa } \\
\text { podríamos hablar? } \\
\text { [What else can we talk } \\
\text { about?] }\end{array}$ & Teresa Margolles \\
\hline & 2011 & $\begin{array}{l}\text { Palazzo } \\
\text { Rota } \\
\text { Ivancich }\end{array}$ & $\begin{array}{l}\text { Jose Luis } \\
\text { Barrios }\end{array}$ & $\begin{array}{l}\text { Cuadro rojo impossible } \\
\text { rosa [Red Square } \\
\text { Impossible Pink] }\end{array}$ & Melanie Smith \\
\hline & 2013 & $\begin{array}{l}\text { ex-chiesa di } \\
\text { San Lorenzo }\end{array}$ & Itala Schmelz & Cordiox & Ariel Guzik \\
\hline & 2015 & Arsenale & Karla Jasso & Possessing Nature & Tania Candiani and Luis Felipe Ortega \\
\hline & 2017 & Arsenale & $\begin{array}{l}\text { Pablo León } \\
\text { de la Barra }\end{array}$ & $\begin{array}{l}\text { La vida en los pliegues } \\
\text { [Life in the folds] }\end{array}$ & Carlos Amorales \\
\hline
\end{tabular}




\section{Bibliography}

Aguilar Camín, Héctor; Meyer, Lorenzo (1993). A la sombra de la Revolución Mexicana. Texas: Cal y Arena.

Barrios, Jose Luis (ed.) (2011). Melanie Smith: Cuadro Rojo imposible rosa = Catálogo de la exposición (Pabellón de México, 56. Esposizione Internazionale d’Arte, Venezia, 06 giugno-27 novembre 2011). Ciudad de México: INBA, CONACULTA y Turner Publicaciones.

Bermejo Mora, Edgardo (2015). "Imagen de México: Diplomacia cultural vs. marca país, una falsa encrucijada”. Villanueva Rivas, César (ed.), Una Nueva Diplomacia Cultural para México. Ciudad de México: Universidad Iberoamericana.

Camacho, Lidia (2017). "Foreword". León de la Barra, Pablo (ed.), Carlos Amorales: $L a$ vida en los Pliegues $=$ Catálogo de la exposición (Pabellón de México, 55. Esposizione Internazionale d'Arte, Venezia, 1 giugno-24 novembre 2013). Ciudad de México: INBA, CONACULTA y RM.

Crespo de la Serna, Jorge (1952). Exposición del Grabado Mexicano Contemporáneo = Catálogo de la exposición (Palacio de Bellas Artes, Septiembre-Noviembre). Ciudad de México: INBA.

Danto, Arthur (2009). Después del Fin del Arte. Trad. por Elena Neerman. Barcelona: Paidós.

Del Conde, Teresa (1979). Un pintor mexicano y su tiempo: Enrique Echeverría (1923-1972). México: UNAM.

Del Conde, Teresa (2014). "Ruptura y sus antecedentes: Gráfica”. Boletín del Museo Fernando García Ponce, 74, abril-junio, 21-5.

García Canclini, Néstor (2014). Imagined Globalization. Transl. by George Yúdice. Durham (NC): Duke University Press.

García Cepeda, María Cristina (2015). "Foreword”. García Cepeda, María Cristina; Jasso, Karla (eds). Tania Candiani and Luis Felipe Ortega: Possessing Nature = Catálogo de la exposición (Pavellón de México, 56 Esposizione Internazionale D’Arte La Biennale di Venezia, 2015). Ciudad de México: INBA, CONACULTA y Turner Publicaciones.

García Cepeda, María Cristina (2013). Schmelz, Itala (cur.) (2013). Ariel Guzik: Cordiox = Catálogo de la exposición (Pabellón de México, 55. Esposizione Internazionale d'Arte, Venezia, 1 giugno-24 novembre 2013). Mexico City: INBA, CONACULTA and Turner Publications.

García, Gomez (2009). Manuel Felguérez. México DF: Conaculta: Instituto Nacional de Bellas Artes.

Jasso, Karla (2015) (ed.). Tania Candiani and Luis Felipe Ortega: Possessing Nature $=$ Catálogo de la exposición (Pavellón de México, 56 Esposizione Internazionale d'Arte, 9 maggio-22 novembre 2015). Ciudad de México: INBA, CONACULTA y Turner Publicaciones.

León de la Barra, Pablo (ed.) (2013). Carlos Amorales: La vida en los Pliegues = Catálogo de la exhibición (Pabellón de México, 5. Esposizione Internazionale d'Arte, Venezia, 1 giugno-24 novembre 2013). Mexico City: INBA, CONACULTA and RM.

Lozada, Priamo; Perea, Barbara (eds) (2007). Rafael Lozano-Hemmer. Some Things Happen More Often than All the Time = Exhibition Catalogue (Pavellón de México, 52. Esposizione Internazionale d'Arte, 10 giugno-21 novembre 2007). Mexico City: INBA, CONACULTA and Turner Publishers. 
Luna Arroyo, Antonio (1962). Panorama de las artes plásticas mexicanas 1910-1960: Una interpretación social. México DF: Gráfica. Panorámica.

Martínez Martínez, Germán (2008). "The Changing National Identity in Contemporary Mexican Cinema". Carpentier, Nico; Spinoy, Erik (eds), Discourse Theory and Cultural Analysis: Media, Arts and Literature. New York: Hampton Press.

Medina, Cuauhtémoc (ed.) (2009). Teresa Margolles: ¿De qué otra cosa podríamos hablar? = Catálogo de la exposición (Pavellón de México, 53. Esposizione Internazionale d'Arte, 7 giugno-22 novembre 2009). Mexico City: INBA, CONACULTA and RM Editorial.

Molina, Carlos A. (2013). "1821-1921-1951: La mexicanidad y su arte”. Zepúlveda, Luz María (ed.), Artes plásticas y visuales en los siglos XIX y XX. México: CONACULTA.

Molina, Carlos A. (2014). "Fernando Gamboa y su particular versión de México". En Marcin, Mauricio (ed.), Las ideas de Gamboa. México: Fundación Jumex.

Paz, Octavio (1986). "Introduzione". Biennale 42, 1986, 300.

Ramirez Feltrin, Gastón (2011). "Pabellón de México". Barrios, Jose Luis (ed.) Melanie Smith: Cuadro Rojo imposible rosa = Catálogo de la exposición ( $\mathrm{Pa}$ bellón de México, 54 Esposizione Internazionale d'Arte La Biennale di Venezia, Venezia, 06 Junio-27 Noviembre 2011). Ciudad de México: INBA, CONACULTA y Turner Publicaciones.

Schmelz, Itala (ed.) (2013). Ariel Guzik: Cordiox = Catálogo de la exposición (Pabellón de México, 55. Esposizione Internazionale d'Arte, 1 giugno-24 novembre 2013). Mexico City: INBA, CONACULTA and Turner Publications.

Tenorio-Trillo, Mauricio (1998). Artilugio de la nación moderna: México en las exposiciones universales, 1880-1930. México DF: Fondo de Cultura Económica.

Ugalde, Alejandro (2014). "Renacimiento Mexicano y vanguardia en el Nueva York de entreguerras”. Zepúlveda, Luz María (ed.), Artes plásticas y visuales en los siglos XIX YXX. México: CONACULTA.

Ventimiglia, Dario (1996). La Biennale di Venezia: le esposizioni internazionali d'arte, 1895-1995: artisti, mostre, partecipazioni nazionali, premi. Milano: Electa.

Villanueva Rivas, Cesar (2014). "Crónica de un declive anunciado: La diplomacia cultural de México en el sexenio de Felipe Calderón". Garza Elizondo, Humberto, Balance y perspectivas de la política exterior de México 20062012. Ciudad de México: El Colegio de México. 


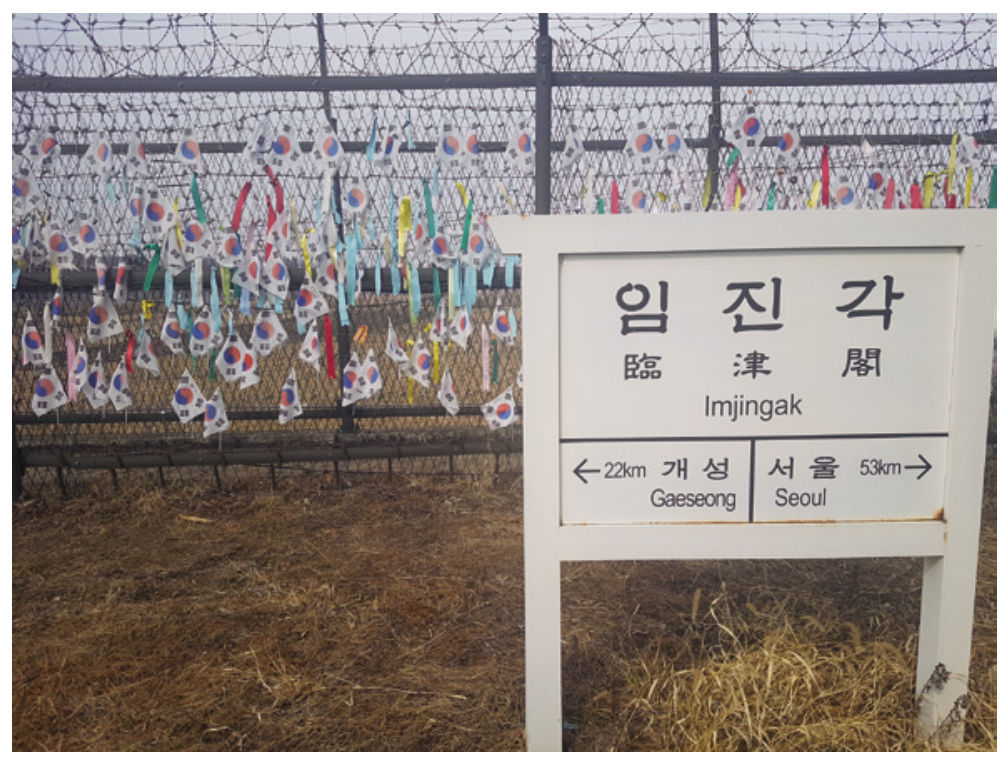

DMZ (La zona demilitarizzata coreana), barriera di confine tra Corea del Nord e Corea del Sud (foto: Samatha Chia) 


\title{
Il Padiglione coreano a Venezia: una questione identitaria
}

\section{Samantha Chia}

\begin{abstract}
The Korean Pavilion was held at Venice Biennale's Centenary, in 1995. This essay reconstructs historical events that led to the creation of a National pavilion for the whole Korean peninsula, which eventually found its place in the traditional site of the Biennale: the 'Giardini' area. The analysis of its exhibitions and the story of the pavilion mesh and intertwine. During these twenty-four years, between 1995 and 2019, the Korean contemporary art changed and today it is so different from the typical Orientalist idea of Korean traditional art. Identity issue is leitmotiv of those art shows and of a generation of artists born after the Korean war, traumatised by political fast changes, in a different economical and social scene. The Korean Pavilion at the Venice Biennale is a proof of this artistic and social journey.
\end{abstract}

Keywords Korean Pavilion. Korean identity. Korean contemporary art. Venice Biennale. Identity issue.

Sommario 1 Il Padiglione della Corea. - 2 Per una storia delle mostre al Padiglione della Corea. - 3 Conclusione. 


\section{Il Padiglione della Corea}

Il Padiglione nazionale della Repubblica di Corea, collocato nella sede storica dei Giardini della Biennale di Venezia, è stato finora l'ultimo Padiglione ad essere edificato, nel 1995. La costruzione di questa struttura si inserisce in un contesto storico e politico ben preciso, motore della proposta del progetto.

Il 1995 è stato un anno simbolico per la Biennale e per la Corea del Sud: a Venezia si celebrava il centenario della rassegna, in Corea ricorreva il cinquantesimo anniversario della liberazione dalla colonizzazione giapponese e veniva inaugurata la Prima Biennale di Gwangju. L'apertura di una struttura permanente all'interno di una delle più importanti mostre internazionali di arte contemporanea sanciva il riconoscimento formale dell'affermazione dell'arte coreana sulla scena mondiale.

L'accettazione della proposta di costruzione del Padiglione all'interno dei Giardini è stata incredibile, se si tiene conto delle norme relative alla costruzione di nuovi edifici a Venezia e alle leggi sulla protezione del patrimonio culturale. Di fatto, essendo Venezia un unicum e essendo designata patrimonio dell'umanità tutelato dall'Unesco, le norme sono assai restrittive e non permettevano di costruire nuovi edifici ai Giardini. Questo è un punto importante da tenere a mente nel racconto delle trattative per la costruzione del Padiglione, iniziate nel 1991.

La Corea partecipava alla Biennale veneziana sin dal 1986, in qualità di Paese ospite. All'epoca i Paesi privi di un Padiglione nazionale venivano ospitati nel seminterrato del Padiglione centrale (allora Padiglione Italia) il cui spazio messo a disposizione era di nemmeno cinquanta metri quadrati e, per regolamento, le spese di partecipazione dei Paesi stranieri - proprietari o meno di un Padiglione - sono a carico del Paese. Questa condizione limitava notevolmente la partecipazione delle Nazioni che non detenevano uno spazio individuale, impedendo di mostrare a pieno le potenzialità e i mutamenti della loro arte: non c'era letteralmente spazio.

La volontà di avere una struttura propria era dunque comprensibile, soprattutto per quelle nazioni che potevano sostenere economicamente l'impresa, e questo era ancora più importante a livello simbolico per quei Paesi considerati 'periferia del primo mondo', come la Corea. In quest'ottica la Corea del Sud aveva - e ha tutt'ora - uno status ambivalente, dal punto di vista politico ed economico, essen-

Questo saggio prende avvio da Chia, Samantha (2018). Il padiglione della Repubblica di Corea alla Biennale di Venezia. Arte come identità [tesi di laurea magistrale]. Relatore Stefania Portinari; correlatore Nico Stringa. a.a. 2018/2019. Venezia: Università Ca' Foscari Venezia. 
do un Paese democratico e capitalista è considerata parte del 'primo mondo', ma dal punto di vista culturale è un paese che non condivide tradizioni e valori con il mondo occidentale, perciò collocata idealmente nel 'terzo mondo'. Avere un terreno di rappresentanza artistica permanente a Venezia significava essere riusciti a entrare a far parte di quel sistema dell'arte e avere opportunità di incontro e confronto con le tendenze mondiali contemporanee, che avrebbero permesso lo sviluppo della stessa arte coreana.

Oltre all'impossibilità materiale di edificare nella sede storica, c'era l'ulteriore ostacolo della 'lista d'attesa', ovvero dei delicati rapporti con le altre nazioni che aspettavano da tempo il permesso di costruire. Tra questi paesi c'erano la Cina, il Portogallo e l'Argentina.

Il caso dell'Argentina è interessante poiché le trattative ufficiali per ottenere il permesso di costruzione di un Padiglione iniziarono già nel 1991. Esiste una notevole quantità di corrispondenza all'archivio della Biennale tra i membri della delegazione del governo argentino in Italia e i rappresentanti della Biennale e del Comune di Venezia. Le richieste si fecero sempre più insistenti nel 1994, con l'obiettivo di ottenere uno spazio in tempo per la celebrazione del centenario. Quell'anno, vista l'eccezionalità della ricorrenza, il Padiglione Centrale sarebbe stato dedicato interamente all'arte italiana, dunque non vi era posto per ospitare le nazioni prive di una struttura. Oltre a vedersi negata la possibilità da tempo auspicata di avere un Padiglione nazionale ai Giardini, l'Argentina perdeva anche l'opportunità di uno spazio nella sede storica in occasione di un anniversario così importante e veniva invitata a trovarsi autonomamente una sede al di fuori.

Tra le ragioni che il Paese sudamericano portava per legittimare la propria richiesta vi erano i premi vinti alla Biennale da artisti argentini, il fatto di essere stato il primo Paese dell'America Latina a partecipare alla mostra d'arte e, soprattutto, come reclamano «i fraterni ed indissolubili legami che sussistono tra l'Italia e l'Argentina, dalla grande influenza culturale che le grandi emigrazioni italiane hanno esercitato in Argentina al Patto Bilaterale di Cooperazione firmato dai due Paesi nel 1987». ${ }^{1}$

L'Argentina faceva quindi leva principalmente su questioni politiche e diplomatiche.

Nel 1995 invece sarà la Corea a ottenere il permesso di costruzione dal Comune, nonostante partecipasse alla mostra da nemmeno dieci anni e la sua richiesta ufficiale fosse giunta anni dopo rispetto a quella del Paese sudamericano. La differenza sostanziale è nelle ragioni presentate dagli interlocutori coreani, ragioni forse meno concrete, ma sicuramente più attraenti e originali. 
Quando nel 1991 il critico d'arte e intellettuale Achille Bonito Oliva visita la Repubblica di Corea, incontra il curatore e storico dell'arte coreano Youngwoo Lee, che gli domanda come poter ottenere un Padiglione nazionale ai Giardini della Biennale. La risposta è categorica: avere un Padiglione ai Giardini è impossibile.

Nel 1992 però Achille Bonito Oliva viene nominato direttore artistico della Biennale del 1993. Questo è un momento di svolta per il raggiungimento dell'obiettivo di Youngwoo Lee, che coglie l'occasione per invitare nuovamente il critico in Corea a visitare una mostra al Recycling Pavilion durante il Dejeon Expo, dove espone anche l'artista americano-coreano Nam June Paik. Il critico Lee, insieme a lui, riapre la questione del Padiglione. Al neodirettore artistico viene riferito che se avessero avuto il sostegno della Biennale per il progetto avrebbero provveduto loro a risolvere le problematiche che sarebbero eventualmente emerse con il governo centrale, con il Comune di Venezia e con il Ministero dei beni e delle attività culturali.

Nam June Paik propone di costruire un unico Padiglione per l'intera penisola coreana, ovvero che potesse essere condiviso tra Nord e Sud Corea in attesa della futura riunificazione e che il Padiglione stesso diventasse un mezzo tramite cui raggiungere la pace. Questa proposta, vista in un'ottica contemporanea, appare assolutamente utopica e in un primo momento venne considerata tale anche da molti sostenitori del progetto. Dalla prospettiva di quel periodo storico però l'idea ci appare meno idealista. I primi anni Novanta infatti erano gli anni successivi alla caduta del muro di Berlino e alla riunificazione della Germania, una fase caratterizzata da un eccessivo ottimismo. Questo ottimismo aveva contagiato anche la Corea che era rimasta l'unica nazione divisa al mondo e la cui separazione era stata spesso comparata alla situazione della Germania Est e Ovest, seppure in realtà diversa sotto molti aspetti. Quello che andava tenuto presente era che la riunificazione della Corea non si poteva dare per scontata in virtù degli eventi, ma la riunificazione della Germania era sembrata a molti un evento del tutto inaspettato.

Bonito Oliva durante quell'incontro non dà alcuna garanzia sulla realizzazione degli intenti, ma rimane affascinato dalla proposta e, dopo aver avvisato che sarebbe stata un'impresa difficile, accetta di fare da mediatore e porta il caso al consiglio della Biennale.

Lo slogan «political healing through art» spiega il progetto e il desiderio di Paik di rendere Venezia lo scenario della riunificazione (Kimsooja to breathe: Bottari 2013, 13). La realtà dei fatti però era decisamente più complessa e sia il Governo italiano che il Comune di Venezia erano scettici riguardo all'efficacia di un simile piano, l'idea era sicuramente interessante, ma le parole di Paik vengono considerate in un certo senso vuote, in quanto affermate non da un rappresentante ufficiale del Governo coreano, ma da un rinomato artista visionario. Non è dato sapere fino a che punto la proposta di Paik fosse 
davvero sostenuta da una reale convinzione, o se fosse nient'altro che un'adulazione nei confronti del Comune di Venezia, ma ha funzionato.

Le possibilità che il Nord e il Sud Corea condividessero un unico Padiglione erano ben remote, ciò nonostante anche solo la più piccola chance che potesse verificarsi venne considerata motivazione sufficiente. Youngwoo Lee, Nam June Paik e l'architetto Seok Chul Kim (che sarà poi incaricato del progetto) sostennero questa motivazione come la più pertinente e valida per la realizzazione del Padiglione e forse se alla base del progetto non ci fosse stato questo desiderio idealista le possibilità di riuscire nell'impresa si sarebbero notevolmente ridotte. A questo punto arrivò il sostegno del Governo coreano e del Comune di Venezia (di cui dal 1993 era sindaco il filosofo Massimo Cacciari) che iniziò a prendere seriamente in considerazione la proposta, quello del Ministero dei beni e delle attività culturali, e dell'Unesco. Tutti giunsero alla conclusione che questa potesse essere un'opportunità unica per il mondo dell'arte: dare un contributo alla storia dell'umanità.

Un bell'esempio dell'entusiasmo dilagante riguardo alla proposta del progetto, dopo un primo momento di comprensibile incertezza, è la lettera inviata il 7 luglio 1994 dal ministro degli affari esteri Antonio Martino al presidente della Biennale Gian Luigi Rondi, in cui si legge:

Desidero assicurarle fin d'ora che l'iniziativa - che sta particolarmente a cuore al Ministero della cultura coreano ed a tutto il gruppo di promotori - gode dell'appoggio di questo Ministero, soprattutto in considerazione dell'impatto sull'opinione pubblica in un momento così delicato nelle relazioni tra le due Coree.

Credo fermamente che operazioni in campo culturale e artistico - ove sono meno sentiti i conflitti etnico-politici - possano essere validi strumenti per superare le tensioni. [...]

Confido nel suo aiuto al fine di accelerare l'iter per la concessione dell'autorizzazione necessaria all'avvio definitivo dei lavori. ${ }^{2}$

Nel 1993 Nam June Paik viene invitato insieme ad Hans Haacke ad esporre al Padiglione Germania. Ciò accade in un momento particolarmente propizio essendo sia il sindaco Cacciari, che il direttore artistico Bonito Oliva, sostenitori del progetto. Grazie all'organizzazione della mostra Paik quell'anno si trova spesso a Venezia, ciò gli permette di fare pressioni in prima persona, avvalendosi anche dell'aiuto dell'architetto Seok Chul Kim che in quel periodo insegna come visiting professor all'Università IUAV di Venezia. Kim viene incaricato da Paik di trattare con il Comune fino a che il progetto non fosse stato approvato.

2 ASAC, FS, AV, b. 6120: Biennale 1994 (provvisorio). 
Dopo la vittoria del Leone d'Oro come migliore partecipazione nazionale assegnata al Padiglione della Germania, Paik sulla scia del riconoscimento stringe maggiormente i rapporti con le istituzioni veneziane e ha un ruolo attivo nella risoluzione dei momenti di crisi e di stallo con esse.

Il progetto inizia a concretizzarsi nel settembre del 1993, viene organizzato un incontro tra la delegazione del Governo coreano composta da Kim Soon-Kyu, direttore del dipartimento di arte del Ministero della Cultura, e l'addetto culturale coreano in Italia. Segue una seconda visita nel novembre 1993 a cui partecipa anche l'architetto responsabile del progetto Kim Seok Chul designato dal Ministero della Cultura insieme a rappresentanti della Soprintendenza e del Comune di Venezia. Durante queste visite si individua la possibile ubicazione del Padiglione, che viene poi dichiarata accettabile.

È nel settembre del 1994 che arriva l'autorizzazione del Comune per l'avvio dei lavori, dopo la delibera della commissione Salvaguardia Venezia dell'8 agosto 1994. Si autorizzava la costruzione di un Padiglione provvisorio sul progetto degli architetti Kim Seok Chul e Franco Mancuso, ${ }^{3}$ alle condizioni che il Padiglione sia smontabile, che non vengano abbattuti alberi o variato l'andamento altimetrico del terreno e che il Padiglione sia smontato entro il 31 dicembre 1998, a meno che entro quella data non venisse approvato uno specifico strumento urbanistico che ne riconfermasse la permanenza. ${ }^{4}$ Con la soluzione del Padiglione provvisorio e smontabile si fa così fronte al divieto di costruzione.

Nel novembre del 1994 viene organizzata dal Ministero della Cultura e dello Sport della Repubblica di Corea e dal Comune di Venezia la mostra dei bozzetti del progetto del nuovo Padiglione nel negozio Olivetti in piazza San Marco. La mostra del centenario viene inaugurata l'11 giugno del 1995, così come il Padiglione coreano: il ventiseiesimo Stato e il secondo Paese asiatico ad avere una rappresentanza ai Giardini.

Il Padiglione è posizionato su una collinetta artificiale situata tra i Padiglioni della Germania e del Giappone, è una posizione un po' nascosta, che non attira subito l'attenzione dei visitatori, in disparte rispetto agli altri Padiglioni che costeggiano il Gran Vialone che si conclude con quello della Gran Bretagna. Anche le dimensioni non sono particolarmente grandi se confrontate con quelle di altri Padiglioni nazionali, quali Russia, Germania, Francia.

Al problema dell'impossibilità di costruire un nuovo edificio si sopperisce attraverso il recupero di una costruzione in mattoni già

3 Mancuso, Franco, «Padiglione della Corea a Venezia», Spazio e Società: rivista internazionale di architettura e urbanistica, 76, 1996.

4 ASAC, FR, AV, b. 6120: Biennale 2004 (provvisorio). 
presente, ormai in stato di degrado, al fine di integrarla a una nuova struttura, creando così un edificio composto da due parti unite. La costruzione preesistente era un antico bagno pubblico che è stato ampliato e corrisponde ora al retro del Padiglione coreano. La nuova struttura sorge a nord dell'edificio esistente, è leggera e trasparente, progettata per non interferire con le alberature che la circondano ed è sorretta da supporti per non modificare l'andamento del terreno. La costruzione è in acciaio, aperta, permeabile e leggera, dall'interno si ha piena visibilità sul bacino di San Marco e sugli alberi. L'accesso principale è sul lato orientale, attraverso un'ansa nel punto di contatto tra i due volumi che affiancano l'edificio esistente. Rispetto agli altri Padiglioni è dotato di un sistema di protezione esterno per l'inverno con aperture e chiusure veloci e semplici, che non è un accessorio ma è parte integrante dell'edificio. Questo sistema di protezione si basa su elementi in legno, ripiegabili e appesi a cornici esterne (quando sono aperti) riprendendo una tecnica tipica dell'architettura tradizionale coreana.

La composizione e l'aspetto dell'edificio sono semplici, caratterizzati da un andamento longitudinale dello spazio espositivo principale (orientato sull'asse est-ovest) in modo da proiettarsi visivamente sul bacino di San Marco. Questo nuovo spazio e l'edificio esistente sono uniti tramite un corpo cilindrico, leggero e trasparente a sua volta. La copertura dello stabile è piana e accessibile (ma non dal pubblico) e dunque utilizzabile per esposizioni all'aperto ed eventi, visibili dal giardino. Il legno viene utilizzato nelle balconate, nella parete nord con configurazione ondulata dettata dalle alberature e nelle chiusure esterne. Questo materiale è stato scelto per raccordarsi in maniera naturale con il contesto circostante, tanto che l'edificio è pienamente integrato con l'ambiente vegetale che lo circonda.

La posizione periferica di cui si è detto è inaspettatamente vantaggiosa, il fatto di essere circondato dal verde dà il beneficio ai visitatori di apprezzare maggiormente l'intimità che si crea tra la natura e lo spazio. Quando durante il giorno la struttura è illuminata dal sole, essa sembra dissolversi, smaterializzarsi, mentre rimane solo il paesaggio esterno, grazie ai materiali che dominano l'edificio: vetro e acciaio.

L'interno è irregolare, composto da forme diverse: rettangoli, cerchi, mezzi cerchi, elementi ondulati, sono tutte forme che non hanno alcuna aderenza ad un preciso schema mentale ma sono dettate dall'ambiente esterno e dalla costruzione preesistente. Nell'insieme l'effetto è quello di una irregolarità organica, che dona al Padiglione una rappresentazione di intimità ambientale combinata all'architettura contemporanea. È in questo senso che il Padiglione può essere paragonato all'architettura tradizionale coreana: le vetrate che racchiudono nel fronte e nel retro la struttura consentono all'ambiente circostante di pervaderne l'anatomia stessa, in modo che l'interno 
diventi l'esterno e viceversa, creando una sorta di dialogo circolare, ciò riprende la composizione aperta del tradizionale Padiglione coreano dove non si ha distinzione tra il dentro e il fuori, tutto dialoga. Questo è un luogo da cui è possibile contemplare ciò che si ha intorno, è possibile il dialogo con la natura, come quello rappresentato in molte opere calligrafiche coreane, le sansuhwa (letteralmente dipinti di montagna e mare).

È per la creazione di questo rapporto intimo dovuto alla struttura aperta che l'architettura del Padiglione Corea è stata descritta come «an expression of the Asian spirit through Western architecture» (Landscape of Differences 2003, 63).

Questa struttura non convenzionale del Padiglione non è rimasta tuttavia esente da critiche, tanto che molti in patria lo ritenevano non adatto a fini espositivi. Gli elementi strutturali e architettonici ritenuti non adeguati all'allestimento di mostre di arte visiva sono stati modificati e celati in molte edizioni, coprendo le vetrate, rimodellando lo spazio interno, oscurando gli altri muri della costruzione. Solo di recente i coreani hanno rivalutato la singolarità della loro struttura, mettendola in evidenza piuttosto che coprendola. Ne è un esempio l'esposizione del Padiglione alla Biennale del 2003 intitolata Landscape of Differences, che parte proprio da una riflessione strutturale sulle qualità specifiche del Padiglione. Infatti, piuttosto che volergli dare una forzata identità asiatica, legata agli elementi tradizionali dell'architettura coreana, si è deciso di costruire la sua identità partendo dallo site-specific del Padiglione stesso.

Il Padiglione in sé non è soltanto un involucro, un contenitore di oggetti artistici, ma è ricco di significati semantici e simbolici, è parte attiva delle esposizioni, rimane sempre elemento determinante nel pensare all'esposizione artistica, sia quando le sue caratteristiche architettoniche vengono nascoste, che quando vengono esaltate. A differenza di altri Padiglioni nazionali, con pompose forme neoclassiche, non è stato costruito per autocelebrare l'architettura stessa. Quando i riflettori della Biennale sono spenti il Padiglione Corea somiglia più ad una casetta tra tanti mausolei, è un oggetto insolito, nascosto tra gli alberi, ambiguo e pacato.

Le due Coree non hanno condiviso il Padiglione, la Corea del Nord non ha mai partecipato alla Biennale e l'unificazione non è avvenuta. Solo la Corea del Sud è riuscita ad ottenere uno spazio permanente nella vetrina d'arte internazionale, mantenendo comunque l'iscrizione 'Corea' sulla porta d'ingresso. Così facendo il governo Sudcoreano non ha voluto ignorare una divisione geopolitica che è ormai permanente, ma ha desiderato creare uno spazio unitario per tutta la penisola, sollecitare il popolo ad una riflessione, quella relativa ai legami di sangue, come possono coesistere quando non si condividono più gli stessi valori culturali. 


\section{Per una storia delle mostre al Padiglione della Corea}

Dal 1995 la Repubblica di Corea ha partecipato ufficialmente a tredici mostre internazionali d'arte. Nel ripercorrerle tutte ci si imbarca in un viaggio attraverso il rapido cambiamento della Corea tra la fine degli anni Novanta e il Duemila: un mutamento politico, economico, culturale e sociale raccontato attraverso il filtro dell'arte, che è anche racconto del rapido cambiamento dell'arte coreana stessa. La scena artistica nazionale ha raggiunto in un brevissimo tempo una dinamicità, originalità e varietà tale da rendere difficile per il pubblico esterno categorizzare e definire un'arte che sia specificamente coreana. La successione delle edizioni mostra il risultato di una lunga lotta degli artisti coreani per affermare la propria individualità, una lotta che nell'ultimo secolo e mezzo è stata segnata dallo scontro tra la tradizione e l'ibridazione culturale e artistica.

Nei ventiquattro anni di esistenza del Padiglione si possono intercettare delle tematiche comuni che ritornano e si evolvono nel corso delle mostre. Tra queste vi sono: la critica della società capitalista, il complesso e contraddittorio rapporto con l'Occidente e con la tradizione, la guerra fratricida del 1950-1953, il veloce sviluppo economico e tecnologico, un possibile futuro distopico e apocalittico. Questi temi dialogano tra loro grazie a molteplici fili conduttori, uno di questi è la questione identitaria.

Le caratteristiche di una specifica identità culturale e la crisi identitaria che può derivarne variano in base alle specificità del luogo in cui questa identità si è plasmata e trasformata. Gli sconvolgimenti storici, politici e sociali attraverso cui la Corea è passata nel secolo scorso (il colonialismo giapponese, la divisione della penisola, la guerra, la rapida modernizzazione, l'afflusso indiscriminato della cultura occidentale) hanno dato vita a fenomeni di discontinuità culturale, tra quella tradizionale coreana e quella contemporanea, causando un'inevitabile crisi identitaria nella popolazione ed erodendo e trasformando quella che veniva percepita come 'tradizione'. Ciò è stato particolarmente significativo e traumatico per un popolo che vanta una cultura ultra-millenaria, rimasto territorialmente unito dal VII secolo fino al 1948 e pressoché non intaccato da punto di vista etnico.

Questi avvenimenti hanno fatto emergere la problematica identitaria, che non è solo sentita concretamente dagli individui, ma è diventata prioritaria nelle politiche culturali dei governi che si sono succeduti sin dalla prima Repubblica. Tali politiche si sono incentrate sulla costruzione di un io coreano, che non riusciva più a collocarsi nel mondo contemporaneo, come se attraverso l'identità fosse possibile creare delle suture che rattoppassero gli strappi della società.

Tuttavia, se da una parte ci sono le politiche culturali, dall'altra ci sono l'arte e gli artisti. Le nuove generazioni sentendosi sempre più ostacolate e limitate dai dogmi estetici che finivano per tar- 
pare le ali alla creatività e alla sperimentazione, in nome di un'arte che esprimesse una fantomatica identità coreana, hanno intrapreso un viaggio sempre più introspettivo rivendicando l'importanza delle identità singole.

Il Padiglione coreano alla Biennale di Venezia può essere una chiave di lettura per analizzare questa formazione in continuo divenire di un'identità coreana, non come la descrivono le politiche, ma come la vivono le persone e i protagonisti della scena artistica. Negli anni sono mutati i modi con cui gli artisti hanno mostrato e raccontato questa problematica, come l'hanno percepita e vissuta in base all'età, al genere, all'esperienza personale. Ne consegue che la questione dell'identità culturale finisce per relazionarsi con tante realtà differenti, e in base a con chi e con cosa si confronta assume sfumature diverse.

Il filo conduttore della questione identitaria che lega - forse inconsapevolmente - le mostre non si è mai spezzato. Talvolta lo si nota chiaramente, esce in maniera vistosa, urgente, inequivocabile. Altre volte si fa più sottile, si nasconde, e se non ci si ferma ad un'analisi superficiale delle forme, ma si indaga il contenuto, ci si accorge che è sempre onnipresente.

Un breve focus su alcune delle singole edizioni restituisce un'istantanea della trasformazione avvenuta e una prima storia del Padiglione Corea.

Nelle primissime mostre, quelle tra 1995 e il 1999, i commissari puntavano alla rappresentazione di un'estetica coreana contemporanea legata alla storia e alle tradizioni, tuttavia già da queste edizioni emerge una commistione di elementi tradizionali e nuovi media. Le personalità dei singoli artisti non vengono represse ma la selezione di essi ricade su quelli che riescono a tradurre l'animo più puro e antico dell'arte coreana di fronte alla platea mondiale. Queste edizioni a primo impatto trasmettono ancora quella sensazione di esotico e orientaleggiante che gli occidentali associano all'estremo Oriente.

Siamo qui sulla soglia di un cambiamento radicale, se gli artisti coreani vengono selezionati in base a quanto sanno esprimere la 'coreanità', ben presto verrà chiesto loro di essere 'personali' in quanto in loro stessi c'è anche la componente coreana, che non è un fattore totalizzante ma solo una parte del loro essere. Il Padiglione coreano può essere inteso come testimonianza di questo passaggio, da un'arte che secondo le politiche culturali doveva esprimere l'essenza di un popolo, a un'arte che è espressione del singolo, prodotto di un io che è necessariamente diverso dal tu. La Corea si conquista una 'casa' a Venezia proprio quando questo processo di slittamento, dal popolo all'individuo, è all'apice.

Nelle edizioni in cui era figurata come Paese ospite la Corea era sempre intervenuta con opere pittoriche, solo nel 1990 aveva partecipato con un'opera installativa. Questa scelta fu dettata unicamente dal limite di non avere uno spazio proprio. Adesso che il Padiglio- 
ne era stato costruito, gli artisti coreani potevano esprimere a pieno le loro potenzialità attraverso medium differenti, non solo quello pittorico. Per questa ragione tra i quattro artisti selezionati per la prima mostra solo uno è un pittore: Yun Hyong-keun, il più anziano del gruppo, nato nel 1928.

Yun è forse l'animo più antico che il Padiglione coreano ha ospitato, ultima testimonianza di una generazione dimezzata e segnata dagli eventi più cruenti del secolo scorso, portatore del clima artistico del dopoguerra, prima che tante voci diverse, nuove ed originali lo popolassero. L'artista nel suo rapporto ambivalente con l'arte tradizionale ha continuato a praticare una «pittura coreana», ibridandola con metodi e tecniche non disponibili nella stessa tradizione, ma finendo in questo modo per recuperarla, così come era richiesto dal clima artistico dell'epoca. È un pittore delicato, interprete di un sublime che emerge da uno specifico contesto coreano, il suo scardinare la rappresentazione, lo stile e i materiali tradizionali proviene da una solida consapevolezza: le vecchie maniere non sono più in grado di parlare al presente. Egli cerca attraverso la non-figurazione, le immagini non definite e il vuoto pittorico di attirare l'attenzione sulla crisi sociale e ontologica derivata dalla catastrofe della guerra civile e della sommersione della società tradizionale causata dallo tsunami della modernizzazione. Il rapporto con la tradizione è ambivalente: da un lato la ritiene superata e non più utile al contesto attuale, ma vi attinge costantemente mescolandola con nuovi stimoli. Quello di Yun non è uno spirito postmoderno, ma ha un eco antico. È una figura di transito tra generazioni d'artisti.

Nell'ottica di un'arte coreana che si muove sempre di più verso la rappresentazione dell'individuo, la mostra del 2001 segna in qualche modo un punto di svolta: si riporta al centro l'idea del singolo e si denuncia la pericolosità di una collettività forzata a condividere un'identità imposta.

Gli artisti che espongono nel 2001, Michael Joo e Do Ho Suh, risiedono entrambi negli Stati Uniti. Il primo è nato in America, figlio di immigrati coreani, il secondo è un immigrato di prima generazione nato in Corea. Nell'analisi del loro lavoro è importante tenere in considerazione questa differenza generazionale in quanto l'esperienza dell'immigrazione ha delle peculiarità specifiche in base a se si è nati nel Paese di origine e ci si trasferisce successivamente in terra straniera, oppure se si è nati nel 'Paese ospite'. Questa è una differenza che va considerata nell'analisi del lavoro degli artisti che affrontano il significato di shock culturale e di identità transnazionale. Questi temi emergono in maniera prepotente nella mostra: Joo con un approccio scientifico porta avanti un'indagine tra i diversi rapporti che animano il mondo postmoderno, quello tra la natura e l'artificio, la distruzione e la ricostruzione, l'Oriente e l'Occidente, il frammento e l'intero, l'individuo e il gruppo, ma sono le installa- 
zioni di Do Ho Suh che esprimono in maniera lampante il mutamento di espressione artistica in atto. Suh affronta direttamente e apertamente la questione della massa e dell'individuo con le loro dinamiche di potere, insieme a quella della società coreana e dell'identità militare e collettiva.

Quella del 2003 è la prima mostra ad avere un titolo proprio, che risulta essere emblematico: Landscape of Differences. Partendo dalle differenze che contraddistinguono il Padiglione dagli altri, si vuole mostrare la peculiarità coreana, ed elevarla nel contesto internazionale al pari degli altri, in tutta la sua diversità. Tra gli artisti è evidente la differente visione estetica, formale e concettuale. Tuttavia, c'è un terreno comune: l'approccio astratto e concettuale di affrontare la realtà, che è il Landscape comune che unisce le diverse e numerose dimensioni create dagli artisti.

Nel 2005 il Padiglione coreano celebra il suo primo decennio e per l'occasione si fa contenitore di una policromia di voci. La mostra Secret Beyond the door è stata pensata come una sorta di retrospettiva storico-artistica di questa ultima decade. Per mostrare le tante finestre e realtà che si sono aperte negli anni appena trascorsi vengono selezionati ben quindici artisti, l'esposizione risulta così essere un variopinto e variegato insieme di scenari, piuttosto che un'accurata ricostruzione storica, in cui viene rimarcata l'unione delle diversità.

Dal 2007 fino al 2015 è il momento invece delle personali. Quella di Hyunjkoo Lee è la prima solo exhibition allestita al Padiglione, dal titolo The Homo Species. L'artista trasforma la struttura in una sorta di museo di storia naturale e in uno sterile laboratorio per raccontare il complesso d'inferiorità culturale del suo popolo, filtrato dalla sua esperienza personale, attraverso un lavoro comico e al contempo misterioso.

Haegue Yang nel 2009 allestisce il Padiglione sulla scia del titolo generale della mostra della Biennale Fare Mondi/Making Worlds. Il direttore artistico del 2009 Daniel Birnbaum voleva che gli artisti invitati non approdassero alla Biennale come difensori dei propri mondi inseriti all'interno di confini politici, sostenitori della propria lingua e cultura, parte di un sistema espositivo suddiviso in nazioni di cui si ritrovano inevitabilmente a fare da portavoce. La sua idea era invece che costruissero i loro personali mondi e li condividessero, ispirando un tipo di riflessione che va al di là di ogni qual si voglia differenza culturale e aspettativa sociale. Haegue lo fa esplorando i luoghi della sua vita e della comunità, riproducendoli e filmandoli, dando maggior enfasi agli spazi privati, spesso considerati marginali e secondari.

Lee Yongbaek, che rappresenta la Corea nel 2011, è uno di quegli artisti che si è tenuto distante nel corso della sua carriera dalle strategie di mercato dell'arte asiatica, quali l'utilizzo di motivi considerati esotici derivati dall'arte buddista, dall'Oriental Painting e dall'Asia Folk Culture per richiamare i riflettori internazionali sul proprio 
operato ed ottenerne il conseguente consenso. In The Love is Gone but the Scar Will Heal Lee si rifiuta di creare nuovi lavori appositamente per il circuito Biennale, o di attirare l'attenzione costruendo gigantesche installazioni, preferisce esporre completamente il suo lavoro che è in un continuo work in progress. Egli è uno sperimentatore di materiali, tecniche diverse, dalla video art, interactive art, installazioni sonore, alla ricerca di sinestesia e sperimentazione robotica. E noto per questa versatilità e per aver trattato importanti questioni culturali dell'era digitale come la questione della soggettività decentrata nell'epoca della realtà virtuale, il cambiamento epistemologico, i nuovi simulacri digitali e l'incombere del paganesimo culturale. Il suo lavoro al Padiglione coreano mostra quattro differenti serie realizzate con tecniche diverse ma interconnesse tra loro dallo stesso tema. È l'affascinante titolo della mostra che convoglia tra loro le diverse opere: The Love is Gone but the Scar Will Heal, un titolo che esprime il dolore sofferto dalla popolazione coreana per la sua recente storia, ma anche la speranza che dopo tutto rimane.

Nel 2013 le caratteristiche strutturali del Padiglione sono il punto di partenza del ragionamento espositivo. Prerogativa fondamentale doveva essere quella di non modificare in alcun modo l'architettura, mantenere un rispetto quasi religioso per essa. Lo stesso tipo di logica era stata utilizzata nel 2003, ma in questa situazione l'intervento è ancora più radicale: nulla viene aggiunto, nulla viene sottratto. Quel che si cerca è una fusione tra l'architettura e la visione dell'artista.

La visione che viene scelta è quella di Kimsooja che nell'installazione To Breathe: Bottari mantiene intatta la forma dell'edificio ma lo muta profondamente a livello visivo. Una pellicola costituita da un reticolo di diffrazione ricopre il vetro delle finestre, dei muri, del tetto e pannelli in alluminio specchiato vengono fissati al pavimento e al soffitto. L'ambiente viene rimodellato, i materiali utilizzati sfruttano la luce naturale, la pellicola di diffrazione fa scaturire un arcobaleno di colori che si infrange in un'infinità di riflessi mai uguali a loro stessi, crea così un ambiente di luce caleidoscopica, un'esperienza altamente percettiva arricchita dal sottofondo sonoro. Opposta a questa luminosa danza visiva e uditiva è la camera buia. Questa stanza è priva di illuminazione e anecoica, l'assenza di eco e di rumori permette di entrare maggiormente in contatto con il proprio corpo, di sentire il ritmo del proprio respiro, il sangue che pulsa nelle vene, i battiti del cuore. La parola coreana Bottari del titolo è il richiamo alla tradizione. I Bottari sono fagotti di tessuti colorati cuciti dalle donne, un'immagine che Kimsooja utilizza spesso nel corso della sua carriera, ricordo della sua terra d'origine. Qui i Bottari non sono concretamente inseriti, c'è solo la loro memoria, il Padiglione stesso è un Bottari metaforico di luce e colore.

To Breathe: Bottari fa vivere l'esperienza dell'individuale e dell'universale, in sottofondo gli echi della tradizione che hanno dato nu- 
trimento all'artista, un lavoro originale che si amalgama completamente con il corpo dinamico della struttura.

Moon Kyungwon \& Jeon Joohon in The Ways of Folding Space \& Flying accolgono l'invito del direttore artistico Okwui Enwezor della Biennale 2015, dal titolo All the World's Futures, di provare ad immaginare ed esplorare uno dei tanti futuri potenziali e il ruolo che l'arte avrà, o non avrà, in questa realtà possibile. Il duo artistico realizza una videoinstallazione multicanale che non ha una continuità storica e narrativa. In una sorta di asettico laboratorio - che ci accorgiamo poi essere il Padiglione coreano sopravvissuto a una qualche apocalisse in un futuro indefinito - un personaggio dall'aspetto androgino prende coscienza. Nel momento in cui apre gli occhi inizia la sua infinita routine, ripete inconsapevolmente gli stessi gesti negli stessi momenti ogni giorno, non c'è differenza tra ieri, oggi e quel che sarà domani. Ci appare come una figura apatica, priva di emozioni, incapace di comunicare e bloccata in un volgere del tempo circolare. La durata della sua giornata sembra essere quella di una vita. Guardando contemporaneamente e parallelamente momenti diversi trasmessi in loop non siamo in grado di ordinarli cronologicamente. Il futuro, il presente e il passato si mischiano, sono tempi possibili, nulla è definitivo e determinato.

In questi anni, avendo a disposizione lo spazio necessario, i singoli artisti hanno avuto l'opportunità di mostrarci la contemporaneità e la Corea così come la vedono loro e di indagare insieme a noi la loro identità.

Nelle ultime due edizioni, invece, assistiamo di nuovo alla convivenza di più personalità.

Counterbalance: The Stone and the Mountain del 2017 parte dal contesto coreano per approdare al contesto globale: dalla Corea all'Asia, dall'Asia al mondo. Gli artisti Lee Wan e Cody Choi hanno preso coscienza delle disparità esistenti nel loro Paese di origine, così come ovunque nel mondo. Partendo da questo presupposto raccontano un'esperienza che è banalmente umana: la loro esperienza, che può essere anche quella del singolo, che può essere anche quella di una moltitudine. In questa esistenza segnata da forti sbilanciamenti gli artisti attribuiscono all'arte una funzione benefica di contrappeso, in grado di risanare i disequilibri.

La mostra del 2019 History has failed Us, but no Matter esplora la storia della modernizzazione in estremo Oriente attraverso le lenti del genere e del ruolo della tradizione. L'edizione risulta essere particolarmente innovativa se inserita nella storia delle mostre del Padiglione: lo sguardo sul mondo è interamente femminile, dalle artiste alla curatrice, e non è un dato di poco conto per una democrazia patriarcale come la Corea del Sud. Per la prima volta alla Biennale d'Arte di Venezia le coreane parlano apertamente di transgender, di queer, di non-genere e lo fanno attraverso il mezzo della videoinstallazione. 
Infine, quel che è ancora più interessante, è la scelta di analizzare il presente e parlare di questi temi per mezzo della tradizione e del mito. Quando si pensa alla 'tradizione' la si associa con molta facilità a un pensiero conservatore, limitato, antico. In quanto contemporanei ci riteniamo tendenzialmente evoluti nel pensiero rispetto ai nostri predecessori, ma la storia non è una linea retta in continua progressione e avanzamento, si va avanti ma si torna anche indietro, ed è così che la tradizione può talvolta rivelare un potere emancipatorio, che va al di là dei canoni stabiliti dalla modernità occidentale, come il fatto che il genere sia qualcosa di non strettamente definibile ma che sia un discorso più complesso e sfumato.

Le artiste ci raccontano storie e miti di donne del passato, intrecciandole e sovrapponendole con le storie delle donne del presente, tutte abbandonate, emarginate e condannate dalla storia. Infine, è la tradizione a fornire l'antidoto alla tradizione facendo emergere la complessità e la diversità, sostituendo le nozioni di identità, statonazione, patriarcato con quelle di genere 'altro', modernità postcoloniale, transnazionalità.

\section{Conclusione}

La partecipazione della Corea a manifestazioni artistiche internazionali - tra il 1989 e la prima metà degli anni Novanta - e l'apertura di un Padiglione permanente a Venezia ha significato per il Governo coreano entrare in maggior contatto con la comunità internazionale, con l'obiettivo di ricavarne benefit dal punto di vista politico e diplomatico. Per gli artisti invece è stata un'occasione di confronto, un modo per divincolarsi dalle ferree regole imposte da musei, gallerie e scuole d'arte coreane, e per ottenere il atteso interesse per il loro lavoro oltre confine, interesse che spesso era assente a livello nazionale. Non sono pochi gli artisti che hanno esposto a Venezia e che in patria erano pressoché sconosciuti ed oggi godono di maggiore notorietà all'estero.

Per noi pubblico occidentale, invece, ha significato la scoperta avvincente di un'arte sostanzialmente sconosciuta che si stava liberando dagli stereotipi, un aiuto nel distinguere la cultura coreana (la 'vera' cultura coreana, vale a dire 'qualcosa che esiste': un gruppo di persone che hanno una storia comune, vivono in un'area geografica definita e hanno in comune determinati comportamenti,...) dalla 'cultura coreana' (quello che noi, ovvero gli osservatori esterni, intendiamo come 'cultura coreana', vale a dire 'quello che abbiamo creato nella nostra mente': le immagini, le strutture, i concetti. La 'cultura coreana' è quello che noi pensiamo che essa sia e che insegniamo agli altri) (Sasse 1996) per mezzo delle forme e delle voci degli artisti contemporanei. Probabilmente non arriveremo mai a distinguerle nettamente, entrambe mutano costantemente e si influenzano a 
vicenda, le confonderemo e mischieremo sempre, così come vengono mischiate dagli stessi artisti, un po' con consapevole ironia, un po' con naturale contraddittorietà.

La scena artistica coreana è oggi eclettica, fatta di singole personalità diversificate ed ostinate a rimarcare la loro unicità di individui, a Venezia si è aggiudicata una piattaforma sicura per essere ammirata con occhi curiosi dal mondo, trasmettendo in questi anni tutta la sua dinamicità.

\section{Bibliografia}

Condensation Haegue Yang (2009). Condensation Haegue Yang = Exhibition catalogue (Venezia, Giardini della Biennale 7 giugno-22 novembre 2009). Seoul: Art Council Korea.

Counterbalance (2017). Counterbalance: The Stone and the Mountain Cody Choi Lee Wan = Exhibition catalogue (Venezia, Giardini della Biennale 13 maggio-26 novembre 2017). Seoul: Arts council Korea.

Do Ho Suh (2001). Do-Ho Suh = Exhibition catalogue (Venezia, Padiglione della Corea, Biennale di Venezia, 9 giugno-4 novembre 2001). Seoul: The Korean Culture and Arts Foundation.

History Has Failed Us (2019). History Has Failed Us, but No Matter = Exhibition catalogue (Venezia, Padiglione della Corea, Biennale di Venezia, 11 maggio-24 novembre 2019). Milano: Mousse Publishing.

Hyungkoo Lee (2007). Hyungkoo Lee the homo species = Exhibition catalogue (Venezia, Padiglione della Corea, Biennale di Venezia, 10 giugno-21 novembre 2007). Seoul: Art council Korea.

Kim, Youngna (2005). Modern and Contemporary Art in Korea: Tradition, Modernity and Identity. Seoul: Hollym.

Kimsooja to breathe (2013). Kimsooja to Breathe: Bottari = Exhibition Catalogue (Venezia, Padiglione della Corea, Biennale di Venezia, 1 giugno-24 novembre 2013). Dijon: les presses du reel.

Landscape of Differences (2003) = Landscape of Differences. Exhibition catalogue (Venezia, Padiglione della Corea, Biennale di Venezia, 14 giugno-2 novembre 2003). Seoul: the Korean Culture and Arts Foundatio.n

Lee Yongbaek (2011). Lee Yongbaek the Love is Gone but the Scar Will Heal = Exhibition catalogue (Venezia, Giardini, 4 giugno - 27 novembre 2011). Seoul: Art Council Korea

Michael Joo (2001). Michael Joo = Exhibition catalogue (Venezia, Padiglione della Corea, Biennale di Venezia, 9 giugno-4 novembre 2001). Seoul: The Korean Culture and Arts Foundation.

Morley, Simon (2015). «The Paintings of Yun Hyong-Keun as 'Emergent Blended Structures'». Third Text, 33(2), 482-3.

Sasse, Werner (1996). «Teaching Korean Culture through Korean Studies». Korean Journal, 36(3), 20-39.

The Pavilion of the Republic of Korea (1995). La Biennale di Venezia - The Pavilion of the Republic of Korea = Exhibition catalogue (Venezia, Padiglione della Corea, Biennale di Venezia, 11 giugno-15 ottobre 1995). Seoul: The Korean Culture and Arts Foundation. 



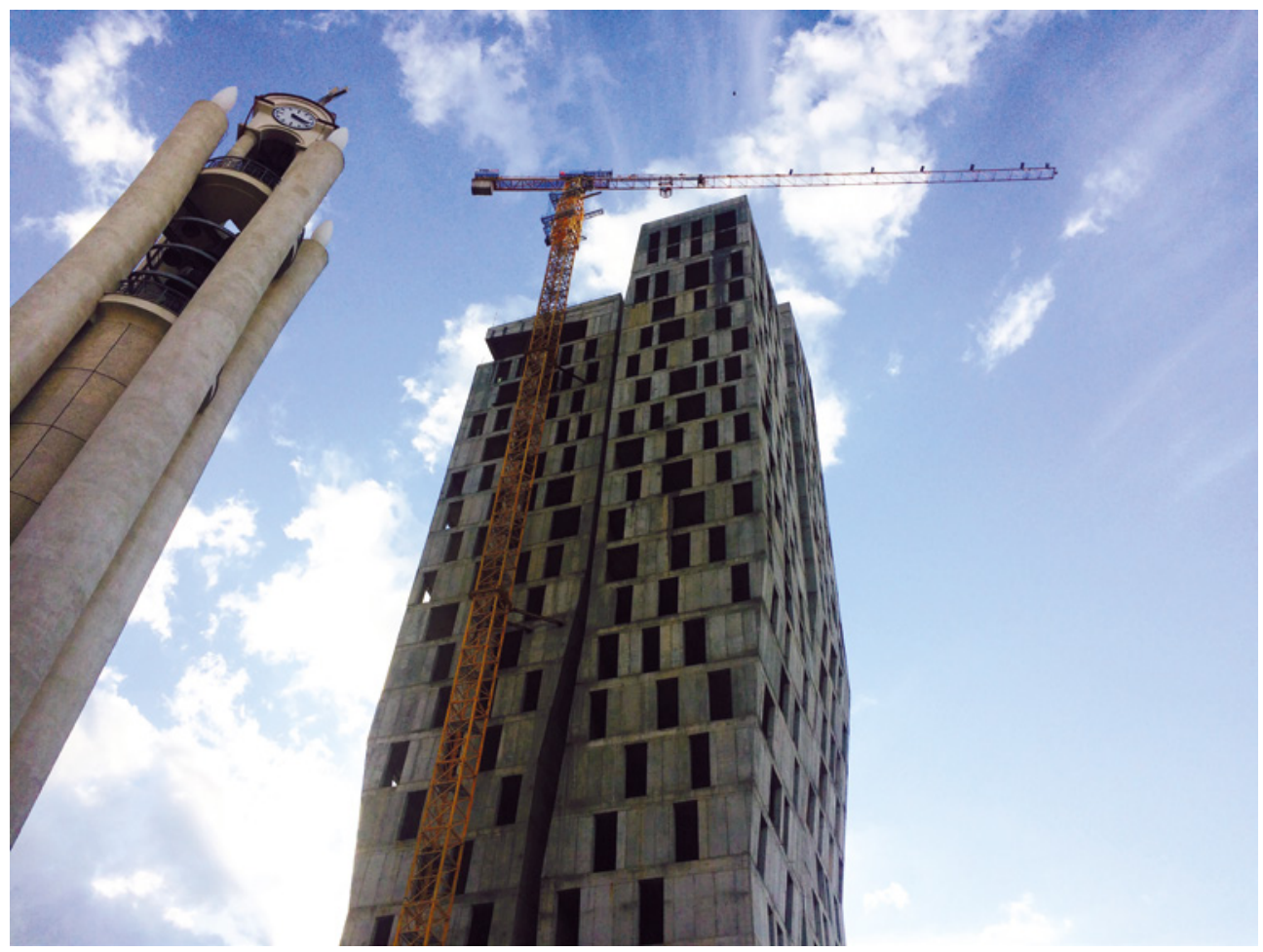

La 4 Ever Green Tower in costruzione (2018; progetto Studio Archea, Firenze) e il campanile della Nuova Cattedrale Ortodossa a Tirana, Albania (foto: Margherita Fochessati) 


\title{
Oltre l'Adriatico La partecipazione albanese all'Esposizione Internazionale d'Arte di Venezia
}

\author{
Margherita Fochessati
}

\begin{abstract}
Albania joined the International Art Exhibition in Venice only in 2005, twenty years later the collapse of the communist dictatorship of Enver Hoxha. The building of a democratic state had consequences also on the expressive research of several artists who had to face a substantial change of their working environment. At the beginning of XXI century the artistic and cultural heritage of Socialist Realism was still the only relevant cultural reference for the construction of a new Albanian cultural identity. Reflecting on the troubled past of the their nation the new generation of artists focused their research on a intimate and personal elaboration concerned the current improvement of the Albanian society. The purpose of this essay is to illustrate the role that the Venice Biennale has been in the realization of the new Albanian artistic scene as landmark for the European and international contemporary cultural tendencies. All the exhibition settled in the Albanian Pavillion since 2005 reveal the different approaches to the national past and to the new cultural identity of the artist that have represented the Balkan country at the Venice Biennale during the last fifteen years.
\end{abstract}

Keywords Albania. Biennale. Identity. Post-socialist art. Immigration. 
Per lungo tempo rimasta in disparte, isolata e soffocata dalla martellante ideologia di Enver Hoxha, il dittatore che per oltre quarant'anni aveva rinchiuso la popolazione all'interno di confini blindati e invalicabili, l'Albania è riemersa sulla scena artistica contemporanea a partire dai primi anni Novanta, conseguentemente alla morte del suo leader avvenuta nel 1985. La difficile eredità del dittatore venne infatti colta dal suo pupillo, Ramiz Alia, che eletto nel 1986 segretario generale del Partito del Lavoro d'Albania, inaugurò una fase di distensione volta a una graduale apertura all'Occidente, in particolare nei confronti dell'Italia, atteggiamento che sul piano culturale comportò una maggiore libertà di espressione e la possibilità di entrare in contatto con la cultura visiva e le tendenze espressive europee.

Il crollo del regime che si era instaurato al termine del secondo conflitto mondiale, unitamente al collasso delle piramidi finanziarie verificatosi nel 1997 che aveva ridotto il Paese in condizioni di estrema miseria e precarietà, costituirono le principali cause dell'ondata migratoria che dai primi anni Novanta vide riversarsi sulle coste del sud Italia migliaia di cittadini albanesi che attraversarono l'Adriatico alla ricerca di migliori condizioni di vita.

Dopo circa quarant'anni trascorsi imprigionata in un ghetto ideologico la popolazione schipetara identificò nuovamente l'Italia, che nel corso del primo Novecento attraverso disposizioni e ordinamenti di carattere economico-politico si era posta come sostenitore e alleato della nazione balcanica, quale meta privilegiata dove poter stabilirsi e dare avvio a un futuro più stabile e sicuro.

Il fenomeno migratorio, l'estenuante e pericolosa traversata intrapresa dai 'boat-people' e il difficoltoso processo di inserimento all'interno della società italiana ebbero un notevole impatto sull'evoluzione delle arti figurative in Albania e sull'operato di molti artisti che si erano ritrovati in prima persona a dover abbandonare il Paese delle Aquile per poter continuare la propria ricerca artistica in un contesto più confacente alle proprie necessità.

Nel corso degli anni Novanta e al principio del nuovo millennio le espressioni artistiche albanesi si connotarono e costituirono principalmente per una riflessione sulle coeve condizioni economiche e sociali in cui versava il Paese, esprimendo i dubbi e gli interrogativi che il dipanarsi del nuovo e incerto scenario politico poteva sollevare.

In questo contesto la maggioranza degli artisti schipetari documentava e interpretava la realtà che li circondava conducendo un'attenta indagine sulla quotidianità del Paese in ogni suo aspetto, anaa.a. 2018/2019. Venezia: Università Ca' Foscari Venezia. 
lizzando la transizione verso il governo democratico e la graduale riconquista delle libertà personali e con esse la volontà e il bisogno della migrazione verso i limitrofi Paesi dell'Europa occidentale. Nonostante le loro opere rispecchiassero e costituissero un dettagliato affresco della contemporaneità, in esse non è possibile riscontrare un'unità tematica dettata da un impegno collettivo; ogni artista conduceva la propria ricerca espressiva indipendentemente dalle tendenze artistiche a lui tangenti, in un'ottica di introspezione e riacquisizione del proprio ruolo di artista all'interno della società. Se infatti negli anni della dittatura gli artisti albanesi potevano muoversi e operare esclusivamente all'interno dei rigidi confini di un sistema di comunicazione predefinito che non parlava mai propriamente del presente, esaltando il glorioso passato nazionale e dipingendo contestualmente l'immagine di quello che avrebbe dovuto essere un «radioso avvenire» (Piretto 2001, 159), successivamente al crollo del regime e all'instaurazione della democrazia la nuova generazione di artisti ebbe modo di confrontarsi direttamente con la contemporaneità, sottolineando, sovente non senza una velata ironia, le problematicità e le contraddizioni della società e della politica schipetara. Gli artisti emergenti posero particolare attenzione alle criticità di un sistema culturale non più oppresso dalle imposizioni governative, ma contemporaneamente meno solido e protettivo nei confronti della figura dell'artista a cui invece negli anni della dittatura socialista era stato attribuito un importante e fondamentale ruolo professionale.

La conquista della libertà espressiva, che in molti casi comportò l'isolamento dal resto della società dell'artista, non più cantore del popolo ma ideatore di una poetica privata e personale, si tradusse in opere autoreferenziali dove le complesse condizioni della vita quotidiana e il rapporto con il regime, le cui attestazioni visive erano ancora ampiamente percepibili, costituivano le tematiche principali. L'utopia collettiva del passato lasciò dunque spazio all'utopia individuale del presente dove i decantati progetti per il glorioso e sereno futuro del Paese vennero sostituti dai più concreti desideri personali volti alla conquista di una stabilità economica e al riconoscimento del proprio operato e del proprio ruolo di artista in una nazione dove ogni certezza era venuta a mancare.

La desolazione e il crescente degrado di molti degli edifici e monumenti eretti durante gli anni del regime costituirono il nuovo universo visivo all'interno del quale si trovò a muoversi l'artista contemporaneo che, ormai libero dalla miope adesione ai principi ideologici del Partito di cui si era dovuto fare interprete durante la dittatura enveriana, nei primi anni del governo democratico di Sali Berisha ebbe modo di sviluppare un nuovo universo narrativo caratterizzato da una pluralità tematica.

In tale ambito la considerevole e impegnativa eredità costituita dal Realismo socialista influì significativamente sullo sviluppo delle 
arti figurative negli anni successivi al crollo del regime, costituendo un costante rimando e confronto sul quale articolare le nuove tendenze espressive.

L'opprimente dottrina socialista che per oltre quarant'anni aveva rappresentato l'unico riferimento culturale per i pittori e gli scultori schipetari e che aveva permeato ogni aspetto della vita quotidiana della popolazione divenne, nel nuovo contesto politico e sociale, l'elemento su cui innestare un linguaggio artistico basato sulla riflessione e sul rapporto con il passato, un passato troppo ingombrante per essere dimenticato le cui testimonianze visive ancora costellavano il territorio nazionale, dove le migliaia di bunker in cemento armato fungevano da perenne ricordo della spietata tirannia del dittatore che per circa mezzo secolo aveva imprigionato il Paese all'interno di invalicabili confini.

La nuova generazione di artisti dovette dunque rapportarsi con l'evoluzione delle dinamiche politiche che avevano condotto l'Albania fuori dall'isolamento fisico e ideologico in cui era stata segregata sino agli esordi del nuovo millennio e che avevano finalmente fornito l'occasione di poter conoscere il mondo occidentale e in particolar modo l'Italia, quell'Italia fino ad allora immaginata e idealizzata attraverso la visione clandestina dei suoi palinsesti televisivi i cui programmi di intrattenimento avevano consegnato una visione distorta e troppo ottimistica della realtà nazionale (Polovina 2002, 9).

L'emancipazione dalla retorica propagandista imposta dal governo enveriano si espresse non solamente attraverso il cambiamento delle tematiche affrontate, ma anche mediante una diversificazione linguistica atta a rispondere alle esigenze dei giovani artisti schipetari che progressivamente indirizzarono la propria ricerca estetica verso nuove tecniche, abbandonando gradualmente la pittura, unico medium espressivo insieme alla scultura concesso e promosso dal regime. I manufatti pittorici risalenti agli anni della dittatura, spesso non di altissima qualità a causa della connotazione dogmatica che impediva ogni sperimentazione individuale da parte dei pittori, hanno rappresentato un punto di riferimento per l'evoluzione delle arti figurative nella nuova Albania democratica dove gli artisti ebbero modo di sperimentare nuovi linguaggi artistici in linea con le coeve tendenze espressive occidentali.

Alle soglie degli anni Duemila il retaggio culturale determinato da oltre quarant'anni di regime socialista ha dunque costituito per molti artisti schipetari un ulteriore impulso per esplorare nuovi linguaggi espressivi ai quali si sono avvicinati indirizzando principalmente la propria ricerca artistica verso il panorama culturale della vicina Italia che divenne nuovamente, dopo circa mezzo secolo, il principale interlocutore della Terra delle Aquile e dei suoi rappresentanti nel campo delle arti visive. 
Il nuovo assetto politico e sociale delineatosi con la democrazia può essere compreso e analizzato attraverso l'osservazione della presenza dell'Albania alle recenti edizioni della Biennale di Venezia.

La prima partecipazione ufficiale risale al 2005, in occasione della 51. Esposizione Internazionale d'Arte durante la quale venne rappresentata da Sislej Xhafa (Biennale 51 2005).

Nel contesto della rassegna curata dalle critiche e storiche dell'arte di nazionalità spagnola María de Corral e Rosa Martínez, articolata in due mostre distinte ma tra esse complementari intitolate rispettivamente L'Esperienza dell'Arte e Sempre un po' più lontano, l'Albania ha esordito presentando un'opera la cui struttura costituiva essa stessa lo spazio del Padiglione.

Parzialmente celato dal verde degli alberi che circondano l'area dei Giardini, l'imponente costruzione, la cui forma ricordava una torre, si stagliava fronteggiando la laguna e generando un'iniziale senso di confusione nel visitatore. Intitolata Ceremonial Crying System Pv, in albanese Sistemi Cerimonial i Vajtimit, l'opera di Xhafa, artista originario del Kosovo ma residente a New York, rappresentava e incarnava il carattere insurrezionale della sua ricerca artistica caratterizzata dall'utilizzo del diffuso pregiudizio sull'Albania quale terra di violenza e criminalità, come strumento di indagine e sfida alle istituzioni.

A Venezia la torre-scultura, ricoperta da materiale plastico a connotarne la sua natura effimera e segnata da due tagli a forma di occhio, assumeva la fisionomia di una gigantesca maschera piangente rappresentante il tipico copricapo dei membri del Klu Klux Klan, un'immagine il cui significato crea immediata repulsione e confusione in chi vi si imbatte ma che contestualmente risulta legittimato da quella stessa società che permettendone l'esistenza ne ha favorito la crescita e la nefasta azione. É in tale contraddizione che si muove l'operato di Xhafa, caratterizzato da una vigorosa carica polemica e da un'analisi delle trasformazioni sociali in atto nella sua terra natale così come nel resto del mondo, esaminate attraverso l'occhio critico di un artista che della sua condizione di clandestino e viaggiatore ha fatto il proprio principale strumento di indagine artistica.

La figura di Xhafa rappresenta in maniera esemplare la nuova tendenza espressiva abbracciata sul finire del Novecento dagli artisti albanesi nel cui operato l'esperienza culturale assimilata nel $\mathrm{Pa}$ ese d'origine, così come le problematiche scaturite dallo scontro tra la stessa e le tradizioni sociali e culturali dell'Europa Occidentale, costituiscono il sottofondo e la radice della propria ricerca artistica condotta attraverso i più vari strumenti espressivi, dalla pittura alla fotografia alla performance.

A quindici anni di distanza dalle elezioni politiche tenutesi in Albania nel 1990 l'iniziativa che segnò la prima partecipazione del Paese balcanico alla Biennale di Venezia si inseriva compiutamente nel contesto dell'Esposizione, rimarcando e sottolineando il ruolo arti- 
stico e sociale che l'Albania democratica aveva iniziato a ricoprire nel contesto internazionale.

Il progetto della mostra, curata dai commissari del Padiglione Andi Tepalena e Cecilia Tirelli e realizzata con il sostegno della Galleria Nazionale delle Arti d'Albania, ha costituito una delle prime significative interazioni tra le nuove dinamiche culturali albanesi e le tendenze espressive internazionali, dimostrando come la recente produzione degli artisti schipetari, rappresentati in tale occasione da Sislej Xhafa, aprisse degli inediti percorsi volti alla dialettica e al confronto con le coeve realtà culturali occidentali (Lambrecht 2005, 87).

L'autorappresentazione della propria identità nazionale, espressa attraverso la struttura del Padiglione le cui sembianze fungevano esse stesse da prodotto artistico, rappresentò la direzione in cui, al principio del nuovo millennio, iniziava a volgere la ricerca artistica albanese che nell'instaurare un dialogo con l'arte internazionale e specialmente europea manteneva intatta la propria individualità.

Se nel corso della sua prima partecipazione ufficiale alla Biennale l'Albania aveva proposto il suo progetto non inserendosi all'interno del contesto dei Padiglioni nazionali, ma esponendo l'opera di Xhafa nell'area antistante all'ingresso principale dei Giardini, in occasione della 52. edizione dell'Esposizione Internazionale le venne invece assegnato uno spazio istituzionale all'interno delle Artiglierie dell'Arsenale, in cui allestire la propria mostra.

Nell'ambito della manifestazione curata dallo statunitense Robert Storr e intitolata Think with the Senses and Feel with the Mind, titolo che alludeva a un'inversione della normale percezione della realtà, caratteristica della poetica dadaista, la presenza dell'Albania si inserì coerentemente nell'ottica di comunicazione e dialettica tra le diverse ed emergenti realtà culturali proposta dagli organizzatori dell'Esposizione.

Diretta dalla curatrice Bonnie Clearwater e dal commissario del Padiglione Rubens Shima, la mostra si proponeva come una riflessione sui recenti sviluppi in campo artistico che si erano manifestati in Albania in seguito al collasso della dittatura comunista, ponendo particolarmente in risalto la comune tendenza dei giovani artisti all'abbandono dei dettami propri dell'arte di regime in favore di nuovi paradigmi espressivi capaci di interpretare compiutamente una transizione non solo generazionale ma anche linguistica e di contenuto (Biennale 52 2007, 14, versione in lingua inglese). La cristallizzazione di tali stilemi, che nel contesto dell'Albania democratica si era sviluppata come una rivendicazione della libertà espressiva in contrapposizione alla lezione accademica imposta durante gli anni del potere socialista, nell'ambito della Biennale veneziana del 2007 risultò decisamente percepibile attraverso i differenti registri formali proposti dai cinque artisti chiamati a rappresentare il proprio Paese. Helidon Gjergji, Genti Gjkola, Alban Hajdinaj, Armando Lulaj e 
Heldi Pema incarnavano infatti, attraverso la loro poetica, la diversificazione dei linguaggi espressivi adottati in Albania a partire dai primi anni Novanta: le installazioni video di Lulaj e Hajdinaj, così come le elaborate opere grafiche di Gjkola costituivano tutte prodotti concettuali mirati a proporre un'attenta lettura della storia recente presentata al pubblico internazionale della Biennale mediante peculiari e diversificati approcci espressivi.

Dopo l'assenza all'edizione del 2009 l'Albania torna ad esporre negli spazi dell'Arsenale in occasione della 54. Esposizione Internazionale d'Arte curata da Bice Curiger.

La mostra diretta dalla critica d'arte svizzera intitolata ILLUMInazioni, termine decisamente simbolico ed evocativo della poetica di autori quali Arthur Rimbaud e Walter Benjamin così come della stessa struttura espositiva della Biennale, composta da diversi Padiglioni nazionali, si proponeva di fare luce e di rivelare gli aspetti più pregnanti delle azioni artistiche contemporanee, dove l'incontro e la dialettica tra culture e tendenze espressive differenti emergevano quali componenti fondamentali, specialmente nel contesto internazionale della manifestazione veneziana (Curiger 2011, 43).

In tale ottica la mostra allestita nel Padiglione albanese, curata da Riccardo Caldura, docente all'Accademia di Belle Arti di Venezia, e coordinato dal commissario Parid Teferici, si offriva come una riflessione sulle manifestazioni artistiche dell'Albania contemporanea condotta mediante diverse chiavi interpretative.

Intitolato Geophaties, termine che può essere inteso sia letteralmente come «sofferenza della Terra», sia come una condizione che permette all'essere umano di avvertire l'intima connessione tra la sua esistenza e ciò che lo circonda, il progetto della mostra suggeriva come tale locuzione potesse parimenti essere associata alla funzione che le opere d'arte ricoprono nel mondo contemporaneo, dispositivi estremamente sensibili in grado di percepire le complesse relazioni tra luoghi, storia e dimensione sociale. Nel caso dell'Albania, Paese in continua trasformazione, la possibilità di definire e tracciare tali relazioni risulta particolarmente complessa: il crollo del regime e il rapido dipanarsi di un futuro politico e sociale incerto avevano infatti portato a una ridefinizione dei legami con molti Paesi europei e con le loro tradizioni culturali, legami sovente facilitati e veicolati dal massiccio fenomeno migratorio che aveva profondamente colpito il Paese sin dai primi anni Novanta (Caldura 2011, 318).

Proprio sulla ricerca e l'elaborazione di tali rapporti si innestava e snodava l'operato dei giovani artisti albanesi chiamati a rappresentare la propria nazione: Anila Rubiku, Orion Shima, Gentian Shkurti, Eltjion Valle e Driant Zeneli, attraverso linguaggi espressivi differenti tra loro, si proposero dunque interpreti di quella generazione che ancora oggi vive in una condizione di incertezza e al tempo stesso di scambio e ricezione degli influssi socioculturali provenienti da quei 
Paesi dell'Europa occidentale in cui numerosi cittadini schipetari si sono trasferiti alla ricerca di migliori prospettive.

Nel contesto internazionale della Biennale di Venezia gli artisti del Padiglione albanese si interrogavano sulla natura e sull'identità del proprio Paese d'origine, martoriato da una diaspora che solo recentemente ha accennato a placarsi, domandandosi inoltre se tale idea di nazione potesse ancora esistere ed essere condivisa dai suoi abitanti.

Non presente alla Biennale del 2013 l'Albania si ripresenta a Venezia in occasione della 56. edizione della mostra, terzo capitolo di quella che Paolo Baratta ha definito una trilogia composta dall'edizione del 2011 curata da Bice Curiger, da quella del 2013 diretta da Massimiliano Gioni e infine dalla mostra del 2015 che ha visto come direttore artistico Okwui Enwezor. La ricerca espressiva proposta dal critico d'arte nigeriano verteva su una riflessione del rapporto tra arte e realtà umana, sociale e politica e sull'influenza esercitata su di esse da tutte le forze e i fenomeni esterni che rendono il mondo attuale lacerato da incertezze e fratture. Nel contesto di questa mostra globale, dal simbolico titolo All the World's Future, gli artisti erano stati invitati e chiamati a fornire una personale interpretazione delle nuove dinamiche internazionali, non formulando giudizi, ma offrendo una ponderata valutazione del rapporto tra la propria condizione di artista e la complessa realtà in cui si trovavano ad operare.

In tale ambito a rappresentare l'Albania, nella sua ormai definitiva sede alle Artiglierie dell'Arsenale, è stato Armando Lulaj, che a distanza di pochi anni dalla sua prima partecipazione alla Biennale nel 2007 tornava a Venezia con un progetto elaborato insieme al curatore Marco Scotini.

Da sempre impegnato nell'interpretazione del difficile passato dell'Albania, indagando in particolare sugli anni del regime socialista e sulla svolta neoliberista verificatasi in seguito al crollo della dittatura enveriana, in occasione della 56. Biennale Lulaj ha raccolto ed esposto insieme per la prima volta, all'interno della mostra intitolata Albanian Trilogy: A Series of Devious Stratagems, un ciclo di film elaborato nel corso degli anni e ultimato nel 2015.

La serie di «equivoci stratagemmi» dell'artista tiranese costituiva un percorso nella memoria collettiva della popolazione schipetara mirato a incentivare nel visitatore internazionale un riflessione sui rapporti di potere e sulla relazione tra ciò che è stato e il suo graduale anche se criptico riemergere nell'odierna quotidianità.

Il progetto presentato a Venezia, elaborato sotto l'egida del Ministero della Cultura Albanese, grazie alla collaborazione di eminenti personalità del panorama culturale internazionale quali Borys Groys e Adrian Paci, si connetteva al tema scelto da Enwezor in relazione all'idea di storia collettiva e condivisa che il curatore della manifestazione proponeva facendo riferimento all'interpretazione di Walter Benjamin contenuta in Tesi di filosofia della storia rispetto alla poe- 
tica di Paul Klee (Scotini 2015, 9). Nelle installazioni di Lulaj l'opera del grande artista svizzero diventava metafora della memoria del popolo albanese che nel contesto della manifestazione veneziana veniva analizzata attraverso i tre film che compongono Albanian Trilogy: It wears as it grows del 2011, Never del 2012 e Recapitulation, realizzato appositamente per la Biennale del 2015, lungometraggi che insieme al mastodontico scheletro di una balena e a diverso materiale d'archivio offrivano un'interpretazione del passato e una riflessione sul futuro del Paese balcanico (Scotini 2015, 20).

Il primo video raccontava di uno storico incidente avvenuto in Albania nel 1963 quando, in seguito alla rottura dei rapporti con l'Unione Sovietica e al conseguente dilagare della paura di un possibile attacco da parte dell'ex alleato, i soldati della marina militare albanese spararono e abbatterono un capodoglio scambiandolo per un sottomarino. In seguito a tale operazione i resti del cetaceo vennero recuperati ed esposti al Museo di Storia Naturale di Tirana dove sono stati conservati fino al 2015 quando, in occasione dell'Esposizione veneziana, sono stati trasferiti ed allestiti all'interno del Padiglione nazionale per interagire e dialogare con la sopra citata l'installazione video.

In Never veniva narrato lo spettacolare gesto di un gruppo di studenti e militari che nel 1968 sulla montagna di Shpirag, vicino alla cittadina di Berat, realizzarono con massi e pietra un'immensa scritta con il nome di battesimo del dittatore: Enver.

Nel 2012 un'azione condotta da Lulaj, ripresa e documentata nel video, condusse gli abitanti del luogo a tramutare tale scritta in «Never», scambiando semplicemente le prime due lettere, operazione concettuale estremamente potente volta a negare il passato totalitario e monito per la popolazione affinché gli orrori compiuti nel secolo scorso non si ripetano mai più.

L'ultimo video riferiva la vicenda storica che vide protagonista un aeroplano dell'aviazione statunitense che nel 1957 entrò nello spazio aereo albanese e che, una volta intercettato, fu costretto ad atterrare all'aeroporto della capitale. Dopo essere stato trattenuto per circa un anno all'interno dei confini nazionali il pilota venne rilasciato, mentre il velivolo non abbandonò più l'Albania dove venne esposto nel parco del castello della cittadina di Gijrokastër.

Attraverso Recapitulation Lulaj ha reinterpretato l'azione avvenuta circa sessant'anni prima, ipotizzando di liberare l'aereo americano, facendone sollevare e volar via la carcassa ormai divenuta un oggetto museale.

Attraverso la sua trilogia Armando Lulaj, formatosi in Italia all'Accademia di Belle Arti di Firenze in un contesto di rinnovata mobilità culturale che a partire dagli anni Novanta ha visto l'Italia affermarsi nuovamente come meta di studio privilegiata per le nuove generazioni di artisti schipetari, trasmetteva e condivideva con il visitatore internazionale un'elaborata riflessione sul passato albanese e 
sul pervasivo ruolo che la dottrina comunista aveva ricoperto nell'esistenza quotidiana della popolazione; un'influenza che appare ancora oggi presente e tangibile in alcuni significativi reperti, come la carcassa dell'aereo o lo scheletro del capodoglio (Groys 2015, 94).

Il tema di un'intima e a tratti onirica riflessione sul passato e sull'influenza che la memoria collettiva può ricoprire nella gestione dei ricordi condivisi da un intero popolo ritorna e viene sviluppato ulteriormente in occasione della 57. Esposizione Internazionale d'Arte.

Nel contesto della Biennale del 2017 curata da Christine Macel dal titolo Viva Arte Viva, incentrata sulla celebrazione dell'esistenza dell'arte e dei suoi interpreti, l'Albania, rappresentata da Leonard Qylafi, ha proposto un'interpretazione degli sviluppi politici e culturali del Paese elaborata attraverso la riscoperta e lo studio di materiale bibliografico e d'archivio (Biennale 57 2017, 10).

Il progetto, diretto dalla curatrice tedesca Vanessa Joan Müller e coordinato dal commissario Mirela Kumbaro, ministro della Cultura della Repubblica d'Albania, si poneva di indagare sulla natura retrospettiva della memoria e sull'influenza che le immagini storiche ricoprono nella creazione dei ricordi personali. In tale ambito libri e riviste di epoche passate hanno costituito, nella loro qualità di depositi della memoria popolare, il punto di partenza del progetto artistico di Qylafi e della sua analisi sul processo di mutazione percettiva che nel tempo contribuisce a costituire la comprensione del nostro passato.

I dipinti dell'artista tiranese esposti al Padiglione nazionale in occasione della mostra, intitolata Occurrence in Present Tense, rappresentavano un'indagine sulle trasformazioni nella nostra percezione dei ricordi che, spesso influenzati da avvenimenti pubblici importanti, se osservati da una certa distanza appaiono sfuocati e incerti. Tali peculiari processi di emersione del ricordo appaiono espressi nelle grandi tele di Qylafi mediante immagini che paiono poco nitide, dai contorni confusi, caratterizzate da una vaghezza che incarna la metafora della dissoluzione della memoria e dunque dell'esperienza ad esso associata (Drake 2017, 60).

Nell'ambito della manifestazione diretta da Christine Macel, in controtendenza con le riflessioni portate avanti da Qylafi nello spazio del Padiglione albanese alle Artiglierie dell'Arsenale, all'interno del Padiglione Centrale ai Giardini erano proposte le opere dell'artista albanese di fama internazionale Edi Rama. Sindaco di Tirana dal 2000 al 2011 e attuale primo ministro dell'Albania, Rama presentava un'elaborazione dei numerosi schizzi eseguiti durante riunioni e incontri ufficiali e tracciati sulle pagine della sua agenda così come sulla carta intestata ministeriale. Quelli che inizialmente erano degli scarabocchi disegnati a penna si trasformavano in colorate composizioni equilibrate e sinuose che a Venezia popolavano la carta da parati con la quale erano ricoperte le pareti di una sala della sezione voluta da Macel intitolata 'Padiglione degli Artisti e dei Libri' (Biennale 57 2017, 68). 
La spontaneità della pratica di Rama, strettamente integrata alla propria attività istituzionale, rappresentava nell'accezione della curatrice francese una tangibile testimonianza delle potenzialità espressive dell'arte contemporanea anche in momenti di crisi, conflitti e inarrestabili trasformazioni della società.

Una diversa prospettiva sul tormentato passato della nazione viene invece fornita dall'installazione video di Driant Zeneli, intitolata Maybe the Cosmos is not so Extrordinary, presentata alla 58. Esposizione Internazionale d'Arte curata da Ralph Rugoff; l'artista di Scutari, alla sua terza partecipazione alla Biennale di Venezia, si rapporta alle complesse tematiche proposte dal direttore artistico esplicate mediante l'evocativa espressione che costituisce il titolo della mostra, May You Live in Interesting Times, esponendo alle Artiglierie dell'Arsenale un'opera multimediale che combina insieme video e scultura (Biennale 58 2019, 6).

Elaborato a partire dal progetto del 2015 Beneath a surface there is just another surface, realizzato al Kombinati Metalurgjik, un complesso industriale distopico situato nel distretto di Elbasan e ispirato al racconto di fantascienza del 1983 Drejt Epsinolit të Eridanit (Sulla via dell'Epsilon Eridani) dello scrittore e fisico albanese Arion Hysenbegas, l'installazione presentata a Venezia è costituita da un film a due canali ambientato nelle miniere di Bulqizë, cittadina a nord-est del Paese dove dal 1918 viene estratto il cromo; tale significativa risorsa per l'economia e lo sviluppo industriale albanese in questo specifico contesto fornisce una riflessione e rivela come l'estrazione mineraria abbia costituito per secoli una delle principali cause di conflitti economici e politici nel Paesi del Sud del mondo (Dibra 2019, 87).

Il film proiettato nel Padiglione albanese, allestito e trasformato per l'occasione in un ambiente la cui sembianza evoca gli spazi in cui si svolge la narrazione, mette in scena la scoperta da parte di un gruppo di adolescenti di Bulqizë di una capsula cosmica che segue il percorso del cromo, dalla sua estrazione e lavorazione all'interno della fabbrica sino all'esportazione verso diversi Paesi dell'Europa. Attraverso il connubio tra l'aspetto visivo e sonoro e l'allusiva coreografia, la fabbrica diviene espressione della drammaticità del contesto sociale in cui è ambientato il film e del suo carattere ambivalente, in bilico tra la ripresa e un possibile tracollo. Ponendo enfasi sull'ipnotica fase di estrazione del cromo Zeneli mira a creare una tensione tra una realtà oppressiva, nascosta e sotterranea e un'utopica condizione di libertà e potenzialità.

Curato da Alicia Knock e coordinato dal Ministero della Cultura della Repubblica di Albania il progetto esposto al Padiglione Albania in occasione della 58. edizione della Biennale si propone di rivelare i modi in cui l'ordinario, anche nei suoi aspetti più tetri, possa essere in grado di amplificare la percezione dell'esistenza. 
Le diverse partecipazione dell'Albania all'Esposizione Internazionale d'Arte di Venezia mettono in luce ed evidenziano la tendenza condivisa da molti artisti albanesi che sul finire del XX secolo indirizzarono e focalizzarono la propria ricerca espressiva su un'attenta e diversificata elaborazione del passato nazionale volta alla comprensione e all'interpretazione delle attuali dinamiche politiche e sociali.

Tale inclinazione risulta parimenti percepibile e riscontrabile nell'evoluzione delle partecipazioni del Paese balcanico alla Mostra Internazionale di Architettura, manifestazione cui l'Albania ha preso parte per la prima volta nel 2010.

A tal proposito appare significativa la mostra allestita in occasione della XIV edizione della rassegna biennale diretta da Rem Koolhaas intitolata Fundamentals in cui i curatori del Padiglione albanese, Jonida Turani e Stefano Rabolli, hanno proposto una lettura del singolare e discontinuo sviluppo urbano di Tirana, conseguenza della travagliata storia della nazione, esplorando i diversi modi in cui l'architettura elaborata nel corso del Novecento risulti oggi profondamente mutata, modificata e adattata alle contemporanee esigenze abitative attraverso l'opera di due artisti, Edi Hila e Adrian Paci (Fundamentals. 14 Mostra Internazionale di Architettura 2014, 24).

Mediante tali narrazioni artistiche il visitatore internazionale veniva proiettato in un universo urbano e sociale dove l'eredità del passato - l'occupazione italiana nella prima metà del Novecento e successivamente gli anni del regime comunista - ha dato vita a una stratificazione architettonica indicativa delle complesse condizioni di vita della popolazione che al crollo del regime enveriano si era ritrovata a dover ricostituire la propria identità nazionale anche attraverso la ridefinizione della fisionomia della capitale.

Documentata da un video, l'opera di Paci, artista originario di Scutari ma residente in Italia, si focalizzava sulla trasformazione di un blocco di marmo in una colonna, elemento architettonico universale che, una volta realizzato, non era stato eretto ma disposto orizzontalmente nello spazio antistante il Padiglione. Avulso dal contesto circostante e in uno stato di potenzialità che non era in grado di concretizzarsi, la colonna di Paci si proponeva come manifestazione visiva di quei «potential monuments of unrealised futures» che potrebbero costituire la conformazione architettonica dell'Albania del nuovo millennio e che, nel contesto della Biennale veneziana, facevano riferimento al titolo programmatico della mostra.

Rilevante nell'ambito della riflessione sul proprio passato nazionale e sulle nuove dinamiche scaturitesi in seguito al crollo del regime e al conseguente processo di democratizzazione dell'Albania risulta inoltre la mostra allestita nel Padiglione albanese in occasione della XV edizione della rassegna diretta dall'architetto cileno Alejandro Aravena e intitolata Reporting from the front. 
Curata da Simon Battisti, Leah Whitman-Salkin e dal collettivo di grafici inglese Abäke la mostra, dall'evocativo titolo I Have Left You the Mountain, presentava dieci testi sull'architettura relativa al fenomeno della migrazione, che dagli anni Novanta ha riguardato un'ampia parte della popolazione albanese, commissionati a poeti e pensatori contemporanei e musicati da alcuni dei pochi interpreti ancora viventi del canto albanese iso-polifonico, espressione artistica riconosciuta nel 2005 Patrimonio culturale immateriale dell'umanità ( $R e$ porting from the front. 15 Mostra Internazionale di Architettura 2016, 8). L'iso-polifonia, caratteristica del sud del Paese, è stata tramandata oralmente di generazione in generazione sino ai giorni nostri, rimanendo immune dalle numerose influenze costituite dalle diverse occupazioni che si sono susseguite in Albania; nel contesto del Padiglione albanese il canto infatti costituiva uno strumento attraverso cui elaborare ed esprimere il processo culturale della partenza e dell'abbandono della propria terra e i conseguenti effetti della migrazione sull'ambiente edificato (I Have Left You the Mountain 2016, 6).

Osservando le diverse partecipazioni del Paese delle aquile alla Biennale d'Arte di Venezia risulta evidente come le tematiche connesse alla travagliata storia del Paese e in particolare agli anni del regime socialista costituiscano i principali elementi di riflessione su cui si sono innestate ed evolute le diverse mostre allestite all'interno del Padiglione albanese.

Dalla predilezione per questi temi si evince dunque come il passato nazionale abbia ricoperto, a partire dai primi anni Novanta, un ruolo predominante nella produzione artistica delle nuove generazioni di artisti albanesi che, ormai liberi dai vincoli espressivi imposti dalle direttive governative, hanno potuto orientare il proprio operato verso un'intima analisi delle coeve condizioni politiche e sociali del Paese e del pervasivo retaggio culturale del regime enveriano.

Ai primi decenni degli anni Duemila la cultura visiva socialista appare quindi costituire ancora la principale fonte tematica per le nuove espressioni artistiche albanesi che proprio nel confronto e nella dialettica con il passato nazionale trovano la propria fonte di ispirazione.

Come evidenziato dalle significative partecipazioni dell'Albania alle biennali veneziane, caratterizzate da una tendenza e da un'aspirazione a instaurare un dialogo con le espressioni artistiche europee e internazionali, il lungo e complesso processo di elaborazione e metabolizzazione dell'eredità socialista continua dunque a rappresentare, con accezioni differenti, uno strumento di indagine e una componente fondamentale nella creazione di una nuova identità culturale albanese. 


\section{Bibliografia}

Caldura, Riccardo (2011). «Geophaties». Biennale 542011.

Curiger, Bice (2011). «ILLUMInazioni». Biennale 542011.

Dibra, Avni (2019). «There would be no wars if it weren't for chromium». Biennale 582019.

Drake, Cathryn (2017). «Present Perfect: Meme and Memory». Occurrence in present tense $=$ Exhibition catalogue (Venezia, Padiglione Albania, Esposizione Internazionale d'Arte, 13 maggio-26 novembre 2017). Berlin: Sternberg.

Fundamentals (2014). Fundamentals. 14 Mostra Internazionale di Architettura (2014) = Catalogo della mostra (Venezia, Padiglione Albania, Esposizione Internazionale d'Arte, 7 giugno-23 novembre 2014). Venezia: Marsilio.

Groys, Boris (2015). «Memories of Hybrid Communism». Scotini, Marco (a cura di), Albanian Trilogy: a Series of Devious Stratagems = Exhibition catalogue (Venezia, Padiglione Albania, Esposizione Internazionale d'Arte, 9 maggio-22 novembre 2015). Venezia: Marsilio.

I Have Left You The Mountain (2016). I Have Left You The Mountain = Exhibition catalogue (Venezia, Padiglione Albania, Esposizione Internazionale d'Arte, 28 maggio-27 novembre 2016). S.l.: s.n.

Lambrecht, Louis (2005). «ll mondo secondo Sislej». Tepelena, Andi; Tirelli, Cecilia (eds), Sislej Xhafa. Ostfildern-Ruit: Hatje Cantz.

Piretto, Gian Pietro (2001). Il radioso avvenire: mitologie culturali sovietiche. Torino: Einaudi.

Polovina, Ylli (2002). Rai \& Albania: Una grande presenza nella storia di un popolo. Roma: Rai Eri.

Reporting from the Front (2016). Reporting from the Front. 15. Mostra Internazionale di Architettura (2016) = Catalogo della mostra (Venezia, Padiglione Albania, Esposizione Internazionale d'Arte, 28 maggio-27 novembre 2016). Venezia: Marsilio.

Scotini, Marco (2015). «Spettri non rovine. La Albanian Trilogy di Armando Lulaj». Biennale 56 2015. Venezia: Marsilio.

Scotini, Marco (2015). «The science of Whales: narratives of power and the invention of enemy». Scotini, Marco (a cura di), Albanian Trilogy: a Series of Devious Stratagems = Exhibition catalogue (Venezia, Padiglione Albania, Esposizione Internazionale d'Arte, 9 maggio-22 novembre 2015). Venezia: Marsilio. 



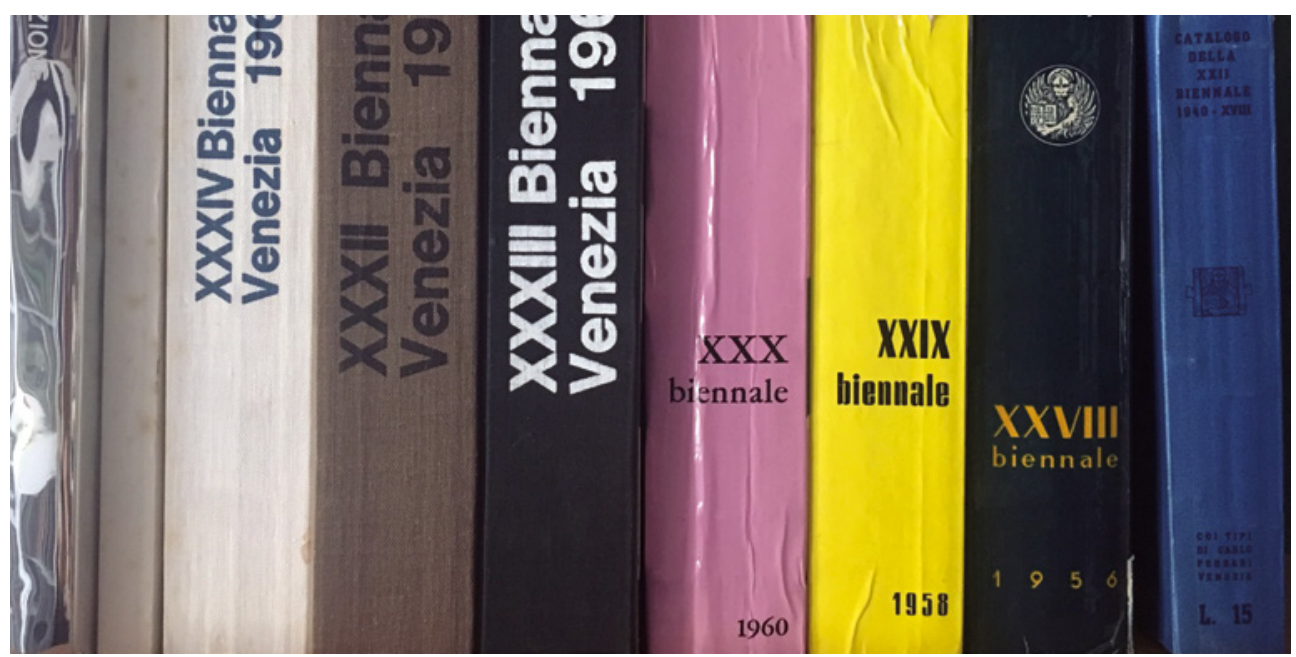




\section{Nota sui cataloghi della Biennale}

Andrea Masala

Università Ca' Foscari Venezia, Italia

I cataloghi della Biennale di Venezia si presentano come pietre miliari dei cambiamenti intercorsi nell'istituzione.

L'elenco che segue contiene più informazioni: le nozioni riguardano innanzitutto l'esposizione vera e propria, di cui viene riportato il titolo, l'anno, il periodo d'apertura e, nei casi successivi al 1976, il titolo-tema della Biennale, oltre all'eventuale curatore del volume. A questo si aggiunge l'editore, il luogo e l'anno di stampa.

La rassegna riporta i titoli originali dei volumi non come indicati sulla copertina, in quanto spesso essa ha carattere meramente estetico, bensì nella prima pagina interna del libro.

Sono numerose le considerazioni che si possono evincere e suggerire a partire da questo elenco. Emerge, ad esempio, l'indicazione in lettere, anziché in simbolo numerico, delle prime otto edizioni, nonché l'adozione dei numeri romani fino alla 34esima esposizione. Da qui segue un'alternanza tra i due sistemi di numerazione a seconda dell'annata e del titolo del catalogo.

Tutto ciò introduce l'idea che il catalogo della Biennale di Venezia non sia solamente un resoconto della mostra. Da un'analisi comparata si ottiene l'impressione di come sia cambiato nel tempo e abbia incarnato di volta in volta l'epoca, lo spirito e l'arte in esso contenuti e documentati. A consolidare questa situazione contribuiscono gli aspetti più tangibili di questo importante oggetto librario. Il formato, per esempio, lo vede trasformarsi nel tempo da libretto tascabile a grande tomo, talvolta diviso in più volumi; la quantità di informazioni e fotografie riportate nelle pagine, invece, mostrano la progressiva crescita delle partecipazioni, dei temi e dei contenuti dell'esposizione veneziana. 
Risulta più complicato sviluppare delle riflessioni sui curatori dei cataloghi: essi rimangono dei protagonisti fantasma di questa macchina espositiva almeno fino al 1932, data in cui appare esplicitamente la figura di Domenico Varagnolo. Al contrario, per quanto riguarda i cataloghi più recenti, spesso ai nomi dei curatori della rassegna si affiancano assistenti o giovani colleghi del curatore della Biennale che seguono la pubblicazione a livello redazionale. Viene apposta anche l'indicazione dell'editore, dell'uso dei monotipi, del luogo di stampa.

Come incarnazione della mostra in un oggetto cartaceo, il catalogo stesso è dunque oggetto d'interesse, come conferma il libro Biennale di Venezia. «Il catalogo è questo» (2012) di Tiziana Migliore, al quale si rimanda per approfondimenti su questo tema.

Accostati l'uno all'altro sopra lo scaffale, i cataloghi ricordano i mattoni di un'architettura più ampia composta, oltre che di materiali concreti, di contenuti, funzioni, aspetti e storie: una miniera di informazioni.

Avvertenza L'elenco delle Biennali qui apposto è utile strumento di riferimento per le citazioni dei saggi riportati in questa collana: a questo scopo si è provveduto a uniformare le edizioni della Biennale secondo un criterio redazionale composto dalla successione di numeri arabi e anno a cui poi poter fare riferimento come lemma breve nelle note degli scritti. 


\section{Bibliografia relativa ai cataloghi della Biennale di Venezia}

Biennale 11895 = Prima Esposizione Internazionale d'Arte della Città di Venezia 1895 = Catalogo (Venezia, 22/04/1895 - 22/10/1895). Venezia: Stabilimento tipo-litografico Fratelli Visentini.

Biennale 21897 = Seconda Esposizione Internazionale d'Arte della Città di Venezia 1987 = Catalogo (Venezia, 22/04/1897 - 31/10/1897). Venezia: Stabilimento tipo-litografico Carlo Ferrari.

Biennale 31899 = Terza Esposizione Internazionale d'Arte della Città di Venezia 1899 = Catalogo (Venezia, 22/04/1899 - 31/10/1899). Venezia: Stabilimento di Carlo Ferrari.

Biennale 41901 = Quarta Esposizione Internazionale d'Arte della Città di Venezia 1901 = Catalogo (Venezia, 22/04/1901 - 31/10/1901). Venezia: Stabilimento di Carlo Ferrari.

Biennale 51903 = Quinta Esposizione Internazionale d'Arte della Città di Venezia 1903 = Catalogo (Venezia, 22/04/1903 - 31/10/1903). Venezia: Stabilimento di Carlo Ferrari.

Biennale 61905 = Sesta Esposizione Internazionale d'Arte della Città di Venezia 1905 = Catalogo (Venezia, 22/04/1905 - 31/10/1905). Venezia: Stabilimento Carlo Ferrari.

Biennale 71907 = Settima Esposizione Internazionale d'Arte della Città di Venezia 1907 = Catalogo (Venezia, 22/04/1907 - 31/10/1907). Venezia: Officine Grafiche C. Ferrari.

Biennale 81909 = Ottava Esposizione Internazionale d'Arte della Città di Venezia 1909 = Catalogo (Venezia, 22/04/1909 - 31/10/1909). Venezia: Stabilimento Carlo Ferrari.

Biennale $91910=$ IX. Esposizione Internazionale d'Arte della Città di Venezia 1910 = Catalogo (Venezia, 22/04/1910 - 31/10/1910). Venezia: Stabilimento Carlo Ferrari.

Biennale $101912=$ X. Esposizione Internazionale d'Arte della Città di Venezia 1912 = Catalogo (Venezia, 23/04/1912 - 31/10/1912). Venezia: Stabilimento Carlo Ferrari.

Biennale 111914 = XI. Esposizione Internazionale d'Arte della Città di Venezia 1914 = Catalogo (Venezia, 15/04/1914 - 31/10/1914). Venezia: Stabilimento Carlo Ferrari.

Biennale $121920=X I^{a}$ Esposizione Internazionale d'Arte della Città di Venezia 1920 = Catalogo (Venezia, 15/04/1920 - 31/10/1920). Venezia; Milano: Istituto Veneto di Arti Grafiche; Casa Editrice d'Arte Bestetti \& Tuminelli.

Biennale $131922=$ XIII ${ }^{a}$ Esposizione Internazionale d'Arte della Città di Venezia 1922 = Catalogo (Venezia, 15/04/1922 - 31/10/1922). Venezia; Milano: Istituto Veneto di Arti Grafiche; Casa Editrice d'Arte Bestetti \& Tuminelli.

Biennale $141924=$ XIV ${ }^{a}$ Esposizione Internazionale d'Arte della Città di Venezia 1924 = Catalogo (Venezia, 01/04/1924 - 31/10/1924). Venezia: Officine Grafiche Carlo Ferrari.

Biennale $151926=X V^{a}$ Esposizione Internazionale d'Arte della Città di Venezia 1926 = Catalogo (Venezia, 01/04/1926 - 31/10/1926). Venezia: Officine Grafiche Carlo Ferrari.

Biennale $161928=X V I^{a}$ Esposizione Internazionale d'Arte della Città di Venezia 1928 = Catalogo (Venezia, 01/04/1928 - 31/10/1928). Venezia: Officine Grafiche Carlo Ferrari. 
Biennale $171930=X V I I^{a}$ Esposizione Internazionale d'Arte della Città di Venezia $1930=$ Catalogo (Venezia, 04/05/1930 - 04/11/1930). Venezia: Officine Grafiche Carlo Ferrari.

Biennale $181932=X V I I I^{a}$ Esposizione Internazionale d'Arte della Città di Venezia 1932 = Catalogo (Venezia, 28/04/1932 - 28/10/1932). A cura di Domenico Varagnolo. Venezia: Officine Grafiche Carlo Ferrari.

Biennale $191934=X I X^{a}$ Esposizione Internazionale d'Arte della Città di Venezia 1934 = Catalogo (Venezia, 01/05/1934 - 31/10/1934). A cura di Domenico Varagnolo. Venezia: Officine Grafiche Carlo Ferrari.

Biennale $201936=X X^{a}$ Esposizione Internazionale d'Arte della Città di Venezia 1936 = Catalogo (Venezia, 01/06/1936 - 30/09/1936). Venezia: Officine Grafiche Carlo Ferrari.

Biennale $211938=X X I^{a}$ Esposizione Internazionale d'Arte della Città di Venezia $1938=$ Catalogo (Venezia, 01/06/1938 - 30/09/1938). Venezia: Officine Grafiche Carlo Ferrari.

Biennale $221940=X X I^{a}$ Esposizione Biennale Internazionale d'Arte $1940=\mathrm{Ca}$ talogo (Venezia, 01/05/1940 - 31/10/1940). Venezia: Officine Grafiche Carlo Ferrari.

Biennale $231942=X X I I I^{a}$ Esposizione Biennale Internazionale d'Arte $1942=\mathrm{Ca}$ talogo (Venezia, 21/06/1942 - 20/09/1942). Venezia: Officine Grafiche Carlo Ferrari.

Biennale $241948=$ XXIV Biennale di Venezia $=$ Catalogo (Venezia, 01/05/1948 - 30/09/1948). A cura di Umbro Apollonio. Venezia: Edizioni Serenissima.

Biennale $251950=$ XXV Biennale di Venezia $=$ Catalogo (Venezia, 08/06/1950 15/10/1950). A cura di Umbro Apollonio. Venezia: Alfieri Editore.

Biennale $261952=$ XXVI Biennale di Venezia $=$ Catalogo (Venezia, 14/06/1952 19/10/1952). Venezia: Alfieri Editore.

Biennale $271954=$ XXVII Esposizione Biennale Internazionale d'Arte (Venezia, 19/06/1954 - 17/10/1954). A cura di Umbro Apollonio, Adriana Albini e Antonio Gnan. Venezia: Lombroso Editore.

Biennale $281956=$ XXVIII Esposizione Biennale Internazionale d'Arte (Venezia, 16/06/1956 - 21/10/1956). A cura di Umbro Apollonio, Adriana Albini e Antonio Gnan. Venezia: Alfieri Editore.

Biennale 291958 = XXIX Esposizione Biennale Internazionale d'Arte (Venezia, 14/06/1958 - 19/10/1958). A cura di Umbro Apollonio, Flavia Pilo Casagranda e Antonio Gnan. Venezia: Ente Autonomo La Biennale di Venezia.

Biennale $301960=$ XXX Esposizione Biennale Internazionale d'Arte (Venezia, 18/06/1960 - 16/10/1960). A cura di Umbro Apollonio, Flavia Pilo Casagranda e Antonio Gnan. Venezia: Ente Autonomo La Biennale di Venezia.

Biennale 311962 = Catalogo della XXXI Esposizione Biennale Internazionale d'Arte Venezia (Venezia, 16/06/1962 - 07/10/1962). A cura di Umbro Apollonio, Antonio Gnan e Vania Luzzetti. Venezia: Ente Autonomo La Biennale di Venezia.

Biennale 321964 = Catalogo della XXXII Esposizione Biennale Internazionale d'Arte Venezia (Venezia, 20/06/1964 - 18/10/1964). A cura di Umbro ApolIonio, Fernando Coletti e Antonio Gnan. Venezia: Ente Autonomo La Biennale di Venezia.

Biennale 331966 = Catalogo della XXXIII Esposizione Biennale Internazionale d'Arte Venezia (Venezia, 18/06/1966 - 16/10/1966). A cura di Umbro Apollonio, Biancamaria Borri e Antonio Gnan. Venezia: Ente Autonomo La Biennale di Venezia. 
Biennale 341968 = Catalogo della XXXIV Esposizione Biennale Internazionale d'Arte Venezia (Venezia, 22/06/1968 - 20/10/1968). A cura di Umbro Apollonio, Antonio Gnan e Ernesto Rubin de Cervin. Venezia; Firenze: Alfieri Editore; Centro di.

Biennale $351970=$ Catalogo della $35^{a}$ Esposizione Biennale Internazionale d'Arte Venezia (Venezia, 24/06/1970 - 25/10/1970). A cura di Luciano Caramel e Sergio Pozzati. Venezia: Ente Autonomo La Biennale di Venezia.

Biennale 361972 = Catalogo della $36^{a}$ Esposizione Internazionale d'Arte Venezia (Venezia, 11/06/1972 - 01/10/1972). Venezia: Ente Autonomo La Biennale di Venezia.

Biennale 37 1976 = La Biennale di Venezia 1976. Ambiente, partecipazione, strutture culturali $=$ Catalogo generale (Ambiente, partecipazione, strutture culturali) (Venezia, 18/07/1976 - 10/10/1976). A cura di Barbara Radice e Franco Raggi. Venezia: La Biennale di Venezia; Alfieri Edizioni d'Arte.

Biennale 381978 = La Biennale di Venezia 1978. Dalla natura all'arte, dall'arte alla natura = Catalogo generale (Dalla natura all'arte dall'arte alla natura) (Venezia, 02/07/1978 - 15/10/1978). A cura di Ziva Kraus. Milano: Electa.

Biennale 39 1980 = La Biennale di Venezia Settore Arti Visive = Catalogo genera le 1980 (Venezia, 01/06/1980 - 28/09/1980). A cura di Gianfranco Dogliani e Teresa Ricasoli. Milano: Electa.

Biennale 401982 = La Biennale Settore Arti Visive = Catalogo generale 1982 (Venezia, 13/06/1982 - 12/09/1982). A cura di Enzo di Martino, Maria Teresa Sega e Giorgio dal Bo. Milano: Electa.

Biennale $411984=$ XLI Esposizione Internazionale d'Arte La Biennale di Venezia. Arte e Arti · Attualità e Storia (Arte e Arti · Attualità e Storia) (Venezia, 10/06/1984 - 09/09/1984). A cura di Marie-George Gervasoni. Milano: Electa.

Biennale $421986=X$ XII Esposizione Internazionale d'Arte La Biennale di Venezia. Arte e Scienza = Catalogo Generale 1986 (Arte e scienza) (Venezia, 29/06/1986 - 28/09/1986). A cura di Marie-George Gervasoni. Milano: Electa.

Biennale $431988=$ XLIII Esposizione Internazionale d'Arte La Biennale di Venezia. Il luogo degli artisti = Catalogo Generale 1988 (Il luogo degli artisti) (Venezia, 26/06/1988 - 25/09/1988). A cura di Marie-George Gervasoni. Milano: Fabbri Editore.

Biennale $441990=$ XLIV Esposizione Internazionale d'Arte La Biennale di Venezia. Dimensione Futuro L'artista e lo Spazio = Catalogo Generale 1980 (Dimensione Futuro. L’Artista e lo Spazio) (Venezia, 27/05/1990 - 30/09/1990). A cura di Marie-George Gervasoni. Milano: Fabbri Editore.

Biennale $451993=$ La Biennale di Venezia XLV Esposizione Internazionale d'Arte. Punti Cardinali dell'Arte (Punti cardinali dell'arte) (Venezia, 14/06/1993 10/10/1993). A cura di Achille Bonito Oliva. Venezia: Marsilio Editori.

Biennale 461995 = La Biennale di Venezia 46. Esposizione Internazionale d'Arte (Identità e alterità: figure del corpo, 1895-1995) (Venezia, 11/06/1995 15/10/1995). A cura di Jean Clair. Venezia: Marsilio Editori.

Biennale $471997=$ XLVII Esposizione Internazionale d'Arte La Biennale di Venezia (Futuro Presente Passato) (Venezia, 15/06/1997 - 09/11/1997). A cura di Germano Celant e Antonella Soldaini. Milano: Electa.

Biennale 481999 = La Biennale di Venezia $48^{a}$ Esposizione Internazionale d'Arte. dAPERtutto APERTO over ALL APERTO par TOUT APERTO über ALL (dAPERtutto APERTO over ALL APERTO par TOUT APERTO über ALL) (Venezia, 12/06/1999 - 07/11/1999). A cura di Harald Szeemann e Cecilia Liveriero Lavelli. Venezia: Marsilio Editori. 
Biennale $492001=$ La Biennale di Venezia $49^{a}$ Esposizione Internazionale d'Arte. platea dell'umanità = plateau of humankid = Plateau der Menscheit = plateau de l'umanité (Platea dell'umanità = plateau of humankid = Plateau der Menscheit = plateau de l'umanité) (Venezia, 10/06/2001 - 04/11/2001). A cura di Harald Szeemann e Cecilia Liveriero Lavelli. Milano: Electa.

Biennale 502003 = 50esima Esposizione Internazionale d'Arte. Sogni e Conflitti - La Dittatura dello Spettatore (Sogni e conflitti. La dittatura dello spettatore) (Venezia, 15/06/2003 - 02/11/2003). A cura di Francesco Bonami e Maria Luisa Frisa. Venezia: Marsilio Editori.

Biennale 512005 = 51. Esposizione Internazionale d'Arte. Sempre un Po' Più Lontano La Biennale di Venezia $51^{a}$ Esposizione internazionale d'arte (L'esperienza dell'arte, sempre più lontano) (Venezia, 12/06/2005 - 06/11/2005). A cura di Rossella Martignoni e Martina Mian. Venezia: Marsilio Editori.

Biennale $522007=52$. Esposizione Internazionale d'Arte. Pensa con i sensi - senti con la mente. L'arte al presente (Pensa con i sensi, senti con la mente. L'arte al presente) (Venezia, 10/06/2007 - 21/11/2007). A cura di Robert Storr. Venezia: Marsilio Editori.

Biennale 532009 = 53. Esposizione Internazionale d'Arte. Fare Mondi Making Worlds (Fare Mondi. Making Worlds) (Venezia, 07/06/2009 - 22/11/2009). A cura di Daniel Birnbaum e Jochen Volz. Venezia: Marsilio Editori.

Biennale $542011=54$. Esposizione Internazionale d'Arte. ILLUMInazioni (ILLUMInazioni) (Venezia, 04/06/2011 - 27/11/2011). A cura di Bice Curiger e Giovanni Carmine. Venezia: Marsilio Editori.

Biennale $552013=55$. Esposizione internazionale d'arte. /l Palazzo Enciclopedico Biennale Arte 2013 (Il palazzo enciclopedico. The Encyclopedic Palace) (Venezia, 01/06/2013 - 24/11/2013). A cura di Massimiliano Gioni. Venezia: Marsilio Editori.

Biennale 562015 = La Biennale di Venezia 56. Esposizione Internazionale d'Arte. All the World's Futures (All the World's Futures) (Venezia, 09/05/2015 22/11/2015). A cura di Okwui Enwezor. Venezia: Marsilio Editori.

Biennale 572017 = Viva arte viva, 57. Esposizione internazionale d'arte (Viva Arte Viva) (Venezia, 13/05/2017 - 26/11/2017). A cura di Christine Macel. Venezia: La Biennale di Venezia.

Biennale $582019=58$. Esposizione Internazionale d'Arte. May You Live in Interesting Times (May you live in interesting times) (Venezia, 11/05/2019 24/11/2019). A cura di Ralph Rugoff. Venezia: La Biennale di Venezia. 

Atlante delle biennali intende essere un portolano per attraversare la storia e il presente di mostre che diventano epicentri di incontro e confronto anche tra nazioni e identità, che come mappe complesse e mesmerizzanti segnalano molteplici prospettive e punti cardinali. Uno sguardo widestream sul sistema dell'arte inizia puntando il periscopio su delle nuove Storie della Biennale di Venezia: rivelando altri intrecci di questa istituzione, ma interpretando pure le sorprendenti rotte che percorrono i padiglioni, in un gioco combinatorio che conduce verso avventure curatoriali e sorprendenti immaginari.

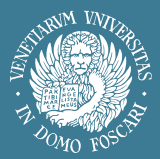

Università Ca'Foscari Venezia

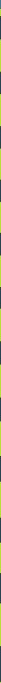

